


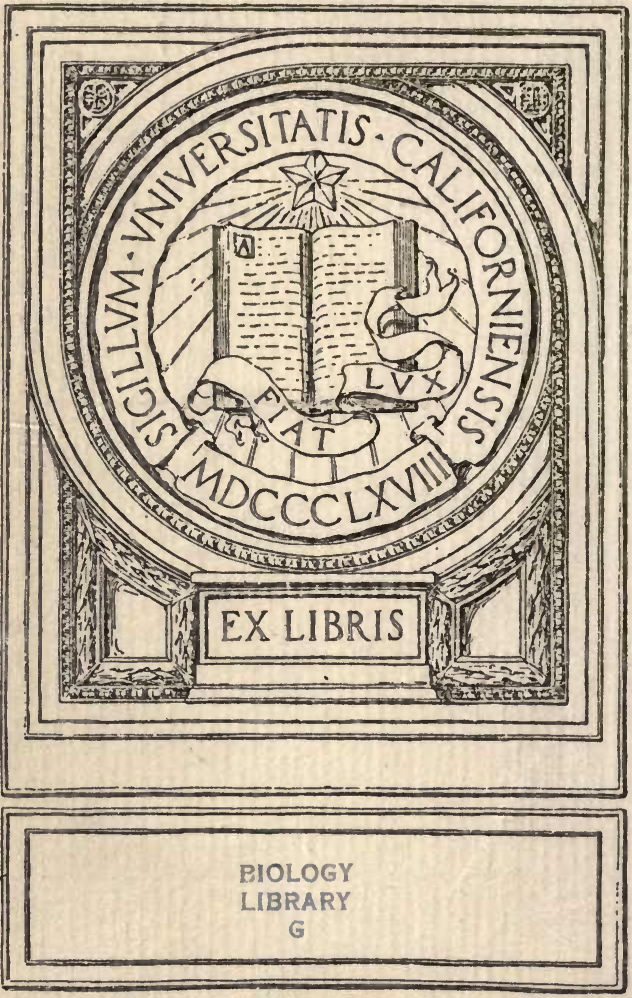





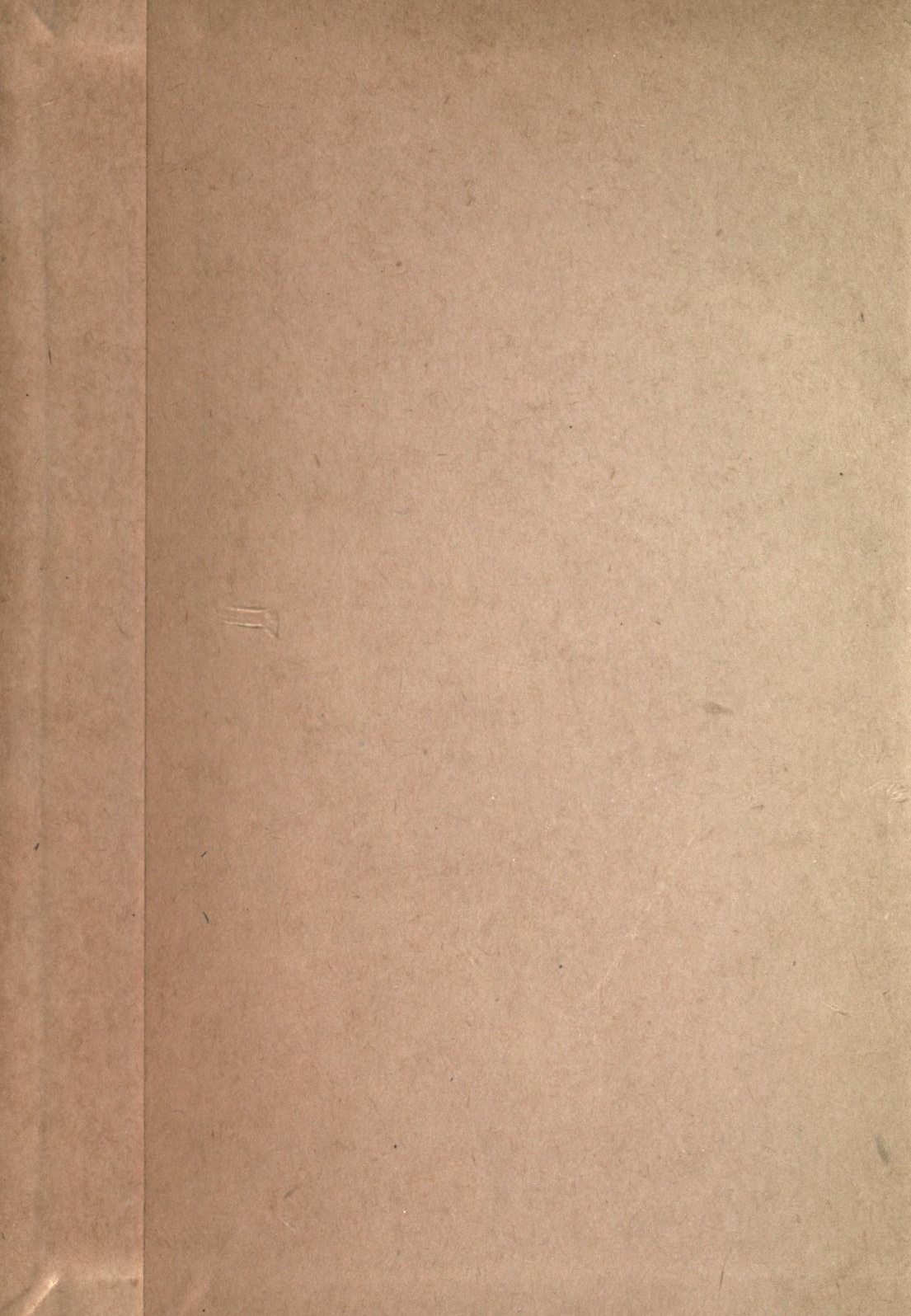


W.

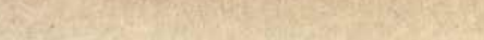





\section{APPLIED BIOLOGY'}

AN ELEMENTARY TEXTBOOK AND LABORATORY GUIDE

BY

MAURICE A. BIGELOW, Ph.D.

PROFESSOR OF BIOLOGY IN TEACHERS COLLEGE, COLUMBIA

UNIVERSITY; CO-AUTHOR OF THE " TEACHING OF

BIOLOGY IN THE SECONDARY SCHOOL"

AND

ANNA N. BIGELOW, M.S.

TEACHER OF HIGH-SCHOOL BIOLOGY

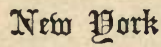

THE MACMILLAN COMPANY

1911 


\section{Coprriant, 1911, BY THE MACMILLAN COMPANY.}

Set up and electrotyped. Published October, rgrr.

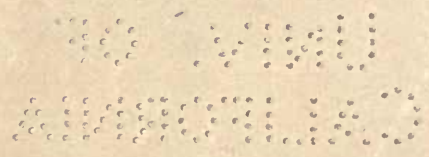

Norbood 看ess

J. S. Cushing Co. - Berwick \& Smith Co. Norwood, Mass., U.S.A. 


\section{PREFACE}

THIs book is intended for use as a combined textbook and practical guide for a year's course of five hours per week. It attempts to select from the fields of botany, zoölogy, and human biology the essential facts and especially the great ideas of the science of life which are of interest to the average intelligent person who has no time and reason for more extensive study of biology.

The word "applied" in the title of this volume should not be understood in the limited sense of economics, nor solely with reference to human physiology and hygiene. It is true that in these two lines biology has vastly important applications to human life, but it must not be overlooked that in certain phases the science has value in the intellectual and æsthetic life of cultured citizens. Hence, in the most liberal interpretation, "applied biology" must present those facts and ideas of the science which apply to human life in its combined intellectual, æsthetic, economic, and hygienic outlook. It has been the aim of the authors to select for this book the facts and ideas in these lines which seem best adapted to that stage of education which for the vast majority of students is the climax of formal education. In other words, it has been attempted to present the science of biology applied to the daily life of the average intelligent citizen.

This book presents an order of study and selection of materials which have long appealed to the authors as a very helpful answer to the widespread criticisms of the common elementary courses of botany and zoölogy from the pure- 
science point of view. It is far from being merely a theoretical attack upon the problems of an introductory course in biology, for a large part of the radically new arrangement of subject matter has been worked out in practice by the authors and by several other teachers who have worked under suggestions from the authors.

The authors trust that the critical readers whose attention may be attracted to parts of this book which seem to depart radically from the traditional introduction to biology, especially by means of separate courses in botany and zoölogy, may be so kind as to frame their criticisms in the light of the explanations and defense which have been written for an accompanying "Teachers' Manual of Biology." See foot-note on the first page of Chapter I.

A noticeable feature of the book is omission or at least lack of emphasis upon several hundred technical terms commonly used in elementary books of botany and zoölogy. Italics have been used for emphasizing the most important scientific terms when introduced and defined, while technical words in the plain type should be understood by students as important for special study, for temporary use in the text, or for comparison with other books. The authors realize that in selecting the biological terms for emphasis they may have omitted some really useful terms, and it may be that certain italicized words which have a narrow range of application do not deserve the emphasis given them. A further discussion of this question of technical words will be included in the "Teachers" Manual."

This book also departs widely from the traditional teaching of biological sciences, in that it presents much laboratory work in the form of demonstrations by the teacher instead of entirely as individual work for the students. This introduces an important problem which can be adequately presented only in the "Teachers" Manual"; but the experience of many 
science teachers in secondary schools and colleges is leading towards some golden mean between the old-time lecture and demonstration method and the more recent laboratory work for individual students. The authors have suggested for demonstrations all practical work which experience shows is ill-adapted for individual study by students with limited time and training. See foot-note on page 10.

One of the authors has discussed the meaning of the movement towards a course in introductory biology, instead of separate courses in botany and zoölogy, in Chapter V of Part II in Lloyd and Bigelow's "Teaching of Biology in the Secondary School," and also in School Science and Mathematics for October, 1908; and the principles there stated have been held fundamental while making this book of "Applied Biology." See also the "Teachers' Manual."

While it would be easily possible to select from this book material for a very elementary course of biology in the first year of some high schools, the authors have planned to issue a smaller book especially arranged with reference to the present peculiar conditions obtaining in high schools where a course of biology comes in the first year.

The authors acknowledge the useful suggestions and constructive criticisms which they have received from many teachers of biology; and especially are they grateful for the help on numerous problems which have been discussed with Professor H. M. Richards and Professor C. C. Curtis, of Columbia University, and with Miss Jean Broadhurst and Miss Caroline Stackpole, of the Department of Biology in Teachers College, Columbia University. Also, acknowledgments are due publishers and authors who have granted permission for the use of numerous illustrations from standard books. As far as sources were known to the authors, credit for figures has been given in the legends.

The authors cordially invite correspondence from teachers 
who have suggestions and criticisms, or who need help in conducting courses along the lines laid down in this "Applied Biology" and the accompanying "Teachers' Manual of Biology."

M. A. B.

NeW YoRK CITY,

A. N. B.

September, 1911. 


\section{CONTENTS}

\section{PART I}

INTRODUCTORY STUDY: PRINCIPLES OF BIOLOGY

CHAPTER

I. Biology: The Science of Life . . . . . . 1

II. Changes and Composition of Lifeless and Living Matter 5

III. The Characteristics of Living Things . $\quad . \quad 10$

I. Chemical Composition of Living compared with Lifeless Things. II. Life-Activities : (a) Animals, (b) Plants

IV. The Structure and Life of an Animal: Introduction to Animal Biology . • • . • • • 23

The Structure (Anatomy) of the Frog . . . . 25

The Tissues of the Frog: Introduction to Microscopic Study 37

The Work of Organs of the Frog: Introduction to Animal

Physiology . . . . . . . . . 44

Development of the Frog : Introduction to Embryology $\quad 57$

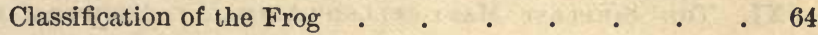

V. The Structure and Life of a Plant: Introduction to Plant Biology • The Structure of a Bean Plant . . . . . 67 The Reproduction of the Bean Plant . . . . . 76 The Work of the Organs of a Plant: Introduction to Plant

Physiology . • • • • • . • 85 Classification of the Bean Plant . . . . . . 121

VI. Comparison of Animal and Plant Biology . • 122

VII. Classification of Animals and Plants . . . . 133 


\section{PART II}

PRINCIPLES OF BIOLOGY ILLUSTRATED BY TYPES OF PLANTS

CHAPTER

VIII. Studies of Seed-Plants

Seeds and Seedlings .

Roots of Seed-Plants . . . . . . . . 156

Stems of Seed-Plants . . . . . . . . 163

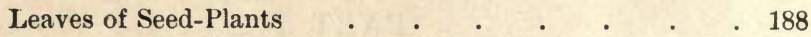

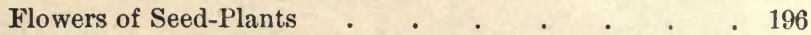

Seed-Plants without True Flowers : Gymnospernis . . 213

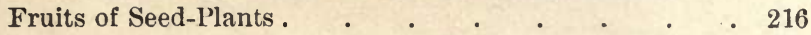

Seed-Plants Reproducing without Flowers . . . 225

General Notes on Seed-Plants . . . . . . . 228

IX. Studies of Spore-Plants . . . . . . . 232

I. Higher Spore-Plants - Ferns, Mosses . . . 233

II. Lower Spore-Plants - Algæ, Fungi . . . . 245

III. Bacteria . . . . . . . . 276

\section{PART III}

PRINCIPLES OF BIOLOGY ILLUSTRATED BY TYPES OF ANIMALS

X. The Simplest Animals: Protozoa . . . . 300

XI. The Simplest Many-celled Animals: Porifera and

Colenterata

- 320

XII. The Worm-like Animals . • . • . • 340

XIII. The Echinoderms . • • • • . . . 355

XIV. The Arthropods . . . . . . . . . $35 \hat{\imath}$

Crustaceans . . . . . . . . . 358

Arachnids . . . . . . . . . 376

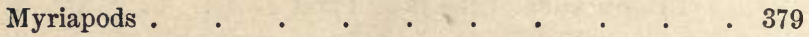

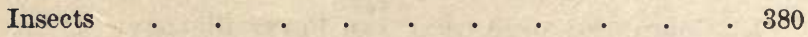

XV. The Shell-Animals : Mollusca • . • • 405 
CIAPTER

XVI. The Vertebrates

Fishes . . . . . . . . . . 419

Amphibians . . . . . . . . . . 424

Reptiles . . . . . . . . . 426

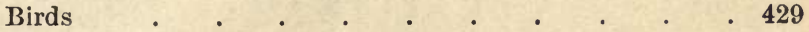

Mammals . $\quad . \quad . \quad . \quad . \quad . \quad . \quad . \quad .436$

Life-Histories of Vertebrates . . . . . . 442

\section{PART IV}

\section{PRINCIPLES OF BIOLOGY APPLIED TO HUMAN} STRUCTURE AND LIFE

Introduction. Human Biology and Classification of Man 4455

XVII. Human Structure And Life-Activities . . . 457

XVIII. Biology applied to Healthful Living . . . 525

Personal Hygiene . . . . . . . . 525

Effects of Stimulants and Narcotics _ . . $\quad$. 539

Bacteriology applied to Human Health . . . . 554

\section{PART V}

XIX. Evolution And Heredity of Animals and Plants - 561 



\section{PART I}

\section{INTRODUCTION TO BIOLOGICAL STUDY

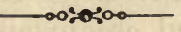

\section{CHAPTER I}

\section{BIOLOGY: THE SCIENCE OF LIFE}

1.* Living and Lifeless Things. - Since the science of biology deals with living things, it is of importance that at the beginning of our study we should stop to consider that all things which we know through our senses are either living or lifeless. This will be evident if we attempt to write the names of some common things, grouping them according to whether they appear to have life or not. It is not difficult to decide that air, soil, minerals, and water belong in the list of lifeless things and that the most common animals and plants are examples of the living; but we are puzzled by such objects as dry seeds, undeveloped eggs of animals, some plants in the winter condition, and many microscopic animals which show no signs of life when dry. Are such things living or lifeless? Usually it is not possible to answer until time and proper conditions have given an opportunity for changes which suggest life-activities. However, such uncertain cases must be left undecided until after a careful study of the differences between living and lifeless things.

*In the "Teachers' Manual of Biology" designed to accompany this book there will be found, in paragraphs numbered to correspond with those in this textbook, notes on books, materials, and methods of interest to teachers, or to advanced students in normal-school classes. 
2. Organisms, Organic and Inorganic Matter. - Except in science study, we rarely stop to think of the facts brought out in the problem above; but for the purposes of our later work in science it is important that we stop and make such a survey as above suggested, and recognize clearly that in this world of ours there are two kinds of things, - the living* (collectively called animals and plants) and the lifeless (e.g., air, soil, water, minerals, etc.). Living things are in science commonly called organisms, and the substance of which their bodies are composed, or which they form, is organic matter. Lifeless substance which has not been formed by organisms is called inorganic or mineral matter. All substances, then, in living and lifeless things are composed of matter which, as we learn through our five senses, exists in many different forms.

3. The Sciences. - Knowledge regarding the living and lifeless things of nature is systematically arranged in the natural sciences. A common division of these sciences is that into (1) the physical sciences (chemistry, physics, geology, mineralogy), and (2) the biological sciences, which are concerned with living things. We shall see later that there is much chemistry and physics used in the study of living things, and hence it will be made clear that these two sciences deal not only with lifeless things, but also with all substances and changes which are found in both living and lifeless things.

4. Biology, Botany, and Zoölogy. - The study which this book will direct deals primarily with living things, both plants and animals. Biology is the science which treats of

* Throughout this book italics are used for words and phrases which are very important in biology, and especially for scientific words where they are first introduced and defined. Such emphasized words and their meanings should be given special attention by students. Technical biological words which have a very limited use, perhaps applicable to only a few animals or plants, are printed in plain type; and also they are in parentheses if not important except for reference to other biological books. 
life and all living things - plants, animals, and man. There are two subdivisions of biology; namely, zoölogy, treating of animals, and botany, treating of plants. Zoölogy is often called animal biology, and botany, plant biology. The division of biology into botany and zoölogy does not mean that plants and animals are entirely unlike; but, on the contrary, we shall find later that these two kinds of living things have many points of remarkable similarity in both structure and activities. This similarity is especially striking in many microscopic living things which so combine both plant and animal characteristics that biologists have not decided whether they are animals or plants. But although there is often a great similarity between animals and plants, it is sometimes convenient to study the two kinds of living things separately, and so biological science is subdivided into botany and zoölogy. These two subdivisions of biology are most important for advanced students; but the best and most interesting beginning study is that which directs attention to the great facts common to all living things. Hence this book for beginners is called "Biology," to indicate that it uses both animals and plants to illustrate facts and ideas which are true of all living things.

5. Applied Biology. - More important than the similarity of animals and plants is the fact that many animals are in structure and activities very much like the human body; and hence the study of animals and plants helps us to understand better the human body and its life-activities. Moreover, thousands of animals and plants profoundly affect human life. For example, they provide all the food-supply for mankind; many harmfully influence human health; and some, such as pet animals and ornamental plants, contribute much to the pleasures of life. Clearly, some knowledge of the science of living things ought to be of great interest to educated citizens, because it applies in so many ways directly or indirectly to human life. Recogniz- 
ing this fact, it is the aim of this book (1) to call attention to the most important facts and principles to be learned by the study of selected animals and plants, and then (2) to show how biological science applies to everyday human life. This book is therefore entitled "Applied Biology"; that is to say, biology, the science of all life, is applied to, or looked at from the standpoint of, human life as represented in the experiences and interests of intelligent people in general. 


\section{CHAPTER II}

\section{CHANGES AND COMPOSITION OF LIFELESS AND LIVING MATTER*}

IN our later studies of living things (animals, plants, and man) we shall often need to have in mind some important facts and principles relating to the composition of both living and lifeless things and to the changes which occur in them; and these are outlined in this chapter.

6. Three States of Matter. - Soil, water, and air are forms of lifeless matter which are examples of the three states, solid, liquid, and gaseous, in which matter exists.

Matter in one of these states may be transformed into either of the other states. Thus water, which is ordinarily liquid, may be cooled and frozen into ice (the solid state), or it may be heated and changed into vapor or steam (the gaseous state). Iron and other common metals, which are ordinarily solid, may be melted into the liquid state and at an extremely high temperature may even change to a gaseous state. Liquid air is made by reducing the temperature to $-312^{\circ} \mathrm{F}$. by subjecting air to great pressure in powerful machines.

7. Physical Change. - In all such changes of matter from one state to another (from solid to liquid, or to the gaseous, etc.) the same substance continues to exist. Ice is only solid water, steam is a gaseous state of water, molten iron cools into solid iron, and sugar and salt will dissolve in water. In these cases there has been a change in the state of matter,

* Students who have previously taken courses in chemistry and physics should read this chapter as a review of familiar facts, but from a new viewpoint. 
but not a change in composition. Such changes of state which do not affect the composition of substances are called physical changes.

That branch of science which treats of the form and physical changes of matter produced by heat, light, sound, electricity, gravitation, etc., was formerly called natural philosophy, but is now usually known as physics.

8. Chemical Change. - All matter is subject to another kind of change in which the composition is affected and new substances are formed. Ordinary burning of wood or gas, and dissolving baking soda in vinegar or other acid, are common examples. The substances burned, or dissolved in acids, are changed to other substances. Such transformations which affect the composition of matter are chemical changes.

9. Elements. - The nature of chemical change will be clear after some further consideration of the composition of matter. In chemistry, the science which treats of the composition and chemical changes of substances, we learn that all forms of matter - all living and lifeless substances in land, air, and water - are composed of about 80 elements, of which about 20 are very common. Some of these elements exist naturally in the solid state; for example, iron, copper, lead, sulphur, gold, nickel, silver, platinum, carbon, magnesium, aluminum, tin, and zinc. Some others, like mercury (quicksilver), are liquid; and still others are gases, of which the two known as oxygen and nitrogen constitute the greater part of the air.

Chemical Symbols. - For convenience in writing the names of the elements, chemists have adopted certain symbols or abbreviations. The ones which will be most needed for reference in this book are: $\mathrm{H}$ for hydrogen; $\mathrm{N}$, nitrogen; $\mathrm{O}$, oxygen; C, carbon; S, sulphur; $\mathrm{P}$, phosphorus; $\mathrm{Na}$, sodium (or natrium); $\mathrm{K}$, potassium (or kalium); $\mathrm{Fe}$, iron (or ferrum); Ca, calcium (lime). A table giving the full list may be found in any elementary textbook of chemistry. 
10. Compounds of Elements. - Now, the elements have the power of combining with each other so as to form various compounds. To illustrate: the burning of magnesium is a chemical combination between magnesium and oxygen of the air - two elements are here united to form a new substance, which is a compound of magnesium and oxygen and is known as oxide of magnesium. Such a union of any substance with oxygen is called oxidation. The burning of coal is in part a combination between the elements oxygen and carbon, but coal contains elements besides carbon. All ordinary burning or combustion is an oxidation; that is, the forming of a combination between oxygen of the air and some other elements. In all cases of chemical change there is a combining of elements into new or different substances. Such substances composed of two or more elements are called compounds. Most of the materials in the solid matter of the earth are compounds; water, which is the most abundant substance, is a compound of the elements hydrogen and oxygen; and most of the materials in the bodies of animals and plants are compounds. Air, however, is not a chemical compound; the nitrogen and oxygen are not united into a new substance, but are simply mixed together, just as dry sand and sugar can be mixed without change of composition in either. Other minor constituents of the air will be mentioned later.

Using the chemical symbols for the elements, chemists write $\mathrm{MgO}$ for magnesium oxide formed by burning magnesium $(\mathrm{Mg})$ in the oxygen of the air, and $\mathrm{ZnO}$ for zinc oxide formed by burning zinc in the same way. The formula $\mathrm{MgO}$ expresses the composition of a molecule of magnesium oxide and means that it is made up of one atom of magnesium united with one of oxygen. This is the simplest possible compound. In most compounds of two or more elements there is a greater proportion of certain elements, and this is expressed by numbers after the elements of which there is 
more than one atom in the compound. Examples are: water, written $\mathrm{H}_{2} \mathrm{O}$, meaning that two atoms of hydrogen $(\mathrm{H})$ are combined with one of oxygen $(\mathrm{O})$; and sulphuric acid, written $\mathrm{H}_{2} \mathrm{SO}_{4}$, meaning that two atoms of hydrogen, one of sulphur, and four of oxygen are combined to form the acid.

In the above example of burning magnesium there are two elements; but more than two elements are often involved in a chemical change. One compound of several elements may cause a chemical change in another compound, as dissolving baking soda $\left(\mathrm{HNaCO}_{3}\right)$ in sulphuric acid $\left(\mathrm{H}_{2} \mathrm{SO}_{4}\right)$ illustrates. This dissolving of baking soda in acid illustrates the most common kind of chemical change, for most substances on the earth are compounds. It has been noted that the burning of magnesium involves only two elements, but the ordinary burning of coal and other common fuels is a union of compounds with oxygen, resulting in several new compounds in the smoke and ashes.

Disintegration of Compounds. - Not only may elements unite to form compounds, but these may be separated into simpler ones or even into their constituent elements. For example, water may be formed by burning hydrogen gas so that oxygen of the air unites with the hydrogen as shown by the formula $\mathrm{H}_{2} \mathrm{O}$, but water may also be decomposed by an electric current passed through it in a suitable apparatus and the two constituent elements (hydrogen and oxygen) in gaseous form be collected separately. Such disintegration of compounds into the constituent elements is not so common in nature as is change of compounds into simpler ones.

i1. Composition and Changes of Living Matter. - The principles stated in this chapter have been illustrated by lifeless matter, but we shall see in later lessons that they are also applicable to living matter. Living things are composed of a number of common elements united in very 
complex substances, and in their bodies there are constant physical and chemical changes connected with all life-activities. Especially do foods eaten and oxygen from the breathed air become involved in numerous changes in the living matter of animal and plant bodies. Many times in later lessons we shall need to refer to the principles of chemistry and physics which this chapter reviews.

Summary: (1) Both living and lifeless matter may undergo physical and chemical changes; and (2) all matter is composed of elements, usually combined in compounds, and capable under certain conditions of new recombinations into other compounds. 


\section{CHAPTER III}

\section{THE CHARACTERISTICS OF LIVING THINGS}

\section{CHEMICAL COMPOSITION OF LIVING COMPARED WITH LIFELESS THINGS}

IN order to understand the relations of living and lifeless things, we need to know whether the organic matter of living things is composed of peculiar substances which are not found in inorganic matter. A few simple experiments will show some remarkable similarity of composition.

12. Water in Living Things. - Water, which is itself lifeless, forms a large part of the bodies of animals and plants, as the following experiments show.

$(D)^{*}$ With a delicate balance, weigh carefully a piece of plant stem or a leaf, record the weight, place in a warm, dry place (e.g., over a radiator, lamp, or stove, or in sunlight), and when dry weigh again. The loss in weight represents approximately the amount of

* Directions for practical work are printed in the smaller type throughout this book. Problems for individual work in the laboratory are marked $(L)$. Most of the laboratory work requires the supervision of a teacher, but it will be found that most exercises require little or no apparatus and may be conducted in the ordinary class-room if, as in many schools, there is no special laboratory.

The letter $D$, at the beginning of a paragraph in small type, indicates that the practical work suggested is recommended for demonstration by the teacher; but when marked $D$ or $L$ it may be assigned, if the teacher prefers, as a problem for the individual work of the students.

All subject-matter in the larger type is suitable for recitations. In most cases the laboratory problems and the demonstrations should be taken in regular order, because much of the text in larger type interprets and supplements the practical work. In preparing any section for recitation or examination, students should review the laboratory directions in small type, for many fundamental facts there given are necessary for the intelligent reading of the text in larger type. 
water that has evaporated. Keep the dry piece of plant for a later experiment.

(D) Get a small piece of raw meat, or a frog killed by chloroform. Wipe dry with blotting- or filter-paper, and weigh. Dry and weigh again, as in the case of the plant in the first experiment. What proportion of the animal substance is water? Compare with the plant.

These experiments give us a general idea of the large amount of water in animal and plant bodies. It would require very careful experiments with very delicate apparatus for weighing and special apparatus for drying in order to determine the exact amount of water in plants and animals. Careful investigations by chemists have shown that the body of a higher animal (e.g., a dog) is nearly 70 per cent water. This is believed to be true also of the human body. This water is derived from that which we drink and also from foods. Thus potatoes contain about 78 per cent water, milk 85 per cent, tomatoes over 90 per cent, apples over 80 per cent, and lean meat over 50 per cent.

It is evident that water must play an important part in the life of animals and plants, and in this connection it is interesting to note the abundance of water and its wide distribution over the earth. All good modern textbooks of geography emphasize the close relation between the distribution of water and that of animal, plant, and human life.

13. Gaseous substances, which are themselves lifeless, may be obtained from the bodies of animals and plants.

$(D)$ Place the plant material left after drying in the above experiment in the bowl of a clay tobacco-pipe, close the mouth of the bowl with soft clay or plaster of Paris, support the pipe by a wire or by a retort-stand, heat the bowl in the flame of a gas- or alcohol-burner until it reddens and smoke (gases) begins to issue from the pipestem, then light the smoke with a match. In another pipe heat some splinters of wood or sawdust, and burn the gas in the same way. A piece of dried meat heated in the same way gives off gases, which may be burned. 
Such experiments prove that combustible gases may be obtained from animal and plant substances after the water has been removed. Chemists have shown that these gases are not present as such in the animals and plants, but the elements of which they are composed are united in chemical compounds which change to gas when heated. This will be clear when we recall that coal, wood, or oil (all of which are of organic origin) when heated in the retorts at the gas-factory, give off illuminating gas. Gas as such is not present in the solid coal, but the coal has compounds whose elements recombine when heated and form the gas. So it is in the bodies of animals and plants; the elements to form the gases are present, and the gases are produced by heating, which causes new recombinations of elements.

14. Carbon is a prominent part of the solid substance of animals and plants. After the gases were given off in the preceding experiments a black substance was left in the pipe. The same thing occurs when we burn an ordinary match; that is, certain gases burn and produce the flame and a charred mass is left. This black material we know as charcoal; at the gas-factory a similar substance from heated coal is called coke. The black color of coke and charcoal is due to the presence of an element known to chemists as carbon. The easiest way to get pure carbon is by charring white sugar with intense heat. Chemists know more than one hundred thousand compounds of carbon with other elements. It is especially abundant in the organic compounds of which the bodies of animals and plants are composed, and consequently must be also in the food from which the body substances are made.

15. Mineral substances enter into the composition of animals and plants. If we reheat the charcoal obtained from either the animal or the plant matter in the preceding demonstration, by holding it with a wire in the hot flame of a gasor alcohol-burner, the carbon which it contains will soon 
burn; that is, combine with oxygen to form carbon dioxide $\left(\mathrm{CO}_{2}\right)$, and only mineral matter or ashes remain. Another example is the familiar fact that when wood (or any plant matter) is burned there is formed a mass of red-hot coals or embers, which if allowed to cool quickly become charcoal; but if the coals remain heated, they soon change to ashes, because the carbon is burned. Obviously, the charcoal obtained by heating ordinary animal or plant substances is a mixture of combustible carbon and incombustible mineral matter or ashes. Charcoal made by heating sugar leaves no ashes, because it.is pure carbon, which in burning combines with oxygen and forms carbon dioxide.

A chemist could prove by careful analysis that the ashes from either plant or animal substances are a mixture of several compounds containing the elements calcium, sulphur, iron, potassium, sodium, and several other elements. These are all found in the soils, and in the water of the lakes, rivers, and the sea.

16. Summarizing our inquiry into the composition of living things, we have found that water, carbon, gases, and mineral matters may be obtained by analysis of living things. All these substances are also found in lifeless or inorganic matter. It is evident then that chemical composition alone does not enable us to distinguish between living and lifeless matter. And yet in most cases we have no difficulty in deciding whether a certain thing is dead or alive. How do we know? The answer will be found in the life-activities discussed in the next lesson.

\section{LIFE-ACTIVITIES}

17. Distinguishing between Living and Lifeless. - The conclusion reached in the preceding lesson was that all the materials entering into the composition of living things (animals and plants) are also found in inorganic things, 
such as soil, water, and air; and hence animals and plants cannot be distinguished from lifeless things by the substancez entering into their composition. What, then, does distinguish the living from the lifeless? Let us first try to answer this question by examining a living animal (e.g., a frog) and, if possible, determine just how the living frog is different in behavior from lifeless objects, such as a stone or a dead frog.

\section{LIFE-ACTIVITIES OF AN ANIMAL}

18. The living frog moves automatically or spontaneously. By this we mean that within the animal there is machinery for producing motion. A stone or other lifeless thing can move only through the action of some external agency; it may fall (gravitation), or be moved by swiftly flowing water, by a violent wind, or by some animal. Not only does the frog have the power of locomotion, but also there are internal movements, such as breathing and the beating of the heart, which go on continuously as long as the frog lives.

The statement that the living frog has machinery for producing motion reminds us of the steam-engine, but a little study shows that the engine is not automatic, as is the body of a living animal. For example, the engine requires the attendance of an engineer to supply it with water and fuel (that is, to feed it); but the frog is able to obtain its own food (fuel) and water to feed itself. Careful study of all machines invented by man shows that the movement which at first may appear to be as automatic as that of an animal is really dependent upon regular human attendance. Among common objects there is nothing lifeless which seems to have automatic movement, and in the vast majority of cases it is easy to decide whether an animal is dead or alive by simply testing for evidences of movement.

19. The living frog requires food if it is to continue to live and move and grow. This is a fact so well known that 
it needs no demonstration. There is nothing like animal growth among lifeless things, which increase in size only by addition of particles of substances like themselves. If a saturated solution of common alum (or copper sulphate) be made by dissolving as much as possible in boiling water, and then a stone be dropped into the alum solution, it will be coated by alum crystals, but the mass of stone will not increase. If a lump of alum be dropped into the saturated solution, the mass will increase by the addition of more alum crystals to the surface. Such an experiment with alum, showing that a mass of it can increase in size only by addition of the same substance, illustrates a fact applicable to stones, crystals, minerals, and other inorganic things; they cannot add other substances to themselves and make them really a part of their own bodies. On the other hand, living animals can live and grow on food derived from other animals or from plants. For example, a frog may eat smaller frogs; but it may also eat earthworms or plants, and the substance of these will be changed and built into that of the frog. This power of the living animal to take food unlike itself and to make it over into its own substance is known as assimilation (meaning to make like or identical).

20. The living frog breathes, and the lifeless object does not. If we watch a frog, or a higher animal, we see muscular movements concerned with pumping air into and out of the lungs; and we call this process breathing. In many simple animals there are no lungs, but there are several ways of proving that they require air (only the oxygen, and not the nitrogen of the air), and that they change the air when they breathe it. That animals require air is shown by the fact that land animals die very quickly if placed in a jar from which the air has been pumped out with an airpump, and aquatic animals will soon die in water from which the air has been withdrawn by an air-pump or been 
expelled by prolonged boiling. The following experiment will show one simple method of testing the changes produced in air which has been breathed:-

$(D)$ Pour some lime-water or barium-water into a small bottle and blow air from the human lungs through a small glass tube (or straw) into the water. What happens? The change in the limewater is due to a gas, called carbon dioxide, which is added to the air while it is in the lungs.

The same gas is formed by ordinary burning. Wrap a piece of wire around a small candle, light it, and then lower it into a tall, wide-mouthed bottle. After a time the flame will flicker out. Then lift out the candle and put in a little lime-water. Compare the lime-water with that changed by air from human lungs.

Take a tall, wide-mouthed bottle or fruit-jar, wash and rinse with fresh water, then lower into the jar a frog inclosed in a loose bag of cheesecloth or mosquito-netting with a string attached so that the frog may be lifted out quickly without inverting the jar or leaving it uncovered more than for a moment. One of the preceding experiments suggests that human breathing changes the air of rooms; and hence it is important that the jar be held at an open window where fresh air may enter while the frog is being placed in the jar. A good plan is to keep the jar full of water until the moment when ready to place the frog in it. The water will prevent the jar becoming filled with the air of the schoolroom. A second jar, treated exactly like the first, but without a frog, should be kept beside the first for comparison. Leave the frog in the jar, carefully covered, for two hours, quickly lift it out, pour in $10 \mathrm{cc}$. of lime-water, replace cover, and shake. Has any change occurred in the lime-water when it came into contact with the air which the frog had been breathing? Test the air in the jar without a frog.

The above experiments prove that some change occurs in air when animals breathe it. Until a later lesson we need not take time to consider just what this change is. For our present purpose it is sufficient to have demonstrated that animals do change air when they breathe it and that lime-water makes it possible to demonstrate breathing of animals in which we cannot see breathing movements.

21. The living frog has the power of reproducing new animals like itself. Frogs' eggs gradually develop into new 
frogs. We shall later study the development of some animals from eggs, but for our present lesson it is sufficient to note that books on embryology (the science of development) state that all higher animals develop from eggs, which are small masses of living matter separated from the parent animals. No lifeless object has any such power of separating from itself a small body which is able to take food and grow into a body like the original one. The power of reproduction is, then, a striking characteristic of living animals.

22. The frog after a time loses the power of moving, breathing, etc.; that is, it dies. We are familiar with the fact that animals of a given kind or species live for a certain length of time and then grow old and die. For example, an elephant has been said to live 200 years, a horse 40 , lion 35 , cat 40 , toad 40 , sea-anemone 50 , crayfish 20 , vulture 118 , eagle 100 , pike and carp 200, squirrel and mouse 6 , pig 20, sheep 15, fox 14 , and hare 10 years. However, it is certain that most individuals of these species live a much shorter life; for example, few horses live over 20 years. The life of all individual animals, even though they escape accidents and disease, is of limited duration. They are like machines, able to run a certain period of time. Ultimately the machinery of life stops and the animal bodies rapidly decompose into substances which show no signs of ever having been living.

23. Summarizing, we have seen that a living animal has the following activities: it moves; it breathes; it takes food for assimilation; it reproduces. It has still other peculiar powers which will be considered later. None of these is found in lifeless objects, such as stones or dead animals. All such processes - moving, eating, breathing, reproducing - which are peculiar to living animals, are known as life-activities. These are not permanent in any individual animal, for after a certain length of life the individual animal loses its life-activities - we commonly say 
that it dies - and the lifeless body soon changes to the condition of inorganic substances which make up air, water, and the soil of the earth.

\section{LIFE-ACTIVITIES OF A PLANT}

To one who has never studied botany it may seem that most of the activities named above as characteristic of living animals are absent from living plants; but a careful examination shows that living plants move, breathe, require food, and reproduce, and in still other ways resemble animals in their life-activities.

24. Living plants have movement. It is true that most plants with which we are familiar are not capable of locomotion (i.e., movement from place to place); but the same is true of many lower animals. On the other hand, there are many lower plants (to be studied later) which have locomotion like that of some lower animals.

Locomotion in animals is only one phase of their movements, and much more impressive are the constant movements

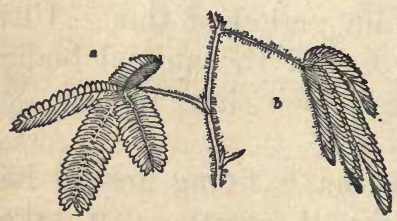

Fig. 1. The "sensitive plant"

(Mimosa). $a$, expanded leaf ; $b$, folded after being touched.

(After Detmer.) of internal organs, such as the heart and the lungs. There are many similar cases of plants able to move certain organs, e.g., the Mimosa (" sensitive plant") moves its leaves and branches when touched (Fig. 1); the Oxalis, the bean (Fig. 30), and certain clovers fold their leaflets at night; the Venus fly-trap (Fig. 2) has peculiar leaves able to snap together and catch insects ; many plants twine their stems around supports ; and plants bend toward the light when growing near a window. All such cases show that animals have no monopoly of movement; for in addition to cases of plants which have locomotion 
and others which move leaves and other parts, there are in plants many movements which can be detected only with the aid of a good microscope.

$(D)$ Examine any plants available which show any of the movements mentioned above. Many will be found at greenhouses. Leaflets of the Elodea (an aquatic plant) are excellent for the movements visible with the microscope. A piece of an oyster's gill will show microscopic movement going on in animals (see § 338).

25. Plants require food if they are to continue to live and grow. It is a wellknown fact that ordinary garden plants will not grow well unless there is a supply of fertilizer (one kind of crude plant food) in the soil. It will be shown, in a later lesson, that green plants do not grow well if kept long in darkness, because light enables them to make use

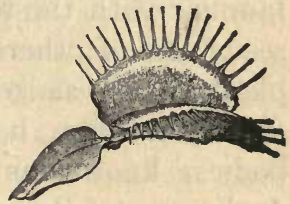

Fig. 2. Leaf of Venus fly-trap adapted for catching insects. (From Strasburger.) of certain food materials.

26. Plants breathe. In no plant is it possible to see breathing movements, as in animals; but it is possible to prove by the lime-water test that changes are produced in the air by breathing of plants.

(D) Take about twenty pea or corn seedlings (directions for raising such seedlings are given in first lesson on germination of bean, $\S 81$ ). Place in a loose bag of netting, such as was used for the experiment with the frog $(\S 20)$. Then lower the seedlings into a wide-mouthed jar filled with fresh air, and after 24 to 48 hours lift out the seedlings and test the air by pouring in some lime-water. A second jar without seedlings, but otherwise treated in exactly the same way, should be kept for comparison (control experiment). Take all the care suggested for the corresponding experiment with the frog. Compare results with that experiment. The breathing in the plants is so much slower than in animals that the same amount of change in the lime-water is not to be expected.

A potted plant, such as a geranium or a begonia, if placed under a bell-jar beside a small dish filled with lime-water, will give the same proof that the plant causes changes in air, just as animals do when breathing. 
In trying experiments with seedlings or with any full-grown plants which have green color, keep the jar covered so as to exclude light. The reason for this precaution will be clear in a later lesson which deals with the effect of exposing plants with the green color to light.

Other experiments connected with later lessons will give further proof that plants breathe, and will help to explain how they breathe.

27. Plants have the power of reproduction. We are familiar with the reproduction of many common plants from seeds and of others from cuttings or slips (e.g., many house plants, such as geranium, coleus, begonia). Many plants (ferns, mosses, horsetails, mushrooms, etc.) form small bodies, known as spores, from which new plants grow. And many of the microscopic plants reproduce by automatically dividing their bodies into two equal parts, and these half-size plants soon grow to the full size.

28. As in the case of animals, individual plants ultimately die, but some plants may live to a very great age. Some of the giant Sequoias of California, the largest of which are nearly 300 feet high and more than 75 feet in circumference, are probably at least two thousand years old, and some botanists estimate over four thousand years. Some of the famous oaks of Europe are believed to be eighteen hundred years old, but our largest American oaks are probably less than five hundred. There are in Europe specimens of chestnut, olive, cypress, yew, and other trees which are probably much more than one thousand years old. It is impossible to estimate accurately even after the trees are cut down and the so-called "annual" rings of the trunk counted; but some of these trees were famous four or five hundred years ago, and we may be sure that they are of far greater age than any animals are known to have reached.

29. Characteristic Life-Activities of Animals and Plants. Our brief studies of a living animal and a plant have shown us the following striking points of resemblance; $(1)$ the power 
of movement, (2) need of food, (3) power of growing, deriving the new substance from (4) power of breathing, (5) power of reproducing. All these activities or changes are found in living plants and animals and none of them in lifeless bodies. These are life-activities characteristic of living things.

We see, then, that in order to distinguish accurately between living and lifeless things we must determine whether there are evidences of life-activities. Every year dealers in seeds, gardeners, farmers, and the scientists at the government laboratories must test samples of seeds in order to determine whether they are living or lifeless. Chemical analysis will not settle such a question; and so the only way to test seeds is to put them under conditions where some or all the life-activities may be manifested. In short, the seeds must be planted under conditions favorable for growth. Likewise dormant animals must be carefully examined for evidences of life. For example, small animals of certain species which are often abundant in soil where pools of water have dried up in midsummer may appear perfectly dead when viewed with the microscope; but they begin to move, eat, and manifest other life-activities soon after they are placed in water.

30. The Machinery of Life-Activities. - We have seen that certain activities in animals and plants make the living things different from the lifeless; and we shall now inquire concerning the stucture and working of the living machinery which in the animal or the plant moves, takes food, breathes, grows, and reproduces. But in order to understand the working of any complicated machinery, we must first take it to pieces and examine its structure, and later find out the use and work of each part. To this end we shall now examine with considerable care the structure of an animal, and later that of a plant.

31. Subdivisions of Biology. - The science of the structure of animals and plants is called anatomy or morphology. 
The science of minute structure as studied with the aid of the microscope is called microscopical anatomy or histology. The science of life-activities or functions of living things is called physiology; and there are special books devoted to the sciences of plant physiology, animal physiology, and human physiology. The sciences named in the foregoing are simply subdivisions or departments of the great science of living things, biology. 


\section{CHAPTER IV}

\section{STRUCTURE AND LIFE OF AN ANIMAL (FROG): AN INTRODUCTION TO ANIMAL BIOLOGY*}

32. Why the Frog is selected for Study. - Other animals might serve as types for this study; but it is better to select a backboned or vertebrate animal, because such an animal, by reason of its great similarity to human structure and functions, will make it easier to apply this introductory study to later lessons on human biology. And among the backboned animals none has been so popular for scientific study as the common frog. Many books, some of them very large, have been written about the biology of this animal, and the scientific knowledge concerning it is greater than that on any other animal. Strange as it may seem, we know far less concerning the human body from direct study; but fortunately the frog and the human are so much alike in numerous ways that biologists have applied to the haman species many facts which were learned first by study of the frog. Since the study of the frog by scientific men has played so important a part in building up the science of animal biology, teachers now regard this animal as valuable for study by those who wish to rediscover for themselves some of the most important facts concerning animal structure and life.

The common green frog is usually most available for study, but the descriptions which follow will fit any other species of frog, or even the common garden toad.

* To the Teacher : This chapter may be studied after the lessons on the plant, Chapter V. See note in Chapter IV in "Teachers' Manual." 
33. Nature-Study of Frog and Toad.-Readers of this book who have not studied, perhaps in elementary-school nature-study, the habits of life of the common toad and frogs should read at least one of the following: "Usefulness of the American Toad," in Farmers' Bulletin No. 196 (free from U. S. Dept. of Agriculture); "Life History of the Toad," in Cornell Nature-Study Leaflets; or Chapter 16 in Hodge's "Nature-Study and Life."

34. Justifiable Use of Animals for Science Study. - Some people think it wrong to kill frogs or other animals for scientific study, but such persons do not seem to have considered points 1 to 6 as follows: (1) Much useful knowledge can be obtained from such study. (2) It is no more wrong to kill a few frogs painlessly with chloroform than to kill cattle, sheep, and pigs for our use as food. It is not absolutely necessary that we should have meat for food; for thousands of people living healthy lives use only plant materials, milk, butter, eggs, and similar foods. This is not to be taken as meaning that animals should not be used for human food; that is entirely another question which must be answered by the tastes of individuals. But one who favors killing animals for food purposes cannot sincerely oppose scientific study of animals, for the scientific use is no less necessary than the use as food. (3) Killing a few animals painlessly for scientific study does not tend to make the student and teacher cruel and hard-hearted. On the contrary, people who have studied zoölogy extensively are usually very kind to animals and would not brutally kill a toad with a stone or step on an earthworm. (4) So few animals are required for scientific study that it does not tend to exterminate any species. It is certainly far more justifiable to kill a few animals painlessly for the purpose of scientific study than to kill or indirectly to hire others to kill animals for the sake of articles of decoration, such as bird plumage for millinery. No sensible person who wears aigrettes (heron plumes) or seal 
skins and who has read the revolting but true accounts of the barbaric cruelty practised by the hunters of the animals which produce these articles of decoration would ever question the painless killing of a few animals for the sake of scientific knowledge which, as we shall see, affects human life in many ways. (6) No one who approves of that relic of barbarism, hunting animals merely for sport and trophies, can conscientiously raise opposition to the scientific study of animals.

Facts such as the above must lead us to take a sensible view of the study of animals. Of course, the dissection* or the taking to pieces of an animal is not as pleasant work as pulling a rose into pieces, but since we want to see for ourselves the most important parts of the machinery inside an animal, we will be sensible, and set ourselves the task of carefully examining first the outside (external structure) and then the inside (internal structure) of the frog.

\section{THE STRUCTURE (ANATOMY) OF THE FROG}

"The first step towards an appreciation of animal life must be taken by the student himself, for no booklore can take the place of actual observation. The student must wash the quartz and dig for the diamonds, though a book may help him find these, and thereafter to fashion them into a treasure." From "Study of Animal Life," by Professor J. Arthur Thomson, of the University of Aberdeen, Scotland.

35. External Structure of the Frog. - $(L)$ Place a living frog in a plain glass tumbler, and cover with paper or mosquito-netting. The ordinary "jelly-glasses" with tin covers are convenient, if

* Dissection is the term commonly applied to the work of separating a dead animal or plant into its organs in order to learn the plan of structure. Careless people sometimes confuse dissection with vivisection. The latter term means operating on living animals which have been rendered insensible to pain by means of ether, chloroform, or other anæsthetics. In short, as now practiced by the greatest investigators, vivisection of animals is exactly the same as the surgical operations on human beings. Such operations on animals are occasionally performed with the hope of improving the methods for surgical work. In fact, most of the great operations which surgeons perform would be unknown had not operations on anæsthetized animals shown the proper methods for operating on the human body. 
small holes are punched in the cover. By looking through the glass, it will be possible to learn many things about the frog's external structure and habits.

Notice that the frog's body consists of head, trunk, and limbs. How many limbs? Is there a neck, or a tail?

Just as in geography we use the terms north, south, east, and west to indicate directions, so in zoölogy we must have terms for directions or positions on bodies of animals. The head-end of an animal is called anterior, the opposite end of the trunk is posterior, the lower surface of the body is ventral, and the back or upper surface is dorsal.

Imagine your own body supported on all four limbs (i.e., walking on hands and feet) and locate anterior, posterior, dorsal, and ventral. Make an outline sketch of the frog as seen in profile and another one of a boy as you imagine him walking on hands and knees, and then write the four terms given above on your sketches so as to indicate the parts of the body to which they are applied. Compare the right and left sides of the frog. Remember that in the study of animal biology right and left refer to the frog's body, not to your own. Hold the tumbler so that the frog will sit with its head pointed away from you, and your right will be the frog's right. If the animal were lying on its back with head pointed away from you, as you look down upon its ventral surface, would your right be right or left of the frog? Are the two sides of the frog's body alike? Are the right and left sides of your own body similar externally?

Any animal having differentiated (meaning made different) dorsal and ventral surfaces, and anterior and posterior ends, might be divided into similar halves (right and left) only by cutting in the median plane from anterior to posterior and from dorsal to ventral. Can you think of any other plane in which a frog can be equally divided? Such an animal is bilaterally symmetrical. Do you know any animal which is not so? Look at a jellyfish in the school-museum or at a picture of one in a book of zoölogy. In how many places could a wheel with eight spokes be divided? Make a rough sketch to prove your answer. Animals, like jellyfishes, which have the wheel-like plan of structure are said 
to have radial symmetry. There are comparatively few such animals, but numerous plants are so arranged.

The word organ will be used frequently hereafter. An organ is a part of the body of a plant or animal fitted for doing a particular work. For example, the heart is an organ for pumping blood, lungs are organs for breathing, muscles are organs for moving. A group of organs doing similar work form a system. Thus all the muscles constitute the muscular system, which is a group of organs adapted to the work of producing movement.

At this point it will be well to have at hand a dead frog, one either recently chloroformed or preserved for some time in formalin. Examine the dead frog for all points not easily seen in the living one kept in the glass tumbler.

Skin. - Note the color of the skin on the dorsal surface of the frog's body. Compare with the color on the ventral surface. In a later chapter it will be pointed out that many zoölogists think that these colors help to conceal the frog in the grass along ponds, among water weeds, and in other places where frogs live. When you make a trip into the country look for evidence that the color helps to conceal the frog. The skin is covered with a slime or mucus. How would this help the animal if an enemy tried to catch it?

Are there hairs on the frog's skin? Look with a hand-lens. Compare with your own skin.

Sense-organs. - (a) Examine eyes. 'Are there eyelids? Can the frog close its eyes? Look into a small mirror and compare your own eyes with those of a frog. (b) Ears - the large, dark round spots just behind the eyes are the membranes of the ears, stretched like a drum-head over the cavities (internal ears), which will be examined later. The frog has no projecting external ears such as the human being has. Also in the human ear the ear-drum (tympanum) cannot be seen from the outside, because it lies deep in a canal or tube which leads to the internal ear.

Limbs. - Compare the anterior pair of limbs with the posterior ones. Are they alike? In the anterior limbs (fore legs) notice three divisions: upper arm, extending from shoulder to elbow; forearm, extending from elbow to wrist; and the "hand." How many "fingers"? Compare the anterior limb with your own; i.e., your arm. What differences in the divisions do you notice? What similarities? In the posterior leg (hind limb or hind leg) of the frog 
notice: the thick fleshy thigh, extending from the hip to the knee; the shank, extending from knee to ankle; and the foot. How many toes? Compare with number of "fingers." The shortest toe corresponds to the big toe of the human foot.

Notice the membrane ("web") stretched between the toes of the hind foot, fitting the foot as a paddle for swimming. When you have an opportunity to observe frogs swimming in a large aquarium, or in the clear water of a pond, compare the uses of fore and hind limbs. The fitting of any structure or organ of an animal or plant to a special function is known as adaptation. Another adaptation

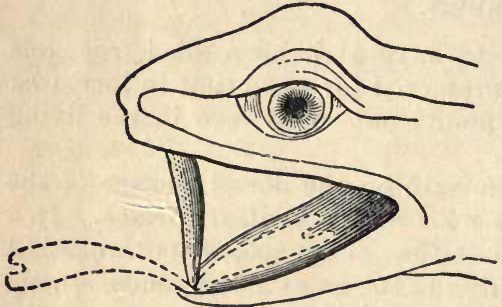

Fig. 3. Various positions taken by tongue of frog when catching an insect. (From Holmes, after Wiederscheim.) of the frog's hind legs is in the great muscles of the thigh, which fit it for jumping.

Mouth. - The opening is the mouth, and inside is the mouth-cavity (also called buccal cavity, meaning cheek cavity). Commonly we speak of the human mouth-cavity as mouth, and the opening as lips; but to be accurate "mouth" should be applied to the opening. Examine the mouth-cavity of a frog which has been chloroformed. Are there teeth? Look for teeth on a mounted skeleton of a frog. Notice two small openings (nostrils) on the dorsal side of the head near the upper lip of the frog's mouth. Pass a shoemaker's bristle or a slender broom-straw into a nostril, and note where the bristle comes out into the mouth-cavity. The human nostrils have no such direct communication with the mouth-cavity. With a needle make a hole in the frog's ear-membrane back of the eyes, and then carefully push a bristle into the opening. Open the mouth and find where the bristle comes out into the mouth-cavity.

To prove a similar connection between the human ear and the back part of the mouth-cavity, close your nostrils with your hand, then swallow once or twice and notice a feeling of pressure in your ears, due to forcing air back into the internal part of the ears. This explains why workmen are told to swallow air when entering the compressed-air chambers used in tunneling under rivers. The tubes connecting the internal parts of the ears with the mouth are known as Eustachian tubes, named in honor of Eustachius, professor of anatomy at Rome, who wrote a book describing the ear in 1754 . 
Examine the frog's tongue, and note that it is attached at the forward end to the tip of the lower jaw, while the free end extends backward towards the throat. How does this compare with the human tongue? In seizing an insect, the frog's tongue is turned quickly forward out of the mouth, and then quickly withdrawn. Figure 3 will make clear the positions of the tongue at various steps of this peculiar movement.

36. Internal Structure of the Frog. - $(L$ when not marked $D)$. Look at the mounted skeleton of a frog, compare with Fig. 4, and note the position of the following bones: backbone (vertebral column); skull; shoulder-girdle, which is a set of bones attaching the fore legs to the body; pelvis, a set of bones which attach the hind legs to the body. Notice that the "ribs" are very short. Now turn to the frog you have been studying, and feel the position of the above-named bones through the skin.

Lay the frog on its back, head pointing away from you. With forceps lift the skin and with scissors carefully cut through it along the median ventral line the whole length of the body. Carefully separate the skin from the underlying parts, cutting the thread-like connections, turn the flaps of skin outward to right and left, and pin to the board or wax in the bottom of a dissecting-pan. Cover the frog with water.

Notice the muscles of the body-wall of the abdomen, and the bones connecting the fore limbs (shoulder-girdle).

Again using scissors and forceps, carefully cut through the bodywall in the median ventral line from the pelvis to the shoulder-girdle, and then cut across the body (transversely) just posterior to the girdle. Separate and spread out the two flaps of the body-wall, and pin down to the dissecting-board. The cavity containing the internal organs thus opened is the body-cavity (cœlome). Now identify the organs exposed - liver, stomach, intestine, egg-organs or ovaries (if the specimen is a female frog), comparing your specimen with Fig. 5 in order to identify the organs.

Now cut out, with strong scissors, the ventral bones of the shoulder-girdle (the teacher will demonstrate how this is best done). As you lift up the bones, notice the heart lying beneath. The posterior, conical, whitish part of the frog's heart is called ventricle (Fig. $5)$; it lies in a depression between two parts of the liver. Anterior to the ventricle are the thin-walled auricles (right and left), usually found filled with dark blood in a dead frog. The ventricle is the part of the heart which forces the blood from the heart into the bloodtubes (blood-vessels); while the auricles are reservoirs for holding 
blood coming back to the heart and collecting for the next "beat" of the ventricle. The extremely thin membrane which incloses the heart is the pericardium (meaning, around the heart).

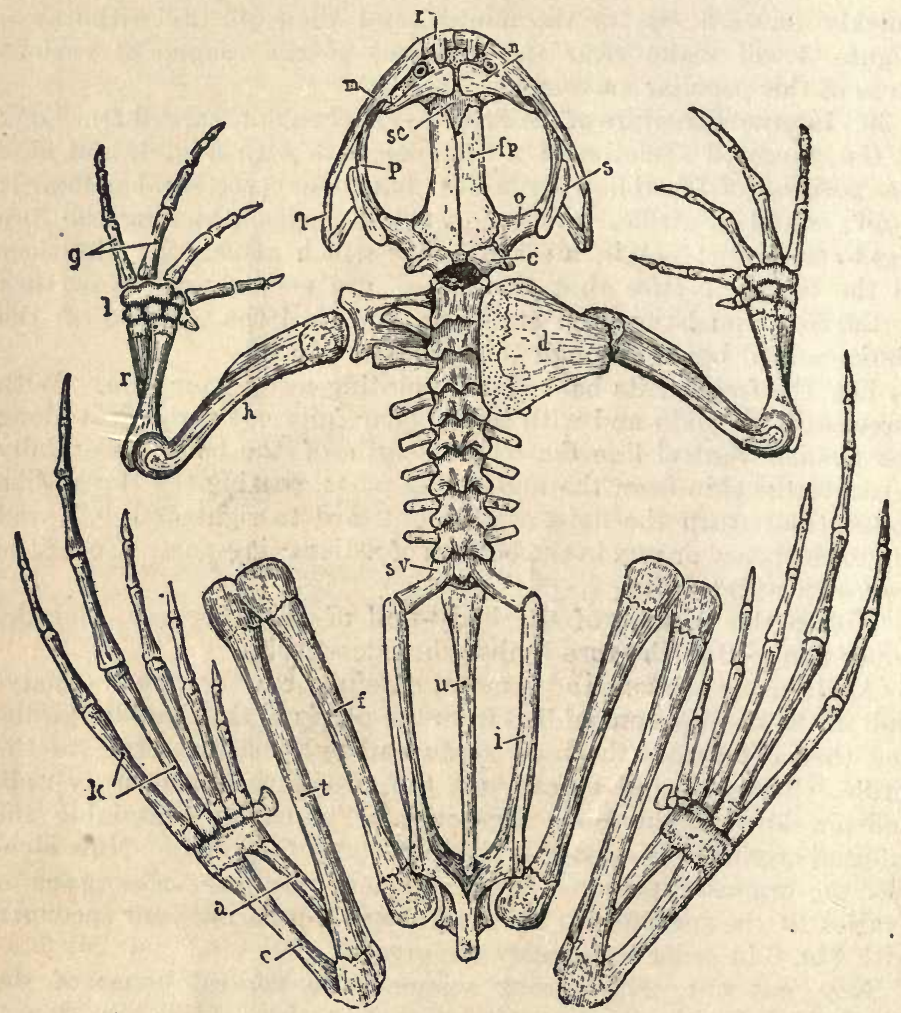

Fig. 4. Skeleton of frog viewed from dorsal. Nine segments (vertebræ) and the urostyle $(u)$ in backbone. $d$, shoulder-blade or scapula, dorsal part of shoulder-girdle; $h$, humerus, upper-arm bone; $r$, one of two bones in forearm ; $i$, pelvis ; $f$, femur or thigh-bone ; $t$, shank-bone. (From Marshall.)

( $D$ or $L$ ) At the anterior end of the heart is a blood-tube (aorta) which branches into two smaller tubes, each of which has several 
smaller branches leading to the right and left* sides of the body. These are the chief blood-tubes through which blood flows away from the heart, and they are called arteries. The branches of the two arteries lead to all parts of the body, and may be seen in many

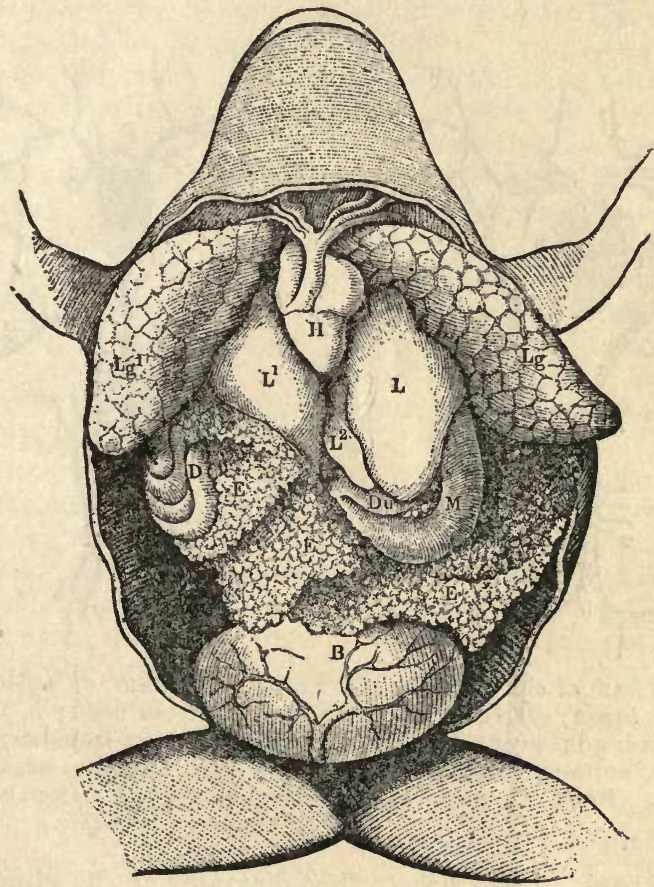

FIG. 5. Frog dissected from ventral surface. $h$, heart; lg, lungs; $l$, liver; $m$, stomach ; $d$, intestine ; $e$, ovaries ; $b$, bladder. (From Ecker.)

organs. Connecting with the anterior end of the heart and on its dorsal surface are three thin-walled tubes, which, in dead frogs, are usually filled with dark-purple blood. These are the blood-tubes in which blood flows back to the heart, and they are called veins.

* The student should remember that with the frog lying on its back the right side of the frog will be on the observer's left. In all descriptions in this book, right and left refer to the animal studied, not to the observer. 
Later we shall see small branches of the three veins in various parts of the body. In fact every organ which has an artery in which blood comes from the heart must have a vein for the return of blood to: the heart; for the blood circulates from heart to arteries, from arteries to smaller tubes called capillaries, from these to veins,

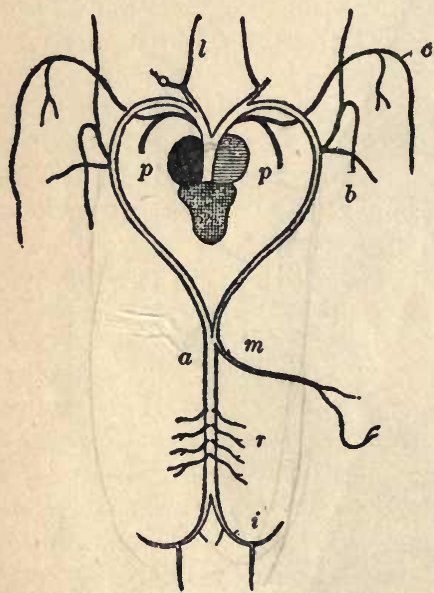

Frg. 6. Diagram of chief arteries of frog. $p$, to lungs ; $c$, to skin; $b$, to arm; $l$, to head; $m$, to digestive organs; $a$, aorta ; $r$, to kidneys ; $i$, to legs. (From Thomson, after Ecker.)

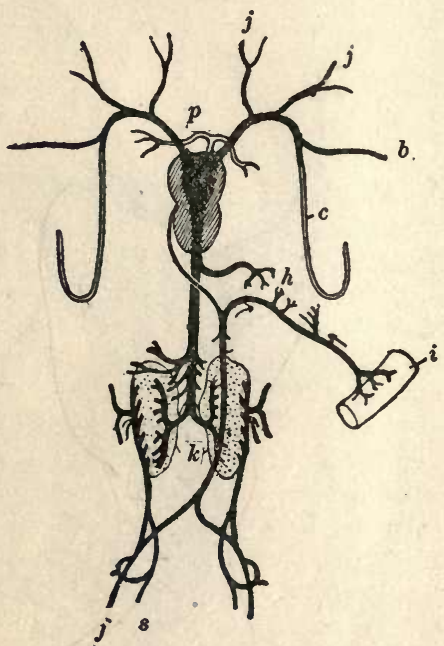

FIG. 7. Diagram of chief veins of frog. $j$, from head; $b$, from arm ; $c$, from skin; $p$, from lungs; $h$, from liver and digestive organs $(i) ; k$, from kidneys ; $f, s$, from legs. (From Thomson, after Ecker.)

and thence back to the heart. See diagrams of the circulation in Figs. 6 and 7.

(D) Capillaries. - Watch the flowing of blood through the capillaries in the tail of a small tadpole allowed to lie on its side on a wet plate of glass, or in the spread web of a frog's foot. Use low power of microscope.

In addition to the system of blood-tubes carrying blood from the heart, through the capillaries in all organs, and back again to the heart, there are in all organs many small tubes which collect a watery fluid called lymph. This fluid is derived from the liquid part of the blood, and it ultimately flows back into the blood. 
The largest organ in the frog's body is the liver, a large bi-lobed (two lobes or divisions) organ behind and at the sides of the heart. Its color is reddish brown in frogs not preserved in chemicals. It will be studied more carefully later.

Ovaries. - In a full-grown female frog, masses of spherical black and white eggs (ova) lie among the other organs (Fig. 5). These ova are attached to, or are really united to form, two organs (the ovaries or egg-organs) which later will be found to be fastened to the dorsal wall of the body-cavity, one on the right and one on the left of the median line; i.e., bilaterally symmetrical. With forceps, pull out the masses of ova, taking care not to injure other structures.

Alimentary Organs. - These are the organs concerned with receiving food and preparing it for the use of the frog's body. The alimentary canal, or food-tube, extends through the body from the anterior to the posterior extremity (Fig. 8). The mouth is the anterior opening; the posterior opening is ealled anus. Turning the organs in the body-cavity, but not cutting, examine the various parts - stomach, intestine, etc. - of the food-tube. The stomach lies dorsal to the left lobe of the liver. The short tube from stomach to throat is the esophagus (gullet). The throat or pharynx connects the esophagus with the mouth-cavity. Carefully push a probe (such as a small stick) down the throat into the stomach. The tube extending from the stomach backwards or posteriorly is the intestine. Note that the first part lies parallel to the stomach. The constriction between the stomach and this first part of the intestine is the pylorus. The small intestine is a slender and much convoluted tube. The large intestine (or rectum) is a short, straight tube, of greater diameter than the small intestine. The expanded end of the large intestine is called cloaca. Tubes from the kidneys and reproductive organs open into the cloaca; but this is not so in the highest animals. The opening (anus) of the large intestine to the exterior is on the dorsal surface of the body near the end of the backbone.

Carefully cut the membrane (mesentery) which holds the alimentary canal in place, cutting close along the canal, and pin the intestine to one side and out of the body-cavity. Cut open the stomach longitudinally. It may contain food, such as softened and disintegrated pieces of worms, etc.; the condition of the food suggests that solid foods are dissolved in the stomach. Wash out the contents, and notice the longitudinal folds which line the stomach and increase the surface with which food eomes into contact. Cut open the intestine in several places. Do you find folds arranged as in the 
stomach? Notice that the food in the intestine is more liquid than the food in the stomach. Also notice that by the time food has passed along the posterior part of the intestine its bulk has been greatly reduced - the stomach's capacity is much greater than that of the intestine. Evidently the greater part of the food disappears

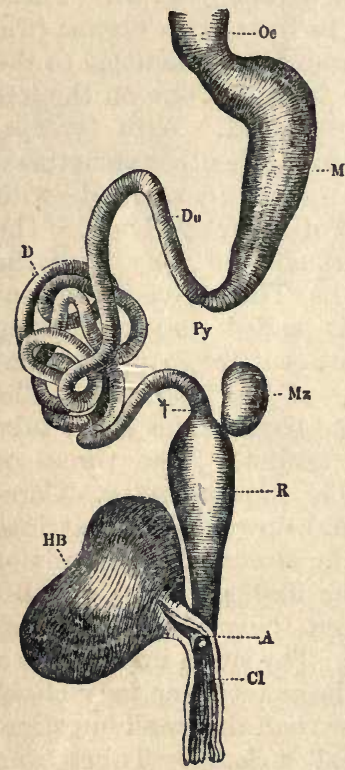

Frg. 8. Alimentary canal of frog. $\propto$, esophagus ; $m$, stomach ; $d u, d$, small intestine; $p y$, pylorus; $m z$, spleen; $r$, large intestine; $h b$, bladder; $c l$, cloaca. (From Ecker.)

(D) The lungs are two thin-walled sacs, which, before removal of the liver, were dorsal to that organ. Cut through the articulation of the jaws so as to allow the mouth to open widely, and demonstrate the slit-like glottis in the pharynx just ventral to the opening of the esophagus. The tube into which the glottis opens is the windpipe or trachea, which has two branches leading to the lungs. Insert the tine. Where does it go?

In addition to the parts of the foodtube, the liver and the pancreas must be considered alimentary organs, because they form or secrete substances needed for preparing foods in the intestine.

The liver, whose position has been already noticed, consists of two lobes, the lobe on the left side of the body being the larger and subdivided into two parts. Notice a small sac, the gall-bladder, between the right and left lobes. The greenish-colored fluid which fills the gallbladder is the bile, which is manufactured by the liver and stored in the gall-bladder until it is needed in the intestine. A bileduct (very small and difficult to see) leads from the gall-bladder to the intestine.

(D) The pancreas lies between the stomach and intestine. It is an irregularly lobed mass of light color in fresh specimens. It secretes a fluid (pancreatic juice) used in digestion of foods. Many of its small ducts open into the bile-duct, which extends along the pancreas on the way from the gall-bladder to the intestine.

Cut the attachment of the liver, esophagus, and intestine and remove these organs, thus exposing other organs nearer the dorsal part of the body-cavity.

as it passes along the stomach and intes- 
end of a small tube (blow-pipe) into the glottis and blow in air to inflate the lungs; or, using a rubber-bulbed pipette, fill them with water.

$(L)$ Watch a living frog in a glass jar, and observe how the floor of the mouth ("throat") moves up and down at regular intervals. Count the movements in one minute. The frog cannot breathe as we do and practically "swallows" air, forcing it down into the lungs by moving the floor of the mouth up while the mouth and nostrils are closed. Many persons breathe while keeping the mouth open, but a frog cannot breathe when the mouth is held open. Not every movement of the throat forces air down into the lungs; most of the movements simply pump air into and out of the mouth-cavity through the nostrils. But watch earefully, and occasionally the sides (flanks) of the body back of the shoulders will be seen to expand greatly when the frog seems to take a big "swallow" of air; this expansion indicates that air has been forced down into the lungs. Then, after a time, the muscular walls of the body help the elastic lungs expel the air, forcing it up into the mouth-cavity, where it is gradually mixed with fresh air pumped in through the nostrils.

(D) The kidneys are flat bodies of a dark-red color. They are attached to the dorsal body-wall in the posterior portion of the bodycavity, one on each side of the backbone. The ureters are the ducts or tubes of the kidneys which lead to the terminal part of the large intestine (cloaca); they are very small and difficult to demonstrate. The kidneys extract water and certain other substances from the blood which flows through them, and then pass these substances to the exterior through the ureters and cloaca. Before these substances from the kidneys are eliminated, they may be stored for a time in a sac known as the bladder, which lies on the ventral side of the cloaca, and opens into it. This structure is usually found as a collapsed membrane if one looks carefully before cutting out the large intestine. Examine a museum specimen prepared to show the bladder expanded.

The spleen is a small, oval, red body attached to the mesentery near the large intestine. Its use will be explained after we have studied the blood and lymph.

Reproductive Organs. - ( $D$ or $L)$ Examine the rgans in specimens of each sex. In the male frog the spermaries are a pair of yellow ovoid bodies attached ventrally to the kidneys. Their ducts lead into the kidneys, and there connect with little tubes leading to the ureters, and thence through the cloaca to the exterior.

In the female, the ovaries are attached at about the same place. 
The oviducts are a pair of long, much-coiled, white-colored tubes, lying close to the dorsal wall of the body-cavity and at the sides of the kidneys. Posteriorly they open into the terminal part (cloaca) of the intestine, while anteriorly they have funnel-shaped openings into the body-cavity dorsal to the liver. There is no direct connection between the ovary and the oviduct, but the ova, when mature, fall into the body-cavity, pass through the above-mentioned openings into the oviducts, and then to the exterior through the cloaca.

The fat-bodies are tufts of bright-yellow masses attached to the dorsal side of the body-cavity behind the liver. The fat is food stored for use in the early spring. Such fat-bodies are found only in frogs and their near relatives, but other animals store fat in various organs.

Nervous System. - $(D$ or $L)$ Remove all the organs which have been studied and cut aw $y$ the floor of the mouth. Notice (1) the skull, which is covered on its ventral surface by the roof of the mouth, and (2) the backbone or vertebral column. The skull contains the brain, and the backbone is a tube which incloses the spinal cord. Looking at the body-cavity side (i.e., ventral), notice the large nerves which extend from the vertebral column to the fore and hind limbs; also some small nerves extending out to the body-wall of the back. Examine brains which have been hardened by chemicals and then removed, and also observe a specimen of a frog dissected from the dorsal side to show brain and spinal cord.

The bones (skeleton) of the frog may be studied later in comparison with those of some other backboned animals. The chief bones of the frog can be identified by comparing a skeleton with Fig. 4 .

The muscles of the frog's legs should be examined as to their attachments to the bones. Also, note how shortening of muscles would affect the movements of bones to which they are attached. There are other muscles in the body-wall, and in the walls of stomach, intestine, and blood-tubes.

37. Organs of Frog. - Summarizing, we have found the frog to be made up of parts or organs as follows:

Alimentary organs: mouth, mouth-cavity, pharynx, esophagus, stomach, small intestine, large intestine (including cloaca), liver, and pancreas.

Breathing organs: nostrils, mouth-cavity, pharynx, trachea, lungs, and skin. 
Circulation organs: heart, arteries, veins, capillaries, lymph-vessels, and spleen.

Excretory organs: kidneys, ureters, bladder, cloaca, lungs, and skin.

Nervous organs : brain, spinal cord, nerves, eye, ear, and nose.

Supporting organs: skeleton (bones and cartilages).

Muscle organs : muscles of body-wall, of limbs (for locomotion), and of internal organs (heart, stomach, etc.).

Reproductive organs : ovaries, spermaries, ducts of ovaries and spermaries, and fat-bodies (peculiar to frog and its relatives or allies).

\section{THE TISSUES OF THE FROG: INTRODUCTION TO MICROSCOPIC STUDY}

38. We have found that the frog's body is composed of many parts which we call organs, examples of which are skin, liver, stomach, heart. We shall now examine more carefully the structure of these organs.

(L) Examine a frog's leg. First on the outside, notice the skin. Remove the skin, and the muscles are brought into view. Compare a piece of skin with a piece of muscle. Is there any difference? In separating the skin from the muscle, or the muscles from each other, notice fine but strong threads binding them or connecting them together. Notice bright, glistening bands (called tendons) which unite muscles to bones. Next, examine the nerves which lie between the large muscles. Scrape the muscle from the bone and attempt to cut into it about the middle and also at the rounded ends. Is there any difference?

In thus examining the frog's leg we find that it is composed of several distinct kinds of building materials. These are tissues. The skin is an example of covering and protective tissue which is called epithelium or epithelial tissue. The strong threads binding skin to muscle, muscles to each other, and (in form of tendons) muscles to bones, is known as 
connective tissue. The muscles contain muscular tissue, and the nerves have nervous tissue. The hard part of bones is bony tissue, and the softer tissue at the ends of bones is called cartilage. In the frog's leg, then, we find epithelium, connective tissue, muscular tissue, nervous tissue, bone, and cartilage.

$(L)$ Examine your own hand. Skin or epithelial tissue covers and protects it. Pull up on the skin, and it is found to be bound (by connective tissue) to the underlying muscles, which you can feel and move. You can also feel bones. Lastly, you have the sense of touch or feeling in the hand; this indicates the presence of nerves. Name the tissues which you find in your hand. Do you find any which were not seen in the frog's leg?

The tissues which have just been examined are the kinds of building materials which form not only the frog's legs, but also its whole body. The same kinds are also in our own bodies. If we were to examine any organ in the frog or in the human body, we should find it made up of two or more of the tissues. For example, the heart is largely composed of muscular tissue, but it has nerves, connective tissue, and epithelium; and the stomach has epithelium, muscles, nerves, and connective tissue. Any backboned animal's body is made up of many kinds of materials or tissues which have different appearances and serve different purposes. And just as the materials (iron, stone, brick, wood, etc.) used in building houses may be put together in various combinations so as to form many different kinds of buildings for different purposes, so the few kinds of building materials or tissues of an animal's body are united to form organs which are quite different in appearance and purpose. A frog's heart does not resemble a leg muscle and their purposes and work are different, but they are chiefly composed of the same kind of tissue (muscular), because muscular activity is needed for movement in legs and in the heart. In like manner, we find epithelium wherever there is a surface, inside or outside, 
to be covered; cartilage wherever flexibility combined with considerable rigidity (e.g., at ends of bones) is needed; connective tissue wherever other tissues must be joined together; bones for supporting framework; and nervous tissue in all places where nervous activity (feeling, sensation, control, etc.) is needed. Each tissue has its peculiar purpose, just as wood, bricks, iron, have their own purposes. Briefly, the purposes or functions of the tissues are as follows : epithelium for covering, connective tissue for uniting, bone for rigid support, cartilage for flexible support, muscular tissue for contraction and movement, and nervous tissue for feeling and control.

So far we have been studying the larger structure of the frog as seen by the unaided eye. We have been able to locate the various organs and to learn something about their general form or position; but concerning the structure of the organs themselves our unaided eyes have been able to discover only the tissues. It is now necessary to make use of the microscope in order to see the minute structure of the tissues which we find in organs.

(L) A lesson on use of the compound microscope should be introduced at this point.

39. Cells. - (D) Mount a small piece of the outer skin (epithelium) of the frog in a drop of water on a glass slide and cover with a cover-glass. Examine this with a compound microscope (magnification 50 to 100). The epithelium is seen to be composed of small (usually hexagonal) blocks, called cells, set side by side like bricks in a wall or pavement (see Fig. 9). A small spherical mass (called nucleus) may be seen near the center of each cell, and in most of the cells the nuclei may be

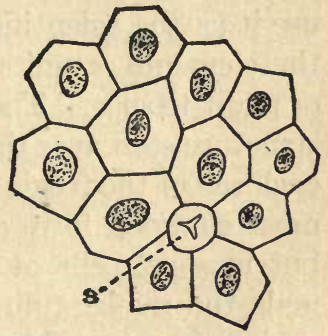

Fig. 9. Group of cells from surface epithelium of frog. $s$, opening of a skin-gland (From Holmes.) brightly stained by dipping a piece of epithelium into a dye such as eosin solution (red ink), then into water, and then mounting on a glass slide for microscopic examination. In the same slide notice 

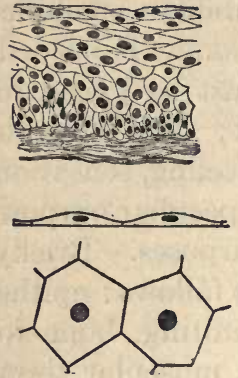

FIG. 10. Upper figure shows that there are many layers of cells in epidermis, those of Fig. 9 being at the surface. The lower diagrams represent two cells in surface and edge views.

that the cells are closely joined together; in fact, there is between them a cement substance, which can be dissolved with some chemicals, and the cells are then easily separated.

Cells. - We have chosen the frog's epithelium as a convenient introduction to cells. All other animal and plant tissues which biologists have examined microscopically have been found to have cells; and so the cells are regarded as the units of which the bodies of organisms are composed. Various forms of cells are shown in Figs. 10, 14, 16, 18, and 20. The word cell commonly means a cavity, and it was originally applied to plant cells (e.g., in cork and elder-pith which have cavities in their substance); but it is now known that most animal cells and many plant cells do not have cavities. Nevertheless, the word cell has become firmly fixed in biological language, and so we must use it as the scientific word for the elements or units of animal or plant tissues. The spherical structure seen near the center of each cell of the frog's skin is the most common form of nucleus; but in some cells of other animals the nuclei (plural) are in various forms - ribbon-like, like a string of beads, or even scattered in fragments in the substance of the cell. Apparently every living cell has a nucleus of some one of these forms. The substance of a nucleus is

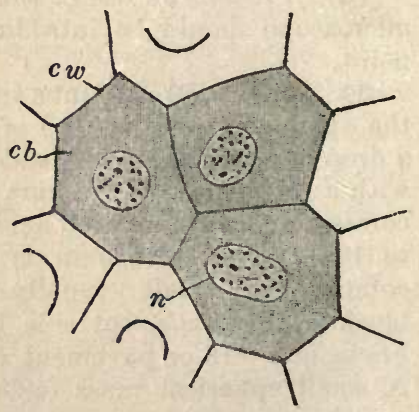

FIG. 11. Diagram of cells. $c w$, cell-wall; $c b$, cell-body; $n$, membrane of nucleus; black spots in nucleus are chromatin. Verworn.)

(From 
called nuclear material. Careful experiments have proved that a cell cannot continue to live without a nucleus. Organisms grow by multiplication of cells, and the nucleus plays an important part in that process of celldivision. The boundary of a cell is called cell-wall or cell-membrane; it is sometimes exceedingly delicate, and sometimes very thick and hard, as in certain plant cells. The substance between the cell-wall and the nucleus is called the cell-body.

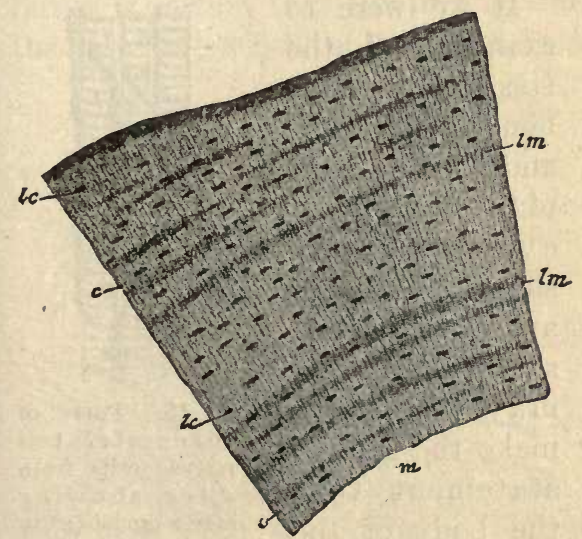

Fia. 13. Cross section of bone from leg of frog. $l c$, lacunæ or spaces in which lie bone cells; $m$, marrow cavity in center of leg bone; $c$, branching tubes from bone spaces and containing branches of the cells; $l m$, concentric layers of bone deposited by the bone cells. (After Parker and Parker.)

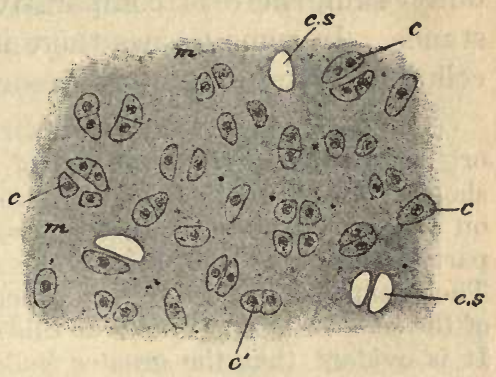

Fig. 12. Cartilage from end of femur of frog. $c$, cartilage cells; $m$, matrix of inter-cellular substance formed by the cells; $c . s$, empty cell spaces. (From Parker and Parker.)

Cells are composed of cell-substance, part of which is living substance and part stored food and other lifeless materials to be considered later.

40. Inter-cellular Substance. - In some animal tissues all the substance does not lie within cell-walls ; some of it is between cells or inter-cellular. In the frog's skin and muscles there is a cement substance between cells which holds them together. This is called inter-cellular substance, and is formed or se- 
creted by the cells. In an epithelial tissue, such as the frog's outer skin, there is comparatively little inter-cellular substance. In some tissues there is a large proportion of intercellular substance, and an example will now be examined.

$(D)$ With a sharp knife or razor cut very thin slices of cartilage or "gristle" from the joint end of a bone procured at a butcher's shop, or from a frog's bone. Mount these slices in a drop of water on a slide. Examine with a microseope. Notice that the greater part of the tissue is a bluish-white substance in which the cells lie. The distance of one cell from another is often more than the diameter of the cells (see Fig. 12). Was this so in the epithelium of the skin? It is evident that the greater bulk of cartilage is not cells, but the substance between the cells (i.e., inter-cellular).

A section of dry bone is similar to cartilage in that there are numerous small eavities in which origi-

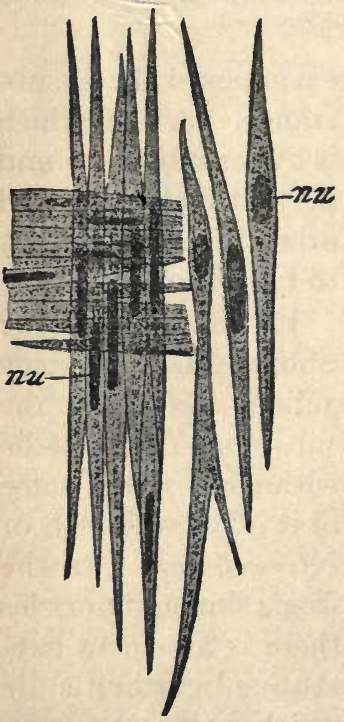

FIG. 14. Unstriated muscle cells from frog's intestine. $n u$, nucleus. Compare with m.c. in Fig. 19. (From Howes.) nally were located the cells that formed the hard bony substance, which is entirely inter-cellular (Fig. 13).

If we were to examine all the tissues besides bone and cartilage and all the organs of the frog's body, everywhere we should find cells and inter-cellular substance. We may therefore Fig. 15. Parts of make the general statement that the body of the frog is composed of cells and intercellular substance.

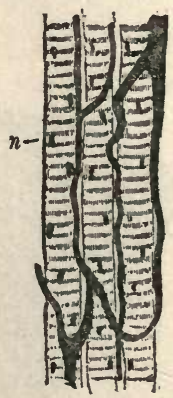
three striated muscle cells from a leg, showing many nuclei $(n)$ in each cell, and blood-capillaries (black lines) between cells.

But the latter is formed or secreted by the cells, and hence the cells are 
the primary units composing the body. These statements are also true of all higher animals and of the human body.

\section{Life-Activities in Cells: Proto-} plasm. - Since the frog's body is composed primarily of cells, we are led to infer that the life-activities $(\$ 29)$ are located in the individual cells. For example, the shortening of a muscle when it contracts and moves is the result of the combined shortening of the thousands of cells of which the muscle is composed. It appears, then, that the living active substance is located in the cells of the body. The technical word for living matter is protoplasm. It is not known how much of the cell-substance is living; but it is certain that the protoplasm is
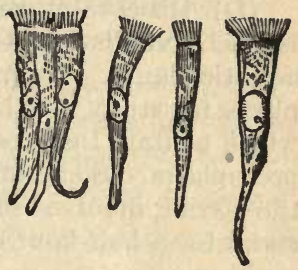

Fig. 16. Ciliated epithelial cells from the trachea of a dog. The hair-like structures on ends of cells project into cavity of the trachea. They have constant lashing movements. (After Sharpey.) the basic or essential substance in both nucleus and cell-body.

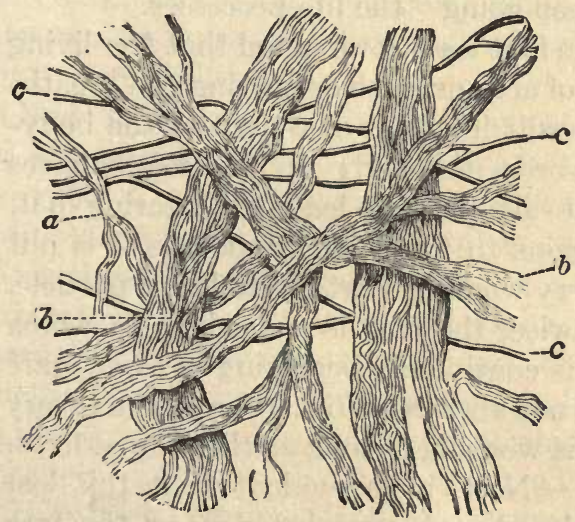

Fig. 17. Fibers of connective tissue. $a, b$, white fibers in bundles; $c$, elastic fibers. These fibers belong to inter-cellular substance. Numerous small cells are present between the fibers in connective tissue (see $c$ in Fig. 18).
The "life" of the frog, then, is not limited to any one organ, such as the heart, the brain, or the lungs ; rather it is located in every living cell of the body.

In order to understand better that protoplasm is living and active, the microscope may be used to examine leaflets of certain a quatic plants in which the 
cells are so transparent that we can see the protoplasm moving (flowing) around inside the cell.

(D) Observe movement (streaming) of protoplasm in leaflets of Elodea, Nitella, or Chara - all of which are widely distributed aquatic plants. Mount leaflets in fresh water, and select transparent places for study with low power of microscope. In Elodea the bodies which contain the green-colored matter (chlorophyll) move with the protoplasm. Sometimes the movements are checked temporarily by the jarring involved in mounting, and it is necessary to let the slide stand for a half-hour or more before the protoplasm shows its most rapid motion.

\section{THE WORK OF THE ORGANS OF THE FROG: INTRO- DUCTION TO ANIMAL PHYSIOLOGY}

42. Need of Food. - We shall now consider the work of the organs of the frog's body, and first we may inquire, Why does the frog need food? Some one may answer, "To keep its body alive," but that is not a scientific answer. In studying science we want to know why and how food is used by the frog so as to "keep going" the life-processes.

Waste. - In one of the first lessons we noted that the living frog performs a number of actions, such as moving and breathing. These activities result in a loss of weight in the bodysubstance. In other words, the body of the frog behaves like a machine in that all activity leads to wearing out. A new steam-engine begins to wear out as soon as it is put into motion, and at every working part, particle by particle, it is worn away. Likewise, the frog is a machine in which wearing out or wasting is continually occurring in every part of the body; for though not always visible, every organ (every living cell) of the body is working as long as the animal lives.

Repair and Growth. - Now, it is evident that this lost substance must be replaced by new substance, or the frog will soon wear out and die. In other words, the waste which is always occurring in the living animal must be made good by the processes of repair. The materials for this 
repair are supplied by the frog's food. And in addition to materials for repair, food also supplies those required for growth in size, which means increase in number of cells.

What would be the result as to the weight of the body if the wasting processes were more rapid than those of repair? What are the conditions with respect to the rate of waste and repair in a young frog which is growing rapidly?

A scientific answer, then, to our question, "Why does the frog need food? " is that food (1) furnishes the new substances necessary for assimilation (see $\$ 19$ ) to replace the bodysubstances which have been wasted owing to activity; and (2) in addition to repairing, food may furnish the materials for growth. Especially do young animals need food for growth.

Energy. - We may look in another way at the question, "Why does the frog need food?" The activities of the frog - breathing, eating, moving, etc. - indicate that the animal is continually doing work or using energy. Energy is the capacity for doing work. For example, the energy of the coal may move the steam-engine, the engine may run a dynamo and produce electrical energy, and the electricity may run a trolley-car.

Now, it has been discovered that energy cannot be created by any machine, plant, or animal ; but energy may be transformed. Thus the energy of the coal is transformed into heat energy and the mechanical energy of the engine, the mechanical energy is transformed into electrical energy, and this into heat energy (electric heater), light energy (electric lamp), or mechanical energy (electric motor). A steamengine does work and expends energy; but it derives its power of doing work (that is, its energy) from the coal burned in the furnace. Likewise the frog, being unable to create energy within itself, requires food as a source of the energy which is to be used in the life-activities. The food of the frog corresponds to the coal of the engine, in that each supplies the energy for its respective machine. 
The value of the food of the frog or of the coal depends upon the amount of stored or potential energy which each contains. Thus a ton of one kind of coal may run a certain large engine eight hours, while another quality from another mine may make the engine work just as hard for ten hours. Likewise, the amount of energy in foods is highly variable. Chemists have methods of burning samples of foods and fuels and determining how much energy they are capable of furnishing; that is, how much stored or potential energy they contain. Such tests of energy value are especially interesting and important in connection with human food, and will be studied later.

In addition to supplying energy for the life-activities, we should note that the food of the frog also supplies the materials for repair and growth of the frog's body, while the coal only furnishes energy and is powerless to repair the continual wear of the engine. Here is the great difference between a living machine, such as the frog's body, and the lifeless engine. Only the living machine, the animal's body, has the power of using food in order to repair the parts wasted by activity. When the animal's body grows old, it loses the power of using food to make repairs, and some of its essential parts wear out and stop the activity of the living machine.

Summary: Uses of Food. - We can now give a complete answer to the question, "Why does the frog need food?" by saying (1) food supplies energy, (2) food supplies the materials for repairing the waste of the living animal body, and (3) food supplies the materials for increase in size (growth), especially in the case of young, growing animals.

43. Changes in Food. - Having learned that food supplies materials for repair, growth, and energy of the frog's body, we now turn to trace it as it is taken into the body and undergoes the changes which take place internally when it supplies energy, builds up the body, and repairs the wasted body-substances. 
In the third lesson we found that an animal body contains water, carbon, gaseous substances, and mineral substances. In order that food may serve for making new body-substance for growth or repair it must contain all these; in fact, all the chemical elements found in the body.

Dissolved Food. - The food which is taken into the mouth of the frog and thence passes through the esophagus into the stomach is largely solid, such as worms and insects. These must be reduced to a liquid condition before they can "soak through" or be absorbed through the stomach lining into the blood. Solid food retained in the stomach cannot fulfil its purpose of supplying the energy and materials for repair, for the reason that energy is being expended and repair is necessary, not only in the cells composing the stomach, but also in all living cells of all the organs of the body, even in the remotest parts, such as the fingers and toes. It is evident that solid food cannot be distributed so widely; but a solution (like sugar dissolved in water) can "soak through " the lining of the stomach into tubes (blood-tubes) through which it can flow to distant organs and then "soak" into the cells. Hence we see the necessity of rendering solid food soluble in the water which is taken with food.

(D) A drop of milk, when examined with the microscope, is found to contain numerous fat droplets. A drop of water containing some starch shows the small particles mixed with the water. Examining with the microseope a drop of water in which sugar or salt has been dissolved, no particles of these substances are evident.

Milk and the mixture of water and starch are examples of fluids or liquids, but they are not solutions. The particles of starch or fat are not dissolved (i.e., not soluble) in the water. On the other hand, the water-and-sugar mixture is a liquid or fluid, and it is also a solution. It is clear that all liquids are not solutions. This distinction should be kept in mind for use in connection with the changes which foods undergo in the alimentary organs, for in them foods are 
chiefly prepared for absorption by being made into a solution in water. Even liquid foods like milk and soup must be dissolved so that no solid particles can be seen when they are examined with a microscope.

44. Digestion. - Some solid foods, such as sugar and salt, readily dissolve (are soluble) in water; but most of the frog's food consists of meat and other things which have to be acted upon by certain substances before they will dissolve in the water which is taken into the food-tube. To this process of changing foods and causing them to dissolve the term digestion is given. Definition: Digestion is the preparation of foods for absorption by the cells. It is chiefly a changing of foods so that they dissolve in water.

In order to understand how a chemical change may make an insoluble substance capable of dissolving (soluble) in water, try the following experiment:

$(D)$ Place a very small piece of marble or limestone (or a piece of zinc) in water. Does it dissolve?

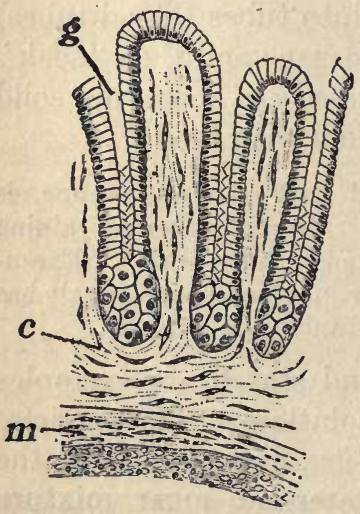

Fig. 18. Lining membrane of frog's stomach. $g$, gastric gland composed of epithelium; $c$, connective tissue; $m$, muscle coat of stomach. (From Ecker.) Pour some dilute hydrochloric acid into the water, and repeat from time to time until the limestone (or piece of zinc) becomes dissolved in the water.

From such experiments we learn that some substances which are not soluble in water will become soluble after a chemical change has been produced by an additional substance. This is just what happens in the alimentary canal of the frog. Small pocket-like tubes on the inside wall or lining of the stomach and intestine (called respectively gastric (stomach) and intestinal glands, Fig. 18), as well as the liver and pancreas, form or secrete peculiar. 
fluids (called secretions), which are poured into the stomach and intestine. These, coming into contact with foods, change them so that they are dissolved in water taken with the food. We say that the secretions of the gastric and intestinal glands and of the liver and pancreas digest the various kinds of foods which the frog eats; by this we simply mean that the foods are dissolved and prepared so that they can be absorbed by a process of "soaking through" the lining of the stomach and

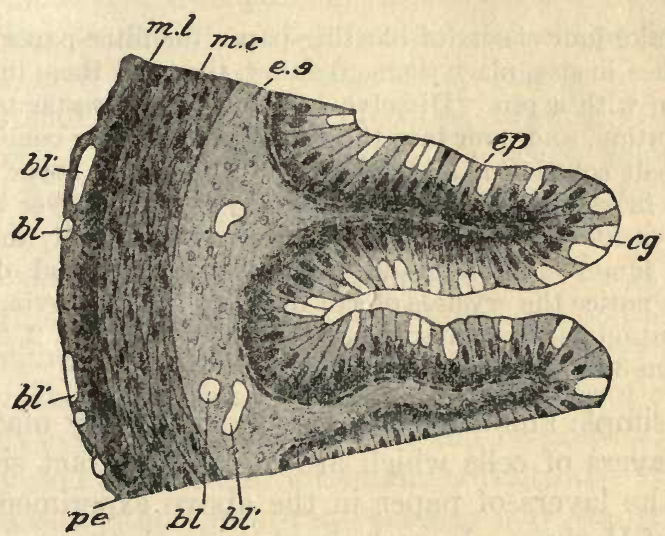

Fig. 19. Part of a cross section of small intestine of frog. pe, peritoneum surrounding the intestine; m.l, outer muscle layer (longitudinal); m.c, inner, circular muscle layer; $b l$, blood-vessels; e.s, connective tissue layer (submucosa); $e p$, epithelial lining; $c g$, one-cell glands. (After Howes.)

intestine into the blood-tubes (Fig. 19). The nature of these digestive secretions and of their action on the various kinds of foods will be taken up more carefully in close connection with similar processes which occur in the human body (Chapter XVII). For our present purpose it is sufficient to point out that digestion is preparing foods for absorption into the cells.

45. Distribution of Digested Foods. - We have noted reasons why digested foods must be distributed to all parts of the body (See $\$ 43$ ). We shall now consider the means by which this is accomplished. 
Absorption. - If the frog were a small animal with all its tissues near the food-tube or digestive-cavity (i.e., the stomach and intestine), the dissolved food could be absorbed through the walls of the food-tube into the surrounding organs. This is the case in some very small animals of simple structure, which will be studied later. We can illustrate the absorption of digested (liquid) food in such a simple animal by the following experiment:

(D) Take four sheets of blotting-paper (or filter-paper) about 4 by 10 inches in size, place them together, then roll them into a cone, and fasten with a pin. Dissolve common salt in water to make a strong solution, and pour into the cavity of the paper cone. Notice that the salt solution is absorbed first by the inner layer of paper, and then in succession by the outer layers, each layer absorbing from the next one nearer the center. Now unroll the sheets, mark the inner one No. 1 and the outer No. 4, and dry them. When dry notice the crystals of salt on sheet No. 4, proving that the salt in solution has been absorbed from sheet No 1 to 2 , from 2 to 3 , and from 3 to 4 .

Some simple animals have a cylindrical body made up of several layers of cells which are in close contact similar to that of the layers of paper in the above experiment. (See Fig. 98, of Hydra.) In such simple animals the cavity in the center of the body is the digestive cavity in which food is prepared for absorption. The cells which line this cavity are in contact with dissolved food and can absorb directly, just as sheet No 1 did in the above experiment; and the cells which form the outer layers must absorb from the inner ones, just as in the above experiment the outer layers of paper absorbed from those nearer the center.

46. Need of Blood for Distributing Food. - Absorption directly from the digestive cavity of the stomach or intestine or both would be possible only in a very simple animal. If in the above experiment we had used twenty or thirty sheets of paper, we should have found that very little salt solution would soak through to the outermost layer; and likewise in 
any animal with many layers of cells the outermost cells would get insufficient food if they had to depend upon absorption by layers, as in the case of the experiment with paper. Obviously absorption directly from the stomach and intestine would not be possible in an animal like the frog, in which, as we have already learned by dissection, many organs are at some distance from the digestive cavities of the stomach and intestine. Clearly, then, there must be some method of transporting digested food from the digestive organs (stomach and intestine) to all parts of the body. This is one work of the blood, which, as we have already found, flows in a set of tubes, leading to all parts of the body. The details of the way in which blood carries dissolved food from the stomach and intestine to all parts of the body will be studied in a later lesson in connection with the same process in the human body. The important point in this lesson is that the frog needs blood and a mechanism (pumping heart) for moving it in order to carry absorbed food from the stomach and intestine to all parts of the body; that is, to every living cell where it can be used as a supply of energy and as material for repair and growth. We shall later find still other important reasons why the frog needs blood.

47. Changes in Living Cells: Oxidation. - We turn now to the changes which occur in the living cells everywhere in the body of the frog. As has already been stated $(\$ 42)$, the continuous activity of the living animal causes a wearing out of the body-substance; that is to say, of the particles of cell-substance in the tissues composing the body. The wearing out changes are largely due to the chemical union of oxygen with the cell-substances, producing a kind of slow combustion or oxidation. We commonly think of combustion as the rapid burning of substances in the open air ; but essentially the same kind of change may take place slowly. For example, a piece of iron wire can be quickly burned (oxidized) 
in a jar of pure oxygen. But in the oxygen of the air, diluted as it is with nitrogen, oxidation of iron is the very slow process of "rusting." Another example: A piece of magnesium wire may be burned quickly at a high temperature $(\$ 10)$; but if we place a piece of magnesium wire in water kept at the ordinary temperature, it will slowly oxidize (turn to white powder) on the surface. Now, the slow oxidation of the magnesium wire in water, or the familiar rusting of iron, illustrates the slow chemical changes which are continually taking place in every living cell of animals and plants. We emphasize the word "continually," for oxidation of the cellsubstances is invariably associated with the activities which we call "living"; and as long as there is life in a cell, oxidation is going on and particle by particle the cell-substance and the food brought by the blood are being burned; that is, they are being combined with oxygen to form new substances.

Heat. - This slow burning (oxidation) results in heat, and this is how the human body is kept warm. In frogs and other lower animals the heat thus generated is lost rapidly, because the surface of the body is not covered with hair or other structures to prevent loss of heat, and so the frog is never perceptibly warmer than the water which touches its skin. We call it " cold-blooded," because it is usually colder than the human body.

48. Oxygen Required. - We have just learned that oxidation is a process necessary to the life of the cells in the frog's body. Oxidation requires oxygen; and therefore there must be in the cells of the frog a continual supply of that gas. In some very small aquatic animals oxygen is absorbed from the surrounding water by all the cells, and there is no need of any special organs for supplying oxygen. But in an animal as large as a frog the cells of the internal organs are so far from the external air and water that some method of distributing oxygen is required, just as it is neces- 
sary that digested food be transported to cells which are far away from the digestive organs. The distribution of oxygen is accomplished by the blood, which absorbs oxygen from the air or water external to the body and then carries it to the internal cells. When the frog is under water, oxygen (a small quantity of $\mathrm{O}$ is always mixed with or dissolved in water exposed to the air) is absorbed by the blood flowing through the blood-capillaries in the skin; but when the animal is on land, oxygen is absorbed directly from the air, partly by the blood flowing in the capillaries in the skin and also by the blood flowing in the capillaries of the lungs. In our own bodies the blood absorbs most of the necessary oxygen in the lungs. In fishes the blood flowing through the capillaries in the delicate membranes of the gills absorbs oxygen from the water just as the frog's skin absorbs some oxygen when the animal is under water.

(D) Examine specimens of frogs' lungs with injected bloodvessels, frogs' skin with injected blood-vessels, gills of a fish injected to show blood-vessels. (These may be obtained by injecting with a hypodermic syringe or sharp-pointed pipette some colored mixture, such as starch and carmine in water, into the large arteries of a frog killed with anæsthetics.)

49. Excretions. It has been stated that the cell-substance (including foods absorbed by cells) is continually being oxidized in every living cell of the frog's body. This chemical change produces a number of substances which are of no further use in the cells of the body; in fact, they would be poisonous if allowed to accumulate. These waste substances are called excretions.

We have already noted $\$ \$ 12-16$ that animal substance may be analyzed into water, gas, carbon, and mineral matters; and also that the carbon may be burned. Something similar takes place when cell-substance is oxidized in the living body, and the chief excretions produced are of four kinds; water, carbon dioxide (a gas formed by burning the carbon of 
cell-substance), mineral substances, and peculiar substances containing nitrogen and called nitrogeneous excretions. Three of these - water, carbon dioxide, and mineral matter are easily shown to be present when animal matter is heated and burned in a pipe or tube $(\S 13)$. Also it could be proved by a careful test that one of the gases given off when the meat is heated is ammonia; and this gas contains nitrogen, which is a constituent of the nitrogeneous excretions formed when cell-substance oxidizes in a living cell. It is true, then, that all the substances found when we analyze animal matter by heating and burning are also present in the excretions formed by oxidation which takes place at the temperature of the frog's body. The chief difference is that oxidation in the test-tube is rapid and at high temperature while in the frog's body it is slow and at low temperature.

50. Removal of Excretions. - The excretions formed in all the living cells of the frog's body are poisonous if allowed to accumulate. Hence they must be eliminated from the body. Kidneys, skin, and lungs are the organs of excretion or excretory organs. Dissection of the frog showed that many cells are at such a distance from these organs of excretion that the poisonous substances cannot be absorbed directly by these organs, thus making it necessary that the blood should absorb excretions from the cells, and, flowing to the excretory organs, give up these excretions to be eliminated from the body of the animal. In the frog, the carbon dioxide is carried by the blood from the cells, where it is formed, to the skin and lungs, where it is given off to the air or to water. The nitrogeneous excretions are first absorbed from the cells by the blood and then carried to the kidneys, where, along with water, these excretions are removed from the blood, passed into the ducts (ureters), and thence to the exterior. In some simple aquatic animals, the excretions are absorbed directly from the cells by the water in which the animal lives; and just as in the case of 
supplying food and oxygen to these animals ( $\$ \$ 4,48)$, there is no need of blood as a carrier of excretions.

51. Respiration. - This is a term which has long been used in physiology as almost a synonym for the popular word "breathing." It includes two of the processes which have been described; namely, taking in oxygen and giving out or excreting carbon dioxide. In most animals the two processes go on at the same time and in the same organs. Air taken into the lungs supplies oxygen to the blood and at the same time absorbs carbon dioxide from blood circulating in the lungs; and hence the lungs are often called respiratory organs. Keep in mind for use in future lessons that respiration includes (1) supplying oxygen, and (2) excreting carbon dioxide.

52. Summary of Functions of the Blood. - We have found that blood and a mechanism for its circulation are necessary in the frog for communication between the living cells and certain organs which communicate with the external world. As we have seen, all cells must have a supply of food and oxygen and must get rid of the substances (excretions) resulting from the oxidation of cell-substances. Some small animals have all their cells near the places where food and oxygen must be absorbed and excretions eliminated; and for this reason such small animals need no blood-system. But in the frog, and in all except the simplest animals, there are cells at some distance from the organs which supply oxygen and food, and also far from those which eliminate excretions; and in all such animals the blood acts as a transporting medium which (1) carries food and oxygen to the cells from the organs (lungs and digestive organs) which obtain these substances directly from the exterior, and (2) carries excretions from the cells to the organs (lungs, skin, or kidneys) which pass them out of the body. In animals higher than frogs and reptiles the blood is also important in distributing heat. 
53. Nutrition. Fundamental Processes. - We have now briefly traced food from its entrance into the frog's body through the changes of digestion, absorption, and distribution to the living cells. These cells are active living machines requiring food (1) for repairing their waste and for growth, and (2) for oxidizing to give the energy which is expended in the activities of the body. Sooner or later most of the materials entering the cells as food become combined with oxygen, and the resulting substances are excretions of no further use to the living cells.

All the changes which food and oxygen undergo, beginning with their reception into the body and ending with. their elimination in the form of excretions, involve the processes of digestion, absorption, circulation, respiration, changes within cells (metabolism), and excretion. It is important to remember that all these processes are fundamental, for one is just as necessary as another. All the processes - taking of food, digesting of food, its absorption by the blood, its transportation to the cells, its absorption by the cells, its use by the cells, the supplying of oxygen, and the removal of excretions - all these processes are linked together, as it were, in a chain, and each process must play its part in the life of the body.

54. Need of Organs Working Together: Coördination.All the organs concerned in the processes named in the paragraph above must work together, for if any organ fails in the proper performance of its work, the result is that the working of all the organs of the body is affected. For example, if the heart beats slower, the blood flows slower, and consequently the supply of food and oxygen to the cells and the removal of excretions will be lessened. We know that if the heart stops beating, or the lungs cease acting, animals die at once; and the reason is that the cells of the body fail to get their necessary food and oxygen and the poisonous excretions are allowed to accumulate. We see then how 
necessary it is that all the organs concerned in the nutrition of the body should work together or coöperate. To accomplish this, there are organs which cause the coördination (working together) of all the organs of the body. These are the nervous organs of the body - the brain, spinal cord, and nerves.

55. Nervous Organs for Coördination. - If we touch a living frog anywhere on its skin, various muscles of the body will contract, causing the animal to jump. A similar result comes from suddenly thrusting a stick before the frog's eyes, or from making a loud noise. The same thing happens when the frog sees food, such as a worm; the muscles contract so that the frog jumps and seizes the worm. These are examples of coördination between the external organs (skin, ears, and eyes) and the muscles which move the body. Likewise, whenever any change takes place in any inter-

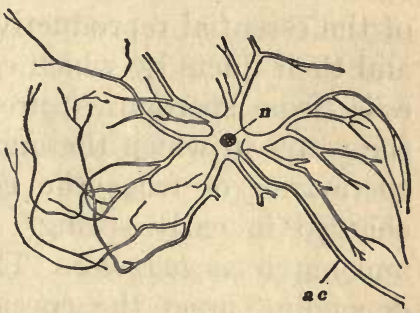

Fig. 20. A nerve-cell and its fibers. $n$, nucleus; $a c$, main fiber (axis cylinder). The other fibers are shorter and with many branches near the cell. nal organs, coördinated changes in other organs, as in the beating of the heart mentioned above, are caused by the nervous organs. The activities of the nervous organs are due to the combined working of the nerve-cells (Fig. 20) and their fibers which connect them with various organs of the body. A large part of the nervous organs consists of connective tissues surrounding the nerve-cells and their fibers.

\section{DEVELOPMENT OF THE FROG: INTRODUCTION TO EMBRYOLOGY}

E6. Reproduction. - So far in considering the work of the organs of the frog, we have given attention to the organs which are necessary for the life of the individual frog, and 
this includes all organs except the reproductive organs. The latter are necessary only for the continuance of the frog species; for, as we have already noted, individual animals of all kinds live a relatively short time. The work of the reproductive organs of the frog (and of all other organisms) is the production of new individuals; and to the general facts in this line we shall now give some attention.

57. Egg-cells and Sperm-cells. - In the study of the structure of the frog, we have noted the position and form of the essential reproductive organs (ovaries and spermaries) and their ducts by which egg-cells (from ovaries) and spermcells (from spermaries) are conducted out of the body into the water in which the animals live. From the ovaries and spermaries of frogs the egg-cells and sperm-cells are discharged in early spring. The eggs develop in the water, and hatch as tadpoles. Then, after a few weeks or many, depending upon the species, they develop legs, lose their tails by a process of absorption, and become small frogs. This change from tadpoles into frogs is called metamorphosis.

The ovaries in a young frog are masses of tissue with numerous small egg-cells. Each egg-cell is a spherical mass of protoplasm with a nucleus near its center. As the eggs grow larger, each one accumulates granules of a material known as yolk; and after a time the yolk comes to occupy one hemisphere of the egg, while the protoplasm is concentrated in the other. Frogs' eggs examined soon after they are laid in water are seen to be black (with pigment) in one hemisphere and whitish (due to yolk) in the other. The black hemisphere contains most of the protoplasm. Each egg is surrounded by an envelope of transparent jelly, which was secreted by the cells of the oviduct as the egg passed from the ovaries to the exterior.

The sperm-cells have the form shown in Fig. 21, A. The thickened part is chiefly nucleus. The tails of living sperm- 
cells vibrate rapidly and enable the cells to swim in the water into which they are discharged.

58. Fertilization. - Before an egg-cell can develop into a tadpole it must be entered by a sperm-cell. Soon after the egg-cells of a frog are discharged into the water they are approached by the swimming sperm-cells; and each egg is entered by a sperm-cell. The sperm-cell then becomes a second nucleus, called sperm-nucleus. This nucleus ap-
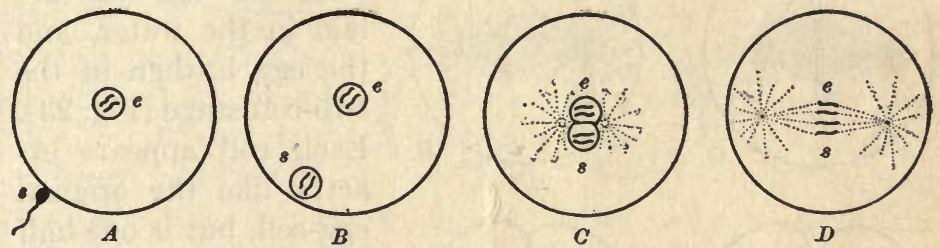

Fig. 21. Diagrams to illustrate fertilization of an egg-cell by a sperm-cell. $A, e$, nucleus of matured egg-cell; $s$, a sperm-cell ready to enter. $B$, sperm-cell entered and transformed into sperm-nucleus $(s)$. $C$, spermnucleus and egg-nucleus united, fertilization complete. $D$, division leading to two-cell stage (compare with Fig. 22, C). The nuclei are represented as having only two chromosomes; but those of most sperm- and egg-cells have more.

proaches that of the egg-cell, and the two nuclei unite. When this has occurred the egg-cell is said to be fertilized, and the entering of a sperm-cell and its union with the nucleus of the egg-cell is called fertilization (see Fig. 21).

59. Division of the Egg-Cell. - As soon as the egg-cell is fertilized, it prepares for division into two cells. Delicate fibers appear and form a spindle-shaped structure with a starlike structure (aster) at either end (Fig. 22). In the center of this spindle are a number of small bodies known as chromosomes. These are masses of granules such as may be seen in any stained nucleus (Fig. 11). Within a few minutes the changes shown in Figs. A, B, C, D occur. These consist first of a division of the chromosomes (Figs. C, D), then a division of the cell-body, and finally the formation of a 

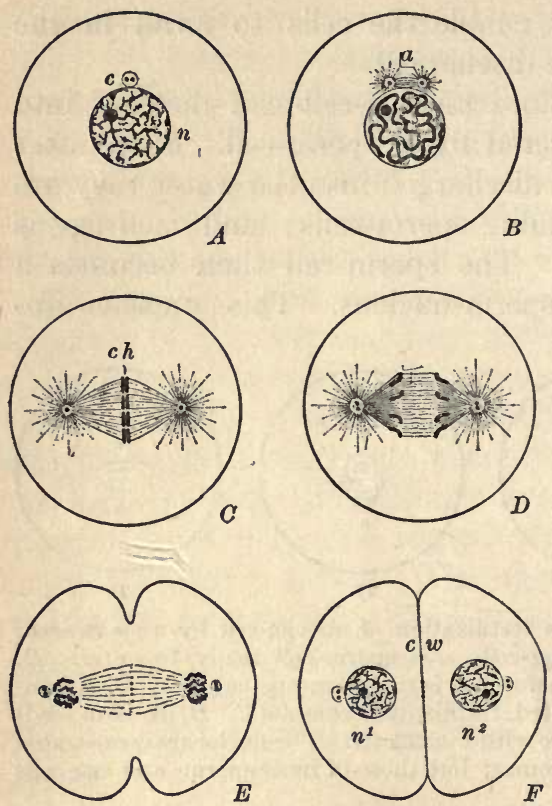

Frg. 22. Diagrams illustrating division of a cell. $A$, resting cell with nucleus $(n)$ and centrosome $(c)$. $B$, preparing to divide, two asters (a) near nucleus, each with a centrosome, chromatin becoming massed into chromosomes. $C$, two asters have formed a spindle with chromosomes $(c h)$ in center. $D$, each chromosome divided and two halves being moved toward the asters. $E$, chromosomes forming the two new nuclei, and cell-body beginning to divide. $F$, division complete, two-cell stage, each cell has the same structure as the one cell in $A$. $c w$, new cell-wall. Compare with cells shown as dividing in Figs. 40, 41 . (From Wilson.)

approximate $64,128,256$ and more cells, multiplying by two when all the cells have divided. Usually within two or three days the egg has reached a stage consisting of a spherical

new nucleus containing the chromosomes in each of the two cells formed by the division.

In warm spring weather the first division is usually completed within two hours after the egg-cells are laid in the water, and the egg is then in the two-cell stage (Fig. 23). Each cell appears exactly like the original egg-cell, but is one half the size. In another hour each cell of the two-cell stage will have gone through the same processes of division, and the four-cell stage will be reached.

Such divisions of cells occur about every hour; and so, in succession, stages of two, four, eight, sixteen, and thirty-two cells are formed (Fig. 23). Later some cells may get slower in division, and hence the stages only 
mass of many hundred cells ; and it is then ready to arrange the cells so as to form the body of a tadpole.

60. Later Development. - The changes in external form of developing frogs' eggs should be observed, either in eggs preserved in formalin-solution, or in living eggs (only in early spring) kept in aquaria (e.g., fruit-jars) and examined daily during development. In brief, the changes are as follows: The spherical mass of cells formed by the numerous
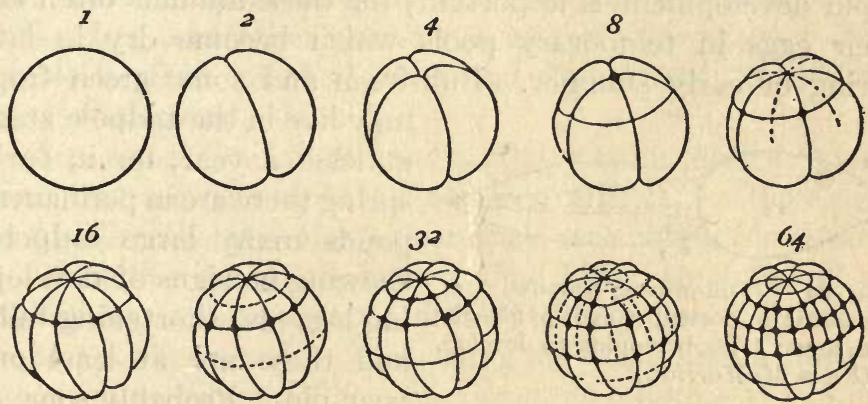

Frg. 23. Stages in division or cleavage of frog's egg. The figures indicate the number of cells. (From Thomson, after Ecker.)

divisions of the egg-cell becomes elongated, the various organs are formed by complicated changes which cannot be described in the limited space of this book, and gradually the embryo becomes a tadpole. Finally, the tadpole hatches, that is, it breaks through the jelly that has surrounded the egg throughout the development.

The tadpole at hatching appears larger than a fertilized frog egg; but if dried would not weigh more, for no food can be taken until after hatching. The larger size of the tadpole as compared with the egg is due to water absorbed during development. !

The time necessary for a fertilized egg-cell to develop into a free-swimming tadpole varies with the temperature of the water. Also, the eggs of some species develop faster than 
others; toads' eggs will hatch within three or four days after being laid and those of wood-frogs develop almost as rapidly.

61. Metamorphosis. - The time necessary for a tadpole to become full grown (Fig. 24) and to metamorphose into a toad or a frog varies with species, and with food and temperature of water. Toad and wood-frog tadpoles may metamorphose within one or two months after hatching. Such rapid development is important, for these animals often lay their eggs in temporary pools which become dry in late spring or early summer. Bull-frogs and some green frogs

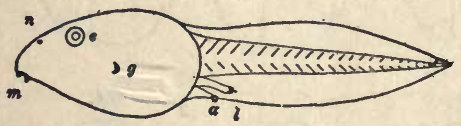

Fig. 24. Full-grown tadpole. $m$, mouth; $n$, nostril; $e$, eye; $g$, gill-slit; $a$, anus; $l$, leg beginning to develop. (From McMurrich.) may live in the tadpole stage at least a year, for in early spring there are in permanent ponds many large tadpoles showing no signs of developing legs and shortening tails, and these are at least one year old. Probably some of these will become frogs in the second summer when somewhat over one year old, while others may live still another year in the tadpole stage.

The nature of the metamorphosis of a tadpole into a frog is popularly misunderstood. The tadpoles are said to "lose their tails," and this is often taken to mean that the tails drop off. This is not true. In collecting tadpoles in ponds one often finds specimens with short stubby tails and welldeveloped legs, and others showing various intermediate stages between these and tadpoles with long tails and no legs. The explanation of these different conditions is that after the legs develop the tail is absorbed. First, the tissues inside the end of the tail are disintegrated by the white blood-cells, which "eat" the particles of tissue and carry them back into the body. In a short time after this process starts, the tip of the tail appears withered. The white 
blood-cells continue this process of disintegration and removal of tissues until the tail has entirely disappeared. Thus instead of the tail dropping off, as is commonly believed, its substance is gradually carried back into the body by internal processes of absorption, and the materials are used in building other tissues.

62. Embryonic and Larval Development. - The development of any fertilized egg to the hatching stage is called embryonic development; and the scientific study of such stages of the frog and other animals is animal embryology. The tadpole is often called a larva, and its changes constitute larval development.

All species of backboned animals have embryonic development; but a large number do not have a larva, for their eggs develop directly into minature animals resembling the adults. For example, it is well known that birds are hatched as small birds, and common mammals at birth show the characteristics of their species. All young animals which at hatching are quite unlike their parents (e.g., as tadpoles are unlike frogs, and caterpillars are unlike butterflies) are said to have a larval development.

63. Oviparous and Viviparous Development. - All such animals as frogs, birds, turtles, fishes, etc., which lay eggs that develop outside the body of the animal that produced them, are called oviparous. Such eggs are usually protected by special coverings or shells. We shall see later that such oviparous development is very common in the animal kingdom.

In many species of animals eggs are retained in the oviducts or other specialized cavities until embryonic development is completed, and the young animals are "born alive" ; by which we mean that as organisms ready for an independent existence they are expelled from the cavity in which embryonic development occurred. Such internal development is viviparous. It occurs in all mammals, except the duck- 
bills of Australia; in some snakes; in some salamanders; in some fishes; in some insects ; and in other kinds of lower animals.

There is a great advantage in viviparous over oviparous development in the protection afforded the eggs and embryos. Hence relatively few eggs need be produced. It is well known that many fishes and other oviparous animals produce an astounding number of eggs, and that vast numbers of the eggs and young are destroyed. Sharks illustrate the advantage of viviparous development for fishes. Few eggs are formed, and these are retained in the oviducts, not only until the young animals are fully formed, but for, many months, until they have grown to be several inches long. They are then born (expelled by muscular contraction of the oviducts), and are well able to shift for themselves. Thus a few sharks' eggs well protected during development will perpetuate the species as successfully as would hundreds of eggs forced to develop oviparously and exposed to attacks of numerous enemies.

A similar case is found among salamanders, which are near relatives of the frogs and toads. Our common salamanders lay each spring large masses of eggs. In a species which lives in the mountains in Europe, each female forms but two eggs in a year, and these are retained in the oviducts until developed into young salamanders ready to care for themselves.

RELATION OF FROG TO OTHER ANIMALS: CLASSIFICATION

64. Classification of the Frog. - By this phrase is meant the position of the frog in the scientific list of the animals of the animal kingdom. In a dictionary or encyclopedia we find frogs placed according to alphabetical order, but in a book of zoölogy they are grouped with the other animals 
which are very similar in structure. This similarity is believed to indicate close relationship, and hence the animals which are most like frogs are said to be relatives or allies.

The nearest allies of frogs (that is, the animals most like them) are the toads. Next nearest are the animals known as newts and salamanders. Toads, frogs, newts, salamanders, and similar animals together constitute a group of animals which have closer resemblances to each other than to any fishes, reptiles, or other backboned animals. To this group the term Amphibia is applied; and we may speak of frogs and salamanders as amphibians, just as we say that salmon and perch are fishes.

The various kinds of common frogs are known by double scientific names, such as Rana catesbiana (bull-frog), Rana sylvatica (brown wood-frog), Rana virescens (the leopard frog), and so on for many other kinds. The common American toad is scientifically known as Bufo Americanus, while certain little tree-toads are specimens of Hyla versicolor.

The system employed in giving scientific names to animals and plants will be explained in Chapter VII.

The Life-Activities of a Plant. - Having now studied in considerable detail the structure and life-activities of an animal which illustrates in a general way the life of all animals, it will be interesting to make a parallel study of a plant in order to see how it performs the functions of moving, breathing, using food, reproducing, etc., - life-activities which have been pointed out in $\$ 29$ as occurring in both animals and plants. Such a study is presented in the next chapter. 


\section{CHAPTER V}

\section{STRUCTURE AND LIFE OF A PLANT: AN INTRO- DUCTION TO PLANT BIOLOGY*}

65. Introductory Study of a Plant. - The lesson on "Lifeactivities of a Plant" ( $\$ \$ 24-28)$ has already called our attention to various processes which are characteristic of living plants. They move, use food, breathe, reproduce, and perform other functions much as do animals. In order to understand how plants are able to carry on these life-processes we shall now enter upon a careful study of a plant selected because it illustrates the structure and work of other plants. We shall see later that much of what is learned from the study of one plant applies to all plants, and that a knowledge of plants helps us to understand many things in the somewhat similar life-processes of animals.

The word "plant" usually calls to mind familiar trees, garden vegetables, and ornamental plants ("flowers"). In fact, most of the plants known to those who have not studied botany belong to the highest groups, which are often called the flowering plants. But the kingdom of plants is not limited to the flowering plants; $\dagger$ for in addition it includes a vast variety of forms known in popular science as flowerless plants. Examples of these latter which will be studied in this book are ferns, mosses, toadstools and mushrooms, sea-weeds, molds, yeasts, and bacteria. Some of these are of micro-

* To the Teacher: This chapter may be studied before the preceding chapter on the frog, if there are reasons for beginning with plant study. See note in "Teachers' Manual," Chapter V.

† It is pointed out later that seed-plants is a better name for the highest group of plants. 
scopic size, and all are in general appearance quite different from the familiar flowering plants; but in many important respects there is much similarity. It is therefore possible by a careful study of some common flowering plant to learn many interesting and useful facts which are common to plants in general.

The common bean plant has been selected for this beginning study, because it can be conveniently grown from seed planted in gardens or in boxes in the schoolroom, and because the seeds themselves are large and easy to study. Many other plants might have been selected for these lessons; in fact, the following account of the structure and life of the bean plant will serve as a general guide for the study of any flowering plants, provided the student has specimens of the plants and uses this book chiefly as a guide to examining the actual plants.

\section{STRUCTURE OF A BEAN PLANT *}

66. Organs of the Plant. - If we examine a fully developed bean plant, we notice first that it has several parts or organs. These are roots, stem with its branches, buds, leaves, flowers, and sometimes fruits with seeds. We call these organs, because an organ of a plant or of an animal is a structure for doing a particular work; for example, lungs are organs for breathing. The roots, stem, leaves, and flowers are the plant's organs for taking food, for breathing, for reproducing - in short, for carrying on all the life-activities which in an earlier lesson $(\$ 29)$ we found to be characteristic of all living things. How can the organs of the plant perform

* The most prominent part of the subject-matter of this division is a description of structure; but, as in the case of the frog, some general reference to the work of the various organs has been coupled with the first study of their structure. A later division of this chapter presents a more careful survey of the life-activities of a plant in a way similar to the lessons on the work of the organs of the frog in Chapter IV. 
these functions? In order to answer such a question, even in part, it is necessary that we study first the structure of the organs of a plant. For this reason we will examine the roots, stem, buds, leaves, flowers, and fruit of the bean plant.

\section{The Bean Roots}

67. General Structur of Roots. - $(L)$ Carefully dig up a young bean plant, and note. how firmly the roots anchor it in the soil, and how the particles of soil cling to the small rootlets. Wash by dipping into water. Note that there is no definite boundary between roots and stem. The small rootlets appear to be attached to the blunt lower end of the stem and also to a central root which seems to be the downward continuation of the stem.

Some of the stem is below ground. That all the parts of the plant below the surface of the soil do not belong to the roots, as is popularly believed, is evident if we compare the roots of a bean plant grown from a seed planted four inches deep with those on a plant from a seed placed one inch deep in the soil. In both plants the roots are grouped at about the same place, and the deeper planted one has more than three inches of stem below the surface of the soil, but at first showing no roots. After growing six or eight weeks, other roots usually start from the stem nearer the soil surface. It is well known that stems of many kinds of plants will form roots if they are kept in contact with moist soil.

Usually the main root is called primary root, and the branches secondary roots.

On roots of some bean plants there may be seen small spherical bodies, some of them perhaps as large as one eighth of an inch in diameter (Fig. 25). These are root-tubercles. In the later lessons on bacteria (a kind of microscopic plants), these tubercles will be referred to as caused by bacteria and useful as makers of a peculiar kind of plant food supplementary to that furnished by manures or fertilizers in the soil. 
In addition to the structures already noted, there are on the roots many delicate root-hairs (Fig. 26). Their great importance in absorbing water will be explained later. They are usually so firmly attached to soil particles that they are broken when the plant is lifted from the soil. It is well known to gardeners that it is easy to transplant many plants if one carefully lifts a mass of soil so as not to disturb that near the roots and thus break the delicate root-hairs.

$(L)$ Examine a bean root and note that a layer of rind or bark (cortex, or cortical layer) is easily scraped or stripped from the central cylinder of wood. Test the strength of this central cylinder of a small root by pulling and by bending. How are strong roots of

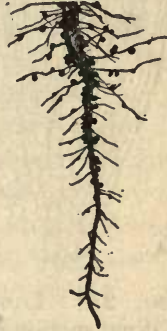

FIG. 25. Roottubercles. advantage to the plant? Tear a root lengthwise and note the fibers which make it strong.

68. Microscopic Examination of Roots. - (D) Mount on an object-slide, with cover-glass, some small roots from Tradescantia

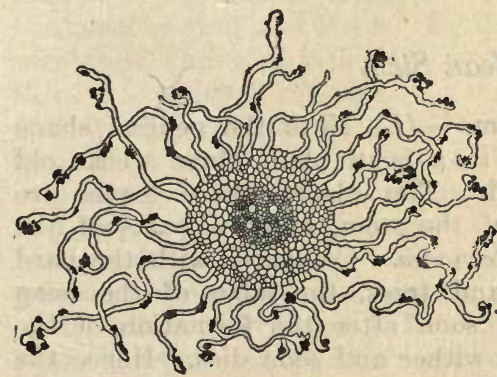

Fig. 26. Cross section of root, showing central woody cylinder; rind or cortex; root-hairs with particles of adhering soil. (After Frank.) stems kept in water, or from seedlings of bean, radish, clover, oats, or barley, grown on moist blotting-paper ( $\$ 81)$. Using first the low power, note (1) a cap-like structure on the tip of the root (Fig. 27), (2) a somewhat opaque central eylinder, (3) a transparent rind outside, (4) the outermost layer of the rind is the very transparent epidermis.

Cells. - Now, using the high power of the microscope, notice that the root is composed of box-like structures, the cells (Fig. 27). Are they all the same shape? Many of the cells contain living matter (protoplasm), but some appear empty. (D) If some iodine-eosin (eosin dissolved in a solution of iodine) be drawn under the cover-glass by means of absorbing-paper touched to the edge of the glass opposite a drop of the stain, the protoplasm in the cells will 
become stained, and in each cell a small rounded body will stain brighter than the surrounding protoplasm. This body is the nucleus,

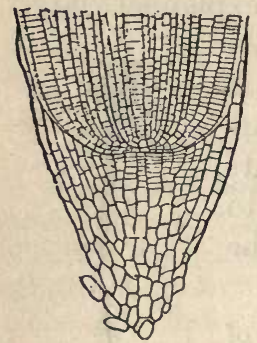

Fig. 27. Longitudinal section of tip of barley root, showing root-cap which covers the rounded end of the root.

a very important part of the living matter of the cell. Each cell has a cell-wall, which is the hard substance of which wood is composed. Some cells appear to have only a cell-wall and to be filled with water or air. Such cells are older and have lost the living matter which originally was contained within their cell-walls.

The above study of a root calls attention to the fact that plants, like animals ( $\$ 39)$, are composed of units called cells, and that the essential living substance in cells is the protoplasm. Plant cells multiply by division similar to that shown for animal cells in Fig. 22. Epidermis stripped from a stem of Tradescantia, and from the leaves of an onion bulb, should be examined and compared with the epidermis of a frog (§39). See also Figs. 40 and 41.

\section{The Bean Stem}

69. General Structure of Stem. - $(L)$ Note the general shape of the stem and its branches in a bean plant three weeks old and in another six weeks old. The places where leaves are attached are joints or nodes of the stem, and the parts of the stem between the nodes are internodes. Compared with the hard and woody stems of shrubs and trees, the stem of the bean plant is soft (herbaceous), and soon after the formation of the pods (fruit) the plant begins to wither and soon dies. Hence the duration of life of a bean plant is one growing season; and such a plant which does not naturally live over winter is an annual. Name five other annuals.

With a sharp knife, cut into a piece of a bean stem from a fullgrown plant and then peel off the soft rind (also sometimes called cortex or cortical layer). Beneath the rind lies the hard wood (xylem), which constitutes the central axis of the stem. Between the rind and the wood is a layer of very soft slippery substance which makes it easy to separate rind from the wood. In early spring 
when the stems of trees are growing rapidly and this layer is soft, boys make whistles at the end of willow sticks by pounding the bark until it is loosened from the wood so that as a ring it can be slipped off, and then certain notches are cut in the wood. Also, the same soft substance between the bark and wood of one species of elm trees is the delicious "slippery-elm bark," which in a dry and powdered form is sold by druggists for alleviating irritations of the throat.

In the center of the soft substance between bark and wood of such stems is a layer of cells which are active in growth and division. This is the growing layer or cambium. On its inner side new wood cells are formed and added to the older wood of the stem, and on its outer side the new cells formed are added to the inner surface of the bark. Hence growth and division of cambium cells adds new cells to both wood and bark, resulting in increased diameter of the stem.

On the outside of the rind of the bean stem is a thin layer of cells, the epidermis. Some cells of the rind are green in color, which is due to a substance common in leaves and known as leaf-green or chlorophyll.

With a sharp knife or section-razor, make a cross cut (transverse section) of the stem. Examine with hand-lens, and note relative thickness of rind and wood. In the center of the wood is a softer substance, known as pith; and in the oldest parts of the bean stem there is a cavity in the pith.

Split open lengthwise (longitudinal section) a stem from an old bean plant, and note the extent of the pith and of the central or pith-cavity. Tear the wood apart lengthwise, and note that it is "stringy." Pay special attention to the arrangement of the wood, bark, and pith in a longitudinal section at a branch. Make diagrams showing position of rind, cambium, wood, and pith.

70. Microscopic Study of Bean Stem. - $(D)$ With a razor or very sharp knife cut a very thin transverse slice or section of the stem, mount in water on a glass object-slide. Examine with low power first. Examine the rind and its outermost layer (epidermis), the wood, and the pith. These are examples of the tissues or building materials of the plant. Use a higher power of the microscope, and note that each of these tissues resembles a piece of honeycomb or hollow blocks set together like bricks in a wall. These are the plant cells. Some of the substance in the cells is the living matter or protoplasm, but some cells in the central part of the stem appear to be empty. The fact is that such empty cells are dead, and only their 
hard cell-walls remain. Examine a stained section (permanent preparation, or stained as suggested in $\S 68$. In some of the cells darkly stained bodies (nuclei) may be seen. These also are composed of living matter, and as far as is known every living plant cell has a nucleus.

Carefully compare the section as seen with the microscope and Fig. 48. Locate the thin-walled sieve-tubes in the inner layer of the rind, and the surrounding bast-fibers. These tubes and fibers make the rind "stringy." The function of the sieve-tubes is to conduct fluids down the stem ( $\$ 103)$.

Note in the wood of the stem certain groups of large empty cells. These are really groups or bundles of tubes (wood-tubes) and strong fibers (wood fibers) extending up and down the stem. These are the bundles of tubes and fibers which make the wood "stringy," as already noted.

The tubes and fibers of the rind are separated from those of the wood by the cambium (Fig. 48). All the tubes and fibers taken together constitute the fibro-vascular bundles, meaning bundles of fibers and tubes. They will be studied more carefully in other kinds of stems later.

The work or function of the fibers of the wood-bundles may be discovered by bending the stem to test its rigidity and by pulling to test its strength. The function of the wood-tubes is shown by the following experiment :

( $D$ or $L$ ) Cut off a young bean stem and stand it in a bottle with red ink (eosin solution with water). After a half-hour, carefully strip off the bark in various places in order to see the red color in the woody part of the stem. Also cut across the stem in various places. It is obvious that liquids go up the stem in the wood-tubes.

\section{The Bean Buds}

71. Position and Kinds of Buds. - At various places on a bean plant are buds, which will unfold later. Some of these buds will form flowers, and hence are called flower-buds; others will unfold as leaves and are therefore leaf-buds. At the ends of the main stem and its branches are terminal buds which, by growth, lengthen the stem and branches. If the terminal bud of the main or a branch stem be destroyed, lengthening of the stem will cease. For this reason gardeners 
often break off the terminal buds of climbing or pole beans (e.g., limas) when they have reached a height of about six feet. This has the effect of sending into the developing fruit (pods) much of the nutriment which would have gone to make the useless extension of the stem.

(L) Examine buds on a young bean plant, (1) with reference to their position, and (2) separate carefully the parts of both a leafand a flower-bud.

\section{The Bean Leaves}

72. Leaf and Leaflets of Bean Plant. $-(L)$ Brief examination of these will show that what we at first take to be the individual leaves are arranged in groups of three (Fig. 28). A study of the development of the bean has led botanists to the conclusion that the three leaves are really three parts or divisions of one leaf. Such a leaf is called compound, and the three divisions are called leaflets. The supporting leaf-stalk (petiole) is attached at a node of the stem and can be easily distinguished from a branch of the stem, because the leaf-stalk has an enlarged and somewhat flexible attachment to
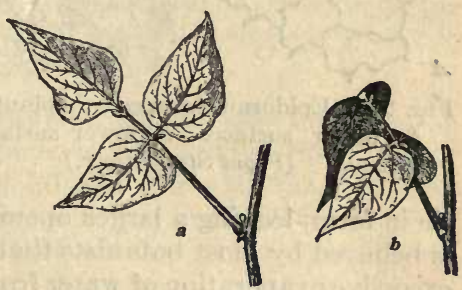

FIG. 28. $a$, leaf of bean, with three leaflets on petiole; $b$, position at night. (From Detmer.)

the stem; and also because a longitudinal cut through the node shows that the leaf is attached chiefly to the rind, while the branch is attached firmly to the wood of the stem. Each of the leaflets is attached by a thickened joint similar to that of the petiole at the stem. These flexible joints of the leaf and leaflets allow the drooping of the leaves at night and on hot days.

Examine any one of the leaflets, and note that the expanded surface (blade) is a thin sheet of tissues supported by ribs and veins. The central rib is the mid-rib. Hold the leaflet up so that light may shine through it, and with a hand-lens examine the delicate veins (veinlets) between the ribs. Make an outline sketch showing the structure of a complete leaf and the detailed structure of one of the three leaflets; and label all the parts named above. 
73. Microscopic Structure of Bean Leaf. - (D) With fine-pointed forceps strip off a very small piece of the thin transparent epidermis from both the upper and lower surfaces of a leaf, and mount for microscopic study. This will present surface views of the leaf. With low power, note the irregular cells of which the epidermis is composed (compare with Fig. 29, which is from other leaves).

Scattered among these irregular cells are pairs of crescent-shaped cells set together so as to leave a small pore or opening through the epidermis. Each pore is a leaf-pore or stoma (a Greek word meaning an opening, plural stomata). It opens into a small cavity (air-
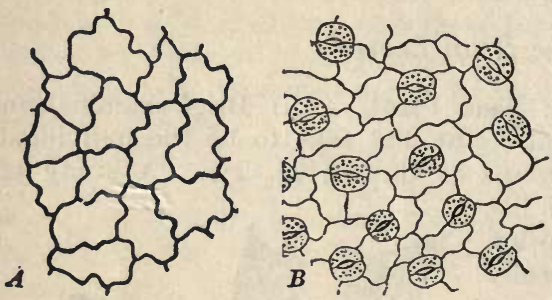

Fig. 29. Epidermis from certain plant leaves. $A$, upper surface. $B$, lower surface with stomata. (From Strasburger.) space) beneath the epidermis. Are the leaf-pores present and equally abundant on both upper and lower sides of the beanleat?

These two crescentshaped cells around the pore are called guard-cells. It has been noticed that when water is abundant in the plant these cells swell and become more crescentic in form, leaving a larger opening than when the leaf is drier. It is believed by most botanists that the guard-cells are able to prevent excessive evaporation of water from the air-spaces, and thus conserve water when necessary.

Make a drawing of a group of cells from the epidermis, including at least one pair of guard-cells.

Cut off a leaf transversely, and with a strong hand-lens examine the cut end. Note the transparent epidermis which covers both sides and the edges; that is, entirely surrounds the leaf. Between the upper and lower epidermis the center of the leaf appears to be filled with a green-colored, somewhat granular material (middletissue or mesophyll). Also notice the cut ends of the colorless veins.

If the hand-lens is a strong magnifier, it is possible to see that the green-colored middle-tissue is more compact toward the upper surface and appears to have small cavities in the part next the lower epidermis. This will be very clearly seen when the compound miscroscope is used for examining the cut end of a leaf.

(D) Examine with a miscroscope a very thin transverse section from the end of a leaf cut like the one described above. [To cut 
such a section hold the leaf between two pieces of elder-pith, or slices of potato, or roll the leaf to form a rather tight cylinder. Keep the edge wet with water and with a very sharp razor shave off a number of very thin slices. Float these off the razor into water and with

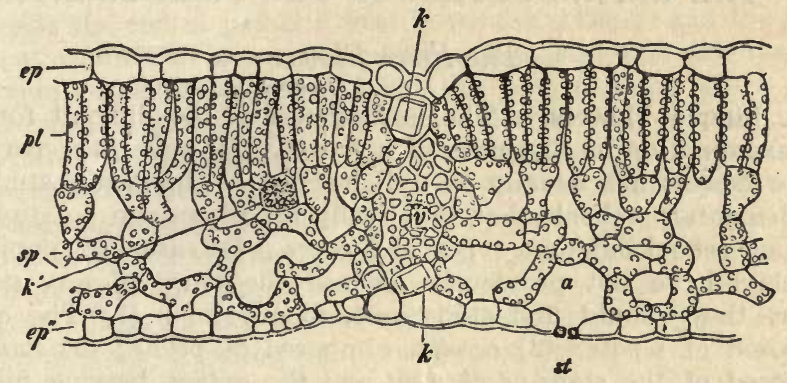

Fig. 30. Cross section of privet leaf. $e p$, epidermis; $p l$, palisade cells in upper part of middle tissue; $k$, deposited crystals; $a$, air-spaces; $v$, vascular mid-rib; st, a stoma. (From Strasburger.)

fine forceps or a small brush transfer these sections to a drop of water on a glass-slide and place the cover-glass in position.] Compare a transverse section with Fig. 30 and locate all the structures in the cut-end of the leaf that was seen with the hand-lens. Note that the epidermis is transparent and without green color, except in the cells (the guard-cells) at the leafpores. Compare with the epidermis which you previously stripped off and examined in surface view.

Most of the cells in the middle-tissue have green bodies (the chlorophyll-bodies, or chloroplasts). The compact upper part of this middle-tissue is seen to be composed of elongated cells (palisade cells) set closely together. In the lower part of the leaf the cells are irregular in shape and there are numerous air-spaces. Some of these spaces communicate with the outside through the leaf-pores, and thus air may enter the leaf and become widely distrib-

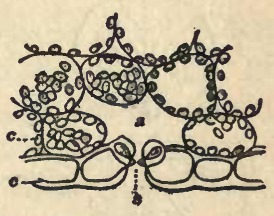

Frg. $30 a$. Magnified view of cells around a stoma $(s)$. a, airspace; $c$, cells with chlorophyll; lower cells (e) epidermis. (From MacDougal.) uted throughout the air-spaces.

Bundles of small transparent cells in the middle-tissue are the cut ends of veins, and the empty cells in them are tubes for earrying 
water. The significance of these details of structure will be made clear in the next part of this chapter, in which the work of the leaf is described.

\section{THE REPRODUCTION OF THE BEAN PLANT}

\section{The Bean Flower}

74. Simple Flowers. - The bean flower is too difficult for the first examination of the parts of a flower. However, the parts of simple flowers are usually so well learned in the nature-study of the elementary schools that it is really an advantage to study in the high school different types of flowers. In case any pupils in the class have not previously learned the parts of any simple flower, they should first study one, identifying: (1) The calyx, composed of sepals; (2) corolla, composed of petals; (3) stamens, composed of the stalk or filament and the anther, bearing minute grains of pollen; (4) in the center of the flowers the pistil, composed of the ovary, and the style, which extends upward and has the stigma at its end.

Flowers which have separate petals (scillas, tulips, sedum, lilies, etc.) are good for such preliminary study of the parts of simple flowers.

75. Study of Bean Flower. - $(L)$ Bean plants six to ten weeks old will furnish all the stages needed for this study. Note how the flowerstalks (pedicels) are attached at the nodes of the stem and often in the axils of the leaf; that is, in the angle between leaf and stem. Identify: (1) two green leaf-like structures (bracts) at the base of the flower. On very young flower buds on the same plant these bracts may be seen inclosing the flower. (2) Between the bracts and the corolla is the calyx, composed of five sepals united into a cup. This can be seen best in an old flower from which the corolla is ready to fall. (3) The petals (white, pink, or red) of the corolla, five in number and unequal in size, are arranged as in the diagram in Fig. 31. The three largest petals are so prominent that at first sight the flower appears to have only three; but two smaller petals are united and coiled so as to lie between the three largest petals. The largest petal of a flower like 
that of the bean plant is called the "standard"; the two petals at the sides of the flower are called the "wings"; and the united and twisted petals form the "keel," which lies between the "wings." In a bud just about to open note how the largest petal ("standard") incloses the others.

Inside the coil of petals ("keel") are ten stamens and the pistil. Look at a flower in its natural position on a plant and note that the two side petals (the "wings") are the only ones on which a bee could easily alight. If you have opportunity, watch bees visiting bean flowers in the garden. Hold a flower by its stalk in the natural position, and with a pencil press downward on the two side petals, and carefully watch the end of the coiled petals ("keel ") as you press. Note how the stigma and part of the style appear when the side petals are pressed, and disappear within the coiled petals when released. Imagine how a bee could cause the same exposure of the pistil when alighting on these petals. Hold these side petals down so as to keep the style protruded and with a hand-lens examine the stigma, and also notice rows of hairs along the style.

Now, carefully uncoil the twisted petals and note how the style and the ten stamens are inclosed by the coiled petals. The filaments of nine of the stamens are joined together at their base. The stamens are firmly fastened in place, but the style is not attached to the spiral tube formed by the twisted petals. The stigma does not touch the anthers, but the hairs just below the stigma brush over the anthers, and some pollen-grains cling to them.

The meaning of this remarkable apparatus is this: When a bee alights on a bean flower the stigma and the upper part of the style is pressed out, as we have seen, and pollen is brushed on the bee's body by the hairs on the style. Then the bee goes to another flower, and when its style touches the bee's body the stigma will touch some pollen from the first flower; and at the same time the hairs of the style will brush on to the bee some pollen from the second flower. And so, as the bee goes from flower to flower, it will brush pollen-dust on stigmas and get pollen-dust brushed out from the anthers by the hairs on the styles below the stigmas. There is very little chance that the stigma of a flower will get pollendust from anthers in the same flower. This may sometimes 
happen when a bee leaves a flower and at once goes back to it, carrying pollen-dust received on its first visit.

It seems probable from the arrangement of the bean flower that insect visits are necessary to distribute the pollendust of this kind of flower. Darwin, the famous English biologist, made many experiments by keeping beans covered with netting so that insects could not reach the flowers, and the result was that seeds rarely formed. Pea flowers are very similar to those of the bean, but the stigma is so near the anthers that it often gets pollen-dust before the flower is visited by insects. Botanists call such a flower self-pollinated. The bean, then, is not often self-pollinated, but cross-pollination (meaning pollen from other flowers) usually occurs.

We now see the significance of the peculiar irregular arrangement of the petals of the bean flower: the three big petals are arranged so as to make bees or similar insects alight in a certain way; and the other petals form a protective covering for the stamens and pistil, and at the same time are a curious mechanism for preventing pollen-dust from reaching the stigma of the same flower, thus insuring cross-pollination.

Many plants closely related to the bean - clover, locust, pea, wistaria, peanut, are common examples - have similar flowers. If opportunity offers, observe insects visiting such flowers. There are many other types of flowers arranged or adapted for the transfer of pollen-dust by insects, and some of these will be described in the lesson on flowers ( $\$ 192)$.

Ovules. - $(D)$ Carefully remove the corolla and thus expose the pistil of a bean flower. Hold up to the light and notice a row of opaque spots in the ovary. Then with a sharp knife or a razor split the ovary lengthwise. A low-power miscroscope or a handlens will make clear that the opaque spots are "seeds." In this early stage, however, the term ovule should be applied to each one of these structures which later grows into a seed. Inside each ovule an embryo develops, and later when the seed sprouts the embryo grows into a new plant. 
Pollen-Grains. ( $D$ or $L$ ) Examine some pollen-grains mounted in a drop of water, using first low power and then high power of microscope.

Fertilization. - When pollen-grains have been lodged on the stigma of a bean flower they soon swell, and each one sends out a delicate tube, which grows down through the tissues of the style and into an ovule in the ovary (Fig. 32). There the pollen-tube meets a cell known as the egg-cell. Then a mass of protoplasm (really a cell) passes from the end of the pollen-tube into the eggcell. The union of the two cells is fertilization. Soon the fertilized egg-cell divides into many cells, and these form an embryo within the

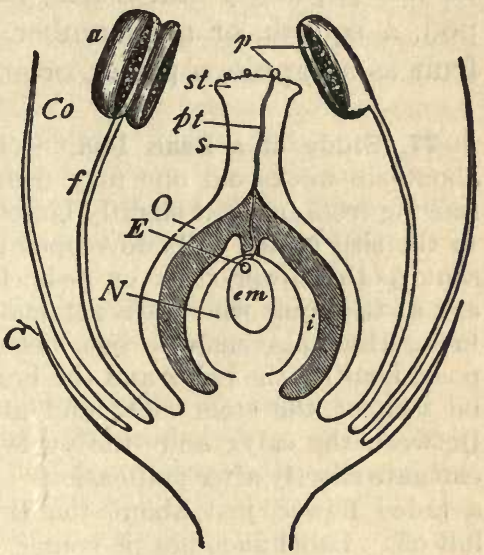

FIG. 32. Diagram of a flower. $C$, calyx; $c o$, corolla; $a$, anther on filament $(f)$; $p$, pollen-grains; $s t$, stigma; $p t$, pollentube; $s$, style; $O$, ovary; $e m$, egg-cell in ovule; $c$, fertilizing cell. (From Bessey.)

ovule, which grows into a seed. This process of fertilization in plants will be described more in detail in the section on the reproduction of flowering plants in the next chapter.

Pea Flower. - $(L)$ If material is available, make a brief examination of the flower of a pea, preferably of a sweet pea, in order to see better some parts which are larger than in the bean flowers.

\section{The Bean Pod or Fruit}

76. Fruits. - After the stigma of the bean flower has been pollinated by insects, the ovary of the pistil soon begins to develop into the pod containing the seeds. Botanists call the pod with its seeds a fruit, and apply this name to the 
structures containing seeds which develop from flowers. Hence many things are known in biology as fruits which we do not popularly call fruits; for instance, a tomato, a bean pod, a squash, or a cucumber is in botany just as much a fruit as an apple, a peach, or an orange.

77. Study of a Bean Pod. - $(L)$ On a full-grown bean plant about six weeks old one may usually find pods of various sizes, ranging from one but slightly larger than the pistil of the flower up to the size of the fully developed pod. The stalk of the pod is the same as the flower-stalk or pedicel of the flower, and its expanded end at the point where it is attached to the pod (fruit) is the receptacle, which also may be seen beneath the flower. In the younger pods, identify the calyx and the bracts of the flower, which can still be seen at the stem end; and at the other end find the style. Between the calyx and the style is the ovary, which begins to elongate shortly after pollination. If possible, examine the ovary in a faded flower, just about the time that the corolla is ready to fall off. Label sketches of young pods so as to show what parts of the flower develop into the fruit.

Study a full-grown bean pod (use green pods, known as "stringbeans"). Sketch and label, naming the parts by comparing with younger pods. The pointed end is the base of the style, most of which, with the stigma, was pulled off by the falling corolla. Note that the pod is composed of two similar valves fastened together along the edges, which are ealled sutures (meaning seams). The bean pod is bilaterally symmetrical. The position of the style and the concave curvature of the pod mark the edge or suture where the seeds are attached. This is called a ventral suture, because it is down or toward the ground in the natural position of the flower. The opposite edge is dorsal. These terms are applied in the same way to animals, the upper side or back always being the dorsal, and the ventral the opposite side toward the earth.

Carefully split open a green pod along the dorsal suture and note that some beans (or seeds) are attached to each half of the pod. Is there any regularity as to the number attached to either half? Note that each bean is held in place by a short seed-stalk (funiculus). Undeveloped ovules may be seen near the ends of the pod. The part of the inner lining of the pod to which the seeds are attached is known as the placenta. Make a labeled sketch of the opened pod, showing the seeds in position. 
(Optional.) Cut transverse sections of the pod in several places, and identify the layers in the wall of the pod, the seed-stalk, placenta, dorsal and ventral sutures. Sketch and label.

Break a pod transversely in several places, and notice strong fibers. On which edge of the pod? Do you see any relation between the position of the strongest fibers and the natural curvature of the pod? Why are the green pods called "string-beans" by gardeners?

(D) Cut a fresh bean branch having young pods, and place cut end in red ink. After a time note where the pods are colored and draw your conclusions as to the functions of the fibers in the pods. Is there any reason why these fibers should be more abundant on one edge of the pod?

Look at specimens of pods which have matured, dried, and split naturally. Does the splitting (dehiscence) usually occur on the dorsal or ventral edge, or both?

Take out a bean and note its markings where it was attached to the seed-stalk. The scar left when the seed-stalk is pulled off is called the hilum. At one side of the hilum (toward the stalk of the pod) is a small translucent elevation with a slit-like marking. On the opposite side of the hilum is a very minute pit known as the micropyle. When pollen-grains touch the stigma, as previously described in $\$ 75$, the very small tube that grows from each pollengrain extends down the style and along the placenta of the ovary to the micropyle of an ovule. Later, when fertilization is completed as described in $\S 75$, each ovule develops into a seed, and the entire ovary into the fruit or bean pod. Look at an opened bean pod with the seeds in position, and note whether the micropyle is above the seed-stalk or below (toward the style). Now, make a diagram of a pod with seeds, and by a broken or colored line show the path a pollen-tube must take from the style to an ovule.

\section{The Bean Seed and its Germination}

78. Varieties of Beans. - $(L)$ Compare color, markings, and size of specimens of some of the common varieties of beans grown in gardens. (The school-museum should have a collection of the most common varieties of beans arranged in small labeled bottles or boxes.)

The variations in color, size, etc., of the seeds are no greater than the variations of all parts of the plants which grow from them: (1) Bean plants may be low (dwarf beans), or climbing (e.g., lima beans); (2) they may have flowers of 
various colors; (3) the leaves may differ in shape, size, and color; (4) the pods may be rounded or flattened, short or very long (in one variety two to three feet), green or yellow (so-called "wax beans"), with "strings" ( $\$ 77)$ or almost "stringless," and with many flavors; (5) some bean plants form edible pods in six or seven weeks after planting (the early and extra-early varieties), while others take a longer time (lima beans are often killed by frost before the seeds

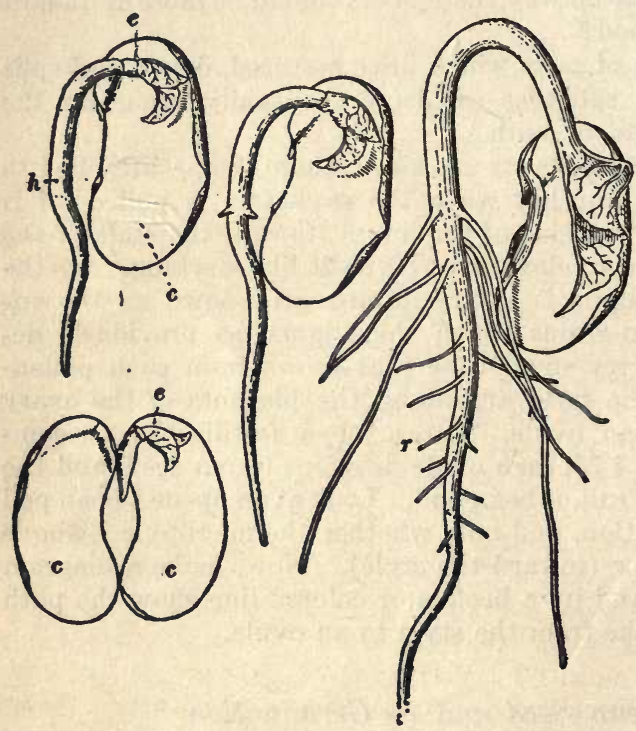

Fig. 33. Bean seed and seedlings. c, cotyledons; $e$, epicotyl; $h$, hypocotyl; $r$, roots. (From Atkinson.)

are full size). These are a few of the variations of beans which interest gardeners, because all these are qualities sometimes desired. Look over the descriptions of beans in a seed-catalogue, and note the points emphasized in the descriptions of varieties offered for sale.

79. Structure of a Bean. - (L) Use any large beans (limas, "yellow sixweeks," "scarlet runner," and "golden-eyed wax" are excellent); some dry, and some which have been soaked in water over night.

Examine the surface markings. Locate the scar or hilum and the micropyle, which were described in the preceding lesson on the pod. The translucent elevation seen in green beans near the hilum and opposite the micropyle is colored in dry beans of many varieties 
(dark brown in "yellow six-weeks," light brown in "golden-eyed wax" beans). These conspicuous marks will aid in locating the micropyle on the opposite side of the hilum. Make outline drawings of a bean, looking down upon the hilum, and also as seen from the side.

Strip off the seed-coat from a water-soaked bean. The seed-coat has two layers, which are easily seen in a green bean. The main body of the bean consists of two thickened halves (seed-leaves or cotyledons). These are stored with food for the early use of the young plant that will grow from the seed.

Carefully separate the two cotyledons, and notice a pointed rodlike body which is joined to the cotyledons. This is called hypocotyl (Fig. 33, $h$ ). It is best seen in a bean which has begun to sprout, for the hypocotyl then pushes through the seed-coat at the micropyle. The part of the hypocotyl next the cotyledons will form the beginning of the stem, while the pointed end will form the first root. Before sprouting begins, it is difficult to see any line between the stem and the root part of a hypocotyl ; and we simply call the entire structure hypocotyl until growth makes it easy to distinguish between stem and root. By placing the hypocotyl in certain dyes (e.g., solution of permanganate of potash) the root part quickly takes up the stain and makes it easy to see that the pointed end (root) of the hypocotyl is different in its cells from the upper or stem part.

The words caulicle and radicle which are used in some botanical books are practically synonymous with hypocotyl.

Joined to the hypocotyl where this is united with the cotyledons is a very short stem with a pair of small leaves. Between these leaves is a small bud. This short stem with leaves and bud constitutes the epicotyl. It will form the stem and leaves above the cotyledons. Some books call the bud with the small leaves a plumule, but for the beginning of the stem and leaves above the cotyledons the word epicotyl is preferred.

80. The Bean Embryo. - Cotyledons, hypocotyl, and epicotyl together constitute the embryo, which may develop into a bean plant. The part of the plant which develops from each part of the embryo is as follows:-

Cotyledons - not very useful as working leaves of bean seedling, but stored with food.

Bean embryo Hypocotyl - forms stem below cotyledons and consists of root at its lower end.

Epicotyl - forms stem and leaves above cotyledons. 
As we shall see in later studies, the seed-coat is lost as the embryo develops; it is therefore simply a structure for protecting the embryo after the seed is out of the pod and until germination is completed.

81. Germination or Awakening of Seeds. - A dry bean seed shows none of the usual signs of being alive; but it soon revives when placed under proper conditions (with moisture, heat, and oxygen from the air), and rapidly grows into a young plant (called seedling, or plantlet). This "awakening" or reviving and growth into a new plant is commonly called germination; and the seed is said to germinate. The popular word "sprouting" usually means the early stages of germination.

Germination of Beans. - $(L)$ Beginning about two weeks before this exercise, some beans should have been planted every other day in soil (preferably in small boxes or flower-pots which can be taken to the schoolroom). Plant about two inches deep, and keep the soil moist and warm. Plant a few beans four, five, and six inches deep, and some near the soil surface. Mark the position of these with wooden stakes on which figures have been written with lead-pencil. When some, young plants (seedlings) are two inches above the soil, and others just emerging, the materials are ready for the following lesson.

Without pulling up any plants, carefully examine and compare the various stages in order to determine where the parts of the embryo seen in the seed are located in the seedling. What becomes of the seed-coat? The first joint or node of the stem is where the cotyledons are attached; the second at the leaves of the epicotyl. Does the internode between these two nodes lengthen? Do the cotyledons of the bean become leaf-like? Compare the size and shape of the leaves of the epicotyl with those you have seen on a large bean plant.

Make a series of sketches showing stages in the emergence of the seedling from the ground. Leave space in note-book for adding other and larger sketches of later stages as the plant develops for several weeks.

In order to find out what happens between the time the seed is planted and the seedling emerges from the soil, we must either dig up seeds planted at various periods of time or we must study these 
early stages of germination in seeds which are germinated without contact with soil. This latter is best because the particles of 'soil cling to the seeds and make them more difficult to study.

To germinate seeds without soil, place them between layers of cotton batting, sphagnum moss, sawdust (some kinds are too acid), blotting-paper, filter-paper, or other soft papers. Keep moist, not wet, and warm. Or simply place seeds on a few layers of soft paper on a flat dish (such as a dinner-plate) and ke p moist, warm, and covered. Sand or cotton under the paper will help hold moisture.

$(L)$ Examine various stages of bean seedlings grown without soil. Compare carefully with seedlings growing with their roots in soil, and make sketches of the chief stages.

$(D)$ In order to determine whether any part of the hypocotyl grows more rapidly than others, make equidistant marks with waterproof India ink, and observe changes of distances between marks as growth proceeds (see Fig. 34).

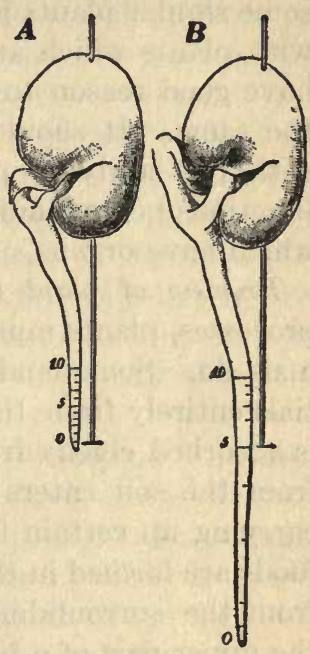

Fig. 34. Hypocotyl of bean seedling marked to show region of greatest growth (between 0 and 5). (From Strasburger.)

\section{THE WORK OF THE ORGANS OF A PLANT: AN INTRO- DUCTION TO PLANT PHYSIOLOGY}

The preceding lessons have dealt largely with the structure of the various parts or organs of the bean plant; and all the facts to which special attention has been given are true of the vast majority of the plants which have roots, stems, and leaves. In short, the bean plant has been studied as a type of the plants which we most often see in everyday life. In connection with the study of structure, some brief mention has been made of the use or work of each organ, but fuller explanations have been reserved for this section. As far as possible, the following physiology lessons are based upon the bean plant, but sometimes we shall use 
some similar plants for illustration and try some experiments with plants which are so much like the bean plant that we have good reason to believe that the work of their organs is the same. It should be remembered, then, as we proceed with the study of plant physiology that the processes or functions being studied are essentially the same in all plants which have organs such as we have found in the bean plant.

Preview of plant life. - In order to carry on their lifeprocesses, plants must have food, water, and oxygen, as animals do. Some land plants get the first two of these essentials entirely from the soil and others in part. The oxygen is absorbed chiefly from the air through the leaves. Water from the soil enters the roots and ascends to the leaves, carrying up certain food-materials. In green plants certain foods are formed in the leaves from water and carbon dioxide from the surrounding air. Sap containing foods made in the upper part of a plant may flow down certain tubes in the stem. The details of these processes will now be considered.

82. The Need of Water. - In one of the first experiments we found that plants contain a large amount of water. Moreover, any one who has ever cultivated plants knows that unless the soil is kept moist the plants will wither and die. Evidently water must be of great importance; and so it will be of interest to study (1) how water gets into the plant organs (root, stem, and leaf) and (2) what work water does in these organs.

83. Source of Water. - It is obvious that ordinary plants which have roots must get most of their water from the soil. It might be supposed that some water from rain and dew which wets the leaves is absorbed; but that this is exceedingly small in amount and insufficient could be proved by taking a potted plant and covering the soil with waterproof cloth so that rain and dew touch the stem and leaves but not the roots. Under such conditions most kinds of ordinary plants would soon wither. 
84. Water in Soil. - How can plants get water from soil which appears to be very dry? The answer to this question is that the apparently dry soil is not without water. It is not necessary, or even desirable, for many plants that the soil be wet; that is, contain free water which might be drained off. On the contrary, it is best for most plants if the soil contains moisture in the form of thin films adhering to the particles of the soil. We have already noticed that the root-hairs adhere to the soil particles (Fig. 26), and therefore they are in the best possible position for absorbing the moisture of the soil.*

85. Rise of Water in Soil. - For our plant studies the most important points concerning soil moisture are as follows: The water stored in the deeper layers of the soil supplies moisture to the layers nearer the surface. This is due to capillary attraction, of which good illustrations are the rise of oil in an ordinary lamp-wick and of coffee into a lump of sugar which just touches the liquid. Water is continually being lost at the surface of the soil, owing to evaporation and to absorption by plants, and it is as constantly coming up from below. But it may be lost by evaporation from the surface soil more rapidly than it can come from the deep layers, especially if the surface is hard-packed, as on a road, or if the surface is covered with large numbers of plants, such as weeds or grass, which take much water from the soil.

Mulching. - Excessive loss of water from the surface of the soil may be prevented by mulching, two kinds of which may be illustrated by two ways of raising potatoes:

(1) In the usual way the soil is cultivated on the surface in order to kill weeds, which use water needed by the growing

* If the students working with this book have not in some previous science work had lessons on water in soil, it is suggested that some work in this line be here introduced. Read Osterhout's "Experiments with Plants," pp. 103-121 ; and Burkett, Stevens and Hill's "Agriculture for Beginners," pp. 10-15; and perform the experiments suggested. 
potato plants, and also in order to keep the surface of the soil in a dusty condition. This checks the capillary attraction a few inches below the surface and moisture comes up to within reach of the roots of potato plants but not to the surface where it will be wasted by evaporation. Such a condition is known in agriculture as a dust-mulch. On many farms which are conducted according to modern science one may see on hot dry days in the summer the cultivatingmachines at work pulverizing the surface of the soil in order to keep in the moisture. This is the main secret of success in the "dry farming" in many western states.

(2) The second kind of mulch is illustrated by the following method, often successfully used in growing potatoes in dry regions and seasons. The potatoes are planted shallower than in the usual method and the entire field is covered with several inches of straw. The potato plants grow up through the straw, but most of the ordinary weeds do not. The straw prevents evaporation of water from the surface, and in dry weather the soil is found to be moister than soil treated by the dust-mulch method. In fact, a great objection to the method is that the soil often becomes too moist after heavy rains. The same method is often used in orchards, cutting the weeds and grass and spreading them over the ground around the trees, instead of cultivating the soil to keep in the moisture.

It is obvious from the foregoing discussion of water in soil that this is one of the most important problems connected with growing useful plants, and horticulturists and agriculturists have found it important to understand the scientific facts concerning the water of the soil and its use by plants.

86. Absorption of Water by Roots. - That pressure is developed when water is absorbed by the roots may be demonstrated as follows :-

(D) Cut the stem of a plant near the ground, and attach a glass tube by means of rubber tubing as shown in Fig. 35 ; but use a 
straight tube and put in a small quantity of water instead of mercury, which is used with the S-tube. Geranium, dahlia, tomato, sunflower, corn, many young shrubs and trees, and grape-vines have stems which make it easy to attach the tube. Make marks on the tube and note the rise of water in the tube for several days.

Instead of a straight tube, botanists often use an S-shaped tube, and fill the lower bend of the tube with mercury. Since mercury is 13.6 times heavier than water, it is easy to compute the height to which a column of water might be forced. It has been found that in the grape the root-pressure is sufficient to force a column of water up 10 meters (how many feet?).

87. Work of the Root-Hairs. - How does the water concerned in root-pressure get into the root from the soil? This is the question which naturally arises in our minds when we observe the preceding experiment. Scientists have studied the microscopic structure of the roots of many plants and have found no openings or pores through which water can get into the root, and so they have concluded that the water must soak through or be absorbed through the walls of the surface cells of the root and especially through the walls of root-hairs.

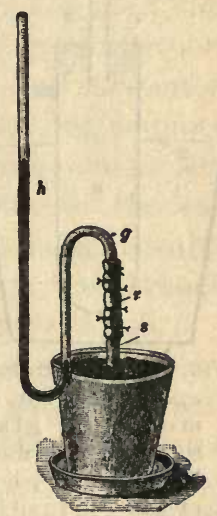

FIg. 35. Glass tube $(g, h)$ with mercury, attached to a stem (s) by rubber $(r)$, to measure rootpressure. (Detmer.) In order to make this method of absorption clearer we must try some experiments.

88. Osmosis. - (D) Select a cork that will fit firmly in the mouth of a "diffusion shell" (a membrane bag which may be purchased from dealers in scientific apparatus), bore a small hole in the cork, and into the hole fit a glass tube of small ealiber and 18 to 40 inches long. Fill the shell with dark-colored molasses, insert the cork in the shell, wrap and tie around the cork a strong coarse thread, and support with a retort-stand, wooden tripod, or otherwise, so that the diffusionshell will hang in water (Fig. 36). Note the movement of the 
molasses up the tube for several days. Change the water when it becomes discolored by exuded molasses. If the shell does not break, or leak at the cork, the column of fluid may rise to a height

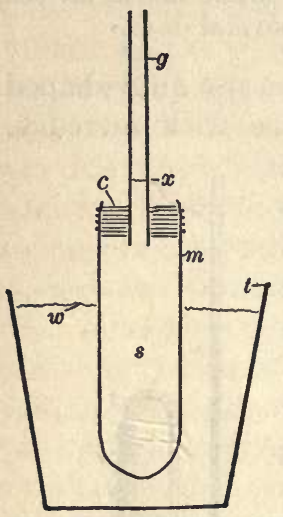

Fig. 36. Diagram of apparatus for osmosis. $t$, glass tumbler; $m$, membrane bag; $w$, level of water in tumbler; $s$, sugar-solution in bag and up to level $x$; $c$, cork tied into mouth of the bag; $g$, glass tube of $\frac{1}{8}$ inch bore fitted into hole in cork. Rise of fluid higher than $x$ indicates osmosis of water into the sugar solution faster than that of the sugar solution out into the water. of 8 or 10 feet. The glass tube may be made longer by joining on other tubes with pieces of rubber tubing for the joints.

Or a piece of gold-beater's membrane, or of fish-bladder, may be tied over the funnel of a thistle-tube, or even on one end of an ordinary tube, filled with molasses in the same way and placed in water.

The above experiment shows that sugar solution (molasses) "attracts" water through a membrane so strongly that the pressure developed will raise a column of water many feet in height. Like roots, as stated above, the membrane used for the experiment has no visible pores. The water must pass through spaces which are far too small to be seen with the aid of the strongest microscope. Such diffusion or absorption of water through a membrane without visible pores is called osmosis (sometimes osmose) in the science of physics. The verb to osmose will be used in this book. For an explanation of osmosis one must study the advanced books on physics; but for the purposes of plant study it is sufficient to remember the above experiment showing osmosis through a membrane which has no visible pores.

Another point needed for our later studies is that water having substances in solution may osmose. For example, in the above experiment the water became discolored by the exuded molasses (solution of sugar in water), proving 
that some of the molasses osmosed. Evidently the molasses did not osmose outward as rapidly as the water osmosed into the molasses, otherwise the column of fluid would not have been forced up the tube.

This outward osmosis of molasses in the experiment simply illustrates the fact that solutions will osmose through a membrane; but it must be understood that sugar solution in a plant root would not osmose out into the soil, for the living plant cell can prevent outward osmosis of its constituent substances. However, the osmosis into roots of substances dissolved in soil water might be illustrated by adding common salt to the water outside the membrane in the above experiment, and later it would be found that some of the salt solution had osmosed into the molasses. A large number of substances in solution will osmose through membranes, but others (e.g., white-of-egg, and glue) will not osmose.

89. Osmosis or Absorption by Roots. - The above principle of osmosis applies to the absorption of water by roots as follows: The cells of the roots (especially the root-hairs, Fig. 26) contain cell-substances which attract water very much as the molasses did in our experiment. The walls of the cells allow osmosis as the membrane of the diffusionshell did. In short, water from the soil, containing mineral substances in solution, osmoses into the cells on the surface of the root, especially the root-hairs. There is one difference between this osmosis in the root and that in our experiment; namely, that the cells of the root are filled with substances which attract water but do not themselves osmose out, as did the molasses. Hence water continually osmoses into the root, while the chief cell-substances do not osmose out into the soil. Some substances in root cells do pass into the soil, but in very small quantities.

Recalling the pressure made evident by the height of the water column in the last two experiments $(\$ 88)$, we see that in both cases pressure is the result of osmosis or absorption of 
water, in one case into the root by the cell-substance, and in the other case into the diffusion-shell by molasses. Roots, then, get the water from the soil by a process called absorption or osmosis, which is due to the power of cell-substances to "attract" water through the delicate walls of the root cells, especially of the root-hairs.

90. In what Part of the Root does Water ascend to the Stem? - Our experiment on root-pressure ( $\$ 86)$ showed that water passes from the soil through the root into the stem. It has been stated that water gets into the root through its surface, particularly through the root-hairs, which greatly increase the amount of surface cells for absorption. What is the path of water through the root on its way to the stem? The following experiment gives the answer.

(D) Cut off the small end of a slender root of carrot or parsnip, or any other plant large enough for convenience in cutting sections; place cut end of root in a bottle of red ink (eosin in water); after several hours cut transversely in several places, and note the position of the red-colored water in the tubes in the woody part (in fibro-vascular bundles, $\$ 70$ ). Some of the ink after a time soaks out (osmoses) into the cells in the bark and pith of the root. Thus water from the soil is distributed to cells by the wood-tubes.

91. Why Water ascends Stems. - There are many evidences that water is continually ascending the stems of plants to the leaves. For example, if we cut the stem off anywhere between the root and the leaves, the result is that the leaves soon wither and dry up, while similar leaves with the natural connection to the root through the stem remain fresh and well supplied with water. We have already studied the rise of water from the soil into the root and from the root into the stem. What causes the water to ascend the stems of plants? This is still one of the unsolved problems of botany. Root-pressure, as we have seen ( $\$ 86)$, is enormous. In a young birch sapling it has been found to be great enough to raise a column of water 18 meters (how many feet?). But 
root-pressure alone is not enough to force water to the top of tall trees, and it does not move water upward fast enough to make good the loss by evaporation from the leaves on a hot day. There must be other factors in the elevation of water.

Another possible explanation is suggested by the following experiment :-

(D) "Suction" Force of Evaporation from Leaves. - Cut off * a shoot (stem with leaves) of geranium or other potted plant and, with a short piece of rubber tubing, attach to a glass tube. Fill the tube with water and hold under water while attaching the plant stem. See Fig. 37. The rubber must be firmly wrapped with cord so as to bind it to the shoot and to the glass. It is well to spread a little vaseline around the two ends of the rubber. Support tube with a retortstand so that the lower end dips into mercury in a small dish or bottle. As water is evaporated from the leaves, the mercury will rise in the tube. If mercury is lifted one inch, it indicates a force able to lift water 13.6 inches. In this way it has been determined that some shoots will lift water several meters. Evidently there is great lifting power or suction force caused by evaporation from the shoot.

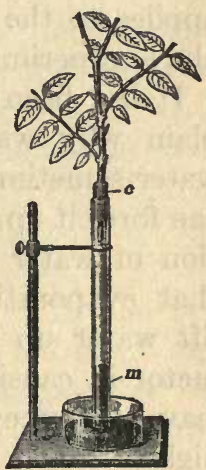

Frg. 37. Mercury $(m)$ lifted in glass tube by evaporation of water from leaves. Stem closely fitted to tube by cork at $c$. (From Detmer.)

That the lifting power seen in the leafy shoot is due merely to evaporation and not to some force peculiar to living plants may be demonstrated by tying a bladder or other membrane over a thistle-tube filled with water and suspended vertically so as to dip into a cup with oil or mercury. As

* For this and similar experiments it is best to bend the stem so as to have the part where the cut is made under water at the moment of cutting. This prevents the entrance into the tubes of the stem of air bubbles which impede the passage of water just as they can be seen to do in a glass tube of small bore. Flowers cut in this way will not wilt as soon as those cut off in the air. (Cut five flowers, or leafy shoots, each way, stand in water and compare as to their wilting after some days.) 
rapidly as the water evaporates through the membrane, the oil or mercury rises in the tube. The apparent "suction" is the same as in a pump, and is to be explained by atmospheric pressure on the mercury or oil forcing it up the tube as rapidly as the water evaporates. Possibly the same explanation applies to the evaporation of water from the leaves in the above experiment.

It has been stated that root-pressure alone does not explain why water ascends stems of high plants, because water sometimes evaporates more rapidly than root-pressure can force it up. The last experiments, showing that evaporation of water may exert great lifting force, suggest to us that evaporation from the leaves of plants may suck or lift water up the stems. This is certainly one important factor in causing the ascent of water from the roots to the leaves; but evaporation alone could not lift water up the highest trees.

Root-pressure and evaporation from the leaves are the two best suggestions concerning the rise of water up the stems of plants; but botanists admit that these together do not explain how a plant can lift water as high as do the highest trees. This is only one of many hundreds of things in science which it has not been possible to explain satisfactorily; and in the attempt to get more knowledge hundreds of scientific men are constantly trying experiments in new ways.

92. In What Part of Stem does Water Ascend? - We have already examined $(\$ 69)$ a stem of bean plant and found rind or bark, wood, and pith. Does the water go up in all these regions of the stem? We can best answer this question by an experiment.

(D) Cut off a shoot (stem with leaves) of a nasturtium, balsam, corn, bean, or other plant, and place the cut end of the stem in a small bottle with red ink (solution of eosin in water). Watch the rise of the ink along the stem into the veins of the leaves. Now cut across the stem in various places and note that the red color is 
in the woody part. Cut a thin section and note that the red color is in the wood-tubes of the fibro-vascular bundles which were mentioned in $\$ 70$.

If some stems be left in the ink for several hours, the sections will show that the red ink soaks (osmoses) from the wood-tubes into the bark and pith.

From such experiments as those above we conclude that the path of water up the stem is through the fibro-vascular bundles in the woody part of the stem. It was through these tubes that the water passed in our experiment on rootpressure (see $\S 86$ ) and in that on evaporation ( $\$ 91)$. From the wood-tubes water slowly osmoses into the cells of the bark and pith.

93. Water in the Leaf, and Evaporation. - In the last experiment the red-colored water ascended the stem and passed along the veins of the leaf. This was possible because the veins of the leaf are bundles of tubes directly connected with the wood-tubes in the stem.

$(D)$ Take a leaf from the bean or other shoot which was used in $\S 92$. Scrape the petiole and veins so as to show the bundles of tubes colored by the red ink. Note (especially good in the bean and in celery) the connection of the bundles of tubes in the veins of the leaf with the bundles of the stem; this is easily done by carefully scraping away the surface tissue from one side of the stem and petiole until the colored bundles of tubes are uncovered.

It is evident from the arrangement of the tubes and the path taken by the ink that water can pass directly from the wood-tubes of the stem into those of the veins of the leaf, and thence into the numerous veinlets. In this way water coming up the stem from the root is distributed throughout the leaf, which is thin and greatly expanded so as to expose as much surface as possible to sunlight and air, and thus promote rapid evaporation. It is obvious that one purpose of the veins of the leaf is to distribute or spread the water so that it may be evaporated rapidly. That the arrangement is very efficient is indicated by: the fact that the leaves of a 
sunflower plant six feet high have been proved to evaporate a liter (how many pints?) of water per day; and it has been carefully estimated that a large birch tree with about 200,000 leaves gave off 500 liters of water ( $2 \frac{1}{2}$ barrels) on a dry, hot day and probably averaged 60 to 70 liters per day during the summer.

Estimating Loss of Water from a Plant. - (D) Take a potted geranium, or other convenient plant, surround the pot and cover the soil with a sheet of rubber, tin-foil, or waterproof cloth, and tie earefully around the stem of the plant. Or set the pot in a battery-jar and cover the top with sheet rubber tied tightly around both jar and stem of plant. Thus only the upper stem and leaves will be exposed for evaporation. Now place the potted plant on a small platform scale (preferably one with two equal platforms which balance each other), and add the weights until there is an exact balance. Note from day to day the loss of weight from the plant. A glass funnel may be inserted through the waterproof covering into the soil, and a/weighed amount of water poured in daily to replace the amount lost by evaporation.

In order to make evaporation more rapid than the slow drying from the surface cells of the leaf, the leaf-pores (stomata) are sometimes opened (as described in $\$ 73$ ), allowing watery vapor to escape from air-spaces in the leaf (Fig 30). These cavities are surrounded by cells from which evaporation of water takes place rapidly. Thus the giving off of water is more rapid when the leaf-pores are open, and less when they are closed. The leaves of many kinds of plants seem to have a very complete control of the amount of water evaporated, because in these species the covering cells of the leaves are thick and sometimes coated with waxy substances or hairs, and in still other ways are unfavorable for the evaporation of water from the surface of the leaf. In such plants evaporation appears to take place chiefly through the leaf-pores. When we remember that an ordinary cabbage leaf has ten million and a sunflower leaf thirteen million leaf-pores, we can easily understand how 
the opening and closing of these pores may control the amount of evaporation.

The process of evaporation of water from the leaves of plants is in botany commonly called transpiration, and we say that leaves transpire, meaning that they give off water by evaporation. For all practical purposes the words evaporation and transpiration are equivalent as applied to the work of leaves.

94. Soil-Water and Sap. - Throughout this lesson we have referred to water as ascending in the plant, but it is never pure water. Water absorbed from the soil always contains some mineral materials in solution. Also, as the water passes through the plant it absorbs or dissolves other substances and becomes sap. However, a large part of the water which goes directly up the stem in the woodtubes is not very different from the water of the soil; and travelers in tropical countries often cut off certain vines and drink the water which runs from the stems.

One other point should be emphasized; namely, that in the evaporation of water there is left behind in the plant, especially in the leaves, all the substances brought in solution from the soil. Leaves which fall in the autumn give by burning much more ashes than do leaves which fall in the early summer; and the explanation is that evaporation during the long summer season has left behind in the leaf various materials carried up in solution in water from the soil, and not needed by the plant.

95. Use of Water in the Plant. - So far in our study of water and its movement through root, stem, and leaves, and its final loss through evaporation, we have considered the purely mechanical processes and without reference to the use of the water while passing through the plant. Now, plants are not elevating water simply for the sake of evaporating it from the leaves; on the contrary, evaporation is necessary to make place for more fresh water and so keep up the 
current from root to leaves. This is necessary for the following reasons: (1) The water carries up in solution indispensable food materials obtained from the soil. (2) Water is necessary in many parts of the plant in order to give turgidity and rigidity. Without plenty of water, the plant wilts, which is due to lack of water in cells and consequent loss of turgidity. A Windsor bean plant grown in a pot is excellent for showing this. Allow the soil to dry until the plant wilts, then water the soil. (3) Water is necessary in order to dissolve sugar (e.g., maple sap and other sweet juices of plants) and other food-substances which must often be transported in solution from one part of a plant to another. (4) Water is needed because it is used in the chemical combination of such food-substances as starch, sugar, and oils which are made by the cells of the plant. (5) Water in large quantities is needed by growing plants because such a large proportion of the substance of new cells is water. (6) Evaporation of water results in cooling the plant, thus preventing a dangerous amount of internal heat.

In addition to these special reasons why plants need water, we must remember that all living matter requires water ( 12 ). Without water there is no life, so far as we know. Even seeds which are apparently dry contain a certain amount of water ( 8 to 15 per cent of their weight).

96. Food Requirements of Plants. - In one of the first lessons ( $\$ 16)$ we found that a plant is made up of water, carbon, and mineral matters (in ashes), and also some carbon and other elements making up the gases which were burned. When chemists carefully analyze these substances from plants they find the following ten chemical elements : carbon, $(\mathrm{C})$, hydrogen $(\mathrm{H})$, oxygen $(\mathrm{O})$, nitrogen $(\mathrm{N})$, sulphur $(\mathrm{S})$, phosphorus $(\mathrm{P})$, iron $(\mathrm{Fe})$, potassium $(\mathrm{K})$, calcium $(\mathrm{Ca})$, and magnesium $(\mathrm{Mg})$ in every plant; and still other elements are found in many plants, but are not absolutely necessary for plant life. The first four $(\mathrm{C}, \mathrm{H}, \mathrm{O}, \mathrm{N})$ form the chief part 
of the combustible matter of all plants. These ten elements which are always found in plants must also be in their food, and in the next three sections we shall consider how plants get the food which will furnish these necessary elements for making plant structure.

97. Food-Materials from the Soil. - It is a matter of common observation that the growth of plants is largely influenced by the nature of the soil. Every farmer and gardener learns through experience to distinguish between barren and fertile soils, and that the addition of manures and various chemical "fertilizers" increases the growth of plants. The relation of plant growth to the materials available in the soil may be well illustrated by the following experiment.

(D) Growing Plants without Soil. - This may be done by germinating seeds of oats, beans, peas, lupines, and other common plants on moist sawdust, cotton, sand, crushed stone, or other materials into which roots can penetrate, but which contain no plant food. When the seeds are well germinated, begin to moisten the sawdust or cotton daily with water in which has been dissolved some chemical tablets containing the materials such as are found in good garden soil; that is, the necessary elements $(\$ 96)$. If the roots are kept moist with such a solution of chemicals, some plants will develop flowers and seeds. By trying various chemicals in such experiments, it has been possible for botanists to prove that only certain elements are necessary in the soil for plant growth. Most of the elements are common in agricultural soils, but commercial fertilizers rich in nitrogen, potassium, calcium, and phosphorus are needed on many farms. The tablets may be purchased from the Agassiz Association, Sound Beach, Conn., for ten cents a box, post paid.

Other interesting experiments in the same line may be performed by growing plants in different kinds of soil in pots, fertilizing the soil with various kinds of plant foods and fertilizers sold for garden use (see catalogues of seed-dealers).

98. Food of Plants without Chlorophyll. - We can better understand how a bean plant or other green plant gets its 
food if we first study the nutrition of plants like the mushrooms and the Indian pipe (Monotropa), which have no chlorophyll. Such plants get their food-materials entirely from the soil in the form of (1) certain inorganie or mineral substances which are commonly found dissolved in water in good soils, and (2) organic materials absorbed from the decomposing matter, such as the leaf-mold, on which mushrooms and the Indian pipe commonly grow.

The water obtained from the soil by mushrooms contains compounds with the elements nitrogen, and also in various combinations are other elements (e.g., calcium, phosphorus, sulphur, potassium, magnesium, iron) which chemists find in analyzing such plants. These necessary elements may come in part from the decaying organic materials in the soil.

It is from this decaying matter only that the mushroom can get the indispensable foods known as carbohydrates, a term which means a compound containing carbon $(\mathrm{C})$ and water $\left(\mathrm{H}_{2} \mathrm{O}\right)$, and therefore composed of the necessary elements carbon, hydrogen, and oxygen $(\mathrm{C}, \mathrm{H}, \mathrm{O})$. Examples of carbohydrates are starch, sugar, and cellulose (the chief substance in plant cell-walls).

Now, mushrooms and other plants without chlorophyll cannot make such foods as starch and sugar, and so they must use these foods made by some pre-existing plant which had chlorophyll; that is, they must live on leaf-mold or other decaying plant matter from which they can absorb carbohydrate food in a dissolved form, usually as sugar. These foods which are absorbed by the cells of the mushroom are used by its protoplasm in the life-activities of the cells, especially in making new cell-materials into which the nitrogen and the other necessary elements named above are also combined.

Saprophytes and Parasites. - Plants which get their carbohydrate foods from decaying organic matter are often called saprophytes (from Greek words for rotten and plant). 
Most plants without chlorophyll are saprophytes; but some of them absorb the necessary food from living plants or animals, and such plants are called parasites (e.g., dodder, and mistletoe to some extent).

99. Food of Plants with Chlorophyll. - Such plants have in their bodies at least the same ten essential elements ( $\$ 96)$ as plants without chlorophyll. Nine of these elements (all except carbon, and some oxygen) are obtained from the soil, the hydrogen and oxygen in the form of water $\left(\mathrm{H}_{2} \mathrm{O}\right)$ and the other seven in solution in water absorbed by the roots. The one fundamental difference between the nutrition of plants with and without chlorophyll is that the chlorophyll is a special substance with the aid of which the protoplasm of leaf-cells is able under the action of light to make the carbohydrate food needed by the plant, while plants without chlorophyll must absorb, by their roots, such food from the decaying bodies of other plants or animals.

Cells containing chlorophyll are able in light to make carbohydrate food in the form of starch or sugar, obtaining the neçessary elements from water, from the soil, and from carbon dioxide from the air.* This production of sugar and starch takes place chiefly in the leaves. Probably sugar is first formed and then changed into starch; but since starch is usually demonstrable in green leaves exposed to light, we shall give special attention to its formation. However, it makes no difference to the plant whether sugar or starch is formed in the leaves, for they are of equal value as food for all plant cells.

The stomata allow watery vapor to escape, in transpiration, and they are also important in allowing air with carbon di-

* The atmosphere contains on the average about three parts of carbon dioxide in 10,000 of air, measured by volume; and yet from this exceedingly small amount of carbon dioxide the green plants get the necessary carbon for making all carbohydrates, which compose a large part of the solid matter of plants. 
oxide to enter the spaces of the leaves, whence the carbon dioxide passes into the cells with chlorophyll (Fig. 30). Water reaches these cells by osmosing from near-by veinlets, which receive water from the roots through the stem.

100. Photosynthesis. - That light is necessary for carbohydrate-making (sugar and starch), the following experiment shows. Since the process is a synthesis or a combining depending upon the action of light, it is commonly termed photosynthesis (meaning, combining by light). Briefly, it is starch-making or sugar-making in green leaves exposed to light.

Test for Starch. - (D) Put a small quantity of eorn starch in a tube with water, add a few drops of iodine solution (crystals of $I$ in 70 per cent alcohol). Note the blue color. Try effect of iodine on powdered sugar in water. If we had time, we might test in the same way all the various substances found in plants and animals; but we must accept the records of science that no one has yet found any other substance which with iodine solution gives this peculiar blue color seen in starch. Hence we use iodine as a test for the presence of starch in animal and plant substances. Sometimes iodine is mixed with chloral hydrate in order to make it stain more.

Starch Formed in Leaves in Light. - (D) Take two potted plants (bean, nasturtium, or other convenient plants), leave in a perfectly dark room (or box) one day, and on the morning of the second day take one of the plants from the dark and set in strong sunlight. Near the close of the day take leaves from both plants, dip into boiling water for a minute, and place in hottles (labelled "dark," "light") containing strong alcohol. When the class meets again, note that the green color (chlorophyll) has been extracted by the alcohol ("bleached"). Take a leaf from each bottle, rinse in water, and place in a beaker or a small saucer containing some iodine solution (or better, use saturated solution of chloral hydrate mixed with enough iodine solution to color). In which leaf does the iodine test show presence of starch? Remembering that the two leaves have been treated exactly alike except that one came from a plant left all day in the sunlight, while the other remained in the dark, state your conclusion as to the importance of sunlight in starchformation. 
Another way of showing the effect of light on starch-formation is as follows: Take a piece of sheet cork, or thick pasteboard, or tin-foil, about two inches square, and cut out a triangle, star, or any figure preferred, from the center. By means of pins or paper clamps fasten this on the upper side of a leaf of a potted plant, and a piece of black cloth or sheet of cork on the lower side; or cut the opening desired in a strip of tin-foil and then fold this around the middle of the leaf. Set the plant in bright sunlight with this leaf supported so that the upper side will get full illumination. Near the close of the day, cut off the leaf, dip into boiling water, then place in alcohol for a day or two. Finally, test for starch with the iodine (or chloral hydrate and iodine) as in above experiment. Make a sketch of the leaf, and by colored pencil or shading indicate where starch was formed. Label the portions of the leaf " exposed to light" and "in dark.".

Variegated Leaves. - Many varieties of plants grown in greenhouses have "variegated "leaves with large white areas due to the absence of chlorophyll. That starch is not formed in these areas can be shown by taking a leaf which has been in sunlight all day and testing for starch as in the above experiments. Plants grown for a long time

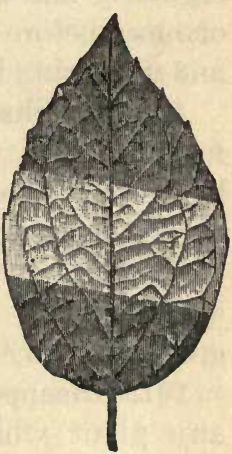

Fig. 38. The darker ends of this leaf were colored by iodine, indicating starch. The center was protected from sunlight. in a dark room lose their chlorophyll (e.g., sprouting potatoes in a cellar), and after a day in sunlight their leaves show no starch.

Intensity of Light Required. - It must not be inferred from the preceding experiments that light must necessarily be in the form of bright and direct sunlight. Starch-formation is most rapid in direct sunlight, but probably goes on in light of all intensities, even in moonlight. This explains why some species of plants can live and grow slowly in shaded spots in the woods; they apparently make use of the diffuse and weak light which reaches them. Moreover, 
light from other sources than the sun will serve for photosynthesis; for example, electric lights are sometimes used in greenhouses. The blooming of Easter lilies may be hastened from four to ten days; and lettuce grown within fifty or sixty feet of a 2000-candle-power arc-light, used regularly half of the night, will be ready for market a week or more before plants not so treated. Incandescent electricand gas-lights have a similar effect, but in lesser degree.

Another illustration of the effect of light is in the rapid maturing of plants under the influence of the intense light of the short arctic summer. Also, plants in greenhouses in winter do not grow as rapidly in a given number of hours of sunshine as they do in summer when the light is so much more intense. And this is so even when the interior of a greenhouse is constantly at the average summer heat.

101. Disappearance of Starch from Leaves. - If we take any plant which has been standing in sunlight, clip off some leaves, and place in alcohol for later testing to make sure that starch is present, and then set the plant in a dark room or box for a night and take other leaves before light reaches them, there will be no blue color with iodine solution, thus indicating that starch has not remained in the leaves kept in darkness. Where has it gone during the night? There are two answers:-

First, some of the starch has probably been used in the cells of the leaf, either changing to other cell-substances composed of the same elements (e.g., sugar), or combining with the elements brought in water from the soil and forming compounds containing, besides the $\mathrm{C}, \mathrm{H}, \mathrm{O}$ of the starch, some $\mathrm{N}, \mathrm{S}$, and $\mathrm{P}$, and perhaps other essential elements. The compounds thus formed (containing C, H, O, N, S, P) are called albuminous substances or proteins; and some of these may form some new living matter (protoplasm) in the cells of the leaf.

The second explanation of the fact that the starch dis- 
appears from the leaf at night is that it goes down into the stem or root, or into the developing flowers or fruits. That this is what occurs in part is proved by the fact that starch and other foods (e.g., sugar, with the same elements) are found in these parts of the plant, while all experiments ever tried have failed to show that these substances can be formed in cells without chlorophyll. The carbohydrate foods needed in all living cells of a plant must come from the cells with chlorophyll, most of which are in leaves.

Microscopic study shows that starch is in the form of grains inside of cells in leaves.

Starch in Cells. - $(D)$ Thin sections of a potato will show the starch-grains in the cells. Stain some sections with iodine solution before mounting, or by drawing iodine under the cover-glass with blotting-paper. Note the form of the starch-grains.

102. Digestion of Starch to Sugar. - How can such solid grains get through the cell-walls in going from the leaves and then into cells in other organs? The answer to this question is that starch is easily changed or digested to sugar, which is soluble in water and can osmose from cell to cell. This change from starch to sugar is caused by a substance known as diastase, which is present in cells of the leaves and other organs of plants. This substance may digest starch at all times during the day and night, but the starch is formed during sunlight more rapidly than it can be digested. At night when starch is not being formed the diastase succeeds in digesting all the starch which was left in the leaf at the close of daylight. Hence, starch-grains are not found in leaves in the morning after being in darkness for several hours; but sometimes it is possible by chemical analysis to find in leaf cells sugar into which the starch has been changed by enzymes, and which either will be used in the leaf or will pass down the petiole into the stem.

Sugar Test. - (D) Boil a few grapes, raisins, or prunes in a testtube with a little water in order to extract some of their sugar. 
Pour some of the extract into another tube, add a few drops of Fehling's solution (a mixture of eopper sulphate and Rochelle salts, used by chemists for testing certain kinds of sugar), heat tube in a flame, and note the red color of the contents. Test some glucose or corn-sirup. Try Fehling's solution on a little starch in water; does the red color appear? Only sugars like glucose give the red reaction. White granulated sugar from cane and beet does not.

Change of Starch to Sugar. - (D) Boil a small quantity of starch in water in a test-tube, thus making a very thin starch paste. Put half of the paste in a second test-tube and add some diastase (obtained by extraction from plant tissues, and sold at drug-stores). After a half-hour, take some liquid from each tube and apply the starch and sugar tests. Results? Conclusions?

To test regarding osmosis : pour the contents of the two test-tubes into two gold-beater's bags and hang the bags in tumblers or beakers containing some water, or use the osmose-apparatus described in §398. After allowing an hour or more for osmosis, pour some water from each tumbler into test-tubes, and test with a few drops of Fehling's solution. Also test some of the water for starch, using iodine. Does the starch paste osmose? Is there sugar in the water having the starch without diastase? Conclusion?

103. Path of Foods Down the Stem. - It has been stated above that the foods (chiefly sugar) derived from the starch of the leaf may go down into the stem or into other parts of the plant connected with the stem. Also, the proteins (compounds of $\mathrm{C}, \mathrm{H}, \mathrm{O}$, with $\mathrm{N}$ and other elements) which are mentioned in $\S 101$ as being formed in the cells of leaves, may go down into the stem and thence into roots, flowers, fruits, or new branches. Obviously the sugar and proteins in solution cannot go down in the wood-tubes, because in them there is the strong upward current produced by transpiration. Botanists are now agreed that the downward current is in the bark part of the fibro-vascular bundles, and through the tubes previously described $(\$ 70)$ as sieve-tubes. The movement is so slow that it cannot be demonstrated with red ink, as in the case of the wood-tubes ( $\$ 92$ ). However, the evidence obtained from other experiments is no less convincing. 
Effect of Girdling. - If a rose bush or other shrub be girdled (cutting away a ring of bark down to the cambium $\S 69)$ and the injured region covered with wax to prevent drying, a thickened band of bark will slowly develop above the girdle, but never below. Evidently the tissue above the girdle obtains more food for growth, and this must come down the stem in the sieve-tubes of the bark, for the wood continues to have the upward current of water from the soil. A plant so treated will die after some months, for the parts of the stem below the girdle and also the roots cannot get the necessary foods from the leaves.

Another example: Trees often die because an iron wire has been left wrapped around them until the stem has grown so large that the ring of wire becomes embedded in the bark down to the cambium, thus cutting off the sieve-tubes of the bark and preventing foods from reaching the roots. Farmers often girdle trees in early summer in order to prevent them from shading crops; on some kinds of trees the leaves will remain green all summer and sometimes die next year if only the bark is girdled. But even the hardiest trees, like the honey-locust, will fail to put forth leaves after two or three summers, because the supply of food, which came down . from the leaves and was stored in the roots before the girdling, is gradually used up, and no more can get down to the roots. In order to make the leaves of such trees wilt soon after girdling, it is necessary to chop through the bark and several inches into the wood. Anticipating the lessons on stems in Chapter VIII, it may be stated here that only the wood-tubes in the light-colored outer layers of wood, known to lumbermen as sap-wood, are important in the ascent of water ; and therefore cutting deep into the sap-wood of a tree will cut so many wood-tubes that the leaves cannot get water to supply loss by evaporation, and hence soon wilt.

Still another interesting proof that the prepared foods from the leaves go down the stem in the bark next to the 
cambium is that a branch of a grape-vine girdled between a bunch of grapes and the root will produce better fruit on that branch, because the foods from the leaves are kept from going on down the stem. Of course, such a girdled branch will be useless in the next fruiting season, but since grapes are always developed on new shoots, it is the common practice to prune away each winter most of the branches which bore fruit in the preceding summer.

It is possible for foods to go up a stem. The leafy branch above a bunch of grapes might be cut off and the grapes continue to grow from foods coming up the stem in the woodtubes from other branches with leaves. In the spring before leaves appear on trees and shrubs, foods go up the stem from the cells in root or stem where they were stored during the winter. If a branch of a tree be cut off in winter just above a bud, this bud will usually grow rapidly in the following spring and form a large branch. Orchardists make use of this fact when they prune trees. All these cases prove that the foods must be able to go up the stem. By removing girdles of bark it can be demonstrated that the upward movement is in the wood-tubes.

It is evident from the experiments described in the above paragraphs that foods in solution in water may be transported either up or down the stem, depending upon where the supply is located and where needed.

104. Use of Foods Transported from Leaves. - The dissolved sugars and other more elaborated foods made in the leaves may be used in the plant in two ways, as follows:

(1) Used by Cells. - We have seen that living plants require food, and this is true of every living cell. Some of the food from the leaves is at once used by cells in roots, stem, flowers, or developing fruits. Part of the food undoubtedly goes to make new particles of living matter (protoplasm) to replace that continually being worn out and made lifeless. It should be kept in mind that a living plant 
is like a moving machine in that activity causes wear or waste, and hence some food must be used for repair or the entire plant will soon die. In short, some food must be used continually in making new protoplasm. If more protoplasm is made than is needed for repair of waste, the result will be growth; and usually growth means the formation of new cells. This is especially true at the growing tips of plant stems. The making of new protoplasm is known as assimilation, or constructive metabolism.

(2) Stored in Cells. - If the foods received from the leaves are not needed for immediate repair, they are stored in various parts of the plant (stem, roots, leaves, or seeds). In later lessons (Chapter VIII) we shall study various modifications of plants adapted to storing foods ; but for our present purposes it will be sufficient to mention that carrots, turnips, and sugar-beets are examples of plants that store large quantities of plant food in their roots; sugar-cane and sago-palm store food in their stems; the head of a cabbage is a bundle of leaves stored with food; and beans, corn, and nuts are seeds stored with food. These are examples of various plant organs in which food is stored for the future use of the plants. It has happened that man and the herbivorous (plant-eating) animals have found it convenient to appropriate many of these reserves of plant food for use in their own food-supply.

These reserved foods of the plant are commonly stored as oil and starch, which are easily seen (with low power of microscope) in thin sections of various stems and roots, especially in the late autumn or winter after storage has been going on during the entire growing season. In cases of such starch storage, the sugar solutions derived from starch in the leaves osmose into the cells of the root, stem, or fruit, and is then changed and stored as starch-grains. When the plant needs this stored starch, the starch-grains are changed back again into sugar, which is able to osmose out of the cell and into other cells. 
But all cells do not store starch. Sugar, from which our ordinary granulated sugar is obtained, is stored in large quantities in the cells of sugar-beets and in sugar-cane. Sometimes the sugar which enters cells is changed to oil, as in nuts ; and when the plant needs the oil elsewhere it is changed (sometimes to sugar again) and osmoses out of the cell. In still other cases the sugar from the leaves enters cells and is used in combination with nitrogen and other elements from the soil to form protein substances.

Enzymes. - All these remarkable changes which take place when foods are stored in plant cells have long puzzled chemists. No one yet understands fully how the plant cells are able to make these changes; but it is known beyond doubt that these changes do occur regularly in the life of plants, and further it is known that many peculiar substances called enzymes (e.g., diastase) are present in plant cells, and that in some way not understood and not yet imitated in the chemist's laboratory these enzymes can change sugar into starch, oil, or albuminous foods, or these back into sugar. A peculiarity of enzymes is that they can change other substances without undergoing change themselves, and that a small quantity of enzyme can produce a large amount of change. The most familiar example of an enzyme is pepsin, which in the stomach of animals digests protein foods.

Whatever the forms in which foods may be stored, it appears that they are commonly in the form of sugar when passing (by osmosis) into or out of any living cell.

105. The Oxygen-Supply of Plants: Respiration. - It has been pointed out that plants breathe or respire and have the same effect upon air which animals have; namely, they absorb oxygen from the air and give off carbon dioxide $\left(\mathrm{CO}_{2}\right)$. The term respiration is in both plants and animals usually applied to this combined process; but it is simpler to consider first the obtaining of oxygen.

In a plant without chlorophyll (e.g., a mushroom), oxygen 
is absorbed at all times of the day and night by the surface of the plant in contact with the air; and the gas then diffuses or osmoses from cell to cell, for all living cells must have a continual supply of oxygen. If some mushrooms be placed in a closed jar for many hours, it can be demonstrated by chemical tests that the air in the jar has lost oxygen. This will be the same no matter whether the jar is kept in light or in total darkness.

A bean plant kept in darkness in a closed jar will act like the mushroom at all times; that is, it will take up oxygen. But the same plant kept in a closed jar for several hours in sunlight will give off oxygen. Apparently this is the reverse of the mushroom or the green plant in darkness; but let us withhold our conclusion until we have reviewed previous lessons dealing with carbon dioxide in leaves.

We have noted ( $\$ 99)$ that carbon dioxide is used rapidly in making carbohydrates (in photosynthesis) while the plant is in light; but in darkness the plant makes no carbohydrates and consequently uses no carbon dioxide. Now, in the combining of the elements of carbon dioxide and of water to make sugar or starch, the carbon of the carbon dioxide is used, but the oxygen is not needed and is set free in the cells of the leaves.* The amount of oxygen thus freed is very much more than the plant needs for its oxygen-supply; that is, it is very much more than the same plant in darkness would absorb from the air; and the result is that there is excess oxygen to be given off to the air. The truth is that the living cells throughout the plant require oxygen the same in light as in darkness; but while the starch-making goes on

* Readers who have studied chemistry will be interested in the proportions of $\mathrm{CO}_{2}, \mathrm{H}_{2} \mathrm{O}$ and $\mathrm{O}$ in starch-making as follows: $\left(6 \mathrm{CO}_{2}+5 \mathrm{H}_{2} \mathrm{O}\right)^{x}=$ $\left(\mathrm{C}_{6} \mathrm{H}_{10} \mathrm{O}_{5}+60_{2}\right)^{x}$, which means that to every six molecules of carbon dioxide and five of water there will be one molecule of starch $\left(\mathrm{C}_{6} \mathrm{H}_{10} \mathrm{O}_{5}\right)$ and six molecules of free oxygen. This formula simply gives the proportions; for starch is some unknown multiple of $\mathrm{C}_{6} \mathrm{H}_{10} \mathrm{O}_{5}$. 
so much oxygen is freed from carbon dioxide that the plant cannot use it all.

Oxygen Liberated by Photosynthesis. - (D) Place some water plants, such as Elodea, in a glass funnel which is then placed with tube upwards in a glass battery-jar filled with water. The water must be deep enough to more than cover the funnel and its tube. Fill a test-tube with water, and keeping its mouth below the water in the battery-jar, invert it over the end of the funnel tube. Set in sunlight. Bubbles of gas (chiefly oxygen) will rise from the plants and displace the water in the test-tube. The gas may be tested for oxygen by stoppering the test-tube before lifting from the water, and then quickly inserting a glowing taper when the stopper is removed.

Critical study has proved that all kinds of plants, as well as animals, require oxygen constantly. They may get the necessary amount directly from the air, or green plants may get it from carbon dioxide when the carbon of that compound is used in starch-making during daylight. There is, then, no real difference between the breathing of mushrooms or animals and plants with chlorophyll. The increase in oxygen and decrease of $\mathrm{CO}_{2}$ in the air around green plants during daylight is obviously due to the independent process of photosynthesis, not to their breathing.

There are good reasons for believing that roots of plants absorb oxygen from the air, which is abundant in good soil. In fact, one scientific reason for cultivating or tilling the soil is to mix air with the soil particles. The water of the soil contains oxygen in solution, just as the water in a river contains oxygen which fishes can absorb by their gills. When the water is taken up into the plant stem it probably carries along with it some oxygen, which is absorbed by the cells with which the water comes into contact.

Also, some of the large tubes of the fibro-vascular bundles are filled with air, probably taken in chiefly at the leafpores and also at the bark-pores (lenticles), which are slitlike openings in the bark leading to internal air-spaces. 
The following experiment shows how air may enter the leaf and pass through the stem.

(D) Select a wide-mouthed bottle and a cork or rubber stopper with two holes. Take a leaf with a small round petiole and push the petiole into a hole in the cork and almost to the bottom of a bottle. Also fit a glass tube into the other hole of the cork. Fill the bottle half full of water. Use vaseline to make the apparatus air-tight. Apply suction to the glass tube (a small bicycle pump with the leather on the plunger reversed, so as to "draw" air out, and connected by rubber tubing with a reversed valve from a bicycle tire will answer, if an air-pump is not at hand). Air will exude from the cut end of the petiole and appear as bubbles in the water. The experiment may be reversed by attaching the petiole to rubber tubing leading to a bicycle pump, and forcing air from the pump into the tubes of the petiole and out through the leaf, which should be held under water so as to make air-bubbles rise from the leaf surface.

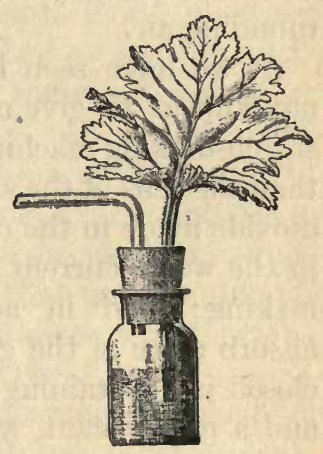

Fig. 39. Apparatus to show that air can enter the leaf. (From Strasburger.)

106. Excretion of Carbon Dioxide from Plants. - The oxygen absorbed by plants and distributed to all their living cells is used in the cells in a process of slow oxidation or chemical union of oxygen with foods and other substances in the cells. This is the same as in animal cells. Such oxidation is constantly going on among the particles of living plant cells, and one of the substances formed is carbon dioxide $\left(\mathrm{CO}_{2}\right)$. When we remember that substances containing carbon are abundant in cells, we can understand why oxidation of cell-substances should form a compound of carbon and oxygen $\left(\mathrm{CO}_{2}\right)$. For example, when sugar is highly heated the effect is first to drive off the water and leave carbon, which then burns and disappears in the air as a gas $\left(\mathrm{CO}_{2}\right)$. Something similar occurs in living cells when any substance made from carbohydrate foods burns. The result is carbon 
dioxide $\left(\mathrm{CO}_{2}\right)$ and water $\left(\mathrm{H}_{2} \mathrm{O}\right)$. The water thus formed cannot be distinguished from the other water which is abundant in plant cells. The carbon dioxide is transported (probably chiefly in solution in the moving liquids in plants), to the surface, especially of the leaves, and then diffuses to the surrounding air.

As we have seen in $\S 105$, a plant with chlorophyll does not appear to give off carbon dioxide in light, because the starch-making machinery is using that gas much more rapidly than the cells of the same plant are making it. If any carbon dioxide made in the cells of the roots or of the stem is carried in the water current to the leaves, it may be used in starchmaking; and in addition, the leaves must continually absorb more of the gas from the air. As has been shown, a closed jar containing a measured quantity of carbon dioxide and a green plant, and placed in sunlight, will have less of the gas after a few hours; while a similar jar and plant kept in total darkness will have more carbon dioxide in the air of the jar. In the first jar the plant must have used for starch-making all the carbon dioxide produced by oxidation from its own cells and in addition some of the gas taken from the air.

107. Other Excretions of Plants. - The term excretions is commonly applied to such substances as carbon dioxide, which are produced by oxidation in the cells of plants and animals, and which are eliminated because they are of no further use in the cells, and are sometimes actually poisonous. Carbon dioxide and water have been mentioned above as two excretions formed by oxidation of cell-substances containing the elements carbon and hydrogen. The water formed by oxidation mixes with the water taken into the plant by the roots, which is being eliminated continually by evaporation.

In addition to carbon dioxide and water, plant cells form other excretions and also have an excess of certain substances containing the elements absorbed with water from the soil. In 
higher animals all excretory and excess substances containing nitrogen, sulphur, phosphorus, calcium, etc., are in solution in the water eliminated by the kidneys. Land plants usually give off water only by evaporation, and certain excretory and excess matters are left behind in the plant (in the leaves) just as lime is left in a tea-kettle. Plants living in water may have some of these substances absorbed by the surrounding water. Also, plants may eliminate some substances by the roots, for the roots of some plants (e.g., oat seedlings) will etch or corrode polished marble or limestone by the action of acid substances which osmose from the roots. Possibly these corroding substances enable a plant to dissolve useful minerals. Also, it has been shown quite recently that grasses give off from their roots to the soil some substances which poison and check the growth of young orchard trees.*

It seems certain that some of the substances formed by the oxidation of cell-substances are stored in the plant, but often changed so as to render them harmless, or even useful, to the plant which produces them. Our knowledge of a large number of peculiar substances found in plants is still very incomplete; but it seems probable that many of them are modified and stored waste products which could not be eliminated along with water as animals eliminate similar substances by the kidneys. Examples of such substances which are not known to be of further use to the plants which contain them, are the active constituents of tea (in leaves), coffee (in seed), cocoa (in seeds), the poisonous substance in the pits (seeds) of almonds, peaches, and plums; quinine from the bark of a tree; nicotine in tobacco plants; strychnine and many other poisons in various plants. These and many other peculiar products of plants contain the element nitrogen, and some of them are chemically similar to the

* For an account of this see bulletins published by U. S. Dept. of Agriculture. 
nitrogenous excretions (those containing nitrogen) formed in animals. While the plant cannot eliminate these substances directly, it is interesting to note that many of them, along with mineral substances taken up with water from the soil, become stored in leaves, fruit, seeds, and bark - all of which parts of plants are frequently detached, resulting in the elimination from the plants of useless and possibly sometimes harmful substances. In many other cases substances of no further use to the plant may be stored permanently in harmless forms in stem or roots.

This habit of storing certain useless substances is peculiar to plants. As plants grow older the amount of stored substance, especially mineral matter from the soil, increases. Thus a leaf collected and burned in early summer leaves little ash as compared with a similar one taken in late autumn. Throughout the growing season water has been evaporating and leaving behind in the leaves the mineral materials carried up from the soil.

Animals do not store excess or useless mineral substances and excretions, but eliminate them daily, dissolved in water which is discharged by means of the kidney-system. Since most plants cannot discharge water in liquid form, storage of useless mineral matter and some excretions is the plant's only possible way of doing the same kind of necessary work which the kidneys of animals do.

108. Irritability of Plants. - Irritability in either plants or animals is the power of responding to a stimulus. For example, if a frog be touched suddenly (mechanical stimulus), the leg muscles contract and the animal jumps. The frog also responds to heat stimulus (goes into shade when the sun's heat is too great); to light stimulus (sees enemies and jumps); to sound stimulus (hears sounds and jumps); electrical stimulus (jumps if touched by a slightly charged electric wire). These are phases or kinds of irritability common in animals which have a brain, spinal cord, and nerves. 
We shall later study some microscopic animals which respond to mechanical, heat, light, and electrical stimuli; but they have no visible nerves or nerve organs. These lower animals have irritability or nervous functions without special organs to perform the functions. This is essęntially the case in plants. In recent years there have been many magazine articles discussing "the nerves of plants." The truth is that no one has seen any nerves or brains or similar nervous organs in plants, although they do exhibit the various forms of irritability and respond to the different kinds of stimuli which affect animals, as the following examples will show:-

Mechanical or touch stimuli (such as a jar by passing animals) causes the sudden folding of the leaflets and drooping of the branches of the Mimosa or sensitive plant (see Fig. 1). The leaf of the Venus fly-trap (Fig. 2) closes quickly and grasps insects which happen to touch it.

Response to light stimulation is seen in the familiar growing of house-plants toward the window, and also in the opening and closing of many flowers. Flowers of the dandelion and others of the same family, California poppy, etc., open in the light and close when placed in darkness.

Heat stimulus also causes many flowers to open and close; tulip, crocus, and "star of Bethlehem " are familiar examples. The combined effect of heat and light stimuli upon the opening of flowers is so marked in many species of plants that it is possible to make a flower-clock, which is simply a gardenbed arranged to imitate a clock-dial, in which for each hour of daylight there is a selected group of plants whose flowers are commonly open at that hour. Of course, the flowers do not open exactly on the hour, for the controlling temperature and sunlight vary from day to day. However, many flowers are open in early morning; certain flowers (example, "teno'clock") open in the middle of the forenoon; others (like "flower-of-an-hour") open only in the mid-day sunshine; 
"four-o'clock" and others open in late afternoon; and the eyening primrose at sunset.

Home-work: Keep a list of the plants which you have an opportunity to observe, and note the hours when the flowers are seen open.

Leaves of many plants (oxalis, clovers, bean, etc.) droop or fold in darkness and assume the so-called "sleep" position. Some so-called " compass plants" avoid the intense noonday sun by moving their leaves so that the edges are vertical and in the north-south direction.

Another form of external stimulus affecting plants is that of gravitation: That the stems of most plants ordinarily grow upward and the roots downward is a familiar fact. Experiments made by growing young plants attached to rotating wheels prove that this direction of growth of plants in a state of nature is due to gravitation.

Plants also respond to water. Roots will turn away from dry soil and grow in the direction of greater moisture.

Still other forms of the responses of plants are the numerous cases of the twining of stems and the movements of tendrils and special roots in order to aid in climbing.

Tropisms. - We see that in many different ways plants have irritability and respond to external stimuli. The responses in plants are much slower than in animals, but they are none the less definite. These reactions of plants to stimuli are often known as tropisms (from a Greek word meaning to turn). Turning in response to light is heliotropism (literally, turning to the sun), or phototropism; to heat is thermotropism; to gravity is geotropism (literally, turning to the earth); to water is hydrotropism ; to chemicals is chemotropism; to electricity, which seems to have little influence on plants in nature, is electrotropism. The same terms are used in describing the reactions of animals to the various kinds of stimuli. 
Although these responses of plants to various stimuli have been observed and studied for a long time, we do not understand them. Neither do we know why a frog responds. when touched. We simply know that it has the power of such response and that in some way it is connected with the protoplasm in certain special nervous organs of the frog. Likewise, we know many facts about plants responding in ways very similar to animals and that this power of response is connected with the protoplasm of plants, but apparently in no particular organs like nervous organs. We use the word irritability to mean that animals and plants respond to stimuli. We do not know what it is, but there is plenty of evidence that such a power exists, and we know much about how it works. Likewise, we know that electricity exists, very much about how it acts, and can make great use of it; and yet no one knows what electricity is. These are merely examples out of hundreds of cases in science where we must accept the facts and make use of the knowledge which it is possible to discover even when we cannot find a satisfactory explanation.

109. Summary of Work of Plant Organs: Life-Processes. - Most plants require, as food-material, carbohydrates, nitrogen compounds, and certain other elements. Plants with chlorophyll can make the necessary carbohydrates by combining the elements from carbon dioxide and water. Light and chlorophyll are essential for making carbohydrates. Plants which have no chlorophyll must absorb the carbohydrates (probably as sugar) from decaying organic matter. The nitrogen, in the form of compounds with other necessary elements, are commonly absorbed from the soil or water in which the roots grow.

All plants require oxygen for use in the oxidation which is going on constantly in all living cells. Plants with chlorophyll may get oxygen from that which is freed from carbon dioxide when the carbon is used in starch-making. For this reason 
such plants do not appear to take oxygen in the daytime from the surrounding air, as they do at night, and as plants without chlorophyll do both day and night.

All plants produce excretions by the oxidation going on in their cells. Most prominent of these is carbon dioxide, which all plants give off at night to the air or water in which they live. Plants without chlorophyll give off carbon dioxide in daylight also ; but the green plants use this gas so rapidly when making starch that during the daytime none appears to be given off to the surrounding air.

All plants have assimilation or constructive metabolism of some foods into new protoplasm. This takes place only in living cells. Much of the food containing only carbon, hydrogen, and oxygen (i.e., carbohydrates and oils) is believed to undergo oxidation in cells or is stored for future use, but does not become protoplasm. Probably only a small part of the contents of an ordinary plant cell is living matter, and much of the cell-substance which we see with the microscope consists of food-materials, water, and other lifeless substances.

Digestion of foods is necessary whenever insoluble foods (starch, oil, proteins) require transfer from cell to cell or to distant organs of the plant.

Moving liquids in the higher plants serve to transport the foods, oxygen, and excretions; but these liquids do not make a complete circuit as do the animal circulating liquids (blood and lymph), whose function is also transportation of foods, oxygen, and excretions ( $\$ 52$ ).

Some form of irritability, or power of responding to stimuli, is present in all plants. But there are no special nervous organs, such as are connected with irritability in higher animals.

All plants have the power of reproduction, either from parts of their bodies which can grow into complete plants (asexual reproduction), or from egg-cells which usually require union 
with other cells (i.e., fertilization by cells derived from pollengrains) before developing into new plants.

110. Classification of the Bean Plant. - By this we mean the relation of the bean plant to other plants. In the first place, the bean plant is a member of the great division of flowering plants or seed-plants. Within this division are many families, one of which is the family of leguminous plants (including peas, beans, vetches, clovers, locusts, and numerous other plants which have an irregular flower similar to that of the bean plant). In this family are included a number of bean-like plants which are somewhat different from ordinary garden beans (e.g., Windsor beans and horse beans). The common garden beans belong to the genus Phaseolus and to the species vulgaris, hence the scientific name is Phaseolus vulgaris. The various kinds of ordinary beans are varieties of the same species. The Windsor and horse beans belong to another genus.

The meaning of classification of animals and plants will be discussed in Chapter VII. 


\section{CHAPTER VI}

\section{COMPARISON OF ANIMAL AND PLANT BIOLOGY}

IN an earlier lesson ( $\$ 29)$ we noticed that living animals and plants have certain characteristics which lifeless things do not have. Most evident of these characteristics are movement, taking food and assimilating it, breathing, reproducing. We have now studied these and other activities more carefully, and are ready to compare the animal and the plant. Without careful study, a frog and a bean plant seem to have nothing in common, except that they are both living; but

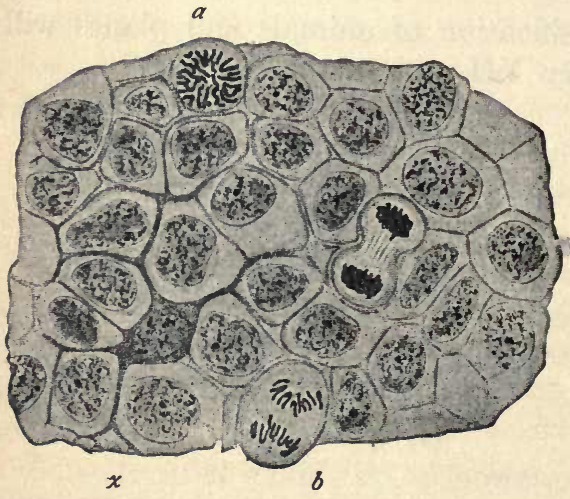

Fra. 40. Epidermis of salamander tadpole.

Three cells undergoing division. (From Wilson.) detailed study has shown us the following remarkable similarity between the animal and the plant.

111. Similarity of Structure of Bodies of Animals and Plants.In both the frog and the bean the essential living substance is protoplasm, and this is found in units of structure called cells (see Figs. 40, 41). Chemical analysis has shown no essential difference between animal and plant protoplasm. Both animal and plant cells have nuclei, and they multiply by an automatic process of division. Biologists 
whose specialty is the study of cells are continually making discoveries with the microscope which make still more impressive the similarity of cells in animals and plants.

In both animals and plants the cells are grouped to form tissues, and the tissues to form organs, which are simply groups of cells for performing a particular work necessary in the life of the living thing.

112. Food of Animals and Plants. - Both require food containing the elements found in

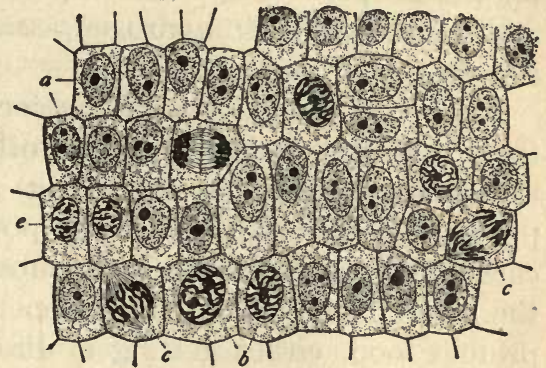

Fia. 41. Epidermis from leaf of onion bulb. Seven cells are in various stages of division. (From Wilson.) their bodies. That the necessary elements are the same is evident from chemical analysis and from the fact that many animals (the herbivorous) live on plants as food. Both animals and plants require foods' containing carbon, hydrogen, and oxygen. Plants which have no chlorophyll, and all animals, must depend for such food upon the sugar, starch, and oils which have been made from carbon dioxide and water by green plants ( $\$ 99)$. Herbivorous animals get carbohydrates and oils directly by eating plants; but carnivorous animals get similar food from the fat, sugar, and other substances in the flesh of herbivorous animals. Thus when a frog eats an earthworm it gets materials which the worm got from its plant food. In short, all animals, and plants like the mushrooms, depend upon those plants which are able to combine the elements of carbon dioxide $\left(\mathrm{CO}_{2}\right)$ and water $\left(\mathrm{H}_{2} \mathrm{O}\right)$ to form such foods as starch, sugar, and fat.

In addition to foods containing only three elements $(\mathrm{C}, \mathrm{H}, \mathrm{O})$, animals and plants require foods containing also the element nitrogen. Most plants are able to get nitrogen by using 
very simple substances absorbed from water in soil (e.g., sodium nitrate used as a soil fertilizer), and to unite elements from these with those of sugar to make the substance known as protein or albumen, which contains nitrogen in addition to carbon, hydrogen, and oxygen. Most plants can do this.

Some very simple plants (bacteria) can use free nitrogen from the air. Animals, on the other hand, must get their nitrogenous (nitrogen-containing) food in the form of proteins, for no animal has the power to make these from carbohydrates and such simple substances as plants get from the soil. Hence all animals depend upon plants for their protein food, either getting it directly by eating plants as food or indirectly by eating flesh from animals which derive their protein from plants.

Animals, then, depend upon plants for all their food-supply. They require as foods carbohydrates, fats, and protein; and only plants are known to make these.

Especially should we note that both animals and plants depend upon sunlight, which is necessary for combining elements from carbon dioxide and water into carbohydrates, which in turn are necessary for making proteins. Hence the energy manifested in the activities of animals comes from food formed by plants and indirectly from sunlight. This is what is meant by the statement in popular books that the " energy in foods is stored sunlight."

Stored Energy. - It should be noted further that plants by photosynthesis of carbohydrates store energy which may later be converted (by oxidation) into energy of action by either plants or animals. Besides foods, we may name as examples of stored energy such cases as gunpowder, a waterfall, coal, a wound-up watch-spring, etc. Such stored energy is said to be potential; that is, it has latent power. Energy being liberated from burning coal, exploding powder, etc., is called kinetic or energy of action. 
All the above applies to our human food-supply, for our dependence upon plants as food-producers is exactly the same as that of all animals. We may eat food from plants, or we may eat meat from animals which acquired their store of foods from plants. So, directly or indirectly, all our food comes from plants and depends upon the action of sunlight in the photosynthetic processes of green plants.

113. Oxygen Required by both the Animal and the Plant. - Oxygen is necessary in all living cells, because oxidation of cell-substances, chiefly absorbed foods, goes on continuously as long as the animal or the plant lives. In other words, oxidation in cells is inseparably connected with lifeactivities. In both animals and plants there are special breathing mechanisms for getting the required oxygen from the air and for distributing it throughout their bodies. (Describe these mechanisms in frog and bean plant.) The nitrogen of the air seems to play the part of a diluting substance, for oxidation goes on too rapidly when an animal or a plant is placed in a jar of pure oxygen. Animals require more oxygen in proportion to weight than do plants, because in animals life-activities (especially movement), are more intense, and oxidation is more rapid.

114. Metabolism Occurs in Both Animal and Plant Cells. - In each case foods which reach the cells may be oxidized to furnish energy, or may be used in forming new particles of protoplasm (repair and growth), or may be stored until needed. Plant cells require less food than do animal cells for supplying energy and for repairing protoplasm; and hence more is available for growth and for storage. This enables plants to grow rapidly and to store up great quantities of foods (sugar, starch, proteins, oils). Animals take advantage of this food stored by plants. The great difference between animal and plant metabolism is that plant protoplasm can make such foods as sugar, starch, oils and proteins from simple materials like carbon dioxide, water, and nitrates. 
This is constructive metabolism. On the other hand, animal protoplasm cannot construct foods out of such simple materials; but can only oxidize the foods made by plants. This is destructive metabolism, and its result is the formation of excretions. Plants do oxidize a limited portion of the foods they construct, and thus they form some excretions (e.g., $\mathrm{CO}_{2}$ and $\mathrm{H}_{2} \mathrm{O}$ ) similar to those formed when animals oxidize foods. On the whole, however, plants have a limited amount of destructive metabolism, and they are characteristically constructive.

Heat. - It should be noted that oxidation in plant cells, as well as in animals, produces heat; but the amount is small and not evident except in careful experiments.

115. Excretions of Animals and Plants. - Since oxidation is less rapid in plants, they produce less excretions than animals do. Since both animals and plants must oxidize foods made by plants, it is to be expected that the excretions of animals and plants will contain the same elements. As we have seen, the most evident excretions of animals and plants are carbon dioxide, water, nitrogenous, and mineral substances. These contain all the elements which a chemist could find by analyzing the cell-substance of either animals or plants.

The Cycle of Organic Matter. - Since plant cells are primarily constructive in their metabolism, they are able to use the excretions formed by animals, which oxidize foods derived from plants; or plants can use substances similar to excretions which are formed by micro-organisms from decaying animal and plant matter. Hence simple matter is constantly being built into foods by plants and reduced to simple conditions by animals.

116. The Cycle of Carbon. - Some of the carbon dioxide in the air comes from the breathing of animals; some comes from decaying of dead plant and animal matter; and some also from the burning of wood, oil, and coal (all 
of which fuels are of organic origin). Thus there is a neverending cycle of carbon dioxide being taken up from the air, built up into plant substance, which in turn is oxidized or becomes food for animals, then is oxidized to form excretions or built into animal substance, which ultimately dies and decays and is again taken up by the plant. In short, the foods made in plants are by animals changed back into excretions which plants use over again in making new foods.

Without chlorophyll and sunlight much of the carbon dioxide in the world now stored in the organic compounds of plants and animals would ultimately become free in the air, and be so abundant as to poison all life. Certainly all animals would perish because of the lack of the foods which plants with chlorophyll must make primarily for themselves, and which secondarily are used by other plants and also by all animals. The cycle of carbon, then, is as follows: Carbon from the carbon dioxide in the air enters into the composition of plant substances. This may be oxidized (in the living plant) or decayed (in the dead plant) directly back to carbon dioxide, which gets free in the air ; or the plant substance may be used by animals and thence oxidized (in living animals) or decayed (after death of the animal's body) back to free carbon dioxide.

Since animals depend upon plants in this food relation, philosophical biologists have concluded that plants with chlorophyll must have existed on this earth before animals did. In fact, with the exception of a few of the lowest bacteria (a group of microscopic plants), all animals and plants are dependent upon the plants which are able to use light and carbon dioxide in making foods. Some of the simplest bacteria can make food materials without light and from purely inorganic materials. Obviously such organisms could have lived on this earth before all other living things which we know and at a time when there were conditions which were not adapted to plants with chlorophyll. 
117. The Cycle of Nitrogen. - Parallel with the cycle of carbon from air to plants, from plants to animals, and from animals back to the air, there is a corresponding cycle of the element nitrogen. There is an abundance of this in the air, but only certain species of bacteria can use it in its free form. It has been pointed out that ordinary plants get nitrogen in compounds absorbed from the soil and use this in making proteins. Also, only plants can make the proteins required by animals as food. Proteins in animals and plants, then, depend upon the nitrogen in simple compounds such as are found in fertile soil. The nitrogen in the bodies of plants and animals gets back to the soil either by the decay of dead animals and plants, or as excretions produced by animals; while animals obtain protein foods from plants directly or indirectly through other animals. Animal excretions furnish nitrogen to the soil, from the soil the nitrogen goes to plants, from plants it goes to animals as protein food, and then animals reduce the food to nitrogenous excretions, which in the soil may again be taken up by plant roots.

Such is the cycle of nitrogen, which, like that of carbon, must be continuously undergoing repetition. If dead animals and plants did not decay and living animals not change protein foods to nitrogeneous excretions, all the once available nitrogen would soon be bound in the bodies of dead animals and plants and life would cease. A marked tendency toward such a result is evident in certain agricultural regions where farmers have wasted manures (animal excretions) until the soil has lost its primitive fertility; that is, has suffered exhaustion of the nitrogen compounds.

-118. Interdependence of Animals and Plants. - We have noted that animals depend upon food from plants for both carbon and nitrogen, and that animals return these elements to plants. Thus the cycle of organic matter is a double one, including a cycle of carbon and one of nitrogen.

The explanation of a "balanced" aquarium, one in which 
water snails and aquatic plants live together for a long time without change of water or addition of food, is to be found in the existence of the double cycle of organic matter. The snails feed on the plants. (Fishes which would eat the plants might be kept in the same way.) The carbon dioxide excreted by the snails is used by the plants in making carbohydrates. Plants also absorb from the water the nitrogenous excretions of the snails. Thus the excretions of the snails furnish the important elements, carbon and nitrogen, for the growth of the plants. When the plants use the carbon dioxide excretion in starch-making there is an excess of oxygen, and this, absorbed by the water, is breathed by the snails. A small quantity of the oxygen in the water is absorbed by the plants, especially when in darkness. The plants and the snails when properly balanced in an aquarium so supplement each other that they may live together indefinitely.

Such a "balanced" aquarium wherein animals and plants supplement each other is a sort of miniature of the world in general, for all plants and animals have the relations of food, oxygen, and excretions illustrated by the plants and animals in the aquarium.

119. Irritability or Nervous Reaction. - We have previously noted that higher animals have a system of nervous organs; but many of the lowest animals and all plants have no special organs corresponding to the nervous organs of higher animals in which are concentrated the powers of responding to stimuli. Nevertheless, there is in all living things the power of responding to various kinds of stimuli. This power is known as irritability, and it seems to be one of the fundamental characteristics of all living matter.

120. Digestion occurs in both animals and plants, and in each case it is a process of rendering foods soluble in water in preparation for osmosis through cell-walls. In both plants and animals digestion is accomplished by peculiar substances (enzymes), and there is a great similarity between these sub- 
stances. Thus the enzyme (diastase), which digests starch to sugar in plant cells, is very similar to enzymes secreted in the salivary glands and pancreas of higher animals, and which digest starchy food to sugar in preparation for absorption. Both in plants and animals there are enzymes which have the power to change starch, sugar, oil or fat, and proteins in various ways to fit them for absorption, transportation, or storage.

121. Water as a transporting medium for foods, oxygen, and excretions plays an important part in the life of higher animals and plants, being the basis of the blood in animals and of sap in plants. In each of these liquids, water is the medium in which are dissolved the materials to be transported. The blood of animals contains more oxygen and excretions than the same amount of sap of any plant, because greater activity in animals means that more oxygen is required, and more oxidation means that more excretions are produced. The amount of dissolved food in a given quantity of sap of a plant and blood of an animal might be the same, that is, as much sugar or other substance as can be dissolved in water at a given temperature.

There is one striking difference between the transporting liquids in animals and in plants; namely, that in animals the liquids (blood and lymph) move in a circuit from the heart around through the tissues of the body and thence back to the heart. Hence there is a circulation of the blood in most of the animals which have blood, and there is an organ (heart) for keeping up the circulation. In plants, on the contrary, there is no circulation. Water from the soil moves up in the wood-tubes of plants, and water holding in solution foods and other matters moves down in the inner bark (sieve-tubes) of plants; but there is no direct connection between the two sets of tubes, and hence no possibility of a complete circuit. However, the important point is that both plants and animals use water for transporting foods, oxygen, and excretions ; 
and whether the water moves in a circuit, as in animals, or up and down, as in plants, is a matter of secondary importance. The complete circuit in animals has for them the advantage that a swifter movement of water (of the blood) makes possible more rapid distribution of foods, oxygen, and excretions. But plants, because of the sluggish life-activities of their cells, can get along with a less rapid distribution of the necessary foods and oxygen, and excretions.

We see, then, that while sap in our common plants and blood in animals appear at first sight to be widely different, careful study of their respective functions shows that they are really doing similar work in connection with the lifeactivities of protoplasm.

122. Reproduction. - This process has many similarities in animals and plants. The frog and the bean plant are typical of the great majority of living things in that they reproduce by means of two cells which unite to form a fertilized egg-cell able to develop into a new organism. In both animals and plants the egg-cells are very similar in their microscopic structure, and in each case fertilization causes the egg-cell to begin division into a number of cells which form the embryo. The cell from the pollen-tube which fertilizes the egg-cell in flowering plants ( $\$ 75)$ corresponds to the sperm-cell of animals ( $\$ 57)$. In some flowerless plants (e.g., sea-weeds) there are motile sperm-cells which swim in water just as those of fishes or frogs do; but these plants live where water surrounds the ovaries producing eggcells and the spermaries producing sperm-cells, and so the sperm-cells can readily swim to the egg-cells and fertilize them. The absence of water surrounding the flowers of the higher plants evidently makes such a method of sperm-cells reaching egg-cells impossible; and so the method of communication by means of pollen-tubes has been developed as an adaptation to the peculiar dry conditions under which fertilization must take place in most flowers. . However, it 
should be noted that the important point concerning fertilization is that it is the union of two cells to start the development of an embryo.

The method of reproduction by generative cells or germcells (sexual reproduction), which has been illustrated by the frog and the bean plant, is the only method in a large number of species of plants and animals; but in both there are many species which may at times reproduce without germ-cells. Reproduction of certain plants from roots, stems, etc., will be described in the next chapter; and many lower animals reproduce by automatically dividing themselves. Such reproduction of plants and animals without germ-cells is termed asexual (i.e., independent of sex). 


\section{CHAPTER VII}

\section{CLASSIFICATION OF ANIMALS AND PLANTS}

123. The Scientific Names of Animals and Plants. - The student who turns over many pages of any textbook of botany or zoölogy must discover that the scientific names are quite different from the common ones. Thus our ordinary garden toad is in zoölogical terminology Bufo americanus, a dog is Canis familiaris, a house-cat is Felis domestica, a honey-bee is Apis mellifica; and so for all the known animals there is such a double name. Likewise among plants, the spring-beauty is Claytonia virginica, the garden beans are Phaseolus vulgaris, pansy is Viola tricolor, blood-root is Sanguinaria canadensis, and the liver-leaf is Hepatica triloba. These are simply examples, taken at random, of names of some common plants and animals.

This use of double scientific names is known as binomial nomenclature; and this system of naming was introduced by the celebrated Swedish naturalist Linnæus, ${ }^{*}$ who lived between 1707 and 1778. It has a number of advantages over any other system of naming. For example, the pansy is only one of many kinds of violets which are known; and it is very convenient to have the word Viola for all kinds of violets, and then tricolor to distinguish the pansy from other kinds of violets, each of which has its own special name, e.g., Viola rotundifolia (round-leaved violet), Viola odorata (sweet violet), Viola lanceolata (lance-leaved violet), and so on for about fifty species of violets which grow in America.

* See any standard encyclopedia for account of the life and work of Linnæus. 
It is obvious that the advantage of the double name is the same as in a human family. The names Smith, Jones, etc., designate families; but we must have a second name, such as John, Thomas, etc., to indicate individuals. Likewise in biology, names like Viola serve as family names to include all closely related animals or plants, and a second name is needed to distinguish the different kinds of violets. In writing the scientific name we put the family name first; but we also do the same thing with human family names when making an alphabetical list of names, as for example in a biographical dictionary like "Who's Who in America."

But why should scientific names not be in English, as for example, violet sweet, violet yellow, and violet lance-leaved, placing the family name in English form first and adding a second name to distinguish different kinds of violets? The answer is that scientific language must be cosmopolitan so that students in any language can understand; and so it has been agreed to base scientific names on the classical languages, making the names Latin in form, and largely of Latin and Greek words. Viola tricolor (violet three-colored) is understood all over the world among scientific men as meaning the plant which we call pansy, which some people call "heart's ease," and for which there are other local names in many of the languages of Europe.

Many of the scientific names are simply the Latin name. For example, Bufo americanus is Latin for American toad, Phaseolus vulgaris for common bean, Felis for cat, and Canis for dog. Sometimes the words are descriptive, e.g., all the second names of violets mentioned above, Sanguinaria referring to the juice of blood-root, and Hepatica to the shape of the leaf of that plant. Some names are in honor of the discoverers of certain plants or of prominent botanists ; e.g., the spring-beauty, Claytonia, was named for Clayton, an early botanist; and Viola brittoniana is a species of violet named for Professor Britton, the director of the New York 
Botanical Gardens. Many names of species are of geographical origin; e.g., canadensis, virginica, americanus, europea, etc.

The student of animals and plants should remember that a large number of names are no longer descriptive. For example, Claytonia virginica is found in many states besides Virginia; and many species originally named vulgaris, meaning common, have become relatively uncommon. However, this does not interfere with the use of the names as definite designations for particular kinds of animals and plants. There are many names in the human families which do not correctly describe their bearers, e.g., Mr. White, a negro; and Mr. Black, a white man; but these meaningless names serve to mark the individuals.

124. Genera and Species. - In many earlier lessons of this book the word species has been used in its popular sense, meaning a kind of animal or plant. It is now time for a more accurate definition of the word as used in biology.

The pansy, Viola tricolor, has been referred to as a species of violet. One might buy pansy seed from hundreds of dealers in seeds, and from these seeds grow thousands of individual plants which by the form of stem and leaves, and by the form and colors of the flowers, would be recognized by any gardener as pansy plants. In short, pansies constitute a species, a group of closely similar individuals.

Now, many specimens of violet plants producing yellow, blue, and white flowers might be collected; and comparison would show that the individual plants with yellow flowers are closely similar, but differ from those with white and blue flowers and from pansies. Hence the similar individual plants with yellow flowers would be named as a species distinct from the others. Note, however, that all parts of the plants must be compared, and not color alone, as the above illustration might suggest. For example, a blue violet with rounded leaves would be considered one species, and a blue violet with lance- 
shaped leaves would be considered another species. Other points of structure, such as shape and color of seeds, size of the plant, etc., enter into consideration; and so there are known to be many species of each color of violet.

All the species of violets taken together constitute the genus Viola; and in giving the double names to species the generic name Viola is followed by the names of the species, e.g., Viola tricolor.

Summarizing the above, we may define a species as a group of closely similar individuals; and a genus as a group of similar species. As an illustration of an animal genus and species, we may take the dog genus, Canis, which includes dogs, wolves, and jackals. We have no difficulty in recognizing dogs, because they have certain characteristics which distinguish them from wolves and jackals. Hence all dogs are included in the one species, familiaris. Likewise, wolves differ from dogs and jackals, and so wolves are classed in other species; and jackals in still other species.

125. Families, Orders, and Classes. - Genera (plural of genus) are grouped into families. For example, fox, wolf, $\mathrm{dog}$, and other dog-like mammals are grouped together in the dog family, Canidæ. Similarly, cat, lion, tiger, leopard, lynx, and many other cat-like animals, belonging to several genera, are grouped together in the cat family, Felidæ. The marten, mink, otter, weasel, skunk, and badger belong to a related family, the marten family. Then there is a bear family, and a raccoon family. All the animals of the dog, cat, marten, bear, and raccoon families have some general resemblances, particularly in that their structure is adapted to a flesh diet, hence they are called the flesh-eaters or carnivores. Such an assemblage of similar families constitutes an order.

Another familiar order is that of the hoofed animals, such as cow, horse, pig, sheep. Another is that of the gnawers or rodents (rats, mice, squirrels, rabbits, gophers). Another 
order contains the opossum and kangaroo, animals with a pouch for carrying the young. Now, all these animals have certain common characteristics. For example, a cow, dog, rat, and kangaroo all have hair, all feed their young with milk, and all have a diaphragm for breathing. None of these important things is found in birds, frogs, fishes, worms, and other low animals. Hence the animals which have these characteristics are considered related in one large group. And to such a group of orders the name class is applied; and the particular class to which the flesh-eaters, the gnawers, the hoofed animals, and several other orders belong is Mammalia, or mammals, a name referring to the fact that the young are fed with the secretion of milk-glands or mammary glands.

126. Phyla, or Divisions. - Classes in turn are united into larger groups. The class Mammalia and the classes to which birds, reptiles, frogs and fishes belong are all composed of animals which have a backbone or vertebral column. This larger group, which contains all animals with a backbone (fishes, amphibia, reptiles, birds, and mammals), is one of the great divisions of the animal kingdom, and has long been known as the phylum or division of vertebrates (Vertebrata, but some authors prefer Chordata).

Other primary divisions or phyla (plural of phylum) of the animal kingdom are those represented by the shelled animals (oysters and snails), by the animals with jointed legs (crayfish, lobster, crabs, spiders, insects), by the worm-like animals (earthworm, tapeworm, leech, etc.), by the jelly-like animals (jellyfish, coral-animal), by the sponge-animals, and by the simplest microscopic animals. The technical names are given in $\$ 133$, but for our present purpose it is sufficient to state that there are about a dozen such primary groups or phyla in the animal kingdom. Each such primary division may be called a division, phylum, or branch ; but phylum is used by most zoölogists. 
127. Summary of Animal Classification. - Reversing the above order of presentation, the animal kingdom is composed of divisions or phyla, each phylum o1 classes, a class of orders, an order of families, a family of genera, a genus of species, a species of individuals. And sometimes there are varieties of individuals in a species; e.g., various well-known breeds or varieties of dogs in the dog species.

The full classification of a bulldog is as follows: Kingdom, animal ; phylum or division, Vertebrata (vertebrates); class, Mammalia (mammals); order, Feræ (carnivores); family, Canidæ (dog family); genus, Canis; species, familiaris; variety, bulldog; and individual dogs, which are not exactly alike.

128. Summary of Plant Classification. - In a similar way botanists have classified plants into groups. The primary divisions of the plant kingdom are into lowest plants, mosslike plants, fern-like plants, and seed-plants. (For the technical names see $\S 133$.) Taking the division of seedplants, it is subdivided ( $\$ 209$ ) into gymnosperms (flowers in form of cones, like pines) and angiosperms (with true flowers). Taking the higher group, the angiosperms, it is subdivided into the classes of monocotyledons and dicotyledons ( $\$ 141)$. Each of these classes is composed of orders, which are subdivided into families (e.g., composite family, $\S 207$ ), families into genera (e.g., Viola, $\S 124)$, genera into species, and sometimes species into varieties (see long lists of varieties of pansy species in seed-catalogues). It is evident that the general plan of classification is essentially the same for both animals and plants.

129. Classification Based on Resemblances. - It is evident from the foregoing illustrations that the classification of animals and plants is based on the fact that there are certain resemblances in structure. Thus all vertebrates are alike in having a backbone and some other structures not found in lower animals ; all mammals are alike in having not 
only a backbone, but also hair, milk-organs, and diaphragm; all carnivores are alike in having mouth and digestive organs adapted to meat-eating in a way not found in other mammals ; and dogs and wolves are nearer alike in all respects than they are like any other animals. It is all a question of resemblances - resemblances in detail between individuals of a species, and more and more general resemblances as we pass from species to larger and larger groups (genera, orders, classes), until we find in divisions or phyla only such general resemblances as the backbone, which characterizes vertebrates. These resemblances, or points of structure which mark the animals or plants belonging to any particular group, are the characteristics. For example, the backbone is a characteristic of vertebrates, for no lower animals have it; and hair, milkglands, and the diaphragm are characteristics of mammals; the formation of seeds in a peculiar manner is a characteristic of seed-plants; the arrangement of flowers in a peculiar head is a characteristic of composites. So for each large or small group of animals and plants, from species to primary divisions, there are distinguishing marks based on resemblances.

130. The Reasons for Classification. - Probably the first reason why men of science have classified animals and plants is that it has seemed natural to group together the similar things in nature. The fact is that we seem naturally inclined to associate together things which are alike, and thereby distinguish between the unlike. Without any idea of classifying in a scientific way, we are constantly noting the characteristics of people we see; and we compare and discinguish between people of different races (white, black, red, etc.), of nations (German, English, French, Swedish, etc.), of people from different regions of a country (Northern, Southern, and Western in United States), and of individuals in families. If we see two or more strangers who look alike, we think of them as closely related, and in the same family. If we see that the members of several families have some general simi- 
larity of form, manners, language, etc., we naturally classify the families as members of the same nation. And we think of Germans, French, English, and Swedish nations as belonging to the white race; and we think of Chinese and Japanese as belonging to a different race. Also we classify men according to their political and religious beliefs and their business in life. When we stop to consider the matter, it becomes evident that we are all the time classifying things which interest us in everyday life.

Now, naturalists (biologists) have followed the same tendency in grouping similar animals and plants. Noting that dogs and wolves and foxes are very much alike, naturalists have long regarded these animals as in one family, just as we think of two similar human individuals as in the same family. And then, noting that the members of other families of animals (e.g., bear and raccoon) have some of the same characteristics, naturalists have united the families into an order, just as we unite similar human families into nations. And orders are united into classes, as nations into races of men. In some such way naturalists have been at work from before the days of Aristotle (B.c., 384-322), who wrote the first books on animals and plants, and they have gradually grouped similar forms together into species, genera, families, orders, and larger groups. Many of these groups are not yet complete, for many animals and plants have not yet been studied carefully enough to show what other species they most closely resemble.

Another reason for classification is that it is convenient for references, just as in a library it is useful to have all the books on a given subject placed together. Since there are several hundred thousand species of animals and plants, it would be quite impossible to find the descriptions and names if we had no classification. But with classification identification is easy. A new animal was found in Africa recently. It had a backbone, and was therefore a vertebrate. It had 
hair and other structures, which showed that it was.a mammal. It had hoofs and other structures similar to the cow, deer, etc., and it therefore belonged to the order of hoofed animals. And finally it had close similarity to members of the antelope family. Closer comparison with the named species of antelopes, of which descriptions have been published and specimens stored in the great museums of the world, will make it possible to determine the genus to which this new specimen belongs. And then the naturalist who writes a description of the specimen will give a name to the species, so that the next man who looks for the name of this animal can find it in the proper place in the scheme of classification.

Such, in essentials, is the story of the use of systems of animal and plant classification whenever any one wants to give a name to a new specimen or to find out whether it has been given a name. It is simply necessary to begin with the characteristics of the larger divisions, and then work down through classes, orders, families, genera, to species ; and since the great books on classification (e.g., Gray's "Manual of Botany," or Jordan's "Manual of Vertebrates") are arranged in this order, it is easy for one who carefully observes and compares to trace out the name of a plant or animal specimen. This practical use of classification is made every day by numerous people who seek the names of organisms for the pleasure of feeling acquainted with them, or by biologists whose professional work makes it necessary for them to find the names of their specimens.

131. Practical Work in Classification. - (D) A small collection of crayfishes, shrimps, lobster, and various species of crabs may be used to illustrate some of the chief principles stated above regarding species, genera, and larger groups. A collection of insects in alcohol is especially excellent for the same purpose. The specimens of a mixed collection should be arranged in groups, according to obvious similarity to grasshopper, butterfly, beetle, dragon-fly, and house-fly. This exercise can be made to illustrate the leading principles of classification without going into detailed study of the in- 
sects at this time. Later, after study of insects in Chapter XIV, the students should have practice in identifying common insects with reference to the orders represented by those named above. For the present purpose it will be sufficient to speak of grasshopper group, beetle group, butterfly group, crayfish group, crab group, etc., leaving the technical names of these until later lessons.

For plant classification, a set of leaves, fruits, branches, bark, etc., of two or more species of some common plants, e.g., oaks, maples, or others available. In the spring of the year, members of the rose family (apple, pear, peach, plum, cherry, quince, wild rose, etc.) will furnish some interesting materials for classification; and in the autumn there are many composites which might be used in the same way.

132. Learning Scientific Names. - It is not necessary that all the technical names given in the following tables of classification be memorized at one time. The best way to learn them is through use. Only the more important names have been given, and many of them are rapidly becoming common terms. In fact, few people realize that many common names are also the scientific ones. For example, among common plants grown in home gardens: asparagus, spinacia (spinach), aster, alyssum, calendula, centaurea (sometimes called bachelor's button), chrysanthemum, phlox, crocus, cosmos, iris, smilax, narcissus (sometimes called daffodil), tulipa (tulip), hyacinthus (hyacinth), yucca, lilium (lily), viola (violet), petunia, portulaca, salvia, thymus (thyme), zinnia, verbena, heliotropum (heliotrope), - all these and many more are the scientific names of the genus in each case; but since for most of these plants there is no common name, the scientific name has become also the common one. This use of scientific as common names should be encouraged whenever there is confusion. Such a case is that of "marigold," which has been applied to so many yellow-flowered composites that even gardeners who have not studied botany are learning to use the scientific names calendula, coreopsis, and tagetes as common names. Seed-dealers are helping 
popularize such names by putting the scientific names on packages of seeds.

It is evident from the above list of scientific names made common by popular usage that there is no truth in the statement that scientific names are hard to learn. Any one who can learn such a name as chrysanthemum can surely learn most other scientific names when they are needed. And in order to be definite, they are often needed. Therefore the student should drop all prejudice arising from the supposed difficulty of scientific names, and aim to make frequent use of the most common ones.

133. Chief Group of Animals and Plants. - The tables below are intended to show the most important of the primary subdivisions of the animal and plant kingdoms. The lowest group is named first and the highest last, but in the case of the animals there is doubt as to the relative position of some of the intermediate groups ; for example, whether annelids are higher than echinoderms, or arthropods higher than mollusks.

\section{ANIMALS}

(A primary group of animals usually called a phylum, plural phyla.)

Phylum

Phylum

Phylum

Phylum

Phylum

Phylum

Phylum

Phylum VIII. Arthropoda (lobster, erab, spider, insects).

Phylum IX. ^Mollusea (clam, oyster, snail, cuttle-fish).

Phylum X. Vertebrata (backboned animals).

The large textbooks of zoölogy include several other phyla of animals not belonging to any of the ten groups named above. Most of these are small marine worm-like animals 
which few except zoölogists have occasion to examine. The most common animals belong in the ten phyla named above.

The subdivision of most of the above phyla into classes is given at ends of the chapters in Part III of this book.

Phyla I-IX inclusive are often called Invertebrates or backboneless animals.

\section{PLANTS}

(There are four grand divisions or primary groups of plants).

Division I. Thallophyta (simplest plants).

(1) Algæ (with chlorophyll). Examples: seaweeds, many minute aquatic plants.

(2) Fungi (without chlorophyll). Examples: molds, mushrooms.

Division II. Bryophyta. Examples : liverworts and mosses. Division III. Pteridophyta. Examples : ferns, horsetails, lycopods.

Division IV. Spermaphyta (or Phanerogamia)

(1) Gymnospermæ. Examples : cycads and conifers.

(2) Angiospermæ.

(a) Monocotyledones. Examples: palms, grasses, lilies, orchids.

(b) Dicotyledones. Examples: most of the common trees, vegetables, and "flowers."

The plants in Divisions I, II, III, are of ten termed "SporePlants," "Flowerless Plants," or "Cryptogams" ; while those of IV are "Seed-Plants" or "Flowering-Plants." 


\section{PART II}

\section{PRINCIPLES OF BIOLOGY ILLUSTRATED BY TYPES OF PLANTS*}

PART I has given an introduction to many of the general facts and principles concerning animals and plants. It has been possible to do this by studies of one animal and one plant, because in a general way there is great similarity among living things. But the introductory study will lose much of its value as useful science unless the reader goes on to study illustrations and applications of the general principles. Especially would one who had studied but two living things have a very limited knowledge of the various forms of animal and plant life, and of the many remarkable modifications of structure of organisms which adapt them for carrying on the life-processes in various ways. In order to extend our knowledge of the principles of plant biology, we shall now make a series of comparative studies of seed-plants or floweringplants, and later we shall study some of the most common flowerless plants (moss, fern, mushroom, etc.).

* To the TeAcher: For possible changes in order of study of this and later chapters see suggestions in the preface of this book and especially Chapter VIII of the "Teachers' Manual of Biology," which accompanies this book. 


\section{CHAPTER VIII \\ STUDIES OF SEED-PLANTS}

134. This chapter will direct the study of the parts of seedplants which are most modified in adaptation to various habits of life. Since many seed-plants are peculiar only with regard to some one organ, it is most interesting to study and compare corresponding parts of various plants rather than compare entire plants with each other. Accordingly, we shall study (1) types of seeds and their germination, (2) roots, (3) stems, (4) leaves, (5) flowers, (6) fruits - in each case giving attention to special uses of these organs in the lifeactivities of the plants which possess them. Prominence is given to the seed-plants because they are so common and so important economically.

\section{TYPES OF SEEDS AND SEEDLINGS}

135. The bean seed has served as an introduction to the structure and germination or awakening of seeds; but without examination of such seeds as pea, squash, castor-oil plant and corn it will be difficult to understand the peculiarities of germination which one may see in any garden where many different kinds of plants are grown. In $\S 140$ is given a special list of seeds for optional study by members of the class who work rapidly and at the same time carefully, or who are in advance of the class because some of the germination studies are reviews of nature-study lessons taken in the elementary schools.

136. Pea Seed. - $(L)$ Materials: dry peas, both smooth and wrinkled varieties; some soaked in water 24 hours; seedlings of 
various stages grown on moist paper ( $(81)$, which may be preserved for years in 5 per cent formalin solution; some peas planted one week, and some two weeks before the lesson, some in soil in pots and some in a tumbler or lamp-chimney arranged as described in $\S 81$; and some seedlings with hypocotyl marked with waterproof ink to determine the region of most rapid growth (see Fig. 34, bean).

In connection with this lesson on seeds consult figures in Atkinson's "First Studies of Plant Life."

Examine pea seeds, following the directions given for the bean (\$79). Examine seedlings of various stages and compare with those of the bean. Especially compare the ways of the two plants in getting out of the soil. Make sketches showing structure of the pea seed and stages in its germination. Write in your note-book a short paragraph comparing the germination of pea with bean seeds.

Recall that the stem part of the hypocotyl (that is, the stem between root and cotyledons) elongated rapidly during germination of bean seeds and pushed the cotyledons out of the soil (consult your labeled drawings); and then try to explain why the cotyledons of the pea seed remain where they are planted in the ground. Examine specimens of pea seedlings whose hypocotyls have been marked with waterproof ink (see Fig. $34, \S 81$ ), and determine whether the root has been growing downward, or the stem just below the cotyledons elongating rapidly, or both. Make sketches and compare with corresponding sketches of marked bean seedlings.

137. Squash Seed. - $(L)$ Materials : dry squash seeds; seeds soaked in warm water for 12 hours; seeds soaked for 24 hours; seeds in various stages of germination (may be preserved in formalin); seeds planted in soil two or three weeks in advance of lesson; and also seeds placed in various positions in a lamp-chimney or tumbler as in $\S 81$, so that the students can follow the changes from day to day.

Examine first a dry seed, and notice the color and texture of its covering (seed-coat, or testa). At the pointed end is the hilum and inside this is a small hole, the micropyle - this is best seen in a soaked seed.

Open a soaked squash seed (this can easily be done by first cutting around the edge of the flattened seed, and then inserting the knife and prying it open). Remove the green covering from the "kernel," and examine the embryo. At the small pointed end is the hypocotyl. Separate the two cotyledons from each other and note that they are fastened together only at the hypocotyl. Follow development of squash seeds planted in soil, in tumbler as in $\S 81$, and laid on paper 
kept moist. Notice a peculiar hump (the peg) which develops on the hypocotyl and when that grows longer the seed-coats are pried open. Notice how the stem part of the hypocotyl is looped as it comes from the seed-coat. In specimens planted in the ground this loop is the first part to appear, soon dragging the cotyledons out after it, and then the looped stem straightens out and lifts up the two green cotyledons.

Compare cotyledons of squash and bean seedlings at various stages until the second pair of leaves develop, and write a brief account.

Take a squash seed that has sprouted, and open as before. Between the cotyledons, and fastened to the upper end of the hypocotyl, is the epicotyl, which is too small to be easily seen in a dry seed and one must look for it after it has grown larger. Examine the epicotyl in seedlings of various sizes.

Compare a squash seed with a bean seed in the following points : texture of seed-coat, number and thickness of cotyledons, the more leaf-like cotyledons, and relative size of epicotyl. Record these facts in tabular form.

138. Seed of Castor-Oil Plant. - $(L)$ Materials : dry seeds (poisonous). Seeds soaked in water for at least 24 hours. A series

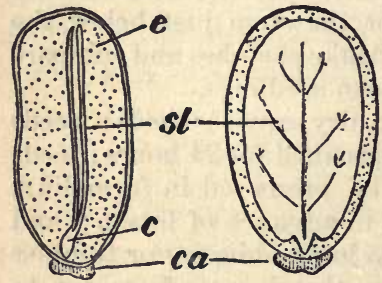

FIG. 42. Castor-oil seed. $c$, hypocotyl; st, cotyledon: $e$, endosperm. (From Osterhout.) of sprouted specimens to show the development of the seedling up to the time the epicotyl is well developed.

Examine dry castor-oil seeds and note color and markings. The meaning or use of these is not known. In fact, it is not necessary to assume that they have a use; for hundreds of beautiful minerals are buried, and therefore their color as seen in the light cannot be of use. Some authors have imagined that castor-oil seeds resemble beetles or ticks so closely that they would be avoided by birds, but there is no scientific evidence to support this theory. Examine a castor-oil seed which has been soaked in water. The point of attachment in the ovary (that is, the hilum) and the micropyle are near the rounded projection at the small end of the seed. Crack the shell-like seed-coat, and carefully remove it. The "kernel" thus exposed is covered with a delicate membrane (inner seedcoat). Carefully remove this. The double "kernel" will at first be taken to correspond to the embryo of a bean with very thick cotyledons; but by carefully separating the two halves of water- 
soaked seeds, and especially some which have begun to germinate, it will be found that most of the two halves taken to be the cotyledons like those of the bean are masses of stored food (really numerous cells stored with food) and that between them, in the center of the seed, are two delicate white cotyledons joined to the hypocotyl, which is at the hilum end of the seed. Carefully separate the cotyledons from the food-masses (called endosperm), taking care not to break off the hypocotyl. The embryo will thus be freed from all other parts of the seed. Examine the parts of the embryo, especially in seeds which have begun to sprout. Notice the epicotyl.

Examine a series of seedlings from castor-oil seeds planted in soil ; some placed in a tumbler as in $\$ 81$; and some kept on moist paper. Find out how the embryo gets out of the seed-coat; how it comes out of the ground; what becomes of the endosperm ; why the hypocotyl remains bent so long; development of the cotyledons ; compare the seedlings with those of bean and squash previously studied. Make a series of sketches showing stages in germination of castor-oil seed.

139. Corn Grain. - $(L)$ Note : By corn is here meant Indian corn or maize. In the United States the word is always so understood; but in other countries the word is used in its original sense, meaning grains of any cereals - corn, wheat, oats, barley, rye. It is called Indian corn because found under cultivation by the Indians when America was discovered by Columbus. The name maize is from the Indian name, and the scientific name is Zea Mays.

Materials : dried grains or kernels of sweet, "field," and popcorn; dried ears of corn; "green" corn on the cob (fresh or in formalin solution); some ears with husks; very young ears showing the attachment of the silk; grains of yellow "field" corn soaked in water for several days; sprouted grains in all stages of development (may be preserved in formalin).

Examine specimens of ears of corn with and without the husks removed. The central axis or cob is really the mature form of a sort of flower-stalk on which there were as many flowers as there are grains on the ear. Each individual flower which forms a grain has only the pistil, the petals and sepals being absent and the stamens in separate flowers in the tassels at top of the corn plant. Since each pistil develops into a grain, this is therefore a fruit in the botanical sense ( $\$ 212)$; and each flower produces one fruit containing one embryo. In the bean each flower produces one fruit (the pod), but this fruit happens to have several seeds, each with an embryo, that 
is, one bean flower produces several embryos. A bean pod with one seed would be equivalent to a grain of corn because it would be one seed produced by one pistil.

Examine arrangement of grains on the cob. The attachment of each grain corresponds to the flower-stalk or pedicel of the beanpod. At the opposite (free) end of the grain is the withered base of the style, best seen as a sharp point in some varieties of popcorn. In immature ears it is easy to see that a thread of so-called "corn silk" (each thread is really a greatly elongated style) is attached to the free end of each grain. The "silk" extends from each grain in the groove between two rows of grains to the end of the cob where the surrounding "leaves" or "husks" are arranged so that the ends of the "silks" are exposed. These exposed ends are the stigmas, one for each flower (represented by a pistil, and in the mature ear by a grain). Pollen-dust from the tassels (which are flowers with stamens only) falls or is blown by the wind upon the exposed "silks" or stigmas. Down each "silk" or style a pollen-tube grows to the ovule in the ovary. Then the ovary with its ovule develops into a grain of corn with an embryo corn plant.

Examine grains of field, sweet, and pop-corn and notice on one of the flat sides of each grain an oval patch (usually a groove in dry grains) which marks the position of the embryo beneath the covering of the grain. Look at an ear of sharp-pointed pop-corn and determine whether the embryo is on the side toward the stalk or toward the free end of the ear, and also note in which direction the remains of the style is turned. Do you see any reason for this in reference to position of the "silks"? Now look at an ear of "field" corn and compare. The style is not so prominent in this variety, but it is a small elevation between embryo and end of grain.

Imagine an ear of corn split longitudinally, and make a diagram showing relation of grains to cob, and also indicate by dotted lines the position of "silks" extending from a few grains.

Remove the covering from a water-soaked grain or from "green" corn (fresh or preserved in formalin). This covering is the "hull" which is removed when making hominy or samp. In the oldfashioned method of preparing hominy the grains were first boiled in lye from wood ashes which loosened the hull, and then soaked in water to remove the lye.

With the sharp point of a knife, lift out the corn embryo (sometimes called the "germ"). The remainder of the grain (endosperm) is food for the embryo. In the yellow varieties of corn the endosperm is yellow and the embryo whitish in color. 
In order to identify the parts of the embryo, it is best io examine first some stages in germination.

Examine a corn seedling just coming out of the soil. Only the epicotyl appears as a coil of leaves rolled up with the younger leaves within. Dig up such a seedling, or study a similar one grown on moist paper, and notice that the root or roots and the epicotyl grow from the embryo part of the grain. Make a drawing of such a stage. The part of the embryo which still remains embedded in the endosperm is thought to be the single cotyledon, which remains within the seed-coat as an organ to digest the endosperm and then to absorb the digested substances and transfer them by osmosis to the growing parts of the seedling.

The first root appears at the end of the grain which was attached to the cob. Look for it in grains before germination begins and label it hypocotyl in drawings. Recall that the hypocotyl in bean and squash seeds consisted of two parts or regions, stem and root (consult your drawings). Could the corn grain remain in the ground if part of the hypocotyl grew into an elongated stem (that is, a stem between root and cotyledons) as it does in bean and squash? Write a brief paragraph comparing the corn grain and pea seed with regard to behavior of epicotyl, cotyledons, and hypocotyl.

Make transverse and longitudinal cuts through water-soaked (or preserved "green") grains of corn. Identify endosperm, hypocotyl, epicotyl, and cotyledon by comparing with germination stages studied as above. Make sketches.

140. Other Seeds. - The following are suggested for study by pupils who satisfactorily complete the preceding lessons in advance of the class. Use dry and water-soaked seeds and seedlings in various stages of germination as in preceding lessons. Grow some in flower-pots. Look for the same parts of embryos and compare their development as the seeds germinate. Make drawings and compare similar seeds.

Sunflower Seed. - The so-called "flower" of the sunflower plant is really a group or head of small flowers, and each flower forms one "seed." The hard seed-case is wall of ovary, corresponding to pod of bean. The inside delicate coat is the real seed-coat corresponding to that of a bean. Find two cotyledons, hypocotyl, and epicotyl. Compare germination with that of squash seed.

Four-o'clock Seed.- One flower produces one seed and the outer coat is wall of ovary. The two large cotyledons are folded around a central mass of white endosperm. Compare with position of endosperm in castor-oil seed. 
Buckwheat Seed. - Compare with the four-o'clock.

Morning Glory. - Embryo with two cotyledons is embedded in the endosperm. Epicotyl not apparent until germination.

Seeds of Oak (acorn), Horse-Chestnut, Windsor- or Horse-Bean, and Lupines. - Compare with the pea seed, especially behavior in germination.

Peanut. - A nut-like pod, comparable to pod of the closely related bean.

Onion Seed. - Embryo is embedded in endosperm. Peculiar in that the one cotyledon lengthens in germination and raises the seed-coat with endosperm out of the ground. When endosperm is all absorbed cotyledon withers at top and epicotyl appears near the surface of soil. Compare with germination of the corn grain.

Wheat and Oats. - Germinate on moist paper and in soil, and compare with corn.

141. Number of Cotyledons in Seeds. - Some of the seeds studied have one and some two cotyledons. This difference in number has been made the basis of classification of a large number of the flowering or seed-plants into the monocotyledons (one cotyledon) and dicotyledons (two cotyledons). In addition to the number of cotyledons, plants of these two groups are marked by certain characteristics of stems and leaves and flowers which will be described later $(\$ 161$ and $\S 197)$. Among the monocotyledons are all true grasses such as timothy, June-grass, lawn-grasses - and all the lilies, tulips, daffodils, iris, orchids, banana-plants, trillium, pineapple, palms, etc. Among the dicotyledons are the familiar trees of our deciduous forests, the fruit-bearing trees and shrubs of our orchards and gardens, all our most common garden vegetables (except onions and asparagus), and most of the common wild flowers which do not resemble lilies in leaves and flowers.

Many of the common cone-bearing evergreen trees (pine, yew, fir, cypress, hemlock, etc.) have seeds with more than two cotyledons (from 3 to 15). The cone-like flowers (as well as other parts) of such plants are different from the 
flowers of the ordinary flowering plants, and they will be described later $(\$ 211)$.

142. The Work of Cotyledons. - It is evident from the seedlings studied in the laboratory that cotyledons have several kinds of work. (1) They may become leaf-like and for a time serve as leaves (squash, sunflower). (2) They may be simply store-houses of food materials and without the functions of ordinary leaves, not even getting out of the soil (pea, acorn). (3) They may be of use only as an organ for digesting and absorbing food stored in endosperm around the cotyledons (corn). (4) Some cotyledons combine functions 1 and 2. As an example, the bean cotyledons become green and do some work as leaves, and also are stored with food. This is true in all seedlings which have very thick green cotyledons rising above the ground and no endosperm. (5) Functions 1 and 3 may be combined. For example, the cotyledons of the castor-oil seed and onion are leaf-like, but they remain in contact with the endosperm until it has been absorbed. This is the case in all seedlings with cotyledons which rise above the ground, with endosperm in the seed, and with the seed-coats pushed out of the soil and remaining around the endosperm until it is absorbed.

Observe, when opportunity offers, seedlings of various wild and cultivated plants. It will be interesting to reserve a page in your note-book for "Work of Cotyledons" and make records in five columns headed as follows: (1) As Leaves; (2) For Food-storage; (3) As Absorbers; (4) As Leaves and storage; (5) As Leaves and absorbers. (The numbers refer to those used above.)

Cotyledons are usually equal in size, as in bean and squash seedlings; but in some species of plants one is larger than the other. In some cacti both cotyledons are exceedingly small (rudimentary); and in still other seeds only one cotyledon is rudimentary.

143. The Epicotyl. - The epicotyl (sometimes called plumule) is found well developed in some seeds before germina- 
tion, especially in seeds like bean with so much stored food that the cotyledons cannot do well the work of leaves and in seeds like corn and pea with cotyledons that do no work as leaves. Under such conditions it is important that the seed have a well-developed epicotyl ready to put forth leaves at an early stage of germination. In seeds like the squash with cotyledons which are leaf-like, the leaves from the epicotyl are not needed so early and there is time for further development of the small epicotyl after germination begins.

When you notice either a large or small epicotyl in a seed, look for such relations of the work of the cotyledons as is suggested in paragraph above.

In cases like corn and pea where the hypocotyl does not push up the cotyledons, the epicotyl lengthens and rises above the ground.

144. The Hypocotyl. - It has been noted in the laboratory work that in the seeds of squash and bean the hypocotyl of the ungerminated seed develops into part stem and part root. In these cases the stem part of the hypocotyl grows in length and pushes the cotyledons upward out of the soil, while the root is growing down into the soil. The stem part of the hypocotyl of the pea and corn does not lengthen, and so the cotyledons remain below ground, their place as first leaves above ground being taken by the epicotyl.

145. Conditions of Germination. - It is easy to demonstrate that water, oxygen (from air), and proper temperature are necessary for germination.

Water. - That water is necessary requires no special experimental proof, for every one knows that seeds kept dry remain dormant, and that seedlings do not "come up" in gardens when the soil is dry, as in midsummer. Many hard-coated seeds have special ways of letting in the necessary water, such as the spongy mass at end of castor-bean, and the holes in a cocoanut. Other seeds must remain in the soil 
for months until freezing or decay of seed-coats cause cracks into which water can enter. Gardeners often crack certain hard seeds, like peach and various nuts, or file or cut notches in others, and still other kinds are soaked and softened in hot water before planting. By soaking seeds for various lengths of time in red ink it is possible to trace the paths taken by entering water, usually the same as that taken by the sap while the seed was developing.

Proper Temperature. - This also requires no special demonstration. Any one who has ever been interested in a garden knows that warm weather is necessary, and that some kinds of seeds will germinate in early spring when the soil is still cold and that others require warm soil. By simply placing some seeds on a moist paper in an ice-box and others of the same kind in warmer places one could show the effect of temperature, and after many trials it would be found that there is a best or optimum temperature for each kind of seeds. Seed-catalogues and books on gardening usually give the necessary information regarding best temperatures for germinating common vegetable and "flower" seeds.

Air, Oxygen. - That the oxygen of the air is necessary might be expected because a germinating seed is a living plant, and we have learned that all protoplasm must have oxygen. It can be proved by placing seeds in a bottle and pumping out the air with an air-pump, or by filling the bottle with pure nitrogen gas made with a chemist's generator. That carbon dioxide is formed in germinating seeds can be proved by the lime-water test - simply stand a small cup or vial containing lime-water in a larger, carefully stoppered bottle containing germinating seeds. Or use the method described in $\$ 26$, suspending seeds in a bag of netting during germination, and then after lifting out the bag, pour limewater into bottom of the jar.

The student is advised to consult Chapters I and II of Osterhout's "Experiments with Plants" for numerous experiments and 
information regarding many points concerning the physiology or activities of germinating seeds.

\section{ROOTS OF SEED-PLANTS}

146. Structure and Kinds of Roots. - The structure of all roots of seed-plants is essentially like that described for the bean plant; namely, bark or rind with its outermost layer of epidermis, a central woody axis with tubes for conducting water upward, and the epidermal root-hairs near the ends of the young rootlets. See $\$ 67$.

As to the kinds of roots, some plants have a main root which grows downward and gives off smaller roots laterally. This is a tap-root; carrot and radish are good examples. In many plants no such main root can be distinguished. Examples are the much-branched roots of Indian corn and many grasses; and such roots are called fibrous. Taproots when thickened by food-storage are said to be fleshy (e.g., carrot). It should be noted that a carrot or radish as seen in markets is not all root, for the upper end is thickened stem with a bud for next season's growth.

$(L)$ Pull up as many different kinds of cultivated plants and weeds as possible, examine the roots, and make sketches of the different forms of roots seen.

147. Roots as Anchors and as Absorbers. - The two primary functions of roots, anchoring plants in soil and absorbing water with useful substances in solution, are carried on in all roots essentially as described in the preceding chapter on the work of the bean plant (see $\S 86$ ).

148. Roots Fitted for Growth in the Soil. - The root-cap, already studied ( $\$ 68$ ), protects the growing tip from injury as the root pushes between the particles of the soil. The fact that increase in length takes place near the growing tip (Fig. 34) may be of advantage in curving to pass around stones and other obstructions, and in turning towards moisture and food. The primary root grows downward. 
Its branches (secondary roots) grow out sidewise, and the branches grow in various directions, so that they finally result in a complicated root-system with distribution of roots through a large mass of soil. This better fits roots for absorbing from the soil and for anchoring the plant.

149. Extent of Roots. - If one pulls up a plant, such as most common weeds, it becomes evident that if all the little roots were cut and placed end to end, the total extent would be very great. But this would not give an adequate idea of the total extent, for hundreds of small roots are broken in pulling up a plant, as can be proved by comparing a plant pulled from the soil of a pot with one which has been grown without soil. It has been estimated that the total length of roots of a corn plant is over a thousand feet and that a large squash plant has many miles of roots. In ordinary garden soil roots are often found several feet below the surface. In dry regions roots of alfalfa (a kind of clover) are said to go down twenty to thirty feet. However, the great majority of roots of most plants are not far below the surface of the soil, but they extend long distances horizontally.

In addition to the great length of roots, the root-hairs may increase the absorbing surface so much that a plant with roots of a total length of one foot would equal roots of from twenty to fifty feet without root-hairs. These are estimates made by botanists who have carefully examined roots of various plants.

The tendency of many roots to extend far from the stem makes transplanting more difficult because so many small roots are necessarily cut off and left in the soil. Gardeners and nurserymen overcome this by transplanting young plants several times, thus forcing the growth of small roots in a cluster near the stem. In order to prepare large trees for moving, it is often necessary to begin several years before and cut off annually a few large roots several feet from the stem so that new branch roots will be grown near the stem 
before the last long roots are cut at the time of removal to a new location. One firm of tree dealers keeps on its grounds large trees worth several hundred dollars each, which for many years have had their roots cut back in preparation for sale and removal.

150. Food Storage in Roots. - It is a well-known fact that, in addition to the two primary functions of anchoring and absorbing, many roots (turnip, beet, carrot, parsnip, sweet potato, etc.) become thickened and thereby useful as food for man and some animals. However, the primary purpose is not storage for man and animals, but for the plant's use later. Many plants which thus store food in roots use it in the second year for the development of flowers and seeds, and then the plants die. Such are biennials or two-year plants. Other plants which live many years (e.g., rhubarb and asparagus) store food every year during the summer to be used for early growth in the next spring. In fact, when one sees either cultivated or wild plants growing rapidly in very early spring, it is very probable that food stored in the roots furnishes the materials for such rapid growth. This is the explanation of the early flowering of crocuses, daffodils, tulips, hyacinths, snowdrops, spring beauty, and numerous other plants which bloom in very early spring.

Roots of many species of plants have long been one source of human food; and in recent years the European practice of feeding roots of turnips, mangels (a kind of beet), ruta bagas (a kind of turnip), and carrots as a part of the food for farm animals has been adopted in many places in America.

151. Adventitious roots are those given off by the stem or parts of the plant other than roots. The aërial roots of poison ivy, the brace-roots of corn, and parasitic roots of dodder are good examples. Cuttings of stems of willow, poplar, and garden tradescantia placed in bottles with water will soon form adventitious roots. Gardeners start many plants like geranium, coleus, begonia, and numerous other 
greenhouse plants from slips or cuttings of stems which form such roots when set in moist sand. In order to prevent erosion along river banks, stakes cut from branches of willows are often driven into the soil and soon form adventitious roots and put forth leaves, forming young trees. Strawberry runners (which are branches of stems trailing along the ground), blackcap raspberry stems, and numerous common weeds "take root"; that is, form adventitious roots at the nodes. Bryophyllum leaves and those of some begonias form roots when placed on moist soil, and develop new plants.

152. Root-tubercles. - These were mentioned in connection with the bean root ( $\$ 67)$. Only by the aid of the bacteria which live in the root-tubercles is it possible for plants to make use of the nitrogen which constitutes over two thirds of the air. These bacteria have the peculiar power of combining nitrogen with other elements derived from the soil, and the nitrogen compounds thus formed are absorbed by the roots and used by the plant just as it uses the nitrogen in manures and in such soil fertilizers as nitrate of soda.

153. Special Adaptations of Roots. - The great majority of plants have roots whose functions are (1) anchoring the plant, and (2) absorbing water and dissolved substances from the soil; but some roots have interesting modifications of structure to adapt them to other kinds of work. Such modified structures which are fitted to special work or functions are called adaptations. Some of the most important of such specialized root structures are aërial roots, prop-roots, water roots, breathing roots, and parasitic roots. These will be described in this order.

(1) Aërial Roots. - Many plants develop roots in the air; that is, above the soil. Such roots may serve as clinging organs, as in poison ivy, trumpet-creeper, and English ivy; and on some plants they are important absorbers of water. Many so-called "air-plants," from tropical countries, such 
as some orchids and ferns common in greenhouses, are attached to branches of trees, but do not have parasitic roots penetrating and absorbing sap from the supporting tree. Instead, their roots absorb water from the moist air and also that which drips from leaves and branches.

(2) Prop-Roots. - On some plants roots start from the stem above ground and grow down into the soil so as to form bracing or supporting structures. Every one who has cultivated Indian corn must have noticed the brace-roots or prop-roots which start from nodes above the soil and grow down into the soil. These help the ordinary roots in bracing the tall stalk (stem) against winds, and also it is probable that they advantageously increase the number of rootlets available for absorption of water while the plant is maturing. Other examples of roots forming supports or braces are in the famous banyan trees of India, whose aërial roots descend from branches to the ground and form many additional supports and absorbing roots for the tree. A single tree may cover a space 300 feet in diameter. Mangrove trees, which grow along the sea-coast in tropical regions, form braceroots from both main stem and branches, and these roots grow down into the mud.

(3) Water Roots. - Roots of many plants cannot live in water. This is the reason why many trees die when a new dam has raised the water level. Some plants, however, have roots which can live in either soil or water, e.g., willows. Many aquatic plants have roots adapted to living in water only, e.g., duckweed and water-hyacinth.

Many aquatic roots have no root-hairs. Such roots are usually much branched and have the required amount of absorptive surface without root-hairs, Some aquatic plants can live entirely without roots, absorbing by their submerged stems and leaves.

(4) Breathing Roots. - Reference has already been made $(\S 105)$ to the fact that roots get some oxygen from air in 
the soil ; but the mangrove trees mentioned above often grow in mud covered with water, which interferes with aëration. Certain species of mangroves overcome this difficulty by the development of branch roots which grow up into the air out of the mud and water. These roots have special air-passages for conducting air down into the roots, and they take up oxygen and discharge carbon dioxide as do other plant tissues exposed to the air; that is, they breathe.

(5) Parasitic Roots. - Many species of plants have peculiar roots adapted for penetrating the epidermis of other plants and thus forming a close attachment for absorbing the necessary foods. Examples are the mistletoe, which grows on the bark of oaks, apple, and other trees; and the common dodder, which has root-like branches penetrating other plants from which it can absorb suitable foods. These are cases of plant parasites; and this condition in which one plant (the parasite) absorbs its food from another plant (the host) is parasitism. The mistletoe has pale green leaves and can make some starch, so it is only in part a parasite. The dodder has no roots in the soil, only some very small scale-like rudiments of leaves without chlorophyll, and a yellow, string-like stem with very little chlorophyll. Evidently it must depend upon other plants for the foods which ordinary plants get from the soil and make in their green leaves. In recent years the dodder has been accidentally planted in many fields by seed mixed with those of clover and alfalfa', and has caused great damage by absorbing food from the plants with which it makes a root-like connection. For more facts concerning the relation of this weed to agriculture, read Farmers' Bulletin 306, "Dodder and its Relation to Farm Seeds."

154. Binding Action of Roots. - After any heavy rainstorm one can see that bare soil, such as on roads and cultivated fields, has been washed away where water has flowed rapidly, while grassy sod has not been so affected. This 
illustrates an important work of roots, that of holding particles of soil together. Cutting a forest from a hillside or sloping land allows rapid washing, and large tracts of valuable land have been ruined by removal of fertile soil and by formation of deep gullies. See books and pamphlets on forestry.

The same erosion often occurs when cultivated fields are bare during the winter. Even if deep furrows or gullies are not formed, it is evident that the muddy water which always flows down bare slopes is carrying away large quantities of the fertile top soil. This has not been sufficiently understood by many farmers in the past, but its importance has been so clearly demonstrated that the scientific farmer of to-day avoids leaving bare during the winter cultivated soil which is sloped so that water tends to wash it away. Protection against erosion is accomplished by planting in the late summer some winter crop, either after harvesting a cultivated crop like corn and vegetables, or between the rows after the last cultivation, or in cultivated orchards and vineyards. This winter crop may be wheat, rye, or grasses to be harvested in the following summer, or a " cover crop " of certain clovers, vetches, rye, and other plants which make great root growth in the autumn, largely prevent erosion by the rains of the winter and early spring, and add to the fertility of the soil if plowed under in the next year's cultivation.

155. Propagation from Roots. - While many stems readily develop roots, most roots do not form stems. Exceptions are sweet potato, which is a thickened secondary root, and roots of the osage-orange tree. In order to start new plants of sweet potato or osage-orange, it is simply necessary to bury root-cuttings in favorable soil. A sweet potato will form stems on any part of its surface; but roots like carrot, beet, horse-radish, and turnip soon die when the stem end or bud is removed. Some plants (e.g., dahlia) form a cluster 
of roots at the base of the stem; but each one of these can grow a new stem only at the upper or stem end. Many of the root-like structures from which new stems originate are really underground stems (see $\S 176$ ). A familiar example is the ordinary potato.

\section{STEMS OF SEED-PLANTS}

We have seen in the case of the bean plant that the stem has certain definite functions in the life of the plant. Most of the statements made concerning the structure and functions of the stem of the bean plant ( $\$ 69)$ are true of higher plants in general. However, stems of many plants have modified structure and special functions; and we should have a very limited knowledge and appreciation of plant stems if we confined our study to one kind of stem. Hence, the following lessons provide for study of the more important facts concerning stems which have not already been presented in connection with the bean plant.

156. Twigs. - This is a popular term applied to pieces of stems, or to small branches of trees and shrubs. They are very convenient for study, because they illustrate on a small scale the general structure of the entire stem of the plant from which they are taken.

Horse-chestnut Twig. - $(L)$ Or twigs of hickory, ash, box-elder, ailanthus, linden, sycamore, etc. Note that the bud at the end of the twig (terminal bud) is larger than the buds along the sides (lateral buds). Observe the positions of the buds along the stem are they alternate or opposite to each other? What is the relation of the arrangement of each successive pair of buds to each other? Observe the large horseshoe-shaped scars immediately beneath the buds. These are leaf-scars. What is the meaning of the markings on the scars? What is the position of a bud with reference to the leaf? Look for other kinds of sears on the twig. The very small scars scattered irregularly over the stem are lenticels or breathing pores $(\S 105)$. The nature of the rings of scars will be made evident after a study of the bud. Often one can find a large sear in the fork 
of a branch; this is a flower-sear. On a much larger twig, observe the arrangement of the branches - how does it compare with the arrangement of the buds already noted? This is what we should

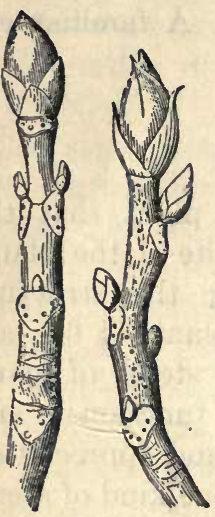

Frg. 43. Left twig, horse-chestnut ; right one, hickory. (From Gray.) expect if the branches are merely developed buds, as stated in the definition of a bud in the next section.

Make a sketch of the twig which has been studied, showing as many of above points of structure as possible. Compare with as many twigs as possible - lilac, apple, cherry, tuliptree, butternut, beech, and others named above as possible substitutes for the horse-chestnut branches.

157. Buds. - A bud is a growing point on a plant which when conditions are favorable will develop into a leafy branch, a flower, or both. In temperate regions where plants must withstand a long cold winter, trees and shrubs have formed the habit of covering and protecting their growing points with scales, hair, or gummy substances. The horse-chestnut bud is a good example of a scaly or winter bud, and similar ones can be seen on any of our hardy shrubs or trees. In annuals and herbaceous perennials of temperate climates and in tropical countries where the growing season is not interrupted by a winter season, with its sudden changes of temperature and moisture, the buds as a rule have either very little covering of scales or hairs, or none at all (naked buds).

Buds of Horse-chestnut. - $(L)$ Or hickory, lilac, elm, or any large bud. Examine horse-chestnut buds and note that they are all covered with sticky brown scales which overlap as do the shingles on a house. Examine a bud cut longitudinally. Suggest a use for the sticky substance. Carefully remove the scales from a bud and observe the ring of scars left on the stem. This marks the end of the year's growth of the stem. Look along the stem for other similar rings of scars - how many do you find? What relation do the number of such rings of scars bear to the age of the twig? 
Note that the bud-scales are arranged in pairs, and that they become softer and more leaf-like as you approach the center. When all the scales have been removed, there remains a soft woolly object, the young stem and leaves. Suggest a use for the hairy substance. Sometimes the terminal bud is a flower-bud. Compare what you have found in a closed bud with what you find in an opening bud on a twig which has been standing in water in a warm room. Observe that such a bud has swelled, some of the outer scales have fallen off, leaving a fresh ring of scars on the stem, the inner scales have become larger and more leaf-like, the shoot has elongated, the leaflets are separating and unfolding.

Compare buds from various trees and shrubs.

Leaves are formed folded in buds. This is necessary for two reasons: (1) leaves in a bud must take up as little space as possible; (2) young tender leaves must have as little exposed surface as possible to the dry and cold air of winter. The folding of the leaves in the bud is known in botany as vernation.

Examine buds of various trees and shrubs such as lilac, tulip-tree, hickory, beech, currant, etc., for types of vernation. Also examine naked buds.

Bud-like Structures. - The cabbage head and the onion bulb both illustrate the structure of a bud; but it should be noted that they are not true buds, for they represent fully developed shoots with very short stems. Examine both of them in longitudinal sections obtained by cutting through the centers.

158. Position of Buds and Branches. - In the study of the horse-chestnut twig, attention was called to the fact that buds appear in the axils of leaves and are either terminal or lateral. But buds often occur on other parts of plants, for instance, on the root or along the trunks of trees and even on the notches along the margin of leaves (e.g., in Bryophyllum). Such buds are abnormal in position and are known as adventitious. They develop on some trees when large branches have been cut off, and advantage is taken of this 
fact by pollarding willow trees and thus causing them to produce from adventitious buds the straight and slender branches suitable for making baskets. Some trees are pollarded in order to get ornamental trees with rounded bushy tops. There are many trees and shrubs which have more than one bud in the axil of each leaf; but only one of these is axillary, while the others are supernumerary or accessory. This is well shown in the box-elder and butternut. On currant bushes there are usually three buds at the end of each twig, the central one being the terminal bud and the other two accessory.

Opposite Branching. - When the buds are in pairs at each node and opposite each other, there is a four-rowed arrangement on the twig; but owing to the failure of some of the buds to develop and the death of some young twigs, this arrangement is seldom perfect on fully grown branches. As examples, observe maple, ash, horse-chestnut, and lilac.

Alternate Branching. - When the buds are arranged one to each node, there may be two, three, five, or more rows. In passing from any bud to the next above on the stem we go spirally around the stem. Examples are hickory, elm, beech, ailanthus, and walnut. Alternate is more common than opposite branching.

159. Tree Forms. - If the terminal bud takes the lead in growth, the result is a tree like the spruce or hemlock, in which the straight main trunk can be traced to the very top of the tree, and with relatively small lateral branches. When the branches from the lateral buds develop rapidly, the result is a round top tree, like the apple, in which the main trunk cannot be distinguished from main branches. Most of our common forest trees are intermediate between these two types. Observe various trees and decide to which of these classes they belong. Also notice that the characteristic contour of a tree or shrub depends both upon its mode of branching and upon the angle which the branch makes 
with the main stem, e.g., compare Lombardy poplars with ordinary trees of our forests and parks.

160. Elongation of Stems. - Careful studies have shown that elongation is most rapid just below the growing tip of stems and their branches, but that elongation occurs elsewhere to some extent so long as hard woody tissue has not developed in the internodes of the stem. For example, the distance between the first true leaves and the cotyledons increases for some time while the tissues in this internode are soft. If one measures the distance between marked branches on a young fruit-tree in early spring and again in autumn, it will be found that the older branches have not grown farther apart or higher from the ground; but the branches which appear during the growing season grow farther apart during some weeks while the tissues are soft. This is a matter of practical importance for one who cultivates trees. In pruning $(\$ 166)$ branches must be left at the height where it is desired to have them when the tree is fully grown. Also by cutting the ends (terminal buds) of the main stem and of its branches it is possible to control the height of the tree, and thus keep fruit-bearing branches near the ground for convenience in collecting fruit.

161. Stem of Monocotyledons. - The bean plant has the type of stem found in all dicotyledons and cone-bearing plants. The stem of monocotyledons is different in that its fibro-vascular bundles (bundles of fibers and wood-tubes) are arranged irregularly in the pith (Fig. 44), and not in rings as in stems of dicotyledons (Fig. 48). Moreover, the monocotyledon stem does not increase in diameter each year by addition of layers to the outside of the wood, but instead grows to the full diameter during its primary growth. Thus the trunks of palms and cocoanut trees are nearly the same diameter almost to the leafy top; and although the trees continue to grow for many years, there is little increase in diameter except near the growing top. Such increase as 
does occur, as in some palms, is due to expansion of the first-formed cells, not to new layers of cells added between the

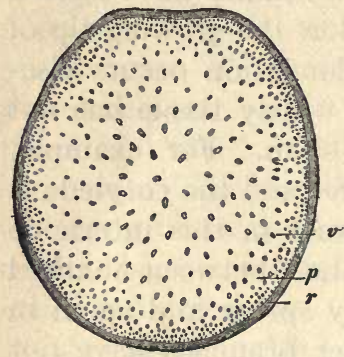

-FIg. 44. Transverse section of corn stem showing rind $(r)$, pith ( $p)$, and numerous fibro-vascular bundles $(v)$. bark and wood, as in the dicotyledons (\$162).

Structure of Corn Stalk. - $(L)$ Cut a dry corn stalk (stem) transversely. Note the smooth rind (not true bark) on the outside, and inside the rind is the soft pith throughout which the fibro-vascular bundles are scattered irregularly. Where are the bundles largest? Where are they most abundant? Suggest a reason for such arrangement.

Split a stalk lengthwise, and the fibrovascular bundles appear as long threads which are easily pulled out. Trace these long threads through nodes. Do they all continue upward or do some of them turn toward the rind at the nodes? Try to trace some of the bundles out into the bases of leaves left on the nodes.

Stand a piece of green cornstem for a short time in red ink, and then eut both transversely and longitudinally in several places, and find out where the colored liquid ascends the stem.

Examine with a microseope a thin cross section cut from a green stem or from one preserved in alcohol or formalin solution. Observe that all parts of the section are composed of cells, most of which are thin-walled pith-cells. Scattered irregularly among these are denser areas of cells, the fibro-vascular bundles. Notice that the bundles are very small and closely packed near the margin, thus making the firm rind, while the bundles toward

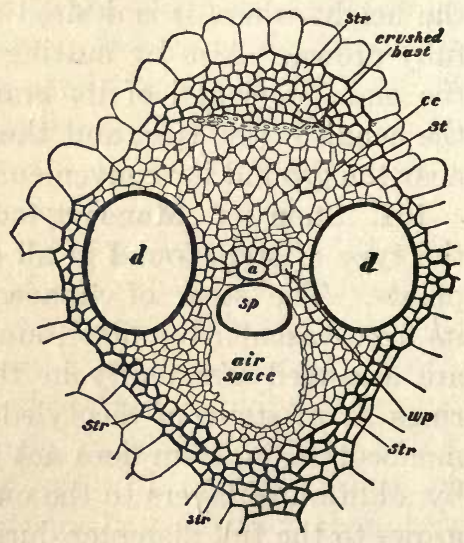

Fig. 45. Transverse section of a bundle in corn stem. Lower part of the figure is towards center of stem. $a, d, s p$, ducts or wood-tubes ; $s t r$, strengthening fibers ; $s t$, sieve-tubes : $w p$, woody pith. (From Osterhout.) 
the center are larger and more scattered. Make a sketch showing the above points. Carefully examine a bundle near the center of the section of the stem, and note that it consists of two parts, (1) that directed toward the center of the stem is made up of wood-cells surrounding the air- and water-tubes, while (2) the outer part of the bundle consists of wood-cells surrounding tubes known as sieve-tubes. Carefully observe the structure of the bundle, and compare with Fig. 45.

162. Stem of Dicotyledons. - While a growing stem is elongating in a given internode, the arrangement of the tissues remains.practically the same as described for the young bean stem $(\$ 70)$. This is the primary growth, and is the condition in many annual dicotyledonous plants. But in many plants which live for a number of years the stem increases in diameter long after the elongation has ceased in a given internode, and this increase in diameter after the first woody tissue has been formed is due chiefly to the formation of new woodcells between the cambium and the first-formed wood. At the same time that the cambium is adding new wood-cells, it adds new cells to the inner layer of the bark. These additional layers of wood and bark constitute the secondary growth of the stem. It is the common method of increasing diameter of the hard stems of perennial dicotyledonous and cone-bearing plants. This growth of new layers commonly occurs annually and results in the rings of wood seen in transverse sections of many trees. These annual rings are clearly marked in many trees by the extra-large wood-tubes formed during the rapid growth in the beginning of the growing season.

The age of a tree cannot always be accurately determined by counting the annual rings, for it has been found that sometimes two rings are formed in a single year. This may happen if growth is checked for a time in early summer, as by drought or destruction of leaves by caterpillars, and begins again later in the summer. In tropical regions, where conditions for growth are uniform throughout the year, it may happen 
in certain cone-bearing trees that no annual rings are marked off, because at no time is there extra-rapid growth such as produces the large wood-tubes in trees of the temperate zone in the spring.

163. Gross Structure of Dicotyledon Stems. - $(L)$ Materials : Stems of oak, pine, beech, maple, and as many others as obtainable,

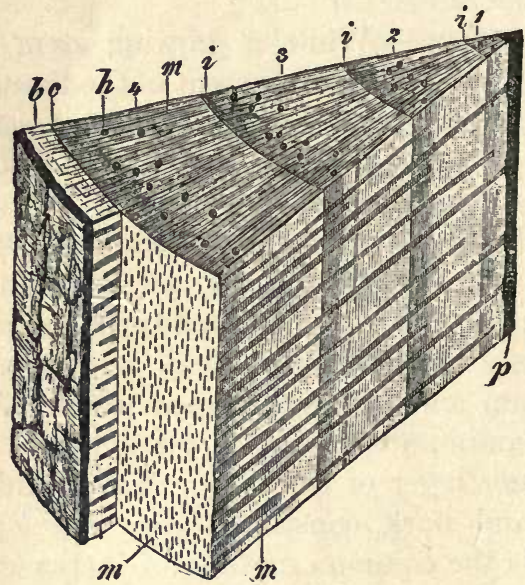

Fig. 46. From four-year-old pine stem cut in winter. Annual rings $(1,2,3,4)$; pith-rays $(m)$; cambium $(c)$; bark with bast $(b)$; lines between wood of successive years $(i)$; center of stem ( $p$ ). (From Strasburger.) selecting stems of various diameters, from half inch to two inches or more. These should be cut off with a fine-tooth saw and then smoothed by rubbing on sandpaper which has been glued or tacked to a board. Also, some sections or cut-ends of twigs, one, two, and three years old. In many high schools the boys who know how to use carpenters' tools prepare a neat collection of woods. Dead and well-dried ("seasoned") branches are best.

The hand-lens shows that the annual rings are marked off by large pores (the wood-tubes). The large wood-tubes of the inner ring were formed while the stem was soft and still elongating (during its primary growth). Usually only this ring will be found in stems taken during the first season's growth. Compare stems of varying diameters, and count the rings. Each season's growth begins with the large tubes, formed when growing rapidly in spring, and extends outward to the next ring of large wood-tubes. In many woody stems some pith remains in the center.

Most woody stems have delicate radiating lines, starting from the pith at the center; and in older stems some of these radiating lines start in annual rings and do not extend to the center. These lines are the pith-rays or medullary rays, which are so named because they are composed of the same kind of cells as those found in the central 
pith or medulla. In some woods, if examined with the unaided eye, the rays appear to extend only to the bark; but in other stems (as beech) the rays extend out into the bark. The fact is that the same kind of cells extend from the pith out into the bark in all dicotyledon stems, but only in cases like beech are they compressed in the bark into plates visible to the unaided eye.

Notice the difference in color between the outer and inner annual rings. The lighter colored outer part of the wood is the sap-wood, so called because it is active in transporting water and in freshly cut stems appears to be filled with sap. Some of its cells contain living substance. The darker central wood is the heart-wood, composed entirely of dead cells. How do you explain the fact that many large trees are hollow (because the heart-wood has rotted or been burned away), and yet they appear to be healthy trees?

$(D$ or $L)$ Split blocks from stems of various kinds of trees (especially oak, chestnut, beech, ash, pine) through the center and plane the cut surfaces. The section thus made is radial. Parallel to the cut surface of one of the halves plane off the bark until some heart-wood is exposed. This surface will be a tangential section. In the radial section the pith-rays will appear as glossy plates of wood, and in the tangential section it will be evident that each ray extends some distance up and down the stem. Evidently the delicate radiating lines or rays seen in the transverse section were-simply the

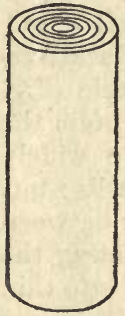

$a$

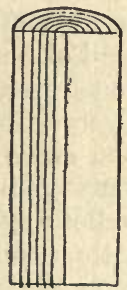

$b$

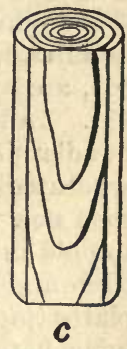

Fig. 47. $a$, transverse section; $b$, radial ; $c$, tangential. (U.S. Bureau of Forestry.) edges of flattened plates which are placed vertically and radially in the stem.

Make diagrams showing structure seen in radial and transverse sections.

( $D$ or $L$ ) Examine the inner and outer layers of bark in various woody stems. Soften pieces of bark by long soaking and boiling in water, tear it into pieces and observe the position and direction of the fibers. Some of these are bast-fibers (elongated cells). The bast-fibers of many plants are of great value in the textile industries, e.g., flax (linen), jute, ramie, and hemp are all obtained by rotting the bark of certain plants and then separating the bast-fibers. 


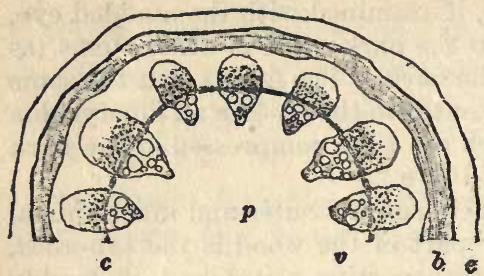

Fig. 48. Half of transverse section of dicotyledon stem (Aristolochia). $p$, pith (white background of figure); seven fibro-vascular bundles $(v)$; cambium $(c) ; e$, epidermis. All outside the cambium is bark, its middle region (b) is composed of hard bast-fibers. (Modified, from Strasburger.)

Some of the fibers found in the bark are sieve-tubes, which have already been mentioned $(\S 103)$ as the path of sap down stems.

164. Microscopic Structure of Dicotyledon Stems. - $(D)$ Examine one-, two-and three-year stems in very thin transverse sections. Identify the parts which can be seen without a microscope, and then note the appearance of the cells composing them. Compare sections seen with the microscope and Fig. 48. A cross section of a very young dicotyledon stem (e.g., bean or geranium) consists of thin-walled cells (pith-cells) and fibro-vascular bundles; but the bundles, instead of being scattered irregularly as in corn stem (Fig. 44), are arranged in a circle (Fig. 48). In the very young stem the bundles in the circle are widely separated by many pith-cells; but soon new bundles appear between the old ones, thus completing the circle and forcing the pith into thin plates (pith-rays or medullary rays) lying between the bundles (Fig. 46). If a single bundle be examined, it will be seen to consist, as in the corn, of wood-cells and large tubes on the inner side, and sievetubes and wood- or bast-cells on the outer side (Fig. 49); but unlike the monocotyledon (corn) bundle, the dicotyledon bundle has between these two regions a layer of cells (cambium) which are capable of dividing, forming new bark (consisting of bast-and sievetubes) on the outer side and new wood-cells and tubes on the inner

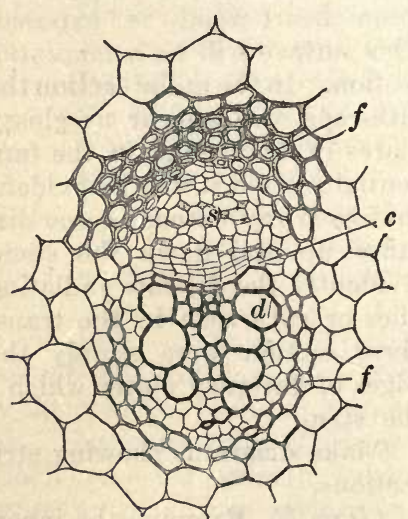

FIG. 49. Transverse section of vascular bundle of a dicotyledon. Lower part of figure is towards center of stem. $f$, woody fibers which surround the bundle; $s$, sieve-tubes mingled with other cells; $d$, wood-ducts; $c$, cambium layer. 
side. It is in this way that the new rings of wood and bark are formed in the dicotyledonous stem.

Longitudinal sections should be examined with the microscope and compared with Fig. 50, which will serve to identify the parts seen.
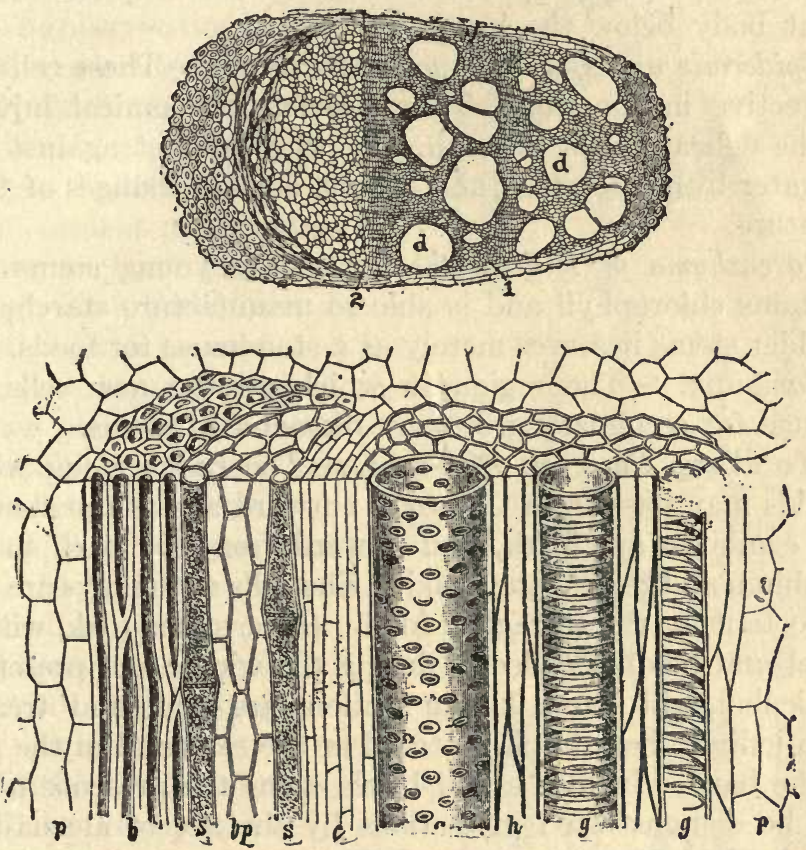

FIg. 50. Upper figure represents a transverse section of a fibro-vascular bundle of moonseed. Bast is at left of 2 , wood is at right. Lower figure is a longitudinal section of a sunflower bundle. Cambium at 2 in upper figure, $c$, in lower one ; $d$ and $g$, wood-tubes; $h$, wood cells between tubes ; $s$, sievetubes; $p$ and $b p$, parenchyma or pith; $b$, bast. (From Bailey, lower figure after Wettstein.)

165. Functions of Parts of Stem. - Wood-cells and bastfibers are very rigid cells because of their thick walls, and thus are able to give the support which is so necessary if a plant is to grow very large. They also serve as a protection to the tubes and more delicate cells which they surround. 
Wood-tubes and Sieve-tubes. - The tubes in the wood conduct the materials collected by the roots up to the leaves; and the sieve-tubes of the inner bark distribute the food materials prepared by the leaves to various parts of the plant body below the leaves.

Epidermis and Corky Layer of the Bark. - These cells are protective in function. They prevent mechanical injuries to the delicate cells beneath, and they protect against loss of water by evaporation, and against sudden changes of temperature.

Parenchyma or Pith of the Bark. - In young stems this contains chlorophyll and is able to manufacture starch, but in older stems it serves merely as a storehouse for foods.

Cambium. - The region in which all the new cells are formed for secondary growth in diameter of stems.

Medullary Rays or Pith-rays. - The paths along which liquids may pass from the wood outward across the stem to the cambium and bark, and inward from the bark to the cambium and cells of the wood. The cells of the rays are also important for the storage of food. Some stems (oak, willow, hazel, and lilac), if collected during the winter and preserved in alcohol, will show, if thin sections are made and treated with iodine, that starch is stored in the rays and in the pith of the bark. In linden and birch stems the presence of fat can be demonstrated in sections by the use of alcana-root dissolved in 70 per cent alcohol, which stains fat red. In still other trees sugar is stored in the pith-rays.

166. The Cambium in Grafting and Healing Wounds. The power of growth of the cambium is well illustrated in these two processes. If two branches of a tree be scraped down to the cambium and the wounded surfaces brought together, the rapid growth of cambium cells will cause a grafting or union of the two. Examples of this can often be seen in a forest or orchard, for branches often grow so near together that the swaying by the wind wears away the bark, exposes 
the cambium, and allows the two branches to grow together. Sometimes two trees may so grow together when they are small.

The observation that stems will grow together or naturally graft probably led to artificial grafting of fruit trees. Essentially all grafting consists in bringing the cambium layer of a piece of twig with a bud (scion) from one plant into contact with the same layer on stem of another plant of the same or sometimes a closely related species. Various ways of doing this are illustrated in Fig. 51. Grafts are usually placed on young plants near the roots ("root-grafting") so that after the graft grows and the original stem is cut away, only the variety of the graft will be represented in the stem above ground. Old trees are grafted by cutting off branches(" top-grafting") and inserting scions or grafts. If a new branch grows below the graft, it will bear fruit of the original variety.

Budding is essentially the same as grafting, but a single bud with a slice of bark (Fig.
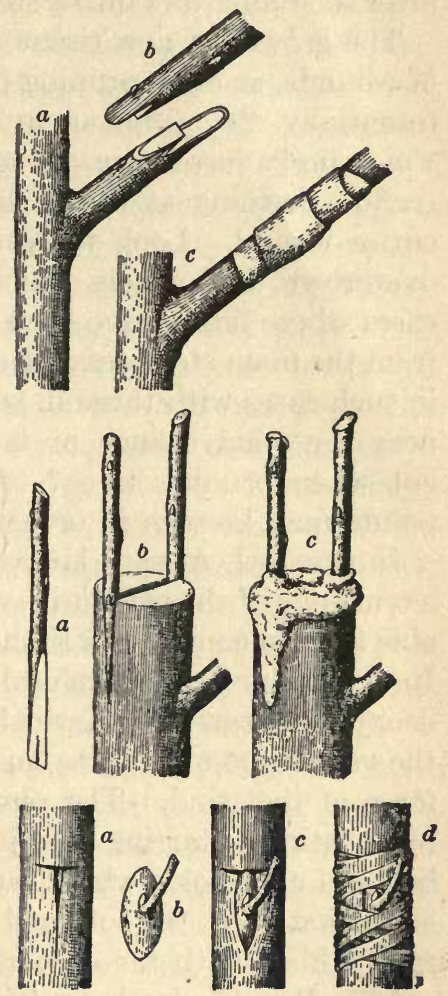

Fig. 51. Upper row-whip- or tongue-grafting. $a$, stem or stock ; $b$, scion or graft; $c$, united and wrapped. Middle row-cleft grafting. $a$, scion; $b$, two scions inserted in cleft; $c$, waxed. Lower row - budding. $a$, stem cut to receive bud $(b)$; $c$, bud inserted beneath bark; $d$, wrapped. Note in all the figures that cambium of scion and stock meet. (U.S. Dept. Agriculture.) 
51 ) is placed in contact with the cambium of another tree, and after the bud grows into a stem the original stem is cut away.

The growth of new tissue which takes place in the healing of wounds, as after pruning or breaking of limbs by winds, is essentially the same as in grafting. Examine trees from which limbs have been cut and notice the ring of new tissue (callus) forming at the bark and tending to grow over the entire wound. Look for cases where the wound is almost overgrown, and others which are completely so. Look for cases where limbs have been broken or cut off some distance from the main stem, leaving a stub; and compare the healing in such cases with those in which the branch has been cut off near the main branch or trunk. How should branches be cut when pruning trees? Numerous illustrations of these points may be seen in orchards and in shade trees.

In cases where very large limbs have been cut off, there is a tendency of the wood to decay before the cambium has been able to form enough new tissue to cover the wound completely. In old and neglected orchard trees many holes are formed by decay thus started, and which, after many years, extends into the very heart of the tree, making it unable to withstand the force of the wind. The remedy is (1) prevention of decay by painting or tarring all injured places so as to keep out the bacteria and molds which cause decay; and (2) if decay has already started, the softened wood should be cut out and the cavity filled with tar or portland cement.

167. Pruning is the cutting out of unnecessary and injured branches of trees, shrubs, and vines. It is necessary in orchards because trees standing alone develop too many branches, and grow too high for convenience in spraying against fungi and insects or in picking fruit. Also, too many branches result in small and imperfect fruit.

Pruning has been developed so well that the gardener can now make a young tree take almost any desired shape by simply removing buds and branches which are not wanted, 
and thereby forcing growth in other directions. For example, pruning off a terminal bud makes the lateral branches grow. Farmers' Bulletin No. 181 should be consulted, and a field-trip taken to some well-mamaged orchard or tree-nursery.

Nature's method of pruning by crowding out superfluous branches is well illustrated in any dense woods. The shaded branches soon die and decay, and the result is the growth of long straight stems with living branches near the top only. Why are trees set close together in young forest plantations?

Pruning trees and shrubs at planting time is of great value, in that it reduces the number of leaves until roots can get well established in the soil. Unless the leaf-surface is thus reduced, evaporation is likely to be greater than can be replaced by absorption of water by the roots. It is difficult to transplant evergreens, because pruning would spoil the form of these ornamental trees, and the leaves cause excessive evaporation before new roots are established in the soil.

168. Duration of Life of Plant Stems. - It is a matter of common observation that some plants live only one year and that others live many years. All plants of which the life of a generation, beginning with the seed and ending with death of stem and roots, comes within a year are called annuals. That is to say, an annual plant is one which completes its lifehistory by starting from seed, producing seed and dying, root and all, within a single year. Some plants (e.g., beet, cabbage, thistle, mullein) start from seed one year, the roots and base of the stem live over the winter, and in the second summer they blossom, produce seed, and then die. Such twoyear plants are called biennials. Still other plants (especially trees and shrubs) live more than two years; and such plants are perennials. Some perennials have a soft stem which cannot withstand the winter, but the underground parts are hardy and new stems form above ground in each growing season. Examples are peony, golden-rod, hollyhock, asparagus, violet, dandelion, buttercup, and most common 
grasses of our lawns and meadows. Such plants are called herbaceous perennials. Many of them are short-lived; for example, farmers know that the best crop of red clover is grown in the first and second years, and after that the roots are unable to produce luxuriant growth of stem and leaves and gradually die out, giving place to grasses and weeds. There are many such cases among plants grown for their flowers.

Cultivation has changed the duration of life of some plants. A plant which is a perennial in the tropical regions may be an annual in a country with cold winters. Go into any vegetable garden when the frost comes and you will see many plants, such as tomato, pepper, egg-plant, which were started very early in the spring and would have lived much longer if not killed by frost. These are naturally warm-climate plants, but in the temperate zone the winter season limits their duration to a single summer. There are many other examples of changes in duration due to cultivation. Parsnips have been changed from annuals to biennials. Wheat and rye (both annuals) are commonly planted in September, live over the winter, and mature grain in July. Likewise, gardeners often start annual vegetables and "flowers" in the late autumn, cover them up over winter, and have them mature in early spring or summer. The length of life of such doubtful plants could be determined by planting seed in very early spring in order to learn whether the plant will mature seed within a single summer. As an example, certain kinds or varieties of wheat and rye are planted in the spring in the extreme north (Dakota and Manitoba), where cold winters would kill the roots of the winter varieties, and this spring wheat is harvested in the autumn. This could be done whereever wheat and rye grow; but the crop is usually not so heavy as with the variety planted in autumn. Also, it is of advantage to the farmer to be able to plant wheat and rye on the same ground after corn is harvested in the autumn, 
because proper cultivation of corn has left the soil in condition for planting wheat without replowing, and also because in the corn-growing regions oats and corn must be planted in the spring and there would be little time for planting spring wheat and rye. Still another example : many of our garden biennials (carrots, beets, cabbage, onion, turnips) will bloom and form seed in one long season in a warm climate, or sometimes if forced with rich soil and plenty of water. The above are simply examples taken from a long list of cultivated plants whose duration of life man has been able to change.

Home-work : Make lists of the common plants you know, classifying them as annuals, biennials, or perennials.

169. General Functions of Stems. - The primary work of ordinary stems is (1) support of the leaves in positions adapted to their work of food-making and transpiration, (2) conduction of materials between roots and leaves, and (3) breathing.

(1) In its work of support stems make use of the elasticity and rigidity of woody tissues and also of turgidity due to water in the cells. A very young plant depends largely upon turgidity, for if water is withheld from the soil, the leaves and stems become wilted. A young Windsor-bean plant growing in a pot will illustrate this. If the soil be allowed to dry, the stem will lose its turgidity (become wilted) and will gradually bend over until the leaves touch the soil. If then water is added to the soil, the stem will become turgid and rigid with remarkable rapidity, and within two or three hours will be as erect as ever. Stems of older plants of most species do not lose their rigidity when water is wanting, for woody tissues furnish the necessary rigidity. A straw of wheat or rye is a splendid example of rigidity due to woody tissues arranged in cylindrical form.

(2) The work of conducting materials is, as we have seen in the bean plant, due largely to the tubes in wood and bark which are specially fitted to conducting liquids up and down 
the stem. Other cells of the stem also play a part in conduction of liquids, especially in the transverse or horizontal direction.

(3) Breathing by stems occurs through certain openings in the outside, layers of tissue. The epidermis of herbaceous stems has stomata similar to those of leaves. Examine with microscope epidermis from such plants as bean, tradescantia, and begonia. In plants with corky bark the stomata are replaced by openings known as lenticles (meaning lens-shaped) or stem-pores. Good examples may be seen on young stems of cherry and plum. Notice that on the older parts of these stems the lenticles are elongated by growth. The holes in cork used for bottles look like holes made by worms, but they are lenticles. Examine a bottle cork and note the parallel holes which originally extended through the thick corky bark to the cambium, thus allowing air to penetrate to the active cells.

170. Special Adaptations of Stems. - In addition to the primary functions discussed above, many stems have become adapted or fitted to special kinds of work such as climbing, propagation, living underground, and in still other ways to be described in the paragraphs which follow.

171. Propagative Branches. - Strawberries, red raspberries, currant, and gooseberry are examples of numerous kinds of plants which have some branches either lying on the surface of the soil or creeping underground, and which form roots and develop new plants. When these branches are underground, gardeners call the new plants which come up "suckers."

172. Branches for Climbing: Tentrils. - Slender branches, without leaves and buds, are in mâny plants adapted for climbing. Examine specimens of tendrils from grape-vine, Virginia-creeper, Boston-ivy, cucumber, squash-vines, passion-flower plant, or gourd-plants. Note that some are single tendrils, some are branched, and some have flat 
disks at the tips. If possible, observe the growth and movements of tendrils on some plant which can be watched from day to day.

Leaves of certain plants, as those of the pea, form tendrils; but these are easily distinguished from branches because they are connected with leaves and not in the usual positions of branches.

173. Branches as Thorns. - Examination of specimens of thorns from trees of pear, honey-locust, hawthorn, buck-thorn, and others shows that they are branches, or tips of branches. Prickles on many plants are simply elevations of the bark. Peel off some bark from stems of blackberry or rose and note that the prickles are not

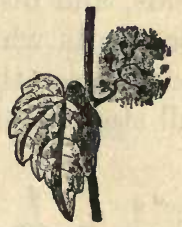

FIG. 52. Leaf and stem-tendrils $(r)$ of Ampelopsis (Boston-ivy). attached to the wood as branches of stems are. Barberry prickles are modified leaves, and those of black locust are stipules of leaves.

The uses of thorns and prickles are sometimes doubtful. While they appear to be adaptations for protection against grazing animals, it is well known that often they do not so protect. Cows and sheep often eat the thorny branches, especially young and delicate shoots when the thorns or prickles are soft. Certainly these sharp structures are not absolutely necessary, for many plants without them appear to flourish as well as their " armed " relatives. It is probable that prickles and thorns developed first without any reference to defensive use (e.g., the spines of cacti, which are leaves reduced in adapting to dry climate), and that now they may sometimes be of slight advantage to the individual plants that possess them.

174. Twining Stems. - Some stems climb without the aid of tendrils. Study plants of pole-beans, morning-glory, hop, on any wild plants available. Lay your watch on the ground with the hour-mark pointing north, and note whether plants 
observed twine in the direction the watch-hands move (i.e., clockwise, west-north-east) ; or in opposite direction (counterclockwise, east-north-west). Plants of some species appear to move always in one of these directions.

175. Creeping Stems. - In walking across pastures, sandy fields, and other places where tall plants do not grow, look for plants which have their stems prostrate on the ground. Do you see that such plants have any advantage over erect tall stems under the conditions in which they live?

176. Underground Stems. - Many

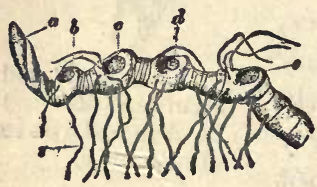

Fi G. 53 . Underground stem of Solomon's seal. $a$, terminal bud; $b, c, d$, $e$, leaf-scars of former years ; $r$, roots. plants have their main stems underground. Three common forms of such stems are rootstocks, tubers, and bulbs.

Rootstocks. - These are rootlike stems which grow in the soil. Many ferns, grasses, sweet flag, goldenrod, quack-grass, peppermint, iris, Solomon's seal, and trilliums are examples of common plants with rootstocks (rhizomes). That these are not true roots is shown by the presence of buds and by the formation of branches and leaves. Solomon's seal (Fig. 53) is especially good for study. Its rootstock sends up an erect branch every spring which becomes the above-ground stem with leaves. All the above-ground part of the plant is herbaceous and dies in autumn. The seal-like scars on the rootstock mark the positions of the above-ground branches in successive years. The oldest portion of the rootstock dies and decays ; and some plants have short rootstocks, because the part more than a few years old has died and decayed.

Tubers. - Many of the rootstocks mentioned above store food, but do not undergo noticeable thickening at any particular point. In some plants storage of food in stem underground causes great enlargement and produces tubers (e.g., the common potato and Jerusalem artichoke). 
(L) Examine a tuber of potato, or artichoke. Note the point where it was attached to the main stem of the plant. Examine the "eyes," which are buds. Each "eye" is capable of developing a new plant, and hence to avoid having too many plants in one place gardeners cut tubers into pieces, each having two or three "eyes." Dig up a potato plant, and note position of the old and the new potatoes.

Bulbs are short and greatly enlarged underground stems, producing stalks and leaves from the upper surface and roots from the lower. The onion is a good example of a bulb. A longitudinal cut through the center shows it to be a short stem surrounded by the thickened layers, which are modified leaves. Indian turnip (Jack-in-the-pulpit) and crocus have similar short and bulb-like stems, but they are solid and not composed of layers like the onion. Such solid short underground stems are often called corms; but there is no sharp distinction and corms are often called solid bulbs.

"Stemless" Plants.Many common plants (e.g.,

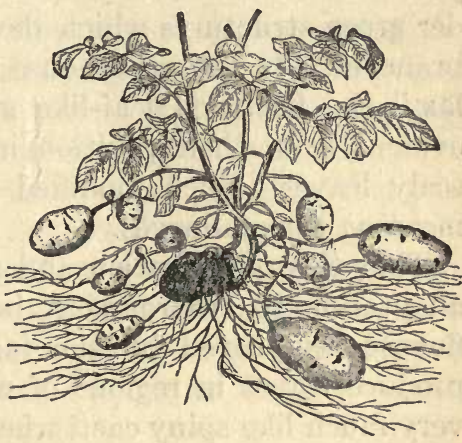

Fig. 54. Potato plant, developed from dark-colored tuber in center. New tubers are thickenings of underground branches of the stem. (From Strasburger.) dandelions, plantains) have short stems and are often incorrectly said to be "stemless."

177. Condensed Stems above Ground. - Tubers and bulbs have been mentioned as examples of condensed underground stems. The various forms of cacti are condensed stems above ground. The flattened leaf-like parts of pricklypear cactus are not leaves, but branches of the stem. The thorns are reduced leaves. The flat and thickened branches not only perform the ordinary work of leaves, but are also 
important reservoirs of water needed in times of drought. The absence of leaves gives the advantage of greatly reduced surface exposed to evaporation.

178. Stems Adapted as Leaves. - In extremely dry countries some plants have formed the habit of producing very small leaves or none at all, in order that there may be as little loss of water as possible. An extreme case of this kind is seen in cacti, described in the preceding section. Our garden asparagus is by nature adapted to very dry conditions. Its only leaves are the scales which appear on the young edible shoot early in the spring, and the mass of slender green structures which develop later in the summer are branches. In the greenhouse plant commonly called "smilax," the flattened leaf-like structures are stems. This is evident from their position in the axils of the very small scaly leaves. Such modified stems are called cladophylls, meaning branch-leaves.

That this modification and reduction of leaf surface is an adaptation to environment brought about by external influences is proved by the fact that certain plants which produce leaves in regions of moderate water-supply become very much like spiny cacti when grown in desert conditions.

179. Economic Value of Wood Structure. - The usefulness and consequent monetary value of lumber depends upon the nature of the various structures seen in the sections of stems ( $\$ 163)$. For many purposes hardness and strength combined are desired (e.g., white oak for wagon-axles, hickory for ax-handles and wheel-spokes). Sometimes a light, elastic, straight-grained wood is wanted; and so ash is most common for the long handles of hoes, shovels, pitch-forks, etc. Woods which resist decay because they contain resins, oils, or other protecting substances are needed for fence-posts and telephone poles; and for such purposes cedar, chestnut, oak, mulberry, and black locust are usually preferred. White and red cedar, cypress, and redwood make the best shingles 
now in the market. For some purposes wood softer than oak is desirable because easy to work - carpenters charge much more for working oak than for soft woods like white pine. Hence pine, hemlock, spruce, redwood, basswood, tulip-wood or whitewood, cedar, sweet gum, and poplar are used for numerous purposes where soft and light wood will serve. For high-grade furniture, woods with beautiful grain and color combined with hardness are desired; and hence the popularity of mahogany, black walnut, rosewood, ebony, and oak.

It often happens that in a given locality a wood is used for a purpose because it is the cheapest available, but not the best. For example, hemlock is largely used in Eastern states for frames of buildings ; but its softness and tendency to split makes it far inferior to spruce among soft woods and not to be compared with the oak, formerly much used for this purpose. White pine and spruce are often used for cheap floors, but are as much inferior to yellow pine as this is to hard maple and oak for this purpose. Such semi-hard woods as elm, chestnut, birch, and basswood are now abundant in cheap furniture, and are often stained to imitate harder woods like oak and mahogany.

Woods like chestnut and red oak, with large wood-ducts, making the annual rings very conspicuous, are said to be "open-grained." In finishing such woods for furniture it is first necessary to use wood-fillers, which are pastes for filling the open spaces left after planing, and thus give a perfectly level surface to receive the varnish. Examine polished oak furniture and compare with an oak board which has been planed but not filled.

A wood which may be split into straight pieces parallel to the central axis of the stem is said to be "straight grained." Ash, chestnut, and hemlock are commonly so ; but irregularity in thickness of annual rings and in position of the medullary rays, vascular bundles and knots makes some woods more or 
less " cross-grained"; that is, they tend to split obliquely and not parallel to the central axis of the stem. Many woods (such as elm, sycamore, apple) are often difficult to split because their wood-fibers are crossed and interlaced. Split some pieces of boards or branches of various trees by driving a wide chisel carefully and notice the direction of the fibers of the wood.

The usefulness of boards for certain purposes depends upon the direction of sawing. Examine pieces of pine, cypress, maple and other boards, especially where they have been subjected to wear, as in floors, and note the direction of the cut of boards which have splintered or in other ways become undesirable. The transverse section is best if a block is to be subjected to great strain, as in pavement-blocks, mallets, etc.

The decorative value of the grain of woods depends in part upon the way the logs are sawed into boards. Compare the tangential and radial sections of oak and other woods, and decide which is the most beautiful cut. Most people prefer the radial cut of oak with the exposed glossy medullary rays capable of excellent polish. In the usual method of cutting boards only a few boards near the center of the log will be radial sections or nearly so. All the others will be more or less tangential (see 4, 5, and 6 in Fig. 55). In sawing oak and other woods with prominent rays for furniture and housefinishing it is best to saw as shown in Fig. 56. Two boards (sometimes four) are taken from the middle (cuts $1,2)$. Next the same number of boards are taken from the middle of each half $(3,4)$. These will show the same grain as the first boards cut, but will be less than half as wide. Then each of the "quarters" of the log is taken separately and sawed into boards cut as radially as possible. There are several possible ways of sawing "quarters," but a little study of the diagram and of blocks of wood will show that cutting as in $A$ and $D$, Fig. 56 , is to be preferred for "quarter-sawing" the choicest woods, because it will re- 
sult in the largest number of radial sections showing the pith-rays.

In some woods the most beautiful sections are tangential. In order to get the greatest number of such sections, such woods are often cut into sheets of veneer by means of a machine with a large knife which cuts an immense shaving,

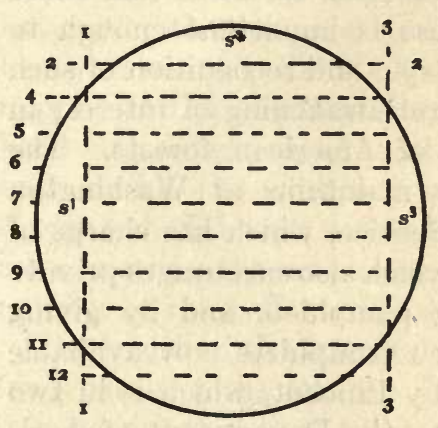

Frg. 55. Ordinary sawing of lumber. $s^{1}, s^{2}, s^{3}$, slabs removed by cuts 1 , 2,3 ; and followed by cuts 4 to 12 .

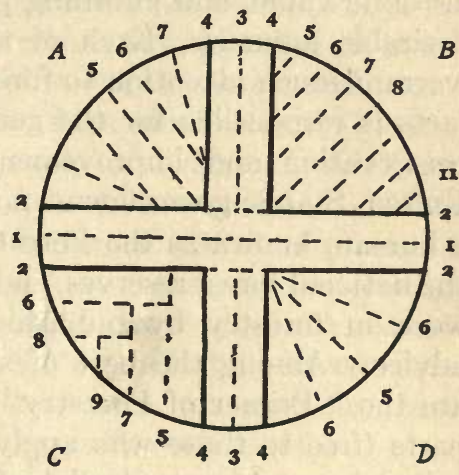

Fig. 56. Four methods of quarter sawing. Cuts 1, 2, 3, 4 remove boards which are perfect radial sections on surfaces 1 and 3 . Then each "quarter" is sawed in order $5,6,7,8$. The method illustrated in "quarter" $C$ is least desirable.

a continuous tangential section, as the log is revolved on its axis. In this way an entire $\log$ can be cut into tangential section. Pieces of the sheet of veneer are then glued on the surface of other and less valuable boards. This method also has the advantage of avoiding the waste of material which results from cutting with saws.

180. Forestry. - The application of scientific principles to the management of forests is known as forestry. Such scientific management has become necessary because the use of tree stems as lumber has greatly reduced the extent of forests ; and in the interest of future supply it is now desirable 
that forest planting and forest conservation be practiced extensively. And not only is it important that forests should be preserved and new ones developed for the sake of a continued supply of lumber; but, also, among many other things which make forests valuable are their effect upon climate, prevention of soil erosion, prevention of sudden floods, æsthetic value, and affording protection for birds and other desirable animals. Each of these is important enough to warrant much attention to forestry; and recognition of such facts is responsible for the general awakening of interest in preservation and improvement of American forests. The United States government now maintains at Washington a bureau, known as the Forest Service, which has charge of the national forest reserves, and which also encourages private work in forestry by publishing pamphlets and by giving advice. Among the best of such pamphlets now available are the "Primer of Forestry" by Pinchot, which is in two parts (free to those who apply to the Department of Agriculture); and many leaflets (also free) giving information as to how to plant special kinds of trees. The "Primer of Forestry" should be obtained and read by all students of biology. Another interesting book in the same line is Roth's "First Book of Forestry."

\section{LEAVES OF SEED-PLANTS}

181. Functions of Leaves. In connection with the study of the bean plant three functions of leaves (breathing, transpiration, and starch-making) have been mentioned. In addition to these functions, which belong to all typical leaves, some plants have leaves fitted for special functions, which will be described in the paragraph on adaptations of leaves.

In order to carry on most efficiently the three main functions, it is important that leaves should be arranged so as to have the most favorable exposure to light and air. This 
requires (1) great expanse of surface, which is secured by the broad blades; and (2) arrangement so that they will shade each other as little as possible. This latter point deserves some special study.

182. Arrangement of Leaves. - One of the most impressive points about leaves is their position on the branches of plants. It would be a careless observer indeed who does not notice that the leaves are commonly arranged in rows, but perhaps only the student of botany observes that there may be two, three, four, five and even more rows. This has been already indicated in the study of twigs, on which the buds mark the positions of future leaves $(\$ 156)$. The student should look at plants having different numbers of rows, viewing the main stem from above, and note how the number of rows affects the shading of leaves by each other.

The length of the internodes of stems affects light exposure by separating the leaves. If the leaves are close together, it is evident that they will tend to shade each other.

The size of leaves and the number of leaves have a definite relation to the arrangement in many plants. Some plants have a few large leaves, while others have many small leaves. If possible, contrast such trees as catalpa and willow, or hickory and birch, looking at small trees of these species from above and at large trees from below.

Varying length of branches of the stem and of petioles of most leaves of trees aid in making the best adjustment. Numerous plants illustrate this. The lower branches of most trees are longest and so hold their leaves out of the shadow of the upper branches; and also some of the leaves have long petioles so that their blades avoid shading by leaves above.

Twisting of stems and petioles brings some leaves into the best position. This is most strikingly illustrated by the horizontal branches as contrasted with vertical branches on the main stem of the same trees.

Most leaves face the source of light, as may be seen in plants 
growing in window-boxes; but many grasses and other plants have leaves nearly vertical or erect, and others hold their leaves edgewise. In such cases light does not strike the surface directly. Leaves so arranged are probably very sensitive and the indirect light is better suited to their work.

Climbing plants grow so as to arrange their leaves with reference to light as definitely as do plants with erect stems. Many vines spread their leaves over the outermost branches of trees, especially in dense forests. In fact, the climbing habit is believed to have its purpose in securing the best possible exposure of leaves to light.

It is interesting to note that even plants adapted to growing in the shade of forests appear to have leaves arranged for best exposure to light. This shows that while such plants are adjusted to shade, they must have as much of the diffuse light as possible under the conditions.

The leaves of some plants attain the best exposure by movements, such as have been mentioned, adjusting their leaves to the amount of light best suited to them.

Finally, the forms of leaves appear to be of some meaning in connection with best exposure to light. The triangular leaves of some plants seem to fit together better than rounded leaves would on the same arrangement of branches. The numerous kinds of notches, lobes, and branching of leaves allow light to pass through to lower leaves. If one examines plants with such leaves exposed to bright sunlight, it is evident that some light passes through the spaces between leaves.

There are numerous other ways in which certain plants have their leaves arranged in order to get the best possible light. The best for a given plant does not mean the most light, for at times sunlight may be so intense as to be injurious to some plants. However, this is usually provided for by movement in some species and by thick cells which reduce the intensity of light that reaches the more sensitive middle tissues of leaves. 
In field trips taken in connection with this course in biology, attention should be directed to the arrangement of leaves in the light relation whenever good specimens are found. The discussion of the above outline of the most important types of leafarrangement should be taken up in the laboratory with specimens at hand to illustrate the main points, and pupils should make outline sketches showing various types of leaf-arrangement.

Reading for pupils: Chapter II, "Foliage Leaves: the LightRelation," in Coulter's "Plant Relations." The same chapter is in "Plant Studies" and "Plants" by the same author. Or Chapter $\mathrm{X}$ in Bergen's "Foundations of Botany," or Chapter XI in Bergen and Davis's "Principles of Botany."

183. Special Adaptations of Leaves. - While the primary purposes of leaves are starch-making, transpiration, and breathing, many leaves have assumed additional work for which their structure shows special adaptations. The most interesting of these are those concerned with catching insects, storing food, climbing, and protection against animals (leaves modified into prickles).

Leaves as Insect Traps. - Certain plants growing in bogs have curious leaves adapted to catching insects. Some of these plants have leaves in the form of deep cups or pitchers containing water in which insects are drowned (pitcher plants); some species (the sun-dews) have strong bristles coated with a sticky substance which holds fast any insects which happen to touch them (Fig. 57); and a third kind (Venus fly-trap) have at the end of each leaf a folded structure which opens and shuts very much like a steel-trap (Fig. 2). In all these cases the insects caught either decay by action of bacteria or are digested by certain fluids secreted by the leaves, and are absorbed by the cells of the plant. Thus the ordinary food-supply of these plants is supplemented by animal food, and such plants are called carnivorous (flesh-eaters), or sometimes insectivorous (insect-eaters).

Leaves for Climbing. - The leaf-stalk or petiole acts as a tendril in some plants (nasturtium, clematis, etc.). In 
the compound leaf of the pea and of many other members of the bean family there is a tendril in place of the terminal leaflet of the compound leaf (Fig. 58). There are some plants which have the tips of simple leaves serving as tendrils. In some plants (common green brier or cat-brier) a pair of tendrils are attached at the base of each petiole; and these are modified stipules of the leaf. It is evident that any part of leaves (petiole, blade, or stipules) may be modified

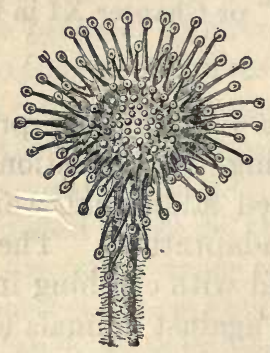

FIg. 57. Modified leaf of a sundew. The sticky bristles catch insects. (From Strasburger.)

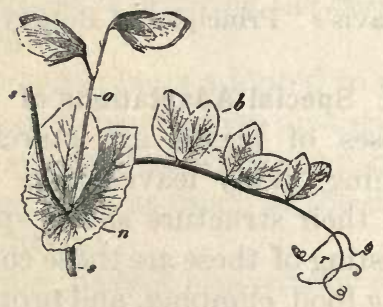

Fig. 58. Leaf of pea. Large stipules $(n)$, leaflets of the compound leaf $(b)$, terminal leaflets modified into tendrils (r).

to serve as tendrils. It has already $(\S 172)$ been pointed out that some tendrils are modified branches. A comparison of position and structure will usually make it easy to decide whether particular tendrils are from leaves or branches of stem.

Leaves for Food-storage. - It has already been stated that the concentric layers of bulbs (onion, tulip, etc.) are the bases of leaves thickened by food stored for use in the next season's growth. The use of the food becomes evident if one plants an onion or other bulb in a pot, for after a time the layers become reduced to thin and dry sheets. The thick cotyledons of bean, pea, acorn, etc., are also examples of food-storage in leaf-like structures.

Leaves as prickles occur in some plants. Examine branches 
of common barberry and notice gradations between the spiny leaves and the prickles. Also notice that buds are in the axils of the spines, and compare with the position of buds with reference to leaves $(\S 157)$.

Stipules of Leaves. - These are often absent, and when present are variously modified. Compare those of leaves of clover, pea, rose, India rubber tree, buckwheat. In some plants (e.g., pansy) the stipules are so large that they are not easily distinguished from leaves. The outside bud-scales in beech, tulip-tree, and magnolia are stipules. The prickles at base of leaves of black locust tree are obviously modified stipules (Fig. 60).

Scale-leaves. - This name is applied to

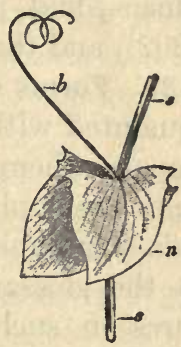

Fig. 59. The leaf of yellow vetch is reduced to a tendril $(b)$, while the two stipules $(n)$ are large and serve as leaves. the small scale-like structures which do not have the usual functions of leaves, but which are chiefly for protection in bulbs and buds. The inner scales of the horse-chestnut bud have a structure which suggests that scale-leaves are modi-

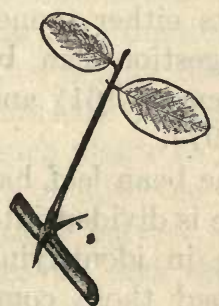

Fig. 60. The spines at base of the petiole of a black locust leaf are modified stipules. fied or reduced leaves. The structures termed bracts which are at the base of flower-stalks (e.g., the petal-like bracts of the clusters of dogwood flowers and the sepal-like bracts on the hepatica flowers) are of similar origin.

184. The Parts of a Leaf. - In the study of the bean leaf, the petiole, blade, and stipules were mentioned as the parts of a typical leaf; but many leaves have some of these parts lacking. Especially are there many leaves where we cannot distinguish between petiole and blade (grasses, lilies, etc.), and leaves of many species of plants have no stipules. 
185. Floral Leaves. - Careful study of the development of flowers has led botanists to regard the sepals, petals, stamens, and pistil as highly modified leaf-like structures $(\S 202)$, and they are sometimes called floral leaves.

186. Forms of Leaves. - Students who have not become acquainted with the various common forms of leaves should compare them whenever an opportunity offers. Special names are applied to the shapes of leaves and to the different kinds of notched and lobed margins. For these names consult the glossaries of books for identifying plants, and the figures in such books as Gray's "Lessons with Plants." Such names are not worth memorizing unless one makes frequent use of manuals for identifying plants.

187. Veins of Leaves. - The type of veining found in the bean leaf is known as netted veining, while that seen in the corn plant is parallel veining. Most monocotyledons have parallel veining, and most netted-veined leaves are found on dicotyledons. The arrangement of the veins in these two types appears to have nothing to do with the work of the leaves, for the two kinds of leaves perform essentially the same functions. The kind of veining is chiefly of interest in classifying plants, for it at once suggests either monocotyledons or dicotyledons; and this suggestion can be tested by examining the structure of the stem $(\$ 161)$ and certain points with regard to the flowers ( $\$ 197)$.

188. Simple and Compound Leaves. - The bean leaf has been described as compound because its blade is divided into separate parts or leaflets. For convenience in identifying and describing plants, botanists have termed those compound leaves with leaflets arranged on sides of the leafstalk (Fig. 58), as in honey-locust, black locust, elder, ash, pea, parsnip, etc., pinnate (meaning, arranged like a feather); while the term palmate (meaning, like a hand) is applied to those leaves like clover, horse-chestnut, buckeye, in which the leaflets are all attached to the end of 
the leaf-stalk, similar to the attachment of fingers to the palm of the hand.

Either type of compound leaves may have the leaflets again divided one or more times, as on young shoots of honeylocust, in meadow-rue, carrot, and parsnip. Compare carrot and parsnip leaflets.

Compare pinnately compound leaves with the pinnate veining in simple leaves, such as elm. Compare a palmatelyveined simple leaf, as that of maple, with a palmately compound leaf with five leaflets. Such comparisons suggest that the leaflets of compound leaves correspond to the lobes or divisions of simple leaves, of which there are all gradations from small notches as in elm leaf and lobes as in oak leaves to such extreme division as in the celandine, French marigold, and ragweed. These latter are almost compound, but show narrow strips of the blade connecting the divisions.

Obviously, it is often difficult to distinguish between simple and compound leaves, for there are many simple leaves so deeply divided as to resemble closely compound leaves. The only reason for attempting to distinguish in such cases is for convenience in describing and identifying plants. So far as functions are concerned, the compound leaves and the deeply divided simple leaves are apparently equally efficient in permitting light to reach lower leaves. Observe such plants as carrots and ragweed in bright sunshine, and note how the light filters through the clefts and reaches lower leaves. Moreover, since breezes frequently sway the leaves, and the position with reference to light constantly changes with that of the sun, the result is that lower leaves are quite sure to get more or less direct illumination each day. Undivided leaves which exposed the same surface on the same branch arrangement would certainly keep many of the lower leaves in shade most of the time. 


\section{FLOWERS OF SEED-PLANTS}

189. The Functions of Flowers. - Our studies of the bean flower ( $\$ 75)$ may now be applied to flowers in general. The petals and sepals of flowers serve the purpose of inclosing and protecting the stamens and pistil, which are the parts of flowers essential to development of seeds. The important parts of the stamens are the anthers or pollen-cases which contain pollen-grains. There may be one or more pistils. If we open the ovary of the pistil in a common flower, we see one or more rounded bodies, called ovules; and by comparing flowers of different ages it is made evident that from each ovule a seed develops. Inside of each ovule is an egg-cell, the part from which the embryo of a new plant will develop. The ovules are usually easily seen by the unaided eye, but egg-cells are microscopic. An ovary in some species contains a single ovule, with a single egg-cell; and hence a flower with one such ovary could form only one seed and one plant. Examples are buckwheat and "four-o'clock." In most flowers each ovary contains more than one ovule, and hence could produce as many seeds as there are ovules. Examples are the bean pod, which grows from a single flower, and has six to ten seeds, and many flowers (e.g., poppy) with numerous seeds from one flower.

The pollen-grains form cells which are often called male reproductive cells or fertilizing cells, and the egg-cells are called female. Neither kind of cell alone is able, as a rule, to produce a seed; and usually there must first be a union of the two kinds of cells as a preliminary to development of seeds (see $\S 75$, on bean flower). There are a few cases of flowering plants in which the egg-cells have the peculiar power of developing without the aid of the pollen-grains. This condition is parthenogenesis.

Pollination. - The fertilizing cells produced by pollengrains must be brought near the egg-cells in the ovary. 
The first step in this transfer is the placing of the grains upon the stigma or upper end of the pistil. This transferring of pollen-grains, called pollination, is a purely mechanical process, sometimes done by insects, sometimes by wind, sometimes the pollen falls from anthers to stigma, and often gardeners make the transfer by means of a small brush which is first touched to anthers and then to stigmas of flowers. A large number of flowers have their sepals, petals, anthers, stamens, and pistils modified to fit them for the visits of insects, or to provide for pollination by other means; and such modifications will be considered later. Fertilization. - When pollination has occurred, that is, when pollen-grains have lodged on the stigma of the pistil, the wall of the pollen-grain grows out into a tube (pollen-tube) and this grows down between the cells composing the style and ovary until it reaches the egg-cell in the ovule (see Fig. 61). One pollen-cell is required for each egg-cell, so that in a flower with a dozen eggcells there must be a dozen pollen-tubes from as many pollen-grains growing down the style. Many more pollengrains may be on the stigma and each may grow a pollen-tube, but only one is needed for each egg-cell.

When the pollen-tube has nearly reached the egg-cell, as shown in Fig. 61,

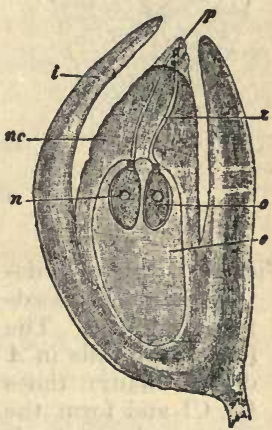

FIG. 61. Longitudinal section of ovule of pine, with two eggcells $(n, o)$, each with a nucleus. $p$, pollengrains ; $t$, pollen-tube; $e$, endosperm ; $i$, integument. The entire ovule forms a seed with one embryo, one of the egg-cells not developing. (From Strasburger.) the end of the tube opens and some of the contained protoplasm and a nucleus from the pollen grain slips down the tube and goes into the egg-cell. This mass which enters the egg-cell is the fertilizing cell or sperm-cell, and its nucleus is the sperm-nucleus. The sperm-nucleus soon unites with the 
egg-nucleus, and the combined nucleus is the nucleus of the fertilized egg-cell. This process of fusion of sperm-cell with
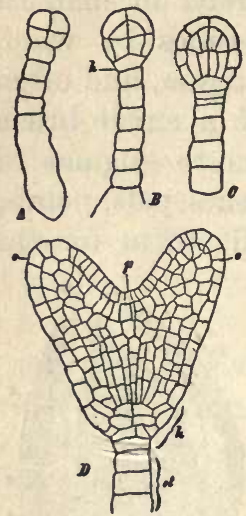

FIG. 62. Stages in development of a seedplant embryo. The four upper cells in $A$ divide many times $(B, C)$ and form the embryo shown in $D$ with two cotyledons $(c)$, epicotyl $(p)$, and hypocotyl $(h)$. The row of cells shown below the embryo in each figure holds it in position in the ovary. (From Strasburger.) egg-cell is fertilization. Distinguish between pollination and fertilization as defined above.

Cell-division. - The result of fertilization is to stimulate the egg-cell to begin a series of cell-divisions to form a group of many cells (Fig. 62, $A, C$ ). These cells become the embryo, which is the part of the seed able to develop into a new plant. While the egg-cell is developing into the embryo, the surrounding cells of the ovule are forming other parts of the seed. In all seeds there is the outer covering or seed-coat of one or more layers; and in many seeds there is more or less tissue lying between the seed-coat and the embryo. This is composed of cells stored with food to be used later when the seed is sprouting or germinating. The bean seed is an example of one with an embryo and seedcoat only.

A castor-oil bean ( $\$ 138)$ is one with an embryo, a seed-coat, and a large mass of food-storing tissue between embryo and seed-coat. Such food tissue outside of the embryo is called endosperm (meaning within the seed).

In flowers like four-o'clock and buckwheat, which have one ovule and form one embryo, the development of the embryo and its surrounding parts (endosperm and seed-coats) is associated with enlargement and change in the wall of ovary. This forms an outside case close to the seed so that the fullydeveloped seed is inclosed in a sort of double seed-coat. 
In the four-o'clock seed the hard outer wall has developed from the wall of the ovary, the inner coat is the real seed-coat. In the buckwheat the "hull," which is commonly removed before the seed is marketed, is formed from the wall of the ovary. In the bean flower, which is an example of an ovary. with many ovules, a number of egg-cells are usually fertilized and begin development at about the same time. While the ovules are developing into seeds, the wall of the bean ovary elongates and becomes the pod.

In all flowering plants the fully-developed ovary with any part of the flower which develops with it is called a fruit $(\$ 212)$.

190. Special Adaptations of Flowers. - Study of such simple flowers as those of the lily family and of the bean plant have shown the essential organs and work of flowers as reproductive structures. In order to adapt flowers of plants under special conditions to the all-important work of reproduction, there have been developed numerous modifications; and to some of the most interesting of these special adaptations we shall now give some attention. Most important of the adaptations are those which in some way are related to securing proper pollination.

191. Flowers and Insects. - Many of the most wonderful adaptations of flowers are in relation to insects. There is a mutual advantage in this relation. The insect secures food (pollen and nectar). Some insects like moths and butterflies, which have a long " tongue," visit flowers for the nectar; others like the bees and wasps collect both nectar and pollen. On the plant side, the advantage arises from the fact that while getting food the insects brush pollen from anthers upon stigmas, thus producing pollination and very frequently cross-pollination (placing pollen of one flower on stigma of another flower of the same species).

192. Advantage of Cross-pollination. - Many experiments have proved that plants from seeds which originated through cross-pollination are usually better than those resulting from 
self-pollination (pollen on stigma of its own flower). Also in some cases flowers are not fertile with their own pollen, or at least not so fertile as when cross-pollinated. Why these things are so is unexplained by biology; but it is a well-established fact that in all groups of animals and plants there are special arrangements for producing new individuals from egg-cells fertilized by unrelated sperm-cells or pollengrains. The remarkable contrivances, described in the following pages, some to aid cross-pollination and some to prevent self-pollination, can only be interpreted as meaning that for some unknown reason it is important that plant ovules should be fertilized by pollen-grains from other flowers and very commonly from flowers on other plants.

However, it must not be understood that self-pollination is always guarded against, for there are some flowers which do not open and which must be self-pollinated, and yet they produce good seeds. There are some cases of flowers which do not open, but which may possibly be pollinated by insects which bite holes in searching for food.

193. Adaptations against Self-pollination. - Most familiar of these is the fact that in many species certain flowers have only stamens and others have only pistils. In the case of cultivated strawberry plants, some varieties produce only pistils in their flowers and are called pistillate flowers. Other varieties have both stamens and pistils in each flower and are said to be perfect or bi-sexual flowers. There are no varieties of strawberries which have only stamens (staminate flowers); but some other kinds of plants have such flowers. Strawberry plants which are known to produce only pistillate flowers must be planted near plants which have perfect flowers. In buying plants from dealers one must learn the name of the variety and then find out from catalogues or garden books whether the variety is perfect or pistillate. A very instructive experiment consists in planting some strawberry plants of a pistillate variety in several pots or 
boxes, keeping covered with mosquito-netting to ward off insects, and then pollinating the flowers of the plants in one or more pots, using a small brush or a feather to transfer the pollen from stamens of the perfect to the pistillate flowers. Perfect and pistillate plants for such an experiment can be purchased from seed-dealers for about 30 cents per dozen, postpaid, in autumn or spring.

A second common adaptation against self-pollination is found in some flowers in which the pollen and the stigma of a flower are not mature (ready for pollination) at the same time, but since the flowers open at different times it is possible for insects to produce cross-pollination.

Still another kind of protection against self-pollination is illustrated by some flowers which have stamens much shorter than the style so that pollen cannot fall upon the stigma of the same flower, making pollination by insects necessary. Also, there are other flowers (e.g., the bean and orchids) which have their corollas peculiarly modified so that at the same time they guard against self-pollination and favor crosspollination.

194. Adaptations for Cross-pollination. - The simplest of the arrangements for cross-pollination is connected with wind as an agency for carrying pollen. Great quantities of light pollen are produced by many plants, and this floats upon currents of air to the stigmas of other flowers. Indian corn and pines are good examples. Many thousand species of plants are known to be cross-pollinated by the wind.

Some of the adaptations for cross-pollination by insects are among the most remarkable structures in the plant kingdom. The bean flower as an example has been described in $\$ 75$. Probably most flowers which, like that of bean plant, are irregular in shape (not radially symmetrical) are pollinated by insects. In some of the orchids (e.g., lady-slipper) the flower has a peculiar sac surrounding the anthers and the stigma, and through this insects must crawl 
in order to reach nectar. Pollen rubbed on the insect's body while in one flower will be brushed off when crawling past the stigma of another flower. Many such peculiar arrangements are described in Charles Darwin's great work on " Crossand Self-fertilization in the Vegetable Kingdom."

195. Structure of Various Flowers. - $(L)$ In addition to some simple flower, and the bean flower previously studied ( $\$ 74)$, the student should study a number of other types. If this work is left for the spring of the year, a few collecting excursions will furnish an abundance of materials for study (trillium, buttereup, mustard, apple or pear or cherry, dandelion, violet, anemone, hepatica, adder's tongue, dogwood, spring-beauty). Any such flowers may be collected in the proper season and preserved in a solution of five parts commercial formalin in one hundred of water, in fruit-jars. In winter there are scillas, tulips, hyacinths (especially white Roman), snowdrop, narcissus, freesias, fuchsias, and primroses available in greenhouses, some of them at small cost, especially if arrangements are made with gardeners for growing ordinary varieties. Plants with bulbs can easily be brought into flower in schools by planting the bulbs in pots or boxes in early autumn, leaving in a cold cellar or buried outdoors for two months, and then bringing into warm and light rooms. Or if planted in gardens according to directions in dealers' eatalogues, they will bloom in very early spring.

The time available for laboratory study of flowers should be used in careful examination of as many kinds as possible, comparing with flowers previously studied, and making brief notes and simple sketches. Only a small proportion of the time should be used for making notes and sketches; it is better to understand the parts of a dozen types of flowers than to have made a beautiful drawing in detail and have studied only one flower.

It will be well to read the following pages in advance of the laboratory study, and then later study the text carefully in the light of the facts learned by examination of flowers themselves.

It is recommended that, beginning with the first flower studied, a record be made in the note-book of answers to the following questions :-

Are sepals, petals, stamens, and pistil present in flowers examined, and how many of each? Record your observations in tabular form under headings such as the following:-

Name of flower Sepals Petals Stamens Pistil 
196. Petals and Sepals. - The simplest flowers have only the essential reproductive parts, the stamens and pistils, the petals and sepals being absent. Such flowers may have both stamens and pistils (i.e., are perfect); or some flowers may have stamens (staminate), and others pistils (pistillate). Willows are familiar examples of the latter.

In many flowers, particularly of the lily family, sepals and petals are present, but they are all alike in size and color. In such cases where we cannot distinguish between calyx and corolla, the term perianth is often used. In the most common flowers the petals are larger than the sepals, and usually white or colored. The sepals in some flowers are small, scale-like structures, and in others they may be leaflike. Some flowers (e.g., anemone, hepatica, rue anemone, marsh marigold) have no petals, but their sepals resemble petals. Some flowers of this kind are at first puzzling, because the leaf-like bracts (collectively called involucre) on the flower-stalk may be so close to the flowers that they are at first taken to be green sepals. Hepatica is such a puzzle, but in the closely related anemone flower the bracts are not near enough to be mistaken for sepals. In the dogwood flower (really a cluster of small flowers) the bracts are large and petal-like, and are commonly mistaken for petals by those who have not studied botany.

Modification of Petals. - In the simplest flowers the petals are free from one another; but in the highest seedplants they are more or less united into bell-shaped or tubular corollas. The sepals may also be united, as in the tobacco flower; or they may be separate while the petals are united so completely that only the lines of fusion indicate the number of united petals (as in the morning glory). Between these two extremes of fusion of petals and sepals there are numerous intermediate stages which may be found in common flowers.

197. Number of Parts of Flowers. - In some flowers the number of sepals, petals, and stamens is the same (often three, 
four, or five); but many other flowers vary as to the number of these parts.

There is no essential relation between number of parts and the reproductive function of flowers. Three sepals in one flower may inclose the flower bud as well as five or more do in some other flower; a few petals which are large and conspicuous may be more useful in relation to insects than are many small petals; a few stamens may be large and produce more pollen than numerous stamens; and there is

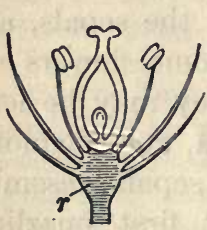

A.

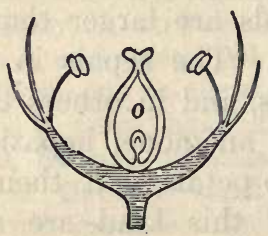

B

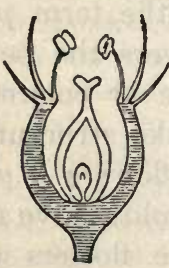

$\mathbb{B}^{\prime}$

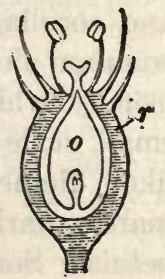

c

Fig. 63. Relation of the ovary and receptacle in flowers. $r$, receptacle; o, ovary. (From Strasburger.)

no necessary relation between number of styles and stigmas and the number of seeds which may be produced.

It is interesting to note that many monocotyledon ( $\$ 141)$ flowers have their parts in threes, and many dicotyledons have their parts in fives or fours. References to such constant numbers in certain families of plants will be found in books for identification.

198. Position of Ovary in Flowers. - In very many flowers the sepals, petals, and stamens appear attached beneath the ovary (Fig. 63, $A$ ). In some cases (e.g., apple, squash), the ovary appears to be entirely beneath the flower; that is, the sepals, petals, and stamens appear attached above the ovary (Fig. 63, C). There are still other flowers which are intermediate, as shown in $B$ and $B^{\prime}$ of the same figure. Special names are often applied to these conditions when describing species. 
199. Simple and Compound Pistils. - In many flowers there is a single pistil, which appears to be formed from a single leaf-like structure known as a carpel. If we imagine a small narrow leaf with one or more ovules attached on its margin, and then this leaf rolled so as to bring its edges together and the ovules inside the inclosed cavity, the formation of a simple pistil from a carpel would be illustrated.

In buttercups there are many simple pistils in a flower. In very many flowers the pistils have united together to form a compound pistil. In some of these the ovaries of the pistils are more or less united, but

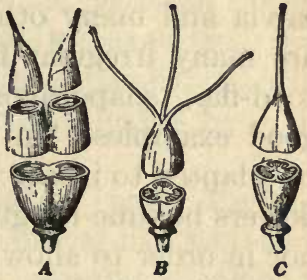

Fig. 64. Compound pistils. $A$, two pistil-leaves or carpels slightly united; $B$, three united as to ovaries, but with separate styles; $C$, three united into one compound ovary and style. (From Gray.) there are as many styles as there were pistils (Fig. 64, $A$ and $B$ ). In others there is complete union so that there is one ovary and one style (Fig. $64, C$ ), and only careful examina-
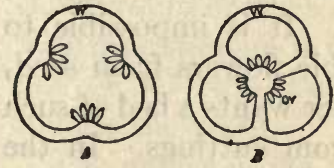

Fig. 65. Transverse sections of two ovaries, showing attachment (placenta) of ovules (ov) in $A$ with one chamber and in $B$ with three chambers. $w$, ovary wall. tion of grooves on the surface and of sections shows that the pistil is compounded of two or more simple pistils. Fig. $65, A, B$, shows in cross section two kinds of ovaries formed from three carpels. In $A$ the carpels appear to have united at the edges, leaving a single cavity in the ovary and the ovules attached to the outer wall; while in $B$ the arrangement suggests that three simple pistils have united completely, so that the ovules are attached to a central column.

200. Irregular Flowers. In the bean flower we have already studied a good example of irregular flowers; that is, 
flowers which are not radially symmetrical, as are those of apple, buttercup, tulip, and many others equally well known. In the irregular flowers of bean, sweet pea, violets, pansy, salvia and many others the petals are separate; but there are many irregular flowers with united petals. Flowers of toad-flax, snapdragon, mullein, catalpa, and any mint are good examples. In most cases irregular flowers appear to be adapted to insect visits. This does not mean that regular Howers became irregular because insects began to visit them, nor in order to allow or to encourage insects to make visits; but rather that flowers which were irregular were thereby fitted to insect visits. Just what caused flowers to become irregular in the first place is unknown.

201. Double Flowers. - A large number of varieties of cultivated flowers have become "double," which means that the petals have become very numerous and the stamens and pistils have largely or entirely disappeared. In some varieties (e.g., the double-flowered portulacas) some flowers do not develop completely "double," but have pistils and stamens which produce seeds. When these seeds develop into new plants, some of the flowers produced will be "double" and others only partly so. It is impossible to continue to raise only completely double flowers from seed, for they would be seedless. If a gardener wants a bed of such flowers, he must propagate entirely from cuttings. In the case of many varieties of roses this propagation from cuttings is the exclusive method used by gardeners, and we never see stamens and pistils in flowers of these varieties.

The explanation of double flowers is often said to be that the stamens and pistils have changed to petals. One fact which at first sight seems to support this view is that in some flowers (e.g., water-lily) there are all stages of transition between petals and stamens. However, this does not prove that stamens and pistils were originally petals. On the contrary, stamens and pistils appeared in the lowest flowers 
before petals did. Moreover, the fact that sometimes green petals are found does not prove that petals were once green leaves. Also, colored sepals and bracts were not once petals. But the fact that sepals, petals, stamens, and pistils all suggest more or less similarity to leaves probably means that they have developed or been evolved from expanded leaf-like structures in lower plants and that foliage leaves have developed from similar structures.

202. Flower Regarded as Shortened and Modified Branch. - Every flower begins as a set of leaf-like structures arranged in whorls or circles on the receptacle, which is simply the conical end of the flower-stalk. As the sepals, petals, and stamens grow up, the receptacle may remain conical (as in Fig. 63, $A$ ), or the receptacle may become more or less cup-shaped, resulting in flowers like $B$ and $B^{\prime}$ in Fig. 63. And if the receptacle both grows cup-shaped and at the same time closely surrounds the ovary, the result is a flower like Fig. $63, C$, in which the ovary is beneath the sepals and petals.

203. Stamens are usually entirely free from each other, but in some flowers they are united either by the anthers or by the filaments into a ring, and in some flowers into two or three groups or more. In the bean flower there are two groups, with nine stamens in one, and one in the other. In the dandelion ( $\$ 207)$ the stamens are united into a ring, which surrounds the pistil.

The anther is the essential part of the stamen. In order to get acquainted with some of the various forms of anthers, they should be carefully examined in the flowers studied. Use a hand-lens, and also remove some pollen for examination with the compound microscope.

Anthers are in some flowers attached directly and without filaments. They are then said to be sessile, just as a leaf attached without a petiole is sessile.

$(D)$ Germination of pollen-grains and growth of pollen-tubes may be studied by placing some pollen, if possible from several kinds of 
plants, in concaved object-slides containing 5,10 or 15 per cent of white sugar in water (sugar-solution, or syrup). The slides should be kept covered to prevent evaporation. Examine from time to time with the low power of the microscope. It is well to use several kinds of pollen and various strengths of syrup, for the grains do not all germinate in the same strength of sugar-solution. Sometimes it is possible to find pollen-grains beginning to germinate on the sticky stigmas of flowers.

204. Introductory Study of Flowers. - The foregoing account is intended to be merely an introduction to the study of flowers. Their forms are so numerous that only brief interpretation of some of the most common modifications can be attempted in a year's course in biology; and so the authors have selected for study the materials which are most likely to be interesting and useful to general readers.

Those who are especially interested in the forms and adaptations of flowers should read the accounts in the standard textbooks of botany by Coulter, Bergen, Atkinson, Bailey, Gray; and then perhaps such famous books as Darwin's "Cross- and Self-fertilization in the Vegetable Kingdom," and the Chapters on flowers in Kerner's "Natural History of Plants."

205. Flower-clusters: Inflorescence. - In many small plants there is only one flower, and in others the few flowers appear singly in the axils of ordinary foliage leaves. But in the great majority of the common seed-plants the tendency is to produce a number of flowers near together in groups or clusters. The term inflorescence refers to such flower-clusters. Fig. 66, $A-G$ illustrates some of the most common arrangements. The main lines in each of these figures represent the main flower-stalk (peduncle), and the branch lines show the position of the individual flower-stalks (pedicels). If time allows special study of flower-clusters, the textbooks of botany by Gray, Bergen, or others should be consulted. 
206. The Meaning of Flower-clusters. - The value of flower-clusters is believed to be threefold; namely, (1) to attract insects which carry pollen, (2) to expose certain flowers for pollination by wind, (3) to aid in distributionof seeds.
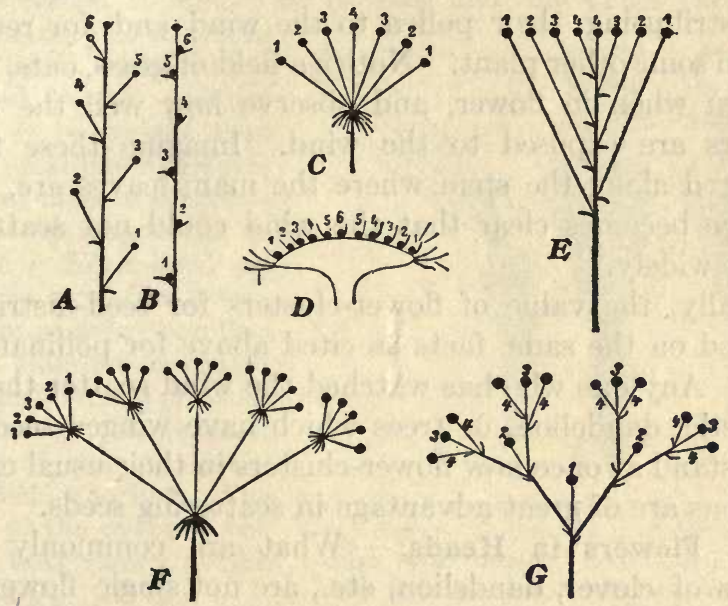

Fra. 66. Types of flower-clusters. The positions of individual flowers are represented by the black spots. The order of flowering is shown by the numbers. $A$, raceme ; $B$, spike ; $C$, simple umbel ; $D$, head ; $E$, corymb ; $F$, compound umbel; $G$, cyme. Note that the first-formed flower is in center of $G$, but at edge of clusters shown in $C, D, E, F$.

With regard to insects, a cluster of small flowers held above the leaves of the plant by the flower-stem appears to be in a particularly advantageous position for attracting attention. Certainly they are made more conspicuous to the human eye, but the truth is that it is not yet certain whether most insects which visit flowers are guided by the brilliant colors of the flowers. It is well known that many inconspicuous flowers are favorites of insects, which are probably led to the flowers by odors more than by colors. However, many botanists believe that conspicuous flowers do aid in attracting 
insects; and if this is true, grouping of flowers in clusters is surely important.

Concerning the suggestion above that flower-clusters favor pollination by the wind, it is evident that flowers held above the leaves of a plant have a most advantageous position both for distributing their pollen to the wind and for receiving it from some other plant. Notice a field of grass, oats, wheat, or corn when in flower, and observe how well the flowerclusters are exposed to the wind. Imagine these flowers scattered along the stem where the main leaves are, and it at once becomes clear that the wind could not scatter the pollen widely.

Finally, the value of flower-clusters for seed-distribution is based on the same facts as cited above for pollination by wind. Any one who has watched the wind scatter the seeds of thistle, dandelion, or trees which have winged seeds, will understand at once how flower-clusters in their usual exposed positions are of great advantage in scattering seeds.

207. Flowers in Heads. - What are commonly called flowers of clover, dandelion, etc., are not single flowers, but clusters of flowers set on the expanded end of a flower-branch (Fig. 66, D). When thus set in heads and crowded closely together, the constituent flowers are very small. A dandelion flower-head (commonly called dandelion flower), a sunflower, or some similar flower-head should be examined.

Dandelion Flower-head. - $(L)$ Dandelion plants taken up in fall or early spring are easily grown in pots or boxes in the schoolroom, and flowers in various stages of development obtained.

Examine the peculiar flower-stem which supports the head of flowers. As the flowers grow old and the head becomes closed by the surrounding bracts, notice whether this flower-stem grows longer, and especially notice its length when the seeds are mature and about to be blown away by the wind.

Notice that beneath the flower-head are two rows of bracts, collectively forming an involucre. Observe the position of these bracts in young heads, and in old ones which have closed. These 
bracts correspond to leaves on stems beneath ordinary flowers, and not to the calyx. The white petal-like structures surrounding clusters of dogwood flowers are also examples of bracts.

Any one of the numerous flowers in a dandelion flower-head has the structure shown in Fig. 67, $a$. The tuft of bristles or hairs (called pappus) and the ring below the hairs represent the calyx. The surrounding corolla is tubular at its base and the upper part is a flattened strap-like structure called a ligule. Five petals are united in this peculiar corolla. It is evident then that the petal-like structures which first attract attention when one casually looks at a dandelion flower-head are simply the strap-like extensions from as many corollas. The five stamens are united at their anthers, and protruding up through the center of this ring of stamens is the style, which is forked. The position of the ovary is the same as that shown at the bottom of Fig. 67, $a, c$.

Sunflower, Aster, Calendula, Coreopsis, Zinnia, Marigold. - $(L)$ These are common flower-heads with

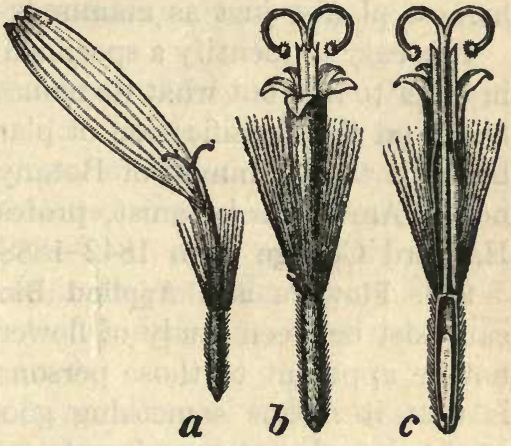

Fig. 67. Composite flowers of Arnica. $a$, ray-flower; $b$, disc-flower consisting of a calyx with bristles, a tubular corolla with five lobes, style divided into two stigmas, stamens united in ring around the style; $c$, longitudinal section of $b$. Some composites have stamens in rayflowers $(a)$. (From Strasburger.)

structure similar to that of the dandelion, except that only the flowers at the margin of the head have the strap-like rays. All the other flowers in the heads have the same structure; they are tubular flowers without rays. The flowers without the straps are called discflowers, those with straps are ray-flowers. It is evident that the conspicuousness of sunflowers and their relatives named above is due largely to the large and colored rays of the marginal flowers.

Flower-heads of Common Thistles, Burdock, Centaurea, Tansy, Ironweed. - $(D)$ These have no strap-like rays on any of the corollas in the head. In thistles all flowers are alike and similar to the disc-flowers of the sunflower head. In centaurea the marginal flowers are enlarged and without stamens and pistils (i.e., they are sterile.) 
The dandelion, sunflower, aster and the others mentioned above are members of the family of plants known in botany as the Composite Family (Compositæ). It is the largest family of seed-plants (phanerogams). There are over five hundred species of composites in the United States east of the Mississippi River. The composites are regarded as the highest plants, just as mammals are the highest animals.

It is easy to identify a specimen flower as a composite, but in order to find out what its name is, one must consult special books on the classification of plants. Most famous of such books is the "Manual of Botany," by Asa Gray, the most noted American botanist, professor of natural history at Harvard College from 1842-1888.

208. Flowers and Applied Biology. - How any relation can exist between study of flowers and applied biology may not be apparent to those persons whose idea of "applied" is that it means something good to eat or something of economic value, as are forests, cereal plants, etc. And yet it would be a mistake to think of flowers as not having economic value, for millions of dollars are invested in land and greenhouses used solely for producing flowers for the market, and in all civilized lands the sale of flowers amounts to an enormous total of dollars annually.

In still another way study of flowers is of direct practical use, namely, in producing new varieties of plants. Each year the catalogues of dealers announce new and attractive varieties. Many of these have been produced by gardeners who have applied their knowledge of flowers to experiments with artificial cross-pollination; and the result of such experiments is often valuable new varieties of plants.

But while some readers may not be interested in flowers because some other persons make great profits in growing and selling them, it is probable that there are few individuals who will admit that they have no interest in flowers for their ornamental value. Have you ever met a person so dead 
to all appreciation of the beautiful that he did not sometimes show interest in flowers? Perhaps you have; but such persons are becoming rare. Study of flowers was once thought to be a subject for girls, but in recent years it has become more and more evident that appreciation of the beauty in nature is just as valuable for men as for women. In many colleges for men there are now courses in æsthetics, the science of the beautiful.

It is evident, then, that some outline study of flowers belongs in applied biology; for besides the application of such knowledge to the business of growing plants and of producing new varieties mentioned above, an understanding of the nature of flowers is useful to every cultured citizen who has any æsthetical appreciation whatever. Flower study is certainly applicable to our daily life, if we mean life in the widest sense of the word.

These comments on the importance of appreciating the beauty of natural things have been suggested in connection with flowers, because to most people flowers are the most beautiful and remarkable of natural objects. However, in many other structures of animals and plants there are things which appeal to our sense of the beautiful (æsthetic sense), and the student of biology should keep constantly on the lookout for them.

\section{SEED-PLANTS WITHOUT TRUE FLOWERS}

209. Angiosperms and Gymnosperms. - So far the descriptions of plant reproductive organs have applied to the flowers found among monocotyledons or dicotyledons. All these have the seeds formed inside the closed ovaries of flowers, and are classified in a great group called Angiosperms (meaning seed-vessel, i.e., ovary). In addition to such seed-plants with closed ovary, there are several hundred species of plants which form seeds, but which have the seeds unprotected by an ovary. Such plants form a group known 
as Gymnosperms (meaning naked seeds). The most familiar examples are the conifers (a word meaning cone-bearers), including pine, hemlock, larch, spruce, and similar trees. Other gymnosperms are the cycads seen in greenhouses, and the Japanese gingko tree in American parks.

The Angiosperms and Gymnosperms together constitute the great division of plants known as Spermaphytes (meaning seed-plants). Seed-plants are by some botanists called Phanerogams (meaning visible organs of reproduction, i.e., flowers). The term seed-plants is better than flowering plants, because gymnosperms do not have true flowers; and, strictly speaking, flowering plants would include only angiosperms. The following table shows the relations of these groups of plants mentioned above.

\section{Groups of Seed-plants}

Spermaphytes

Phanerogams
Gymnosperms

Angiosperms
Monocotyledons

Dicotyledons

210. Gymnosperm Reproductive Organs. - Only these organs of gymnosperms are selected for special mention, because in stem, roots, and leaves there is great similarity to angiosperms already studied. The stems of the conifers have the general plan of the dicotyledons, with secondary growth causing annual enlargement of diameter (Fig. 46). Most conifers have needle-like leaves adapted to severe conditions of weather, cycads have leaves suggesting those of palms (which are monocotyledons), the gingko tree has broad leaves, and the arbor-vitæ has scale-like leaves. Also, most of the conifers are evergreens, that is, their leaves live several years and do not all fall at a certain season; but the common larch or tamarack is a conifer that sheds its leaves annually like most dicotyledon trees, and there are many evergreen shrubs and trees among angiosperms. In short, there is no important characteristic of leaves in gymnosperms 
which is not also found among angiosperms. In the seeds of conifers there may be more than two cotyledons (from two to twelve); but botanists do not attach any special significance to the mere number, since in other respects conifer and dicotyledonous embryos are very similar.

It is then in the reproductive organs that there is the most striking difference between gymnosperms and angiosperms; and hence the two groups are compared in this connection with the lesson on the reproductive organs (flowers) of the highest plants.

211. Cones as Reproductive Organs. - The pine or other common cone-bearing tree may be taken as a type of the reproduction found in gymnosperms. In addition to the foliage leaves, certain leaves are set apart for purposes of reproduction. These leaves (called sporophylls, or spore-leaves) are arranged in the form of cones, of which there are two kinds.

One kind of cone ("male") is small (Fig. 68, $A$ ), and each sporeleaf, corresponding to a stamen of flowers, bears on its lower surface spore-cases or pollen-sacs, which produce pollen-grains. Such a cone is often called staminate,

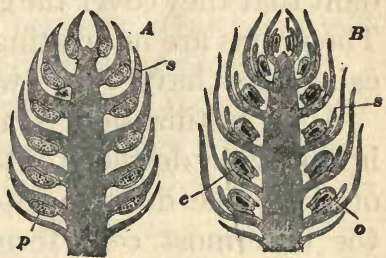

Fig. 68. Diagrams showing structure of cones in section. $A$, male cone; $B$, female cone. $s$, sporophylls or spore-leaves ; $o$, ovules; $p$, pollen-sacs ; $e$, (black) central endosperm part of ovule. (From Parker.) the term commonly applied to flowers which have only stamens.

The other kind of cone ("female ") has a similar structure, but is larger. It is the well-known cone often seen on conebearing trees (Fig. 68, B). Each spore-leaf of the larger cones has on its upper surface spore-cases or ovules (two in pine); and such a spore-leaf is equivalent to a carpel, with its ovules, in true flowers of angiosperms. In true flowers the carpel folds and grows together to form a pistil with an ovary 
inclosing the ovules; but in gymnosperm cones the sporeleaves do not unite to inclose the ovules, and hence these ovules and the resulting seeds are said to be "naked."

Such a cone containing spore-cases or ovules on its sporeleaves corresponds in its function to a flower containing pistils only. Within each ovule is formed an egg-cell from which an embryo develops after fertilization.* This is effected by a cell which enters through a tube (pollen-tube) from a pollen-grain. In short, fertilization is very similar to that described for plants with true flowers (\$189).

Pollination of conifers is due to gravity and the wind, the pollen falling on the spore-leaves and sliding down to the ovules. The pollen-grains in pine forests are often so abundant that they cover the ground, the so-called "sulphur snow." The grains are interesting objects for miscroscopic study, because they have two "wings," which are really air-sacs.

After fertilization is accomplished the fertilized egg-cell in an ovule develops into an embryo, the surrounding cells of the ovule develop into endosperm stored with food, and the outermost cells form the hard seed-coats. When the seeds are fully developed (sometimes two years are required) the leaves of the cone dry and separate so that the seeds may fall out. The seeds of some species of conifers have wings which assist in scattering by the wind.

\section{FRUITS OF SEED-PLANTS}

212. The word fruit in popular usage refers to various edible products of flowers, but in botanical terminology it means the structure, usually containing seeds, which develops from a flower, chiefly from the ovary. In the simple cases one ovule forms one seed, and the wall of the ovary forms the surrounding structure. These may be in part edible (as in

* In some gymnosperms there are two or more egg-cells in each ovule (as shown in Fig. 61); but only one survives and forms the embryo in the seed, which develops from one ovule. 
cherry and plum), or dry and hard (as in the so-called "seeds" of sunflower and buckwheat). In other cases, as in bean pod and poppy capsule, there are many seeds inside the structure formed from the wall of the ovary (Fig. 63, C).

Some fruits are more complex than the above in that they include structures in addition to the pistil. For example, the core only of an apple develops from the ovary, while the outer edible fleshy part is formed by growth of the receptacle which grows up and surrounds the ovary.

Moreover, what is popularly termed a "fruit" may contain several developed ovaries (botanically, fruits). For example, the fleshy part of a strawberry is formed from the receptacle of the flower, and the small hard grains (so-called "seeds") on the surface are each from a pistil (the strawberry flower has many pistils), and hence a strawberry is a mass of aggregated fruits set on an edible receptacle.

For purposes of study and comparison, it is most convenient to group fruits under the following headings: (1) simple dry fruits, (2) simple fleshy fruits, (3) stone-fruits, and (4) complex fruits. The word "simple" is used to mean a fruit developed from pistil only, while "complex" is applied to fruits developed from ovary surrounded by overgrown receptacle, as in case of the apple.

213. Simple Dry Fruits. - Under this heading are included the fruits which are developed from one ovary, and in which the ovary-wall becomes hardened and dry when mature. There may be in the ovary one ovule forming one seed, or many ovules resulting in a many-seeded fruit. Also there may be many pistils in a flower, and each form a fruit (e.g., buttercup). Examples of dry fruits are akenes, grains, nuts, pods, follicles, and capsules.

Akene. - A simple dry unopening fruit (like that of the sunflower, buttercup, buckwheat, dock, bur-marigold) containing but a single seed, is an akene. As in the case of the sunflower and buckwheat, these simple fruits are commonly 
called "seeds," but the outer hull is from the wall of ovary and corresponds to a bean pod, while the seed with its own coat is inside.

$(L)$ Examine a buckwheat or sunflower fruit (" seed").

In the akene fruits of the composites (e.g., dandelion), the calyx usually adheres to the outer wall of the ovary, while the upper edge of the calyx appears on the ripened fruit as a tuft of hairs which aid in the dispersal of the seeds.

Grains. - In the unopened grains or fruits of the rice, oats, barley, etc., the outer hull is formed from the wall of the ovary. This hull is entirely filled with the seed, and the seed-coat adheres to the ovary-wall.

Nuts. - The nuts of the oak (acorn), beech, and chestnut are true fruits, very much like the akenes, except that usually the dried ovary-wall is very hard. The kernel inside is the seed and has its own seed-coat.

In the true edible chestnuts, beech-nuts, and hazel-nuts, two or three nuts are within a bur (involucre); but each nut has developed from one ovary (i.e., is a true fruit). In the case of the oak fruit (acorn), the acorn-cup surrounds the one true fruit (from one ovary). The burs of chestnut and beech, acorn cups, and hazel hulls are formed from bracts of the involucre, which grow up and surround the ovary.

The outer hull of walnuts, hickory nuts, and husks of cocoanut develop from the outer wall of the ovary, while the inner wall forms the shell (see under stone-fruits). The Brazil nut is a seed with a very hard seed-coat, and a number of such seeds are inclosed in a hard-walled shell which comes from the wall of the ovary. This is also the case in the horsechestnut and buckeye, where a fruit has two seeds (so-called "nuts") developed in a single ovary, and the hull is formed from the outer wall of the ovary.

Many-seeded Dry Fruits. The bean pod opens along both edges or sutures. Other pod-like fruits with many seeds open along one edge only, and are called follicles. The fruits of 
poppy and morning-glory have several cavities, each with seeds, and are called capsules.

Aggregates of Simple Dry Fruits. We commonly call a strawberry a "fruit," but botanically considered it is an aggregate of simple dry fruits. The true fruits of a strawberry are the hard bodies (each with a seed inside) which are set in the surface of the berry. Each of these bodies develops from an ovary, and is a single dry fruit or akene ( $\$ 213)$. The fleshy mass in which the true fruits are embedded is the enlarged receptacle. In a strawberry flower a large number of pistils are set on a rounded receptacle. Flowers and berries picked green and preserved in formalin will help to make the mode of development clear.
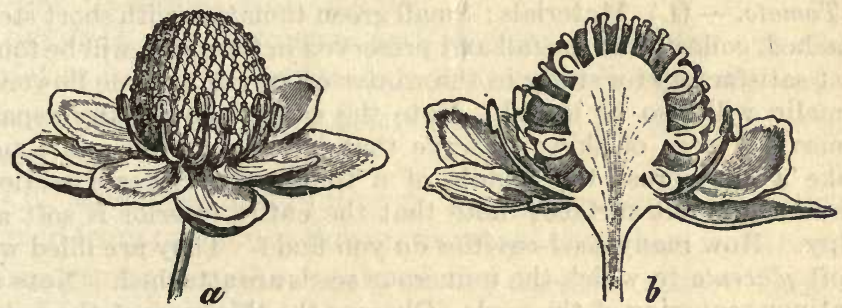

Frg. 69. The receptacle of the buttercup flower bears many simple pistils and each develops into a simple dry fruit (akene). $b$ is a section of a flower showing the globular receptacle (white center).

The flower of buttercups produces a similar mass of simple dry fruits (akenes), but the receptacle does not become as much enlarged as in the strawberry.

A fig develops from a flower which has numerous pistils on its receptacle, as in the case of the strawberry. And in both cases the edible part is from a receptacle, but in the fig flower it is concave, whereas that of the strawberry is convex. As a result of this difference in shape of the receptacle, the fruits of the numerous small pistils (the hard grains) are on the inside of the ripened fig, but on the outside of the straw- 
berry. Each grain is simply a dry fruit, and contains the true seed.

214. Simple Fleshy Fruits. - In all the cases described above the wall of the ovary forms a hard or dry case or shell around the seed or seeds. In the following fruits the ovarywall becomes fleshy, with a relatively thin external skin or rind.

A date is the simplest fleshy fruit, because it contains but one seed, which was formed from a single ovule, while the wall of the ovary developed the edible fleshy part and the thin rind. If one cracks a date seed, it is found solid like a grain of wheat, except that the food in wheat is starch while in the date seed it is a hard woody substance (cellulose).

Tomato. - $(\dot{L})$ Materials : Small green tomatoes with short stems attached, collected in the fall and preserved in formalin, will be found most satisfactory for study in the winter or spring. Some flowers in formalin will also be useful. Note the calyx; how many sepals? Remove a piece of skin; observe that it is very thin and tough. Make a cut across the middle of a tomato (transverse section). Examine the cut surface; note that the entire interior is soft and pulpy. How many seed-cavities do you find? They are filled with a soft placenta to which the numerous seeds are attached. Note the gelatinous covering of the seeds. Observe the thickness of the ovarywall, and also of the partitions.

Other fruits like the tomato, in which the entire wall of the ovary becomes fleshy, are the grape, cranberry, egg-plant, banana, garden pepper, orange, lemon, persimmon, gooseberry, currant.

Lemon. - $(L)$ The lemon is like a tomato in structure, but has a very tough leathery rind. On the stem end of the fruit you may find the dried-up remains of the calyx; how many sepals? At the opposite end is a knob-like projection to which was attached the stigma of the pistil.

Remove the remains of the calyx and observe the ring of little dots left in the depression thus made. These are the ends of the fibro-vascular bundles which passed from the stem (peduncle) of the flower up into the pistil. What was their function?

The surface of the lemon is roughened by numerous pits which, if examined (by making thin sections of the yellow part of the rind, and placing under a lens) will be found to be little pits filled with oil, 
the oil of lemon from which lemon extract is made. Notice that this oil is very volatile and soon evaporates. It can be collected and preserved by dissolving it in strong alcohol, making lemon-extract. If a portion of the white part of the rind is removed and very thin cross sections are made, it will be seen to consist of layers of cells very much like the corky layer of the potato skin. What is the function of this layer?

Cut transversely across the middle of a lemon. Note number and shape of cavities. What relation exists between the number of seed-cavities and the number of knobs left in the depression after removing the calyx ? The cavities are entirely filled with a pulpy mass consisting of little irregular-shaped tubes or bags containing the juice. Make sections at various levels until you find seeds; then observe their arrangement.

The gooseberry and cranberry are simple fleshy fruits similar to lemon and tomato.

Bananas, navel-oranges, and seedless grapes are cultivated varieties in which ovules do not develop into seeds, but the ovary-wall becomes a fleshy fruit as in natural seeded varieties.

The pineapple and mulberry are aggregates of fleshy fruits formed from numerous flowers crowded on a short flower-stalk, which remains in the center of the ripened mass. They are called multiple fruits.

215. Stone-fruits. - Peach, plum, cherry, and apricot. Each of these so-called "stone-fruits" contains a hard stonelike body near the center of the edible fleshy mass, and the whole is inclosed in a thin, tough skin. In these fruits, the inner part of the wall of the ovary develops into a hard, stony substance inclosing the seed and the outer part of the ovarywall forms a pulpy edible tissue. Inside each "stone" or "pit" is a true seed (often called the kernel) with its own seed-coat. This kernel corresponds to a date seed, but the ovary-wall of the date forms fleshy substance close around the seed and no hard stony shell as in the peach and the plum.

The walnuts and hickory-nuts are similar to stone-fruits, except that their outer fleshy part gets hard late in the sum- 
mer and becomes the "hull " or "shuck." Before this hardening occurs, young English walnuts are sometimes used for making pickles. Obviously, a hulled walnut or hickorynut corresponds in origin to a peach or plum stone.

$(L)$ Examine any stone-fruits available. Also flowers and young stages preserved in formalin.

Aggregates of Stone-fruits. - Raspberry and blackberry. Each of the small rounded bodies of which these berries are composed is a stone-fruit, developed from an ovary. The whole berry is an aggregate of fleshy fruits; and each one of these bodies corresponds to a cherry. The numerous pistils of the flowers are crowded so closely together that the resulting fruits become united into the one mass which we know as a blackberry or raspberry. This mass when ripe separates easily from the receptacle. In short, a blackberry or raspberry is equivalent to many small cherries which we might imagine growing together into one solid mass set on one large receptacle. Blackberry flowers and green berries preserved in formalin should be examined.

216. Complex Fleshy Fruits are formed from an ovary plus some adjoining flower parts. All the fruits described in the preceding sections are formed from the ovary only. In some complex cases the receptacle grows up around the ovary as in Fig. $63, C$; the ovary therefore appears to be below the calyx and corolla (inferior ovary), and the fruit formed is composed of ovary and thickened receptacle (apple and cucumber).

Apple. - $(L)$ Materials : Apples, apple flowers, and various stages of the young fruit in formalin. Optional, pears and quinces.

Note the dried-up ends of sepals at end opposite the stem. Compare series of stages from flower to the young fruit. Observe the waxy coating on the skin. Suggest use. Remove the skin from an apple, put aside the peeled apple and examine it after a day or two. Use of the skin?

Cut the apple crosswise midway between the stem and sepal ends. Note the star-like arrangement of the seed-cavities. How 
many? Where are the seeds attached, and how many in each cavity? Note the tough papery walls of these cavities; this is the ovary-wall, commonly called the core of the apple. The fleshy mass which forms the bulk of the edible part is really the thickened receptacle of the flower. In the fleshy part, a short distance from the seed-cavities, is a ring of dots, the cut-off ends of the fibrovascular bundles which carried the food into the developing apple. How many dots are there and what relation is there between their number and that of the seed-cavities and sepals? In a longitudinal section of apple cut from stem end to sepal end, the string-like nature of these fibro-vascular bundles will be more evident; they can be pulled out much as you removed the strings from the pod of the bean in an earlier lesson. Note that these bundles originate in the stem of the apple and pass up to the sepals.

Compare (optional) the fruits of pear, quince, and apple.

Cucumber or Squash, Melon, Gourd. - Examine a series of stages showing development of cucumber flower into the fruit. These may be collected in summer and preserved in formalin. Cut a cucumber crosswise. How many seed-cavities do you find? Where are the seeds attached? The rind and the fleshy mass outside of the seed-chambers is derived from ovary-wall and receptacle. Examine a cross section of an immature squash; observe the seedcavities in the center, and the attachment of the seeds to placentas. The region immediately outside the seed-cavities is the ovary-wall, the remainder of the fleshy mass and the rind is derived from the receptacle. In the watermelon the main bulk and the edible part is the enlarged placenta.

217. Dispersal of Fruits and Seeds. - It is an interesting fact that only by the scattering of fruits and seeds is it possible for most higher plants to become widely distributed. A single plant of the Russian thistle may produce several hundred thousand seeds. If all these seeds were to fall at the base of the plant and there start to develop, it is very likely that none of them would reach maturity, for they would crowd and starve each other to death. Nature has provided against such a calamity and ensured the distribution of the species, for the plant when mature assumes a ball-like form, breaks off at the ground, and is rolled by the wind for miles from the place where it grew, dropping its seeds as 
it is tumbled along. This is only one of many methods of seed dispersal. The success of the common dandelion as a weed depends in a large measure upon its efficient device for scattering its seeds. Here, as in many other composites, the upper end of the calyx forms a tuft of hairs which are caught by the wind. In still other plants like the clematis the style of the pistil becomes a feathery tail by which the seed is wafted about. In the milkweed the little seed-stalk developes into a tuft of silky hairs so light that the slightest breeze may carry the seeds miles away from the parent plant. Plants like the elm, maple, ailanthus, and pine, also depend upon the wind to scatter their seeds; but the simple wing-like outgrowths from these seeds and fruits do not make them very buoyant, and consequently they are not carried far by the wind. In edible fruits like the wild blueberry, pokeberry, currant, etc., the seeds are so hard that probably they are not injured by being passed through the digestive organs of the birds or other animals that feed upon the fruits, and hence fruit-eating animals may serve as distributers of hard seeds far from the locality where they grew. In such cases as the black walnut, wild apple, osage-orange, etc., the fruits, because of their rotund form, may roll some distance from the parent tree. In some plants (e.g., witch hazel) the seeds are inclosed in an explosive pod which when it opens throws the seeds many feet away. The jewel-weed, garden balsam, violet, and morning-glory also belong to this type. In some plants the seeds or fruits develop hooks and barbs which catch hold of the furs of passing animals or the clothing of people and are thus widely scattered. Still others have their seeds or fruits inclosed in waterproof husks or envelopes so that they may be carried for miles by water, and as in the case of the cocoanut fruit, probably thousands of miles. It is probably in this way that the flora (collection of plants) of isolated coral islands originated. Seeds are also scattered in accidental ways, as for instance in the pellets 
of mud on the feet of wading birds, and nuts buried or hidden away by birds or squirrels and then never eaten.

Students of botany should guard against the common tendency to look upon adaptations for dispersal of seeds as having developed because plants required them. That this is not true is indicated by the fact that numerous species of plants have no obvious special methods for distributing seeds. All that we are justified in stating is that plants with seeds specially adapted for dissemination have a better chance of spreading over a large territory. We do not know why or how plants acquired their peculiar methods of seed-dispersal.

\section{SEED-PLANTS REPRODUCING WITHOUT FLOWERS AND SEEDS}

218. While the flowering plants naturally reproduce from seeds, many of them may also reproduce by other methods. Many cultivated plants are examples. Strawberry plants are commonly obtained from branches (called runners) which creep over the ground and take root at the joints or nodes, and form new plants at these points. In this way one plant has formed more than fifty new plants in a single summer. Black raspberry bushes and grape-vines bend over, form roots, and develop new plants where they touch the ground. Red raspberry bushes multiply by forming young plants from underground branches resembling roots. New plants start from roots of osage-orange and some other trees. Some flowering plants may reproduce from leaves; the best example is the "sprout-leaf" or bryophyllum, a garden plant whose leaves fall to the ground, form roots and shoots at the notches on edges of the leaves, and thus each leaf may form a number of new plants. Some begonia leaves will propagate in the same way. Still other flowering plants propagate from bulbs (which are short stems with leaves). This is well illustrated by certain kinds or varieties of onions. The common onions (" seed onions") are grown from seed in one 
season, or else the seeds are started one year and form small onions ("sets") which are planted the next spring. In this case one seed forms one set, and one set forms one onion bulb, which when mature forms flowers and seeds ; hence multiplication can be only by seeds. Some other kinds of onion bulbs behave differently in that the bulb which is planted divides into a group of little bulbs (bulbels). The multiplier or potato onion does this, and garlic is similar. Each of the little bulbels may be planted and will soon grow to full size, and then begin to form more bulbels. Multiplier onions occasionally produce seed, but gardeners commonly use the bulbels for planting. A third kind of onions form "top onions" or bulblets on the flower-stalks, sometimes mixed with some flowers. These small bulblets may be planted next season and soon grow to usable size, but if left in the ground, they will send up flower-stalks which produce bulblets. The so-called "Egyptian onions" and "tree onions" are top-onions. In the wild garlic some of the flowers are frequently replaced by bulblets. The above ways of growing different varieties of onions illustrate the methods of propagating many bulb-producing plants. Hyacinth, narcissus or daffodil, many lilies, crocus, and tulip are examples of flowering plants which are usually grown from bulbs and rarely from seed.

Without the aid of man some flowering plants may propagate from roots, stems, leaves, bulbs, in addition to seeds. By man's help many of them which do not naturally propagate without seeds can grow from parts of the plants like stems and roots. For example, cuttings or slips may be cut from stems of almost any plant and if properly set in moist soil will form roots and develop into a new complete plant. This is the familiar method of starting geraniums, coleus, and other house-plants; and dozens of shrubs (e.g., currant), vines (e.g., grape), and trees (e.g., willow) are commonly started from cuttings of stems. Go into any greenhouse 
and one will find a sand-bed used for starting roots on such cuttings.

Still another artificial method of propagation from cuttings is in the processes called grafting and budding. The details of this method are explained in $\$ 165$, but essentially it consists in taking a small piece of stem, with buds or a single bud, from one plant and attaching it to root or stem of another plant. The transplanted buds grow, and when placed near the root will form the entire plant above ground. This is the method for obtaining standard varieties of apples, pears, plums, cherries, peaches, and many other shrubs and trees.

All such propagation of plants from parts or pieces of fullgrown plants without seeds is known as asexual or vegetative reproduction. The propagation of plants by means of seeds involves sexual reproduction, for most seeds originate through fertilization. As we have seen, in some cultivated plants the method of reproducing by seeds has been supplanted largely by asexual reproduction. This has two advantages: (1) the ease of starting some plants without seeds, and (2) many cultivated varieties will always produce their own variety from parts of themselves, but not from seeds. For example, the seeds from a red apple may grow into trees which will bear green-colored apples and entirely different in flavor, size, etc. The only way to propagate with certainty the desired variety is to take a cutting from a branch of the apple tree known to bear the kind of fruit wanted and graft it on a tree started from seed. The runners of a strawberry plant will form new plants producing the same quality of berries, but the seeds rarely do so. Moreover, in the case of seedless fruits (e.g., navel oranges and seedless apples) the only possible propagation is by cuttings, buds, or grafts. The millions of seedless orange trees in California have descended from a single tree which grew from seed but could not itself produce seed. Without man's help such a variety could not have multiplied. 


\section{GENERAL NOTES ON SEED-PLANTS}

219. Adaptations of Seed-plants. - The preceding lessons on seeds, roots, stems, flowers, and fruits of seed-plantshave shown some of the most important ways in which these organs have become adaptively modified so as to enable certain plants to carry on their life-activities (especially breathing, feeding, and reproducing) better than an unmodified plant could under the same conditions. Write a brief essay on "Adaptations of Seed-plants," showing how at least five adaptations have given plants which possess them special advantage.

Origin of the Adaptations. - Just how adaptations of the various organs of seed-plants have originated is unknown to biologists ; but the fact that modified roots, stems, flowers, etc., are built on the plan of unmodified ones suggests that originally they were all alike. For example, it seems probable that the first flowers were very simple, and that their descendants have developed various modifications which adapt them to such important processes as pollination and seeddistribution. However, it does not seem probable that adaptations have been developed because they were needed; but rather that modified structures have appeared for some cause or reason unknown to us, and have happened to be of special use or value. For example, it seems probable that certain leaves first developed in pitcher-form, not because the plants required such leaves and set about to make them; but having appeared as the result of causes still unknown, the pitchers proved useful as insect-catchers and have been preserved, and probably improved.

This idea that many structures developed and then later found a special use and became adaptations is supported by the observation that many other modified structures have apparently found no use. For example, it is not known that the sharp spines on a chestnut-bur are useful defenses, for when 
the nuts are ripe and attractive to squirrels the burs split open and scatter the nuts on the ground beneath the trees. Obviously the spines were not made to protect the nuts from squirrels, as some books assume, for the spines are not hard until the nuts are ripening, and before that time no squirrel would care for them. What then is the explanation? It appears to be this, that like other modified structures, the spines originally appeared without any regard to possible use, and the splitting habit of the chestnut-bur has made it impossible for them to become useful. If such spines had developed on fruits which do not split and discharge their seeds, we can imagine that they might have been of some advantage and have become a useful adaptation as a protection against gnawing animals. There are many similar cases among animals and plants where structures may at first sight appear to have a use, but closer study shows us that they are not useful adaptations.

If structures appear without any reference to use, and prove to be actually harmful, the plants which possess them will probably disappear in time. For example, if the spines on some chestnut-burs had in any way interfered with the complete development of seeds, the result would have been that the trees having such harmful spines would long ago have disappeared, while the trees without them would have perpetuated their kind, and we should now have only trees producing spineless chestnut-burs. But the fact that the spines continue to exist without serving any use suggests that they are neither harmful nor useful, but simply harmless or neutral. There are many such harmless structures among animals and plants.

The above discussion will suggest that the student of biology who is interested in adaptations of animals and plants must not expect to find an evident use for everything. Apparently some things have use, and many things have none evident at the present time. In some of the apparently use- 
less cases there are reasons for believing that under different conditions of past times some things not now useful may have had a use.

The tendency to look for usefulness in everything is strong in the human mind. Long ago all nature was looked upon as made for the use of man; plants for man to eat and use in other ways, and animals for human food, clothing, beasts of burden, pets, etc. And, after all, it is not very surprising that men got into this way of thinking, for so many animals and plants are useful to us. This fact is impressed upon the reader of such books as Shaler's "Domesticated Animals," Wood's "Dominion of Man," and books dealing with useful plants. But within the past fifty years this attitude has changed, and now scientific men do not look upon all nature as having been made for the direct use of man, but believe that animals and plants have a reason for existence even if there were no human beings in the world. In fact, geology has shown that vast numbers of organisms lived on this earth millions of years before man appeared.

The passing of the view that all the world is arranged with direct reference to use by man has been followed by the tendency to look upon all things in each animal and plant as useful to itself, to its offspring, or to its kind or species. This also was the outcome of the truth that a large number of things are obviously useful. There can be no doubt concerning the usefulness of some adaptations of flowers to insects, of plants to storage of food, of leaves in the light-relation, and of some seeds for distribution; but no one knows a use for many other things (e.g., prickles, thorns, hairs, and the brilliant colors in many plants, or the fleshy parts of some inedible fruits). Hence science does not warrant the conclusion that, even if many things are not arranged for the use of man, they are all of use to the organisms which possess them. The important point in the whole matter is the fact that many, very many, modified structures are 
surely adaptations; that is, fitted to special use ; and so, while the student of biology is kept constantly on the lookout for evidence of such useful structures, he must keep in mind that now and then there will be found things without apparent use. Nature has no hard and fast rule that all things must be useful as seen from our human point of view. A surprisingly large number of things are useful to the organisms which possess them, but there are many puzzling exceptions.

220. The economic importance of the seed-plants is so vast that a special book would be required to discuss it adequately. Here we can only point to the fact that the great majority of plants useful to man are seed-plants. Practically all of our plant food-supply and that of our domesticated birds and mammals ; all of our forests useful for lumber ; all of our plants which produce fibers (cotton, hemp, linen, etc.); most of the plants which produce drugs and other special substances; and almost all of our ornamental plants belong in the great group of seed-plants or flowering plants. The mere mention of food plants, fiber plants, and lumber, calls to mind the vast agricultural and manufacturing industries which have been built up on the basis of seed-plants. In fact, the foundation of the wealth of the civilized nations is in agriculture, which is primarily the business of producing useful seed-plants. With so much to the credit of the seedplants, there seems to be little remaining chance for usefulness of lower plants ; and yet we shall see that many of them have special useful relations to human life. 


\section{CHAPTER IX}

\section{STUDIES OF SPORE-PLANTS}

221. Seeds and Spores. - All the plants mentioned in the preceding chapters are characterized by the development of seeds containing embryo plants, and hence such plants are named "seed-plants." But all plants do not produce seeds with embryos ready to unfold when placed in proper conditions for germination. There are a number of types of lower plants without seeds, but which form spores, simple rounded bodies without embryos but able to develop into new plants. Such plants are called "spore-plants." Ferns, mosses, and mushrooms are examples. It is true that in a seedsman's catalogue we may find "fern seeds" listed; but this is a careless use of the word "seed," for what the seedsman sells are really fern spores.

However, while spore-plants do not form seeds, seed-plants do have spores. These are of two kinds: (1) the pollengrains (microspores, little spores), and (2) a mass (megaspore, great spore) inside each ovule from which the embryo (which is at first egg-cell) and endosperm of the seed develop. We see that both seed-plants and spore-plants produce spores. The chief difference is that in all seed-plants the spores develop inside the ovary into embryos (and endosperm, if present in seed); while in spore-plants the spores may be scattered far from the plant which produced them and then germinate into new plants. We shall be able to understand this point better after studying briefly the life-history of a fern plant. 
222. Cryptogams and Phanerogams. - Spore-plants are often called cryptogams, which means hidden reproduction, and was given when their life-histories were not well understood. The term phanerogams, applied to seed-plants, means evident reproduction, and was applied because the conspicuous flowers are obviously connected with reproduction.

\section{HIGHER SPORE-PLANTS: FERNS AND MOSSES}

The ferns and mosses, and their relatives, are distinguished by the possession of structures which are similar to and perform the functions of the roots, stems, and leaves of seedplants. These are lacking in the lower spore-plants ( $\$ 235)$.

\section{FERNS}

223. General Structure of a Fern Plant.* - Any common kind or species of ferns may serve as material for illustrating this study and for parallel laboratory work. The fern plants are like the seed-plants in having roots, stem, and leaves (also called fronds). The stem of our ordinary ferns is commonly on the surface or slightly buried in the soil ; but the tree-ferns of tropical countries, often seen in greenhouses, have upright stems. The prostrate stem (rootstock) of ordinary ferns grows from a terminal bud, and since the stem lives for many years and the leaves usually only one summer, the stem is continually advancing and sending up new leaves farther and farther away from the position of the first ones. The stem may branch; and since the older part of the stem dies and decays, it frequently happens that the branches get separated from the main stem and become separate plants.

The root, stem, and leaf of ferns are similar in structure to those of seed-plants. In the stems are vascular bundles con-

* Concerning laboratory work on ferns: The teacher should provide the students with materials for demonstrations or laboratory study of the main points in the following description of ferns. See "Teacher's Manual." 
taining wood-tubes and sieve-tubes, which act as in seedplants in conducting fluids up and down the stem. Also, as in the higher plants, the vascular bundles extend into the veining of the leaf, thus providing the leaf with a water-

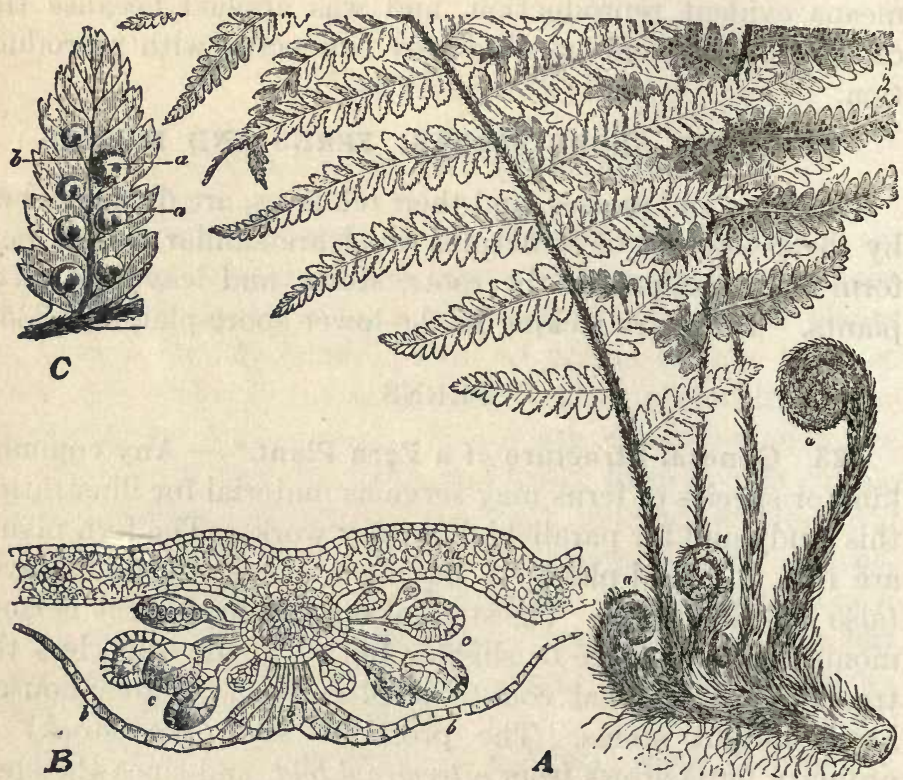

Fig. 70. Aspidium fern. $A$, part of underground stem with three young leaves $(a)$, and parts of three mature leaves, one with numerous clusters of spore-cases. $B$, section transverse of leaflet with cluster of spore-cases $(c)$ on lower side and covered by a cap $(b) . \quad C$, under side of a leaflet with seven clusters of spore-cases. (From Strasburger.)

supply system and a means of transferring elaborated foods to cells of the stem and roots. The general form and structure of the leaves reminds one of those of seed-plants. The leaves of many ferns are much divided and resemble compound leaves of seed-plants.

The one most striking difference as compared with the 
leaves of seed-plants is that fern leaves may have on their lower surface peculiar organs which form spores. In some species of ferns there are on the leaflets rounded spots; in others there are ridges on the leaflets; and in still others there are folds at the margin. Examination with a hand-lens shows that these variously shaped spots are similar in essential structure, for they are clusters of sporecases (sporangia), the organs for producing spores. When immature, these spore-cases are usually protected by a covering (called indusium) in the form of a cap, or a fold of the edge of the leaf.

In many ferns the leaves thus combine the regular work of foliage leaves (breathing, transpiration, starch-making) and that of spore forming; but some species have certain specialized leaves which produce spores (spore-leaves or sporophylls), and thus the foliage leaves are free for the regular work. In such cases the foliage leaves are often called sterile leaves, the spore-bearing ones fertile. In some species these latter become so much modified that they do not resemble ordinary leaves (Fig. 71).

224. Germination of Fern Spores. When the spore-cases (sporangia) are ma-

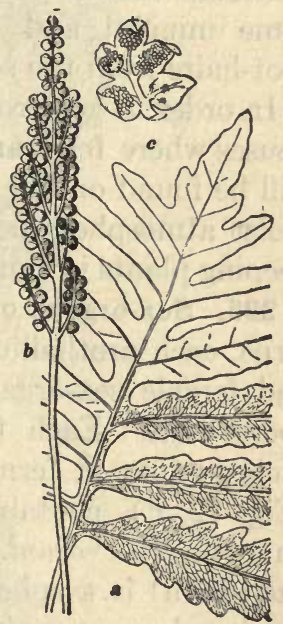

Fig. 71. Specialized spore-bearing fern leaf $(b)$, leaflet magnified $(c)$, and regular leaf (a). (From Gray.) ture and dry, they burst and scatter the spores. The bursting is caused by a peculiar ring (annulus) which almost surrounds each spore-case and acts like a spring. If the spores fall upon favorable soil, they absorb water and germinate. The coats of the spore burst and a delicate tube protrudes (Fig. 72, A). This soon divides into cells (Fig. $72, B)$, and grows into a flat and usually heart-shaped structure, called a prothallium $(C)$. On its under side are 
root-hairs (rhizoids), which act like true roots of seed-plants in absorbing from the soil. The cells of the prothallium are green with chlorophyll-bodies, which are able to make starch as do ordinary leaves. In fact, the great growth from a minute spore to a prothallium, which may be more than one eighth inch in diameter, is due largely to the materials made by the chlorophyll-bodies, supplemented by some mineral and nitrogenous materials absorbed by the root-hairs from the soil.

In order to get prothallia for study, one should visit greenhouses where ferns are grown from spores. Sometimes they will be found on the surface of the soil beneath ferns kept in moist atmosphere, as in a fernery (a glass-covered case for keeping plants in a moist atmosphere).

225. Sex-organs of the Prothallium. - In most species of ferns, each prothallium develops on its under side both male and female sex-organs for the production of egg-cells and sperm-cells. Each female organ (ovary, or usually called archegonium in ferns and mosses) is a tube-like structure (Fig. 72, F), containing in the expanded base of the tube an egg-cell or ovum. Each male organ (spermary, or antheridium) is a spherical body, in whose central cavity are produced many spiral-shaped cells with a group of cilia (Fig. 72, $D, E$ ). These are the sperm-cells (also called spermatozoids), and the lashing movements of their cilia cause them to swim in drops of water on the under side of the prothallium to the mouth of an ovary. The tube or neck of the ovary is filled with a mucilage-like material, through which the sperm-cells swim to the egg-cell. One spermcell unites with an egg-cell, thus accomplishing fertilization. Notice the similarity to the fertilization of the frog's eggs ( $\$ 58)$.

Compared with fertilization in seed-plants (described in $\S 189$ ), the sperm-cell of the fern obviously has the same function as the cell which moves down the pollen-tube to 


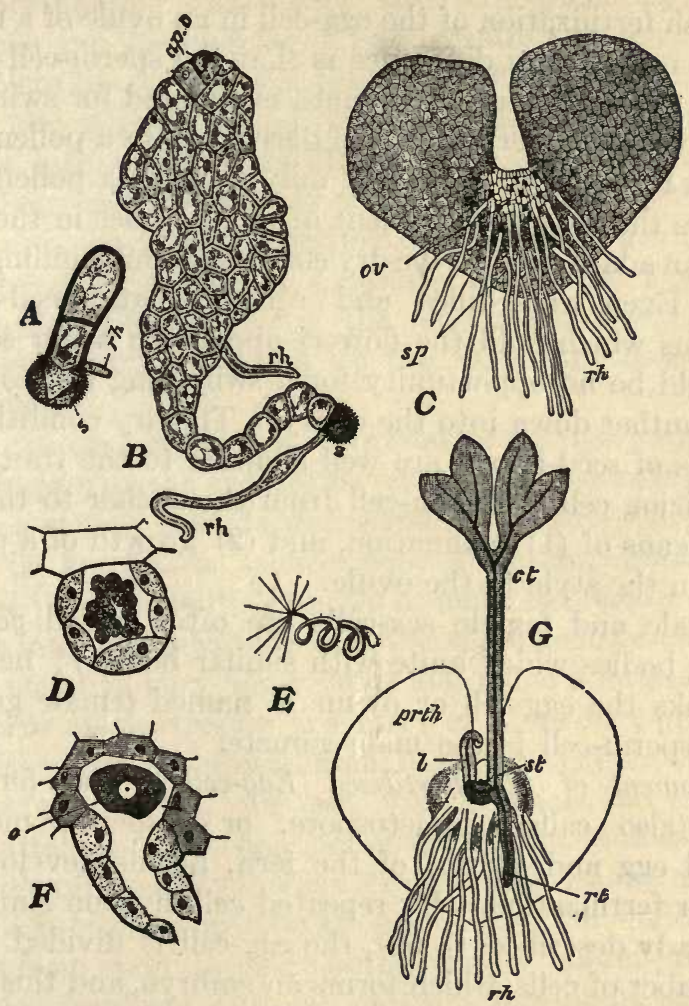

Frg. 72. $A$, fern spore germinating; 8 , spore; $r h$, rhizoid. $B$, a later stage of germination; ap.c, apical cell or growing point. $C$, full-grown prothallium, seen from the lower side, many rhizoids, ovaries (ov), spermaries $(s p)$. $D$, section of spermary, immature sperm-cells in center ; unshaded cell belongs to the prothallium, beneath which spermaries are suspended. $E$, a sperm-cell, coiled body, and a group of cilia for use in swimming. $F$, section of an ovary, with egg-cell $(o)$. The wide-open neck of the ovary is in nature filled with a mucilage through which spermcells swim to the ovum. $G$, prothallium with a young fern plant developed from a fertilized ovum in an ovary. $r h$, rhizoids of the prothallium; $r t$, root of the young plant; $c t$, a cotyledon; st, beginning stem; $l$, first true leaf forming. (From Parker's Biology, with modifications.) 
accomplish fertilization of the egg-cell in an ovule of a flower. The only noteworthy difference is that the sperm-cell of the fern, like the sperm-cell of animals, is adapted for swimming in fluids, while the fertilizing cell derived from a pollen-grain can reach the egg-cell in a pistil only through a pollen-tube. It appears that the development of pollen-tubes in the seedplants is an adaptation to the dry conditions surrounding most flowers. Even water-lilies and other aquatic seed-plants have stems which hold the flowers above the water so that there could be no opportunity for a swimming cell to move from an anther down into the ovary. The dry conditions in all flowers of seed-plants are well adapted to the transfer of the fertilizing cell or sperm-cell from the anther to the eggcell by means of (1) pollination, and (2) growth of a pollentube down the style to the ovule.

The male and female sex-cells are often called gametes, (meaning bodies which unite with similar bodies); hence in some books the egg-cell or ovum is named female gamete, and the sperm-cell is the male gamete.

Development of the Fertilized Egg-cell. - The fertilized egg-cell (also called gametospore, or oösperm, meaning combined egg and sperm) of the fern, begins development soon after fertilization. By repeated cell-division similar to that already described $(\$ 59)$, the egg-cell is divided into a large number of cells, which forms an embryo, and this grows into a plantlet as shown in Fig. $72, G$. In it can be seen cotyledons, stem, and root. Soon afterward, the prothallium withers, and the young fern plant becomes independent. It grows to maturity, produces spores on its leaves, and these spores in turn start another cycle or life-history.

226. Alternation of Generations in Ferns. - It is evident from the above account that the complete life-history of a fern is composed of two individuals quite different in appearance and other characteristics, especially in the method of reproducing. The fern plant reproduces by means of spores 
formed without sex-organs; that is, by asexual reproduction. The prothallium reproduces the fern plant by sex-cells (egg-cells and sperm-cells). The two are necessary to make a complete life-history, for the spores cannot develop directly into a fern plant and the fertilized egg-cell cannot develop directly into a new prothallium. The prothallium and the fern plant each represents what is known in biology as a generation, and the succeeding each other is alternation of generations. It should be especially noted that a generation reproducing asexually (i.e., the fern plant) alternates with the one which reproduces by the sexual method (i.e., the prothallium).

The asexual generation represented by the fern-plant is often known as the sporophyte (meaning spore-plant, because it forms spores); while the sexual generation, the prothallium, is known as gametophyte (meaning gamete-plant, because it develops the gametes or male and female sex-cells).

It is interesting to note that the spore-generation, the fern plant, may live very many years and reach a large size (some tree-ferns of the tropics are forty or more feet high). The sex-generation, the prothallium, is always very small and lives only a few months. In fact, it is so small and attracts so little attention that only by most careful studies were the facts concerning alternation of two generations discovered.

By life-history, or life-cycle, of a fern we understand all stages from spore to spore again; that is, spore, prothallium, sex-cells, fertilized egg-cell, embryo, fern-plant which forms spores starting a new life-history.

227. Alternation of Generations in Seed-plants. - The similarity of structure between roots, stems, and leaves of ferns and the lowest seed-plants suggests that they are related. The fact that the ferns have alternation of two generations (prothallium and fern plant) makes their lifehistory appear at first to be entirely different from seedplants; but careful investigations have shown that even 
seed-plants have two generations alternating in their lifehistory. One of these generations (the sporophyte or sporeforming stage) is the ordinary seed-plant (e.g., an oak tree); and its spores are pollen-grains and ovules. The oak tree then corresponds to the fern-plant in that each is the sporeproducing stage. The other generation (the gametophyte, which produces the sex-cells) in seed-plants is the pollentube, in which is formed the sperm-cell, and a microscopic structure (the embryo-sac) in the ovule, in which is formed the egg-cell. The development of the sperm-cell in the pollentube and of the egg-cell in the embryo-sac of seed-plants is believed to be a great modification of the formation of spermcells and egg-cells in the sex-organs of prothallia of the lower plants. In ferns the spores are all alike, but in seed-plants there are two kinds, - the smaller microspores or pollengrains, and the larger megaspores or ovules. A minor difference is that the spores of ferns grow extensively, forming prothallia, which later produce sex-cells, but seed-plants develop the sex-cells by cell-divisions inside the spores. Thus in both ferns and seed-plants the sex-cells come more or less indirectly from spores, and the united sex-cells of the two kinds develop into ferns and seed-plants, which in their turn form spores again.

228. Allies of Ferns. - In addition to the plants which are easily recognized as ferns, and of which there are numerous species in the United States, there are a number of closely related fern-like plants. These are the water-ferns, the horsetails (Equisetum), and the club-mosses (lycopods). Specimens (fresh or in formalin) should be exhibited in order to give general acquaintance. If time permits, special textbooks of botany should be consulted as guides to very brief study of specimens of these plants.

All the fern-like plants taken together constitute a group known as the Pteridophytes (Pteridophyta), one of the primary divisions of the plant kingdom (see table in Chapter 
VII). The group was much more important in the early periods of the earth's history than at present. During the Carboniferous period, pteridophytes formed the main mass of the land vegetation; but they decreased in prominence in the later period when the gymnosperms and still later the angiosperms appeared. The horse-tails and club-mosses which exist to-day are rather small plants, but some of the ancient species which lived before the end of the Carboniferous period were stately trees.

229. Economic Relations of Pteridophytes. - The ferns and their allies furnished much of the organic material which was transformed into coal. Many leaves and stems of ferns and allied plants are found as coal fossils, splendid specimens of which are on exhibition at natural history museums.

At the present time little use is made of these plants except for ornamental purposes, for which their foliage is unsurpassed. Many tropical species of ferns are kept in greenhouses, and several species have long been favorite houseplants. Various kinds of club-mosses are also used in the same way.

One fern contains in its stem a powerful drug, known in pharmacy as "extract of male fern," and often used for expelling tape-worms and round-worms from the intestines.

The horse-tails were known as "scouring rushes" in the pioneer days in America, because their stems were used to scour pewter and brass utensils. The scouring property depends upon the silicon particles in the stems (easily demonstrated by burning).

\section{MOSSES}

230. Distribution of Mosses. - One of the most interesting points concerning mosses is their wide distribution, which is made possible by adaptations to various conditions of life. They are common everywhere. We may find them on ex- 
posed and barren hillsides, in bogs, in cold northern regions, in forests, and even in water.

One of the most important is the bog- or peat-moss (Sphagnum) which grows luxuriantly where most other plants cannot live, and its growth gradually fills the bogs with dead vegetation called peat. This does not decay rapidly because the water in peat-bogs is somewhat antiseptic (i.e., prevents the development of bacteria which cause decay elsewhere). In Ireland and some other countries the peat which is extensively used as fuel is composed largely of species of peatmoss or sphagnum-moss. The American peat contains many other aquatic plants. The floating islands in some lakes and the quaking soil in marshy regions are masses of dead vegetation, often largely peat-moss, which have collected some silt and thus formed soil on which other kinds of plants grow. Sphagnum moss is extensively used for packing plants for shipping; and in greenhouses for filling hanging baskets for ferns and orchids, for mixing with soil to prevent it from packing and to make it hold water, and for covering the soil in flower-pots.

231. Structure of a Moss. - Specimens of several common mosses should be collected for comparison in connection with the following account made brief by limitation of the time which can be allowed for study of mosses in a year's course of biology. Specimens of common large mosses should be collected in summer and preserved in formalin-solution.

Most of our common mosses have erect stems with leaves arranged radially. Sometimes the older part of the stem is prostrate. A section shows no wood-tubes, as in higher plants (ferns and seed-plants), but only a central axis of cells which serve to conduct fluids. At the base of the stem are rootlets or rhizoids, often twisted together. The leaves have a very simple mid-vein of elongated cells, but no such complex veining as in higher plants. Some mosses growing in dry places (e.g., Polytrichum) have one side of the leaves specially 
adapted to the light-relation, and this delicate side is protected during drought by rolling of the edges of the leaf.

232. Reproduction of Mosses. - Many creeping branches form rootlets, and after decay of the older stem may become independent. This is the chief cause of the mats of mosses often found. But in addition to this vegetative propagation, all mosses reproduce by means of sex-cells and by spores. Sex-organs (ovaries and spermaries) are formed in the budlike apex of the stem or of the main branches. Both kinds of sex-organs are in some species found on the same plant (i.e., it is monocious); but in other species on separate plants (dioccious). A spermary (botanists usually call it an antheridium in mosses and ferns) is an elongated club-shaped mass of cells (Fig. 73, E), the inner ones of which become converted into swimming sperm-cells, which are freed by rupture of the wall of the spermary. An ovary (usually called archegonium, as in ferns) has the shape of a chemist's flask with a long tubular neck (Fig. 73, $F$ ). In the rounded base of the ovary is an egg-cell or ovum. This is fertilized by a spermcell which swims down the neck of the ovary. The fertilized egg-cell (or oösperm) divides into two, four, eight, and more cells, forming the embryo. This soon begins to grow upward as a slender rod, and the end of the rod expands into and forms a vase-like structure (Fig. 73, C). This is a spore-case, usually called by botanists the sporangium, and its inner cells form numerous spores. It usually has a cap-like lid, which falls off when the spores are ripe and ready for scattering. The entire stalk and spore-case formed from the developing egg-cell is called sporogonium.

When a moss spore begins to germinate, a delicate thread or filament grows out and branches. This structure formed by the germinating spore is called a protonema (meaning the first thread).

After a time one or more buds appear on the sides of this protonema, and from them moss plants grow upward. Thus 
a spore may form one or more moss plants. The protonema, then, is a sort of creeping stem able to produce erect branches. It is part of the moss plant.

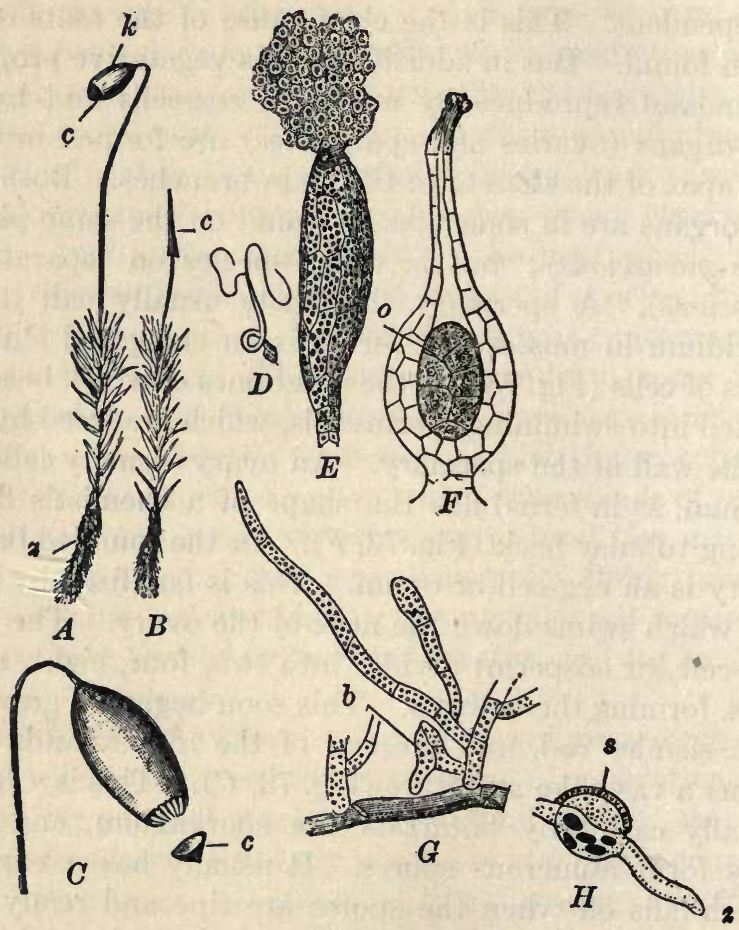

Fig. 73. Moss. $A, B$, plant with spore-case $(k)$ which has a lid $(c) ; z$, root hairs. $C$, enlarged view of spore-case. $D$, sperm-cell. $E$, spermary, sperms escaping. $F$, ovary with embryo $(o)$. $G$, protonema; $b$, bud from which moss plant develops. $H$, spore $(8)$ germinating to form a protonema. (After Parker, Strasburger, and others.)

233. Alternation of Generations of Moss. - It is evident that, as in the ferns, the moss has an alternation of generations. The moss plant is the stage or generation which bears the sex-organs, while the sporogonium produces the asexual 
spores which germinate to produce a new moss plant. Obviously. the moss plant corresponds to the fern prothallium, because each is produced from a spore and each is a gametophyte and produces sex-organs. Moreover, the moss sporogonium and the fern plant both develop from fertilized egg-cells, and produce asexual spores. This relation is expressed in diagram form as follows, the dashes indicating the order of stages.

Fern spore - prothallium (gametophyte) - sex-cells - fern plant (sporophyte) - spores.

Moss spore - moss plant (gametophyte) - sex cells - sporogonium (sporophyte) - spores.

The striking difference is that the spore-forming stage (sporophyte) is the short-lived and relatively inconspicuous sporogonium of the moss and the prominent fern plant. On the other hand, the sex-generation (gametophyte) of the fern is the prothallium which few people ever see, and in the moss it is the well-known plant.

234. Allies of Mosses: Bryophytes. - The only close relatives of mosses are the liverworts, of which Marchantia is an example common in greenhouses. The mosses and liverworts together constitute a group known as Bryophytes (meaning moss-plants). Certain lichens ( $\$ 248)$ and other plants are sometimes mistaken for true mosses. See relation of mosses to other groups of plants in table of classification in $\S 133$.

\section{LOWER SPORE PLANTS *}

235. Algæ and Fungi. - So far all plants studied (seedplants, ferns, mosses) have more or less similarity. Even mosses have the working equivalent of the roots, stem, and leaves as seen in the higher seed-plants. We shall next study some types of still lower and simpler plants, which in fact

* The higher spore-plants are the ferns and mosses, described in the foregoing sections. 
are so simple that there is no differentiation of the plantbody into roots, stem, and leaves. Some of these lower plants have chlorophyll and are called Algæ; and some (the Fungi) have no chlorophyll. All these simple plants belong to the lowest division of the Thallophytes. See table of plant classification in $\$ 133$.

\section{LOWER SPORE-PLANTS WITH CHLOROPHYLL: ALGAE}

236. The lowest spore-plants without root, stem, and leaves and with chlorophyll are often grouped together under the name Algæ.* These are numerous forms, ranging from microscopic plants to the large sea-weeds. Most of the algæ live in fresh or salt water, but a few can live in moist places on soil and surfaces of larger plants, and even occasionally undergo drying.

In this course we cannot take time for more than a brief study of a few common types whose life-activities help us to a better understanding of some of the general principles of biology. But the reader should understand that such a condensed account as that which follows cannot be more than a beginning study of a few examples in a great group of low plants upon which much emphasis is laid in advanced. courses of botany in colleges. In the present study we can do little more than direct attention to the ways in which these simple plants solve the problems of the fundamental life-activities of feeding, moving, breathing, excreting, and reproducing - processes which we have now traced through a series of plants, beginning with the highest or seed-plants. The following descriptions of common types of algæ should be accompanied as far as possible by demonstrations and laboratory study.

* A few of the highest algæ (e.g., the freshwater Chara and Nitella) have structures serving as roots, stems, and leaves very much as might a moss plant entirely submerged in water. 
237. Pleurococcus. - A green coating or stain which is often seen on the shaded (usually north) side of tree-trunks, unpainted walls, etc., will be found upon microscopic examination to be composed of masses of one-celled plants, often arranged in groups of two, four, or more cells (Fig. 74). This grouping is the result of repeated cell-division. Each cell has protoplasm, nucleus, chlorophyll-bodies, and cellwall. Having chlorophyll, they are able to make carbohydrates from carbon dioxide and water. Particles of dust lodged on the wood on which they grow probably supply the necessary materials which higher plants absorb from the soil. Abundance of water favors growth, and they appear a brighter green during rainy weather.

238. Sphærella. - Red stains are often seen in hollows in rocks, in empty urns in cemeteries, in roof-gutters, and in
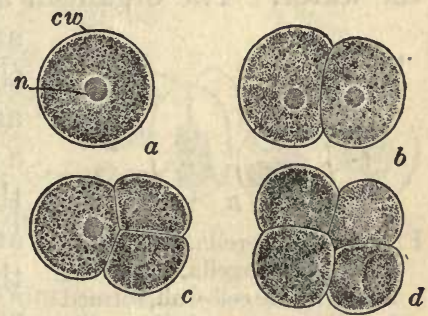

FIg. 74. Pleurococcus. $a$, one cell; $b$, two cells formed by division of $a ; c$, three cells by division of one of two cells in $b ; d$, four cells; $c w$, cell-wall; $n$, nucleus; cellbody between nucleus and cellwall contains chlorophyll. (From Sedgwick and Wilson.) similar places where dust may collect and where temporary pools of water may be formed by rains. If water is present in such hollows, it may be reddish or greenish in color. Collect some of the dry dust, decayed leaves, etc., from such places during the autumn, and keep dry until wanted for study. Then place some of the dry material in water, and expose to light for several days. Examine with the microscope some of the dry material mounted in a drop of water and observe spherical cells with red contents (protoplasm plus a coloring matter), and with thick cell-walls. This is called the "resting stage" of the Sphærella plant. By special methods it is possible to extract the color, and demonstrate a nucleus near the center. It is evidently a one-celled plant. 
Placed in water and light the contents divide into four, eight, or sixteen masses, the cell-wall breaks, and each one of these masses becomes a motile stage, having the appearance shown in Fig. 75, A. The two whip-like structures (flagella) have lashing movements which propel the organism. The color in this stage may be red, green, or a mixture of the two colors. Carbohydrates are made from carbon dioxide absorbed from the water. The organism also absorbs nitrogen compounds

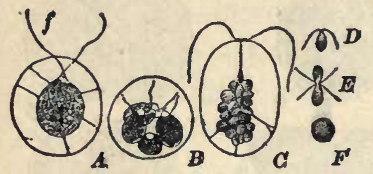

Fig. 75. Sphærella. $A$, motile stage; $f$, flagella. $B$, four like $A$ inside cell-wall, formed by division of a non-motile form similar to Fig. $74 a$. $C$, division into numerous small cells like $D$. $E$, conjugation of two like $D$, forming a spore $F$. (From Strasburger.) and other necessary elements from the water, forms new protoplasm, and soon grows to full size. After a time the flagella are retracted; the plant enters the resting stage; and when conditions are favorable, the protoplasmic contents divide again into four or more masses, which form the swimming stage.

There are numerous interesting details of structure and life-history, but these must be left for special courses in botany. For our present purposes the important facts are that Sphærella is (1) a onecelled (unicellular) plant; (2) it reproduces by a process of cell-division; (3) it is able to make its own carbohydrate foods from carbon dioxide and water by the action of light on the protoplasm containing coloring matter which acts like chlorophyll; and (4), most remarkable of all, it swims in an animal fashion in one stage of its existence.

Plant or Animal? - We classify Sphærella as a plant because its nutrition is the same as that of higher green plants. No known animal can make carbohydrate foods from carbon dioxide and water. The one thing about Sphærella which suggests that it is an animal is that it swims; but we have already noted that reproductive cells of ferns and mosses can swim, and that many higher plants have movements. 
Sphærella is in some books called Hæmatococcus (meaning blood-berry). This is only one of many examples of plants which have two names. The explanation is that it is one of the rules of the botanical societies that the name first given by a scientific man who accurately describes a newfound species should be the accepted one. In the case of the plant under discussion, there is still some doubt regarding the first description; and hence there must be further investigations before it can be decided that one of the names is to be kept in the scientific books of the future and the other allowed to become obsolete. There are many other such undecided problems concerning the naming of other plants, and also of animals.

239. Spirogyra. - In many ponds and streams there are floating green masses of delicate thread-like plants popularly known as pond-scum. These threads are composed of a row of elongated cylindrical cells (Fig. 76), with their chlorophyll arranged in one or more spiral bands. A large nucleus may be seen near the center of each cell. The cells grow and divide so as to form long filaments composed of chains of

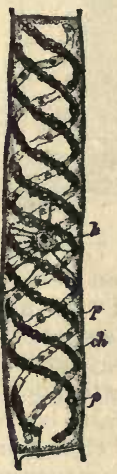

Fia. 76. A cell from a filament of Spirogyra. $k$, nucleus in center; $t w o$ spiral bands containing chlorophyll (ch). cells. Each cell is an individual plant, because it is capable of carrying on all the life-processes of a green plant.

Under certain conditions a tube may grow so as to join two adjacent cells of the same or of separate filaments, and through this tube the protoplasm of one of the two cells passes into the other and the united substance forms a spore (zygospore). This later germinates and forms a cell which by repeated growth and division forms a filament or chain of cells. This process leading to spore-forming is known as conjugation. A similar process occurs in the black mold 
( $\$ 244)$. Like fertilization in higher plants, this conjugation mixes protoplasm from two individual Spirogyra plants, and the mixture appears to have increased vitality (Fig. 77).

The physiology of such a one-celled green plant living in

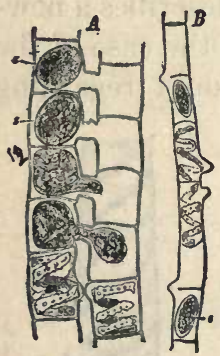

Fig. 77. $A$, conjugation between cells of two adjacent filaments of Spirogyra. Concentration of protoplasm into one cell of each pair, forming a zygospore $(z)$, is complete in two upper pairs, in progress in next two pairs, and a preparatory stage is beginning in lowest pair of cells. $B$, conjugation between contiguous cells of the same filament.

water is briefly as follows: It absorbs water and carbon dioxide, and these are used in the making of starch or other carbohydrate foods. From water also the plants absorb the other elements necessary $(\mathrm{N}, \mathrm{S}, \mathrm{P}, \mathrm{K}$, etc.) for making new protoplasm. These elements are usually the same as in the higher green plants. Also, the plant absorbs from the water the oxygen required for the life-activities. The carbon dioxide produced by oxidation of cell-substances is absorbed by the surrounding water; but during exposure to light is probably used in making carbohydrates by photosynthesis. Probably other excretions besides carbon dioxide are formed in the one-celled plants and absorbed by the surrounding water.

240. Other green algæ are numerous. They appear as greenish coatings on various objects in water, on the bottom of springs and ponds, and on pots and soil in greenhouses. Certain species are likely to grow on the glass of aquaria. In such a position it is easy to show their dependence upon light by shading the glass with black paper in which openings (possibly in the form of letters) have been cut. The algæ will grow well only where they receive the light. For descriptions of the numerous forms which are of common occurrence, the student must refer to textbooks specially devoted to the lower green plants. 
241. Brown and Red Algæ. - These are chiefly marine plants, and are commonly known as sea-weeds. Numerous kinds of these may be seen on any sea-shore. The red and brown colors appear to be due to pigments which conceal the chlorophyll. At any rate these plants make their own carbohydrate foods from carbon dioxide and water just as higher green plants do. Studies of the structure and lifehistories of these interesting algæ must be left for college courses of botany.

242. Economic Relations of Algæ. - Probably the greatest value of the algæ lies in the fact that they make food for higher organisms. This may be observed in any aquarium where snails rasp off and eat the green algæ on the glass. Numerous small animals eat algæ, and these animals may serve as food for still larger animals. Immense quantities of these low plants are found in the ocean down as far as light penetrates. Even some large animàls, e.g., certain fishes and whales, have strainers in their mouths which enable them to collect large quantities of very small organisms, some of them animals and some plants; but especially do small animals which feed on simple algæ serve as food for the larger animals. It is now quite certain that directly or indirectly the simplest green plants play an important part in the foodsupply of aquatic animal life, much of which is of use to man. In this line the simple algæ have great economic importance.

Many kinds of the larger sea-weeds have some economic uses. Thus iodine is prepared from the ash obtained by burning sea-weeds, and formerly this was also the chief source of sodium carbonate, from which baking soda is made. Certain brown sea-weeds are used as food by Chinese and Japanese and by the natives of the Malay Archipelago. Agar-agar and Irish moss, both used in preparation of jellies, are made from certain kinds of sea-weeds. Finally, in some agricultural countries near the sea the sea-weeds cast up by the waves are used as soil fertilizers. 
Algce in Water Reservoirs. - On the harmful side there is very little to be said against the plants of the algæ group. Probably most important is the fact that certain species grow extensively in reservoirs used for storing water, and impart a very disagreeable odor and flavor to the water. Some of the cities in eastern Massachusetts and New York City have had much trouble in this line. A small amount of copper sulphate dissolved in the water prevents the growth of such algæ; but such chemicals must be used with caution until scientific experiments demonstrate whether or not small quantities in drinking water may not be harmful when used continually.

\section{SPORE-PLANTS WITHOUT CHLOROPHYLL: FUNGI}

243. The most important examples of spore-plants which have no chlorophyll, and hence must be in their food-supply different from green plants, are common molds, mushrooms, and yeast-plants. To these might be added the bacteria; but many peculiarities and their great importance make it best to devote a separate section to them (beginning with $\$ 254$ ).

\section{Molds}

244. Study of Common Molds. - It is a well-known fact that when bread, cheese, or other foods are left for some time in a damp place, there appears over the surface a mass of delicate threads or filaments, and the bread is said to be "molding" or "moldy." Soon the color of the moldy mass changes from white to black, blue-green, or brown, and according to the color, the names black mold, green mold, and brown mold are applied.

$(L)$ Examine pieces of bread on which black mold is growing. Break off pieces and note that delicate threads run through the bread (use a hand-lens). These threads are the hypho, and the entire mass of them is the mycelium. Starting on the surface, the 
mycelium will grow in all directions until an entire loaf of bread is interlaced with the hyphæ. At the same time the hyphæ secrete digestive substances which digest to a soluble form starch and other foods in the bread. These digested or dissolved foods are absorbed by the hyphr and used for growth. The mold is therefore a saprophyte ( $\$ 98)$.

The delicate threads seen above the surface of the bread are called erect or aërial hyphæ; they are branches of the mycelium.

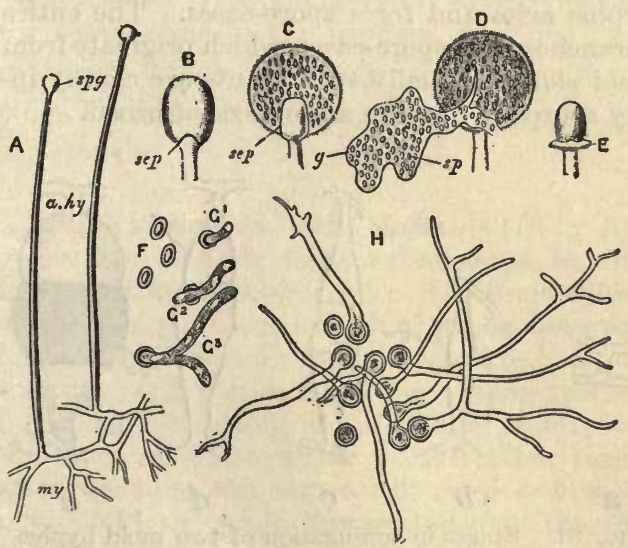

Fig. 79. Black mold. $A$, mycelium ( $m y$ ) with two aërial hyphæ $(a . h y)$, each forming a spore-case $(s p g)$. $B$, immature spore-case. $C$, mature. $D$, liberating spores $(s p) . F$, spores. $G, H$, germination of spores to form new mycelium. (From Parker.)

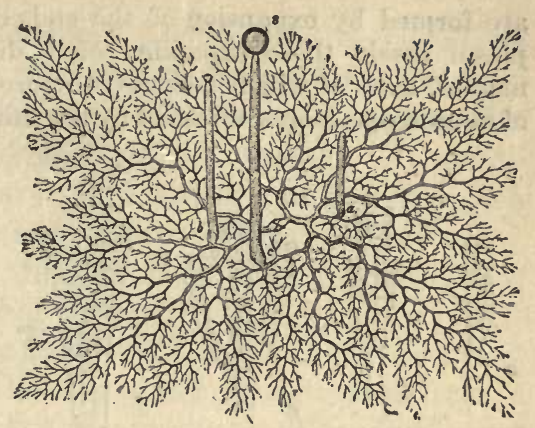

Frg. 78. A black mold plant formed from one spore in center. One mature aërial hypha with spherical spore-case $(s)$, and two immature ones. Root-like structure is mycelium. (From Kny.)

Close examination with a strong handlens will show that the color of the mold is due to bodies attached to the ends of the erect hyphæ, These bodies are the spore-cases, or sporangia, which are filled with spores. The purpose of the aërial hyphæ appears to be production of the sporecases and elevation of them so that wind currents may better distribute the spores.

It is easy, with a low-power microscope, to find various stages in thedevelopment of the spore-cases which 
are formed by expansion of the ends of aërial hyphæ. The protoplasm inside these expanded ends divides into numerous small masses, each of which becomes a spore. Examine with high power of microscope spores obtained by brushing a wet needle or small brush

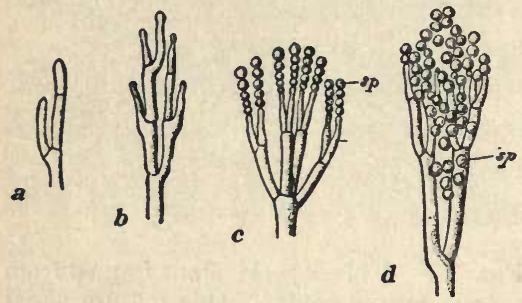

Fig. 80. Unlike the spherical spore-case of the black mold, the green mold forms spores $(s p)$ at ends of branches of aërial hyphæ.

against some sporangia and then rubbing the spores collected into a drop of water on a glass object-slide. Notice the thick cell-wall, which is very protective against heat, drought, and other unfavorable conditions.

(D) If some spores be placed in a dilute sugar solution in a watch-glass, many of them will be found germinating after a day; and after several days much branching will produce an extensive mycelium (Fig. 79). Later some aërial branches or hyphæ arise and form spore-cases. The entire mycelium and aërial branches with spore-cases, which originate from a single spore, is a mold plant. Usually such plants are closely interlaced, because many spores fall on the same piece of bread.

Sometimes a peculiar method of reproduction occurs as follows: From each of two hyphæ which happen to lie near together, c l u b-s h a p ed bodies grow until their ends meet
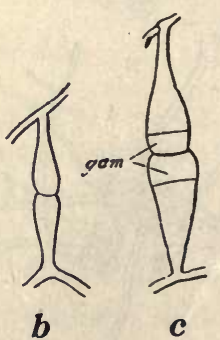

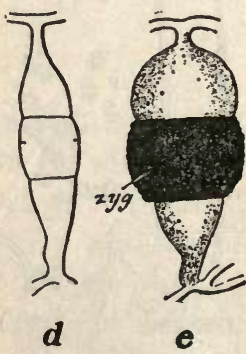

Frg. 81. Stages in conjugation of two mold hyphæ, forming the spore (zyg). (From Parker.)

(Fig. 81). The protoplasmic contents in the ends of the two bodies unite into one mass, and around it a thick wall or coat forms. There is thus formed a combined spore 
(called zygospore, or gametospore), which like the fertilized egg-cells of mosses, ferns, seed-plants, and animals, is composed of protoplasm from two sources. In short, this zygospore is a simple kind of fertilized egg-cell. It germinates much like an ordinary mold spore, and forms hyphæ which develop aërial branches with ordinary spore-cases. Apparently the reason for this process, known as conjugation of molds, is the same as that for fertilization in other plants and in animals; namely, that there is some physiological advantage in new individual organisms beginning from a mass of protoplasm which has two parents. The significance of this is in part a problem of heredity, which will be presented in the last chapter.

As a rule, molds grow best in a warm place, as can be proved by leaving pieces of moist bread in two bottles, one of which is placed near a stove or radiator, and one in an ice-box. However, some molds will grow on foods kept for some time in the ice-box, and one kind forms the slimy material which grows in the drain-pipes of ice-boxes and clogs them unless cleaned frequently by hot water and washing powder.

245. Experiments with Molds. - ( $D$ or $L)$ Test-tubes $\frac{1}{2}$ by 5 inches are best for these experiments, but bottles with mouth at least $\frac{1}{2}$ inch in diameter will do. Cut some strips of bread $\frac{1}{4}$ by 2 inches in size, and place one in each of ten or more tubes, and add three or four drops of boiled water to each tube. Now, plug the mouths of the tubes with cotton-batting or absorbent cotton, making the plugs by rolling the cotton into a cylinder about two inches long. Have the plugs of a size which will fit rather loosely in the tubes, else after steaming the cotton will swell and the plugs will be forced out. Fold the projecting cotton over the edge of the tube, so as to keep dust from falling on the edge. Experiments tried thousands of times have shown that spores and microscopic organisms cannot get through such plugs of dry cotton, and that the tubes are as effectually closed as if the $y$ were hermetically sealed by melting the glass. Also, the cotton has the advantage of allowing the entrance of air, which is needed by molds that grow in the tube. 
Sterilization. - It is probable that both the bread inside the tubes and the cotton have spores of molds on their surfaces, because the spores are so abundant in ordinary buildings that practically everything exposed to air will have them. It is therefore necessary to kill all spores inside the plugged tubes, that is, to sterilize or to make the tubes sterile. This is best done by boiling or steaming $\left(100^{\circ} \mathrm{C}\right.$.) in a sterilizer. There are many sterilizers for laboratory and home use on the market, but one can be easily made from any tin bucket with a cover. A sheet of perforated tin (e.g., an inverted pan two inches deep), or a piece of wire-netting, should be supported about two inches from the bottom, and on this place the tubes to be sterilized. Put in water two inches deep. For convenience in keeping mouths of tubes upward, they may be tied into bunches, or placed in small tin cans perforated with numerous holes. A layer of cloth or cotton between the glass tubes and the metal will prevent breakage.

Keep the water boiling $\left(100^{\circ} \mathrm{C}\right.$.) for a half-hour. From time to time add more water (hot) so that the sterilizer will not "boil dry."

Label some of the tubes "sterilized once, 30 minutes." Set aside for observation from day to day. Do any molds appear on the bread in these? Conclusions?

Sterilize the other tubes again two days after the first sterilizing, and label "sterilized twice, total 60 minutes." Set some of these aside and observe from day to day. Sometimes a third sterilizing is necessary to kill all the spores of molds.

Inoculation. - Take a tube which two or three days after the second or third sterilizing shows no sign of molds on the bread, and hence is probably sterile, and inoculate. it as follows: Sterilize an inoculating needle (or a hat-pin, or piece of wire) by passing it quickly several times through the flame of a gas- or alcohol-lamp. Allow the needle to cool for a moment, and then touch the sterilized end carefully to a spot on moldy bread where there is only one kind of mold. Now, holding one of the sterile tubes in a horizontal position, quickly pull out the cotton plug, insert the needle, wipe it along one side of the piece of sterile bread, and then quickly replace the plug. Inoculate other tubes from other spots of molds, of different kinds if available, but take care to heat the wire of the inoculating needle before and after each tube is inoculated, otherwise you may mix several kinds of spores. Label tubes "black mold," "green mold," etc.

Do not be surprised if with all these precautions two kinds of molds appear in some tubes; for spores may have been left alive 
on the bread even after sterilizing, they may have fallen in when the tube was opened for inoculating, or more than one kind of spores may have been on the moldy spot touched with the needle. If only one kind appears, a "pure culture" has been secured.

Take two tubes which have been sterilized twice, remove cotton plugs, and blow into them some dust from top of furniture, doorframe, or window-casing; or sterilize an inoculating needle and with it transfer some of the dust to the sterile bread. Record results and conclusions. Why do foods mold quickly in a dusty pantry?

Leave the plugs out of two sterile tubes for several hours, and then replace. If molds develop, consider the possible source of the spores. A better way for this experiment is to use a flat dish, known as a Petri dish, made for such experiments (Fig. 82). Place a slice of moist bread in such a dish, sterilize twice, wait several days to make sure that the bread is sterile, then remove the cover for several hours so as to expose the sterile bread to the air of the schoolroom.

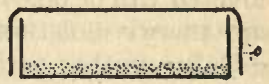

Fig. 82. Section of a Petri dish, for growing molds and bacteria. c, glass cover. The dots in bottom show position of gelatin, bread, or other food.

A small tumbler or wine-glass may be used instead of a Petri dish. Place a piece of moist bread inside on the bottom of the tumbler. cover with a circular sheet of cotton-wadding which by two inches exceeds the diameter of the top of the tumbler, fold the edges of the cotton down, and snap a rubber band around it so as to hold it close to the top of the tumbler. Sterilize as in case of the Petri dish.

A wide-mouthed fruit-jar might be used in the same way, sealing the jar with the rubber as in canning fruit. The Petri dish will by contrast show that the rubber is not necessary to keep out spores. Also, the Petri dish admits air. The secret is that the spores respond to gravitation and do not fall upward as they must do in order to fall into a Petri dish. A fruit-jar could be used with the cap and no rubber, if the contents were thoroughly sterilized, the edge of the cap kept dry and never turned upside down. But in ordinary household canning of fruits the sterilization is not perfect (usually only once), and the keeping out of air by the rubber tends to prevent the development of molds which require air in order to grow. Also, the rubber prevents hyphæ from starting on the outside and growing beneath the cover into the contents of the jar. This would surely happen if fruit juice ran down around the edge of the cover, for the juice would furnish excellent food for the growing hyphæ. This is why fruit in jars of ten remains sterile for months and even years, and then 
suddenly shows molds. The rubbers have softened, or loosened, or become moist, so that mold hyphæ have grown into the jars. Hence jars of fruit should be kept in a cool and dry place unfavorable for growth of molds. Aside from this relation to molds, rubbers of fruitjars also prevent evaporation of the fruit juices.

Rotting of fruit has been mentioned as due to molds. Take a perfect apple and inoculate it by sticking in several places with a needle which has been plunged into a very rotten apple. Take another perfect apple, bruise the skin on one side, and place it in a covered can or box in contact with a rotting apple. In both cases be sure there is moisture - the apples may be kept on wet cloth or cotton.

Take another perfect apple, gently rub the skin in several places with a smooth stick which has been plunged into a rotting apple, so as to distribute spores over the uninjured surface of the apple, and set this one in a cool and dry place for comparison with the others. What are the conclusions regarding apples infecting each other? Why should injured apples be separated from perfect ones before packing? Why are apples bored by worms (larvæ of codling moth) and "windfalls" so liable to decay? Why should fruit-cellars be cool and dry? Since the spores usually mature after rotting is well advanced, it is evident that removal of windfall fruits, as by pigs living in orchards, prevents the enormous multiplication and distribution of spores of various molds which injure fruit.

Instead of pieces of bread called for in above experiments, a dilute sugar solution (three or four tablespoonfuls of sugar in a pint of water) may be used. Or use some diluted juice from canned fruits. It is interesting to prepare some tubes with the sugar solution and try all the experiments both with sugar and bread. Of course, any other organic material on which molds grow could be used in place of bread or sugar solution.

Write a short essay on "Molds in Relation to Preservation of Food for Human Use." Read Section I in Conn's "Bacteria, Yeasts, and Molds in the Home."

246. Economic Relations of Molds and their Allies. Many fungi popularly known as molds, mildews, rusts, rots, blights, smuts, scabs - all of them more or less closely related to the common molds which grow on bread and other foods are very important economically, because of their injurious effect on many cultivated plants. The following are common examples. 
The common molds (black, green, brown) make preservation of some foods for human use difficult, for as the experiments have shown, brief exposure to the boiling temperature does not kill the spores.

During a rainy season the mildews form a white coating on books, paper, clothing, carpets and other organic objects in our homes. Other mildews form their mycelia on the lower sides of many leaves, and some species cause serious diseases of the plants. The mildews attacking grapes grow on the leaves and fruit and cause enormous financial loss in America and Europe. Mildews often destroy lettuce and other vegetables, and severely injure gooseberries and other small fruits.

The rusts, so-called because they appear as streaks or patches of yellow or black color on leaves and other parts of some plants, are very destructive. Rust of the cereal grains, particularly oats and wheat, causes damage in this country to the amount of more than fifteen million dollars every year. The rusty yellow-brown spots seen on wheat leaves in early summer are masses of spores from mycelia in the tissue of the leaf. These spores are distributed by wind, and thus infect other plants in the same field. The foodsupply of the rust is drawn from the sap of the wheat plant, with the result that food for the proper formation of grain is not available. 'Some kinds of rusts require two plants as hosts during their life-history. As an example, one kind of wheat rust has spores that germinate on barberry leaves in the spring, and on the barberry plant the rust produces special spores which infect wheat plants. Of course, this is not the only kind of wheat rust, for in many parts of this country barberry bushes are rarely found. Wheat may suffer from rusts which require no other plant for completing their life-history.

Smuts. - Everybody who has cultivated Indian corn must have noticed the "smutty" ears and tassels, on which 
the smut plants appear first as white masses; but soon these burst open and expose masses of spores. These have the blackness of lamp-black or soot; hence the name "smut." These spores may infect other corn plants in the same season. Examination of a smutty ear of corn shows that the grains have been destroyed wherever the mycelium of the smut has penetrated them.

The blights, rots, and scabs are numerous and destructive. Common examples are potato blight of the leaves and rot of the tubers (caused by mycelium growing from leaves through stem to tubers); the blight which causes leaves of many orchard trees and vegetables to wither and dry up in part or whole; peach-leaf curl ; the black rots of grape and tomato; the scabs of apple, grape, and potato; the leafspot of pear and other trees; and black knots of plum and cherry branches.

The above-mentioned examples are but a small fraction of the plants affected injuriously by fungous diseases. Most of the enemies of cultivated plants are either these diseases or insects. One who attempts to grow any special kind of crop should become familiar with the known diseases and the remedies. For such information consult the bulletins of the United States Department of Agriculture and of state experiment stations. Farmers' Bulletins 91, 219, 243, 250, 284 (all free) describe some important fungous diseases.

Remedies. - In general, the remedies against fungous diseases consist in (1) destroying all infected materials, such as leaves and stems which may carry spores; (2) in destroying spores on seeds before planting (e.g., soaking wheat and rye in formalin solution to kill smut spores, and potato tubers to kill potato rot or blight); and (3) spraying the stems and foliage with solutions containing lime, copper sulphate, copper carbonate, or iron sulphate. The spraying method has within the past two decades come into extensive use, and hundreds of special machines have been invented for spreading 
the liquids over plants in fine mist so as to touch all parts. A mixture of lime and copper sulphate, called Bordeaux mixture because discovered in about 1885 by scientists who tried to check the mildew which was destroying the vineyards near Bordeaux in France, has proved an important remedy for most fungous diseases which are on the surface of plants, and is extensively used by growers of fruits, potatoes, many vegetables, and ornamental plants.

Since insects may attack the same plants, an insecticide (insect-killer) is often mixed with the fungicide. For example, Bordeaux mixture (for the potato blight and rot) and Paris green or other poisonous compounds of arsenic (for killing the potato-beetles) may be mixed. Likewise, Bordeaux mixture (for apple scabs, rots, and blights of either the fruit or the leaves) and arsenical poisons (for the larvæ of codling moth, which makes wormy apples) are commonly sprayed as a mixed liquid.

For information concerning the proper solutions and times for spraying a particular crop in order to get best results write to the agricultural departments either at Washington or at a state experiment station and apply for the latest pamphlets on sprays for plant diseases. Also every spring the best agricultural newspapers review the latest methods for preventing plant diseases.

Water-molds. - Some aquatic molds live on dead animals and plants in water; and some of them are very destructive parasites on living fishes. The fluffy white masses often seen around dead insects in water and on fish in aquaria are examples. The spores lodge on the skin of fishes, germinate, and some hyphæ penetrate the tissues of the fish so that nutriment is absorbed from the blood and lymph. It has been found that brief immersion of trout and other fishes in sea-water will check the growth of the fungus.

Fungi and Diseases. - Some of the fungi allied to common molds may produce diseases in man and domesticated 
animals. The danger from poisonous mushrooms is well known. Certain molds may grow in the lungs of birds, other animals, and very rarely in man. The smut of rye and other plants produces a poisonous substance known as ergot, which sometimes poisons farm animals. In very small quantities ergot is used in medicine. There is always danger in the use of moldy foods by men or farm animals, for molds often form poisonous substances under conditions not yet understood in science. There is good reason for thinking that a large number of small chicks are killed each year by moldy food; and several scientists believe that the mysterious disease pellagra, which has recently attracted so much attention in the southern states, is caused by some form of mold growing in corn meal, which is extensively used as food. Certainly it is best not to use corn meal or other cereal foods if there is about them the least evidence of mold or a musty odor, which indicates some kind of molding.

Other diseased conditions due to molds are thrush, a disease of the mouth-cavity of infants; ringworm, often seen on human faces and scalps; and other skin diseases caused by mold-like plants whose mycelia grow in the human tissues. Most of these are easily prevented. The spores of all the mold-like plants which cause skin diseases may be distributed by towels, sponges, combs, and barbers' tools. Such articles should be carefully washed in boiling water and strong soap after being used by diseased persons. The laws in many states now require such precautions in barbershops.

Useful Molds. - So many of the molds and their allies are injurious from our human point of view that we must guard against the erroneous impression that all relatives of the molds are harmful. The following examples will suggest some useful aspects of the life of these lower fungi. The common molds which cause decay oftentimes destroy 
materials useful as human foods and in other ways, but the same decay processes reduce useless organic matters to a condition which makes them available for plant food. For example, the vast quantities of leaves, stems, and other plant materials seen in late summer must be decayed before their elements can be used over again by plants, and in this decay many of the decomposing fungi aid.

Moreover, some fungi are useful because they destroy insects. House-flies are sometimes seen with delicate white threads projecting from their bodies. These threads bear spore-cases and a mycelium grows inside the flies. The spores which fall on other flies germinate, and so the disease may soon become epidemic. Other similar fungous diseases attack other insects, and millions of grasshoppers, chinch-bugs, and other destructive species are thus killed. An attempt has been made to cultivate some of these insect diseases artificially in order to infect a few insects before turning them loose to spread the diseases to other insects. The chief difficulty is in selecting weather favorable for development of the fungi.

It should be credited to the molds that some of them produce the peculiar flavors of cheeses, such as Roquefort and Camembert. The manufacturers take particular pains to cause the growth of almost a pure culture of the proper kind of mold.

But although some of the molds and their allies may be useful from the standpoint of human interests, it must be admitted that their usefulness is far overbalanced by their harmfulness, especially to the numerous plants which are important to the human food-supply.

\section{Mushrooms}

247. Molds have been studied as examples of lower and simple fungi. The mushrooms are more complicated, 
and yet are made up of hyphæ and have other characteristics showing relationship to molds.

The term mushroom is here used as a general term including all forms popularly known as mushrooms and toadstools, for there is no scientific distinction between them. Formerly all edible fungi were called mushrooms, and all others toadstools; but many so-called toadstools are now known to be edible. Practically and scientifically, then, the two words are now used quite synonymously.

Structure of a Mushroom. - $(L)$ Any common mushroom or toadstool will serve for this study. Specimens may be collected when available and preserved in 5 per cent formalin-solution. Attempt to identify the points of structure described below, and make sketches in note-book.

Examine a well-expanded specimen. Above ground it consists of a stalk and a cap. On the under side of the cap in most species are the gills, delicate plates radiating from the center. In some species there are numerous holes instead of the gills, and in others there are projections in the form of spines. If the cap be cut from the stalk and left for a few days with gills downward on a sheet of white paper, numerous dark-colored bodies (spores) will fall on the paper, leaving a print of the underside of the cap and showing the arrangement of the gills. Closer examination of the under side of the cap will show the spores on the sides of the gills.

Below ground the mushroom is attached to thread-like structures which are popularly called the rootlets, but they have no resemblance to real roots of higher plants, except that they are in the soil. These thread-like "rootlets" are hypho and the entire mass of them is known as a mycelium (see mold, §244). These hyphæ branch and grow in the soil, and from time to time new mushrooms or toadstools grow upward from the mycelium. At first the young mushroom is a small, compact, rounded body, concealed just below the surface until ready for expansion, when it absorbs water rapidly and may reach its full growth in a night.

Microscopic study shows the stalk and cap to be com- 
posed of closely packed and interwoven threads or hyphæ, which have the same structure as the hyphæ below ground. The part above ground is, then, a sort of branch of the subterranean mycelium.

Reproduction. - A new mycelium is produced by germination of a spore which falls in a favorable place. The stalk and cap raise the spore-producing hyphæ above ground so that the spores may be more widely disseminated.

"Fairy-rings." - A mycelium once started in the ground may continue to grow and produce new mushrooms for some time. Frequently mushrooms are seen in pastures arranged in circles. These were once popularly supposed to have been caused during the night by fairies in their dances, and were called fairy-rings, or fairy-circles. This interesting explanation was especially applicable to rings formed by certain minute relatives of the mushroom which give the soil the appearance of having been sprinkled with ashes. But science has in many ways given us rational explanations of many puzzling things in nature, and we no longer need to assume the existence of fairies and other mythical beings. Scientific study of the "fairy-circles" long ago showed that they are caused by the regular growth of a mycelium from a center, and as the older and central portions die, the mushrooms will appear in continually widening circles.

Cultivated mushrooms are grown from "spawn" which comes from seed dealers in the form of dry bricks composed of partially decayed vegetable matter. The spawn is really nothing but mycelium, either taken from old mushroom beds, or started by scattering spores over materials out of which the bricks are to be made as soon as the spores have germinated and formed an extensive mycelium. It is simply necessary to break such bricks into pieces and plant them in a bed of suitable soil with abundance of decaying organic matter. Concerning the cultivation of mushrooms see Farmers' Bulletin No. 204 (free). 
Ants raising Mushrooms. - One of the interesting members of the mushroom group is a species which is regularly cultivated by the leaf-cutting ants of the tropics. Pieces of leaves are chewed by the ants and then packed away to decay and form a proper substratum for the growth of a mycelium, which is eaten by the ants.

Physiology of Mushroom. - As described in $\$ 98$, plants without chlorophyll are like animals in that they must get their food from other plants, either as parasites on living plants (e.g., dodder), or as saprophytes on decaying plant matter. The common mushrooms are usually saprophytes, but some of them grow as parasites on living trees (e.g., shelf-fungi).

In their breathing mushrooms are like animals in that they take in oxygen and excrete carbon dioxide.

248. Lichens. - The familiar plants known as lichens, which in the form of grayish scales or moss-like masses grow on rocks and bark of trees, consist of two kinds of plants living together in intimate connection. One of the plants is a member of the Algæ ( $\$ 236)$, and able to make carbohydrates from carbon dioxide and water; while the other is a fungus, which obtains its carbohydrate food from its associate. The main mass of a lichen is a dense mycelium similar in microscopic structure to some mushrooms, and in this are the cells or filaments of the associated green plant.

Such a living together is an example of symbiosis. A similar association between a plant and an animal is described in $\S 285$.

It is possible to collect the spores and grow separately the two kinds of plants included in a lichen.

Reindeer-moss (a valuable food for reindeer) and Iceland moss (used in cooking) are lichens. Litmus (used for testing acids and alkalies) comes from a lichen. On the mountains lichens assist in weathering rocks, and thus forming soil. An edible form found on sandy deserts is called manna-lichen. 
249. Economic Relations of Mushrooms. - Food Value. The words "economic relations" will probably lead most readers to think instantly of edible mushrooms. There are many thousand species of the group to which the common mushrooms belong, and many of them are edible. The fact is that thay have little value as human food, but they are delicious as relishes. The wild forms are not commonly used because of the difficulty of distinguishing with certainty between edible and poisonous species. There is no safe general rule; and botanists who have specially studied mushrooms advise that no species should be eaten unless identified by one who is competent. Especially should people avoid mushrooms with a ring or cup at the base, for this is one mark of the deadly Amanita, one of the most poisonous plants known to science. Such books as Atkinson's "Mushrooms"; Marshall's "Mushroom Book "; "Mushroom Poisoning" - a circular of the United States Department of Agriculture; and Farlow's "Edible and Poisonous Mushrooms" - also issued by the United States Department of Agriculture, give pictures and descriptions of many species pronounced edible. But even with these books one should be exceedingly cautious before deciding to eat a mushroom which resembles those said to be poisonous.

Effect on Timber. - More important economically than their food value is the destructive action of some mushrooms on trees valuable for lumber. In sawing trees into lumber the heart-wood is often found filled with white threads (mycelium) which have so softened the wood as to make it worthless. Hollow trees are usually due to such decay. The mycelium seen in the wood is connected with a shelf-like structure (often called shelf-mushroom or shelf-fungus) on the bark of trees. In walking through woodland one can easily find pieces of dead branches with small toadstools attached, and if these be split open, the white thread-like mycelium may be seen. These threads (hyphæ) enter trees 
through some injured tissue, as a broken branch, gnawing by animals, or careless pruning which leaves large surfaces exposed. The reason for painting all injured surfaces of trees with tar, cement, or paint is that these substances will keep out mycelia. Spores are abundant in the air and may at any time fall upon an injured surface, germinate, and form a mycelium which penetrates between the cells of the wood. It is an easy matter to keep mycelia from entering a tree at an injured place, but quite impossible to check them when once they have penetrated deeply into the plant.

The "dry rot" which often attacks the foundation timbers of houses and makes them unable to stand the strain of supporting buildings is due to the mycelium of a near relative of common mushrooms. The remedy for "dry rot" is ventilation of spaces beneath buildings and coating timbers with tar, crude oil, or creosote.

Some mushrooms have their mycelia penetrating the roots of trees, causing decay and weakening so that heavy winds uproot them.

Puff-balls, named because they puff out a mass of spores when ripe, are near relatives of the common mushrooms. Their mycelia grow in rotten logs and humus (decaying vegetable matter). They contain numerous small cavities in which spores are formed. Some of the puff-balls are edible in the young state. One kind of puff-balls, called earthstar, has its outer skin split to form a number of star-like projections.

References for pupils. - In addition to those given in the text above, Atkinson's “ Elementary Botany," pp. 326-339 ; Marshall's "Mushroom Book" (excellent illustrations); Coulter's "Plant Structures," pp. 68-74.

\section{Yeast Plants}

250. The Cause of Fermentation. - It is a well-known fact that liquids containing sugar, such as juices of grapes, 
apples, and other fruits, commonly undergo a change called fermentation. The result of this process is the production of an invisible gas (carbon dioxide), which causes effervescence of the fermenting liquid, and alcohol. No other method of producing alcohol is known to occur in nature; and so for thousands of years fermentation has been used in making wines and other alcoholic beverages from fruit juices. It was not until the nineteenth century that the microscopic yeast plants were recognized as the cause of fermentation; and the experiments performed by the great French naturalist, Louis Pasteur, between 1855 and 1865 will always be famous as having placed our knowledge of fermentation processes on a thoroughly scientific foundation.

251. Study of Yeast Plants. - $(L)$ Scrape some small pieces from a cake of compressed yeast, mix with a drop of water on an objectslide, and gently lower a cover-glass into position. Also, if brewer's or baker's yeast in liquid form is available, mount a drop on another slide. Prepare a third slide by mounting a drop of molasses-solution ) water 10 parts, common dark-colored molasses 1 part) in which some scrapings of compressed yeast or some drops of brewer's yeast were placed on the previous day and the liquid kept in a warm place $\left(70^{\circ}\right.$ to $\left.90^{\circ} \mathrm{F}\right)$. It is best to draw up with a rubber-bulbed pipette, or with a dipping tube, some of the white sediment in the bottom of the bottle containing the molasses-solution.

Examine the slides with a high power of microscope. Small oval translucent bodies, some isolated, some united into groups or chains, will be seen. Each

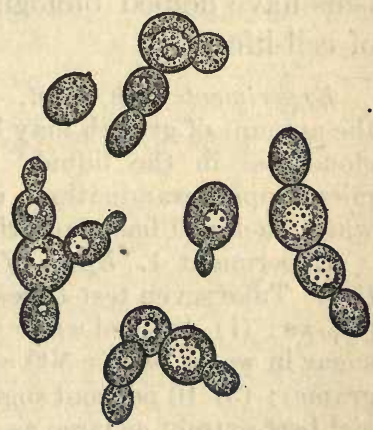

Fig. 83. Yeast cells, two cells beginning to bud, and three chains of cells, formed by repeated budding. Clear centers are cavities. Nuclei not shown. (From Sedgwick and Wilson.) oval body is a yeast plant; and it is a single cell. The chains are due to reproduction by formation of buds. By comparing the sizes of the cells in a chain, it is possible to determine the order in which the buds were formed (Fig. 83). Soon 
the cells of a chain become isolated, and each may form a chain of new cells when food for growth is abundant. An abundance of long chains indicates that the yeast has been growing rapidly; compare that taken directly from the compressed yeast with that which has been in molasses-solution.

The yeast cells are variable in size and shape. The contents consist of protoplasm, cavities containing a clear fluid, small droplets of oil, and a nucleus which is invisible in ordinary living yeast cells. The appearance is different in the "resting" cells, as in the yeast-cake, from that of the actively growing cells. This is visible evidence that changes occur in protoplasm when it is especially active. Of course, slight changes are taking place constantly as long as the yeast cells are living, but during the great activities attendant upon growth the changes become distinctly visible. Many such observations on living transparent cells of lower organisms have helped biologists gain very important knowledge of cell-life.

Experiments with Yeast. - ( $D$ or $L)$ In the following experiments the amount of growth may be roughly estimated by the turbidity or cloudiness in the liquid. It may be determined accurately by microscopic examination, especially by the number of buds to which each cell has given rise.

Experiment 1. Effect of food-supply upon growth and fermentation. Take seven test-tubes, or small bottles, and fill one-half full as follows : (1) distilled water or rain-water; (2) 10 per cent solution of sugar in water (water 500 cc. or nearly 1 pint; granulated sugar 50 grams) ; (3) 10 per cent sugar, and add to the tube a mass of commercial beef-extract as large as a pea; (4) molasses-solution (any dark colored molasses, such as New Orleans, 1 part to water 10 parts); (5) wheat flour in water to make very thin paste; (6) Pasteur's solution;* (7) Pasteur's solution without sugar.

* Pasteur's solution is water containing sugar (C, H, O), ammonium tartrate $(\mathrm{N})$, potassium phosphate $(\mathrm{K}, \mathrm{P})$, calcium phosphate $(\mathrm{Ca}, \mathrm{P})$, and magnesium sulphate $(\mathrm{Mg}, \mathrm{S})$. The symbols in parentheses show the elements (total nine) which the yeast plant gets from each compound used in making the solution, and the amount of these used was originally determined by chemical analysis of yeast. 
Tubes 6 and 7 may be omitted if materials are not at hand. Carefully label each tube with the numbers (on paper labels, or with wax-pencil). Put one drop of washed yeast* into each, shake the tubes thoroughly, tightly plug the mouth of each with a wad of clean cotton-batting or absorbent cotton, and set them in a warm place near a stove or radiator.

Examine the tubes 1 to 7 after several hours and again on the next day and judge from the turbidity, and if possible also from microscopic examination, (1) in which fluids the yeast grows best, and (2) in which fermentation takes place. In which are the most bubbles of gas formed? Does the formation of gas bear any relation to the growth? (Compare 2 and 4.) Keep careful notes, and at the close of the experiments write an account of them, giving your observations and conclusions regarding the substances necessary for growth of yeast and for fermentation.

Experiment 2. (D) Effect of heat on yeast. Three test-tubes half full with molasses-solution. Put equal amounts of yeast into each, and plug tubes with cotton. Label 1, 2, 3. Heat No. 1 to the boiling temperature by holding tube in flame of gas-burner, and leave Nos. 2 and 3 unheated. Keep tubes No. 1 and No. 2 together in a warm place (between 25 and $35^{\circ}$ C.), and No. 3 on ice in an ice-box. After a day or two examine first as to turbidity and indications of fermentation and then open tubes and examine microscopically. Write conclusions as to effect of high and low temperature on yeast.

Experiment 3. (D) Gas evolved by yeast plant. (1) Take one large test-tube, about $\frac{1}{2}$ by 5 inches, and pour in lime- or bariumwater about $\frac{1}{2}$ inch deep. Take a smaller tube, about $\frac{1}{4}$ by 3 inches, and tie a thread around its neck. Provide a cork for the larger tube. Pour vinegar into smaller tube ( $\frac{1}{3}$ full), get it ready to lower into the larger tube. Then drop into the vinegar a small lump of baking soda, and by the thread quickly lower the small tube, and stopper the mouth of the larger one. Results? Compare change of limewater with that produced by breathing through a straw or glass tube into a bottle containing lime-water.

* The washed yeast may be obtained by taking with a long pipette some white sediment from bottom of bottle with molasses-solution in which yeast has been growing for several days. Wash this sediment by placing on filterpaper (preferably in a funnel) and pouring water through it to wash out the soluble food (sugar, etc.). Then place the paper in a small bottle of water and shake to dislodge the yeast cells. They settle to the bottom and may be easily transferred by a pipette to tubes of solutions mentioned above. 
(2) Now, wash out the tubes with clean water. Again put limewater in the bottom of the larger tube. Into the smaller tube put some molasses-solution (about half full), and put in some yeast. Lower the small tube into the larger one, and insert the stopper. Set in a warm place, and after the fermentation has continued for an hour or more look for changes in the lime-water. (If the teacher

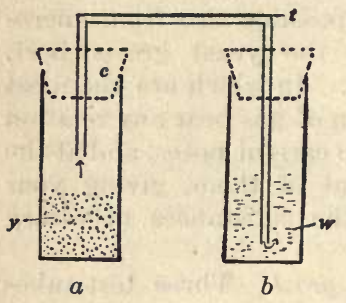

Fia. 84. Two glass bottles connected by a bent glass tube $(t)$, inserted through the cork $(c)$. Tube $a$ contains yeast in molasses-solution $(y)$, and tube $b$ contains lime-water $(w)$. has ready a pan of water, heated as much as the hand will bear with comfort, and the tubes are prepared from growing yeast at the beginning of a two-hour session and placed in the warm water, it will be possible to get results before the close of the session; but examine the tubes again after they stand a day or more in a warm place.) What does this experiment prove? Compare with similar experiments previously performed ( $\$ 26)$.

Experiment 4. (D) Conducting the gas intolime-water. Prepare apparatus shown in Fig. 84. In tube $a$ put lime-water, in tube $b$ put some molasses-solution with yeast. Set in a warm place, and when active fermentation begins notice bubbles of gas rising through the lime-water and its effect. Compare with Experiment 3.

Experiment 5. (D) Collecting gas from fermentation. (1) Arrange apparatus shown in Fig. 85. In the flask $y$ put molasses-solution with yeast; fill the bottle with water before inverting and suspending in position shown in the figure. Set in warm place. When gas is evolved from the flask it will be collected in the bottle, the bubbles rising and displacing the

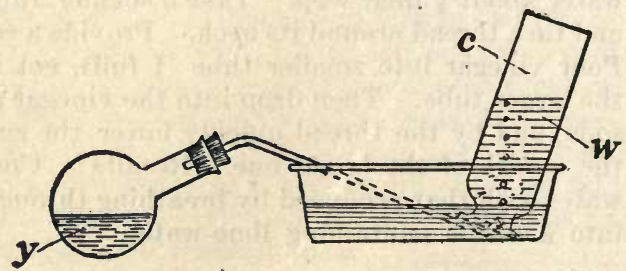

Fia. 85. Apparatus for collecting gas evolved by yeast. $y$, flask or bottle with yeast in molassessolution; $w$, water partly displaced by gas $(c)$.

water. When the bottle is full of the gas, that is, when all the water has been displaced, carefully insert a stopper before raising the mouth of bottle above the surface of the water.

(2) Holding the bottle in 
upright position, quickly remove the stopper and plunge a glowing taper into the gas. Result? (3) Instead of testing with flame, the invisible gas may be poured into tube having lime-water.

Experiment 6. (D) Effect of the gas on a flame. Take a pint bottle or fruit-jar, pour in some molasses-solution, add yeast, cork tightly, and allow fermentation to go on for several days. Then remove cork and quickly insert a glowing taper. Result?

Experiment 7. (D) Alcohol produced by fermentation of sugar. (1) Put a few drops of strong alcohol in a test-tube half full of water, add a crystal of iodine, and heat over gas- or alcohol-lamp. Then add strong solution of caustic potash, or baking soda, until the iodine color fades. Notice the crystals and odor of iodoform, especially after cooling. This is a test for alcohol. (2) Take some molassessolution in which yeast has been growing for several days, add iodine, and test as above. From a large quantity of fermented molasses some alcohol might be collected by distilling.

Experiment 8. (D) Effect of dense sugar solution. (1) Place some yeast in undiluted molasses, keep in a warm place, and compare with results previously obtained with molasses-solution. Write your conclusions concerning the fact that fruit "preserves" do not ferment. (2) Take some "preserved" fruit long kept free from fermentation, place in test-tube or bottle with distilled or boiled water, add some yeast, keep in warm place. Results? Conclusions?

Experiment 9. Bubbles formed by gas from fermentation. Place some yeast in molasses-solution in a test-tube, and pour on the surface of the molasses a small quantity of flour paste. The paste will form bubbles as the gas is evolved.

Experiment 10. Yeast in bread-making. (Optional.) These experiments may be prepared by some pupils at home and demonstrated to the class with full explanations. Mix some actively growing yeast with flour and water to make dough. Bake some lumps of the dough at once and others after leaving in a warm place until "rising" occurs. At the same time mix some baking powder with flour and water, and bake some of the lumps of the dough. Cut across the lumps, and compare the cut surfaces. What has been the effect of the yeast? Of the baking powder? Compare with the previous experiment with bubbles.

Experiment 11. Wild yeasts. (D, optional.) (1) Crush some fruit (apples or grapes), put the juice into a test-tube, plug with cotton, and leave in a warm place. (2) Put some molasses-solution into another tube, and leave exposed to the air in a warm place. Does fermentation occur? Examine a drop of the fluid with the 
microscope. Are yeast cells present? Where could they have come from in 1 and in 2? Why does bottled cider or grape-juice ferment soon after a bottle is opened? Why do we suspect the presence of some antiseptic when grape-juice does not ferment in an open bottle?

Experiment 12. "Salt-raising bread." (Optional.) Add enough common salt to make some milk taste slightly salty, and set in a warm place until foam appears on the surface, i.e., fermentation begins. Yeast cells come from the air. The salt prevents the growth of certain other microscopic organisms (bacteria) which would cause souring of the milk. Such fermenting milk mixed with dough causes it to "raise," but less than when yeast is used. Hence, bread made in this way is heavier than yeast bread.

252. Physiology of Yeast Plant. - The foregoing experiments have given information on which we may base some general statements concerning the physiology of the life-activities of the yeast plant. The plant is a one-celled organism, without chlorophyll, and for its food dependent upon materials built up by other plants. It is therefore a saprophyte like the molds, mushrooms, Indian pipe, and many other plants without chlorophyll. The studies which Pasteur made with the solution which bears his name showed that yeast plants must have nine elements in their food; namely, carbon, hydrogen, oxygen, nitrogen, calcium, sulphur, phosphorus, potassium, and magnesium. These are necessary for the formation of new yeast protoplasm, that is, for growth; and if fermentation is also to take place, sugar (or starch convertible into sugar) must also be present. Fermentation results in changing sugar into alcohol and carbon dioxide. Within recent years it has been demonstrated that this is due to an enzyme secreted by yeast plants. Under great pressure this enzyme has been extracted and used to cause fermentation without the presence of living yeast cells. This is in principle similar to the diastase extracted from plant cells and used in digesting starch to sugar in testtube experiments, or to pepsin from animal stomachs. 
The usual method of reproduction is by budding, but under certain conditions a yeast cell may form spores within itself, and later each of these spores may grow into an ordinary yeast cell capable of budding.

As to relation to temperature, the yeast plants grow best at about 20 to $30^{\circ} \mathrm{C}$. They are quickly killed at the boiling temperature, and hence it is quite easy to bottle sweet (unfermented) cider or grape juice. There is apparently little growth at the temperature of ice-boxes, and hence fermentable foods may be preserved at low temperatures; but only temporarily, for other organisms may develop, except at the freezing temperature (which is colder than in common ice-boxes).

Yeast plants can withstand partial drying. This explains their living in the cakes of dry yeast; but after some months all the cells are dead in such cakes. It also explains how the wild yeasts can live in the dust on fruits, or on the surface of the soil in orchards and vineyards, ready to begin growth as soon as the bruised or crushed fruit allows the yeast plants access to the juices. In the dry condition, the yeast cells float with dust in the air, and this explains why sterile fruitjuices (e.g., boiled cider) or sugary solutions will soon ferment after exposure to ordinary air.

253. Economic Relations of Yeasts. - It is only necessary to allude briefly to the vast industries of manufacturing bread and alcoholic beverages which are dependent upon production of alcohol and carbon dioxide by yeast plants. So great is the importance of pure yeasts in these two industries that enormous factories, costing immense sums of money, are devoted to raising yeasts.

Apart from alcohol in beverages and in medicine, it has long been invaluable in varnishes and numerous other compounds used in mechanic arts. Recently it has been coming into prominence as a cheap source of energy for engines, burners, lamps, etc. It is possible to make alcohol cheaply 
from much plant material which, on the ordinary farm, goes to waste (unmarketable fruits and grains); and it is probable that before many years pass the making of alcohol for mechanical purposes will be a vast industry in agricultural regions.

Vinegar, which is used so extensively as a condiment and for preserving certain foods for human use, is best made from alcohol derived from fermented fruit juice. For example, yeast plants ferment the sugar of apple cider and form alcohol ; and the vinegar bacteria, of which there are millions in " mother of vinegar," ferment the alcohol into acetic acid, which gives vinegar its acid qualities.

On the harmful side, we must mention the undesired fermentation of fruits and sugary mixtures prepared for human food. However, we have seen that heating to near boiling will check such action of yeast. Sometimes yeasts cause undesired fermentation in milk and cheese in dairies.

Certain kinds of yeasts (not the varieties used commonly) have caused skin diseases, but rarely.

We must conclude, then, that on the whole, yeast plants are decidedly useful as producers of alcohol and carbon dioxide. Most of the bread-making since human civilization reached a stage where attention began to be paid to preparing the best possible foods, has depended upon yeast plants. Quite apart from the question of the harmful use of alcoholic liquors, to be discussed in the last part of this book, there can be no question concerning the value of alcohol for many other purposes. The usefulness of yeasts forms a decided contrast to the harmfulness of the molds ( $\$ 246$ ).

Reading for pupils: Section II in Conn's "Bacteria, Yeasts, and Molds in the Home."

\section{BACTERIA}

254. Bacteria, Germs, Micro-organisms, Microbes. The word bacteria (singular, bacterium) is the biological name for a group of one-celled plants without chlorophyll, which 
are lower than any we have yet studied. Since they have no chlorophyll, some authors class the bacteria with fungi; but they are probably not closely related to yeasts, molds and other fungi. Popularly, the four words which stand in the above heading are used as if synonymous; but the fact is that the last three may properly be applied to some microscopic organisms which are not bacteria, and in certain cases are one-celled animals. In other words, the last three are general words meaning microscopic organisms; while bacteria are a special kind of such small organisms, belonging to the plant kingdom. Sometimes it is very convenient to have the general terms. For example, the statement that micro-organisms affect our everyday life in many important ways includes other organisms as well as bacteria. Some of these other organisms will be referred to briefly at the close of this section, because the practical relations to our daily lives of many of them are similar to those of the bacteria.

From our studies of yeasts and molds it is evident that foods prepared for human use are likely to ferment (that is, favor growth of yeast), if in a liquid form containing a certain amount of sugar (e.g., fruit juice, or dilute sugary syrup). If the foods are solid and contain a large amount of starch or sugar (e.g., bread or preserves), molds are likely to develop first. If the foods contain considerable protein (e.g., soups, such vegetables as peas and beans and meats), it is a wellknown fact that decay characterized by disagreeable odors begins quickly. This change is caused by bacteria, which may grow so rapidly as to prevent development of the yeasts or molds. In fact, there is a tendency among the bacteria, yeasts, and molds for the kind which gets the first start to crowd out the others. Thus bacteria do not usually get a chance to develop in fruit juice until after yeasts have used up the sugar ; bacteria get the first start in protein foods; and molds predominate on starchy foods. An apple may first start to rot because filaments or hyphæ of molds pene- 
trate it; but yeasts and bacteria may develop later. This opposition of different kinds of organisms is often called antagonism. It is important, for harmless micro-organisms often prevent the multiplication of other kinds.

255. Study of Bacteria. - ( $L$ or $D)$ Clean by washing with soap or soap-powder and rinsing some small bottles or test-tubes and plug with cotton as described in $\S 245$. Place the plugged tubes in a sterilizer ( $\$ 245)$ and keep the water boiling for, a half hour. Protect the tubes from dust until needed.

Any clear soup or bouillon will serve for the following experiment. The clear concentrated soups commonly sold at ten cents per can may be used after diluting with water. Test with litmus-paper, and if acid add some baking soda (or $\mathrm{KOH}$ solution), little by little, until the soup is slightly alkaline in reaction. When thus prepared, the bouillon may be kept in a bottle or flask by simply plugging the mouth with cotton and sterilizing after each opening of the bottle.

Fill sterile test-tubes half full with the bouillon; and replace the cotton plugs, taking care that they do not become wet. Place the tubes in the sterilizer for a half hour, and repeat on the following day. The sterile tubes may now be kept indefinitely. It is best to keep them covered so that dust from the air may not fall on the cotton and thus increase the chance of bacteria getting into the tube when the plug is removed.

Examine tubes which have remained sterile for many days. If bacteria develop, the bouillon will become turbid or cloudy.

Take the plugs from some tubes with bouillon, and leave the tubes open for several days. Do bacteria develop? What is the explanation of the fact that similar tubes with cotton plugs remained sterile? The cotton certainly cannot keep air out of tubes; what then can be its effect on the air which enters the tubes?

Mount a drop of bouillon from a tube which has become turbid. Examine with a high power. It is well to have some cotton threads under the cover-glass to assist in finding the focus. Examine with a high power. Look for exceedingly minute, transparent bodies, much smaller than yeast cells and mold spores; some rod-shaped, some spherical, some twisted rods (Fig. 86). By shifting the mirror of the microscope, it is possible to see them better. Some of these organisms (bacteria) swim rapidly by means of eilia so small that they cannot be seen with the ordinary microscope. Watch the swimming of some of the largest bacteria visible. Remember that the apparent rate of speed has been magnified as much as have 
the bacteria, and that the distance apparently covered must be divided by the magnification of the microscope used in order to learn the actual speed. Glass slides with delicate ruled lines have been used to estimate the size and speed of the bacteria. Some of those of ten seen are so small that 50,000 placed end to end would make a row one inch long, and a bacterium ${ }_{10 \frac{1}{000}}$ of an inch long belongs to a giant species.

In order to make bacteria more distinctly visible, it is customary to stain them. A simple method is as follows. Place a drop of fluid with bacteria on a coverglass, evaporate the water slowly by holding the glass above a gas-burner (a special kind of forceps is made for holding, but ordinary forceps will serve), then pour over the dry bacteria a few drops of stain (gentian-violet, or other aniline dyes dissolved in water). After twenty or more minutes, wash off the stain by dipping the slide into clean

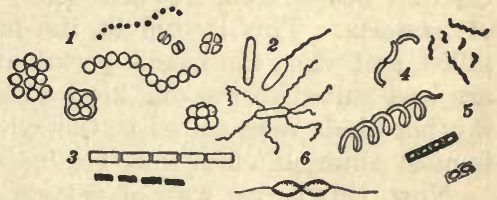

Frg. 86. Forms of bacteria. 1, micrococci, some in chains (streptococci). 2 , bacilli, two flagellated. 3 , bacilli in chains. 4 , spirilla. 5 , bacilli with spores. 6 , a dividing bacillus with flagella.

water, and then place the cover-glass on a drop of water on an objectslide for microscopic examination. Be careful to get the stained side of the cover-glass down. Instead of mounting with water, the coverglass may be carefully dried after washing off the stain, and mounted with a drop of glycerin or Canada balsam. The glycerin will preserve the preparation for several days, and the balsam makes a permanent preparation which may be kept for years.

Make brief examination of bacteria obtained from various sources, such as sour milk, "mother-of-vinegar," and any organic materials found in a state of decomposition. All bacteria seen will be spherical, rod-like, twisted, or spiral in shape, but they may be found united together in groups.

Bacteria may be found which show an enlargement due to the formation of a spore (Fig. 86). These spores appear as dark spots when the microscope is slightly out of focus, and very glistening when in focus. "Hay-tea" made by pouring hot water over some good hay, and filtering through a layer of cheesecloth, is excellent for growing bacteria for study of spores. Fill several test-tubes half full of "hay-tea," plug with cotton, heat to $100^{\circ} \mathrm{C}$. by holding tubes in gas-flame until boiling occurs, or for a short time in a sterilizer. 
Sterilize half of the tubes again on second and third days. The tubes sterilized once will probably become turbid within a fow days and microscopic examination will show that practically all the contained bacteria are rod-like. Later they will form spores; and this is the secret of the bacteria appearing after once boiling. The spores were able to withstand the heat; but before the second or the third heating they had germinated and the bacteria thus formed were killed by the later heating.

Pure Cultures. - It often happens in the above experiment that the tube heated once has practically a pure culture of a certain kind of bacteria. This is due to the fact that all active bacteria are killed, and when the spores germinate the conditions (food, acidity) are best suited to the one kind of bacteria which flourishes. Even if other kinds were suited to the existing conditions, the form which is most abundant first may gradually crowd out the other kinds.

Now, this is one way of getting approximately pure cultures of certain bacteria; but for more accurate work and for separating many species which live together, it is necessary to have a method for isolation of individual bacteria. This is afforded by Koch's culture-plate method, which uses a solid medium like gelatin instead of a liquid medium like bouillon. In a liquid the bacteria are constantly moving, and hence mixing ; but on a gelatin plate a bacterium is fixed at a certain spot, and by division large colonies of its descendants are developed.

Gelatin Plates. - $(D)$ Run a thin layer of some melted gelatin medium (gelatin melted in bouillon; see directions in "Teachers" Manual") into the bottom part of some Petri dishes, cover, and sterilize for a half-hour. Repeat sterilization on second and third days. Take care to keep the dishes level so that the melted gelatin does not touch and glue the cover fast. After the last sterilizing, allow the gelatin to cool so as to form a plate in the bottom of the Petri dish. Wide-mouthed bottles, stoppered with cotton, can be used in same way, simply adding enough gelatin to form a thin plate on the side (Fig. 87). Small bottles and test-tubes may be used, but they should be left inclined after the last sterilizing, so that the cooled gelatin will be an inclined plate against a side of the tube or bottle.

(D) Select some Petri dishes in which the gelatin plates have remained sterile for several days. Remove the covers, and leave the gelatin plate exposed to the air of the room for five, ten, or twenty minutes. Then replace covers, and label. Look at the dishes from day to day. If spots (white, yellow, or red) appear, notice that they increase in size from day to day. Each spot is 
usually made up of the descendants of one bacterium which happened to fall on the gelatin. Of course, two kinds of bacteria might happen to be on the same dust particle which falls on the plate of gelatin exposed to the air; but microscopic examination of some of the bacteria removed by a sterile needle would disclose that fact, and it is easy to select spots which are pure cultures ; that is, made up of one kind of bacteria.

Now, if a sterilized inoculating needle be touched to one of these spots and then stroked across the gelatin on a new sterile plate, or

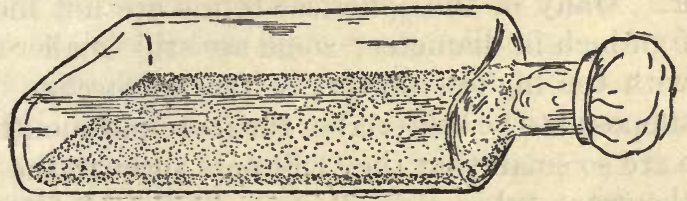

FIG. 87. A flat bottle may be used in place of a Petri dish for gelatin plates. The mouth is stoppered with cotton. (From Osterhout.)

in test-tubes, the bacteria transferred to the new plate will probably be all of one kind, and when they multiply a pure culture will be obtained.

When once a pure culture is obtained, sub-cultures to new plates, or to bouillon or gelatin in tubes, are easily made with a sterile inoculating needle ( $\S 245$ ). Great care must be taken when opening plates and tubes, in order to avoid dust which may contaminate the cultures with other bacteria. In research work with bacteria, special glass cases are often used for keeping dust from tubes while transfers of bacteria in pure cultures are being made.

In case it is desired to separate the bacteria in a liquid, as in water analysis, one or more drops of the liquid are poured on a sterile gelatin plate, and the liquid is allowed to run across the plate. The contained bacteria will start colonies along the path of the drop, and after selecting colonies to be cultivated, some bacteria from them are transferred to new sterile plates. Allow a drop of water from a hydrant to flow across sterile gelatin in a Petri dish, cover quickly and examine next day. Sometimes the number of colonies of bacteria will be so great that it is impossible to select a colony that is pure. In such a case the drop of water to be examined must be mixed with many drops of absolutely sterile water (distilled or boiled) in order to scatter the contained bacteria, and a drop from this run on a gelatin plate will give fewer colonies. 
In similar ways bacteria from any source may be obtained in pure cultures by means of solid culture media. In addition to gelatin, a thin slice of potato, carefully sterilized, may be used ; and for certain bacteria which must be grown in incubators agar-agar is added, because it is not so easily melted as gelatin.

256. Important Facts concerning Structure and Functions of Bacteria. - (a) Size. The average rod-shaped bacteria are not more than $\frac{1}{10000}$ of an inch in length and $\frac{1}{50000}$ in diameter. Many of the spherical forms are not more than $\frac{1}{50000}$ of an inch in diameter; some are still smaller and just visible with the highest powers of the microscope, and the germs (supposed to be bacteria) of the foot-and-mouth disease of cattle are so small that they will pass through the pores of the earthenware tubes in a Pasteur-Berkefeld filter, which will remove all bacteria large enough to be seen with the microscope. In fact, bacteria are so small that fractions of inches are inconvenient measures; and biologists commonly state dimensions in micromillimeters or microns, one of which is $\frac{1}{1000}$ of a millimeter, or about $\frac{1}{25000}$ of an inch. A bacterium $\frac{1}{25000}$ inch long would be 1 micron long.

(b) Growth and Division. Under favorable conditions, a bacterium rapidly grows to full size and then divides into equal halves. Each of these absorbs food, grows, and soon divides again. Certain bacteria have been observed to divide every twenty minutes. Some one has computed that if the descendants of a single individual bacterium were to keep on dividing once an hour for two days there would be more than 280 millions. You can easily verify these figures by applying the mathematical formulas for geometrical progression. At the rate of one division every half hour, there would be 4772 billions in 72 hours, and the weight would probably be more than 7000 tons. These figures simply illustrate the enormous reproductive powers of the bacteria and help us to understand how a few bacteria introduced may soon produce enough to cause disease or the decompo- 
sition of foods. But the truth is that the half-hour or hour rate of division is never maintained for long at a time. Soon food-supply is exhausted, their own excretions accumulate and exert a poisonous influence, or other unfavorable conditions tend to check the rapid rate of growth.

(c) Spore-formation occurs in some bacteria. Only a few human diseases, and those not very common, are caused by bacteria which produce spores. This is a fortunate circumstance, for as we have seen, spores are much more difficult to kill than are the active bacteria. As a rule, one bacterium produces only one spore, and this germinates and forms one bacterium. There is therefore no multiplication, and sporeformation is simply a device for adapting the organism to unfavorable conditions. Spores taken from cattle dead from the anthrax disease have been found capable of germinating many years after the animals were buried.

(d) Temperature. Some bacteria are able to multiply near the freezing point, and some live in hot springs in water at a temperature which will kill most kinds of bacteria and other living things. Between these two extremes there are all gradations. Contrary to the popular belief, freezing does not kill all bacteria. Bacteria of several species, including those which cause typhoid and diphtheria, have been kept several days at the temperature of liquid air (about $-190^{\circ} \mathrm{C}$.), and when thawed out, appeared to multiply normally. However, a very large percentage of common bacteria die when frozen in ice, and comparatively few are living after the ice has been kept five or six months. A few years ago an epidemic of typhoid fever was traced to ice which had been stored seven months, so that all ice from waters contaminated with sewage should be regarded with suspicion. While most of the bacteria will probably die, the few which remain may multiply rapidly when taken into the human body.

The thermal death-point varies. Ten minutes' exposure to a temperature of $70^{\circ} \mathrm{C}$., or one minute at $100^{\circ} \mathrm{C}$. (boil- 
ing), will kill all bacteria of typhoid and tuberculosis. Spores of other bacteria may survive the boiling point for hours; but boiling for a short time on successive days (known as discontinuous sterilization) will cause the spores to germinate, and then they are easily killed by heat. Fortunately, the disease-producing (pathogenic) bacteria likely to be in drinking water and milk do not form spores.

Sterilization of substances containing bacteria is usually accomplished, as already described for molds ( $\$ 245$ ), by discontinuous steaming or boiling on two or three days. The first boiling $\left(100^{\circ} \mathrm{C}\right.$.) swells the spores, and the later heating kills them. Steam under about $30 \mathrm{lb}$. pressure and having a temperature of about $130^{\circ} \mathrm{C}$. will kill even resistant spores in half an hour. Dry hot air at about $350^{\circ} \mathrm{C}$. is also efficient as a sterilizer of such articles as clothing.

Pasteurization (discovered by Pasteur) means heating milk to a temperature of 60 to $70^{\circ} \mathrm{C}$., and keeping at this temperature for 10 to 20 minutes. Its advantage is that it does not change milk as does heating to boiling point $\left(100^{\circ} \mathrm{C}\right.$.), while it kills the disease bacteria likely to be present in impure milk. In some cities much milk is now pasteurized.

(e) Light. - Strong sunlight kills all bacteria which are directly exposed, as on the surface of soil. Some species are killed in a few minutes, and few can withstand hours of exposure. This is important, for it suggests the value of sunlight for killing bacteria in sunny rooms, and especially on clothing, carpets, etc., which can be exposed out-of-doors. It also suggests the importance of building houses so as to get the maximum of sunlight; for example, shade trees should not stand too near houses. The value of sunlight in disinfecting streets cannot be too strongly emphasized. Sprinkling with water increases the action, for wet bacteria stand less light. It is unfortunate that all streets in cities cannot be in the north-south direction so that all their surfaces will get the full force of sunlight at mid-day. 
(f) Chemicals. - Many chemicals kill bacteria, and are called germicides or disinfectants. Some chemicals when much diluted do not kill bacteria, but prevent their multiplication. Such are called antiseptics. Corrosive sublimate (mercuric chloride), and carbolic acid are two powerful germicides, killing bacteria even when much diluted. Most strong acids and alkalies are also germicides. Chloride of lime and washing powders are two efficient germicides for general household cleaning. Among gases, sulphur gas from burning sulphur and formaldehyde from evaporated formalin are now regarded as most powerful germicides. The formalin is better because it does not bleach colored articles, is not so poisonous to human lungs, and is easily evaporated in a room either by a special lamp or by the heat generated by a bucket of slaking lime on which the formalin is quickly poured before closing the door of the room. By exposing cultures of bacteria in such rooms, it has been shown that the bacteria are killed by the gas generated. Among common antiseptics are very dilute solutions of formalin, carbolic acid, boric acid, common salt, and many substances produced by plants (menthol, thymol, eucalyptol, camphor, cloves, cinnamon, etc.). The preservative action on foods of common salt, vinegar, creosote (on smoked meats), and spices is due to the antiseptic power of these substances. The more powerful preservatives, such as boric acid, sodium benzoate, formalin, and salicylic acid are sometimes used in foods; but there is danger of injuring the digestive organs by amounts so small that the sense of taste does not guard against them. There is no such danger with vinegar and common salt.

257. Where Bacteria are Found. - They may be said to be almost ubiquitous. They are abundant in soils ; water of seas, rivers, and lakes; in the bodies of animals and plants; in all dead organic matter in nature; and in the air (except on high mountains, in polar regions, and in uninhabited desert 
areas of such immense size that bacteria could not be carried on dust from other regions). In fact, bacteria are distributed wherever they can get food and proper temperature for growth and multiplication. Unlike other organisms, certain bacteria do not require oxygen from the air, for they can get it by decomposing the organic substances on which they live. Such bacteria are called anaërobic (living without air). Some of them can live deep in the soil ; but at depths below where roots of plants and earthworms penetrate, the soil is usually without bacteria. Hence water from deep wells is usually pure, unless there are openings between strata which somewhere communicate with lakes or other surface water.

The wide distribution of bacteria, especially on or in everything connected with the inhabited surface of the earth, makes it extremely difficult to eliminate them. In fact, only in closed bottles, etc., in which all bacteria can be killed by heat or chemicals, is it possible to keep any substance free from bacteria (i.e., sterile). This is the reason for the careful work necessary in preserving many foods.

258. Useful Bacteria. - The popular impression is that all bacteria are harmful in that they decompose our foods or cause dangerous diseases of man or of useful animals. This is far from true, for very many bacteria are directly useful.

(a) Bacteria in Soils. - Most important of the useful bacteria are those which deal with the nitrogen compounds in the soil. We have already noted that the nitrogen excretions of animals are in turn used by plants $(\$ 117)$; but these excretions must first be prepared by bacteria. These organisms first decompose the rather complex animal excretions like urea, and then unite the contained nitrogen into simple compounds (nitrites and nitrates) which the roots of plants can absorb and use. The guano from Peru, which has long been a valuable agricultural fertilizer, was formed by bacterial action on the excreta of countless thousands of sea-birds which lived in prehistoric times. It contains concentrated nitrogen com- 
pounds readily available for absorption by roots of plants. Nitrate of soda from Chile was probably formed by bacterial action which united the nitrogen from organic matter (seaweed, or guano) with sodium derived from sea-water. Similar bacterial changes take place in all manures used on farms. The odor of ammonia around stables is evidence that the decomposing bacteria are at work, and that valuable nitrogen in the ammonia is escaping into the air, because other kinds of bacteria are not working properly and fixing the nitrogen in nitrites and nitrates. Agricultural books give rules for handling manures so as to avoid such wasteful decomposition; that is, they teach how to cause both kinds of bacteria to work together so that the products of decomposition will be built into available crude plant foods with little loss of nitrogen (e.g., in ammonia) to the air. This is a problem of such great financial value that every farmer should study it carefully in the special books on soil fertilizers.

In still another way certain bacteria are useful in soils. The free nitrogen in the air is not usable by animals and plants, with the sole exception of certain bacteria known as the nitrogen-fixing bacteria, which live in the root-tubercles on certain plants $(\$ 152)$. These bacteria unite the nitrogen absorbed from the air into nitrogen compounds which can be used by the plants that have the tubercles. In recent years, the importance of this method of increasing the amount of nitrogen fertilizers in the soil has been recognized, and now every scientific farmer gives special attention to raising clovers, alfalfa, peas, vetches and similar plants whose roots are favorable to the nitrogen-fixing bacteria. By growing such plants soils can be much improved in fertility at an expense much lower than by use of nitrate of soda and other commercial fertilizers. The only other known way of preparing nitrogen from the air for use by plants is an expensive method of combining the nitrogen with sodium by 
means of electrical action. So far the nitrate fertilizers produced electrically are even more costly than the nitrate of soda from Chile.

(b) Bacteria in Milk. - A number of kinds of bacteria are useful in milk, butter, and cheese. The most common bacteria in milk are those which produce lactic acid, which causes milk to sour and coagulate. While this process may be undesirable in milk intended for drinking, it is useful in the making of certain kinds of cheese. Moreover, the presence of the lactic bacteria in milk prevents the growth of certain species which cause putrefaction. If milk be heated to 70 or $75^{\circ} \mathrm{C}$. (pasteurized), the lactic bacteria are killed and others capable of producing putrefaction remain. This fact leads to the belief that lactic bacteria are useful after milk is taken into the stomach in that they oppose other bacteria which may be harmful. There is no reason to think that the lactic bacteria in milk are harmful when taken into the digestive organs; but, of course, most people do not like to drink sour milk. In recent years, we have had many newspaper articles on Professor Metchnikoff's prepared milk for curing and preventing disease caused by excessive development of decomposing bacteria in the intestines. Such bacteria produce poisonous substances which may be absorbed by the blood and cause ill health (autointoxication, meaning self-poisoning). The milk prepared by the Metchnikoff method is believed to contain substances secreted by certain bacteria whch will prevent excessive multiplication of putrefying bacteria in the intestine. These products of bacteria are sometimes concentrated into tablets to be taken like pills or dissolved in milk before drinking; but most of the tablets on the market are absolutely valueless in physiological effect. In fact, physicians are not yet agreed as to the medicinal value of such prepared milk; but one who wishes to try it should purchase the milk in which the proper bacteria have been grown, for it seems 
probable that only in this way can their secretions be abundant enough to check other bacteria in the intestines.

(c) Bacteria in Butter and Cheese. - The peculiar flavors of butter and cheese are due to certain species of bacteria, and hence the scientific dairyman gives special attention to methods which will prevent the growth of undesirable species and favor the desirable ones. By using pure cultures of bacteria (and sometimes certain molds), it is possible to produce the desired quality of butter and cheese. The disagreeable flavor of butter made in many farm-houses is due to undesirable bacteria. See Conn's “Bacteria, Yeasts, and Molds"; and pamphlets of the United States Department of Agriculture on butter and cheese making.

(d) Bacteria in Vinegar. - The change of alcohol into vinegar by bacteria has been mentioned in connection with yeast. "Mother-of-vinegar" is a mass of bacteria imbedded in a gelatinous substance which they secrete.

(e) Bacteria and Textile Fibers. - Many fibers, such as those of flax, hemp, and others obtained from the stems of plants, are obtained by rotting the stems until the surrounding tissues are soft. Bacteria do this work.

(f) Useful Decomposition. - While the action of bacteria in decomposing foods prepared for human use is harmful and financially a source of great loss, we should not forget that this same process of decomposition is absolutely necessary in order to keep up the cycle of organic matter ( $\$ \$ 116$, 117). We repeat for emphasis the statement made elsewhere that but for the decomposing action of bacteria, aided by other micro-organisms, the world would soon be so filled with dead bodies of animals and plants that the carbon and nitrogen available in the food-supply of living things would be bound up in dead organic matter. Hence as decomposers of organic matter the bacteria are indispensable.

A splendid example of the usefulness of bacteria in causing decomposition is found in the latest methods for disposal of 
sewage by distributing it in concrete tanks or reservoirs, arranged so as to allow decomposing bacteria to grow rapidly. The result is that sewage is changed so that it will be harmless. Even pathogenic (disease-producing) bacteria are thus killed. Many cities now purify their sewage by this method before discharging it into rivers. For private sewer systems in villages and in rural districts, the best method consists in allowing sewage to run into porous drain-tiles laid a short distance below the surface in loose soil. In the soil around the tiles the decomposing bacteria flourish and convert the sewage into harmless substances which may be used by roots of plants. The old-fashioned cesspools are being superseded by the modern bacterial methods, for the cesspools do not favor rapid growth of bacteria, and moreover they are often deep enough to allow escape of dangerous sewage into subterranean water courses.

Summarizing the useful aspects of bacteria, it is evident that their usefulness far exceeds their harmfulness. A few individual organisms may die from bacterial diseases, but the continuance of life on this planet depends directly upon the decomposing action of bacteria, which allows a cycle of organic matter $(\$ 116)$. And quite apart from this allimportant aspect of our relation to bacteria, their value in the other ways we have noted above far outweighs their harmfulness as producers of disease. Moreover, in the not distant future, when civilized people will carefully apply the already well-known biological laws ( $\$ 482-490)$, so that bacterial diseases will be kept under control, the usefulness of bacteria will attract more popular attention than at present, when the average citizen thinks of "germs " only as producers of disease.

Most of the useful aspects of bacteria are well treated in Lipmann's "Bacteria in Relation to Country Life," and more briefly in Conn's "Bacteria, Yeasts, and Molds in the Home." 
259. Diseases Caused by Bacteria. - We are so familiar with the idea that many diseases are caused by "germs" or bacteria, that it seems scarcely possible that less than thirty years ago no disease had been shown to result from the growth of bacteria in the human body. It was in 1876 that Robert Koch, of Berlin, made pure cultures of rodshaped bacteria found in the blood of cattle and sheep suffering from anthrax, or splenic fever, and gave the first proof that bacteria caused disease. Six years later (1882) Dr. Koch demonstrated beyond question that human tuberculosis is caused by a bacterium which he named Bacillus tuberculosis; and in 1883 he proved that the dreaded disease, Asiatic cholera, is caused by another specific bacterium. These discoveries aroused the interest of many investigators, with the result that in less than thirty years there has been built up the new science of bacteriology, which has already been of inestimable benefit to the human race and bids fair to lead soon to absolute control of some of the most dangerous diseases.

To-day the list of diseases known to be caused by bacteria is a long one and each year becomes longer. The following are some of the best known: tuberculosis of any organ, cholera, diphtheria, typhoid fever, blood-poisoning, syphilis, pneumonia, meningitis, gonorrhea, influenza, tetanus, leprosy, bubonic plague, and numerous others of rarer occurrence. These are all infectious, or germ diseases; and some of them are also contagious, or liable to be transmitted by contact with patients.

260. Diseases Caused by other Micro-organisms. - A number of diseases are now known to be caused by microorganisms which are animals, while the bacteria belong to the plants. These animal parasites are one-celled, and belong to the group of the Protozoa ( $\$ 273)$. The terrible African disease known as sleeping sickness, malaria, and a form of dysentery are the best known human diseases cer- 
tainly due to animals which produce effects similar to those of bacteria in other diseases.

261. Infectious Diseases not yet Understood. - The following diseases are almost certainly due to micro-organisms which are readily transmitted from one person to another, thus making them infectious; but the organisms have not been discovered. The diseases of unknown causation are yellow fever, hydrophobia, smallpox, whooping-cough, measles, scarlet fever, and mumps. The last five of these infectious diseases are highly contagious. In all these diseases it has been possible for medical men to do much towards controlling their spread by working on the assumption that some undiscovered micro-organism is the cause, and that therefore cases of the diseases should be handled according to principles based on diseases known to be caused by bacteria. For example, yellow fever has been shown to be transmitted by bites of certain kinds of mosquitoes which have previously sucked blood from a yellow-fever patient; and this has suggested the desirability of applying the rules first worked out for malaria when it was discovered that the cause is microscopic animals which are injected into human blood by mosquito bites. The rules simply require destruction of the mosquitoes ( $\$ 329)$, and preventing them from biting healthy persons and especially those sick with malaria or yellow fever. Similarly, in dealing with smallpox, scarlet fever, mumps, measles, and whooping-cough, physicians have assumed the existence of some undiscovered micro-organisms, and have required isolation of patients, quarantining of exposed persons, and disinfection of rooms and of all articles on which bacteria from patients might have lodged.

262. How Bacteria cause Disease. - We may take diphtheria as an example in showing how bacteria cause disease. We all know that the events connected with this dreaded disease are as follows: A child is " exposed " to diphtheria, 
perhaps in school where other pupils have shown signs of the disease, and after a number of days, the symptoms of the disease may appear; examination of the throat discloses certain peculiar spots, and microscopic examination of material from the surface of these spots shows countless thousands of bacteria of the diphtheria species. The reason why the disease did not appear immediately after exposure is that it required time for the one or few bacteria which first lodged in the throat to multiply and form a colony.

Toxins. - Now, the symptoms of diphtheria are not simply those of a sore throat, for the patient is evidently affected in many other organs. This seems surprising, for the bacteria are not so widely distributed; but the explanation has been found in the fact that the colony of bacteria secrete a poison (toxin) which is absorbed by the blood and then distributed widely in the body. Thus bacteria growing in one organ (local infection) may profoundly affect other organs in the body.

263. Antitoxins. - If the vitality of the patient is strong enough to endure the diphtheria toxin for some days, the climax of the disease is passed and convalescence ensues. The explanation of this conquering of the disease is that the cells of the patient's body have gradually secreted a substance which counteracts the toxin; this is an antitoxin. Those patients whose bodies are not able to secrete enough antitoxin succumb to the disease.

Every one who reads newspapers must have learned that doctors now treat diphtheria with an antitoxin obtained from horses. The explanation is as follows. A great many children are not strong enough to make in their tissues antitoxin sufficient to overcome the poisons of the diphtheria bacteria; and it cannot be known at the beginning of the disease whether the patient is going to be strong enough. Hence, it is desirable to give some artificial aid. No drug has been found to do this. But taking advantage of the fact 
that diphtheria toxins will cause the horse tissues to form antitoxin, which appears in the blood, physicians now inject into the blood of the human patient some antitoxin from horse blood, and this saves the child from being seriously ill while his own cells develop antitoxin. Thus it is possible to put into the blood of a child on the first days of diphtheria more antitoxin than the child might develop in his own cells after days of illness.

Some health laboratories keep inoculated horses constantly so as to have a supply of antitoxin ready for use of doctors who discover cases of diphtheria. The toxins injected into the horses does not make them appear sick. The withdrawing of comparatively small quantities of blood for extracting the antitoxin is done by an instrument which does no serious injury ; and more antitoxin may be obtained from the same horse every month.

264. Other Diseases are Similar. - This story of the relation of bacteria to diphtheria is very similar to that of many other diseases now known to be caused by bacteria. The bacteria enter the body, multiply and form toxins. Then antitoxins, or other opposing substances, appear, counteract the toxins, and the patient recovers. In only a few cases has it been possible to find in other animals antitoxins which can be injected into the human tissues to cure or prevent human diseases.

Ant toxins and other antibacterial substances are specific. For example, diphtheria antitoxin will not cure or prevent any other disease, and the antitoxin which surgeons use after injuries by Fourth-of-July pistols is obtained from animals into whose bodies the toxins of tetanus or lockjaw bacteria have been injected, causing the animal's tissues to make tetanus antitoxin.

265. Immunity. - One of the most interesting things connected with bacterial diseases is the fact that some people never have certain diseases even when often exposed to infec- 
tion; and also it is a rule that one is not likely to have the same diseases a second time. Furthermore, adults are not likely to have the diseases which commonly affect children. This lack of susceptibility to diseases is known as immunity. Immunity which is present in human or animal individuals who are not susceptible to a certain disease is known as natural immunity, while that which follows an attack of a disease is said to be acquired.

Natural immunity is much more common than is susceptibility to germ diseases. Probably the bacteria cause disease in only a small percentage of the individuals which they enter. It is known that germs of pneumonia and other diseases are often present in persons who show no signs of disease. The reason why we do not develop disease every time a pathogenic organism enters our bodies is due to (a) destruction of bacteria by the white cells of the blood and lymph, (b) killing of bacteria by opposing soluble substances in the blood, (c) prevention of growth of bacteria by antiseptic conditions in the body, and $(d)$ counteracting of toxins of the bacteria by antitoxins secreted by the cells of our bodies. The relative value of these four methods of protection varies with health and with individuals. In general, all four are most efficient when there is good health, and hence hygiene by improving general health helps the human body in opposing pathogenic organisms. A good illustration of this is the fact that building up the general health is so all-important in the cure, as well as in the prevention, of consumption or tuberculosis.

Vaccination is an example of acquired immunity produced without an attack of smallpox, but by substitution of a similar and harmless disease known as cow-pox. . In fact, cowpox seems to be smallpox which has been weakened by developing in cows; and so when vaccine matter is taken from the pustules on cows and rubbed into a cut or scratch on the human skin, the result is a mild development of cow-pox. 
This causes the human tissues to produce some opposing substance which effectually prevents the disease smallpox for many years, the length of immunity varying with individuals.

No scientific man questions that vaccination against smallpox has been one of the means (isolation and disinfection are others) which has made smallpox one of the rarest diseases; and that it should be practiced whenever, in a particular locality, there are cases which may have spread infection widely. For example, when a case appears in a school, all teachers and pupils in that school should be immediately protected by vaccination performed by competent doctors.

Protective Inoculation. - Similar to vaccination is protectively inoculating by giving weakened doses of toxins. Pasteur discovered that if the bacteria of cattle anthrax, or those of chicken cholera, be grown in pure cultures in testtubes, the toxins get weaker as the cultures grow older. In other ways, also, it is possible to weaken toxins of bacteria. Now, if weakened toxins are injected first, there is a mild attack of the disease. Then a stronger toxin will produce no more effect; and using in succession in a series of days or weeks, stronger and stronger toxins, the animal into which they are injected finally becomes unable to take the disease and is said to be protectively inoculated. Many recent experiments on thousands of soldiers seem to prove that it will be possible to inoculate against typhoid in a similar way. The destructive cholera of pigs and the distemper which annually kills thousands of dogs are being studied in an attempt to find a successful way of protectively inoculating.

Hydrophobia. - The treatment for this disease is another well-known example of protective inoculation. Persons bitten by dogs believed to have rabies go to a Pasteur Institute, and receive frequent injections of weakened toxins obtained from the spinal cords of rabbits which have died with the disease. The result of these weakened doses, which 
are increased in strength with each successive injection, is that the person injected acquires immunity; that is, he probably develops an anti-toxin faster than the toxins introduced by the saliva of the rabid dog can act. At present no other method of dealing with this terrible disease is known, and hence it is important that protective inoculation be applied as soon as possible. But much common sense is needed in dealing with dog bites. There is no foundation for the idea that dogs are liable to go " mad " in " dog days." Most dogs which bite or appear sick are not rabid. Hydrophobia is a rare disease. One who is bitten by a dog should have the wound properly treated by a surgeon, for bloodpoisoning bacteria may get into any deep wound. If the dog shows signs of illness, it should be kept in confinement for some days; for if it is rabid, the disease will develop rapidly. If the dog has been killed, its body should be expressed, packed in ice, to the nearest Pasteur Institute or health laboratory. Microscopic examination of the nervous organs will show whether the dog had hydrophobia, and it can be quickly determined whether protective inoculation should be given to the person who was bitten.

References: Limited space has made it necessary to confine this chapter to statements of general principles regarding bacteria. Some practical notes on bacteria and human health are given in the next part of this book ( $\$ \$ 48-490)$. Sternberg's "Infection and Immunity" is a good popular account of bacteria and disease. Jordan's "General Bacteriology" is an excellent semi-technical book. 
19.

-

a.

$3243-25=250$

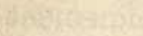

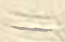

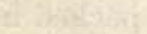

- 


\section{PART III}

\section{PRINCIPLES OF BIOLOGY ILLUSTRATED BY TYPES OF ANIMALS}

WE have seen in Part II how types of plants illustrate the principles of biology which were introduced by study of the bean plant, and also give us a wider acquaintance with representatives of the various groups of plants which, while widely different in form, are remarkably similar in their fundamental life-activities. We shall now make a series of parallel studies on the animal side of biology. In studying plants we began with the bean, which belongs to the highest plants; and then in succession we passed down the scale of plant life to conifers, ferns, mosses, algæ, fungi, and the simplest plants. In our study of types of animals it will be interesting to reverse this order, and instead of going gradually down to the simplest animals, we shall pass directly from the frog to the one-celled animals, and afterwards examine a number of types of animals representing the gradually increasing complexity of animal structure up to the highest vertebrates. 


\section{CHAPTER X}

\section{THE SIMPLEST ANIMALS: THE PROTOZOA}

266. One-Celled Animals. - The simplest animals consist of one cell; that is, they consist of a small mass of protoplasm with a nucleus. Within this one cell must be carried on all the processes connected with the fundamental lifeactivities (feeding, breathing, excreting, reproducing), for each of which processes an animal like a frog has special organs with thousands of cells. In order to understand how an animal with one cell can carry on the same life-activities as does an animal with thousands of cells, it is necessary to study some examples of the simplest animals.

267. Paramecium. - ( $D$ or $L)$ If some chopped hay be placed in water in a fruit-jar or other convenient vessel, and then to this be added some decaying sticks, leaves, and other objects taken from a pond where aquatic plants are growing, there will probably develop within a few weeks large numbers of transparent animals appearing to the naked eye as minute whitish specks. With a rubber-bulbed pipette take a drop of water from the surface and near the edge of the vessel, and place on an object-slide. Then place a few shreds of cotton on the slide, and put on the cover-glass.

Place the slide on a piece of black eloth or paper, and notice the moving white specks. Use a hand-lens. Now examine with a microscope, using first the low-power objective. Look for rapidly moving objects having the form shown in Fig. 88, $A$. These are specimens of paramecium, sometimes called the "slipper-animalcule." In size, they are about ${ }_{1 \frac{1}{0} \sigma}$ inch long. Several other similar animals are of ten abundant in the same water.

Study the following points: form of body, movements, response to stimuli (e.g., when the animals bump against obstructions). Is there evidence that one end is anterior (i.e., goes forward in locomotion)? 
Mount and examine another slide with some paramecia which have been swimming for a half-hour in carmine-water (powdered carmine mixed with water, or lamp-black in water). The particles of carmine appear to be in droplets of water in the protoplasm of the animal. Notice the action of the lashing cilia upon the particles

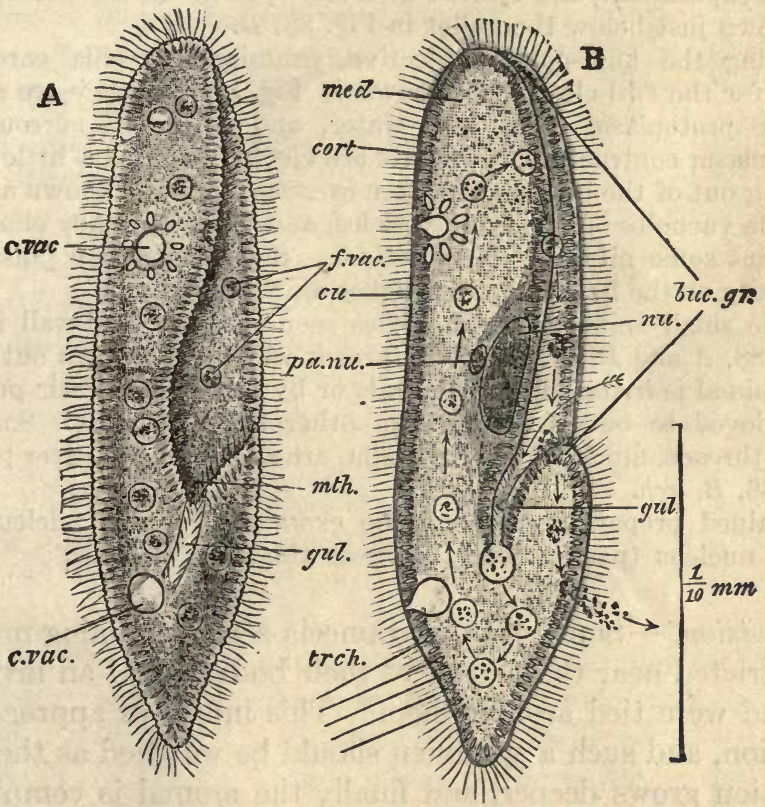

FIg. 88. Paramecium. $A$, from lower surface. $B$, in optical section. c.vac, contractile vacuoles; f.vac, food vacuole; $n u$, nucleus; buc.gr, buccal groove; gul, gullet; $m$ th, mouth; cu, cuticle; cort, cortex, outer layer of protoplasm; med, inner layer. (From Parker.)

of carmine surrounding the animal, and especially the collection of particles in the groove on one side of the body and then their entrance into the body through a tube or gullet (see arrows in Fig. 88, $B$ ). This taking of carmine illustrates how the animal gets food, chiefly bacteria and other small plants or animals. Notice that there is no stomach or other eavity into which food is taken, but simply particles of food surrounded by a film of water are foreed into the protoplasm, which is soft and semi-liquid. These food 
masses (called food-vacuoles) move around as indicated by the arrows in the figure; digestive juices in the paramecium slowly dissolve the digestible food; the protoplasm absorbs the dissolved foods, and the indigestible remains (such as hard parts of some small animals and plants often eaten, or the carmine particles in above experiment) are ejected at a soft spot in the cell-membrane, as shown just below the gullet in Fig. $88, B$.

Using the high-power objective, examine the cilia carefully. Look for the two clear spots shown in Fig. 88, $A$; they are spaces in the protoplasm filled with water, and when the surrounding protoplasm contracts the contents are ejected through a little canal leading out of the body. They are excretory organs, known as contractile vacuoles or pulsating vesicles, and the water they eliminate contains some nitrogenous excretions. Sometimes they pulsate as regularly as the heart beats in higher animals.

The small spindle-shaped bodies seen near the cell-wall in the Figs. 88, $A$ and $B$, contain long threads which are thrown out when the animal is irritated by chemicals or by pressure. Their purpose is believed to be defense against other lower animals. Some of these threads, much longer than cilia, are shown in the lower part of Fig. $88, B$, trch.

Stained preparations should be examined for the nucleus. A small nucleus (paranucleus) lies near the main nucleus.

Division. - Some of the paramecia seen swimming may be constricted near the middle of their bodies, as if an invisible thread were tied around them. This indicates approaching division, and such a specimen should be watched as the constriction grows deeper, and finally the animal is completely divided into two new and young individuals which swim independently. The parent animal merges its own individuality into that of its two equal offspring. Contrast this with higher forms, as the frog, which produce young, while the parent continues to live until it grows old and dies. It is evident that, barring accidents and disease, there is no chance for a paramecium to grow old and die; for when it grows old, it simply divides into two young animals, which in turn take food, form new protoplasm, grow to the full size, and divide. This is the characteristic method of reproduction 
among one-celled animals. It is simple cell-division, but the two new cells separate, instead of remaining together as in the tissues of higher plants and animals. This is asexual reproduction.

Conjugation. - Under certain conditions, not yet entirely understood, paramecia reach a state when they are unable to continue to divide. Two such individuals come into contact, and through their delicate cell-walls some of the nucleus of each one passes over to join the nucleus of the other, the result being in each individual a new nucleus, half of which has come from another paramecium. Then the two animals swim away independently, each soon divides, and their offspring may continue to divide

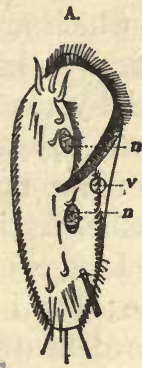

Fra. 89. Division or reproduction of a Stylonichia similar to that of Paramecium. $n$, nucleus; $v$, vacuole. (From Hatschek.) for a long series of generations before this exchange of nuclear substance again takes place.

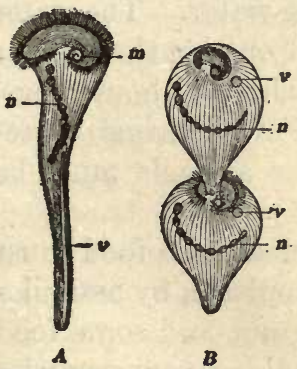

Frg. 90. Division of a Stentor (trumpet-animalcule). $n$, bead-like nucleus; $v$, vacuole. (From Hatschek.)
This process is known as conjugation and in essentials is similar to conjugation in molds ( $\$ 244)$ and Spirogyra ( $\$ 239$ ), and is believed to have the same effect on heredity as has fertilization in higher animals (e.g., frog, $\S 58$ ). In short, the conjugation of two paramecia is the simplest form of fertilization known among animals, just as that of Sphærella, molds, and Spirogyra is the simplest in plants. Other kinds of one-celled animals are known to undergo conjugation.

268. Physiology of Paramecium. We are now ready to survey the life-activities of Paramecium. 
Foods. - Like every other animal, it requires foods which have been made, directly or indirectly, by plants. These foods are taken in through its mouth and gullet, are digested by enzymes which are believed to be similar to those which cause digestion in higher animals, and the digested foods are absorbed by the protoplasm. Finally, the indigestible substances are ejected from the body. And for all these processes there are no special organs like stomach, intestine, liver, and pancreas in a frog.

Oxygen. - Like every other animal, it requires oxygen. It must breathe; but it has no lungs, as have higher animals. However, its delicate cell-wall allows the absorption of an abundance of oxygen from the surrounding water. Paramecia soon die in water from which the oxygen has been removed.

Oxidation and Excretion: - Like every other animal, a paramecium's food is constantly being oxidized to furnish energy to run the living machine $(\S 42)$, and this union of oxygen and foods forms excretions. As in a frog, these are chiefly water, carbon dioxide, and nitrogenous excretion. The carbon dioxide is absorbed by the surrounding water. The water and the nitrogenous excretion is pumped out by the regular pulsations of the contractile vacuoles. Thus, without lungs, kidneys, or skin, a paramecium gets rid of the same excretions which in the frog and other higher animals must be eliminated by these organs.

Assimilation. - As in every other animal, some food must be used continually for making new protoplasm, by assimilation. Protoplasm is continually wearing out and some food (especially proteins) must be used for making new particles of protoplasm to replace those worn out or destroyed. Also, the animals are frequently dividing, and every new individual is half the usual size and must make enough new protoplasm to double its size before it can reproduce. Hence, much food must be used to make new protoplasm for growth $(\$ 42)$. 
No Circulation. - Especially is it noteworthy that, unlike the frog and other higher animals, a paramecium carries on its life-activities without organs for circulation (heart and blood-vessels). Turn back and review the reasons why a frog needs these organs ( $\$ 52$ ), and it will be evident that none of the reasons given applies to a paramecium, for that animal is so small that digested food, oxygen, and excretions do not require transportation to and from distant parts of the body. It is only a short distance from the surface of the body to the innermost particles, and so oxygen and carbon dioxide can diffuse as easily as they go through the wall of a bloodcapillary in a frog. Also, the movement of the inner layer of protoplasm in a paramecium helps distribute food while it is being digested.

Irritability. - A paramecium shows no nerves or other structures which appear to be substitutes for the nervous system in a frog; nevertheless the animal responds to stimuli caused by chemicals, touch, light, and electricity. It has no nervous organs, but it has irritability (response to stimuli) in a simple form.

Motion. - A paramecium has movement, and yet there are no visible muscles. Instead, the protoplasm of its entire body seems to have the power of movement or contractility. This is especially developed in the protoplasm composing the cilia.

Reproduction. - And finally a paramecium has the power of reproducing, without which its species would soon cease to exist. Since it consists of a single cell, its only possible way of producing new individuals is by cell-division a simple process of asexual reproduction. And to provide for frequent mixing of protoplasm from two individuals, which is of great significance in heredity, Paramecium also has a simple process of fertilization or conjugation.

Summarizing, we find within a single cell of this simple animal all the life-processes which we find in higher animals; 
namely, movement, digestion, absorption, respiration, assimilation, oxidation, excretion, irritability (simple nervous activity), and reproduction. All these life-processes are within one cell in a paramecium, and require tens of thousands of cells in an elephant. But there is a difference in that the paramecium cannot perform any process as well as can a higher animal. In fact, to a large extent a paramecium is doing just what every individual cell in any higher animal is continually doing in carrying on its own life-processes.

269. Physiological Division of Labor. - The fact that the cells in higher animals are specialized to do certain work (e.g., muscle cells to contract or cause movement, stomachcells to digest, kidney cells to excrete, nerve cells for coordination, etc.), is known as physiological division of labor. The advantage of such specialization is shown by an analogy from human society. Each pioneer in the country regions in America had to be his own baker, miller, carpenter, blacksmith, cobbler, etc., because special workers in these lines were not near. But such a man never became expert in any of these lines ; he was " a Jack of all trades, master of none." Nowadays in civilized communities a physiological division of labor has led men to become specialists and learn to do one thing excellently. But there must be a coördination or a working together. One man may specialize as a carpenter; but he must depend upon other men to be his cobbler, grocer, baker, farmer, and so on through a long list of people who must do things for the one who confines his work to one special line. In our great cities we do not often stop to think of this physiological division of labor which has grown up in. our complex human society; but if all the grocers were to close their shops, or as has actually happened, the railroad engineers should stop work and leave us without supplies, then we could realize the complex way in which we have become dependent upon other workers in special lines of work.

All this from human society illustrates the division of labor 
in higher animals. For example, muscle cells have specialized for movement; but for them other cells must do the digesting, absorb the oxygen, discharge the excretions, etc. In short, the cells of every organ depend upon the cells of all the other organs ; and all must work together harmoniously, because there is mutual interdependence. And just as a man specializing in one business becomes expert, so a cell specializing in movement, secreting, or in any other one necessary function can do that work better than it is done in a paramecium, for that animal has so many things to do in its one cell that it does none of them as well as they are done by the specialized cells in higher forms of life.

270. Amœba. - In many ways the most interesting of one-celled animals is one known to biologists as amœba, which lives on the surface of submerged objects in ponds and other bodies of water.

$(D)$ If one scrapes the ooze from the lower surface of lily leaves, and from various other aquatic leaves and stems, or collects some of the green felt-like growth which often forms a coating on the mud in the bottom of shallow stagnant ponds or streams, it is probable that some amoebas will be obtained. But they are rarely as abundant as paramecia, and so it is usually necessary to spread the collected material in soup-plates or other shallow dishes and allow a few days in which the amobas may crawl to the surface, and perhaps also increase in numbers. From time to time eollect, with a rubberbulbed pipette, some of the scum, especially near the edge of the dish, spread on glass slides, and with a low-power objective look for little specks having the appearance of ground glass, and irregular in form, as represented in Fig. 91. Sometimes it is necessary to examine dozens of slides with material from different parts of the dish before an amœba is located. It is only in this way that amobas can be found, for they are rarely larger than $\frac{1}{100}$ inch in diameter and usually very much smaller.

Having located a good specimen, look at the cover-glass from the side of the objective and estimate the approximate position of the animal, so that if the slide is moved, it will be easy to find the place again. Then carefully adjust a higher power objective, and study according to the following description. 
The body of an amœba consists of protoplasm, imbedded in which are many food particles that have been eaten and not yet digested. The outermost protoplasm is perfectly transparent and colorless, while the central portion is very granular and resembles ground or frosted glass. The microscope and drawings give the impression that the animal is a thin sheet of protoplasm, spread out like a liquid poured on

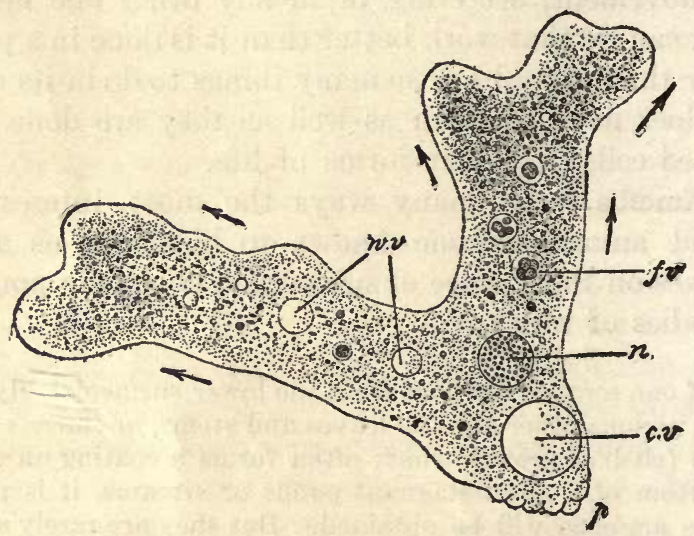

Fig. 91. Amoba. Arrows show direction of flowing of the protoplasm $n$, nucleus ; $f v$, food vacuole ; $w v$, water vacuole ; $c v$, contractile vacuole; $p$, temporary posterior end. (From Wilson.)

a flat plate; but when the animal is disturbed (as by jarring) it rolls up into a rounded mass.

Movement. - The most noticeable thing about an active amœba is that it is continually changing its shape. Close observation will show that this is due to the fact that the animal is composed of semi-fluid protoplasm which is constantly flowing. This movement of the protoplasm is not like the flowing of water down an incline, but is due to contractions originating within the amœba itself. It is possible for the animal to crawl up the stem of a water plant, and this could not be explained by the laws of gravitation. 
When the animal is actively moving, long arm-like projections, called pseudopods (false feet), are formed, and then granules from the main body continue streaming in the same direction. In fact, the motion reminds one of the flowing of lifeless liquids, and we can imitate it by pouring some thick liquid (molasses, melted gelatin, or mucilage) on a plate, and then by tilting cause the liquid to flow in various directions. Notice that when a streamlet starts in one direction, the liquid all tends to flow in the same direction.

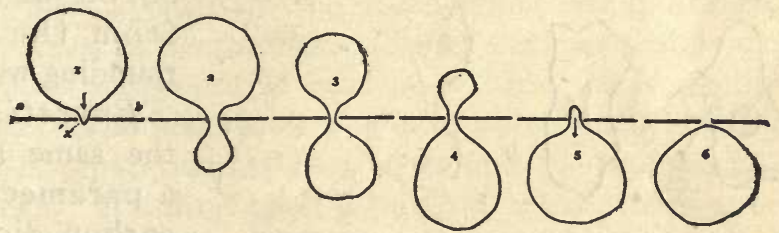

Fig. 92. Diagrams 1-6 show how an amœba, or a similar white cell from blood of higher animals, passes through very narrow openings.

Sometimes a nucleus can be seen in a living amœba, but it is most evident in a stained preparation.

In large specimens it is easy to see the one contractile vacuole, which acts essentially like those seen in a paramecium, and has the same function of excreting water containing nitrogenous waste.

Food. - The taking of food can sometimes be observed when an amœba is moving actively. If it comes in contact with a small animal or plant, two pseudopodia flow out and gradually surround and inclose the food particle in the protoplasm of the amœba. A small amount of water surrounds the food particle, just as in a paramecium. Sometimes an amœba is seen so filled with food particles that the body substance is opaque. The protoplasm of amœbas secretes digestive enzymes which dissolve the proteins, etc., in the animals and plants which are captured. The digested foods are then absorbed by the surrounding particles of protoplasm, 
and when the amœba moves the particles being digested are circulated so that dissolved food is widely distributed. Indigestible particles (hard parts of small animals and cell-walls of plants) are from time to time ejected; the protoplasm of the amœba appears to flow away from them as water on a board might for a time surround and inclose some grains of sand and then flow away and leave them behind.

Respiration. - Amœba breathes like a paramecium; that
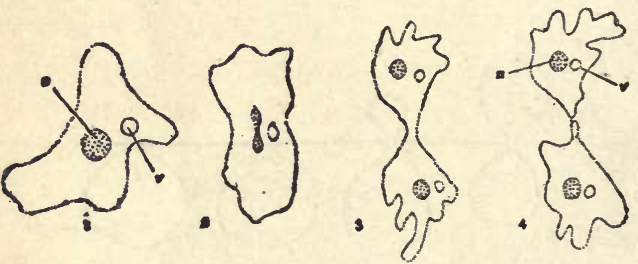

is, simply ab-

Fig. 93. Diagrams showing division of an amoba into two. $n$, nucleus ; $v$, vacuole.

sorbs oxygen from the surrounding water. Excretion is the same as in a paramecium; carbon dioxide is absorbed by the surrounding water, and water and nitrogenous excretions are pumped out by the contractile vacuole.

Reproduction.-Amœbas are rarely seen dividing, but if kept in watch-crystals they multiply rapidly, making it evident that they must undergo division frequently. Figure 93 shows the process of division. Conjugation of two amœbas similar to that of the paramecium ( $\$ 267)$ has been seen. Under conditions unfavorable to the usual activity, amœbas will sometimes become rounded, and secrete around themselves a hard wall or cyst. They are said to be encysted. In this condition they are able to live for some time without food and in a dry state. Such a habit insures their existence in dry seasons when the ponds are dry. Also, in the dry condition they may be widely scattered by winds.

271. Simple Life of Amœba. - Compared with the paramecium, the amcba is exceedingly simple in structure and functions. The paramecium has a constant and definite 
form, a firm but delicate cuticle over the whole surface, special organs of locomotion (the cilia), special organs for defense (the trichocysts), a definite organ for receiving food (groove and gullet), and differentiation into anterior and posterior ends. Since none of these is found in amceba, paramecium is obviously a much higher type of animal. The amœba is the simplest animal known, and we cannot imagine how it could be made simpler except by the absence of a contractile vacuole. Such an amœba has been described, in which apparently the surrounding water absorbs such excretions as the vacuoles of other amœebas pump out.

Amœba appears to have no cell-wall or bounding membrane. The line visible where the water touches it is simply a line of separation between water and the protoplasm of the animal. If a drop of oil is put into water, there appears to be a line where the oil and water meet; but there is no special membrane there, simply a surface film between the oil and water. Likewise, we see insects striding on the surface of water, and it, is easy to make a fine sewing needle float on the surface of water; but no one thinks of the water as covered with a specially differentiated membrane.

In short, an amcba is a mass of simple protoplasm, very similar to the inside material in a paramecium, and to that inside the cells of higher animals. The animal has long been a favorite with biologists because it shows the appearance of protoplasm, which cannot be seen living in the opaque cells of higher animals.

272. Economic Relations of Amœbas. - There are many species of amcbas, and most of them are not known to be of any special economic interest; but one species common in tropical countries produces a serious form of intestinal disease known as dysentery.

273. Allies of Amœba and Paramecium: Protozoa. These animals are members of the lowest division of the animal kingdom, a group of animals which are characterized 
by the fact that their bodies consist of one cell. Any onecelled animal is a protozoan (meaning first animal), and the entire group of them is named Protozoa. There are numerous species, but most of those known are like the amœba and paramecium in being of little interest except that study of them has helped much towards explaining the life of higher organisms, including man himself. However, a few protozoans are now known to be of very great practical interest, and some of these will be briefly described.

274. Malarial Organism. - Malaria in its severest forms has long been one of the worst diseases affecting the human race. Vast territories in some parts of the world have been left practically undeveloped by civilized men because of malaria. Little was known as to the cause of the disease until after 1880 , in which year a protozoan parasite was discovered in red blood-cells. Before that time it was commonly supposed to be caused by some poisonous vapor or miasm which arose from swampy land. In 1897, Ross, an officer of the British army, demonstrated that the malarial parasites first develop in the stomachs of certain mosquitoes ( $\$ 329$ ), from blood sucked from persons who have malaria; and a year later other investigators showed that mosquitoes which have obtained blood from a malarious patient are able to transmit the parasites while sucking blood from perfectly healthy persons. Many later studies have made it absolutely certain that the Anopheles mosquito is the carrier of the disease; and this is one reason for the recent attempts at exterminating mosquitoes as far as possible.

The effect of the malarial parasite upon the red bloodcorpuscles is as follows: Small bodies injected into the blood by sucking mosquitoes attach themselves to blood-cells and begin to burrow (they have amœboid (amœba-like) movements). Within the blood-cell a parasite grows, at the expense of the cell, and soon divides into a number (6-24) of small bodies called spores. Then the blood-cell disintegrates 
and frees the spores, which fasten themselves to new bloodcells and repeat the development. Each time the spores are freed by breaking of the blood-cells the patient has the chill and fever which are characteristic of the disease. It is well known that malarial attacks commonly occur on alternate days, that is, every forty-eight hours. This means that a common form of malarial parasite requires two days for development in a blood-cell. Another variety of malarial parasite takes three days for development into spores, and so the chill of the patient comes at intervals of three days. Still another form of malaria, most common in the tropics, and exceedingly difficult to cure, may appear daily; and the parasites take twenty-four hours for their development in blood-cells.

All these oft-repeated cycles of development in human blood-cells are simply growth and cell-division, typical asexual reproduction. When human blood is sucked into the stomach of a mosquito, pairs of certain spores unite (a true case of fertilization) and the zygote (combined cell) penetrates the stomach-wall of the mosquito and becomes encysted. Sometimes there are several hundred such cysts in the stomach of a single mosquito. Each of these encysted parasites eventually divides into thousands of slender threadlike bodies (sporozoites), which by way of the lymph-tubes get scattered in all parts of the mosquito's body, especially in the poison (salivary) glands. It is these sporozoites which are carried along with the poison when a mosquito plunges its beak into human blood-vessels; and each sporozoite enters a red blood-cell, where it divides into 6 to 24 spores.

The full development in a mosquito's body need not take over ten days in warm weather.

When once started in human blood the repeated cycles may go on for a long time, or spores remain which may long afterwards start a new attack of disease. This can be prevented by the use of quinine, which kills the parasites, especially 
if taken in large doses just as the fever is subsiding, which is while the young spores are free in the blood-plasma. Killing the parasites in human blood prevents infection of mosquitoes; and if all people could be treated with quinine systematically, it is probable that the disease could be stamped out. This has been tried under the direction of Dr. Koch with great success in some small islands. Preventing the spread of the malarial parasite by preventing the breeding of mosquitoes and by protection against bites will be discussed under insects and disease $(\$ 329)$. In the ten years since it was found that mosquitoes are responsible for the spread of malaria, a great effort has been made to exterminate the insects in many localities, with the result that there has been a decided decrease of malaria wherever the mosquitoes have become rare. Protection against bites by sleeping in screened houses and by wearing mosquito-proof clothing and a veil when outdoors at night has greatly reduced the number of cases of malaria among the employees of certain railroad systems along the Mediterranean shore.

275. The Parasite of Sleeping Sickness. - The terrible South African disease known as the sleeping sickness, from which more than half a million natives in the Congo region have perished in the last ten years, is now known to be due to a parasitic protozoan, which is injected into human bloodplasma by the bite of a peculiar African fly. The parasite swims freely in blood-plasma, its movement being due to long whip-like structures, called flagella. It belongs to a group of Protozoa known as the trypanosomes. Many experts on parasitic diseases are now engaged in studying the sleeping sickness; but so far no satisfactory remedy has been discovered. It is spreading rapidly, and has become one of the greatest problems in Africa.

A similar parasite, injected by a similar fly, causes the disease of horses, mules, and oxen called "nagana" by the natives, and by them correctly charged to the tsetse-fly. 
Those who have read the "Travels" of Livingstone and other African explorers will recall how their journeys were so often delayed by death of their oxen. The disease also caused much trouble among horses during the South African war a few years ago. The fly does not develop the parasite, as mosquitoes do in the case of the malarial organism, but simply transmits the parasites from diseased to healthy animals. It is believed that the blood of some large game animals may infect the flies.

The surra disease, which destroys large numbers of horses, camels, and cattle in India and the Philippines, is due to a similar parasite transmitted by bites of flies.

276. Other Protozoan Diseases. - Dangerous diseases, caused by protozoan parasites which are introduced into the blood by the bites of ticks ( $\$ 319$ ), affect cattle, dogs, horses, and sheep. The parasites enter the red blood-cells. Texas fever is one form of the cattle disease, and it has caused enormous financial losses to stockmen in the United States and elsewhere.

A common disease of the liver in rabbits, diseases which seriously affect fish, the silkworm disease made famous by Pasteur's studies, - these are some of the more important cases in which parasitic one-celled animals have great economic importance.

There are some suggestions that yellow fever, scarlet fever, smallpox, and hydrophobia are caused by protozoan parasites similar to those which cause malaria ; but there is yet no convincing proof. In the case of yellow fever, it has been proved that a mosquito (not the species concerned with malaria) transmits the unknown germ of the disease; and so at present the only known way of checking the spread of yellow fever is to check the mosquitoes. This has been very successful in Cuba and in Panama.

Fortunately, most of the protozoans which live in water in lakes, rivers, and sea are unable to live in the bodies of animals, and hence are entirely harmless. 
277. Useful Protozoa. - The emphasis upon certain protozoans as causes of disease tends to leave the false impression that all protozoa are harmful, or at least useless.

A large number of common protozoans are important in the food-supply of somewhat larger animals, these in turn of still larger animals, and so on to aquatic animals such as fishes.

Thus even the minute bacteria eaten by some protozoans may indirectly, through a series of animals of increasing size, finally come to be of use in the food-supply of man himself. We cannot accurately estimate the relation of the smallest animals in the food-supply of larger animals ; but vast numbers of animals certainly depend directly and indirectly upon protozoans as food.

Many of the protozoans are important as scavengers, assisting bacteria in breaking up dead organic bodies ( $\$ \$ 116$, 117) and preparing the organic material for use again in the cycle of organic matter. For example, a protozoan may eat some particles from the body of a dead animal or plant, build these particles into its own protoplasm, which may later serve as protein food for some larger animal; or these particles may soon be oxidized to excretions which may serve as materials for food of plants.

Another example of the usefulness of protozoans is that a large amount of chalk in the great deposits in England and elsewhere is composed of the shells of certain protozoans. In some places these shells are very abundant in the mud of the ocean bottom.

278. Colonial Protozoa. - Amœba and Paramecium represent the common kinds of protozoans in that all individuals live free and independently of each other. We shall examine briefly groups or colonies of certain protozoans.

One of the most common colonial protozoans is a tree-like form. It consists of a delicate and much-branched stalk, which is attached to some object, and at the end of each branch is a one-celled animal of bell-like form (similar to Fig. 
$94, b)$. Some of these individuals can take food (by the action of cilia which drive food particles into the mouth, as in a paramecium), and can divide to form new individuals on new branches. Some of the individuals do not feed while attached to the stalk of the colony, but digested food comes to them through the hollow stalk from others which do take and digest food. These specialized non-feeding individuals break loose, swim freely for a time, attach themselves to some object, develop a mouth, and soon divide to form two new individuals. Then division is repeated again and again and finally results in a new tree-like colony.

Note that in such a colony there is a slight division of labor in that some of the individuals are nutritive, i.e., adapted to feeding and digesting

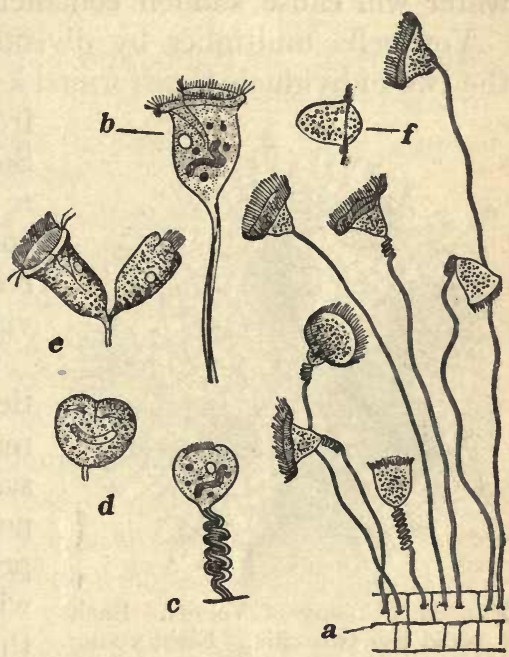

Fra. 94. Vorticella, the bell-animalcule. $a$, group of seven vorticellas attached to the surface cells of a water-plant. The contractile stalk is thrown into a spiral when shortened (see $a$ and $c$ ). $b$, a single individual ; $d, e$, division ; $f$, a free-swimming individual formed by division as in d,e. (From Wilson, and Parker.) for the benefit of the whole colony ; and others are specialized for reproducing new colonies.

Vorticella. - These are similar protozoans, the individuals of which live separately. An example is the beautiful bellanimalcule (Vorticella), several of which are shown in Fig. $94, a$. The food-collecting groove is at the wide end of the bell-shaped body, and a circle of cilia whips particles around the margin and down into a gullet. The stalk contains a cen- 
tral, highly contractile fiber (the simplest muscle known), which frequently shortens (contracts) and coils the stalk into a close spiral (Fig. 94, c). The slightest jar of the microscope, the touch of another organism, or even a current of water will cause sudden contraction.

Vorticella multiplies by division (Fig. 94, $d, e$ ). One of the two individuals thus formed leaves the stalk and becomes

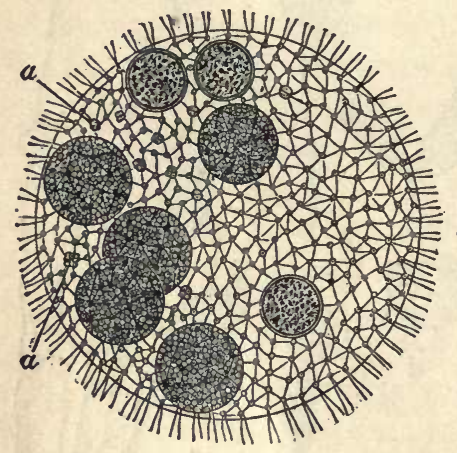

Fig. 95. Colony of Volvox. Each zoöid has two cilia. Eight young colonies are shown in the interior of the hollow sphere. $a$, colonies just beginning to form from certain zoöids at the surface. (From Parker.) free swimming (Fig. 94, f). Soon it settles down, grows a stalk of its own, and becomes a full-grown vorticella, ready to reproduce by division.

If we imagine that the vorticellas formed by division remain on branches of the same stalk, then division repeated many times would produce a tree-like colony with individual animals in the same positions as are the terminal buds on branches of plants.

279. Volvox: Colonial Animal or Plant? - The most remarkable of colonial organisms is the beautiful Volvox (Fig. 95), which lives in ponds of fresh water. The colonies, which are visible to the unaided eye, are hollow spheres of a transparent gelatinous material in which are set numerous individuals (single cells), each with two flagella. Each individual cell has a nucleus, a chlorophyll-body, and a red spot. The combined lashing of all the flagella causes the colony to roll through the water; hence the name Volvox was made from a Latin word meaning to roll.

The eight dark spheres represented in the figure are new 
colonies forming inside the old colony. Each of the new colonies is started by one cell (individual) moving inward and dividing up into a large number of cells, which form a new colony. Finally, the outer sphere breaks and releases the new colonies.

Sometimes Volvox has sexual reproduction. Certain cells enlarge and become egg-cells. Some other cells form spermcells. Each fertilized egg-cell divides into a large number of cells which form a new colony.

Biologists are disagreed as to whether Volvox is an animal or a plant. It is commonly described in textbooks of both botany and zoölogy. The truth is that it is one of many small organisms - some living singly and some in colonies which so combine characters of both animals and plants that they may be said to be near or on the boundary line between the animal and plant kingdoms. Volvox behaves like an animal, but its nutrition is that of a green plant. In fact, each cell of a Volvox colony is comparable to a single Sphærella ( $\$ 238)$. It is probably a colony of one-celled plants, one of the Algæ.

However, whether Volvox is animal or plant is a point of little importance, for it is an organism illustrating colonial life of one-celled living things so well that it deserves to be studied in connection with both botany and zoölogy.

Volvox, like the colonial bell-animalcules, has some cells for nutrition, and some specialized for reproduction. In the next animals to be studied we shall find this division of labor more fully developed.

\section{Classes of Protozoa (One-Celled Animals)}

Sarcodina - simple masses of protoplasm, with pseudopodia. Amœba.

Sporozoa - spore-animals; producing spores. Malaria germs.

Mastigophora - with flagella for swimming. Volvox.

Infusoria - with cilia for swimming and collecting food. Paramecium, Vorticella. 


\section{CHAPTER XI}

\section{THE SIMPLEST MANY-CELLED ANIMALS : PORIFERA AND CELENTERATA}

280. Many-Celled Animals : Metazoa. - All animals higher than the Protozoa consist of many cells. There is no animal with simply two cells or four cells in the adult condition, although all many-celled animals in developing from eggs must pass through two-cell and four-cell stages, as does the frog $(\$ 59)$.

All the many-celled animals taken together are termed Metazoa. This is simply a convenient short term in place of the phrase "animals with many cells." Familiar representatives of the various types of metazoa are sponge-animals, coral-animals and jelly-fishes, worms, lobster and insects, clam and snail, starfish, and frog. These are some members of the larger divisions (phyla) of the animal kingdom. See table in $\S 133$.

All the many-celled animals exhibit more or less physiological division of labor ( $\$ 269)$. In some of those which we shall study first there are some groups of cells assigned to certain functions, but no such separation into special organs as we found in the frog. The most distinguishing feature of the life-activities of the animals described in this chapter is that, although each one of them may have hundreds or thousands of cells in its body, they are able to live without blood or similar circulating medium. We shall see that this is due to their simple plan of structure.

\section{PORIFERA}

281. Sponge-animals. - Simplest of the Metazoa are the animals which produce the fibrous structures known as sponges. 
These are the skeletons of colonies of animals, the flesh having been removed when preparing the sponges for market. Besides these common sponges, there are certain spongeanimals which have skeletons made up of glass spicules, and others which form their skeletons of lime (calcareous) spicules. These various materials found in spongeanimals are secreted by living cells, just as the cells of higher animals secrete bone.

The general plan of one of the simplest sponge-animals is shown in Fig. 96. On the outside are numerous pores. There is a central cavity, which has an opening at the top. This central cavity is connected by small tubes (see the figure) with the pores on the surface of the body, and water flows in through these tubes or canals and out at the top of

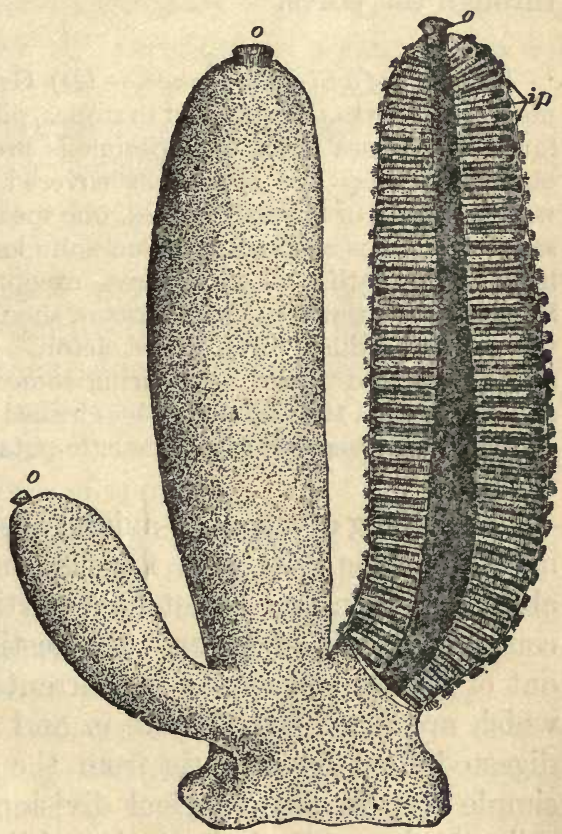

Fic. 96. A simple sponge, the branch at the right cut to show central cavity. o, opening of central cavity; $i p$, inhalent pores. The young branch at left has been formed by budding. (From Parker and Haswell.) the central cavity. As shown in the figure, the canals starting from the external pores do not run quite to the central cavity, and other canals starting from the central cavity extend almost to the surface. Between these inner and outer canals are small openings, so that water entering the outer canal can 
pass through to the inner canal and thence into the central cavity. The inner canal is lined with numerous cells, each of which has a long, whip-like structure (flagellum); and it is the lashing of all the flagella which causes water to flow in through the pores.

Structure of a Simple Sponge. - (D) Grantia is the name of a small marine sponge found attached to stones, piles of wharves, etc. Schools far from the sea must use specimens preserved in alcohol. While observing, keep the specimens covered with alcohol or water in watch-glasses, or in small bottles, one specimen in each bottle. Have some specimens entire, and some split longitudinally. Use a handlens, and identify all structures, except cells, mentioned above. Some cross sections, cut with a razor, should be demonstrated to show the spicules which form the skeleton. That these are calcareous spicules can be shown by pouring some acid on them. The fleshy substance (i.e., the cells) can be cleaned away by boiling a Grantia sponge in a strong solution of caustic potash.

The feeding of a sponge-animal is similar to that of a paramecium. The cells with flagella, in the inner canals, are able to take in and digest food particles brought by the incoming currents of water. Indigestible particles are carried out of the central cavity by currents of water. Those cells which are not fitted to take in and digest food may obtain digested food by osmosis from the other cells. Here is a simple step in physiological division of labor; the outside cells are the protective covering of the body, and some of the inner cells digest food.

The outer cells are collectively known as the ectoderm (meaning outer skin), while the inner layer of cells which line the inner canals and central cavity and do the digesting constitute the endoderm (meaning inner skin).

Reproduction. - Sponges reproduce by outgrowths or buds from the older animals, and when the animals thus formed remain together colonies are formed. Figure 96 shows a colony of three individuals formed by two buds on the 
largest one. Also, some sponges, especially the green ones which live in fresh water, form structures (called gemmules) composed of cells well protected by a hard covering. These gemmules may remain inactive until the following summer, when they grow into new individuals. This kind of reproduction of sponges, like the budding, is asexual; but all sponge-animals also have sexual reproduction. In each animal both ova and sperm-cells are formed from certain inner cells. The ova are usually fertilized by sperm-cells produced and discharged into the water by other individuals and carried into the canals by incoming currents of water. The fertilized eggs develop into rounded embryos covered with cilia and able to swim freely. After a time these settle down on rocks, attach themselves by secreting a cement-like substance, and grow into sponge-animals. Each animal developed from an egg may later by branching, as described above, form a large colony of sponges.

The sponges of commerce (horny sponges) have the same general plan of structure and life as described above for the simplest sponges. The chief difference is that they branch extensively and form immense colonies whose canals and central cavities are united in a complicated network. An ordinary large sponge is the skeleton of a colony of many spongeanimals. Most of the large openings seen on such a sponge are the united central cavities from a number of animals of the colony, and the smaller openings are the united canals for the ingoing of water to many individuals. Each individual animal in such a complicated colony has a structure similar to a simple sponge-animal.

In preparing sponge skeletons for market, the flesh of the masses (colonies) is allowed to decay by the action of bacteria, which have no effect upon the skeletons. These are afterwards cleaned by chemical solutions, which also sterilize them.

Porifera. - Because of the prominence of the pores for the reception and expulsion of water, the sponge-animals are 
known as the Porifera (meaning pore-bearers). This is the lowest group of the many-celled animals. It is a noteworthy fact that the Porifera show much similarity to the colonial protozoa, especially in that food is digested inside of cells, and not in a stomach bounded by cells, as is true in higher animals.

\section{COELENTERATA}

282. Hydra. - Somewhat more differentiated than the sponge-animals, but still exceedingly simple as compared with a backboned animal, are those of the group to which

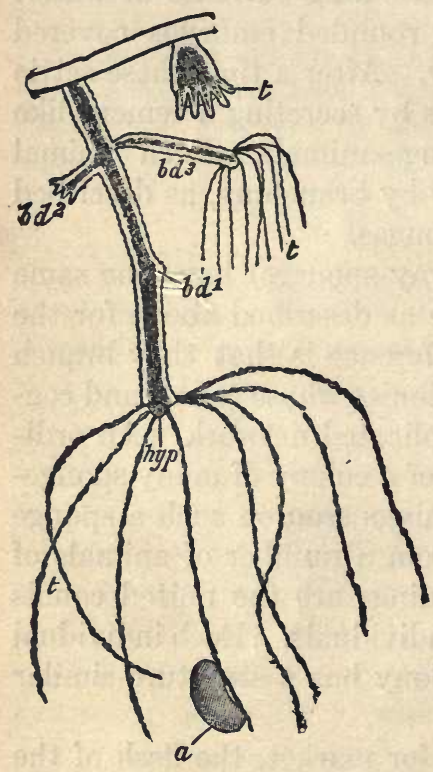

Fig. 97. Two specimens of hydra, one contracted and one expanded. The latter has three buds in stages of develonment. $t$, tentacles; $a$, captured waterflea; hyp, position of mouth. (From Parker.) belong the jelly-fishes and coral-animals. Most of these live in the sea, but among the few species which live in fresh water is the little animal known to naturalists as $\mathrm{Hy}$ dra, and in old books often called "fresh-water polyps." It commonly lives in ponds and streams, where it clings to aquatic plants, dead leaves, and sticks. Such objects should be collected, placed in glass jars with water, and allowed to stand for some days. Many of the hydras will move to the walls of the glass jar.

I. $(L)$ Observe hydras in glass jars (aquaria) near windows. In what part of the aquarium with reference to light and shade are they most abundant? Note the long thread-like arms which are attached at the free end of the animal. Study a hydra which has 
been removed from an aquarium to some water in a watch-glass. Use a hand-lens. What is the shape of the body? Does the shape change? Disturb the animal by touching it with a needle. Describe what happens. How may this reaction be of use to the hydra? Notice that one end becomes attached to the glass; this is the base. Find out if the hydra has a firm hold on the glass dish, e.g., try to wash it off with a gentle jet of water from a pipette. At the free end is a circle of tentacles. How many? These are used for catching the prey. On the summit of a conical elevation in the center of this circle of tentacles is a small opening, the mouth, which is usually very difficult to see. How do the tentacles behave when the hydra is disturbed? Can the tentacles move independently of each other? Is this animal bilaterally symmetrical?

Often young hydras may be seen attached to the bodies of the larger ones, and these are formed by outgrowths or buds (Fig. 97).

Make an outline drawing of a living hydra, showing as many structures mentioned above as possible - (1) fully extended, (2) contracted.

II. (L) Transfer a hydra, with a little water, by means of a clean pipette, to an object-slide. Be careful to support the coverglass (small bits of broken cover-glasses may be used as supports) so as not to crush the animal. Examine with low power of the compound microscope. Notice that there are two layers of the bodywall ; the outer (ectoderm) is colorless, the inner (endoderm) is green or brown. The green is due to the presence of chlorophyll. In the outer clear layer, look for knob-like swellings, especially on the tentacles. These swellings contain the stinging or nettle-threads, which are organs of defense, and also used for spearing and paralyzing water-fleas and other small water animals.

III. (D or L) Study of Sections. - Examine a longitudinal section of hydra with compound microscope and observe:-

The hydra is really a hollow eylinder, the interior of which is the digestive cavity (Fig. 98).

The tentacles are hollow outgrowths of the digestive cavity.

The mouth is the only opening from this cavity to the outside.

The body-wall is composed of layers of cells on both sides of a deeply-stained line in the preparation. The outer layer is the ectoderm; this is the colorless layer seen in the living hydra. The deeply-stained line (middle layer) is not made up of cells, but consists of a gelatinous substance. The inner layer of large cells is the endoderm. It contains the chlorophyll-bodies in green hydras and the brown bodies in brown hydras. 
Look for these three layers in a section of a tentacle. In the ectoderm of the tentacles look for the stinging cells or nettle-cells

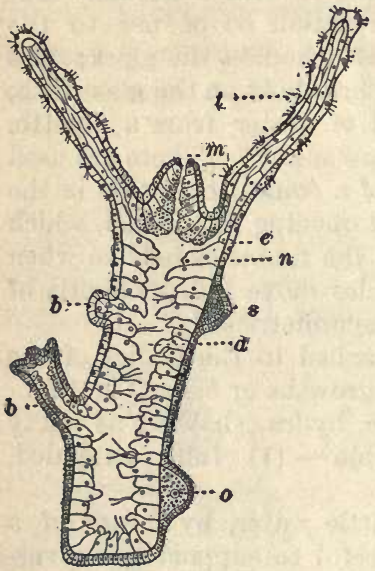

FIG. 98. Longitudinal section of a hydra. $t$, tentacle; $m$, mouth; $e$, ectoderm or outer layer of cells; $n$, endoderm or inner layer; $d$, digestive cavity; $b$, buds; $s$, spermary; o, ovary. (From Parker.)

life-activities of Hydra are slightly more specialized than those of sponge-animals, but they are exceedingly simple as compared with those of an animal like the frog.

Digestion. - Small animals caught by the tentacles and forced into the digestive cavity through the mouth are softened and disintegrated by the action of the digestive juice secreted by the cells of the endoderm which lines the cavity. The digestible particles set free by the disintegrative action of the digestive juice are taken in by endoderm cells, the free ends of which are 
amœba-like and able to take in food in the way that an amœba does. Particles thus taken into the cells are completely digested (dissolved). This is intra-cellular digestion (i.e., within cells), and is the same as the only possible digestion in Protozoa and Porifera. But in Hydra there is a decided advance over the lower animals in that the cells able to digest intra-cellularly are grouped so as to form the lining of a cavity (digestive cavity) into which they pour secretions that cause digestion outside of the cells. This digestion in a cavity, as in the stomachs of all higher animals, is extra-cellular digestion, i.e., occurs outside of cells. The digestive cells in Hydra, then, in part resemble the onecelled animals and the digestive cells of sponge-animals; and in part the lining cells of the stomach of higher animals.

Absorption of Digested Food. - The cells (endoderm) which line the digestive cavity of a hydra can either get food by taking in particles and digesting them inside of cells, or by absorbing foods digested in the cavity. The cells of the outer layer (ectoderm) must get all their food by absorption or osmosis from the endoderm cells. This osmosis of food from cell to cell is as easily accomplished as water soaks through several layers of paper.

Use of food in cells of hydra is the same as in all other animal cells. Some food is used to make new protoplasm for repair and growth, and some serves as a source of energy.

Oxygen is absorbed by all cells in contact with water. This includes the majority of the cells, for even the digestive cavity contains water taken in with the food. But probably the larger part of the oxygen needed is absorbed from the water by ectoderm cells.

No Circulatory Organs. - Although Hydra is multicellular, it is able to live without circulating blood and lymph for the reason that its simple cylindrical body allows digested food and oxygen to reach all cells by osmosis, and all excretions are eliminated by the same process. Compare this with 
the distribution of digested food, oxygen, and excretions in a frog $(\S 52)$.

Excretion is accomplished by osmosis of waste matters from the cells to the surrounding water. Probably the ectoderm cells do the larger share of this work.

Movement is caused partly by contraction of cells and partly by that of muscle-processes which are extensions of some cells in both inner and outer layers.

Irritability is well developed in Hydra. It is in part a function of all the constituent cells; but some cells believed to be simple nerve-cells are near the nettle-cells, which must be extremely sensitive in order to discharge the nettle-threads when stimulated by other small animals.

Reproduction of Hydra is both asexual and sexual. When there is plenty of food and other favorable conditions, asexual budding occurs. A small elevation appears on the side of a hydra, and this soon grows into a small hydra which becomes detached and lives independently. When the animals are growing rapidly, there may be several buds on one animal at the same time (Fig. 97).

Under certain unfavorable conditions, such as lack of food, stagnation of water, etc., hydras form reproductive organs, ovaries and spermaries. These appear as transparent thickenings of the ectoderm, the spermaries near the tentacles, the ovaries near the base of the animal. Any individual may form both kinds of sex-organs; that is, each is both male and female; but usually both kinds do not mature on a given individual at the same time. An animal which can develop both ovaries and spermaries is known as hermaphroditic. Likewise a flower with both stamens and pistil is sometimes called hermaphroditic. Sponges, many worms, many snails, and some other lower animals also exhibit hermaphroditism.

When the spermaries of a hydra are mature, the sperm-cells escape and swim freely in the water. A single ovum or egg- 
cell develops in an ovary, and is fertilized by a single spermcell. The fertilized egg-cell divides into a large number of smaller cells, forming an embryo, and becomes surrounded by a hard shell or cyst. The protected embryo falls to the bottom of the pond. There it may remain for some time. Dry dust scraped from bottoms of ponds during mid-summer drought may contain embryos which will develop into hydras soon after being placed in water.

Hydras have another reproductive process which occurs only when by some accident an individual is cut into two or more pieces. The remarkable fact is that within a few days each piece will grow into a perfect hydra. This is an example of regeneration. Many other lower animals have the same power of forming a perfect body from a part, and some animals as high as frogs can regenerate small parts, such as toes, if they happen to be destroyed. It should be noted that regeneration is not a regular reproductive process in Hydra, but provides against accidents.

284. Division of Labor in Hydra. - All cells must use food, but only the endoderm cells are able to digest food. Hence, ectoderm cells must depend upon the endoderm cells for their food. On the other hand, the ectoderm cells form the external protective covering of the animal, furnish the cells which form the reproductive organs, catch the food, receive impressions from the environment, and probably are the chief causes of the movements of the animal. The tentacles are specialized for collecting food, and their nettle-cells still more highly adapted to a special purpose. The presence of simple nerve-cells suggests the beginning of differentiation of nervous organs.

285. A Case of Mutual Aid: Symbiosis. - The green bodies in the endoderm cells of one species of Hydra have been discovered to be simple, one-celled plants (one of the Algæ), which also live in tissues of some fresh-water sponge colonies and in some other animals. Such a living together, 
known as symbiosis, of a plant and animal is a mutually helpful condition. The green plants use carbon dioxide, an excretion of the animal, and make carbohydrate food, some of which may be absorbed as food by the surrounding protoplasm of the animal. The oxygen freed from the carbon dioxide ( $(105)$, when that is used by the plant, may be of use to the animal's cells. The green plant needs some nitrogenous food, and may get this from the animal's excretions. Thus the plant gets all the materials for its food from the animal, and the animal gets carbohydrate food made by the plant from the animal's carbon dioxide excretion. Here within a single animal we find an illustration of the relation which exists between all animals and plants ( $\$ 115)$.

286. Allies of Hydra: Cœlenterates. - The Hydra is a member of a great group, a primary division of the animal kingdom, known as Cœlenterata, or popularly called ccelenterates. The name is a combination of ccelome (the technical name for body-cavity in all animals), and enteron (technical name for a digestive cavity), and was originally given because it was supposed that in Hydra and its allies the one cavity (the digestive cavity) appeared to combine both the digestive cavity and the body-cavity of higher animals. This was wrong, for in the frog the body-cavity is between the digestive cavity (stomach and intestine) and the bodywall. The cavity in Hydra simply corresponds to the interior of the frog's stomach and intestine, and if it had a space between its endoderm and ectoderm this space would correspond to the body-cavity in a higher animal.

The most characteristic features of the cœlenterates are shown in Hydra; namely, two layers of cells, a digestive cavity, and nettle-cells. Animals of other groups have tentacles, but only cœlenterates have nettle-cells on their tentacles.

287. Hydroids. - Imagine the buds on a hydra remaining attached, as do buds on trees, and budding repeatedly, and 
the result would be a tree-like colony with a hydra-like animal at the end of each branch. This, of course, is impossible for a hydra, for its offspring, formed from buds, always

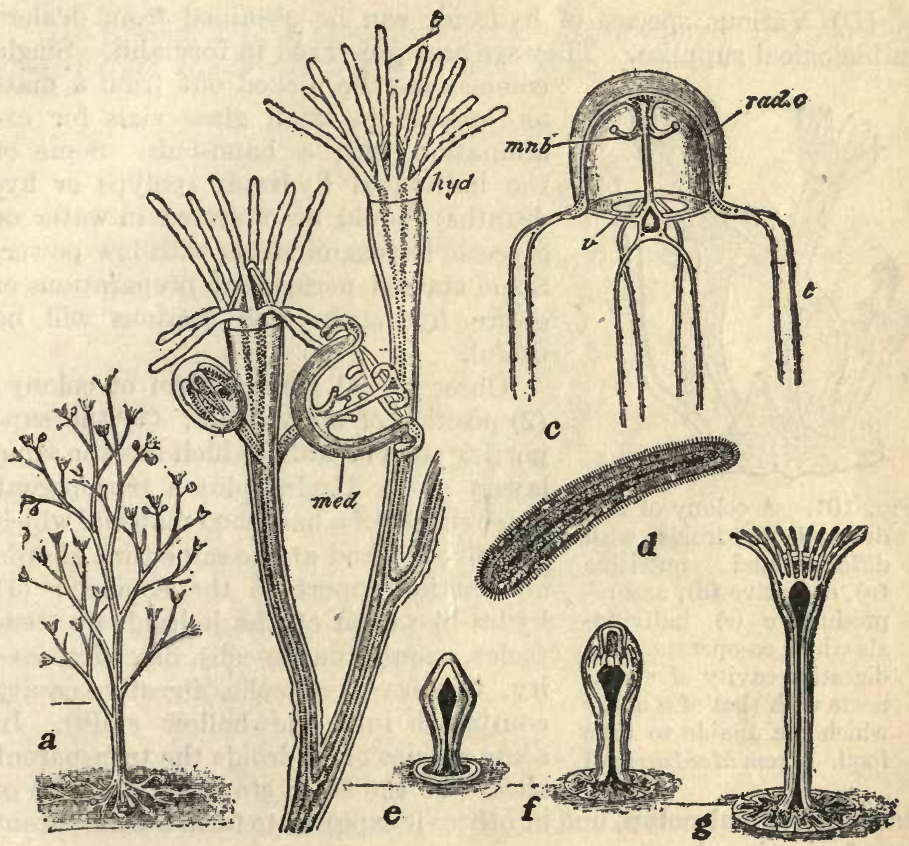

Fig. 100. Hydroids. $a$, colony consisting of root-like base, stem, and a hydranth at end of each branch. $b$, piece of a branch with two hydranths ( $h y d)$ and two medusa buds (med) forming. c a free medusa. $d$, swimming larva developed from an egg produced by a medusa. $e, f$, $g$, stages in development of a larva into an attached hydroid which by budding will form a colony like that shown in a. (From Parker.)

separate quickly; but there are near relatives of Hydra which from one individual form a tree-like colony by such repeated budding. These animals are called hydroids, which means hydra-like. The colonies often grow several inches in height, with hundreds of branches and individual 
hydroids. One who does not examine with a hand-lens would probably mistake a group of hydroid colonies on a rock or other object for a mass of moss-like plants (Figs. 100, 101, 102).

(D) Various species of hydroids ean be obtained from dealers in biological supplies. They are best preserved in formalin. Single

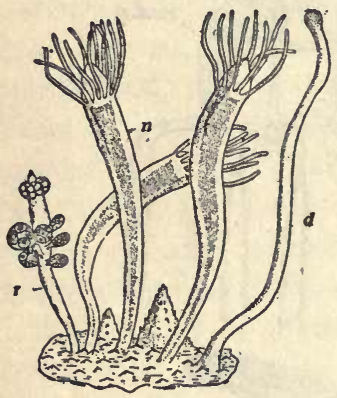

Fig. 101. A colony of $\mathrm{Hy}-$ dractinia hydroids with differentiated nutritive $(n)$, defensive $(d)$, and reproductive $(r)$ individuals which co-operate. The digestive cavity of $n$ connects with that of $d$ and $r$ which are unable to take food. (From McMurrich.) colonies can be picked out from a mass and placed in small glass vials for examination with a hand-lens. Some of the individual hydroids (polyps or hydranths) should be mounted in water or glycerin for examination with low power. Some stained permanent preparations of entire hydranths and sections will be useful.

Observe: (1) general form of colony; (2) position of individuals; (3) the supporting stem or stalk (which has the same layers as in Hydra plus a transparent outer sheath of a hardened material which is protective and at the same time affords an elastic support to the colony); (4) hydra-like form of the individuals (tentacles, mouth, nettle-cells, digestive cavity, two layers of cells, digestive cavity continued into the hollow stalk). In some species of hydroids the transparent sheath of the stalk stops at the base of each individual polyp, but in others it expands to form a transparent cup for each polyp.

288. Medusæ. - Some species of hydroid colonies have certain polyps of a bell-shaped form (Fig. 100, b, c), and in other species there are special polyps which are elongated and without tentacles. Those of the bell-form break away from the hydroid stem and swim freely as medusæ or jellyfishes. Those of the elongated type form a number of buds, and each bud develops into a bell-shaped medusa. All the medusæ are very small when first freed, but they have mouth and tentacles and are able to catch food, as a hydra 
does, and grow rapidly. Some of them may grow to be several inches in diameter. The structure of a typical medusa should be observed.

(D) Specimen of a medusa known as Gonionemus may be purchased from the laboratory at Woods Hole, Mass. They may be

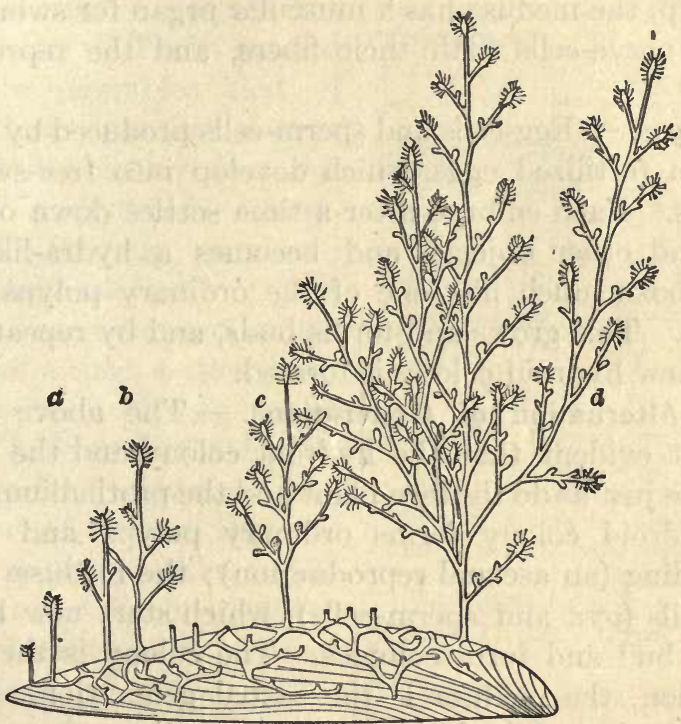

Frg. 102. A colony of hydroids growing on a mussel shell. The erect branches, $a, b, c, d$, show stages in development of branches by budding. The entire colony originated from a single egg developed into a freeswimming larva which attached to the shell and produced the colony by budding. (After Schulze.)

kept in small bottles for convenience in demonstrations, or be placed in water in watch-crystals. Notice: (1) bell-form; (2) tentacles; (3) mouth at end of the central projection, which is in same position as clapper of a bell ; (4) digestive canals forming a conspicuous cross and a ring around the margin of the bell, and connecting with the mouth as may be seen in a specimen into whose mouth some pigment in water has been injected with a pipette; (5) irregular 
bodies (ovaries or spermaries) are attached inside the bell and just beneath the digestive canals; (6) a muscular membrane around the inner margin of the bell is responsible for the locomotor movements of the medusa through the water. See Figure 100, c.

A medusa is more highly specialized than a polyp of a hydroid colony, for in addition to the nutritive functions of the polyp, the medusa has a muscular organ for swimming, a ring of nerve-cells with their fibers, and the reproductive organs.

Embryos. - Egg-cells and sperm-cells produced by medusæ result in fertilized eggs, which develop into free-swimming embryos. Each embryo after a time settles down on rocks, piles, and other objects, and becomes a hydra-like polyp which looks much like one of the ordinary polyps seen in colonies. This grows and forms buds, and by repeated budding a new hydroid colony is formed.

289. Alternation of Generations. - The above account makes it evident that the hydroid colony and the medusæ alternate just as do the fern plant and the prothallium ( $\$ 226)$. The hydroid colony forms ordinary polyps and medusæ by budding (an asexual reproduction); the medusæ produce germ-cells (ova and sperm-cells), which start new hydroids able to bud and form colonies. The colony is the asexual generation, the medusa is the sexual generation. In very many species of hydroids this alternation of generations is just as necessary a part of the life-cycle as it is in ferns and mosses. Medusæ of such species cannot produce eggs which are able to develop into medusæ without the hydroid colony stage; and conversely, the hydroid colony cannot form new colonies without the medusa stage.

There are some species of medusæ closely related to those formed by hydroids whose eggs develop directly into new medusæ; that is, these species do not have alternation of generations.

In one of the common hydroids of the New England coast 
the medusæ sometimes do not develop enough to become free-swimming, and remain attached to the colony. However, they produce embryos in the usual way, and the embryos swim away and start new colonies.

290. The Larger Jelly-fishes. - The term "jelly-fish" usually applies to some of the large medusæ, some of which are from five to eight feet in diameter. Their general structure resembles that of the medusæ described above; but the life-history does not include a hydroid colony. Instead, an egg produced by the large medusæ develops into a ciliated embryo, which swims for a time, settles down and changes into a small animal looking like a hydra. This divides transversely into a number of disks so that the animal now looks like a pile of saucers. Each of these saucer-shaped bodies becomes a medusa, and grows rapidly.

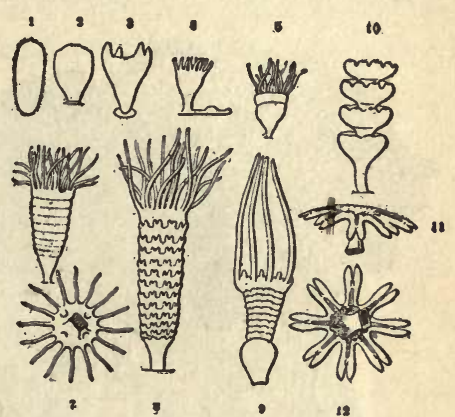

FIG. 103. Development of one of the larger species of jelly-fishes. 1, larva developed from an egg; 2 , $3,4,5$, stages in development of larva into a fixed hydra-like organism; $7,8,9$, division into many disks, each forming a young medusa $(11,12)$. (From Hatschek.) of generations. The large size of the adults is partly due to absorption of water, more than nine tenths of a jelly-fish being water. See Fig. 103.

The Ctenophores or comb-jellies, named because of eight bands of comb-like appearance, consisting of vibrating plates which cause locomotion, are the highest cœlenterates. Museum specimens should be examined. The larger ones are often the cause of the phosphorescent masses seen in sea-water when a boat is being paddled on a dark mid-summer night. Ctenophore eggs develop into young ctenophores; there is no alternation of generations. 
291. Coral-animals. - Most important to man of all the allies of Hydra are the animals whose skeletons form a large part of many coral islands. (Look in a textbook of geography for a list of coral islands.) In order to understand how coralanimals form their hard skeletons, it is necessary to examine a specimen of a sea-anemone; these resemble coral-animals

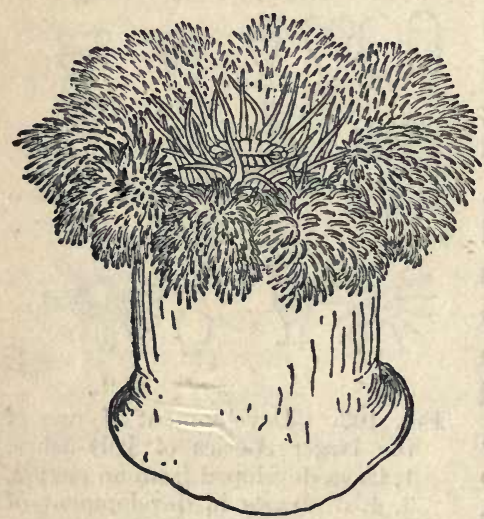

Fig. 104. Common sea-anemone consisting of a cylindrical body with a crown of numerous small tentacles surrounding the mouth. (After Emerton.) in structure, but do not secrete skeletons.

(D) Living sea-anemones are attached to rocks and piles below low-tide mark. Schools near the sea-shore could keep small ones in a salt-water aquarium; but most schools must depend upon museum specimens preserved in formalin. An entire specimen, one split longitudinally, and one cut transversely, will show the essential points of structure.

In an entire sea-anemone, notice: the cylindrical body, the base, the crown of small tentacles, and the mouth in the center of the crown of tentacles.

In a sea-anemone which has been split lengthwise, notice: the mouth opening into a long tube (esophagus) extending down to near the base, and the delicate membranes connecting this tube with the body-wall.

In a specimen cut transversely, notice : the esophagus in the center, the pairs of membranes (mesenteries) which extend from the esophagus to the body-wall, and the pairs of shorter membranes which extend inwards a short distance from the wall. All the spaces between the membranes extend up into the tentacles and down to the opening of the esophagus (compare with the longitudinal section). Foods taken in through the esophagus may pass anywhere between the membranes, and be digested. In brief, the digestive cavity of a sea-anemone is more complex than that of a hydra in that it is subdivided by many pairs of membranous partitions. 
There are many interesting minor points of structure omitted from these brief notes. For supplementary work, consult Linville and Kelly's "General Zoölogy," Chapter XIX.

Formation of Coral Skeletons. - On every piece of coral which has not been corroded by water, there may be seen numerous cup-like depressions, each with many radial partitions. In each of these cups there was once seated a coral-animal, or coral-polyp, which secreted the cup. Im-

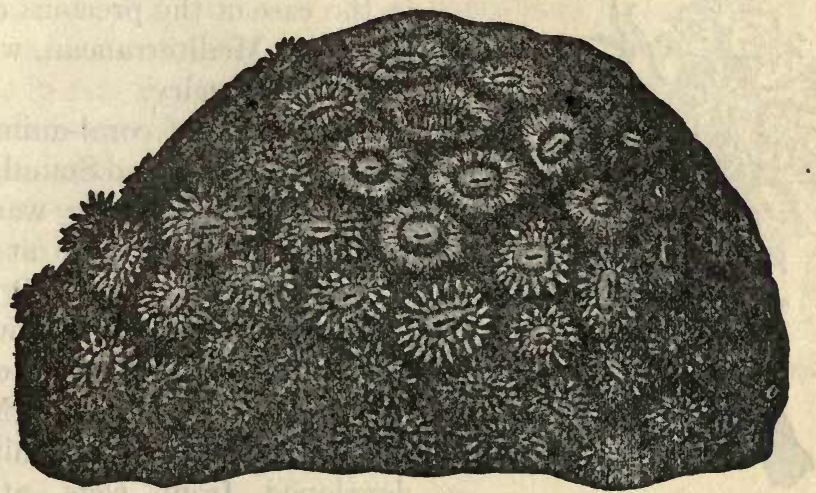

Fig. 105. An Astræa coral colony, with living animals on surface of hemispherical skeleton. Such a colony originates from a single egg which develops into a polyp capable of budding to form a colony. (After Dana.)

agine a sea-anemone able to secrete a calcareous skeleton around itself, and also to secrete a partition of the same material between the two membranes of each pair seen in a transverse section, and then you can understand the relation of a coral-animal to its surrounding cup.

The fact that a piece of coral shows many cups is explained by the multiplication of individuals by budding. A coralanimal which develops from a fertilized egg-cell settles down on a rock or on skeletons of pre-existing corals and begins to secrete a skeleton around itself. Buds are formed, which do not become detached as in Hydra, and these begin to 
secrete skeletons. The final result of oft-repeated budding is a complicated calcareous mass, in tree-like or hemispherical form, with numerous cups representing the number of polyps which took part in the formation.

The sea-fans and sea-plumes are coral skeletons formed in another way. Their outer surfaces are calcareous, and their central axis is composed of a horny material. This is

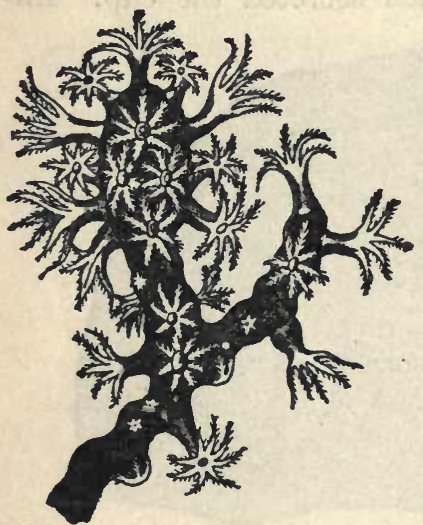

Fig. 106. A colony of coral-animals whose supporting "skeleton" (black in figure) containls the red coral of commerce. (After Lacaze-Duthiers.) black in many species, but red in the case of the precious coral from the Mediterranean, which is used in jewelry.

One species of coral-animal is found in Long Island Sound, but most of them require warmer waters. They can live at any depth down to about 300 feet, but are seldom found below 120 feet. They must have clean and undiluted sea-water. Most commonly the young animals developed from eggs attach themselves to favorable seabottom near land, and form fringing reefs of coral rock near the shore, or barrier reefs when a navigable channel is eroded between the reef and the shore. The formation of an atoll, a peculiar coral island in ring form and inclosing a body of water, may have begun as a fringing or barrier reef around a volcanic island which has later subsided or been eroded away while the deposits of coral rock have been accumulating. Or a small group of corals may have been established on seabottom at a favorable depth, and as the colony grew outwards, the animals in the center may have been killed by coral-sand washed over them by the waves. Erosion takes 
place rapidly when there are no living coral-animals to keep adding to the coral rock; and so the central rock might be worn away, leaving a ring of living animals continually adding new coral rock to the outside.

292. Economic Relation of Cœlenterates. - There are probably more than three thousand species of cœlenterates, but with the exception of coral-animals, none of them are of direct economic importance. Some of them serve as foods for animals of the sea, e.g., small fishes may be seen biting off the branches of hydroid colonies; but the large amount of water in the larger forms makes them of little food value to other animals. However, the cœlenterates prove that human interest is not limited simply to those things which have a money value or are good to eat, for the various forms of hydroids, jelly-fishes, comb-jellies, and sea-anemones have always been great favorites with both amateur and professional naturalists. The great importance of the coralanimals is, of course, that of forming islands and reefs which protect the shores of other islands. Also, there is the red coral, already mentioned as valuable for ornaments.

\section{Classes of Colenterata}

Hydrozoa - Hydra, hydroids, small medusø.

Actinozoa - sea-anemone, coral-animals.

Scyphozoa - large medusæ (jelly-fishes).

Ctenophora - ctenophores (comb-jellies). 


\section{CHAPTER XII}

\section{THE WORM-LIKE ANIMALS}

293. The Worms. - The word "worm" is little more than a name for a shape, meaning an elongated animal with cylindrical or ribbon-like body. The earlier naturalists thought the shape very important, and so they placed all wormlike animals together in a group which they named Vermes (Latin for worms); but later studies have shown that wormlike bodies may belong to animals which in all other points of structure are seen to be totally unrelated. For this reason, the group Vermes is not recognized in the recent textbooks of zoölogy; but instead we find chapters devoted to such groups as flat worms, round worms, segmented worms, and others. There is such a vast assemblage of forms of the worm-like animals that in a short course we can do no more than select a few of the more interesting examples.

294. The Flat Worms. - These are the simplest wormlike animals, and some of them are but slightly more complex than some of the highest cœlenterates. Some of the common species, known as planarians, are brown in color, from $\frac{1}{4}$ to $\frac{1}{2}$ inch long, and very easily found on the under side of stones in fresh water streams and ponds. Small leeches resemble them, but the leeches are easily distinguished by the numerous rings around their bodies. The general appearance of a common planarian is shown in Fig. 107.

295. Other Flat Worms: Tape-Worms. - All the ribbonlike worms known as tape-worms are parasites in the alimentary canal of vertebrate animals. They are simpler than the flat worms, which live independently, for they have no 
mouth or digestive organs, but absorb through their skin the digested food in the alimentary canal of the host in which they live.

A tape-worm preserved and mounted in a jar containing alcohol or formalin will show that the body resembles a long, narrow ribbon or tape, but is unlike a ribbon in that it is divided into segments. The ribbon narrows near the anterior end. The head is a rounded knob, with a circle of hooks and four suckers. There are nerves and excretory tubes in each segment. Each of the larger segments towards the posterior end has a complete hermaphroditic reproductive system, consisting of ovaries, spermaries, and their ducts leading to the surface. The extreme posterior segments become greatly distended with enormous quantities of fertilized eggs, each inclosed in a hard shell; and one by one these "ripe" segments drop off and are carried out of the intestine with indigestible matters or feces. As segments drop off the worm, new ones are formed by new grooves in the segments back of the head. Thus the oldest segments are the most posterior ones.

At the time a "ripe" segment is discharged from the intestine, the egg-shell contains a small embryo with six hooks. If these small embryos, in the case of the

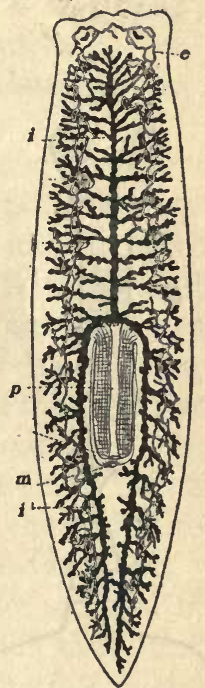

Fig. 107. A planarian, flat worm. The root-like intestine is shown in black. $e$, eye; $p$, pharynx; $m$, mou th. Delicate excretory tubes lie on either side of the body. (From Hatschek.) human tape-worms, happen to fall on grass or other food of pigs and cattle, the digestive fluids in the stomachs of these animals can dissolve the hard shell and free the embryo. Then it bores into some organ and becomes encysted. Then it develops a bladder-like structure with a tape-worm head. 
This stage (called a bladder-worm or cysticercus) remains for some time in the tissues; and if uncooked pork or beef be eaten by man, an encysted bladder-worm may attach itself to the wall of the intestine by means of its hooks and suckers
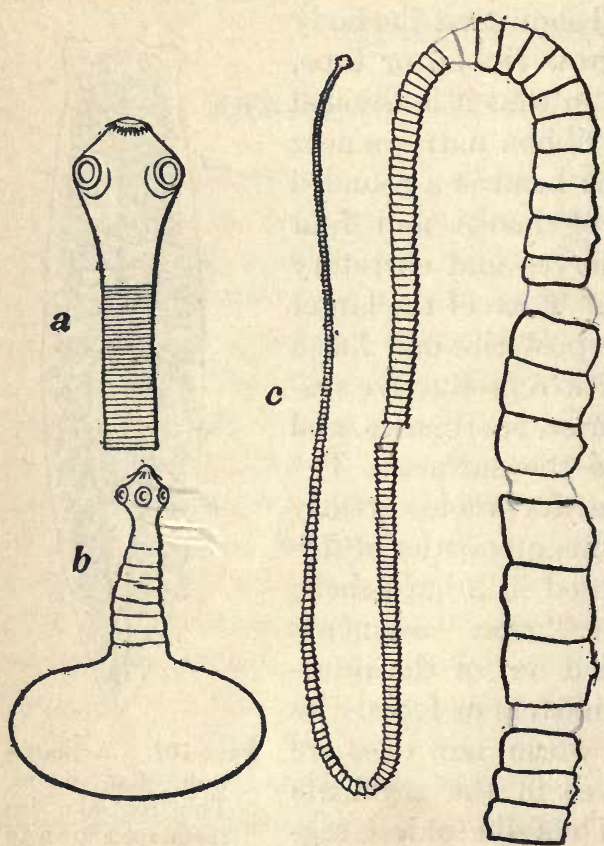

and develop into a tape-worm.

The above account of the human tape-worms, of which one species develops its larval stage in pigs and another in cattle, represents the lifehistory of all the tape-worms which inhabit the intestines of various vertebrates. Two hosts are required, one for the tape-worm, and one for the intermediate bladder-worm stage. One species of tape-worm of dogs has its bladderworm in rabbits; one of sea-gulls has

Fig. 108. Tape-worm. $a$, head; $b$, larval stage found in flesh; $c$, parts of an adult worm from intestine. (From Hatschek.)

its in earth-worms; and one of cats has its in liver of rats and mice. In Oriental countries there is a large human tape-worm whose bladder-worm stage is passed in certain fishes. In a large number of cases, the bladder-worm stage occurs in the particular animal which is a favorite food of the animal which may be the host of the fully developed worm. 
Tape-worms are troublesome parasites in that they interfere with the nutrition of the animals which they inhabit. They are difficult to remove, because the head is so firmly attached to the lining of the intestine. All the segments might be dislodged by powerful drugs, but if the head remained, it might continue to grow new segments.

The bladder-worms embedded in human tissues cause an uncommon disease known as hydatids.

Prevention of tape-worms is a simple matter; namely, eat no meat " cooked very rare." In the case of pork there is an additional reason for this rule, in that the far more dangerous parasite Trichina ( $\$ 297$ ) may be present. With increasing attention to sanitation and the disposal of sewage by bacterial methods described in $\S 258, f$, there will be less chance of tapeworm embryos getting into pigs and cattle and then indirectly entering human beings. The rarity of human tape-worms in United States is probably in part due to the fact that our farms are more sanitary, and being larger than those of Europe farm animals live farther away from human dwellings.

296. Other Parasitic Flat Worms. - One of the best known flat worms whose habits of life resemble those of the tape-worm is the liver-fluke, which lives in the bile-ducts of sheep. It is a flat worm about one inch long and one fourth inch broad, with two suckers for attachment. The eggs escape into the sheep's intestine through the bile-duct, and after being discharged from the intestine, an egg develops into a larva covered with cilia. This larva swims, and if it reaches a pond-snail, bores into it and becomes an elongated sac. Inside this sac are formed many new larvæ, and inside each of these larvæ are formed many more. Thus one larva entering a snail produces a large number of larvæ of different forms; and each one of these may leave the snail, become encysted on grass, and when eaten by a sheep will enter the bile-duct and develop into a liver-fluke. When once a damp pasture along a given stream has become infested with larvæ 
of liver-flukes, the only way to avoid infecting sheep is to keep them away from that stream for a number of years. Ultimately the fluke larvæ would disappear from a pasture if there were no sheep in which the parasites could complete the life-history.

297. Round Worms. - A good example of a harmless round worm is the vinegar-eel, which is often abundant in unfiltered vinegar, and especially in "mother of vinegar." They should be examined with the low power of a microscope.

Many species of the round worms are parasitic in intestines of man and animals. Some are slender threads half an inch long, while others may be a foot long.

The most terrible round worm is the trichina. The adult worms live in the intestine of man, pig, and other mammals. The males are about $1 \mathrm{~mm}$. $\left(\frac{1}{25}\right.$ inch) and the females $3 \mathrm{~mm}$. long. The eggs develop inside the female and the young (1000 or more) are born alive; that is, discharged as young

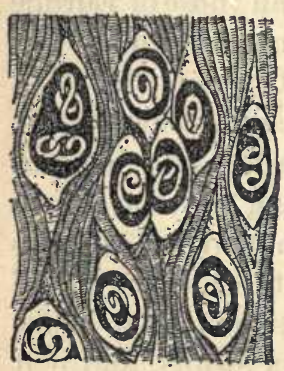

FIg. 109. Trichinæ encysted in muscle. (After Leuckart.) worms. These pass through the walls of the intestine of their host, and reach such muscles as those of the arms, legs, and back. Each worm embeds itself in a muscle-fiber (Fig. 109), and a cyst forms around it. It may remain encysted for years. An ounce of infected pork may have 80,000 such cysts. If the flesh be eaten by another animal or by man, the cysts dissolve in the digestive organs, the young worms develop, form eggs and sperms, and fertilized eggs develop into young worms which become encysted in muscles. In a town in Germany, in 1884, the flesh of one pig infected 364 persons, and 57 died within a month. This large number of infections is easily understood when we calculate from the figures above. A single ounce of pork might introduce into a human stomach 80,000 trichinæ. 
Suppose one half of these are females, each able to produce 1000 young worms, and four million encysted worms might be the result. Thus, at this rate, 100 pounds of pork might contain enough cysts to develop 64 billions of encysted worms, or 160 million for each of 400 persons who might eat the pork. And this is not the whole story, for a female trichina may live in the human intestine and frequently give birth to as many as 1000 young. It is easy from such figures to see how a single infected pig could have caused so much trouble.

There is no way to stop the worms when once they get into the human intestine. If the infected individual does not soon die from the inflammation caused by the encysting in the muscles, the cysts soon become hardened and there is no more danger to the patient. Prevention is very simple; namely, eat no pork which is not well cooked. Government inspectors in the United States and other countries examine meats at the great packing houses and slaughter houses, and condemn as unfit for human food all meat found to have trichinæ. Inspection is not difficult, for if the parasites are present in a pig, they are likely to be so abundant that a small piece of lean meat (muscle) examined with a microscope will reveal the trichinæ. However, the parasites have been sometimes overlooked by expert inspectors.

The above account has not explained how pigs get infected. Since they do not eat human flesh, they must get the parasites from some other animal. Trichinæ are found in rats, and it is well known that pigs will eat dead rats. Also, pigs might eat scraps of pork thrown out in garbage.

298. The Horsehair Worm. - In some rural districts, it is still believed that a long, thread-like worm, looking not unlike a long hair from a horse's mane or tail, and found wriggling in pools of water, has developed from horsehairs which have happened to fall into the water. Hence the names "horsehair worm" or "horsehair snake." The belief seems to have been originated, and is still perpetuated, 
by the fact that the worms are sometimes seen wriggling in troughs where horses drink, and where horsehairs may also be seen. But that there is no connection between the worms and the hairs can be demonstrated by any one who will place in bottles, stoppered with cotton, one or a thousand horsehairs, and await developments.

Scientific men long ago studied the structure and embryology of horsehair worms and solved the mystery of their appearance in pools, watering troughs, etc. The structure of the worm is essentially the same as that of the other round worms. Its scientific name is Gordius, in allusion to its habit of twisting into a tangle like the famous Gordian knot which Alexander the Great cut with his sword. Its eggs develop into minute larvæ, which become parasites in insects, fishes, frogs, and other animals. Later the parasites escape from these animals and develop into horsehair worms. Those seen in horse-troughs have completed their life-history in insects.

299. Spontaneous Generation. - The belief that a living worm might develop from a dead horsehair is opposed to the commonly accepted idea that living things develop only from similar living things. However, this idea that all life comes from life (biogenesis) is rather recent. In former times, even scientific men believed in spontaneous generation of life in dead, or not-living matter. The sudden appearance of numerous earthworms, frogs, mice, insects, etc., was explained by assuming that they had suddenly originated spontaneously. When accurate studies of life-histories began to be made, it soon became evident that all the larger animals and plants originate only from organisms like themselves. Until 1638, it was supposed that maggots developed spontaneously from putrid meat; but in that year an investigator showed that maggots never appear on meat which is screened so as to keep flies from laying eggs on it. Studies of habits have shown that the sudden appearance of earth- 
worms is caused by flooding of their burrows, and that they do not rain down ; and that crowds of toads, frogs, mice, grasshoppers, etc., are due to very favorable conditions for the developing eggs and migrations into new territory. All other such cases which puzzled even the scientific people of a few hundred years ago have been explained so well that for more than a hundred years no scientific man has believed in the existence of spontaneous generation of any organisms higher than the bacteria. But until the studies by Pasteur, supported by the work of the English physicist Tyndall, between 1850 and 1870 , it was believed even by men of science, that certain bacteria may develop spontaneously in sterile bouillon and other foods. Pasteur showed that if proper precautions are taken to make the foods perfectly sterile, that is, to kill all life in the test-tubes used, and to prevent entrance of other germs, no organisms will develop. In short, Pasteur showed that there is no evidence that living matter originates spontaneously from not-living matter. He did not show that it could not happen, for there may be conditions of which we know nothing, as perhaps existed at the first appearance of living matter on the earth; but he showed that the few cases in which some scientific men of his time still believed had not been sufficiently tested by accurate experiments. So far as concerns the origin of new individuals of all known species of organisms now existing, we may summarize the studies which culminated with those of Pasteur in the statement that all living things come from living things, or all life from life.

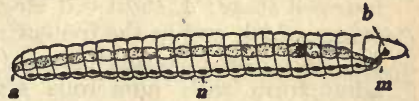

FIG. 110. Plan of annelid's body. $b$, "brain," dorsal to the esophagus; $m$, mouth on ventral surface; $n$, nerve-cord ventral to alimentary canal; $a$, anus or posterior end of alimentary canal; body segmented or ringed.

300. The Segmented Worms: Annelids. - The common earthworms and the leeches are members of a group characterized by division of the body into rings or segments, 
(also called metameres). They are much higher than the worms previously mentioned. There is much diversity of form and habit among the members of this group, and we cannot now take time for more than a brief examination of a few

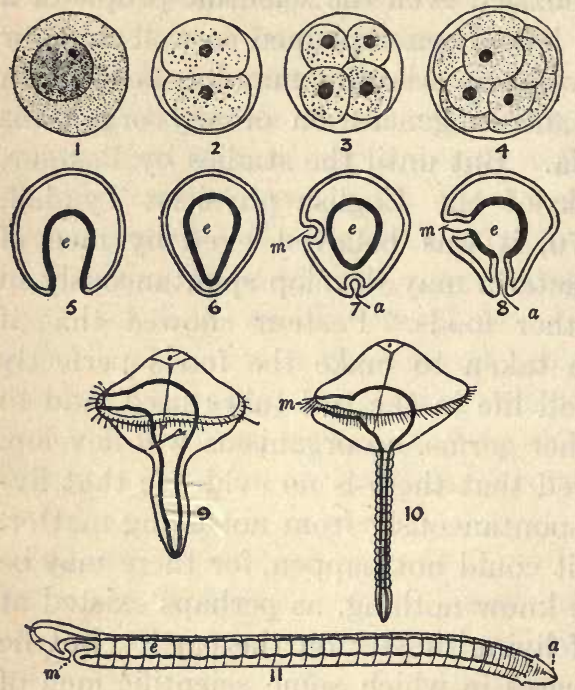

FIG. 111. Development of a marine annelid.

1, fertilized egg-cell; 2, two-cell stage; 3 , four-cell stage; 4 , eight-cell stage; $5-8$, later stages which end in the free-swimming larva, (9), and this metamorphoses (10) into the adult form with numerous segments (11). $m$, mouth; $a$, anus; $e$, alimentary canal. (From Thomson, after Fraipont.) common examples of the segmented worms.

\section{The Sand-} worm.- $(D)$ This worm, belonging to the genus Nereis, lives in sand at the sea-shore near lowtide mark, and thousands are dug and used by fishermen for bait. In certain seasons they are rapid swimmers in the sea. Specimens may be kept and examined in long test-tubes, or in glass tubing stoppered at each end with a cork and then dipped into hot paraffin. Examine a sand worm according to the following notes. It has a head with jaws, small tentacles, two pairs of bead-like eyes, and a pair of cylindrical palps (feelers). On some specimens the proboscis may be found extended from the mouth. There are several pairs of cirri (feelers) back of the head. The body is divided into segments; count the number in an inch of length and then estimate the total number. Are the segments similar? How are the rowing organs or paddles arranged on the segments? Perfect specimens have a forked tail-appendage. The main longitudinal blood-vessels appear through the skin on dorsal and ventral surfaces.

Dissected specimens pinned on pieces of soft wood or on cork may 
be used to demonstrate the presence of digestive, nervous, excretory (kidney), and reproductive systems. The general plan of these organs may be learned from examining an earthworm in a later lesson.

Nereis and other marine segmented worms have an interesting, free-swimming larva in their development. Figure 111 shows the main stages in another segmented worm which has such a stage. The fertilized egg divides into two, then four, eight, sixteen, etc., cells and forms a hollow sphere of cells. Then one pole of this sphere turns inward as one might push in one hemisphere of a hollow rubber ball. This forms two layers, as in Fig. 111, 5, the outer being ectoderm and the inner endoderm. Such a two-layered embryo is called a gastrula. The primitive alimentary canal ( $e$ in Fig. 111, 5-8) soon forms mouth and anus, and the embryo grows into a top-like form. In this condition it begins to lead an independent existence and is called a larva. Gradually the lower end of the larva becomes elongated and seg-

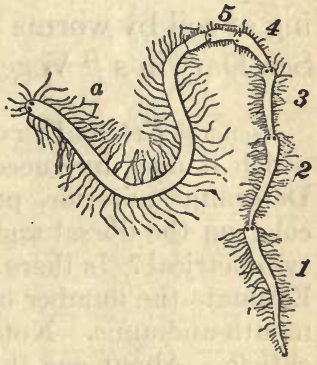

FIG. 112. An annelid worm which has an unusual habit of multiplying asexually by dividing off young worms in the order 1 to $5 . \quad a$ is the original worm. (After Milne-Edwards.) mented (Fig. 111, 10), the head becomes relatively smaller, and the larva is metamorphosed into the adult worm (11), which begins to live in sand. In all cases like this where the young animal which hatches from the egg is quite different from the adult, the young animal is called a larva, and the change to the adult is metamorphosis.

302. The Earthworm. - One other segmented worm deserves more than the brief attention which our time will allow, and that is the land worm, commonly known as earthworm or " fishing worm." It lives in moist soil which con- 
tains decaying organic matter, crawls out at night to feed, or when its burrows are flooded with water. It eats the soil through which it burrows, and its digestive juices dissolve bacteria, leaf-mold, and other organic matter contained in the soil. The indigestible soil is discharged and forms the " castings" which are abundant on the surface of soil where the animals live. Darwin found places where he estimated that the earthworms brought more than 35,000 pounds of soil per acre to the surface in a year. This continual working of soil by worms is regarded as of great agricultural value. See Darwin's "Vegetable Mold and Earthworms."

General External Structure.- $-(L)$ Notice a living worm as it moves, which it does by successively elongating and shortening its body. Determine anterior, posterior, dorsal, and ventral. Compare the color on the dorsal and ventral surfaces. Is the animal bilaterally symmetrical ? Is there a head, thorax, and abdomen, as in the frog? Estimate the number of segments or rings in the body. Locate the mouth and anus. Notice the glistening surface of the skin due to a cuticle. About one third or one quarter the body length from anterior end, there is a swollen region, the girdle or clitellum. It contains gland cells which secrete a cocoon in which the eggs are laid $(\S 303)$. The clitellum is not present on very young worms and on older ones only in spring of the year, the egg-laying season. Four double rows of small bristles (setæ), which aid the worm in locomotion, may be located by pulling a preserved specimen between a thumb and a finger, and by using a hand-lens. How many bristles are on a segment? Make dia rams showing their position.

Living earthworms should be observed with regard to (1) movements on soil and on hard surfaces, and (2) reactions to light and touch.

The dorsal and ventral blood-vessels may be easily seen in a living animal. Movements of the dorsal blood-vessels can be easily seen if a light-colored worm is selected, placed on a moist glass plate, and another piece of moist glass then placed on top of the worm so as to compress the body slightly.

The Body-cavity. - (D) Split a preserved earthworm lengthwise into right and left halves and note that the body-cavity (colome) is divided by partitions into cavities corresponding to the external division into segments. Examine the body-wall and the alimentary 
canal in a transverse section. The body-cavity of a frog is one continuous space, while that of mammals is divided by the diaphragm into the thoracic cavity (containing heart and lungs) and the abdominal with stomach, intestine, liver, kidneys, and reproductive organs.

Internal Organs. - $(D)$ The teacher should point out on preserved earthworms, which have been cut open along the dorsal side and pinned out on a narrow board, the following systems of organs :-

(1) Alimentary system consisting of : pharynx (for seizing food); esophagus; crop (a reservoir for food); gizzard (for grinding food); stomach-intestine (for digesting and absorbing food.

(2) Circulatory System. - A large bloodvessel on dorsal side of the intestine; a number of arch-like branches which extend around the intestine to a ventral blood-vessel. Through the skin of a small living earthworm it is possible to see numerous branches from these larger bloodvessels to all parts of the body. In addition to blood, the space (body-cavity) between the digestive canal and the bodywall contains a fluid which supplements the work of the blood. Blood flows forward in the dorsal vessel and backward in the ventral. The dorsal vessel and the arches have muscular walls which contract at regular intervals, driving the blood forward and then down into the ventral vessel. In the posterior part of the animal the blood gets back to the dorsal vessel, thus making a complete circulation.

(3) Reproductive Organs. - These are large, light colored organs lying on right and left of the alimentary canal between the tenth and fifteenth segments. The animal is hermaphroditic; that is, it has

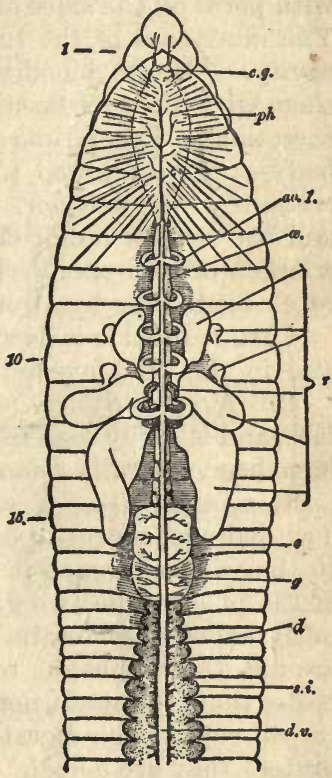

Fig. 113. Diagram of internal organs of earthworm as seen dissected from dorsal side. c.g, cerebral ganglion or "brain"; $p h$, pharynx; $\alpha$, esophagus; ao. 1 , first of five pairs of arches connecting dorsal and ventral bloodvessels; $r$, reproductive organs ; $c$, crop in segment $15 ; g$, gizzard; s. $i, \quad$ stomach-intestine; d.v, dorsal blood-vessel; $d$, septum between two segments. (From Sedgwick and Wilson.) both ovaries and spermaries. Very small tubes, not easily seen, extend to openings on the ventral surface of the animal. 
(4) Excretory Organs. - In each segment, except a few near the mouth, there are two delicate coiled thread-like tubes which connect with pores on the sides of the body between the two rows of bristles. The inner ends of the tubes are funnel-like and open into the bodycavity. Minute blood-vessels in the walls of these tubes supply them with blood, from which the cells of the tubes take nitrogenous excretions. From time to time a small amount of fluid from the body-cavity is allowed to flow through the tubes and wash the excretions to the exterior. These tubes act, then, as simple kidneys ; and the several hundred pairs of such kidney-tubes (nephridia) in a large worm co-operate in doing the work such as the frog's kidneys (each containing hundreds of tubes) do for that animal.

Carbon dioxide is excreted by absorption from the skin, and some also in the fluid ejected by the kidney-tubes.

(5) Nervous System. - The main nerve-cord is on the ventral surface, and is easily seen in a specimen from which the digestive organs have been carefully removed. In each segment there is a thickened portion, known as a ganglion, and containing many nerve-cells. Two pairs of nerves in each segment run from the main nerve-cord to the various organs in that region. Dorsal to the anterior end of the pharynx lie two ganglia (cerebral ganglia), sometimes erroneously called the "brain," and from them two small nerves extend around the esophagus to the main nerve-cord on the ventral side. Note that the main nervous organs of the earthworm are on the ventral side of the digestive canal, while in the frog (and all vertebrates) they are dorsal.

303. Development of Earthworm. - Although both ova and sperm-cells are produced by each individual, self-fertilization is guarded against as effectually as in many flowers we have studied. In each worm there are in segment 10 some small sacs or sperm-reservoirs which are filled with sperm-cells from the spermaries of another individual when two worms pair in early spring. These sperms are stored until time for laying eggs. The eggs are deposited in a case or cocoon formed as follows. A secretion is poured out on the surface of the girdle (clitellum) of the worm, this secretion hardens to form a ring, and then between the ring and the body a jelly-like nutrient substance is secreted. The ring is then worked forward by contraction of the worm's body, and, 
as it slips past segment 14, at least one egg-cell is discharged from an oviduct, and as the ring passes segment 10 , some stored sperm-cells are ejected from the spermreservoirs, which were previously filled with cells from another worm. When the ring slips off the worm's anterior end, its open ends contract and it becomes a closed egg-case. The egg-cell is fertilized by a sperm-cell and then divides into numerous cells, which form the body of an earthworm embryo. There is no larva, as in marine worms ( $\$ 301)$, but a young earthworm about one inch long is hatched.

304. Animals with Blood. - All backboneless animals higher than and including the segmented worms, have blood or similar liquid which is made to circulate, usually by 'a heart. The functions of this circulating liquid are the same as in the frog $(\$ 52)$, for the complexity of the bodies is such that there must be a circulating medium to distribute oxygen and digested food, and to carry excretions from distant cells to the excretory organs.

The blood of these animals lower than the vertebrates is commonly colorless, resembling lymph of vertebrates, and containing only white cells or corpuscles. Red blood-cells occur only in backboned animals. Some of the backboneless forms have blood with a reddish tint, which is caused by dissolved hæmoglobin, the substance which gives the red color to blood-cells of vertebrates. This dissolved hæmoglobin makes it possible for a given quantity of blood to carry more oxygen than could be carried in colorless blood; hence even the reddish tint of the blood is an advantage to the animals which have it. Blood which does not have the coloring matter can probably carry about as much oxygen in solution as water could.

Examples of animals which have blood without red bloodcells are the larger worm-like animals, the crabs, lobsters, spiders, insects, clams, oysters, snails, cuttle-fishes, star- 
fishes, and sea-urchins. Reference will be made to all of these in the succeeding lessons.

$$
\text { Important Groups of "Worms" }
$$

Platyhelminthes - flat worms, tape-worms.

Nemathelminthes - round worms, Trichina.

Annelida - segmented worms, leech, earthworm.

These are phyla or primary groups equivalent to Protozoa and Cœlenterata. For other phyla of worm-like animals, and for subdivisions of each phylum, advanced books of zoölogy should be consulted. 


\section{CHAPTER XIII}

\section{THE ECHINODERMS}

305. Echinoderma. - This group (a phylum) contains the starfishes, sea-urchins, crinoids or sea-lilies, and sea-cucumbers, - all very peculiar animals widely different from those of all other phyla. All members of the phylum are inhabitants of the seas. No other phylum of animals is exclusively marine; and no one knows any good reason why echinoderms have not migrated up rivers. This is another of thousands of well-known biological facts which appear inexplicable. All the forms named above have peculiar spines on the skin (best developed in sea-urchins); and the name Echinoderma means spiny skin. We shall have time in this course for only a brief account of some common members of this group.

306. Starfish. - $(D)$ A starfish illustrates the general plan of echinoderm structure. The most common species of our Atlantic coast has a central dise and five flexible arms, and appears radially symmetrical. In fact, naturalists in the early part of the nineteenth century thought they were related to jelly-fishes and other cœlenterates. Later studies have shown that starfishes and all other

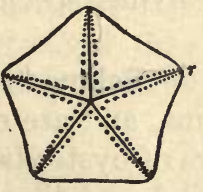

$\boldsymbol{A}$

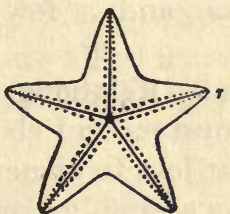

$B$

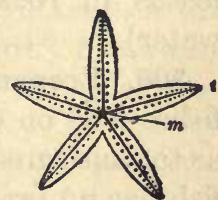

c

Fig. 114. Outlines of three forms of starfishes. $m$, mouth in center of each; $r$, the groove containing the foot-suckers. (After Gegenbaur.)

laterally symmetrical with reference to the internal organs. The median plane is marked by a small pore-plate on the upper surface near the angle between two arms, and a knife passed through this 
plate and the middle of the arm opposite will divide the body into equal halves with two and a half arms each.

On the lower surface of the central dise is the small mouth, and radiating from it is a groove on each arm. Along the grooves are the suckers or "feet" by means of which the animal crawls, clings to objects, and obtains food.

Internally, there is a central stomach and digestive tubes radiating out to the arms. A central nerve-ring has a branch to each arm.

There is a central ring tube with a branch tube to each arm where smaller branches end in the hollow suckers. This tube with its branches is filled with water, which enters at the small pore-plate on the upper side. This water-vascular system is useful in respiration and in locomotion.

307. Other Echinoderms. - It will be profitable to examine specimens and pictures of other types of echinoderms for the sake of general acquaintance. Only extensive study would elucidate their structure and functions. The forms collected along American sea-coasts are "sea-urchins" (hemispherical and covered with long spines, no arms); "brittle stars" (starfish-like, but with long and slender arms); "sand-dollars" or "sand-cakes" (flattened discs covered with small spines, related to sea-urchins); seacucumbers (with tough and leathery skin, and group of tentacles at one end); sea-lilies or crinoids (abundant as fossils in rocks, and a few living species occur in deep water).

308. Economic Relations of Echinoderms. - The starfishes feed on oysters and clams, and are often so numerous as to cause great loss to owners of oyster-beds. Since a starfish has no jaws and an extremely small mouth, it is evident that only by some unusual method of feeding could it eat an oyster. This is accomplished as follows: The starfish stomach is a large thin-walled sac which can be everted through the small mouth, much as one might turn a glovefinger inside out. A starfish fastens its suckers on an oyster, and then the stomach covers the edges of the oyster's shell, 
with the result that the currents of water ( $\$ 338)$ are stopped and the animal within the shell is killed by suffocation. The shell then gapes open, the starfish's stomach pours in its digestive secretion, the tissues of the oyster are dissolved (digested) while in its own shell, then the digested substances are absorbed by the starfish. Finally, the starfish withdraws its stomach into its own body and leaves the empty shell of the oyster.

Against such a remarkable enemy an oyster or clam is completely helpless, for the hard shell which protects against enemies which feed like ordinary animals, is of little avail against an animal peculiarly adapted for suffocating the oyster and then digesting its tissues before taking them as food. It is only among the starfishes that there are animals able to evert their stomachs for the purpose of surrounding and digesting food which is too large to be taken into the mouth.

Owners of oyster-beds now make systematic efforts to destroy starfishes. Formerly, the oyster fishermen used to break the arms from starfishes and throw them back into the sea; but the discovery that starfishes have the ability to regrow or regenerate, and that each of many pieces may soon form a perfect starfish, showed that breaking them into pieces simply multiplied them. Now when starfishes are caught by oyster-dredges, they are killed instantly by boiling water, or they are left on dry land where they die quickly.

\section{Classes of Echinoderms}

Crinoidea - sea-lilies or crinoids.

Asteroidea - starfishes.

Ophiuroidea - brittle stars.

Echinoidea - sea-urchins.

Holothurioidea - sea-cucumbers. 


\section{CHAPTER XIV}

\section{THE ARTHROPODS}

309. Animals with Jointed Legs. - This is the meaning of the name Arthropoda as applied to the group of animals including such forms as crayfishes, crabs, spiders, centipedes, and insects. More than half of the existing species of animals are arthropods, and of insects alone there are more species than in all the other groups of animals taken together.

There are four different types of common arthropods included in the examples named above, and each of these types represents a group known as a class. The important classes are Crustacea (e.g., water-fleas, crayfishes, lobsters, crabs, sow-bugs); Arachnida (spiders and scorpions); Myriopoda, (thousand-legs and centipedes); and Insecta (beetles, grasshoppers, butterflies, flies, cockroaches, etc.). We shall examine examples of each of these types of arthropod animals.

\section{CRUSTACEANS}

310. Structure of a Crayfish, or Lobster. $-(L)$ Either of these animals may be studied in order to gain a good idea of a crustacean. They are so similar that a description written for study of one will serve as a guide for the other, provided that the student keeps a sharp lookout for little points of difference. The crayfish has some advantages in being smaller, and is usually easier to obtain.

\section{External Structure}

Note: In studying the crayfish keep both living and preserved (in alcohol) specimens at hand. The living specimens should be kept in a shallow dish of pure water and examined whenever it is wished to learn the use of any structure seen in the alcoholic specimens, which are more convenient to handle. 
Notice that the animal consists of body and appendages. Are the ends of the body similar? Observe carefully the head end (anterior) and the hinder end (posterior). Notice that the body is divisible into an unjointed head portion (cephalothorax), which means head-thorax, and a jointed flexible hinder portion (abdomen). Are there any indications of joints in the cephalothorax? Is it flexible? Notice that the animal has right and left sides. Examine several individuals and determine whether the sides are exactly alike. Examine the lower (ventral) and upper (dorsal) surfaces. To what surfaces are most of the appendages attached? In how many planes could a knife be passed so as to cut the animal into two similar halves? Use terms anterior, posterior, dorsal, ventral, longitudinal and transverse in describing the position of planes. Is the crayfish bilaterally symmetrical?

Estimate length of the animal and also of the cephalothorax and the abdomen separately. Notice that the body is covered by a hard, outer skeleton or case (exoskeleton). Does the same substance cover the appendages? Notice color of living animal.

Abdomen. - At the extreme posterior end is the tail-fin.

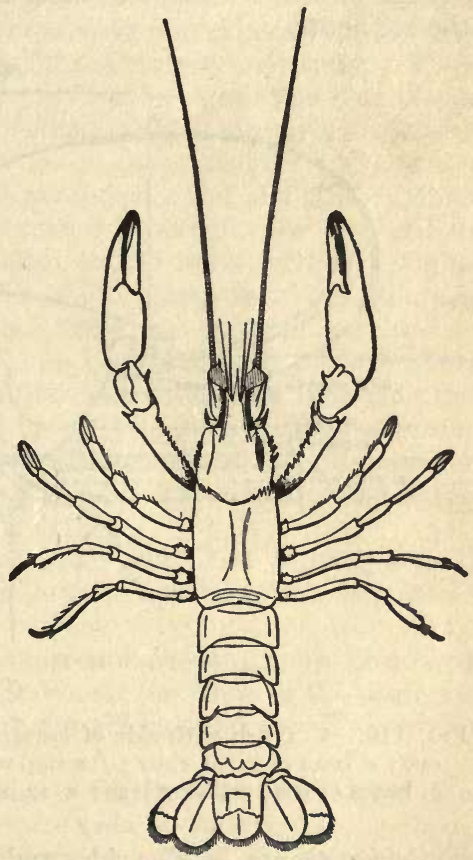

Fig. 115. Blind crayfish from Mammoth Cave. (From Packard.)

Notice that it is composed of a central flattened structure (telson) and on either side a double fan-like plate. Spread the tail-fin and make an outline drawing.

How many segments in the abdomen, excluding the telson? Number the segments of the abdomen, beginning with the anterior segment, and later mark numbers on drawings. Are the segments similar in shape? Sketch abdomen as seen from the left side. 
How many appendages on each segment? Do any segments lack appendages? In what direction can the abdomen be bent? Can it bend laterally? Compare with joints of appendages. Bend the abdomen and make sketch showing the second, third, and fourth segments seen in side view. Now straighten the abdomen and sketch the same segments. Notice the white membrane which unites the segments.

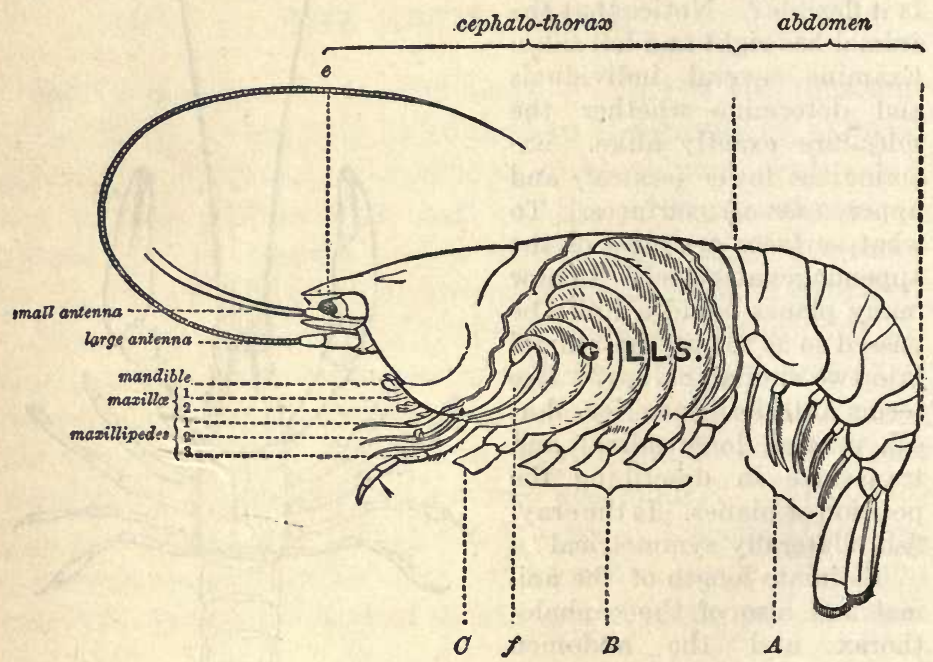

Fig. 116. Crayfish with side of carapace removed to expose the gills. $e$, eye $; c$, base of great claw ; $f$, scoop which drives water out of gill-chamber; $b$, bases of four walking legs; $a$, swimmerets. (From Morse.)

Cephalothorax. - The sides and dorsal surface are in this region covered by a hard shield known as the carapace. Notice the groove which marks the line between the head and thorax. On the head portion notice the beak; the eyes; the mouth on the ventral side (may be seen by separating the appendages).

Make outline drawing of the animal as seen in dorsal or lateral view. (Also ventral view, if time permits.)

Appendages of the Abdomen. - What segments (use numbers) of the abdomen bear appendages? Beginning at the posterior end of the body, carefully use the fine pointed forceps in removing the appendages of one side in order, or examine a mounted set of the 
appendages. Examine all the appendages, and sketch those from the fourth and sixth segments.

Study a living crayfish with reference to the uses of the appendages of the abdomen.

Gills. - Underneath the sides of that part of the carapace covering the thorax are the gills or organs of respiration. Raise with forceps the ventral edge of the carapace on one side and notice that a number of feather-like structures fill the underlying cavity. They are the gills, and the cavity is the gill-chamber, while the part of the carapace which covers it is the gill-cover. With strong scissors, cut away the gill-cover on one side, so as to expose the gills. Cover the animal with water and notice the arrangement of the gills. Move some of the appendages and notice that some gills are attached to their bases, while others are attached to the inner wall of the gillchamber, which is really the body-wall. Note that the gills are outside of the body-wall and are therefore external structures. The gill-cover is simply a fold of the body-wall. It is difficult to count gills in position, and this will be done later after removing the appendages. Make a diagram of the gill-chamber showing arrangement of the gills. In the extreme anterior end of the gill-chamber may be seen a "paddle" or "scoop" which is attached to one of the appendages of the mouth. See Fig. 116.

$(D)$ Observe the movements of this "scoop" in a living animal from which a small piece of the gill-cover has been cut (a painless operation).

Observe the direction of the currents as shown by powdered carmine or indigo placed in the water near the bases of the posterior walking legs. In your diagram of the gill-chamber insert arrows to show direction of the currents.

Appendages of the Cephalothorax. - Observe the locomotion of a crayfish. How many pairs of legs are used in walking?

( $D$ or $L$ ) On the side of the body from which the carapace was cut remove the appendages, beginning with the most posterior walking leg, carefully cutting with strong scissors the muscular attachments to the body. Take care to keep the gills in place and the appendages in order. Examine the appendages in order of removal, comparing with a mounted series in order to make sure that your specimens are complete. Number the appendages, beginning with the first antenna. What appendages bear gills? Count the gills which remain attached to the body-wall. What is the total number?

Do you find the appendages of the cephalothorax arranged in 
pairs? How many? Each pair is believed to represent a segment, as is evidently the case in the abdomen; how many segments, then, compose the cephalothorax? How many in the whole body? Compare: (1) walking legs, and (2) the mouth-parts (appendages used in feeding).

Study of Living Crayfish. - Observe the habits of a living erayfish in an aquarium. In what directions can it walk? What appendages are used in walking? Are there differences in the movements of the appendages? Startle the animal and observe its movements. Can it swim? In what direction? What appendages are used in swimming? Place the animal on its back and observe what appendages are used in turning itself.

Organs of Sense. - Do you find evidence that the crayfish sees? Examine an eye of a dead erayfish with a hand-lens, and later examine with a microscope thin sections eut with a razor. Small sacs in the basal segments of each antennule have been called "ears," but it has not been proved that they are organs of hearing. It has been shown that these organs enable the animal to keep balanced and in the proper position when moving. In short, they perform the function of the semicircular eanals in the human ear, which "make us feel" uncomfortable when in unnatural positions (e.g., head downward).

With a bristle or straw touch a living crayfish in various places. Where is it most sensitive? How does it use the antennæ?

There is some evidence that the crayfish has the senses of taste and smell, but it is difficult to devise simple experiments to test these.

\section{Internal Organs of Crayfish}

$(D$ or $L$ ) Using strong forceps and scissors, break and cut away the dorsal surface of the carapace of a preserved or recentlychloroformed erayfish.

Circulatory System. - Just under the center of the thorax part of the carapace is a cavity (pericardial chamber) in which lies the whitish heart, connected with which are seven arteries whose branches conduct the blood to all parts of the body. The arteries are best seen after injecting some colored fluid into the heart. Blood distributed to all organs by the arteries is collected in irregular spaces in the body, from these it flows into the tubes of the gills, and thence up to the pericardial chamber. Valves in the heart are arranged so that blood from the pericardial chamber can enter the heart, but it cannot go in the reverse direction. 
If a drop of blood be taken from the pericardial chamber of a recently-killed crayfish, it will be found to be a colorless fluid with white cells of amœboid form. These cells are often filled with granules, which have been "eaten" (as an amœba "eats") by the bloodcells. These are similar to the white blood-cells of vertebrates, which also have red cells in their blood.

Digestive System. - In front of the heart lies the stomach (bluish color in fresh specimens), and to the stomach a short esophagus leads from the mouth, which lies between the jaws. The stomach has a oonstriction which marks off an anterior and a posterior portion,

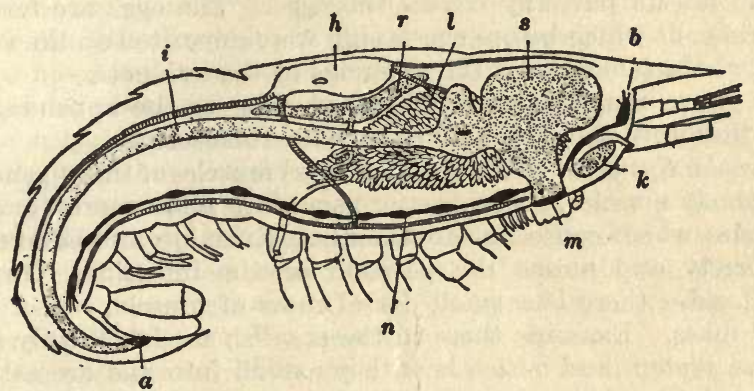

Fir. 117. Internal organs of a crayfish. $b$, "brain"; $k$, kidney; $m$, mouth ; $s$, stomach ; $l$, digestive gland or liver ; $r$, reproductive gland (spermary); $h$, heart ; $i$, intestine ; $n$, ventral nerve-cord ; $a$, anus. (From McMurrich.)

and by cutting open the stomach a peculiar food-grinding apparatus (gastric mill) may be seen in the anterior portion. Food is crushed and shredded before passing into the hinder part of the stomach, and passed through a peculiar strainer before entering the intestine.

On either side of the stomach lies a large digestive gland (greenish yellow in fresh specimens), whose ducts open into the stomach near the intestine. Its primary function is secretion of a digestive fluid which is able to prepare foods for absorption. This fluid is similar in function to the pancreatic secretion in higher animals.

The intestine extends from the hinder end of the stomach, ventral to the heart, and near the dorsal side of the abdomen, to the last segment where its opening (anus) is on the ventral surface. Digestion and absorption of food which begin in the posterior part of the stomach continue in the intestine.

Reproductive Organs. - The male and female crayfishes are easily distinguished because in the female all the appendages on the 
first five segments of the abdomen are similar, except that the first pair is very small; while those on the first and second abdominal segments of a male are modified into twisted and pointed appendages.

The ovaries of a female crayfish lie beneath the pericardial eavity, and the two oviducts open on the basal segments of the penultimate walking legs (third pair). The spermaries of a male lie in the same position and the sperm-ducts open on the basal segments of the last (fourth) walking legs.

At the egg-laying time, eggs issue from the oviducts and become glued to the appendages of the abdomen, which is kept curved so that the tail-fin partially covers the eggs. The eggs are fertilized by sperm-cells which before egg-laying were deposited on the ventral surface of the female near the openings of the oviducts.

The young erayfishes when hatched cling to the appendages of the mother until they are able to care for themselves.

Muscular System. - This consists of (a) muscles of the appendages, and $(b)$ body muscles. The greater part of the abdomen is composed of museles which cause its movements. Examine an abdomen cut transversely and notice the position of the intestine. On right and left sides there is a small dorsal mass of muscle, and a larger ventral mass. Examine these in the crayfish used for study of the digestive system and notice how they extend into and are attached to the skeleton of the thorax. The large ventral muscles bend the abdomen quickly when the tail-fin is used in swimming backwards; the dorsal muscles straighten it.

As an example of how some muscles of the appendages are attached inside the skeleton, move a mandible (jaw) of the erayfish with carapace removed and note the movement of a peculiar conical muscle which extends almost vertically. It was attached to the carapace before dissection. Other appendage muscles can be seen after the digestive and reproductive organs and body-muscles are removed.

Nervous Organs. - Remove the organs already studied, taking care not to destroy the nerve-cord, which lies on the ventral surface of the abdomen between the great body-muscles already mentioned. Note in each segment of the abdomen a thickened portion (ganglion) of the nerve-cord. These ganglia contain masses of nerve-cells, and from them small nerves extend laterally to various organs. In some places the nerve-cord is seen to be double.

On each side of the esophagus is a small nerve-cord which extends anteriorly to a cerebral ganglion (popularly called "brain"), which lies in the median line below the beak. Just back of the esopha- 
gus these two nerve-cords unite to form the double nerve-cord, which continues through a sort of tunnel in the ventral part of the thoracic skeleton to the part of the nerve-cord already seen in the abdomen. In this thoracic part of the nerve-cord there is a ganglion opposite each pair of appendages. Each ganglion and pair of appendages represent a segment.

Respiratory Organs. - As in the frog, respiration of crayfish includes taking in oxygen and excreting carbon dioxide. There is simple absorption of oxygen from the water touching the gills (branchiæ) by the blood eirculating inside the gills, and absorption of carbon dioxide from the blood by the water. The delicate membranes covering the gills are well adapted to such passage of the two gases between blood and water separated by the thin membranes through which a kind of osmosis of the gases occurs.

Obviously, water from outside must continually be coming to the gills, otherwise the available oxygen dissolved in the water would soon be extracted and the carbon dioxide would become excessive in amount. To prevent this, the water in the gill-chamber is continually being changed, entering around the legs and at posterior edge of the carapace and being "scooped" out by a peculiar appendage at the anterior end of the chamber, near the mouth-parts.

Excretory Organs. - The gills, as already mentioned, are the excretory organs for carbon dioxide. The excretion of nitrogenous waste is performed by two organs which lie in the anterior part of the body and just above the bases of the large antennæ. On each antenna note near its attachment to the body a small white tubercle with a central opening. Into this opening inject some colored fluid (using a sharp-pointed pipette), and note the organ into which the fluid goes. This is the antennary gland (a simple kidney). Notice that a similar organ lies above the antenna on the other side of the body, i.e., there is a pair of these glands bilaterally arranged. Owing to their color in fresh srecimens, they are sometines called "green glands." Blood circulating in the tissues of these glands gives out some of the nitrogenous wastes brought from the other organs of the body, just as blood in the kidneys of the frog gives off, through the cells, such wastes into the tubules which lead into the larger ducts of the kidneys.

311. Physiology of the Crayfish. - The crayfish has organs much more highly differentiated than those of the earthworm, but less than those of the frog. In studying the structure of the animal, we have found organs of the following 
systems: supporting (the external skeleton); muscular (muscles of the body and appendages); digestive (mouth, esophagus, stomach, intestine, digestive gland); circulation (heart, arteries, venous blood-spaces); respiration (gills); excretion (gills for carbon dioxide, and antennary glands for nitrogenous wastes, and probably some excretions in the indigestible materials discharged occasionally from the intestine); nervous (cerebral ganglia above the esophagus, nerve-cords from cerebral ganglia to the ventral nerve-cord, a double nerve-cord in ventral part of the body, and nerves extending to various organs of the body); and the reproductive organs (ovaries and oviducts in female, spermaries and sperm-ducts in male).

If we compare with the frog, we find that the same general functions are represented in the crayfish.

(1) The supporting of the body of the frog is accomplished by an internal skeleton, while an external case or skeleton serves the same purpose in a crayfish.

(2) The digestive organs in both animals prepare food for absorption; but the organs for doing this are somewhat different in details of structure.

(3) Both animals are so large that circulating blood must carry digested food, oxygen, and excretions; and in each a heart provides the motive force of circulation. The arteries of a crayfish remind us of those of a frog, but instead of tubes or veins for returning blood to the heart, the crayfish has irregular spaces between the various tissues of its body. The heart of a crayfish is very much simpler than that of a frog, being a hollow muscular organ, with valves arranged to allow blood to enter from the pericardial cavity.

(4) The gills of the crayfish perform the same work as the lungs and skin of the frog. The blood circulating in the capillaries of the gills absorbs oxygen and gives off carbon dioxide, just as does the blood in the lungs and skin of a frog. 
(5) The antennary glands of crayfishes do the work of kidneys of frogs in eliminating nitrogenous excretions.

(6) The nervous system presides over coördination ( $\$ 54)$, and the special senses in both frog and crayfish.

(7) The reproductive organs of both animals have identical work; the ovaries forming ova or egg-cells, the spermaries producing sperm-cells, while the oviducts and sperm-ducts are simply tubes for conducting egg-cells and sperm-cells to the exterior, where each egg-cell may be entered and fertilized by a sperm-cell.

It is evident from above account that in the bodies of a frog and a crayfish the same work is necessary to the maintenance of life-activities, and that there is a system of organs for each function. In other words, the crayfish and the frog both have a high degree of physiological division of labor (§269).

However, there is one important difference; namely, that the organs of the frog are much more complex and more highly specialized. For example, the frog's digestive system consists of mouth-cavity, esophagus, stomach, small intestine, large intestine, pancreas, and liver; while that of the crayfish consists of mouth, esophagus, stomach, digestive glands, and intestine. For another, example of the frog's greater complexity, we might compare its nervous system with that of the crayfish.

However, the crayfish's organs are complex enough for the needs of its sluggish life. With the more highly developed muscular and nervous activities of the highest backboned animals is associated more specialization and complexity of all organs which are essential for the individual life. The great significance of this fact is impressed upon us if we stop to consider that man (the highest organism) is especially distinguished by the complexity of his nervous organs along with remarkably perfect muscular functions; and that to this development of the nervous and muscular systems 
we owe all that has placed man so far above ordinary animal life. But such high development of the nervous and muscular organs, whose activities make human life worth living, would have been impossible without parallel increase in the complexity of those organs (digestive, respiratory, circulatory, and excretory) upon which the muscular and nervous organs depend.

312. Molting of Crayfish. - It is evident that an animal inclosed in a hard external skeleton like that of the crayfish cannot grow rapidly while surrounded by such a coat-of-mail. This difficulty is overcome by periodical shedding of the skeleton, followed by rapid increase in size for a short time while the skin remains soft and extensible. The shedding or molting oceurs several times in the first year of a crayfish's life when it grows rapidly, and less frequently thereafter. The process of molting is in essentials as follows: The membrane on the dorsal side at the joint between the carapace and the skeleton of the abdomen breaks, and through the opening thus made in the skeleton the animal works out the head-thorax and its appendages from the carapace and withdraws the abdomen from its skeleton. As the animal emerges from its old skeleton, it is seen to be covered with a soft new skeleton; and within a short time it expands enormously, largely because of the absorption of water. Gradually lime salts become deposited and cause the hardening of the new skeleton. For a long time new internal tissues may be formed, this new growth displacing some of the water which caused the animal to swell suddenly when released from its old skeleton. It is evident that the sudden absorption of water when molting makes the skeleton large enough to allow for growth (forming new cells) for a long time, perhaps for a year. Owing to the very rapid growth in the first year, a lobster five inches long has probably molted twenty times, while a ten-inch lobster has molted twenty-five times. Such a molting process occurs in all arthropods, but the 
position of the break in the skeleton through which the body is withdrawn varies. If possible, examine "molts" or cast-off skeletons of a crab, king-crab, and an insect (e.g., grasshopper or cicada).

Molting affects crayfishes and all other arthropods so severely that many die from exhaustion, or possibly because blood is lost from broken appendages. However, the disadvantages of molting appear to be more than counterbalanced by the great protective value of the external skeleton.

313. Near Relatives of the Crayfish. - The remarkably close similarity between crayfish and lobster can only be

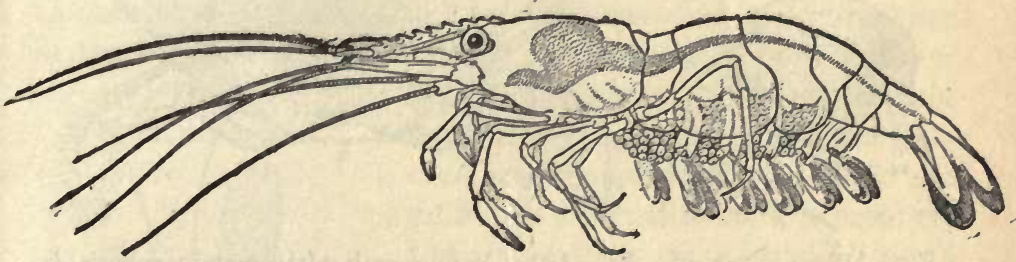

Fig. 118. A common shrimp (Palæmonetes). Eggs shown attached to the swimmerets of the abdomen. Its structure is very similar to that of crayfish and lobster. (From Davenport.)

explained on the assumption that they are closely related. If we see two strange people who look alike, we assume that they belong to the same family; and likewise we believe that similarity among animals and plants indicates relationship.

The crayfishes, lobsters, shrimps, prawns, crabs, - each with numerous species, - are alike in the general plan of the body, the same number of parts, and the same arrangement of appendages. Since all of these have five pairs of large legs, they are grouped together in the order Decapoda (decapods), meaning ten legs.

Now, the crabs are different from the lobsters in that their abdomens (popularly called "tails") have failed to grow as 2 B 
rapidly as has the head-thorax part of the body (see Fig. 120), and hence the greater part of an adult crab's body is headthorax. Because the abdomen is short, the crabs are often called the "short-tailed decapods," while the lobster and others are called "long-tailed." Such facts of structure lead us to think that the various species of crabs are closely related

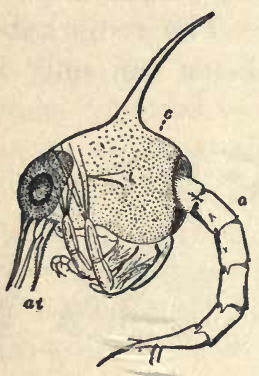

Fig. 119. Larva of crab. at, antennæ; $a$, abdomen; $c$, carapace.

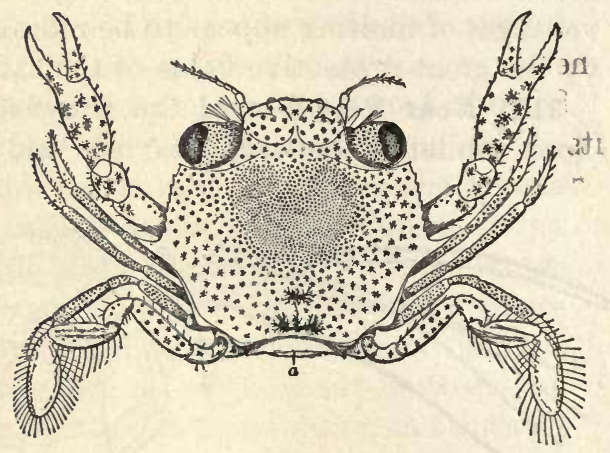

Fig. 120. Young crab after metamorphosis is complete. Note short abdomen in comparison with that of the larva in Fig. 119. (From Brooks.)

to each other, and more distantly related to the long-tailed decapods.

(D) A small permanent collection (in alcohol) of specimens of various species of crayfishes, prawns, shrimps, and crabs would be valuable for illustrating the general facts of structure which seem to indicate relationship between these higher crustaceans.

314. Hermit-crab. - One of the most interesting cases of degeneration among crustaceans is that of the hermitcrabs, one species of which is shown in Figs. 121 and 122. A young hermit-crab takes possession of a small shell (formed by a snail which has died), and backing into it, twists the abdomen so as to hold itself firmly in the shell, which it drags about as it moves from place to place. When growth of the 
crab makes this shell too snug for comfort, the crab goes in search of a larger shell, and having found one, it quickly withdraws its abdomen from the old shell and inserts it in the new one, thus exchanging s' ells.

Since the abdomen and : $s$ appendages are not

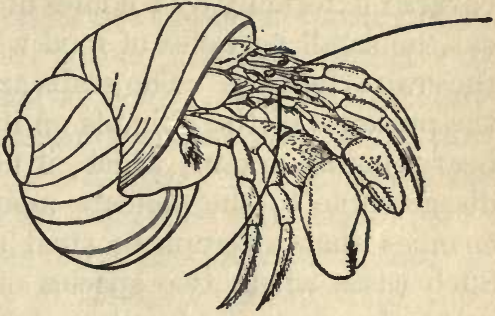

FIG. 121. Hermit-crab in snail shell, abdomen entirely concealed. (After Emerton.)

used as in ordinary long-tailed decapods, degeneration occurs. Compare the appearances of the abdomen and its appendages in the hermit-crab and crayfish (consult the figures, and ex-

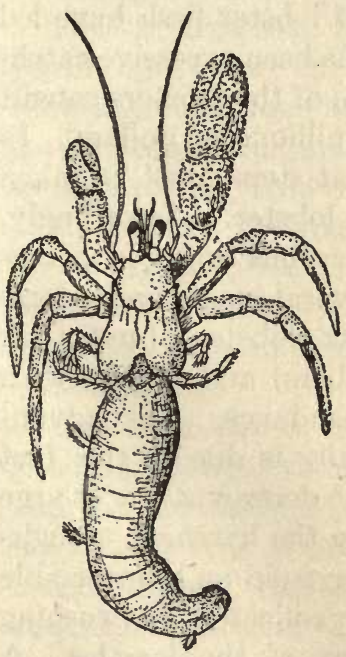

FIG. 122. Hermit-crab removed from snail shell, showing degenerate condition of protected part of its body. (After Leunis.) amine specimens). The abdominal appendages remaining on the hermitcrab are those which help to hold the animal in the snail-shell. Moreover, the abdomen is covered with a soft skin instead of the hard exo-skeleton of other crustaceans. The appendages of the head-thorax are not very different from those of ordinary decapods, and are used in locomotion and feeding. The large claws are used to close the mouth of the snailshell when the crab has withdrawn within, thus protecting the animal. By this peculiar mode of life, the hermit-crab is able to protect itself against the attacks of fishes and other enemies.

A Case of Mutual Advantage: Commensalism. - The snail-shells inhabited by hermit-crabs are frequently 
covered externally by colonies of hydroids (Fig. 101). These eat the small particles of food which float in the water when the crab is feeding. The crabs are believed to be benefited by the presence of the animals on its shell because the hydroids bear stinging organs, which, if not painful, are at least very disagreeable to the delicate mouths of the fishes and other enemies that attempt to steal away the food of the crab. Such cases where two species of animals are associated together for mutual advantage are examples of commensalism, a term meaning mess-mates.

315. Economic Importance of Decapods. - The most valuable of these larger crustacea is the North American lobster, which lives on the ocean bottom within fifty miles of the shore along our North Atlantic coast, and the Norway species, which is supplied to European markets. The large size and the peculiar qualities of lobster flesh have led to such a market demand that there has been excessive catching in recent years. The market value of the lobsters caught each year is estimated to be many millions of dollars. In recent years it has become evident that steps must be taken to prevent extinction of the American lobster. Accordingly, the United States Bureau of Fisheries has engaged extensively in hatching lobster eggs, and several states are enforcing laws which prohibit taking short lobsters (under six inches long in some states, nine in others) and females with eggs attached to the abdominal appendages. The advantage in hatching lobster eggs artificially is due to the fact that fishes and other enemies usually destroy a very large number of eggs or young larvæ, but in the hatching troughs they can be protected until several days old and better able to care for themselves. The eggs are collected by brushing them from the abdominal appendages of the females. A female a foot long may have over ten thousand eggs, and giant specimens over eighteen inches long have been found with more than 150,000 eggs. 
Next to the lobster, the crabs are important as articles of human food. The blue crab is the favorite. Spider crabs and fiddler crabs are not used as food. The "soft-shelled" crabs of our markets are simply individuals which have recently shed their shells (i.e., molted), and the new shell has not had time to harden by deposit of lime salts.

Crayfishes have long been used for food in France and elsewhere on the continent of Europe. More than thirty years ago Huxley wrote that Paris paid over $\$ 80,000$ per year for crayfishes. In the United States they are found in special markets of the large cities. Half a million are shipped annually from the Potomac River. Oregon ships more than 100,000 pounds. The demand is increasing, and it will probably pay to have crayfish farms on which to raise them for market. Land too wet for agriculture might be so used.

The shrimps seen in our markets usually come from the Pacific coast, and the trade in them is worth many hundred thousands of dollars annually. Only the abdomens are commonly seen in the markets, and the bright red color is due to their having been boiled.

316. Other Crustaceans. - Besides the decapods, there are many thousand species of crustaceans, from which we select a few for a brief study which will give us a better appreciation of this type of arthropods.

The wood-lice or sow-bugs, and the similar pill-bugs, which live under stones and logs, are easily kept in a jar containing large pieces of bark. Small bits of bread, butter, apple, and other foods should be placed in the jar occasionally. Keep the bark moist, not wet. The

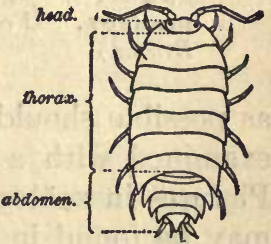

Fig. 123. Sow-bug seen from dorsal side. (From Morse.) most interesting adaptation of these animals is for breathing. The appendages of the abdomen have been modified into breathing organs and covers for them. They are thus fitted 
for land or terrestrial life, while most crustaceans have gills for aquatic breathing.

Lowest Crustaceans. - Most fresh-water streams and ponds contain numerous species of the smaller and lower crustacea known as copepods and water-fleas. These are very important in the food-supply of many fishes. As many forms

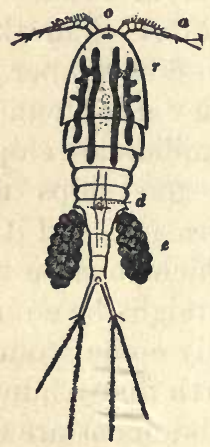

Fig. 124. Dorsal view of a copepod. $o$, ocellus or eyespot; $a$, antenna ; $r$, reproductive organs ; $d$, digestive canal; $e, \quad$ eggmasses. (From Brooks.)

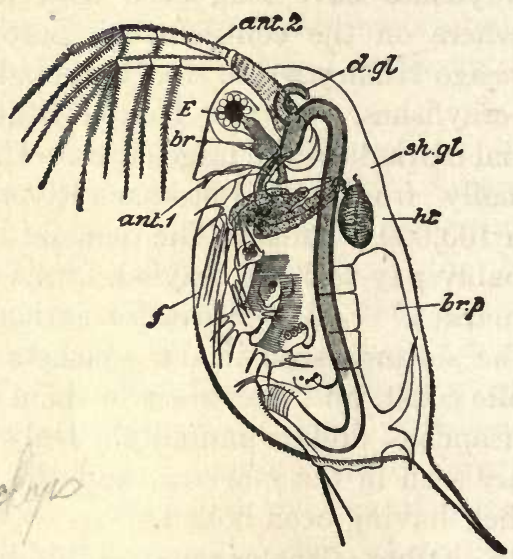

Fig. 125. A water-flea (Daphnia). ant. 1, 2 , first and second antennæ; $f$, feet; $h t$, heart; d.gl, digestive gland; $b r . p$, brood-pouch in which embryos develop. (After Claus.)

as possible should be collected with a fine-meshed net, and examined with a hand-lens and low power of microscope. Pictures in zoölogy books will help to identify those which may be found in pond water.

Barnacles. - The most remarkably modified of lower crustaceans are the barnacles (Fig. 126) which are attached to floating sea-weeds, timbers, and bottoms of ships, and the closely-related rock-barnacles or acorn-shells which cover rocks between high- and low-tide marks at the sea-shore. The barnacles were formerly supposed to be long-necked 
clams, and are still so placed in the popular classification of many fishermen. About 1830 a study of the developing barnacle eggs showed that each egg forms a small triangular larva (nauplius) with three pairs of legs (Fig. 127). In this condition very many crustaceans hatch. This barnacle larva swims for a time and finally settles down on a floating object (or perhaps a rock in case of the rock-barnacles), and metamorphoses into a barnacle. The larvæ literally swarm in tropical waters, and passing ships get their hulls coated with myriads of barnacles. These may grow to be several inches long during a voyage of a few

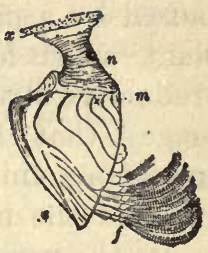

Fig. 126. A goosebarnacle. $x$, piece of wood to which the animal is attached; $n$, neck ; mouth is opposite $m ; s$, bivalved shell ; $f$, feet which move towards the mouth $(m)$. (From Smith.) months; and such a growth, of course, greatly impedes the vessel.

A strange thing about the barnacle is that the point of attachment to rocks and timbers is the

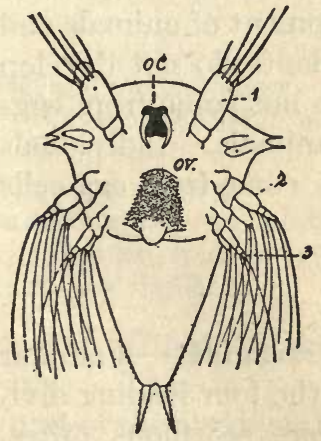

Fig. 127. The nauplius stage of a barnacle. oc, eye-spot; ov, beginning of the ovary; $1,2,3$, the three pairs of legs present at hatching. (From Hertwig-Kingsley.) head; and this led Huxley to define a barnacle as " a crustacean fixed by its head and kicking its food into its mouth with its legs." This is literally true, for the long legs projecting from between the valves of the shell do drive food towards the mouth.

Certain barnacles become attached to crabs, and develop root-like processes which penetrate the tissues of the crab and absorb nutriment. Then the barnacle degenerates until it is nothing but a sac-like tumor on the body of the crab, and the sac contains only the reproductive organs of the barnacle. Such extreme degeneration 
caused by a parasitic mode of life reminds us of the dodder plant, which loses its roots and leaves, and has only its reproductive structures (flowers) fully developed. Such cases suggest that the reproductive function is the all-important one among animals and plants; and it certainly is true that many of the most remarkable adaptations are arranged to secure the perpetuation of a species.

Some of the barnacles which are attached to floating timbers are familiarly known as "goose barnacles." The name originated in Great Britain many centuries ago when it was generally believed that a certain kind of wild goose did not develop from eggs, as do ordinary birds; but that the barnacles along the shore changed into small goslings. Hence, they were named "goose barnacles." This interesting myth was long ago exploded when the life-histories of both the goose and the barnacle became known. Even if we did not now know how these two particular animals develop, we would refuse to believe the barnacle-goose story, unless verified by critical investigation, because it is so well known that "like produces like" in the embryonic development of animals and plants. Every one knows that corn plants do not develop from beans or other seeds, and frogs do not come from eggs of fishes, birds, cats, or other kinds of animals. Individuals of each kind of animal or plant must come from egg-cells formed by one of the same kind.

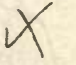

\section{ARACHNIDS}

317. The Spiders and their Allies: Arachnida. - Familiar examples of this group, which is one of the four leading divisions or classes of Arthropoda, are spiders, scorpions, mites, ticks, and the king-crab. Any common spider illustrates the most characteristic structure of the animals of this group.

318. Study of a Spider. - $(L)$ Note that the body consists of a cephalo-thorax (head-thorax), and a large abdomen; in this respect compare with crayfish. 
Appendages: No antennæ. Compare the eyes (6 or 8) with those of erayfish. The anterior mouth-appendages are the mandibles (or cheliceræ), the posterior ones are maxillæ (or pedipalpi). Each maxilla has a long feeler (palpus). The mandibles are hollow and at their tips are the openings of the poison-glands. How many walking legs? Compare number of legs with those of crayfish. Are there appendages on abdomen?

In some species of spiders transverse bands on abdomen are supposed to indicate former segmentation. This may be seen in a scorpion. On ventral side of abdomen: (1) External openings of lungsacs are, in common spiders, one on either side of anterior portion of abdomen. (2) Between these are the openings of the reproductive organs. (3) Two or three pairs of papillæ (spinnerets) are at posterior end of abdomen - note that these are segmented like legs. (4) A spiracle (breathing pore) is just in front of the spinnerets in some spiders. (5) The posterior opening (anus) of the alimentary canal lies behind the spinnerets.

Make drawings of spider from dorsal and ventral views.

Observe the habits of living spiders in fields and in vivaria. Keep spiders' eggs under observation until hatching,

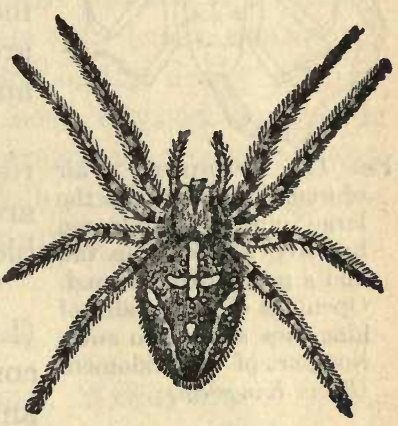

Frg. 128. Common spider (Epeira diadema). Note four pairs of legs, while insects have three. The abdomen is much larger than the cephalothorax. (From Parker and Haswell.) and then observe habits of the young, especially spinning.

How are spiders distinguished from crustacea? In what respects are they similar?

319. Other Arachnids. - The scorpions live in warm countries. They have a sting at the end of the "tail" (which is part of the abdomen), and they use it for poisoning their prey, chiefly insects and spiders. Some of the large ones, six to eight inches long, can seriously poison man, and the sting of even the small ones is painful. This general form (see Fig. 129) suggests a similarity to a lobster; but the large pincers of the scorpion correspond to the jaws or mandibles of crus- 
taceans and insects. When the animal is alive the "tail" is carried over the back. The mouth is very small and the scorpion must suck blood from its prey. All the body back

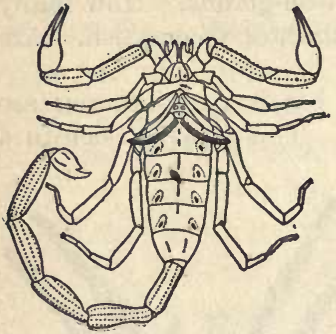

Fig. 129. Scorpion. Pair of mandibles between the large pincers. Abdomen has five segments in tail and a poison-sting at end. Openings of four pairs of lung-sacs shown on anterior part of the abdomen. (From Kingsley.)

southern coast of Asia. of the walking legs belongs to the abdomen. They do not spin webs, as do spiders.

Mites and ticks have the same appendages as have spiders. No segments can be seen. Many of them are parasites on skin of domesticated animals and sometimes on men.

The daddy-long-legs, or harvestmen, resemble long-legged spiders. They are perfectly harmless, and suck the blood from small insects.

The king-crab or horse-shoe crab (Limulus), found along the Atlantic coast, is the last of an ancient race of animals. Another species lives on the The fossil Trilobites were related to the king-crabs which lived in the past ages. Many points in their structure show that the king-crabs are related to scorpions. The spine of the king-crab has no sting.

320. Economic Relations of the Arachnids. - The spiders, scorpions, and daddy-long-legs prey on insects, many of which are injurious to useful plants or in other ways harmful from the human point of view. The king-crabs are not known to have any important harmful relations. Their flesh is not inedible, as is commonly assumed because they are related to spiders and scorpions; and in fact is said to be used to some extent in making a " chowder" in some fishing villages.

Most important of the arachnids in their direct economical relations are the mites and ticks which, as parasites on the skin, irritate and suck blood and thus interfere with the health 
of farm animals. They also, in certain cases, carry the germs of dangerous diseases (e.g., Texas fever of cattle).

The itch-mite which burrows in human skin is a member of this group. The mange of dogs is caused by another mite. The remedy against all the mites and ticks which are skin parasites consists in covering the skin with soap, ointments, or solutions containing sulphur, tobacco, lime, or other substances which are poisonous to these animals. In treating the skin of sheep, cattle, and other farm animals, it is usually most convenient to dip the animals into tanks containing the solutions. Special machines for lifting heavy animals into and out of such a bath have been devised, and sheep in large numbers are often driven through a narrow pathway which leads into a long tank containing hundreds of gallons of the solution through which the animals must plunge.

\section{MYRIOPODS}

321. Centipedes and Millipedes: Myriopoda. - These are worm-like in form, but have the hard exo-skeleton and jointed appendages which are characteristic of arthropods. The segments of the body are similar, and each bears one or two pairs of appendages (one pair in centipedes, two in millipedes). They breathe by means of much-branched airtubes (tracheæ) which open by pores on the surface and ramify through the tissues, thus conducting air directly to the cells.

Centipedes have poison glands, and the bite of some large tropical species is fatal to small animals and causes great pain to men. The bite is inflicted by the hooked ends of the first feet. The common house-centipede is harmless, and destroys insects (see a special circular of United States Department of Agriculture). There is no reason to fear the ordinary centipedes of temperate climates, and the millipedes (" thousand-legs") are also harmless. 
In order to make observations on the habits of myriopods, collect them from under logs and stones in early autumn, and keep in closed boxes with pieces of bark, chips, and leaves under which they can conceal themselves. Keep moist, not
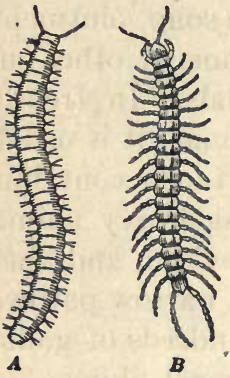

Fig. 130. Two myriopods. $A$, milliped with two pairs of legs on each segment (really two combined segments). $B$, a centipede. (From Thomson.)

wet. Feed with flies, earthworms, or bits of fresh meat for centipedes; various vegetable foods for millipedes.

That myriopods are closely related to insects is shown by many points of structure, both external and internal. But they are evidently lower than insects, for they have no wings and the body does not show differentiation into head and thorax.

References : Davenport's "Zoölogy," Chapter V. Jordan and Heath's "Animal Forms," pp. 111-113.

\section{INSECTS}

322. Prominence of Insects. - Aside from the domesticated animals which belong to the higher vertebrates (birds and mammals), the insects are of more importance in economic relations than are all other groups of animals taken together. But quite apart from practical matters, the insects have long keen favorite objects for study because many are beautiful, many have remarkable adaptations to special conditions of life, and many have wonderful instincts and nervous activities which are surpassed only by certain birds and mammals.

More than half of the known species of animals are insects. From 200,000 to 250,000 species are now known, and many newly-discovered species are named and described each year. Some experts in entomology (that division of zoölogy which deals with insects) believe that there may be living now a million species of insects.

It is a fortunate fact for students of insects that they are all 
built on the same plan of structure, and that a study of a few selected specimens will enable one to understand almost any other insect which may be seen. For such type studies the grasshopper and butterfly will introduce the chief principles of insect structure and life.

323. Study of a Grasshopper. - $(L)$ This animal consists of body and appendages. Notice three regions of the body, - head; thorax, with three pairs of legs; and a segmented or jointed abdomen. Is the grasshopper bilaterally symmetrical? Notice that the body and appendages have a hard covering, the exo-skeleton.

Abdomen. - The tip of the abdomen varies in the two sexes. In the female the abdomen bears two pairs of pointed structures which are of use in depositing the eggs, and together

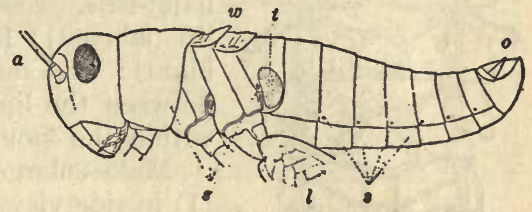

Fig. 131. Grasshopper. $a$, antenna: $w$, wings; $t$, tympanum or ear-membrane ; $o$, ovipositor; $s$, spiracles or breathing pores; $l$, base of leg. (From Hatschek.)

they are known as the ovipositor. The end of the abdomen in the male is turned upward. Count the rings or segments in the abdomen. On the first ring next to the thorax there is on either side a shining oval patch, the organ of hearing. Along each side of the abdomen is a groove, and just above it is a row of pores (spiracles or breathing pores). Watch the breathing movements of the abdomen of a living specimen. The spiracles are connected inside with a system of branched air-tubes (trachea), which ramify through the body and distribute air directly to the tissues. Compare this method of respiration, which is characteristic of insects, with that of the frog.

Thorax. - Judging from the pairs of jointed appendages (legs), how many segments in the thorax? How many pairs of wings? Are they attached to first (anterior), second, or third segment?

Look for the breathing-pores on the second and third segments.

Examine the two wings and compare them as to form, size, texture, color, position, and use. The veins in the wings are hollow tubes which carry blood and air.

The characteristic shrill sound made by katydids is caused by rubbing the upper wing on the lower wing.

Compare legs from each segment of the body which bears thom. 
How many parts in a leg? Are they similar? Observe the hooks and pads on the feet - these are used in clinging when the animal is at rest. Notice how the legs are adapted for jumping.

Head. - The head is attached to the thorax by a soft neck. Examine the large eyes with a hand-lens, and with a microscope examine a slice from one of the eyes. Like the paired eyes of the crus-

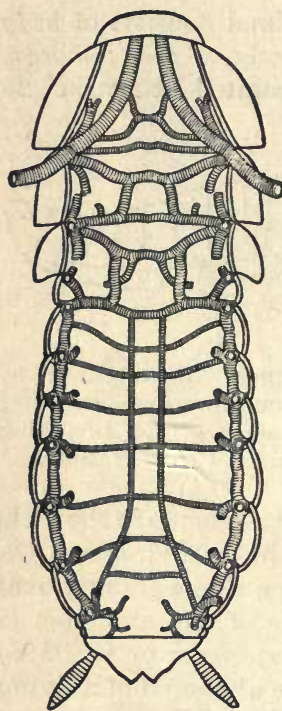

Fig. 132. Arrangement of the larger tubes of the air-tube or tracheal system of a cockroach. (After Miall and Denny.)

taceans, those of insects are compound eyes. Also, several bead-like simple eyes (ocelli) are in many insects situated on the head between the compound eyes (use hand-lens). Examine the long slender feelers (antenna) with hand-lens. Examine the mouth-parts : upper lip (labrum); lower lip (second maxillæ, labium); first maxillæ, and jaws (mandibles) between the lips; and a tongue-like organ within the mouth.

Make enlarged drawings of a grasshopper : (1) in side view with wings in closed or resting position, (2) of front view of head, (3) of a leg, (4) of side view of abdomen.

Examine young grasshoppers of various sizes. How do they differ from the adult?

Observe method of locomotion, taking of food, kinds of food, and breathing movements of grasshoppers. When opportunity offers, carefully observe them in the fields and record your observations.

Read the "Molting of the Grasshopper," Chapter IX in Weed's "Life Histories of American Insects."

Grasshoppers are called locusts in the old world, but rarely in America. The socalled seventeen-year locusts are really cicadas, belonging to an entirely separate order of insects (the Hemipterans).

324. Study of Butterfly, or Moth. - $(L)$ Examine a butterfly with regard to the points of structure already seen in a grasshopper. The chief differences to be observed are: (1) Form and size of wings and presence of scales (use hand-lens and microscope). On some butterflies and the related moths the scales are hair-like.

(2) Legs of butterfly are adapted for clinging, not jumping.

(3) Antennæ are club-shaped on a butterfly, feather-like on a moth. 
parts are adapted for sucking nectar of flowers. There are no jaws for biting solid food, but a long coiled tube consisting of two halves closely applied together is the sucking organ. Obviously the butterfly is, on the whole, built on the grasshopper plan of structure.

Larva. - Most remarkable about butterflies and moths is their peculiar development from egg to larva (caterpillar), then to pupa (which may be in a silky cocoon), and then to perfect insect (imago).

The larva called

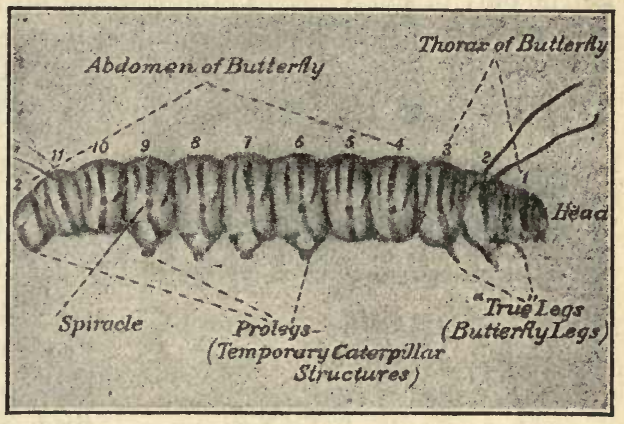

Fig. 133. Butterfly larva. (From Dickerson's "Moths and Butterfies," Ginn \& Co.)

"tomato-worm" (which develops into a hawk-moth) is excellent for study. Note: (1) Head, and its parts. (2) Thorax, with three segments, each having a pair of legs. (3) Posterior to these some

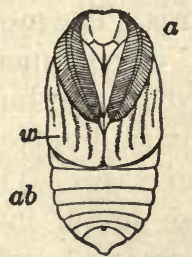

Fig. 134. Pupa of moth. $a$, antenna case ; $w$, wing case ; tongue and leg cases between antennæ; $a b, \mathrm{abdo-}$ men. Kingsley.)

segments have no legs, and then come some segments with peculiar legs adapted to clinging to twigs (prop legs). (4) A curved spine at posterior end. (5) Spiracles or breathing pores along the sides of the body. (6) Color markings in living specimens, if available.

Pupa. - The pupa formed from the tomatoworm is buried in the soil and difficult to find. Pupa from cocoons of Cecropia moth, or other large moths, may be used. The cases or covers of various organs, as shown in Fig. 134, may be seen. Identify the covers of the tongue, antennæ, legs, eyes, and wings. Note spiracles on sides of the body. Examine the movable segments of the abdomen.

The fragments to be found in a cocoon at posterior end of a pupa are from the skin which was molted after the cocoon was spun. The larva molts several times before it is full grown and ready to change to pupal stage.

Cecropia moths will emerge in February or March from cocoons 
kept in a schoolroom, and much later if left outdoors. The habits of the living moths should be observed. They will live but a short time, for their alimentary canal is too imperfect to use food. This is true only of some species of moths and butterflies.

Larva, pupa, and adult of common butterflies and moths can be identified by the figures in Dickerson's "Moths and Butterflies,", which also gives very readable accounts of the life-histories.

325. The Reproductive Processes of Insects. - The eggs of the simplest and lowest insects (e.g., the silver-moth, Fig. 136) form young which at hatching are like the adults except

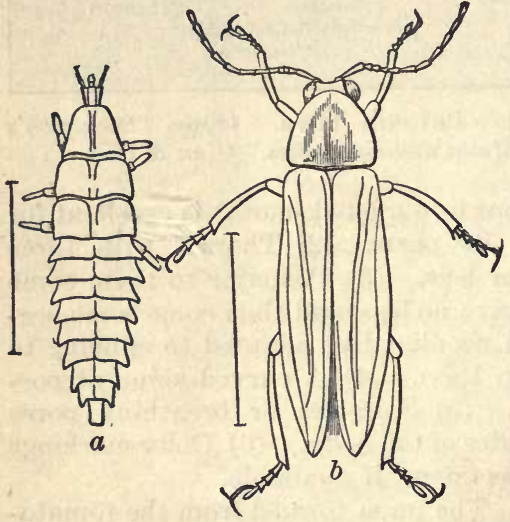
in size. The young of grasshoppers and numerous other species are at first without wings, and they go through a number of moltings before they become adults. This condition is called gradual metamorphosis, or incomplete metamorphosis. The most complicated development is represented by the butterflies, moths, beetles, flies, bees, and wasps. The Fig. 135. Firefly. Larva $(a)$ and adult eggs of these hatch into (b). Really a fire-beetle, for it is not worm-like larvoe (popularly
closely related to flies. (From Packard.) called caterpillars, grubs, maggots). These larvæ are voracious feeders, and usually grow rapidly to full size. Then while transforming from the larva to the adult or imago stage, they undergo a period of quiet in the pupa stage. In the butterflies the pupa stage is often called a chrysalis. The larvæ of some insects, like silkworm, spin a protective cocoon around themselves as they pass into the pupa stage. After a period of quiet, during which vast internal changes (complete metamorphosis) are taking 
place, the pupal case bursts and the perfect insect emerges in full-grown state.

Insects which have larva and pupa stages do not grow in the winged stage. It is popularly believed that the small flies seen around houses are young ones; but the truth is that they are adults of small species.

Many insects live longer in the larval than in the adult stage. Some of the May-flies (also called day-flies and ephemerids) live only a day or two as perfect insects. Vast numbers emerge in the morning, soon lay their eggs, are unable to take food, and perish before the next day. The seventeen-year cicadas (erroneously called "locusts," for this name belongs to grasshoppers) spend the greater part of the seventeen years as larvæ attached to roots of trees beneath ground. In the seventeenth summer they come to the surface, soon pass through the pupal stage, and then emerge as perfect insects. A few of them may remain for a number of weeks, but vast numbers lay their eggs in the twigs of trees and die within a few days after becoming adult insects. The larvæ which hatch from the eggs deposited in the twigs fall to the ground, burrow, and remain in the soil for nearly seventeen years. A thirteen-year variety occurs in southern states.

These insects which have complete metamorphosis are regarded as the highest. The complicated life-history has many advantages. The larval or growing stage is often able to conceal itself and to reach food which perfect insects could not get; for example, the larvæ of numerous species of beetles, of the cicadas, and of flies. In many cases it is possible for the adult insect to supply food for the larvæ; for example, bees supply food to the larvæ in the honeycomb, ants feed and care for their larvæ, some wasps paralyze other insects by stinging and then place them as food for wasp larvæ, and the eggs of many parasitic species are laid in other insects. Still another advantage to some insects is the fact that their larvæ easily withstand the winter storms (examples 
are many moths with cocoons). Also, there is an advantage in that the larvæ live under conditions different from the adult insect; for example, numerous larvæ feed on foliage which the adults never frequent except for laying eggs. These are some of the apparent advantages which have come to insects with a complicated life-history consisting of four stages, - egg, larva, pupa, and adult; and it certainly has been worth while for insects to develop complete metamorphosis in place of such direct development of eggs into miniature adults as occurs in the simplest wingless insects and also in the not distantly related centipedes. No one knows why and how insects have acquired such complicated life-histories; but the known facts have convinced all entomologists that they all once had life-histories even simpler than the grasshopper now has, and that there has been developed greater complexity by introduction of larva and pupa which have certain advantages in that they adapt insects to peculiar conditions of life, as suggested above.

Parthenogenesis. - A peculiarity of the eggs of some insects is that they develop without fertilization. This is called parthenogenesis. Throughout the summer months the individuals of certain plant-lice are all females, and their unfertilized eggs develop into females. There may be many such parthenogenetic generations in a summer. In the autumn both males and females develop from unfertilized eggs, and fertilized eggs are produced which survive the winter and develop into females in the spring. These begin a new series of parthenogenetic generations which continues until the next autumn.

Honey-bee drones (males) develop from unfertilized eggs, and the workers (undeveloped females) and "queens" (mature females) are from fertilized eggs. Thus mating a black drone and a yellow "queen" will result in half-blood or hybrid workers and young "queens," but yellow drones.

Other cases of parthenogenesis are found among insects and 
in some low crustaceans. In some species of gall-insects and scale-bugs males have not been found; and permanent parthenogenesis is supposed to be the rule. This is not known to occur in any other group of animals; but there are well-known cases of flowers whose egg-cells always develop without fertilization. Parthenogenesis of both plants and animals is interesting because sexual reproduction is usually provided for in all types of organisms.

326. Classification of Insects. - The group Insecta is a class, and it is divided into about twenty orders. Most of these, however, are not represented by many common insects, and so will be omitted from the following account.

In classifying insects, as all other animals, it is necessary to consider all points of external and internal stucture in order to determine which species are most alike; but it is a fortunate fact that it is often possible to identify by means of some one structure. In the case of many insects, the wings happen to be the convenient parts for general classification; but similar wings should not be taken as indicating close relationship if other organs are not homologous (i.e., of corresponding structure).

The most common insects in United States are conveniently grouped in the following orders:-

Aptera. - Name means without wings. Example: the "silver-moth" or " silverfish" (Fig. 136) and the "spring-tails." These are the oldest insects now living. They are primitively wingless; that is,

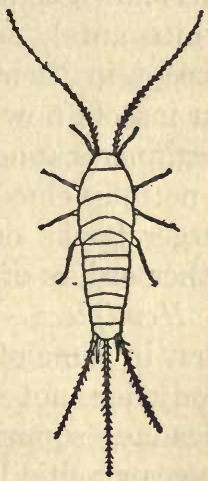

Fig. 136. $A$, " silver-moth," Lepisma. A modern representative of the ancient primitive insects which had three pairs of legs but no wings. there is no evidence that their ancestors had wings. Fleas are secondarily wingless, because of degeneracy caused by parasitism. Entomologists conclude that the ancestors of 
fleas had wings because embryo fleas show the beginning of wings. Hence, such degenerate insects are not apterans, but belong in a higher order.

Orthoptera. - Name means straight wings, referring to main veins of the wings, or to the way they fold together. Examples: grasshoppers, crickets, katydids, stick-insects, cockroaches, mantis, earwig. Two pairs of wings, similar to those of grasshopper. Mouth-parts for biting. At least 10,000 species are known.

Netted-winged Insects. - Old books recognize an order Neuroptera for all insects which have two pairs of nettedveined wings like the dragon-fly and May-fly; but study of their other organs and especially of their embryonic development has shown that dragon-flies, May-flies, termites (often called white ants), and others with netted wings are not similar except in their wings. The netted-veined wings are found on insects now grouped in five or six orders. However, the beginner cannot do better than to use the popular name "netted-veined," and for further information consult the larger books on insects for descriptions of Neuroptera and other orders of insects which exhibit this kind of wings.

Hemiptera. - Name means half wings, referring to the fact that in some of these insects about half the wing next to the body does not show distinct veins. Examples: all true bugs, cicadas, squash-bug, box-elderbug, "stink-bug," chinchbug, parasitic lice, bed-bug, water-bug, cochineal bug, plantlice, scale-bugs. Four wings, overlapping when folded. Mouth-parts for piercing. Incomplete metamorphosis. About 20,000 species.

Lepidoptera. - Name means scale-wings, referring to overlapping scales. Examples: butterflies and moths. Two pairs of wings, covered with flat or hair-like scales. Mouthparts for sucking. Complete metamorphosis. Butterflies usually fly in daytime and have slender antennæ with knob or club at end. Moths are nocturnal and have feather-like 
antennæ. Over 50,000 species of Lepidoptera are known. Consult Dickerson's "Moths and Butterflies" ; Comstock's "How to Know Butterflies"; and Holland's "Butterfly Book" and "Moth Book."

Coleoptera. - Name means sheath wing, referring to the hard anterior wings. Examples : all beetles, - weevils, fireflies, June beetles, blister beetles ("Spanish flies"). Some so-called bugs, e.g., lady-bird " bug " and June "bug," are beetles and not bugs (i.e., not Hemiptera). Front wings of beetles are hard and horny, and often called the elytra. Hind wings are membranous. Complete metamorphosis. It is estimated that over 100,000 species of beetles have been named.

Diptera. - Name means two wings; hind wings absent. Examples: flies, bot-flies, warble-flies, mosquitoes. Mouthparts for sucking (as in house-fly) or piercing (as in horse-flies). Complete metamorphosis. Larvæ commonly called " maggots." Small knobs represent the hind wings. About 40,000 species are named.

Fleas. - Parasitic insects, wingless or with rudimentary wings. Formerly considered wingless flies, but now in a special order.

Hymenoptera. Name means membrane wing, e.g., a bee's delicate wing. Examples: ants, bees, wasps, ichneumons. Mouth-parts for both biting and sucking. Complete metamorphosis.- Four wings, hind pair smaller, few irregular veins. Probably about 30,000 species known.

Practice in Classification of Insects. - $(L)$ Students should examine a set of insects (a mixed lot preserved in wood alcohol or formalin is best); and by comparing with above descriptions, and the well-known examples mentioned, assign the specimens to the proper orders. In an hour of time one can learn to identify as to orders the most common insects. The names of genera and species of the very common insects ean be found in the large books, such as Comstock's "Manual of Insects," but rarer specimens can only be identified by specialists. 
327. Useful Insects. - Honey-bees and silkworms are the only truly domesticated insects. (See Shaler's "Domesticated Animals.") A few others are directly useful for their products, - "Spanish flies" (used in medicine), cochineal bug (cultivated on cactus for the dyes cochineal and carmine which their dried bodies yield), and the lac-insect (which produces the valuable shellac used in varnishes). Some natives of Africa, Australia, and Mexico eat certain insects.

As agents in cross-pollination of flowers insects as a group are worth vastly more than they destroy; but it happens that many of the very destructive insects do not visit and pollinate flowers. Our studies of plants have made it clear that many of our mest useful plants depend upon pollination by insects, e.g., clover, alfalfa, fruit trees, most vegetables. The Smyrna fig is now successfully cultivated in California because an insect imported from Algeria pollinates the flowers.

Some insects are valuable as destroyers of injurious insects. Numerous insects have their insect enemies, some predatory and some parasitic. As an example of predatory insects may be mentioned the history of the fluted scale-bug which once threatened to destroy the orange groves of California. The scale originally came from Australia, and there the entomologists of the United States Department of Agriculture found a natural enemy in a species of lady-bird beetles. Some of these beetles were imported to California and in a few years practically exterminated this species of scale-bug. Specimens of the beetles sent later to other countries have been as successful in ridding orange and lemon trees of the destructive scale-bug. This is one example of the usefulness of a predatory insect. Many with similar habits are constantly keeping harmful insects in check.

Parasitic insects are important .checks on injurious insects. A large number of species of insects belonging to the Diptera and Hymenoptera are parasitic during their larval stage. The caterpillars of many moths and butterflies are frequently 
parasitized by larvæ of certain flies. The ichneumons (Hymenoptera), of which more than ten thousand species are named, are famous parasites. Some of these insects have ovipositors three and four inches long and are able to bore deeply into trees in order to lay their eggs in the larvæ of another insect. "Tomato-worms" (larvæ of hawk-moth) and similar moth larvæ are often seen with their skins covered with small white cocoons. The parasitic larvæ live inside the moth larvæ and crawl out to the surface of the skin when ready to pupate. The result in most cases is the death of the parasitized caterpillars.

The above are simply illustrative examples chosen from the thousands of cases in which insect parasites destroy other in-

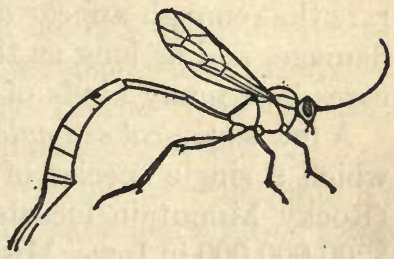

Fig. 137. Ichneumon-fly. One of a group of insects whose eggs are laid, by means of long ovipositors, in the larvæ of other insects. (From Packard.) sects. In all such cases where the insect host is harmful the parasite is beneficial.

Finally, it must be mentioned that insects are useful as foods for numerous species of birds. True it is often stated that the birds are useful as destroyers of insects; but intelligent people are beginning to recognize that many birds would be useful and well worth supporting for æsthetic purposes even if there were no injurious insects to be destroyed. Aside, then, from the great outbreaks of certain insects, a limited number of them are desirable as food for interesting birds.

Also, it should be mentioned that many fishes eat large quantities of insects, both adults and larvæ. This is why imitation insects are made as bait for trout and other fishes.

328. Injurious Insects. - Those insects which injure or destroy useful plants and animals, or organic products (e.g., foods) which are of value to man, or which injure man him- 
self (as by infecting with malaria), are conveniently grouped as injurious insects. The truth is that a very large proportion of insect species tend to be injurious, but usually do not attract attention unless they become excessively numerous, or when man develops a special interest in a particular animal or plant. For example, probably more than eight hundred species of insects attack oak trees; but it is rare that enough appear on any one tree to do any noticeable damage, and as long as there are plenty of oak trees no one cares how many kinds of insects live on them.

A few statistical estimates will give some idea of the damage which a single species of insect can do. The grasshoppers (Rocky Mountain locusts) destroyed crops to the value of $\$ 200,000,000$ in Iowa, Missouri, Kansas, and Nebraska in four years, 1874-1877. Special pamphlets of the United States Department of Agriculture record the enormous damage done by chinch-bug on cereal plants, by Hessian fly on wheat, by scalebugs on fruit trees, by gypsy moth on forest and fruit trees, by cotton-boll weevil, and by numerous others which do great but less damage then those mentioned. Famous entomologists have estimated that insects damage farm crops in United States annually to the extent of $\$ 300,000,000$. Insect damage to valuable forest trees is on good authority estimated at $\$ 100,000,000$, yearly. Add to these figures the enormous loss of animals through disease caused directly or indirectly by insects; the destruction of clothing, foods, and other useful articles; the value of the working time and expense of treatment of people who are ill through disease caused by insects $(\$ \S 329,330)$; and the total annual cost of insect damage in this country is probably more than the combined cost of the army and navy and public-school system. Such general estimates suggest the immensity of the problem of dealing with the injurious insects.

And yet such statistics must not be taken as a declaration of war against insects indiscriminately, but they simply mean 
that efforts must be made to hold in check the ones which are noticeably harmful. The ordinary insects which one meets during a long walk in the country are not likely to appear in such numbers or to develop such new habits as to be of special economic interest. Hence there is no reason why we should destroy them. On the contrary, this old world is of greater interest because of their existence. As an illustration, katydids and crickets do eat some leaves of grass and other plants, but to many a person who is interested in nature-study a mid-summer night's chorus by these insects is worth far more than the trivial damage they do.

The Control of Injurious Insects. - The investigations by entomologists in the past fifty years have made great progress towards controlling injurious insects. Knowledge of habits and life-histories have made it possible to prevent destruction of crops. Here are a few from hundreds of examples recorded in the large works on economic entomology: The discovery that the fruit-moth (codling moth) lays its eggs in the calyx of apple flowers after the petals have fallen suggested the desirability of spraying the trees with arsenical poisons before the larvæ hatch and burrow into the fruit. Grasshoppers lay their eggs a few inches below the surface of the soil, and hence shallow plowing in the autumn will expose the eggs to the winter storms. The scale-bugs and plantlice live by sucking sap from plants, and hence poisons like arsenicals could not reach their stomachs, and the logical conclusion is that they ought to be sprayed with lime, sulphur, or petroleum, which kills by contact. These cases are simply illustrations of the fact that all the satisfactory methods of dealing with injurious insects are based upon careful biological study of the species in question.

329. Mosquitoes and Diseases. - Careful investigations made in recent years have proved that certain insects are responsible for transmission of disease germs. The most famous case is that of mosquitoes of the genus Anopheles 
(Fig. 138), which inject the malarial organism ( $\$ 274)$ into the human blood-system. Numerous experiments have made it certain that one of these mosquitoes must first suck blood from a person who has malarial parasites in his blood, and it is now equally certain that a bite from such an infected mosquito is the only way by which malaria can be acquired.

It is also quite certain that another species of mosquitoes is responsible for transmission of yellow fever, the germ of which is still unknown.

The discovery that mosquitoes are thus connected with certain dreaded diseases has led to a study of the life-histories and habits of these insects, with a view to reducing the number of cases of malaria and yellow fever. The following rules are now agreed upon by competent entomologists.

(1) Multiplication of mosquitoes should be checked by destroying their breeding places; for example, by draining swamps, ponds, and other places containing stagnant water. Even a rain-water barrel, open cistern or tank, buckets, empty fruit-cans, cavities in trees and stones,- in short, any place where a small quantity of water stands for a few days may serve as a breeding place, producing thousands of mosquitoes. As far as possible all such places should be arranged for permanent drainage, and others, such as cisterns and barrels, should be tightly covered or screened with netting so as to exclude mosquitoes which are about to lay eggs.

(2) The larval stages of mosquitoes should be destroyed when it is impracticable to follow the rule above. Many streams, lakes, etc., cannot be drained; but stocking them with fishes will result in destruction of most of the mosquito larvæ. It is important in such cases that the banks of the streams be freed from rubbish and graded so that there will be no small depressions in which mosquitoes may lay their eggs safe from the attacks of fishes.

Oftentimes temporary relief, pending permanent drainage, may be gained by spraying crude petroleum on the surface of 
Culex

$\mathbf{a}$

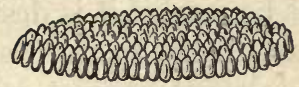

$b$
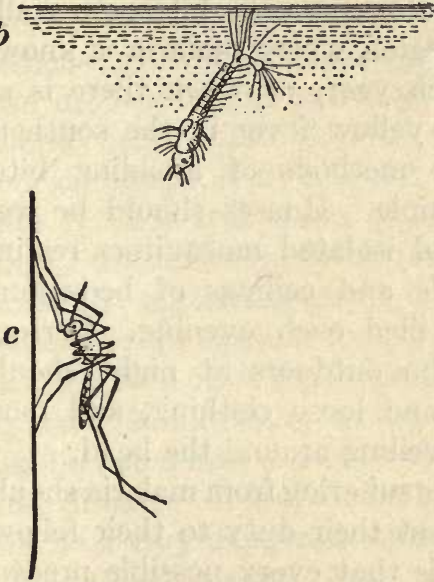

d
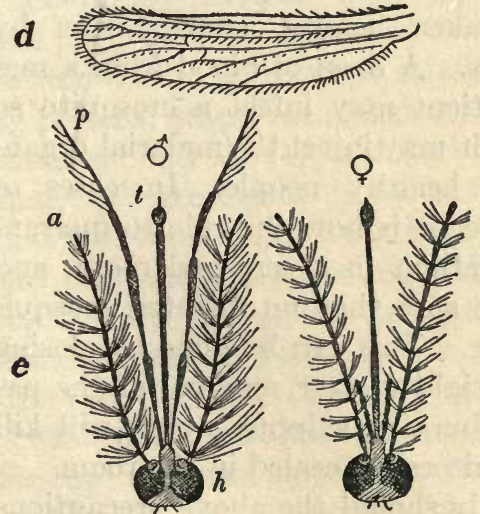

Anopheles
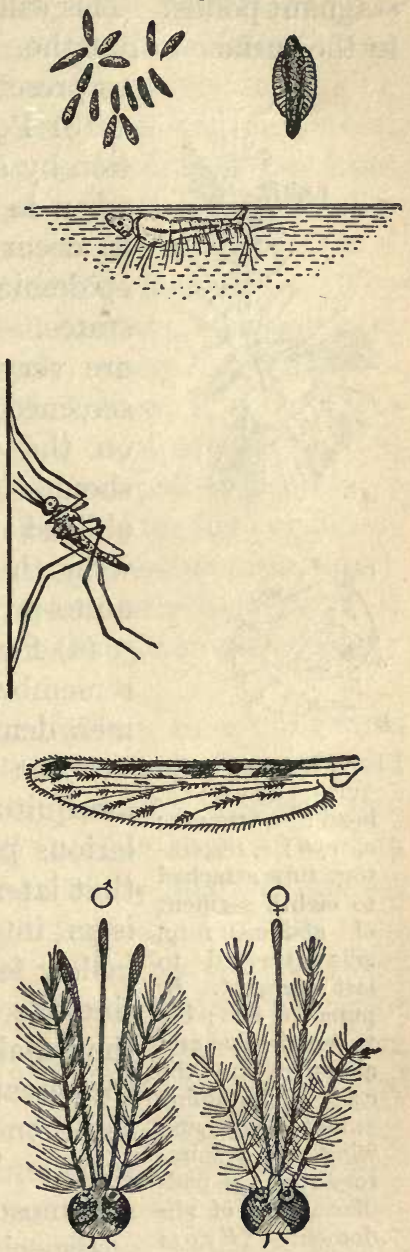

Fig. 138. Comparison of malarial mosquito and common culex. a, eggs; $b$, position of larvæ at surface of water; $c$, position of resting adults; $d$, wings ; $e$, head appendages - $a$, antenna : $p$, palp ; $t$, tongue. Compare lengths of palps of the females. (From Jordan's "Bacteriology," after other authors.) 
stagnant ponds. This will destroy the larvæ when they come to the surface to breathe. Oil cannot be used if it is desired to preserve plants and fishes in the water.
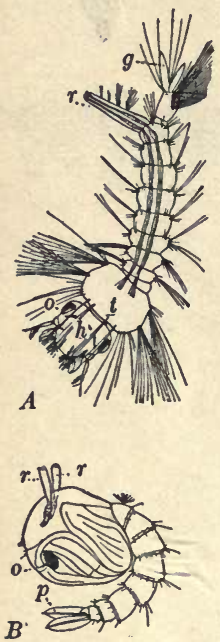

Fig. 139. $A$, mosquito larva; $h$, head; $t$, thorax; $o$, eye ; $r$, respiratory tube attached to eighth segment of abdomen; $g$, gills attached to last segment. $B$, pupa ; $o$, eye ; between the eye and abdomen are the cases containing antenna, legs, and wings; $r$, respiratory tube ; $p$, paddles at end of abdomen. (From Folsom.) mosquitoes are common. Hence, it is important that this species be easily identified. See Fig. 138.

(3) People should guard against infection by avoiding mosquito bites, especially when in a region where malaria is known to occur each year, or when there is an epidemic of yellow fever in the southern states. The methods of avoiding bites are very simple. Houses should be well screened, and isolated mosquitoes resting on the walls and ceilings of bed-rooms should be killed each evening. Persons obliged to be outdoors at night should wear thick and loose clothing, and mosquito-proof veiling around the head.

(4) Persons suffering from malaria should remember that their duty to their fellowmen demands that every possible precaution be taken against being bitten by mosquitoes. A drop of blood from a malarious patient may infect a mosquito so that later it may inject the malarial organisms into healthy people. In cases of yellow fever it is now the rule to quarantine the patient in a screened room, and then make sure that no infected mosquitoes escape. This can be done by closing the room tightly after removal of the patient and burning sulphur which will kill any mosquitoes concealed in the room.

Especially should the above precautions be taken against malaria when Anopheles

A 
330. Flies and Disease. - Probably more important than mosquitoes as carriers of disease germs are the common houseflies. It is a well-known fact that these flies persist in walking on food, and long before disease germs were known, careful housewives made strenuous efforts at keeping them from kitchens and dining-rooms. Recent bacteriological studies have disclosed some startling facts which should lead to a general declaration of war against the house-flies. The facts are these: A fly allowed to walk across a sterile gelatin plate ( $\$ 255)$ will leave in its tracks many bacteria previously acquired by walking on filth. Now, if a fly walks on sewage containing germs of typhoid or of other intestinal diseases, or on sputum from a tuberculosis patient, and later walks on food or on dishes ready to be used for food or drinking water, it may leave in its tracks dangerous bacteria, which may be taken into the body with the food or water, and then cause disease. It is obvious that in this way a single house-fly may be a very dangerous animal.

There are several ways of combating this dangerous pest: (1) Manure piles and similar breeding places should be removed. (2) Houses should be carefully screened and fly-poisons, traps, etc., used to kill the few that succeed in entering. (3) All foods should be carefully guarded against flies. (4) Arrangements for sewage disposal should be such that flies cannot distribute bacteria. For this reason sewers and cesspools that discharge into porous drain-tiles below the surface of soil are preferable for country and village homes. (See a pamphlet on "Sewage Disposal," issued (free) by the United States Department of Agriculture.)

331. Other Insects and Disease. - Mosquitoes and houseflies are the most important insects connected with diseases in America, but other insects may likewise affect human health. The germ of the terrible African sleeping sickness is injected into the blood by the bite of a peculiar fly. The bacteria of bubonic plague is probably transmitted by fleas 
which infest rats in whose blood the germs occur. In fact, any biting insect which gets an opportunity to bite any person or animal whose blood contains disease germs may become a carrier of the germs; and any insect which comes in contact with germs and later with human food may indirectly cause disease.

The above account suggests the general relations of certain insects to human diseases. For more facts on this very important subject read the pamphlets on "Mosquitoes" and "Insects and Health" published by the United States Department of Agriculture.

332. Adaptations of Insects. - No other group of animals is so favorable as the insects for study of adaptations of structures to special uses.

Insect legs are adapted to running (cockroach), leaping (grasshopper), walking (fly), grasping (mantis), burrowing (mole-cricket), clinging (moths and butterflies), carrying pollen (bees), and to still other special uses. However, in all these cases the general plan of a leg is similar.

The mouth-parts are adapted to various kinds of foods; biting and chewing (grasshopper, beetles), piercing (mosquito, hemipterans, and some flies), licking (house-fly), sucking (butterflies), and cutting (carpenter-bee).

The wings are commonly well adapted to flying, but in some species (e.g., fleas) wings would be worse than useless organs and have become degenerate. The front wings of beetles are thickened to form shield-like covers for the posterior wings. In protective adaptation of some insects the wings are remarkably leaf-like in appearance.

The form of insect bodies is strikingly modified in many species. Stick-insects (Phasmidæ) are orthopterans in which the body and legs are stick-like and easily mistaken for sticks, twigs, straws, etc., near which these insects live.

Some of the most remarkable adaptations are connected with the larval stages. Larvæ of May-flies and others 
live in water and breathe by means of feathery gills on the abdomen (Fig. 140). The larvæ of mosquitoes have special breathing tubes which can be extended above the surface of the water. Larvæ of lepidoptera which crawl on plants have false legs for supporting the posterior end of the body; but those of flies, beetles, ants, bees, etc., develop in situations where such extra legs are not required and they have only the three pairs of thoracic legs.

In various ways protective structures for larvæ are formed, and organs are specially adapted for such work. Examples are: spinning silky cocoons (by moth larvæ), making honeycomb (by adult bees), making paper (by adult wasps), making cases or tubes from bits of plants or gravel (by caddis larvæ), and rolling leaves to form tubes (by many larvæ).

Aquatic insects are especially noteworthy for their adaptations. In some species oarlike legs are fitted for locomotion by swimming, and others for "skating" on water.

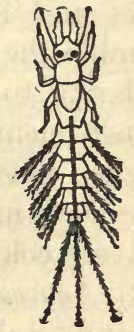

Fig. 140. Larva of May fly, with feathery gills along side of the abdomen adapting to aquatic larval life. (From Parker and Haswell.)

Mosquito larvæ propel themselves by "wriggling" of the abdomen. Some aquatic larvæ (e.g., mosquito pupæ) are so filled with air that they float at the surface and can descend only by swimming. A velvety covering of hairs on the body and legs enables some insects to skate on the surface film of water. The silvery white of the "backswimmers" is due to a layer of air held by delicate hairs. Other aquatic beetles and bugs have air-spaces beneath the front wings. Special insect books describe many adaptations to aquatic life.

Adaptations of insects with respect to color are so important that a separate section of this chapter is devoted to them.

333. Colors of Insects. - Many insects are colored in ways which are apparently useful to them. Such useful 
colors are commonly interpreted as (1) protective, (2) aggressive, (3) warning, (4) mimicry.

(1) Protective coloration is the most common form of useful colors among insects. A green insect on a green leaf is more or less concealed because it so closely resembles its surroundings. Many insects resemble the bark of trees on which they rest, and some both in form and color resemble sticks and leaves. In still other ways numerous insects resemble the objects near which they habitually live. Such similarity to the environment is believed to be protective against enemies. Note that it is not absolutely protective, for such insects are often captured by birds; but probably more such insects escape than would if they were not protectively colored.

(2) Aggressive coloration is the term applied to insects which resemble their environment so that they can lie concealed from their approaching prey. This is found in certain predaceous insects like the mantis, which at the same time are protectively concealed from such enemies as birds.

(3) Warning Coloration.-Many insects are conspicuously colored, and appear to make no attempt at hiding themselves. The common monarch butterfly is an example. The explanation is that such insects often have a disagreeable odor, flavor, or sting which repels their enemies; and hence the conspicuous color is a danger signal.

It has been learned by experiments that insect-eating monkeys soon learn that such a conspicuous coloration is associated with disagreeable things and thereafter will not attempt to catch such insects. This suggests that a species with warning colors would have an advantage in that the comparatively few individuals caught might teach the enemy that such brightly colored insects are not good to eat. It is probable that very many cases of conspicuous coloration in insects are of value as warning colors.

(4) Mimicry. - This means the resemblance of one 
species of insect to another which has warning colors. The American viceroy butterfly is a mimic of the monarch. Young insect-eating monkeys will eat viceroy butterflies, but will not touch them if they have first tasted some unpalatable monarchs. This leads to the view that the viceroy species gains decidedly by resembling and thus living on the bad reputation of the monarch, which has warning colors. In tropical countries there are numerous similar cases where a conspicuous insect which has no special defense against its enemies is similar in appearance to another species whose conspicuous colors are warning signals, advertising boldly to enemies that there is danger.

The word " mimicry" suggests conscious imitation; but, of course, one insect resembles another because it happened to be developed that way. We do not know how the first viceroys came to resemble monarch butterflies, but probably a butterfly appeared which was unlike its near relatives and more like monarchs. This resemblance to the monarchs gave the first viceroy an advantage. Other viceroys developed because " like tends to produce like," which principle of embryology applies especially to the propagation of individual organisms born with some peculiarity. Obviously, any slight advantage gained by the resemblance to the monarchs, which are not so liable to attack as are more edible insects, would have tended towards the continued multiplication of viceroys. It is thus easy to suggest how viceroys might have been preserved and allowed to multiply; but it has not yet been discovered why the first viceroy happened to be so unlike its relatives.

The above explanation of insect colors as protective, aggressive, warning, and mimicry is believed to apply to many insects. It should be understood that these devices for use of colors are not absolutely perfect in their working. For example, green grasshoppers concealed in green leaves are often discovered by birds: but there is reason to believe 
that the green color makes it harder for birds to find the insects, and hence the color gives some protective advantage. Likewise, warning colors are not always efficient danger signals; and mimicry does not always deceive enemies. However, there is probably a decided advantage, on the whole, to species of insects which possess any of these forms of coloration.

334. Colors of other Animals. - Animals other than insects may have useful coloration. Thus fishes, frogs, lizards, and many other animals may have concealing coloration which may work either protectively or aggressively. The white of birds, hares, and other small animals on arctic snowfields is believed to be protective; while the white of polar bears may be aggressive, enabling them to lie in wait for approach of prey. The stripes of tigers are supposed to be aggressive because they harmonize so well with the lights and shadows among the reeds of jungles. Likewise, the tawny color of the lion resembles the sands of the desert.

Some of the most remarkable cases are those of frogs and lizards, which can quickly change colors when placed on new objects, e.g., from green to brown when moved from a leaf to bark of a tree. This is accomplished by a peculiar arrangement of pigment-cells in the skin. Some cells contain green pigment and some have darker colors. When the green cells are expanded so as to expose their maximum surface, and the dark cells are contracted, the animal's skin appears green. Expanded dark cells and contracted green cells make the skin brown in color. Partial expansion and contraction of both kinds of pigment-cells give intermediate shades. The cells are controlled through the nervous system and the eyes.

In the case of those birds and mammals which appear to have concealing colors, it should be remembered that many of the enemies have a keen sense of smell, and so colors cannot always be useful. For illustration, quails and other birds 
which live on the ground are more or less protectively colored. This may help them some in escaping such enemies as hawks, but obviously would be of no protection against weasels, skunks, and other enemies which hunt by night, guided by odors and not by colors.

Finally, it should be noted that some colors of animals are probably not useful. For examples, we may mention the beautiful colors concealed in shells of molluscs (Chapter XV), the "eye-spots" in wings of some moths and butterflies, and the gorgeous color-patterns of many birds (e.g., peacock). For all of these and very many more we have as yet no satisfactory explanations.

335. Instincts of Insects. - The instincts of insects have long aroused the wonder of students of animal life, for under the guidance of inherited instincts many insects exhibit remarkable behavior. It is instinct which leads honey-bees to build honeycomb, care for the young in true nurse fashion, give a different kind of food to the larvæ destined to become "queens," follow the "queen " when she leaves the hive at swarming time, sting intruders, and in various other ways behave almost like intelligent beings. It is instinct which leads parasitic species to lay their eggs in the right kind of larvæ, and other species to place the eggs on plants which will furnish the right kind of food for the young larvæ. It is instinct which leads certain wasps to capture and paralyze other insects, and then place them where wasp larvæ hatching from eggs can later eat them. In short, thousands of cases of striking behavior of insects are apparently due to instinct. Some of the actions of insects suggest that they are intelligent and capable of reasoning; but the most critical studies have led entomologists to the conclusion that insects do not reason, but that they constantly act instinctively and automatically. How they originally acquired their instincts is entirely unknown, but that the instincts are transmitted from generation to 
generation is certain. A bee does not have to learn to build honeycomb, for to do this is as natural as eating and moving. It is an instinctive action.

Sometimes insects seem to have ways of communicating with each other. An ant or bee may, while wandering around, find something good to eat, and soon many others will follow the first one from the nest. This is probably due to the odor of food conveyed to the nest by the first insect. Also, ants can recognize those from another nest; but this is due to peculiar odors, acquired in different nests See article on "Communal Life of Ants," by Adele M. Fielde in Nature-Study Review, December, 1905, Vol. I.

\section{Classes of Arthropoda}

Crustacea - crayfish, crabs, water-fleas.

Onycophora-Peripatus, a rare worm-like arthropod found in troplcal countries.

Myriopoda - centipedes, millipedes.

Arachnida - spiders, scorpions, mites, ticks.

Insecta - insects. 


\section{CHAPTER XV}

\section{THE SHELL-ANIMALS : MOLLUSCA}

336 Examples of Mollusca. - We can best understand what is meant by mollusks by referring to common examples, such as mussels, oysters, clams, snails, sea-snails, garden slugs, cuttle-fishes, and nautilus. At first this appears to be an assemblage of very unlike animals, but closer studies show many points of similarity and lead to the conclusion that these animals are so closely related as to deserve grouping in one phylum, Mollusca. In this group there are more than 25,000 named species.

Snails and clams are usually chosen for first study of the molluscan plan of structure.

337. Study of a Clam. The quahog or little-neck clam and the long-neck clam are common in

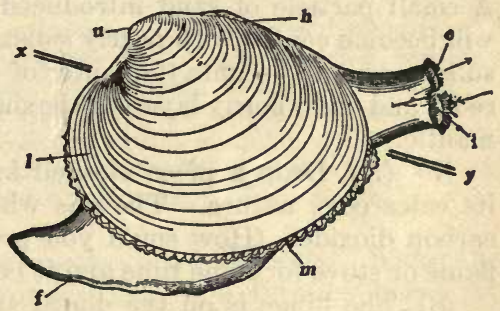

Fig. 141. A marine clam. All below the line $x-y$ is usually embedded in the sand. $h$, hinge of the shell ; $u$, umbo ; $l$, parallel lines of growth of the shell; $m$, edge of mantle; $f$, foot ; $e, i$, exhalent and inhalent siphons, the arrows indicating the direction of currents of water. (From Verrill.) markets in large cities and near sea-coasts; while elsewhere river-clams may be obtained and kept in aquaria. Specimens preserved in formalin are best for most work with the fleshy parts. The river-clams are best for demonstration of structure, the quahog is next best. 
Shell. - $(L)$ Examine some bivalved shells; some empty, and one with the clam inside. Note the following:-

(1) Hinge, which unites the valves. It has an elastic band that pulls the valves apart when the muscles relax, as when the animal dies. Interlocking ridges and tooth-like structures form the hingejoint in some species.

(2) The hump (umbo) is an elevation on the hinge line of the shell. It is the oldest part of the shell.

(3) Concentric lines extend outward from the umbo as a center. These are lines of growth, and represent successive additions to the shell.

(4) The fleshy tissue extending along the free edge of the shell between the two valves is the thickened edges of the two folds of the mantle, each of which lines a valve and secretes new shell material at the edge as indicated by the lines of growth.

(5) On an empty pair of valves, note the two pairs of scars where were attached the two muscles (named adductors) which hold the valves together during life.

(6) The inner pearly lining of the shell is secreted by the mantle. A small particle of sand introduced between the shell and mantle will become coated with pearly substance. Small particles of pearly substance may become the center of solid pearls. The middle calcareous and outer horny layers of the shell are secreted at the edge of the mantle.

(7) (D) Drop a piece of shell into strong acid to demonstrate its calcareous nature. The gas which causes the effervescence is earbon dioxide. (How could you prove it?) Heat a shell in a hot flame or stove for some time and it becomes a mass of lime.

(8) The hinge is on the dorsal side of the animal, the opposite or free edge of the shell is ventral. The siphon or "neck" is posterior, and the umbo and foot are near the anterior end. Holding a shell with hinge upward and the posterior end towards you, the right side of the clam is at your right hand.

(9) Currents of Water. (D) If a living clam is available in an aquarium, drop (with a pipette) some very muddy water near the siphon (popularly called "neck"), and observe directions of currents (see Fig. 141). These currents supply both oxygen and minute organisms as food in the ingoing (inhalent) current, and remove excretions by the outgoing (exhalent) stream. Bivalved mollusks have no other method of getting food and oxygen. Some of them burrow in mud, wood, and even soft rock, and spend their lives connected with the open sea only by means of the siphon, which 
in some species may be many inches long so as to enable the clam to live some distance down in its burrow. The currents are due to numerous cilia which cover organs within the shell (see Oyster, § 338).

Organs within the Shell. - $(D$ or $L)$ The fleshy parts of a clam. which may be seen after removing one valve of the shell, are as follows :

(1) The two great adductor muscles which hold the valves together. One is near the anterior, the other near the posterior end.

(2) Mantle lining each valve, and between the two folds of mantle is the body of the clam.

(3) The foot is a firm muscular structure, which may be projected out from between the folds of the mantle at the an-

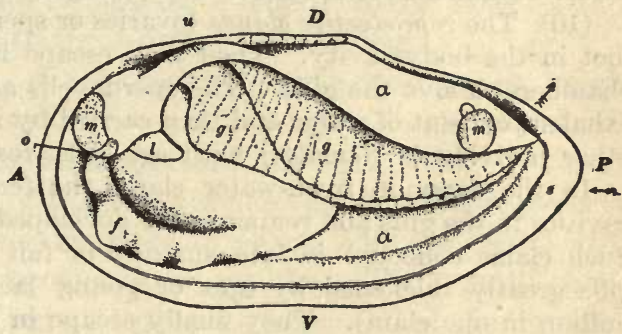
terior-ventral edge. Fig. 142. Organs of a clam exposed by removal of It is large in river clams, and small in the long-necked clams, which do not move much.

(4) Between the left valve of the shell. $A$, anterior ; $P$, posterior ; $D$, dorsal ; $V$, ventral ; $a$, mantle ; $g$, gills ; $m$, muscles that close the valves together; $f$, foot; $o$, position of mouth ; $u$, umbo of shell ; $l$, labial palp. Arrows show direction of ingoing and outgoing currents of water. (From Brooks.)

foot and mantle on either side are two plate-like gills. Water that enters at the siphon flows around these and through pores into eavities in the gills, and thence through the gill-chamber above the gills and out by exhalent current. Blood (colorless) flowing in bloodcapillaries in the walls of the gills is able to exchange oxygen and carbon dioxide with the surrounding water. In short, the gills are the organs of respiration.

(5) Above the foot at the anterior end of the animal is the mouth, just below the anterior adductor muscle. There are no jaws, for the food consists of small organic particles brought by currents of water.

(6) On each side of the mouth are two triangular flaps (called labial palps). These serve to direct the currents of water so as to carry particles of food to the mouth.

(7) From the mouth the alimentary canal (stomach and intestine) extends down into the foot, where it is much coiled, then it runs up 
and along the dorsal side of the animal to its end near the exhalent part of the siphon. A digestive gland (greenish in color) lies near the anterior part of the alimentary canal.

(8) A heart and a pair of kidneys lie in the body of the animal dorsal to the gills. Blood-vessels extend from the heart through all the tissues of the body.

(9) Ganglia (masses of nerve-cells) lie near the mouth, in the foot, and near the posterior end ; and these are connected by nervecords. Small nerves extend to various organs of the body.

(10) The reproductive glands (ovaries or spermaries) lie above the foot in the body-cavity. Their cells escape into the cavities (gillchambers) above the gills. The sperm-cells are carried out by the exhalent current of water and then carried by inhalent currents into other individuals (females) with egg-cells ready for fertilization.

In the common fresh-water clams the fertilized eggs fall into cavities in the gills and remain until developed into bivalved larvæ. Such clams collected in late summer or fall may have the outer gills greatly thickened by eggs or young larvæ (sometimes three million in one clam). They finally escape in the exhalent current. These larvæ have sharp teeth on edges of their shells, and by these they attach to skin or gills of fishes. Having attached, the skin of the fish grows over (encysts) the clam larva, and so it remains while it begins to metamorphose into the clam form. Finally, after several weeks, the larva drops from the skin of the fish, falls to bottom of the stream, pushes the lower edge of its shell into the mud, and begins its life as a clam.

Problem: Can you think of any way by which clam larvæ which are unable to swim might be distributed far upstream in rivers? River clams probably originated near the sea. How have they been distributed even to the very sources of some of the smallest brooks of great river-systems like that of the Mississippi?

The larvæ of marine clams have no such parasitic development in fish, but escape from the gill-chamber as a ciliated larva able to swim freely for some time before it metamorphoses into a clam. Hence, marine clams may be widely distributed by swimming of their larvæ; also by tides and ocean currents.

338. Relatives of the Clams: Lamellibranchs. - The terms "clam" and "mussel" are applied to a number of marine and fresh-water species of mollusks which have a bivalved shell. There are two common types of river clams, one with thick shells and one with thin shells. Of each type there are 
many species indicated by minor differences in shape and markings on the shells.

The two most common marine clams sent to market in eastern states are the hard shell, "little neck," or " quahog " clams of the genus Venus (Fig. 141), which are the favorites in New York markets; and the soft shell, "long neck" clams of the genus Mya, which are demanded by the best trade in the Boston markets. The "little neck" and "long neck" refers to the length of the siphons.

The giant clams of the Indian Ocean sometimes weigh 500 pounds and may be two feet long. The smallest bivalve is a little fresh-water species $1 \mathrm{~cm}$. long.

Most remarkable of the allies of the clams is the ship-worm ('Teredo) which bores burrows in ships' timbers, piles, etc. The body of the teredo is worm-like. The small shell, used for cutting the burrow, is at the inner end of the burrow. The siphons project above the surface of the wood whenever the "worm" is not disturbed, and the ingoing current of water carries in food (small organisms) and provides for respiration. The remedy against these animals is copper sheathing, or soaking timbers with creosote and tar.

The oyster is peculiar in that the eggs develop within a few hours after fertilization into free-swimming larvæ. These finally become attached and metamorphosed into the oyster form. The left valve of an oyster's shell is attached to a stone or to another oyster shell. In consequence of this sedentary mode of life the oyster has no use for a foot (organ of locomotion), and this is absent. One reason why the flesh of oysters is so delicate in texture is that there is no hard muscular foot; and the great development of this organ makes river mussels " too tough " to be palatable. The mouth of an oyster is near the ligament which hinges the shells together; but as in case of clams, currents of water from the opposite side of the shell carry in food particles. 
(D) Locate oyster's mantle, gills, hinge of shell, position of mouth, and one central adductor muscle which holds the valves closed. Mount and examine microscopically a piece of gill from an oyster

a

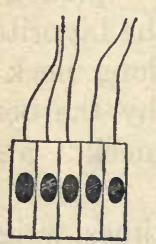

b

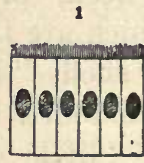

c

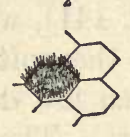
just opened, using for mounting some of the salt water found inside the shell, and observe the movements of cilia, which produce currents of water.

Lamellibranchs.

FIG. 143. Ciliated epithelial cells. $a$, cells with one cilium each; $b$, with many cilia; $b^{2}$ is end of view of $b^{1} ; c$, ciliated cells with underlying cells, the whole forming a stratified epithelium. Similar ciliated cells on oyster gills cause movement of water. (From Hatschek.)

class known as Lamellibranchiata. This name (from lamella, plate; and branchia, gill) refers to the plate-like gills such as the clams and oysters have.

339. Study of Land-Snail. - $(L)$ The common European gardensnail (Helix pomatia), sold in markets, and native snails found in damp woods, may be used for study. When imported from Europe the snails are in the hibernating condition with shell sealed by a plate of hardened slime. If placed on damp sod or paper in a warm room, the animal will "come out" of its shell within a day or two, and begin to move and feed. Specimens for study should be allowed to crawl on plates of glass. Observe the following points of structure and habits : (1) foot, and its movements as seen through the glass; (2) the head, with four tentacles; (3) the position of the spiral shell; (4) the collar at the mouth of the shell; (5) the breathing pore in the collar

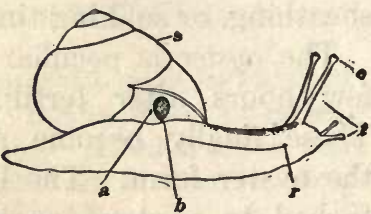

FIG. 144. Diagram of a landsnail (Helix). $s$, shell ; $a$, anus; $b$, breathing pore opening into the "lung"; $r$, opening of reproductive organs; $t$, four tentacles; $e$, eyes at end of longer tentacles. (From Parker and Haswell.)

(watch its movements); (6) the mouth (best seen through the glass from below); (7) the eyes at ends of two of the tentacles. Is the snail bilaterally symmetrical? 
(D) Internal organs. The form of the body must be studied in museum specimens prepared by special methods, and preserved in formalin. The breathing pore leads into the lung-sac. The posterior end of the digestive canal is near the breathing pore, the canal having been twisted so as to bring its opening near the edge of the shell. Heart, liver, kidney, loop of intestine, and reproductive glands make up most of the mass within the shell. Note that this mass of organs has the form of the shell, and that the upper whorls are filled even when the snail's foot is out of the shell. The animal is hermaphroditic ( $\$ 283)$. The nervous system consists of masses of nerve-cells (ganglia), chiefly placed anteriorly in head and foot, and with nerves from these ganglia to various parts of the body. Careful study of the internal organs of a

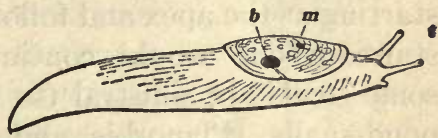

Fig. 145. Garden slug (Limax). $t$, tentacles ; $m$; mantle covering rudimentary shell; $b$, breathing pore. (From Morse.) snail is usually made in college courses of zoölogy, but we have not time in this course.

(L) Study of empty snail-shell. The small end is the apex, the central axis is the columella, and the opening is the mouth of the shell. Notice direction of the spiral as compared with threads on an ordinary wood-screw. At a broken edge may be seen an inner pearly layer, a middle thick layer, and a thin outer layer. The middle and outer layers are secreted by the collar, while the whole inner skin (which is the mantle) secretes pearly lining. Examine a shell from which one side has been chipped away with strong forceps so as to expose the interior.

Garden-slugs, which are usually to be found in gardens and greenhouses, are similar to snails, except that the shell is small and covered with skin. Obviously, such a degenerate shell is of little use as a protecting organ.

340. Snails and their Allies: Gasteropods. - The gardensnails are members of a group of mollusks known as gasteropods (class Gasteropoda). The name means stomach in the foot. Since they have a shell consisting of one piece, they are often called univalves to distinguish them from the bi-valved clams.

While the land-snails and their near relatives, the slugs, breathe air by means of a simple lung-chamber, most gastero- 
pods live in the sea and have in the respiratory chamber feathery gills, which absorb oxygen from the water and give off carbon dioxide.

Very generally the hind-end of the foot of sea-snails bears a hard plate which closes the mouth of the shell when the foot is retracted.

In most gasteropod shells the spiral is dextral ; that is, starting at the apex and following the whorls as down a spiral staircase one would continually turn to the right; but some snails are sinistral (to the left). Two of our common pond-snails (Planorbis and Physa) have this peculiarity. The significance of the direction of the spiral is unknown.

The covered shells of the garden-slugs are colorless. Cowries are polished on the outside because folds of the mantle cover and secrete pearly substance on the outside when the animal is expanded ("out of its shell"). In most gasteropods the shell is covered with an epidermis, but this gets rubbed off as the shell grows old.

Ridges and spines on univalve shells are common. Continuous ridges or lines extending spirally around the shell from its mouth to apex are due to constant and similar ridges on the edge of the secreting collar. Ridges or rows of spines parallel with the mouth of the shell and the lines of growth are secreted periodically (example, harp-shell). Sometimes a wide lip at mouth of the shell is formed only when the shell is full grown, and such a shell will have no such ridges as in a harp-shell.

341. The Highest Mollusks. - The squids, octopus (" devil-fish"), and nautilus represent the highest forms of mollusks. All of them are marine.

The common squid (Fig. 146) has a cylindrical body, tapering to a point at the hind end. The movable head has a group of ten arms surrounding the mouth, and the arms have peculiar suckers adapted to holding prey. A large eye is on either side of the head. 
At the end of the body is a pair of broad fins used for swimming slowly forward. On the lower side of the neck is a siphon or funnel from which water may be ejected with such great force as to drive the animal rapidly backward. At the same time it may eject an inky fluid, so as to cloud the water. From a similar ink of a cuttlefish India ink or sepia was originally made.

The cavity out of which the head protrudes' is the mantle-cavity, and the outer surface or "skin" of the body is the mantle. It contains pigment spots of various colors, which are very changeable during life.

Externally there is no evidence of a shell, but beneath the "skin" on the upper side is a quill-like structure (the pen) four to six inches long. The same structure in cuttlefishes is the cuttle-bone sold for use of caged birds. Comparative studies have shown that the pen and cuttle-bone represent shells such as that of the chambered nautilus.

Giant squids with arms forty feet long and with eyes a foot in diameter have been captured.

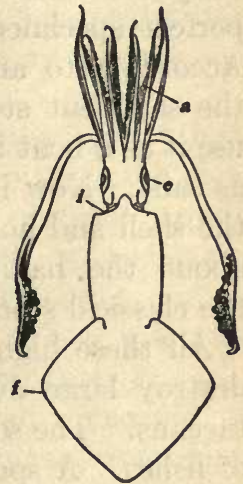

FIG. 146. Male squid. $a$, armstwo long ones in male; $e$, eye; $i$, opening to siphon; $f$, fin. (After Verrill.)

The octopus or devil-fish has a structure similar to a squid; but its body is shorter, there are eight arms and there are no fins. Some species are of enormous size, but not large enough to attack a ship, as described in old books of fiction. Specimens weighing two hundred pounds and with arms five feet long have been seen.

The chambered or pearly nautilus, made famous in the poem by Oliver Wendell Holmes, is similar to an octopus or squid, but develops' a many-chambered shell. It lives in the outer, largest chamber, and from time to time secretes partitions across the shell so as to leave behind an out- 
grown chamber. It lives in the Indian and Pacific oceans, usually in deep water. Some specimens have been found floating on the surface, and so Holmes's reference to sailing may not be entirely poetic license.

The paper nautilus (Argonaut) is a relative of the octopus. Only the female bears the delicate, single-chambered shell, perfect specimens of which are much prized by collectors. According to an old fiction, the argonaut did not secrete the shell but stole it from some other mollusk in order to use it as a boat in which to sail, using its two expanded arms as sails. Now it is known that the two arms help secrete the shell and hold it over the animal. Very little is known about the habits of this remarkable animal. (Look up the classical story of the argonauts.)

All these highest mollusks are exceedingly voracious and destroy large numbers of fishes, other mollusks, and crustaceans. The squids can swim fast enough to pursue schools of fishes. A species of European octopus frequently enters the traps set for lobsters and crabs, and the fishermen find only mangled remains of the crustaceans.

The squids, octopus, cuttle-fish, and nautilus represent a class of mollusks known as cephalopods (Cephalopoda), meaning head-foot, referring to the fact that in the embryo the head develops from tissue which forms the foot in other classes of mollusks.

342. Economic Relations of Mollusks. - The value of the oyster industry is enormous in America and in Europe. Studies by zoölogists connected with government laboratories have vastly improved methods of propagating, and have made it possible to raise oysters where they do not naturally grow. Oyster beds are now regularly leased by states to oyster-men, and oysters are artificially " planted." In many places it is necessary to rake the sea-bottom with steam dredges annually in order to bring the oysters to the surface and free them from the destructive starfishes. The egg- 
laying months are May to August, and the popular saying that oysters are not edible except in the months with the letter " $r$ " in their names, i.e., September to April inclusive, is connected with the fact that in the months without " $r$ " the animals are likely to be filled with eggs. The oysters are the most valuable mollusks, and the business of raising oysters is worth millions of dollars a year. From Chesapeake Bay alone more than twenty-five million bushels of oysters are marketed annually.

The marine clams and scallops are of great importance, and attempts are now being made to cultivate them. Unless this becomes commercially successful, the natural supply will soon be exhausted. Such investigations are under the auspices of the United States Bureau of Fisheries and of certain state experiment stations; and these scientific institutions deserve the necessary financial support.

Certain large land-snails have long been esteemed as delicacies. There were snail-gardens in Roman times, but now the snails are widespread pests in vineyards and gardens of Europe.

Preparations from snails were once used for coughs, consumption, malaria, asthma, dropsy, and almost all other diseases. In some rural regions of England people still believe that snails are of medicinal value.

Numerous marine snails (gasteropods) are used as food in various parts of the world. Squids and cuttle-fishes are eaten by poor people in some countries.

Sepia or Indian ink has been mentioned as obtained from cuttle-fishes. The famous Tyrian purple once used for coloring royal robes came from another mollusk.

Shells of mollusks are of great ornamental value. They have long been sought by conchologists (collectors of shells), and more than $\$ 100$ has often been paid for a single rare specimen.

Natives of the South Sea islands use shells for a great 
variety of purposes, ornamental and useful. In some barbaric tribes of Central Africa shells still pass as money, and the "money cowry" is the standard currency. The North American natives ("Indians") once cut cylindrical beads from the purple spots on shells of the hard-shelled clam (Venus), and this was the "wampum " mentioned in early colonial history of the United States.

Civilized men make extensive use of shells. Some are used for inlaying and for other ornamental work. Vast quantities are used for pearl buttons. Pearls of great value are each year collected from clams of various species. In North America many pearls are found in the rivers of the Mississippi system and in Lower California.

Reference: Roger's "The Shell Book."

\section{Important Classes of Mollusca}

Amphineura - chitons, eight-valved shell. Gasteropoda - snails, univalved shell.

Lamellibranchiata - clams, bivalved shell.

Cephalopoda - squids, nautilus. 


\section{CHAPTER XVI}

\section{THE VERTEBRATES}

343. The Backboned Animals. - Animals which possess a dorsal vertebral column, which is commonly called "backbone," are members of the great group Vertebrata. They are popularly called vertebrates or "backboned" animals. It should be noted that the vertebral column in some of the lower fishes is cartilage, not bone; and hence the popular term "backbone" for vertebral column is not strictly accurate.

The most convenient classification of vertebrates is into five classes: Pisces (fishes); Amphibia (frogs and salamanders); Reptilia (reptiles); Aves (birds); and Mammalia (mammals).

It has been discovered within the past half century that some existing animals lower than the fishes are certainly related to the vertebrates, even though they do not have a backbone. However, these low forms do have a stiffening rod in place of the backbone, and it is similar to a rod which is present in the embryos of all backboned animals as the axis around which the backbone develops. This rod is called the chorda dorsalis (meaning dorsal cord) or notochord, and all animals which possess it in any stage of their existence are called chordates. The recent zoölogical books recognize a Division Chordata, including (1) the very simple forms with the chorda, but having no vertebral column and skull, and (2) the vertebrates. In this course we cannot study in detail any of the simple chordates; but museum specimens of ascidians (sea-squirts), Amphioxus (lancelet), and the 
worm-like Balanoglossus, should be viewed for the sake of general acquaintance. Especially note the general appearance of Amphioxus, for its stucture is in many points so near the vertebrates that some authors so classify it. Nothing about an adult ascidian suggests resemblance to vertebrates, but its larval stage is a tadpole-like animal with some vertebrate characteristics. It degenerates when metamorphosing into the adult stage. All these simple forms

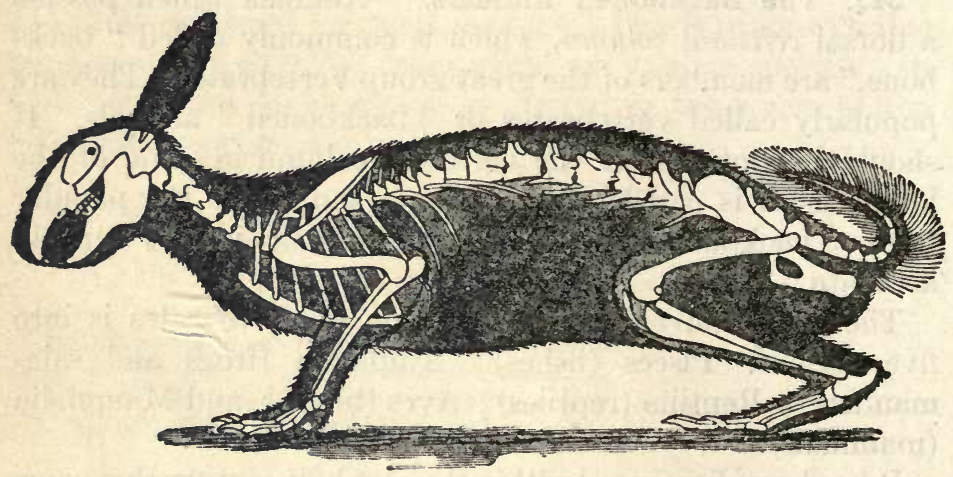

Fig. 147. Outline of a vertebrate (rabbit), showing position of the skeleton. Note that the backbone, which incloses the spinal cord, is in the dorsal part of the body. (From Parker and Haswell.)

will be interesting to the student who elects advanced courses of zoölogy in colleges, or who reads the larger textbooks.

344. General Structure of Vertebrates. - The frog, already studied, is a good example of the general plan of body in vertebrate animals. The important points of structure of vertebrates are as follows: (1) dorsal vertebral column; (2) central nervous system consisting of anterior brain in a skull, and a nerve-cord (spinal-cord) protected by the vertebral column; (3) alimentary canal ventral to vertebral column, and heart ventral to alimentary canal ; (4) bodycavity containing many organs (name them); (5) usually 
two pairs of appendages for locomotion (fins in fishes, fore and hind legs in amphibians and many higher forms, wings and legs in birds, arms and legs in man); (6) red corpuscles in the blood. Review your study of the frog and compare with this list of important points of structure.

If we had time for dissection and careful study of a fish, a salamander, a snake, a turtle, a bird, and a mammal, we should find that they have the same organs and arranged in the same relative positions as those in the frog. Such comparative study must be left for advanced courses in colleges.

\section{FISHES *}

345. External Structure of a Fish. - $(L)$ Any available fish from the market should be examined. Note the two pairs of lateral fins; and the dorsal and ventral fins on the median line. Compare several species of fishes, or pictures of them, as to the arrangement of the fins; and especially note that the posterior pair of fins is in some fishes near the anterior pair. Note arrangement of scales.

Examine the mouth and gills.

Observe (1) the movements of the body and fins, and (2) the mouth and gills in living fishes in an aquarium.

346. Distribution and Habits of Fishes. - Of the more than 13,000 species of fishes now known to exist, about onefourth are found in or near North America.

A remarkable fact regarding the distribution of fishes is that certain species live at various depths in the oceans down to over 2000 fathoms. At this depth there is complete darkness, a constant low temperature, quiet water, and the enormous pressure of over 5000 pounds per square inch of skin. To such conditions the deep-sea fishes are adapted.

* The singular "fish" is commonly used for singular and plural when referring to one or more specimens of a single species of fish, e.g., we may say correctly "a ton of common codfish" which might include hundreds of individuals of the common species. The plural word "fishes" refers to more than one species, e.g., "there are at least 140 species of codfishes among the more than 13,000 different species of fishes now known." 
The internal pressure of gases balances the enormous external pressure; but if brought to the surface, they are killed by the sudden expansion of internal gases.

The spawning habits of some fishes are most remarkable. Some salmon ascend the Columbia River from the sea for more than a thousand miles, at an average rate of 3 to 4 miles a day. After depositing eggs and sperm-cells, they all die; and no old fish lives to lead the young ones down stream to the sea. This single spawning occurs after the salmon are at least three years old. It is not true, as sometimes stated, that they always go back to the river where they were hatched; but they probably do not go far from the mouth of the river, and hence are likely to ascend the same river when fully developed and ready to spawn.

The shad of our Atlantic coast is another example of a fish that ascends rivers to spawn; but this fish lives to return to the sea after spawning.

The common river eels migrate downstream in the fall to spawn in the sea, and after spawning in deep water, the old eels die. Hence adult eels never migrate upstream. In spring, vast numbers of young eels, about one year old, appear below dams and waterfalls. A female 32 inches long may have more than ten million eggs. The life-history of eels was a complete riddle until about twenty years ago, when it was found that the eggs are laid in deep sea-water.

347. Types of Fishes. - There are four distinct types of fishes. Specimens should be examined, if possible.

(1) The hag-fishes and lampreys are distinguished from other fishes by the sucker-like mouth by which they attach themselves to other fishes. The hag-fishes are even able to bore into the bodies of their hosts. The lampreys are found in lakes, rivers, and seas of temperate regions. On the coast of California a species of hag-fish causes much damage by taking baited hooks, entangling fishermen's lines, and boring into captured fish. On the Atlantic coast they are not so 
abundant. Some of the lampreys attain a length of over three feet. They are excellent as food fishes, especially in Europe.

(2) The fishes with skeletons of cartilage include the sharks, dog-fishes, and rays. They are found in sea-water. Some sharks have been caught at depths below 500 fathoms. Their flesh is not esteemed as food.

Dog-fishes are shark-like. Saw-fishes have shark-like bodies with a saw-like structure on the snout. Swordfishes are similar, but with a sword in place of the saw. Sting-rays have a caudal spine, which may cause severe wounds. Torpedoes have electric organs, some of which can disable a man. Skates are harmless.

Specimens of the fishes named above should be viewed at some museum ; or, at least, examine pictures in encyclopedias and zoölogical books.

(3) Most important commercially and most numerous in species and individuals are the bony fishes. The sturgeons and gar-pikes are the lowest examples. They have large plate-like scales which form a strong armor over the skin. The sturgeons are large fishes; and their flesh is valuable as food, and their ovaries are made into caviare. They live in seas and rivers. The gar-pikes of North American rivers are of no value as food.

The vast majority of existing fishes in seas and fresh water are of the type represented by such common fishes as cod, perch, minnow, gold-fish, whitefish, bass, pickerel, salmon, trout, carp, mackerel, and halibut.

The skin of many of the bony fishes is beautifully colored, and some can change their color quickly. Scales are absent in most eels and cat-fishes. Many bony fishes are curiously modified, as the sea-horse, flying-fishes, sword-fishes, searobin, toad-fish, blind-fishes, etc. For accounts of these, see encyclopedias, or Jordan and Evermann's "American Fishes." 
(4) The lung-fishes of South America, Africa, and Australia deserve mention because they have gills and also primitive lungs able to breathe air. This adapts them to life in places where rivers are muddy or dry in certain seasons. The American and African forms are true mud-fishes and bury in mud at the beginning of the dry season. These fishes have attracted much attention from zoölogists because they suggest a connection between fishes and amphibia.

\section{Important Groups of Fishes}

1. Cyclostomata - sucker-mouth (hag-fish, lamprey).

2. Elasmobranchii - cartilaginous skeleton (sharks, rays, and skates).

3. Teleostomi - bony skeleton $\left\{\begin{array}{l}\text { Ganoids (sturgeon). } \\ \text { Teleosts (cod, perch, etc.). }\end{array}\right.$

4. Dipnoi - lung-fishes.

348. Economic Value of Fishes. - A few examples of fishes which are important in the human food-supply will suggest the enormous total value of this group of animals.

Salmon worth more than $\$ 13,000,000$ are annually caught on the Pacific Coast of North America, nearly one-third of these from Puget Sound and Columbia River. The average weight of a full-grown salmon of the Columbia River species is over twenty pounds, and individuals have weighed 100 pounds.

Herrings are probably the most valuable food fishes in the world. Huxley estimated that three billion, each averaging half a pound in weight, are caught in the North Sea and Atlantic annually; and this is now too low an estimate. They swim in enormous groups or "shoals" which sometimes extend over half a dozen square miles. "Sardines" from Maine are simply small herrings, but the true European sardines belong to another species.

The codfish is one of the most important North American fishes. About 7000 men are engaged in the fishery, and the 
annual catch is in some years near $100,000,000$ pounds and worth to the fishermen about $\$ 2,000,000$. The cods live in deep water (20 to 100 fathoms), and are captured only with baited hooks and lines. A cod over six feet long and weighing over 200 pounds was once taken; but from 12 to 40 pounds are the sizes usually caught. They spawn near the shores of New England between December and April. The United States Fish Commission hatcheries liberate more than $75,000,000$ young fry annually. It is easy to collect the cod eggs for hatching, for in a 20-pound female, the ovaries (popularly called " roes") contain more than 2,500,000 eggs, which are so small that a quart bottle will hold about 335,000 eggs. Think of how abundant codfishes would be if all the eggs of a thousand females were to hatch and grow to maturity, and one-half of these were to be equally prolific females. However, since cods do not appear to be either increasing or decreasing rapidly, we are justified in concluding that, on the average, two eggs from each female produce mature individuals (the two sexes about equal in number). The others are destroyed by enemies or die from diseases. This is a good illustration of the intensity of the struggle for existence, which, to a great extent, affects all animals and plants (§ 499).

Next to the Columbia salmon and the cod, the shad is the most important fish caught in waters of North America. It is captured in the spring when it ascends rivers to spawn. The annual catch is about $14,000,000$ fish, weight $50,000,000$ pounds, and worth more than $\$ 1,600,000$. The fact that the shad is taken only at the spawning season would long ago have made the fisheries unprofitable if the United States Fish Commission had not engaged in artificial propagation. More than 200,000,000 young shad are annually "planted" in the rivers of the Atlantic Coast. They have been introduced since 1871 on the Pacific Coast, where they do not naturally occur; and have now become abundant in the 
markets of the west-coast cities. No better proof could be desired as to the value of the work of the government in artificial propagation of fishes. And this is only one of many fishes which has been widely distributed and made more abundant as the result of science applied by the experts on fish culture, employed as agents of the national and of certain state governments.

The three most valuable North American fishes have been selected to illustrate this discussion of economic value; but there are many others worth tens of thousands of dollars annually. The fact is that we have scarcely begun to learn the real value of fishes as a source of meat food. There are hundreds of rivers, lakes, and ponds which might be stocked with fish of selected species and made to produce an abundance of good food, while, at the same time, destroying numerous larvæ of the dreaded mosquitoes. It is certain that the successful methods of artificial hatching and distribution which have been discovered by experts in the government service will ultimately make many useless bodies of water profitable to their owners.

The student who is interested in fishes should refer to Jordan and Evermann's "American Food and Game Fishes."

\section{AMPHIBIANS}

349. The class Amphibia has been mentioned as containing the frogs and toads. It also includes the tailed forms which are popularly called newts, salamanders, mud-puppies (Necturus), water-dogs, and mud-eels. Some of these are often mistaken for lizards; but lizards (e.g., chameleons) are reptiles with scaly skin, while the common amphibians have smooth skin like that of the frog. Some of the tailed amphibians have gills in the adult state, and live in water. Those without gills respire by means of the skin when in water, and by lungs and moist skin when on land. 
The tadpoles of both the tailed and tailless forms of Amphibia are similar; but, as already described in $\S 61$, the tails of frogs and toads are absorbed while legs are developing and the tadpoles are metamorphosing into the adult state. The loss of the tail, and the fuller development of lung-breathing adapts frogs and toads to living on land. Toads are still better adapted by their hard and dry skin, which enables them to live in places so dry that frogs would perish because their skin must be kept moist.

In geology the Amphibians are noteworthy because certain salamander-like forms were the first five-toed vertebrates. The remains of these ancient amphibians have been found in the earliest coal beds (Carboniferous Age). The present-day types of amphibians have not been found until late in geological history (Eocene times).

The name Amphibia means "both kinds of life," referring to the fact that these animals are both aquatic and terrestrial.

350. Myths Concerning Amphibians. - The skin secretion in some toads is disagreeable or even poisonous to their enemies; but produces no serious effect on human skin. Warts on small boys' hands are not caused by handling toads. The truth is that warts may be caused by slight scratches which allow dirt to get into the skin. Moreover, killing a toad will not "cause your father's cow to give bloody milk," for a moment's serious thought shows us the absurdity of the folklore that there is such a relation between cows and toads. Both the wart and the milk legends are absolutely unscientific. There are numerous other absurd beliefs concerning common animals, and one who has read a modern textbook on animals will always demand proof or the authority of some modern scientific book before accepting them.

351. Economic Relations of Amphibia. - It is well known that frogs' legs are eaten; and it has proved profitable to catch frogs for the market. In fact, the annual hunting of 
nearly $\$ 50,000$ worth of wild frogs will soon exterminate them unless "frog-farms" are more extensively developed in the near future. But the chief value of our common amphibians is in that they destroy thousands of insects. The common toad is a valuable inhabitant of gardens. See the bulletin on the American toad which is published (free) by the United States Department of Agriculture, and also see a chapter in Hodge's " Nature Study and Life." We ought to have laws protecting toads as well as birds, but until we get such laws we must depend upon the good sense and fair play of intelligent people who are informed concerning the value of toads. Frogs are likewise useful as destroyers of insects, but have not attracted so much attention as the toad, which is able to go far from bodies of water. They could be kept in gardens without small ponds.

The species of tailed amphibia which spend much time on land are probably useful destroyers of insects.

\section{Important Orders of Amphibia}

Urodela - with tail, usually two pairs of limbs, and may have gills in adult. Examples: newt, salamander. Anura, or Batrachia - no tail and no gills in adults. Two pairs of limbs. Frogs and toads.

For interesting reading and reference : Dickerson's "Frog Book."

\section{REPTILES}

352. The class Reptilia contains four types represented by the existing lizards, snakes, turtles, and crocodiles, of which there are about 3500 living species. All these types of reptiles have scales on the skin. In turtles the scales are large plates (e.g., the valuable tortoise shells), which form the outer surface of a box-like shell. Typically the reptiles have four legs with five toes each. The legs have degenerated in snakes, but certain pythons and boas possess rudiments of the hind pair of legs. Colors are well developed in the skin 
of many reptiles, and some can quickly change color (e.g., chameleons).

Most reptiles are tenacious of life and many can exist for a long time without food and with limited breathing. A winter sleep in cold climates, and a summer, or dry-season sleep in hot climates, is their rule of life.

Fossil reptiles have attracted much attention because of their gigantic size, some of them being over 100 feet long. Dinosaurs (bird-like reptiles), Pterosaurs (flying reptiles), Ichthyosaurs (fish-like reptiles), and Plesiosaurs (lizard-like), are some of the fossil reptiles commonly seen in great museums. So abundant were these and other reptiles that one stage of geological history has been called the Age of Reptiles. In North America the best specimens of fossil reptiles are found in Wyoming and adjoining states.

353. Economic Relations of Reptiles. - Most lizards are harmless, and may be useful as destroyers of insects. Iguanas and other large species are hunted for their flesh. The Gila monster of Arizona may sometimes inflict a poisonous bite. Brilliantly colored lizards are often kept as pets; they should be fed insects, and not starved on sugar and water. (Why?)

Many turtles are valuable as human food. Terrapin turtles are now so very high in price that "terrapin-farms" are profitable.

Snakes feed exclusively on living animals, and hence may be more or less harmful from our human viewpoint, especially those which destroy insectivorous birds and frogs. Poisonous snakes belong to many different families. The American moccasins, rattlesnakes, and copperheads, and the Old World cobra, adders, and vipers are the most poisonous reptiles. Official figures show that in India alone more than 20,000 people die annually from snake bites, but there are few fatal cases in the United States. Pythons, boas, and American blacksnakes are examples of snakes which 
kill animals by constricting. Most of the snakes of the United States are not poisonous. Snake poisons are secreted by glands in the upper lips, and the " poison fangs " are upper teeth with grooves or tubes for conveying poison beneath the skin of the victims.

The remedies for snake bites are briefly as follows :-

(1) Place tight ligature on arm or leg to prevent circulation of blood; (2) enlarge by cutting the punctures made by fangs in order to drain away as much poisoned blood as possible; (3) wash out cuts with wine-colored solution of potassium permanganate; (4) take very small doses of alcoholic stimulants, enough to cause increased pulse-beats; larger doses are dangerous; (5) consult a good surgeon, because blood poisoning may result from the wound, and also it may be necessary to have stimulants and anti-venomous serum injected hypodermically; (6) keep wound covered with a cloth or cotton wet with some antiseptic solution.

Alligators and crocodiles are sometimes dangerous to man. Their tough skin is used for leather. In their embryonic development, all crocodiles, alligators, turtles, most snakes and lizards are oviparous. Some snakes and lizards retain the eggs in the oviducts until development is completed, that is, they are viviparous.

\section{Important Groups of Living Reptiles}

Lacertilia - lizards (iguana, Gila monster, horned toad, chameleons, etc.).

Ophidia - snakes (vipers, python, boa, water-snakes, rattle-snakes, etc.).

Chelonia - turtles (tortoises, terrapins, soft-shelled turtles, boxturtles, etc.).

Crocodilia - crocodiles, alligators.

Reading or reference for students: Dithmar's "The Reptile Book." 


\section{BIRDS}

354. Adaptations of Birds. - No group of animals is so easily defined as that of the birds, for even a small child knows that an animal with feathers is a bird.

Their most important adaptations are those connected with fitting the wings, legs, and bills for locomotion and for obtaining food. The whole structure of birds' bodies is arranged in adaptation to flying. The general outline of the body, the peculiar structure of wings, the great development of internal air-sacs connected with the respiratory organs - all are specialized with reference to aërial locomotion.

However, adaptation of anterior limbs for flying is not limited among vertebrates to birds, for there were ancient flying reptiles (Pterodactyls), and bats are flying mammals. The so-called "flying fishes" do not really fly, but simply use their large fins for gliding through the air for relatively short distances when they leap from the water.

Bats and birds, then, are the only living vertebrates able to fly, and the great differences in their structures make it certain that they have developed independently. Only the birds among vertebrates have proved perfectly adapted to life in the air. That they are well fitted is shown by their success in developing more species than any other group of vertebrates, and also countless numbers of individuals.

Biologists who have studied the flight of birds cannot help marveling at their locomotor mechanism. It is simply astounding that an animal as large as a homing pigeon can fly faster than a limited express train, and average such speed from daylight till dark. The recent attempts at perfecting flying machines have made us wonder more than ever at the flying power of the birds.

It is interesting to note that the largest and heaviest birds 
have lost the power of flight (ostrich group), and it has almost disappeared in many birds (ducks, geese, turkeys, chickens, swans) which under domestication have become much larger and heavier than in the wild state. The bodies of eagles, vultures, and the other largest flying birds are not heavy; and the appearance of great size is chiefly due to great expanse of wings. Evidently lightness of body is essential. The air-sacs, already mentioned, help to provide this in small birds, while in larger birds there are also extensive air-spaces in the bones.

Wings of birds are specially adapted for flight, while the posterior limbs (legs) are adapted for the support and movement on land and in water. Since birds are bi-pedal (twofooted), the legs are usually attached comparatively far forward, so that the body is easily balanced.

Legs. - The modifications of these are correlated with the mode of locomotion and other uses of the feet. The following important types may be noted among common birds; (1) walking feet (ostrich); (2) wading feet (herons); (3) climbing feet, two toes forward and two backward, (parrots); (4) birds of prey (eagle); (5) for perching (pigeon); (6) swimming feet, with toes partly or entirely joined by webs (ducks, geese). Long wading legs may have swimming feet, thus adapting certain birds to both wading and swimming. However, the swimming feet are usually on birds with relatively short legs.

The beaks of birds vary extremely in shape, and are adapted to procuring their special kinds of food. Among the most common forms of beaks, some are adapted to hunting for food beneath water, as is the habit of swimming and wading birds; some are fitted for eating seeds; and some are for insect catching. The pelican bill is adapted both to catching fish and to storing in the attached pouch. The bird-of-prey type, for tearing flesh, has a short, strong, and hooked upper bill. 
355. Feathers of birds deserve special examination. They are closely allied to scales, and sometimes (e.g., on wings of penguins) look like scales.

(L) Examine a feather. Note stem, consisting of quill and shaft. Examine a stem split lengthwise. The side branches of the shaft are called barbs, and their smaller branches are barbules. Examine with a hand-lens and note how the barbs and barbules unite to constitute the vane of the feather. The barbules near the edge of the feather on the side (lower) next to the bird's body have smaller processes (barbicels) with hooks.

The larger feathers of wings and tail (contour feathers) have stiff shaft and firm vane. Down feathers have soft shaft and vane and no hooks. Hair feathers are slender shafts with few or no barbs. Examine a bird and note where each kind of feather is located. What is the use of each kind?

A periodical change (molt) of feathers usually occurs in autumn, to replace the feathers more or less damaged by wear. Many birds also molt in part during the spring when acquiring the breeding plumage; but the change in color commonly seen is due largely to a change in the old feathers. The new feathers are formed in the follicles or feather-tubes in the skin, and first appear as pointed rods ("pin feathers"), which are really the stems inclosing the forming vanes. Examine such young feathers, either fresh or preserved in alcohol.

Color of birds is usually in the feathers, but sometimes in comb and wattles of head and neck. Feather colors are usually due to pigments (blacks, browns, reds, yellows, rarely greens); but metallic luster or iridescent colors are produced by the feather refracting or dispersing light as prisms or thin plates of various transparent substances do (e.g., rainbow colors). Blues, violets, and greens are commonly due to a combination of pigments and light refraction. There is no blue pigment in bird feathers.

356. Internal Organs of Birds. - Time available for this course will not permit dissection of a bird, but students 
who are interested should take the first opportunity to examine the chief organs in a chicken or other large bird which is being prepared for cooking.

The alimentary canal is essentially the same in all birds. Existing birds have no teeth, but some fossil forms had them. The tongue is sometimes specially adapted for seizing food (e.g., insect-catchers). The esophagus often has a crop for storage of food. The muscular stomach (called gizzard) has walls whose strength varies with the food (strong in graminivorous birds). It contains small stones which the bird has swallowed to aid in grinding the food. From the stomach there is a coiled intestine into which a liver and a pancreas pour their secretions. The posterior part of the intestine is expanded into a cloaca, into which the ducts from kidneys and from the reproductive organs open.

The respiratory organs are very peculiar. The voiceorgan is not in the larynx, as in mammals, but is lower down on the trachea or wind-pipe. This voice-organ (also called syrinx) is a complicated structurè, especially in singing birds. Large air-cavities in the bird's body, and even in the bones of some, are connected with the bronchial tubes leading from the trachea. In fact, when a bird breathes by dilating its thorax and abdomen, a large part of the air inhaled rushes into air-sacs and very little distention of the lungs occurs as in mammals. This peculiar mechanism is a more efficient respiratory apparatus than lungs of mammals, and the oxygen-supply to the blood is more complete. This more rapid respiration is necessary because of the great activity of birds, especially in flying and in singing.

Another remarkable fact connected with the respiratory organs is that their great surface eliminates excretory water and excess heat. In mammals, this is accomplished by the kidneys and sweat-glands; but bird kidneys do not eliminate much water, and they have no sweat-glands in their skin.

As a result of the intense respiration and consequent 
rapid oxidation of birds their temperature is higher than that of mammals, some birds reaching $110^{\circ} \mathrm{F}$., which would be fatal in a mammal.

357. Classification of Birds. - No classification of birds yet prepared has been generally accepted, and no two books agree. The difficulty arises from the fact that the 12,000 species of birds are remarkably similar except in details of structure. It is not possible in limited time to describe the groups of birds, and for general purposes it is most convenient to name groups according to some of their wellknown representatives, as in 1 to 16 below. For each of these groups there is a scientific name, for which see special books on birds.

\section{Groups of Birds}

1. Ostrich group - ostriches, emu, eassowaries, kiwi (Apteryx), hotnos. All wingless or with greatly reduced wings.

2. Loon group - loons, divers, grebes.

3. Gull group - gulls, terns, petrels, albatross.

4. Pelican group - pelicans, cormorants.

5. Duck group - ducks, geese, swans.

6. Heron group - herons, bitterns, storks, flamingos, spoon-bill.

7. Rail group - rails, coots.

8. Snipe group - snipes, plovers, woodcock, "killdeer."

9. Pheasant group - pheasants, grouse, quails, chickens, turkeys. 10. Pigeon group - pigeons, doves.

11. Eagle group - eagles, hawks, vultures, falcons.

12. Owls.

13. Cuckoo group - euckoos, kingfishers.

14. Woodpecker group - woodpeckers, flickers.

15. Humming birds.

16. Perching or song-birds, 6000 species - sparrows, warblers, crows, jays, fly-catchers, finches, robins, thrushes, bluebirds, ete. Includes all the most interesting song-birds.

A popular classification arranges birds according to their habits as wading birds, birds of prey, swimming birds, climbing birds, and perching birds. This is very convenient for most people, for they are chiefly interested in how birds live 
and affect human interests. It is important to note that such a grouping of birds with similar habits does,not always correspond to a scientific classification based on similarity of both external and internal structure. For example, there are swimming birds in groups 2, 3, 4, 5 named above; but loons, gulls, pelicans, and ducks show little structural evidence of relationship aside from their swimming adaptations, and hence, in a scientific classification, must be placed in separate groups. However, those who do not specialize in bird study cannot do better than remember the most familiar birds either according to their habits, or as associated with their relatives mentioned in groups 1 to 16 above.

358. Instincts of birds are highly developed. They have sharp eyes and a good memory. Parrots and ravens show an extraordinary power of imitation and ability to take some instruction.

The most highly developed instincts of birds are connected with migration and reproduction. It is well known that most North American birds migrate northward in spring, and after the breeding return southward in autumn. What causes them to migrate, and especially what guides them on the journey, has long puzzled naturalists. A vast amount of information concerning the times and paths of migration of many species in the Northern Hemisphere has been recorded in the special books of ornithology.

The distance covered in migrations varies with species. Some move from the arctic to temperate regions in autumn, and return in spring (e.g., certain "snow-birds "); others migrate between tropical and temperate regions; and still others go from tropical to arctic regions. Some species migrate at night, others by day. Some migrate at great heights. In some remarkable cases, the young birds go southward before their parents; and in other cases, the parents go first. In these cases, we cannot understand how the young are guided, unless by a few old birds which have 
in the previous year had experience in migration. Probably in most cases the young birds hatched in northern regions learn the way southward by accompanying older birds.

Migration southward is believed to be stimulated by absence of food-supply in winter; and a desire to re-occupy their old haunts and breeding places leads to return in spring.

Some birds may be seen at given localities in any month of the year, and such species are said to be resident. Many birds which are resident as species migrate as individuals; and those seen in winter have often come from the north, while the summer residents of the same species have moved southward.

The migration is not always due north and south. Coast lines, mountains, and great rivers may cause eastward or westward deviations. These are not yet well understood.

The instincts connected with nesting habits of birds have long excited the wonder of ornithologists. Many birds build nests exquisite in form. Each species has its peculiarities as to choice of nesting site. Why quails nest on the ground, swifts in a chimney, swallows beneath the house-eaves, and orioles in hanging nests cannot be satisfactorily explained; and we must be content with saying that these birds have inherited their nest-building instincts from their ancestors. How and why their ancestors learned to build nests in certain ways and in specially selected positions is entirely a mystery.

The instincts connected with incubation of the eggs and caring for the helpless young are no less remarkable. In some species the female broods the eggs and her mate brings food to her. In others the male and female take turns in brooding the eggs. Certain cuckoos and some other birds have the peculiar habit of avoiding the trouble of brooding by placing their eggs in the nests of other birds, which incubate them. In many species both male and female coöperate in the work of collecting food for the young nestlings. 
Chapman's "Bird Life" is an excellent introduction to bird study. There are many good books specially adapted for identification of birds in given localities.

\section{MAMMALS}

359. Characteristics. - Mammals (Mammalia) are the animals which in popular language are termed "quadrupeds" or "beasts." They are sharply distinguished from all other vertebrates by three characteristics: (1) milk-glands or mammary glands for supplying food to the young (the name " mammal" refers to this peculiarity); (2) true hair composed of overlapping scales or dry cells; and (3) a diaphragm dividing the body-cavity into an anterior (thoracic) cavity with heart and lungs, and a posterior (abdominal) cavity. No other animals have these characteristics, and by applying them we can quickly decide that whales are mammals and not fishes, and that bats are not birds; for both whales and bats have milk-glands, hair, and diaphragm.

360. Groups of Mammals. - It is easiest to learn the chief groups of mammals by reference to well-known examples such as one may see in any zoölogical garden, menagerie, or natural-history museum. In citing examples below, the plural (e.g., kangaroos) is used in most cases where more than one species is well known under the same popular name.

There are about 2400 species of living mammals, and over 3000 extinct species.

\section{Orders of Mammals}

1. Monotremata (monotremes) - Australian duck-bill and spiny ant-eater. Lowest mammals. Oviparous. All higher mammals are viviparous.

2. Marsupalia (marsupials) - opossums, kangaroos, wombats, and bandicoots. The skin on ventral side of abdomen forms a pouch (marsupium) in which the very weak young are carried. About 180 living species are known. 
3. Edentata (edentates) - sloths, armadillos, most ant-eaters.

4. Cetacea (cetaceans) - whales, porpoises, dolphins. All are aquatic, with fish-like bodies, no posterior (pelvic) limbs.

5. Sirenia - the mantee or sea-cow.

6. Ungulata (ungulates or hoofed mammals)-horses, asses, zebras, tapirs, rhinoceroses, camels, eattle, sheep, goats, antelopes, giraffes, deer, pigs, hippopotami, elephants, and extinct mastodons. Numerous extinct ungulates are found as fossils.

7. Carnivora (carnivors or flesh-eaters) - cats, hyenas, dogs, wolves, foxes, jackals, bears, otters, weasels, seals, walruses.

8. Rodentia (rodents or gnawers) - rats and mice, rabbits and hares, squirrels, porcupines, and beavers. There are about 1500 species of rodents.

9. Insectivora (insectivors) - moles, shrews, and hedgehogs.

10. Cheiroptera - bats and flying-foxes. Several hundred species. 11. Primates - lemurs, marmosets, monkeys, baboons, gibbons, orangs, chimpanzees, gorilla. The highest family of this order of mammals is the Hominidæ, which includes only the human species (Homo sapiens). See $\$ 369$.

361. Adaptations of Mammals. - Space here will allow only mention of some of the most remarkable cases of mammalian structures which are specially adapted.

(1) Fore limbs of bats have been modified into wings for flying, but still retain the same number and arrangement of bones as in ordinary five-toed mammals.

(2) Posterior limbs of whales have become rudimentary in fitting to aquatic life. The remains of the bones are often found several feet beneath the skin. Seals which live partly on land make little use of their small hind legs.

(3) The feet of most hoofed mammals show remarkable adaptations. The typical foot originally had five toes, but some of the toes have either become so small that they do not touch the ground or have disappeared altogether. The following examples will illustrate this point. An elephant's foot has five complete toes (or digits) each with a hoof, and all united by skin. The wild and domesticated hogs and hippopotami have four toes on each foot, the first one (I) on 
the inner side (thumb) being absent, the two outer toes (II and V) are short and do not touch the ground, while the third and fourth are the toes upon which the animal walks. In deer the two outer toes (II and V) are short and in some species absent. In camels and giraffes there are two toes on a foot, the third and fourth, the outer ones (II and V) being absent. In sheep, goats, antelopes, and cattle, the useful toes are the third and fourth, and the outer ones (II and V) are often represented by small hoofs.

The animals mentioned above, except elephant, are eventoed, i.e., with two or four useful toes on a foot. Other hoofed-mammals are odd-toed, with five, three, or one.

The elephant has five toes, the rhinoceros three (some species with four on front feet), the tapir three on hind feet and four on fore feet, and the existing horses have one toe. The three toes on a tapir or rhinoceros foot correspond to second, third, and fourth of an elephant. The fourth toe on a tapir's front foot is the fifth. The one toe or hoof of the existing horses is the third, that is, two toes on either side have disappeared from the typical five-toed foot.

Along with the reduction of toes from five to three, two, or even one, there have been changes in the bones which connect toes to the legs.

Reduction in the number of toes is well shown by the geological history of the horse, as shown by fossils now exhibited in the great museums. Figure 148 shows a series of such fossil feet from a four-toed horse of Eocene times down to the present one-toed type. A five-toed horse-like animal has not yet been found. It should be noted that, as the middle or third toes became the useful ones, the reduced toes were left as small bones at the sides. In existing horses there are two "splint bones" which represent the degenerated second and fourth toes of the three-toed horses.

These modifications of the feet of hoofed animals appear to be adaptations to different habits of life. The horse is 
admirably fitted to swift running on a hard plain, but the three-toed ancestors would have been better adapted to marshy land. It is well known that our present horses cannot travel on very marshy ground because the single hoof is difficult to withdraw from mud. This is why oxen are used

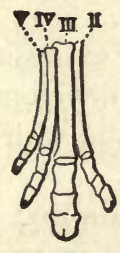

$\mathbf{I}$

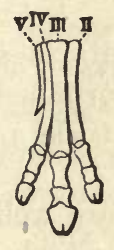

2

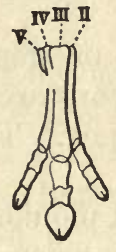

3
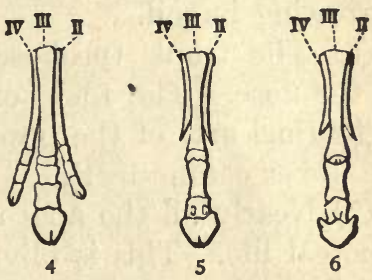

FIg. 148. Fore foot of ancestral forms of the horse. Toes are numbered in comparison with a five-toed animal in which $\mathrm{I}$ is the inner toe and $\mathrm{V}$ the outermost. Only III remains in the present-day horse (6); but there were four toes on the earliest known ancestors. (From Wiedersheim.)

in plowing and otherwise working on very soft soil, for their two-toed feet are easily withdrawn from deep mud. This leads us to think that the three-toed horses of the past ages could have traveled over marshy land as oxen and rhinoceroses now do.

The two-toed animals appear to have special advantages in rough and mountainous countries as well as in low and swampy regions. A deer or an antelope can run swiftly on a hard plain, but its feet are also adapted to other conditions not suited to the horse feet. The feet of camels are specially suited to the sandy regions where they occur, for the two-toed condition allows considerable speed even on yielding sands.

Students who are specially interested in study of adaptations of ungulate feet should examine the illustrations in such books on evolution as Romanes, "Darwin and after Darwin," Vol. I, Figs. 73-85.

(4) There are numerous adaptations of feet and jaws in carnivors. Especially noteworthy are the feet with four or 
five well-formed toes with claws, and the large canine teeth. A rudiment of the toe which does not touch the ground may be seen in dogs. It is the first, i.e., towards middle of the body. In seals and walruses the limbs are especially adapted for swimming.

(5) The head and fore feet of moles are well adapted for burrowing in soil.

(6) The trunk (proboscis) of elephants is an extension of the nose. The tusks of ivory are enormously developed teeth (incisors) of the upper jaw. The tusks of the walrus are upper canine teeth.

(7) Nearly all the apes and monkeys are well adapted to arboreal life. This is obvious when we watch the ways in which they use their hands and feet in climbing.

(8) The pouch (marsupium) of kangaroos and opossums is a special adaptation for protecting the young, which are born in an exceedingly undeveloped condition. Inside the pouch are milk-glands for supplying food.

(9) The horns and antlers of cattle, sheep, goats, and deer are adaptations for defense. Antlers of male deer are shed annually, and new ones grow rapidly. Both male and female reindeer have antlers. The hollow horns of cattle, sheep, goats, and antelopes are not shed.

(10) The skin of rhinoceroses, elephants, and hippopotami is enormously thickened, making them difficult to kill even with powerful guns.

(11) The mass of long hairs forming a mane along the dorsal surface of necks of horses and their near allies protects against bites, for horses can fight more viciously with their jaws than with their hoofs.

(12) The teeth of the rodents are specially modified for gnawing wood, nuts, and other plant materials.

(13) The skin of many carnivors is in winter well protected by close-set hairs which form furs. Examples of great commercial value are seals, foxes, minks, bear. The hairs of 
some hoofed animals develop as a woolly coat, as in sheep, goats, American bison, and Persian lamb.

(14) Adaptations for special methods of locomotion are very common among mammals. Examples are: kangaroos for jumping, sloths for hanging from under side of branches of trees, horses for running on solid ground, seals and whales for swimming, bats for flying, and many mammals of different orders for climbing.

Many other interesting adaptations of mammals may be found described in books on natural history.

362. Economic Relations of Mammals. - No other class of animals approaches that of the mammals in economic importance. The truth of this statement will be obvious to any one who considers the vast monetary value of the common domesticated mammals - horses, cattle, sheep, pigs, goats, and dogs. Also, in some countries, camels, elephants, llamas, and reindeer are important domesticated mammals.

The domesticated species are useful in (1) the human foodsupply, and (2) as beasts of burden.

Many wild mammals are also useful to man. The most valuable of these are the fur-bearers (chiefly carnivors, such as seals, foxes, mink, bear). Whales have long been hunted for the whale-bone obtained from their jaws and the sperm-oil from their "blubber." A large whale of one species may yield over $\$ 10,000$ worth of whalebone and three hundred barrels of oil. Elephants and walruses have been ruthlessly hunted for the ivory of their tusks. Sea-cows are hunted for their flesh, oil, and hides. Beavers have been nearly exterminated because of their valuable skins.

Modern methods have made it possible to utilize every particle of mammals slaughtered for human food. In addition to the meat obtained, the poorer qualities of fat are made into soap; the horns into combs; the hoofs into glue; the best hair into packing for many purposes and the poorer grade is used in plastering walls of buildings ; gelatin is made 
from tendons ; leather from the dermis of the skin; lean scraps and blood are dried to make foods for poultry and other animals; bones are made into hundreds of useful articles and the best small pieces are ground into bone-meal for feeding poultry; and any particles of bone, blood, or flesh, not usable in other ways, is dried and pulverized to make commercial fertilizers for agricultural use.

The dog was the first domesticated animal, and it is interesting to note that primitive dogs were probably chiefly kept as pets and companions, just as many of our modern dogs are to-day. The development of such uses as hunting, guarding flocks of sheep, and drawing sledges and carts seems to have come after the dog's masters began to emerge from the lowest barbarism.

Among very injurious mammals are numerous species of rodents (e.g., rats, mice, gophers, prairie-dogs, certain squirrels, rabbits in Australia); some carnivors (e.g., the dangerous cat-like species, the weasel-like forms, the bears and wolves); and fruit-bats.

Some mammals which are insect-eaters are indirectly beneficial. Many bats, ant-eaters, and the moles are examples; but owing to their subterranean habits the moles do much damage to roots among which they burrow in search of larvæ of insects.

References: Concerning the injurious mammals (rodents, wolves, etc.) there are many pamphlets issued by the United States Department of Agriculture. Two of the most interesting books dealing with domesticated mammals are Shaler's "Domesticated Animals," and J. G. Wood's "Dominion of Man."

\section{LIFE-HISTORIES OF VERTEBRATES}

363. Embryology of Lower Vertebrates. - The lesson on development of the frog's, egg ( $\$ 56-63)$ should be reviewed before continuing the study of the present chapter. 
In all species of vertebrates there are both male and female individuals; and new individuals always originate from egg-cells fertilized by sperm-cells. Asexual reproduction and parthenogenesis are not known among these higher animals.

The eggs of most fishes are laid in shallow water in quiet places, and sperm-cells discharged into the water fertilize the eggs. The enormous loss of eggs and young fishes under natural conditions has led to artificial fish-culture under the control of various states and the United States Bureau of Fisheries. The methods of hatching are a scientific application of nature's way. Fishes are caught by nets at the breeding season, and pressure on their ventral surfaces causes extrusion of eggs and sperm-cells. These are mixed in water and
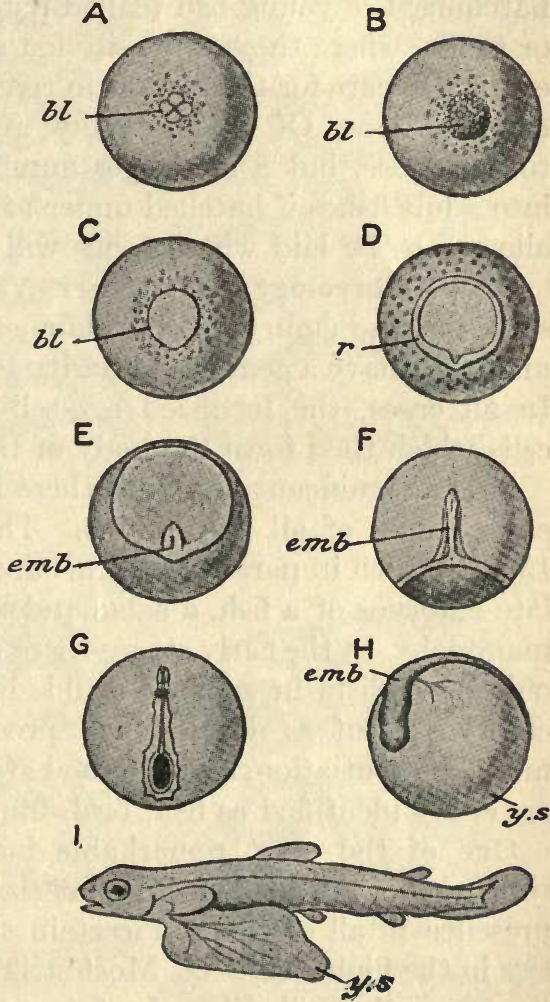

Fig. 149. Stages in development of a fish. $b l$, germ-disc, remainder of the egg is yolk; $e m b$, developing embryo; $y s$, yolk-sac attached to ventral surface after hatching. (From Parker and Haswell.)

allowed to stand in shallow pans or water-tight boxes until the sperm-cells have by swimming reached and penetrated 
the egg-cells (one sperm for each egg), and fertilized them. Later, the fertilized eggs are placed in boxes arranged so that water runs over them while they develop. After hatching, the young fish (called fry) are kept in small pools or tanks, where they are easily fed and protected until large enough to care for themselves in rivers and ponds where their enemies live. Of course many are killed after they are turned loose, but a far larger number of eggs may develop into adult fishes if hatched under artificial conditions than if allowed to be laid where fishes will naturally deposit them.

364. Embryology of Higher Vertebrates. - The early stages of the embryonic development of reptiles, birds, and mammals have a general similarity to those of the frog $(\$ 59)$. In all cases, the fertilized egg-cell divides into numerous cells which then form the body of the embryo.

It is a significant fact that there is great similarity in the early stages of all vertebrates. This is illustrated by Fig. 150 , in which in parallel columns are early, intermediate, and late embryos of a fish, a salamander, a reptile, a bird, and a mammal. In the early stages there is so great similarity that only specialists in zoölogy could distinguish between these embryos; but as development proceeds there is more and more differentiation, and the final stages at birth or hatching are easily identified as fish, bird, etc.

One of the most remarkable facts connected with this similarity of embryos of different classes of vertebrates is the presence in all of them of certain structures which are useless in the higher forms. Most striking of such useless structures are the gill-slits. In fishes these openings from the pharynx to the exterior are exits for water which enters at the mouth. Between the slits are the gills, through whose delicate membranes blood circulates and exchanges oxygen and carbon dioxide with the water. Thus in fishes the gillslits are useful as part of the respiratory system.

The gill-slits develop in very young embryos of fishes and 

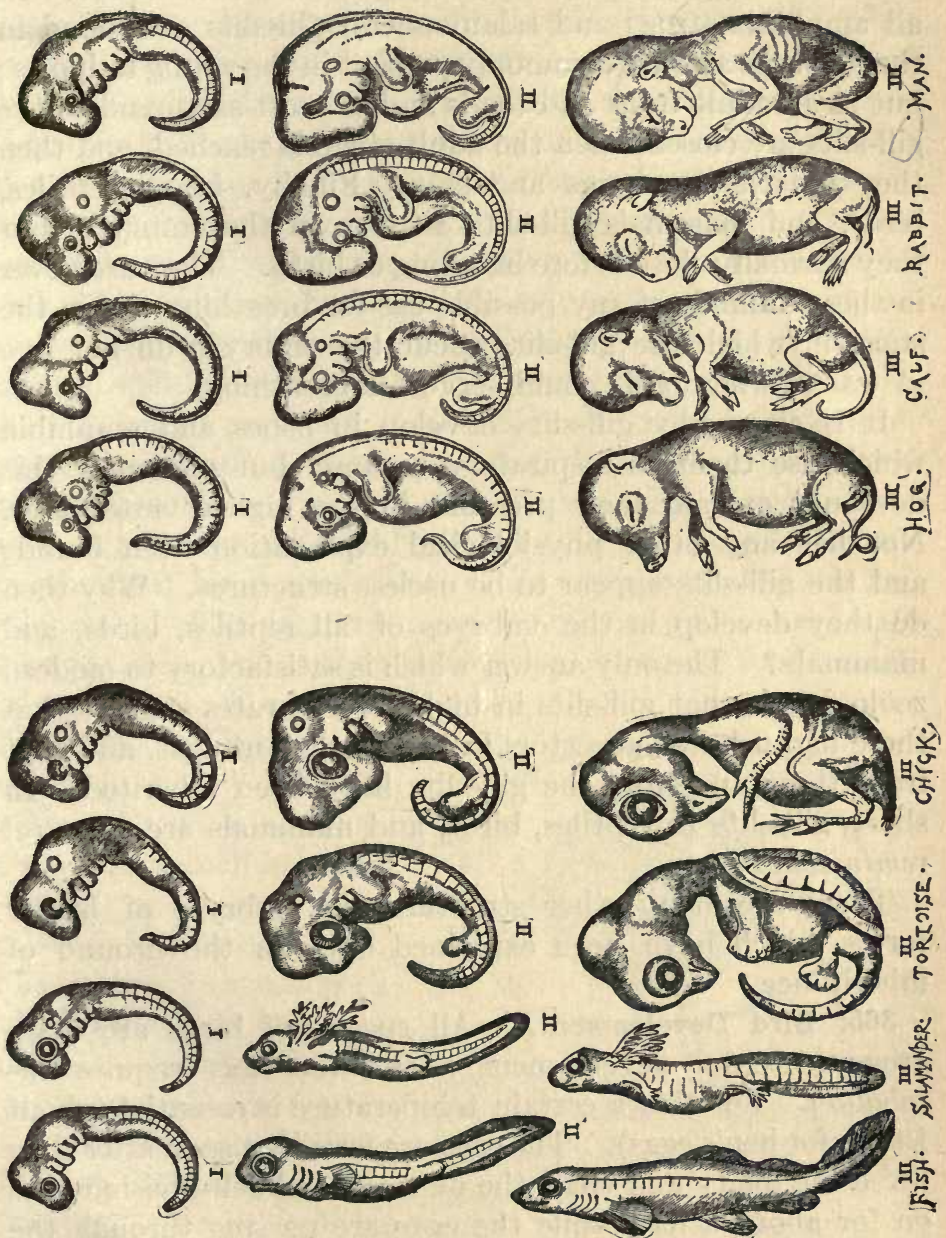

FIG. 150. Similarity of early stages of vertebrate embryos, and later differentiation. (From Romanes, after Haeckel.) 
remain throughout life, functioning as described above. In all amphibia (frogs and salamanders) gill-slits are found in the embryos and are commonly present in the young tadpoles; but in all adult frogs and toads and in most salamanders the gill-slits are closed when the adult stage is reached, and then they breathe by lungs and skin. Finally, in all reptiles, birds, and mammals gill-slits develop in the embryos, but they normally close before hatching or birth. They are never in these animals of any possible use for breathing, for in the stage in which the gill-slits occur the embryos do not live in water, where gills could serve for breathing.

It is clear why gill-slits develop in fishes and amphibia which use them as respiratory organs; but obviously this does not explain their presence in the higher vertebrates. Nor has any other physiological explanation been found; and the gill-slits appear to be useless structures. Why then do they develop in the embryos of all reptiles, birds, and mammals? The only answer which is satisfactory to modern zoölogists is that gill-slits in higher vertebrates suggest that these had fish-like ancestors in the far-distant ages, and that from these ancestors the gill-slits have been inherited. In short, gill-slits in reptiles, birds, and mammals are ancestral reminiscences.

There are many other structures in embryos of higher forms which have been explained only on the ground of inheritance.

365. Bird Development. - All species of birds are oviparous (external development); and the eggs require incubation. For this a certain temperature is essential (about 103 F. for hen's eggs). The eggs are fertilized soon after they leave the ovary and enter the oviduct, and cell-division goes on for about a day while the eggs are passing through the duct to the exterior. But soon after an egg is "laid" it becomes cooled to below the normal temperature and development stops. Within a variable number of days, the de- 
velopment may start again if the egg be warmed to the proper temperature. In natural conditions this is provided for by the instinct which causes female birds (sometimes the males) to sit on or brood the eggs. The feathers prevent rapid loss of the heat afforded by the warm ventral surface of the body of a brooding bird. This brooding instinct usually appears soon after a female bird has laid the eggs which in a given season have developed in the ovary. In many wild birds such a season of egg-laying comes only once a year; in some species two or three broods of eggs may be laid in a summer; and the well-fed domesticated hen may lay from 100 to more than 200 eggs per year, if not allowed to waste time and energy by brooding after each set of 10 to 20 eggs, as they instinctively do. The eggs of birds are large because they have a great store of food (yellow "yolk," and the "white" or albumen) for nourishment of the embryo during the development. The eggs in the ovaries of young birds are small spherical cells, but as they mature the storage of food causes enlargement. For example, in an ordinary hen's egg the "yolk" with its inclosing yolk-membrane is about one inch in diameter, but in a young ovary it is a microscopic cell. The "white" or albumen which surrounds the yolk and also the shell are secreted around the egg as it passes through the oviduct on the way to the exterior. Obviously, the

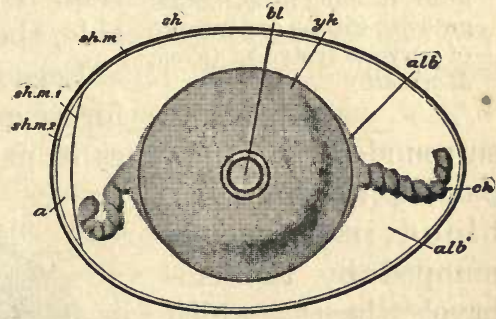

Fig. 151. Diagram of bird's egg. $y k$, yolk ; $a l b$, white or albumen; $b l$, germdisc; $c h$, thickened albumen which holds yolk in position; sh.m, two shell membranes. (From Parker and Haswell.) "yolk" is the real egg, corresponding to a frog's egg, and the "white" and shell are later additions formed like the jelly around frog's eggs. 
Careful observation of the "yolk" of a bird's egg will disclose a white spot on its upper surface. To see this, lay a

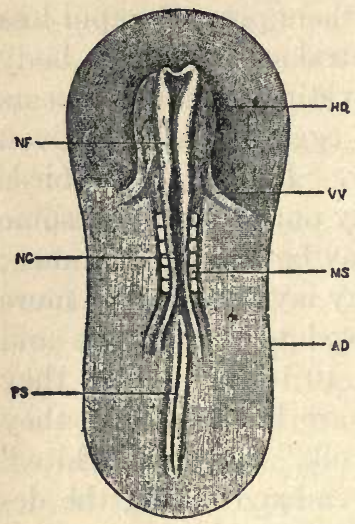

FIg. 152. Germ-disc with chick embryo after about 36 hours incubation. $h d$, head forming; $n g$, neural groove (first appearance of nervous system). (From Marshall.) fresh egg on some cotton or sawdust, carefully chip the shell with forceps, or cut with scissors, making a hole about one inch in diameter. This white spot is the germ-disc from which the embryo will develop; and all the other material inside the yolk-membrane is food. Hen's eggs kept under sitting-hens, or in incubators, for $15,24,36,48$, and 72 hours, and then opened (as directed above) will show a series of stages in the growth of the embryo from the germ-disc. Such stages are sometimes removed from eggs and prepared for microscopic examination. If such preparations are available, they should be examined.

Eggs which have been incubated for several days show the embryo surrounded by membranes in part of which there are bloodcapillaries. The blood in these is pumped by the embryo's heart, which begins to beat on the second day of incubation. The purpose of circulating blood in the blood-vessels of membranes outside the embryo's body is (1) obtaining food

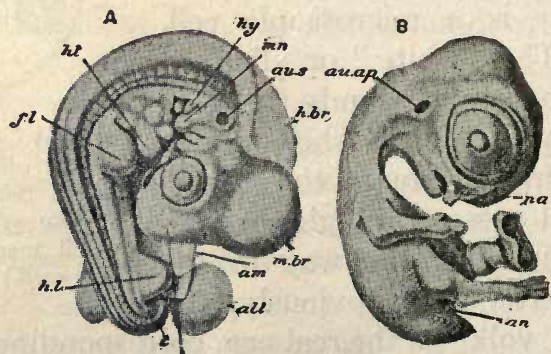

Fig. 153. $A$, chick embryo of about three days. $B$, one of about ten days with all organs formed. (From Parker and Haswell.) 
from the yolk and later from the "white," (2) obtaining oxygen from air which filters in through the pores of the shell, and (3) the discharge of carbon dioxide made by the developing embryo. The importance of the two later processes, which considered together constitute the respiration of the egg, is shown by the fact that the embryo dies from asphyxiation (lack of oxygen and excess of carbon dioxide) if the pores of the shell become filled, as when coated with albumen from another egg accidentally broken. Hence, poultry keepers must take great care to keep eggs intended for hatching clean before and during incubation.

The time for incubation varies. Hens' eggs hatch in three weeks, ducks' eggs in four weeks, and other birds have either shorter or longer incubation. No one knows why the egg of one species of bird requires more time than does that of others.

366. Reptile Development. - The embryology of many snakes, alligators, and turtles is very similar to that of birds, except that incubation is due to the sun's heat and not to brooding by parents. Some snakes and lizards are viviparous, the eggs being retained in the oviducts until fully developed into young animals which are then ejected by muscular contraction of the oviducts. The eggs of these viviparous reptiles are similar to the oviparous ones and contain much stored food to be used by the developing embryos.

367. Mammalian Development. - We have already (in $\S 63)$ noted the advantages of internal or viviparous development over the external or oviparous method; and that while cases of internal development occur among animals of many groups, it is in the mammals that we find vivipary universal, with the one exception of the Australian duck-bill, which lays eggs.

In adaptation to viviparous development the oviducts of the mammals have been modified into a sac-like structure in whose cavity eggs lie during their embryonic development. 
Such a cavity adapted to holding embryos is called a uterus or womb. In some low mammals, like kangaroos and opossums, there is a right and a left uterus formed by expansion of part of each oviduct. These lie in the same position as the oviducts of frogs. In higher mammals the right and left uteri grow together during embryonic life, and so there is a single uterus with a tube (Fallopian) extending to each ovary (right and left). Egg-cells formed and discharged by either ovary pass through the tube into the uterus and there develop into embryos.

In all mammals the egg-cells discharged are fertilized near the ovaries in the Fallopian tubes by sperm-cells which have arrived there by swimming through the secretions on the living membranes of the uterus and tubes. The fertilized egg begins to divide at once and may have undergone considerable development when, after a few days, it slips from the tube into the uterus.

The number of egg-cells fertilized at one time varies in different species. It is well known that many domesticated animals (e.g., sheep, cow, horse) usually have one offspring at a time; but some occasionally produce two (twins) or even three (triplets). Others commonly produce many young at a time (e.g., dog, cat, pig, rabbit, mice). The number of young produced indicates the number of egg-cells which were matured and fertilized.

The period of development in the uterus from fertilization to birth of the young is commonly known as gestation or pregnancy; and the length of time is highly variable. It is approximately 21 days in guinea-pig, 30 days in rabbit and squirrel, 55 days in cat, 62 days in dog, 3 months in lion, 4 months in pig, 5 months in sheep and goat, 6 months in bear, 9 months in cow, over 9 months (280 days) in human species, 10 months in whale, 11 months in horse, 14 months in giraffe, and 22 months in elephant. These are simply illustrations selected from familiar mammals. 
In order to provide for the nutrition and respiration of embryo mammals, a complicated connection is made between the blood-system of the embryo and that of the mother. Figure 154 shows a rabbit embryo with its surrounding membranes. These are abundantly supplied with blood-vessels connected with the embryo's heart. Figure 155 shows the position of an embryo mammal in a uterus. The darkly shaded area around the embryo represents lining tissue (epithelium) of the uterus, and this tissue receives its bloodsupply from the heart and arteries of the mother. The treelike processes shown in Fig. 155 are further outgrowths of the irregular processes shown (in black) on the outer membranes of the embryo represented in Fig. 154. These treelike processes receive their bloodsupply from the embryo's heart through bloodvessels in the

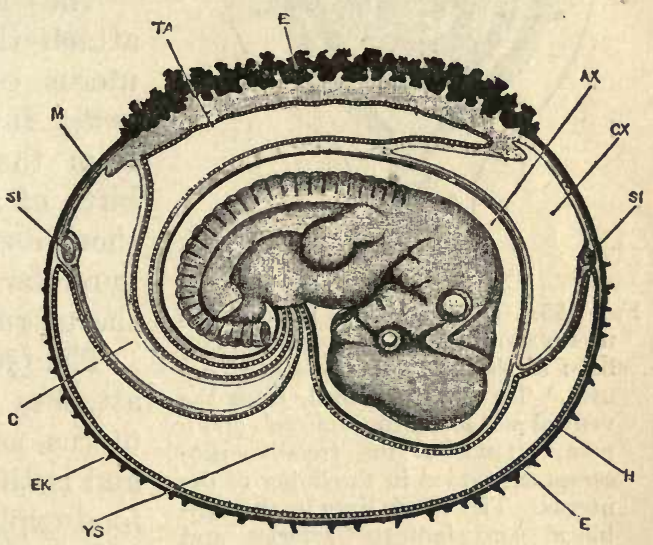

Fig. 154. Rabbit embryo of twelve days' development, with its surrounding membranes, which serve for attachment to the lining of the uterus. (From Marshall.) umbilical cord attached to the embryo at the umbilicus or navel. As a result of this close attachment of the membranes of the embryo and the lining of the uterus, the bloodvessels of the two are near enough to allow osmosis. From the maternal blood-capillaries foods and oxygen osmose into those of the embryo, and excretions of the embryo's cells pass into the maternal blood. Solid bodies, like red blood- 
cells, cannot pass from the maternal to the embryo's blood; and the blood-cells in the embryonic blood-vessels are formed from certain cells belonging to the embryo. However, the important point is that food, oxygen, and excre-

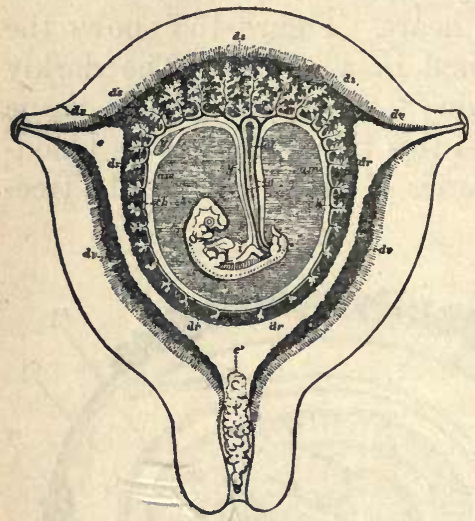

Fig. 155. Diagram of a mammalian uterus showing attachment of an embryo to the lining (black in the figure). The umbilical cord, from the ventral surface of the embryo's abdomen, extends to the tree-like processes embedded in the lining of the uterus. The black lines in the umbilical cord indicate arteries and veins connected with the embryo's heart. The openings of the uterus shown at upper right and left are to the Fallopian tubes leading to the ovaries, while the lower opening is the mouth of the uterus through which the mature embryo is finally expelled by muscular contraction. (From Marshall.)

tions osmose between the maternal and embryonic blood-vessels in the membranes which attach the embryo to the wall of the uterus.

The membranes which attach the embryo to the uterus constitute the placenta. It normally separates from the uterus after the birth of an embryo, and is then itself discharged by muscular contractions of the uterus.

The fact that an embryo attaches to the wall of the uterus as above described and is thus enabled to get food explains the large size of many mammals at birth. The eggs of all mammals are transparent cells of microscopic size, and embryonic growth is due to food supplied by the maternal blood-vessels.

It should be noted that the entire time of gestation is not occupied with the formation of the embryo's organs. For example, an embryo may develop in two or three months so that it has the form and structure of an adult, but may be 
held in the uterus many more months in order to afford protection and nourishment while it grows larger and stronger. This later stage after the organs are formed is often called a fotus, so as to reserve the word embryo for the early stages when the egg is forming organs.

In the limited time available in this course we cannot do more than study the mere outlines of mammalian development as stated in the foregoing. There are many facts in this line which are especially interesting because of the light which they throw on human life, and for these the reader must be referred to special books, and to college courses in embryology of animals. 



\section{PART IV}

\section{PRINCIPLES OF BIOLOGY APPLIED TO HUMAN STRUCTURE AND LIFE}

368. Human Biology. - Biology is the science of living things, and human biology may be defined as the study of man considered as a living thing and interpreted in the light of studies of other living things.

The justification for including study of man as part of biology is found in the fact that the human body in its structure and functions is remarkably like animals, the higher forms in particular. In the body of man are the same organs as in the animals known as beasts or mammals; and the organs of man and the beasts are closely alike even in microscopic details. This similarity also appears when comparing man with still lower animals. In short, when biologists consider the close similarity of structure and function in man and various types of animals, they see no escape from the conclusion that man's relation to the animal kingdom is as stated in the next paragraph.

369. The Classification of Man. - (1) As suggested above, man belongs to the animal kingdom, because his body is built on the plan of structure found in many animals. (2) Man is a backboned or vertebrate animal, because he possesses a backbone or vertebral column. (3) Man belongs to the class of mammals or Mammalia,because he has the three characteristics of this group, - hair, diaphragm, and mammary glands. (4) Man belongs to the order of the Primates, because his body is in numerous respects more similar to apes 
than to other animals. (5) Man belongs to the human family, the genus Homo, and the one species sapiens (a word meaning wise, and referring to the fact that man's intellectual development is characteristic and markedly distinguishes the human species from all other animals). In fact, it is in the highly developed functioning of the nervous system alone that man stands distinctly differentiated from the highest apes and other animals. This, however, is the proper field of the science of psychology (the study of mind or mental phenomena), which should be taken up in college or in private reading after graduation from high school. So far as biology is directly concerned, it simply has to take into consideration the demonstrated fact that man and other animals are remarkably similar in structure and functions. This similarity is fortunate, for it makes possible the application to human biology of many facts which were first learned by the study of various animals. (6) Finally, the human species has five varieties or races, - Caucasian, American Indian, Mongolian, Malay, Ethiopian, - each with certain peculiarities. (Look up these races in any advanced textbook of geography, or in an unabridged dictionary, and report briefly concerning their characteristics and geographical distribution.)

Study of the customs, character, history, and institutions of races of men is the science of ethnology. The science dealing with the laws of human society is sociology. General study of man, combining facts of ethnology, biology, psychology, and sociology, is anthropology. The following brief definitions in parentheses will help the memory : psychology (science of mind); sociology (science of society); ethnology (science of human races); anthropology (science of man). Each of these lines of study of human life is now so highly developed that special books are necessary; but they all are founded on biology to such an extent that a knowledge of that science is important for the reader of any of the special sciences dealing with man. 


\section{CHAPTER XVII}

\section{HUMAN STRUCTURE AND LIFE-ACTIVITIES}

370. General Plan of Human Body. - Like the frog, already studied, the human body consists of head, trunk, and limbs. The trunk is composed of the chest or thorax, and the belly or abdomen. The arms are the upper or anterior limbs; the legs are the lower or posterior limbs. Note that the thigh of a leg corresponds in structure to the upper arm, the shank to the forearm, the ankle to the wrist, the toes to the fingers. Note also that externally the human body is bilaterally symmetrical.

371. Skeleton. - Like the frog and all the other backboned animals, the human body is supported by an internal framework or skeleton composed of bone and cartilage, which is chiefly at the ends of bones. There are more than 200 bones in the human skeleton; 33 vertebræ or segments of the spinal column, about 25 bones in the skull of an adult, 24 ribs, 30 bones in each arm and leg, and the bones in the shoulder-girdle and pelvis. The number of bones varies with the age; for example, the skull bones are more numerous in young children, but they grow or fuse together as the individual becomes older. Each half of the pelvis is composed of three bones which have fused together. Nine bones (vertebræ) at the posterior end of the spinal column are fused together in connection with the pelvis, leaving 24 separate vertebræ. 
(L) The best way to study the human skeleton is to compare a mounted skeleton with labeled drawings in textbooks of anatomy and physiology. At the same time the pupil should locate the position of the larger bones in his own body. If a mounted skeleton is not owned by the school, the pupil should locate as nearly as possible the bones in his own body, using labeled pictures as a guide. The names of the large bones are so frequently referred to that it is desirable to memorize them. Pupils who are studying drawing will find various parts of the skeleton good objects for sketching; but as a rule this work is not possible in the limited time available for the biology class-work.

The most important parts to notice while examining a skeleton are: (1) The backbone or vertebral column, to which all other parts of the skeleton are attached. It is the central supporting axis of the body. (2) The bones of the two pairs of limbs, comparing the anterior with the posterior pairs. (3) The ribs and the bones which connect the arms with the backbone. (4) The pelvis, which connects the legs with the backbone.

(5) The larger bones of the skull.

372. Body-wall and Body-cavity. - As in the frog, the outer, fleshy wall which incloses the internal organs is the body-wall; the internal cavity is the body-cavity. In the frog there is one cavity in which lie the heart, lungs, liver, stomach, intestine, kidneys, and reproductive organs. In the human body the diaphragm forms a partition across the body-cavity, dividing it into the anterior (upper) cavity containing the heart and lungs and known as the thoracic cavity (chest-cavity), and the posterior (lower) abdominal cavity, which contains all the internal organs except the heart and lungs. The thoracic cavity is inclosed by the ribs, while the abdominal cavity is bounded by the muscular walls of the abdomen.

Structure of the Body-wall. - (D) This is essentially the same in man and other mammals, and so we may study any of the animals found in meat-markets. A slice of bacon will serve our purpose. On the one edge of the slice is the skin or "rind." This, of course, was the outside skin of the pig. The streaks of lean meat are muscles of the body-wall. Fat has been deposited between the muscles, and also between the muscles and the skin. The thick- 
ness of the body-wall depends upon the amount of fat; hence very lean bacon is relatively thin. Notice that the skin is fastened firmly to the muscles and fat; this is due to fibers of connective tissue. In some parts of the body of mammals the skin is not fastened down so closely as in bacon; e.g., note the loose skin on the back of your own hand, or on the backs of young puppies.

\section{Relative Positions of Internal Organs in Man. -} Study diagrams, charts, and a manikin, if available; and note the following positions of the largest organs, which are in the same relative position as in the frog. (1) The body-cavity is ventral to the backbone. (2) The alimentary canal extends through the body-cavity from anterior to posterior. (3) The heart is ventral to the alimentary canal (esophagus part). (4) The liver lies ventral to the alimentary canal (stomach and intestine part). (5) The kidneys lie dorsal to the alimentary canal and in the posterior part of the bodycavity (abdominal part). (6) The brain and spinal cord lie in the cavities formed by the bony skull and the backbone.

374. Life-activities of the Human Body. - The characteristics of living things already studied in connection with animals and plants apply to the human body, for it is a living mechanism which performs the functions necessary for life.

The life-activities are located in the cells; and these, as in the case of the frog, are in the tissues (epithelial, connective, muscular, bony or osseous, cartilaginous, and nervous).

Probably the two activities which most attract our attention are those concerned with food and breathing. Moreover, these are the basis of all the other processes occurring in the human body. For these reasons we shall specially consider the taking of foods and oxygen into the human body, and later we shall trace the changes of foods and oxygen in the body. This will lead us on to consider all the essential lifeactivities. 


\section{FOODS}

375. What are Foods? - A convenient definition for our present purposes is that foods are solid or liquid substances which when taken into the alimentary canal are useful in the life-processes of our bodies. In most cases substances able to serve as our foods must be capable of being digested and absorbed as materials for energy, repair, and growth; but a certain amount of plant cell-walls is useful, although not digestible, in the human alimentary canal.

Sources and Kinds of Human Foods. - $(L)$ Write in your notebook a list of some common foods, arranging in three columns those of animal, plant, and mineral origin.

376. Nutrients. - We might consult cook-books and make a very long list of the names of prepared foods which are served on our tables; but these are made by combinations of such common ingredients as meats, vegetables, milk, butter, lard, sugar, flour, starch, chocolate, salt, etc. Chemists have shown that these common things which are used in every kitchen in combining our foods are composed of certain chemical compounds known as sugar, starch, fat (oil), proteins, and minerals. These compounds, from which all the combinations of human foods are made, are known in physiology as nutrients.

In order to prove that various foods are made up of these few nutrients, we need to know some method of identifying each. Fortunately chemists have discovered some simple tests which are easily applied, as directed below.

377. Chemical Tests for Nutrients. - Certain chemicals produce characteristic reactions on nutrients and hence may be used to detect their presence in mixtures of foods.

Starch Test. - (D or $L)$ Repeat the iodine test with diluted starch paste $(\S 100)$.

Sugar Test. - $(D$ or $L)$ Boil a few grapes, raisins, or prunes in water in a test-tube for two minutes, and this will extract some sugar. 
Into another test-tube. put some Fehling's reagent, ${ }^{*}$ the ingredients of which are usually kept in separate bottles and mixed when needed. Heat the Fehling's reagent, and then with a pipette slowly drop into it some of the sugar solution obtained from the dry fruits; or drop some of the reagent into the sugar solution. A red color indicates the presence of one kind of sugar. One such experiment could not prove that when Fehling's reagent causes a red color it means that sugar is present, for we have not yet tried this solution on starch, fat, and other things. However, chemists have tried the Fehling's reagent on all the substances commonly found in animals and plants, and it has been demonstrated that only certain kinds of sugars produce the red color. We have not time to repeat such inves igations, and so we must accept the chemists' statement that Fehling's reagent is a test for a sugar found in grapes and known as grape-sugar or dextrose. The same sugar in corn-syrup is popularly called glueose. Its chemical formula is $\mathrm{C}_{6} \mathrm{H}_{12} \mathrm{O}_{6}$. Ordinary sugars sold in stores are chiefly cane-sugar $\left(\mathrm{C}_{12} \mathrm{H}_{22} \mathrm{O}_{11}\right)$. Milk-sugar (lactose) and malt-sugar (maltose) have the same chemical formulas as canesugar (sucrose).

Kinds of Sugars. - ( $D$ or $L)$ Dissolve some commercial glucose, or corn-syrup, in water and test with Fehling's reagent. Dissolve some ordinary "granulated" sugar in water, and test. This latter will sometimes give no red color until after it has been boiled for some time or treated with strong acids. The explanation is that most "granulated" sugar is chiefly the kind known as cane-sugar, while sugars which give red color in Fehling's test are of the kinds known as grape-sugar and fruit-sugar. The boiling or treating with acid changes the cane-sugar into the other kinds; and these can then be tested with Fehling's reagent. Taste granulated sugar and glucose, and compare as to sweetness. As a practical point, it is interesting to note that granulated sugar should be added to fruits after cooking. If added before, the sweetness will be partly lost by change to the other sugars, which are as good food, but less sweet.

Tests for Fat. - (D or $L)$ Put a drop of olive oil on a sheet of white paper, and note that a grease-spot is produced. Dissolve some beefsuet in a small quantity of benzine or ether (keep such volatile liquids as these far away from a flame), put a drop of the solution on paper, and notice the spot left after evaporation of the benzine.

* Fehling's reagent, a test for certain kinds of sugar, may be purchased from dealers in chemicals or at ordinary drug-stores. It may be made according to the formula in the "Teachers' Manual" which accompanies this book. 
Or lay a piece of suet on a paper and heat slowly. This "greasespot" test is a simple way of finding whether fats or oils are present in foods.

Tests for Protein. - ( $D$ or $L)$ Mix a small quantity of white-of-egg in water in a test-tube, shake well, add some strong nitric acid, boil until the solution turns yellow, then add drops of ammonia until an orange color appears. Or instead of the acid, add to the egg-albumen in water some drops of Millon's reagent (mercury dissolved in nitric acid; obtainable from chemists), heat slowly, and red color will appear, especially after cooling.

Tests for Water and Minerals. - The loss of weight by drying foods is chiefly due to evaporation of water. The ashes left after burning foods represent the mineral contents. By complicated processes chemists can analyze the ashes and determine the kind and proportion of elements present.

378. Sugars and Starches: Carbohydrates. - All sugars and starches are grouped together under the name of carbohydrates. They contain but three elements: carbon, hydrogen, and oxygen. We have already learned that starch is formed in plant cells which have chlorophyll, and that starch is readily digested by enzymes into sugar, or sugar turned back again to starch for storage. The carbohydrates which are used as human foods are chiefly cane-sugar or sucrose and milk-sugar or lactose (both with the formula $\mathrm{C}_{12} \mathrm{H}_{22} \mathrm{O}_{11}$ ); grape-sugar and fruit-sugar (both with the formula $\mathrm{C}_{6} \mathrm{H}_{12} \mathrm{O}_{6}$ ) ; and starch (formula is some multiple of $\mathrm{C}_{6} \mathrm{H}_{10} \mathrm{O}_{5}$ ).

As we have seen, grape-sugar is found in raisins and other fruits. Under the names of glucose and corn-syrup it is common in the markets, and is made by treating the starch of corn grains with strong sulphuric acid. Lactose or milksugar is sold in all drug-stores for use in preparing foods for infants and invalids. Malt- or barley-sugar (maltose) is also sold, especially for flavoring candies, etc. The common "granulated" sugars in our markets are sucrose from the juices of sugar-cane and sugar-beet. The yellow and brown sugars are the crude sugars obtained by evaporating the juice 
pressed from cane and beet; and by refining processes, these dark-colored sugars are made into the "soft white" and "granulated" sugars. Maple sugar, from the sap of maple trees, is chemically the same as cane-sugar, but flavored with peculiar substances found in the maple sap.

379. Fats. - This includes all kinds of fats and oils from animals and plants. Common examples are butter, lard, beef-suet or tallow, olive oil, cotton-seed oil, fat of meats, oil of nuts. Like the sugars and starches (carbohydrates), fats contain only three elements : carbon, hydrogen, and oxygen. The sugars, starches, and fats taken together are often called " non-nitrogenous foods," because they have no nitrogen.

380. Proteins. - Formerly spelled "proteids." Whiteof-egg (" egg-albumen"), lean meat, and milk curds have abundant proteins. They contain carbon, hydrogen, nitrogen, oxygen, and sulphur. Also, some have phosphorus and iron. It appears then that all proteins differ from carbohydrates and fats in having nitrogen and sulphur. As already stated, only plants can make proteins, and animals must get them directly or indirectly from plants.

381. Albuminoids. - This term, which means albumenlike, is applied to certain food substances which have the composition of proteins (formerly called albumens). Gelatin is the most common example. The finer quality used for human food, for photographic plates, and for bacteriological study is obtained by cooking connective tissues, tendons, and marrow of bones. Pigs' feet, which are by many people considered a delicacy, produce much gelatin when boiled. Common furniture glue is a crude gelatin from the connective tissue of the hides and hoofs of animals. Fish glue is a gelatin made from the delicate membranes of the air-bladders of fishes. Common gelatin is the only albuminoid used extensively for human food. Although having the composition of proteins, gelatin cannot take their place as food. Dogs have been found to live well with only protein foods (e.g., lean meat); 
but with gelatin alone they soon begin to lose weight and to show other evidences of starvation. Hence, gelatin must be used with protein foods.

382. Inorganic or Mineral Foods, and Water. - The inorganic foods are the only ones not formed by animals or plants. As we have seen, water plays an important part in all living matter. In the human body it is especially important in dissolving foods during digestion, and also as the circulating medium in the blood- and lymph-systems.

Common salt (sodium chloride, $\mathrm{NaCl}$ ) is only one of a number of mineral salts necessary in the human body. A compound containing iron gives the red color to blood; lime (calcium) is necessary in the bones; and less noticeable quantities of other elements $(\mathrm{P}, \mathrm{K}, \mathrm{S}, \mathrm{Mg})$ are needed in the human body. Most animal and vegetable foods which we commonly use contain these necessary elements, and so we do not have to give any special attention to obtaining them. Common salt is the only mineral food which is regularly added to our diet in addition to what is naturally in our organic foods; and it is probably true that we use this greatly in excess of what the body actually requires.

383. Testing Foods for the Nutrients. - ( $D$ or $L)$ Apply the tests for starch, proteins, and grape-sugar to oatmeal, flour, white-of-egg, egg-yolk, potato, onion, rice, beans, peas, lean meat, apple, honey, corn-syrup, pears, corn-meal, and other common articles of food. Place each food to be tested in some water in a test-tube, boil for a few minutes, and then pour in the testing reagent to be used. Make a table in your note-book and record the results of the tests by making a mark in the proper columns for the nutrient found to be present.

\begin{tabular}{l|l|l|l|l}
\hline Name of Food Tegted & $\begin{array}{c}\text { Contains } \\
\text { Sugar }\end{array}$ & $\begin{array}{c}\text { Contains } \\
\text { Starch }\end{array}$ & $\begin{array}{l}\text { Contains } \\
\text { Protein }\end{array}$ & $\begin{array}{c}\text { Contains } \\
\text { Fat }\end{array}$ \\
\hline & & &
\end{tabular}


384. Foods are Combinations of the Nutrients. - From the foregoing experiments, it is evident that one or more of the nutrients is present in each of the common foods. Chemists have proved that the useful constituents of all our foods are carbohydrates, fats, proteins, mineral salts, and water. The above experiments also showed that some foods have a preponderance of certain nutrients ; e.g., whiteof-egg is chiefly protein, potato is chiefly starch, corn-syrup is almost pure sugar, butter and beef-suet are largely fat. It is evident that if all the nutrients are needed in human diet, they can best be obtained by a mixture of foods; that is, a meal composed of lean meat, potato, bread, butter, and some form of sugar might be arranged to supply equal amounts of protein, fat, sugar, and starch. We shall see later that equal amounts of these nutrients are not needed; but that a mixture of foods is necessary in order to give the proper amount of each of the nutrients of which our common articles of food are composed.

\section{STRUCTURE OF HUMAN DIGESTIVE ORGANS}

In order to understand many points concerning the work of the organs which deal with foods, we must first get a clear idea of the general structure of the alimentary canal and of its attached organs which secrete digestive fluids (liver, pancreas, salivary glands). Therefore, we must for a time turn aside from considerations of function and study the structure of these organs.

385. The Mouth-Cavity. - $(L)$ Turn your back to a window or a lamp, and with a hand-mirror reflect the light into your open mouth. Notice the hard palate forming the roof of the moutheavity. At the back of the mouth-cavity is the soft palate, which separates the mouth-cavity from the post-nasal cavity; and this cavity in turn communicates with the cavities of the nose. Take "short breaths" and notice the effect upon the soft palate. Apply your tongue to the roof of the mouth and slowly move it backward 
and forward until you feel the shape, position, and texture of the hard and soft palates. Press down upon the tongue with a clean (sterile) glass rod, or the handle of a spoon, and examine the small prolongation of the soft palate which touches the tongue when that is not depressed. This is the uvula.

386. The Teeth. - $(L)$ Examine your teeth, again using the handmirror, taking the following description as a guide: Beginning at the middle line at the front of each jaw, there are in order the following kinds of teeth in half of either the upper or the lower jaw : First, two chisel-shape cutting teeth (incisors, meaning to cut into). Next, a tooth with a more pointed edge, which corresponds to the great fangs of dogs and cats and other animals which must hold their prey; hence the name canine or dog-teeth. The tusks of boars and walruses are enormously enlarged canine teeth. Elephants' tusks are upper incisors. Next back of the canine tooth on each side there are in the first or "milk-set" of teeth two grinding teeth (molars). This makes a total of twenty teeth in the first or milk-set, which are deciduous. In adults there are in each half of a jaw two teeth called bicuspids (meaning two cusps or points) in place of the two molars of childhood; and back of these are three molars, often called "wisdom teeth." There are, therefore, twelve molars in adults in addition to teeth in the places occupied by the twenty teeth of the first or deciduous set, making a total of thirty-two for the adult.

The incisor teeth begin to appear in children at six or eight months of age, and the full milk-set is present after eighteen to twenty-four months. The loss or shedding of these, caused by growth of new teeth below, occurs at various times between seven and twelve years of age. The permanent teeth begin with the incisors at seven or eight years and are completed with the appearance of the molars or wisdom teeth at between sixteen and twenty years of age. The growth of the teeth through the fleshy tissue (gums) is often called " cutting teeth."

The structure may be studied by breaking open an extracted tooth, or better by studying a thin section prepared for microscopic use. There is a central cavity which, during the life of the tooth, is filled with a soft mass composed of con- 
nective tissue, blood-vessels, and nerves. This is the socalled pulp. The pulp-cavity extends down into each of the roots of the tooth, and at the tip of a root is a small opening through which nerves and blood-vessels enter. The hard outer part of a tooth consists of the enamel, and the main bulk is dentine, a kind of ivory. The roots of the tooth, which are buried in holes or sockets of the jaw-bone, are covered with a thin layer of bony substance, called cement.

387. The Tongue. - Examine with a hand-mirror. The elevations on the upper surface are papilloe, and nervefibers connect these with the brain. Their function is that of taste and touch. The tongue is chiefly muscular tissue, the muscle-fibers extending longitudinally, transversely, and perpendicularly. Can you think of any relation between such arrangements of the fibers and the possible movements of the tongue?

388. Salivary Glands. - The epithelium which lines the mouth secretes a limited amount of thick fluid known as mucus, hence it is called a mucous membrane. But most of the fluid in the mouth is saliva from three pairs of salivary glands. On either side of the head a gland lies beneath and in front of the ear. These are the parotid (meaning beside the ear) glands, and a disease affecting them is called parotitis* or mumps. A duct from each parotid gland opens into the mouth-cavity on a little elevation on the inside of the cheek near the grinding teeth. The elevation can be seen by using a mirror and holding the cheek away from the jaw. The two other pairs of salivary glands are placed among the the muscles beneath the tongue, and their ducts open into

* Notice that the ending itis added to the name of the organ (parotid) means inflammation or disease of the organ. Likewise, there are in common use such words as appendicitis (inflammation of the appendix of the intestines), gastritis (of the stomach), laryngitis (of the larynx), tonsilitis (of the tonsils), and many other diseases designated by adding itis to the name of the organ involved. 
the mouth-cavity beneath the tip of the tongue. The secretions of the salivary glands will be described later when
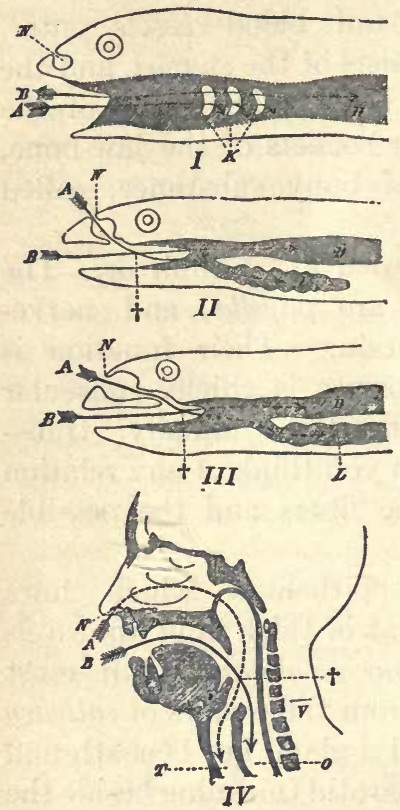

Fig. 156. Diagrams showing relations of respiratory and alimentary passages in a fish $(I)$, amphibian $(I I)$, reptile or bird $(I I I)$, and man $(I V)$. Arrow $A$ marks passage to respiratory organs ; $B$, to alimentary organs ; $N$, nostrils ; $K$, gill-slits ; $D$, alimentary canal ; $L$, lungs ; $O$, esophagus ; $T$, trachea; $V$, backbone. (AfterWiedersheim.)

we study their work in connection with the digestion of foods. 389. The Pharynx or Throatcavity. - Again using the handmirror, notice that at the back part of the mouth-cavity muscular folds extend downward from the soft palate and bound laterally the passage from the mouthcavity to the throat-cavity. Between these folds on each side is a round body, tonsil. These are the organs which often become enlarged during a "cold" in the throat (tonsilitis). They are not known to have any essential function, and surgeons frequently remove them when they become permanently enlarged.

The pharynx opens above into the post-nasal cavity behind the soft palate; and below into the esophagus and the trachea or windpipe. Refer back to your study of the throat of the frog. Also, there open into the pharynx the Eustachian tubes from the ears. The pharynx is the central passage for communication between the nose-cavities and the trachea, and between the mouth-cavity and the esophagus, thus providing for movement of air from the nose to the lungs and of food from the mouth to the esophagus. 
390. The Esophagus or Gullet is a tube extending from the pharynx to the stomach. In the human body, and in all mammals, it extends through the diaphragm, the membrane which divides the body-cavity into thoracic and abdominal portions. In the neck the esophagus is behind (dorsal to) the trachea.

391. The Stomach is a muscular and greatly expanded portion of the alimentary canal or digestive tube between the esophagus and the intestine. It lies on the left side and in contact with the liver. Imagine a membrane (diaphragm) stretched across the frog's bodycavity so as to separate the heart and lungs from the stomach and liver. The human diaphragm lies in the same position; that is, the heart and lungs lie above it (anterior) and the stomach and liver lie just beneath (posterior). The end of the stomach connected with the intestine is provided with a muscular ring (pylorus) which, by opening and closing, is able to control the passage of food from the stomach to the intestine. It should be remembered that the size of a stomach, like that of a rubber bag, depends upon the amount of disten-

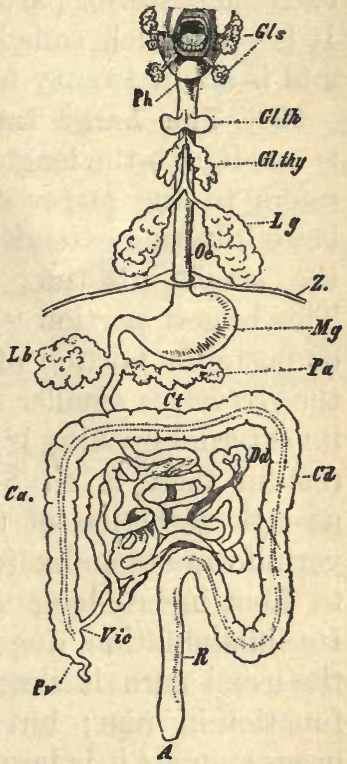

Fig. 157. Human alimentary organs. Gls, salivary glands; $P h$, pharynx ; $G l$. th, thyroid gland; Gl. thy, thymus ; $L g$, lung; $O e$, esophagus ; $Z$, diaphragm ; $m g$, stomach ; $P a$, pancreas ; $L b$, liver ; $D d$, small intestine; Vic, valve between small and large intestine; $P v$, appendix ; $C a, C t, C d$, colon of large intestine; $R$, rectum; $A$, anus. (From Wiedersheim.) tion. When empty, it is contracted so that it has practically no cavity, and when much distended by food, it may hold about a half-gallon. 
392. The Small Intestine. - The part of the intestine connected with the stomach is smaller in diameter than the extreme posterior part, and hence is called the small intestine. It is very much coiled, as shown in the center of Fig. 157, and is about twenty feet long.

393. The Large Intestine is about five feet long, which is one-fourth the length of the small intestine; but the name refers to the larger diameter. As shown in Fig. 157, the large intestine extends upward, from the point of union with the small intestine, then transversely, then downward. This largest portion is often called the colon. The terminal or posterior portion of the large intestine, commonly called the rectum, is smaller in diameter than the colon part; and its external opening is the anus. Between the colon and the rectum there is an S-shaped loop (sigmoid flexure); and near the junction of the large and small intestines is the vermiform appendix (or simply appendix) a tube from two to four inches long and one-fourth an inch in diameter. Its inflammation, due to bacteria, is appendicitis, for which the usual cure is surgical removal of the organ. It has no function in man; but in the rabbit and many lower herbivorous animals it is large and important in digestion.

394. Liver and Pancreas. - These two organs should be named in the list of digestive organs, for they secrete fluids which are poured into the intestine by ducts. The position of the liver has been described in connection with the stomach. The pancreas, which in the case of some animals used as human food is called "stomach-sweetbread,"* lies near the junction of the stomach and the small intestine. Its main duct (pancreatic duct) joins the bile-duct from the liver, and the fluids secreted by the two organs are poured into the intestine a short distance from the stomach.

* The neck- or throat-sweetbread sold in meat-markets is from the thymus, an organ found only in young animals, such as calves and lambs, and lying in the anterior part of the chest-cavity close to the neck. 
395. Glands. - The liver, pancreas, salivary glands, and gastric glands have been mentioned as producers of special secretions useful in digestion. Microscopic study shows that a gland is composed of a layer of epithelial cells which rest on a bed of connective tissue. In this latter tissua are blood-vessels which supply food and oxygen to the cells of the glands; and from the materials thus obtained the cells manufacture their secretions, which they discharge at the free end of the cells (i.e., opposite the end where they may absorb from the blood). See Figs. 158, 159.

(D) Sections of frog's stomach show structure of simple gland. Pancreas sections show numerous tubes cut across at various angles.

\section{THE WORK OF THE DIGESTIVE ORGANS}

We have briefly considered the structure of the digestive organs, and it is now our problem to study the work of these organs, especially with reference to the preparation for absorption of the different kinds of food; i.e., the nutrients. As in the frog, this digestion of foods is caused by secretions; and in the human body these are saliva from the salivary glands, gastric juice from the glands in the wall of the stomach, intestinal juice from the glands in the wall of the intestine, pancreatic juice from the pancreas, and bile from the liver.

396. Mechanical Processes in Digestion. - Various movements, due to the action of muscles in the digestive organs, play two important parts: (1) in taking food and in moving it along through the digestive tube, and (2) in separating more or less solid food into small particles upon which the digestive fluids can act rapidly. The muscular action of the lips and jaws in taking and chewing (masticating) food is so easily observed that no description is necessary. The mastication process is important in that it results in mixing saliva with the food (see $\S 455$, on hygiene of eating).

After mastication comes the swallowing movements, 
which are produced by the muscles of the pharynx and esophagus. These movements are in the beginning voluntary (i.e., under control of the will); but it is well known that when food has started down the esophagus, the muscular walls of that organ contract (narrow the diameter) involuntarily, and force the food into the stomach. The fact that food is forced down and does not run because of gravity explains how an acrobat can perform the feat of drinking water while standing on his head. Horses and cows are examples of animals which regularly drink and eat with the mouthcavity much lower than the stomach.

(D) Take a piece of rubber tubing about two feet long, suspend vertically, plug the lower end with a cork, and fill with water. Now begin at the plugged end and grasp the tubing between a thumb and finger and then move your hand upward so as to contract the tube in succession. It is evident why the water appears to run up-hill in the tube. Similarly, the muscles which are in the walls of the esophagus contracting in succession from the pharynx toward the stomach force the food along the esophagus.

The muscle-fibers in the walls of the stomach are arranged transversely, longitudinally, and obliquely. The part of the stomach next to the esophagus contracts and steadily presses upon the contained food; but the posterior half undergoes a series of wave-like constrictions. The result is that the food is broken up into smaller masses and well mixed with the digestive fluids. These movements have been studied by feeding dogs and cats with bismuth subnitrate, a drug which physicians often prescribe to allay gastric irritation. This drug mixes with the food in the stomach and renders the whole mass so opaque to the X-rays that the shape of the stomach is easily seen with the aid of a fluoroscope, and photographs can be made similar to those often taken of the skeleton within the body.

From time to time the pylorus opens, allowing liquid foods (often called chyme) to pass into the intestine. As 
a rule, the large masses of solid food are held back in the stomach.

The movements of the intestine consist of constrictions. These sometimes appear in series at certain points, and then next at the points which are halfway between the first constrictions. This causes a thorough mixing of the foods with the digestive fluids. Another form of constriction which occurs at intervals moves along the intestine and forces the contents towards the large intestine. The above experiment with rubber tubing illustrates this type of constriction. Such movements are known as peristaltic contractions.

397. Why Foods must be Digested. - We have seen in our study of the frog that foods must be prepared so that they may be absorbed through the lining membranes (epithelium) of the digestive organs into the blood. In other words, foods must be capable of osmosing through membranes; and most foods, as taken into the mouth, are not ready for this process. Sugar and common salt dissolved in water are practically the only cases of common food which can osmose or be absorbed without digestion. Even milk, our most common liquid food, contains droplets of fat too large for absorption and which must be dissolved in preparation for absorption.

(D) Microscopic Examination of Milk.-Mount a drop of milk on a glass object-slide. Examine with (1) low-power, (2) high-power objective. Note the droplets of fat. These are lighter than the water of the milk; and, like oils in general, rise to the surface when milk stands, forming the concentrated layer of fat which we call cream. When the cream is shaken or agitated, as in a churn, these fat droplets fuse together into larger masses of fat, which we call butter.

Since digestion practically means the preparation of foods for absorption, it is convenient to study the action of the various digestive secretions (saliva, gastric juice, bile, and pancreatic fluid) by adding them to foods in test-tubes; and 
after allowing time for a change, try for osmosis of the food through membranes, such as fish-bladder or parchment. There are good reasons for thinking that any food which will osmose through a dead membrane will also be absorbed under conditions which exist in the linings of the stomach and intestine. Hence any digestive changes which will prepare foods in test-tubes for osmosis will serve to illustrate the changes which take place inside the living alimentary canal. But not all the digestive processes can be illustrated by testtube experiments, for the living digestive organs cause some changes in foods which cannot be imitated in the lifeless conditions in test-tubes. However, it is possible to perform a number of experiments which will throw much light on the various secretions and parts of the alimentary canal with regard to their part in the digestion of the various kinds of foods. We shall consider the digestive processes in the order in which foods come in contact with the secretions in passing from the mouth-cavity through the esophagus into the stomach, and thence into the intestine; this means contact, in succession, with the salivary, gastric, intestinal, pancreatic, and hepatic (liver) secretions.

398. Digestion by Saliva. - The fluid secreted by the salivary glands consists chiefly of water and an enzyme called ptyalin, which is very similar to the diastase of plants. The dry ptyalin may be purchased from chemists, or saliva may be collected in a test-tube (when chewing a piece of rubber or gum it is secreted rapidly). The work of saliva is the digestion of starch to a kind of sugar, which is absorbable. This is illustrated by the following experiments:-

(D) Make some thin starch paste, by heating starch in water. Notice that the resulting fluid is not clear, but opalescent. Place a small quantity of the paste in a test-tube and add a few drops of iodine-solution. Note the color.

(D) Place some of the paste in a small bag made from a piece of gold-beaters' membrane, or fish-bladder, and suspend the bag so 
that it dips into water in a small tumbler or beaker. Or put some of the paste in an osmose-apparatus.* After allowing an hour or two for osmosis to take place, transfer some water from the tumbler into the test-tube, and add some iodine solution. Compare with test of starch as directed above. Is there evidence that starch osmoses through a membrane?

(D) Along with the above, test grape-sugar or corn-syrup by placing in water inside a bag or in the tube of the osmose-apparatus. Wait the same time as in the case of the starch, and test water in the tumbler with Fehling's reagent ( $\S 102)$. . Does the sugar osmose?

$(L)$ Mix some starch scraped from a potato in a drop of cold water on a glass slide, put on cover-glass, and examine with low power of microscope. Note the appearance of the starch-grains. Now remove the slide from the microscope and heat slowly over an alcohol- or a gas-burner until the water begins to steam. Examine again with the microscope, and note the broken starch-grains. These broken grains mixed with the water form starch paste, and the opalescent appearance of the thin paste is due to the numerous particles of starch floating in the water. Examine starch scraped from a baked or boiled potato.

(D) Place some thin starch paste in each of two test-tubes. Add to one tube (No. 1) a few drops of clear saliva (filtered through coarse filter paper). Tube No. 2 has only paste. After twenty to forty minutes note the appearance of the paste in the tubes, especially with regard to opalescence. Pour a small quantity from tube with the saliva (No. 1) into a clean test-tube, and add a few drops of iodine solution. Is starch present?

(D) Into another tube pour some starch paste from tube No. 1, and into still another pour paste that has been acted upon by saliva in tube No 2. To each of the tubes apply Fehling's test. Results? Conclusions? Do these experiments suggest why a dry starchy cracker becomes sweet to the taste after being held in the mouth for some time?

(D) If time permits, prepare three tubes with thin starch paste. To No. 1 add boiled saliva; to No. 2 add normal saliva, but add a few drops of acid (vinegar will do) to make the paste slightly acid (test with blue litmus-paper); and to No. 3 add saliva, but keep the tube standing in a tumbler filled with finely cracked ice. After twenty to forty minutes test for starch and sugar as in above. What do these experiments show regarding (1) effect of boiling 
saliva, (2) effect of acid, (3) effect of low temperature? Boiling and low temperature have the same effect on all the secretions of the stomach, intestine, and pancreas.

(D) In order to show that saliva changes starch into sugar capable of being absorbed (i.e., osmose through a membrane), take some starch paste which has been acted upon (digested) by saliva for two or three hours, place in a membrane bag or osmose-apparatus, allow half-hour for osmose, then test water in tumbler for sugar.

The above experiments simply prove that saliva digests starch to a sugar; and that while starch does not osmose, the sugar formed from starch does. Saliva, then, prepares starch for absorption into the blood which flows in bloodcapillaries beneath the lining membrane (epithelium) of the alimentary canal, especially abundant in the stomach and intestines. The sugar derived from starch osmoses through the epithelium into the blood, just as it is demonstrated by the above experiments that it will osmose through the dead membrane of the osmose-apparatus.

It is evident that the above experiments do not prove anything regarding the action of saliva on other nutrients. If time permitted, we might try similar experiments with proteins and fats, using these nutrients in place of starch, and, of course, applying the appropriate tests (§377). Such experiments have been performed many times by physiologists, and their conclusion is that saliva has no digestive power for foods other than starch.

No simple experiment will show that there is in saliva a substance (ptyalin) which causes the digestion of starch, but chemists have proved it to be present, and the cause of the action of saliva. Moreover, it has been shown to be an enzyme, because it acts by its presence, without entering into the sugar formed, and a very small amount of it can digest a large quantity of starch.

The amount of starch food which will be digested in the mouth-cavity depends upon the length of time it is held there. Hence, prolonged mastication is advisable. It has 
been demonstrated within recent years that saliva continues its action on starch for some minutes after the food reaches the stomach, or until the acid gastric juice stops the action.

399. Digestion by Gastric Juice. - Physiologists have found that the human stomach secretes from five to ten quarts of gastric juice in twenty-four hours. This secretion consists chiefly of water containing a small amount of hydrochloric acid and three enzymes - pepsin, rennin, and lipase. The last named seems to be of most importance after food reaches the intestines. The action of pepsin and rennin are most easily demonstrated. Pepsin may be extracted by soaking stomach-membranes in glycerine, but the commercial extract sold at drug-stores is most convenient for experiments. Rennin is especially abundant in the stomachs of calves, and is sold in grocery and drug stores under the names of "liquid rennet" and " junket tablets."

Numerous experiments by physiologists have proved that gastric juice digests proteins, curdles (coagulates) milk, dissolves some minerals in foods, digests a small amount of fat, but it has no effect upon starch. In this course, we have time for only a few experiments which illustrate some of these points.

(D) The action of pepsin on proteins. - (1) Make some albumensolution by mixing white-of-egg in cold water, fill a test-tube half full, add some dry pepsin or glycerine extract of pepsin, add enough hydrochloric acid to make the mixture slightly acid to litmus paper, place the tube in a warm place near a stove or radiator, or in a "fireless cooker," or bucket of water heated to $37^{\circ} \mathrm{C}$. (98 F.) and protected from cooling rapidly. After from three to ten hours, pour the contents of the test-tube into a membrane bag or osmose-apparatus and suspend in pure water. (Start the next experiment at this time.) Allow an hour for osmosis. Test the water for proteins ( $\$ 377)$, using Millon's reagent. Have proteins gone through the membrane into the water?

(2) Pour some fresh undigested albumen-solution into a membrane bag, and after an hour test for osmosis as in above experiment. Does this albumen osmose? What conclusion is to be drawn con- 
cerning the effect of pepsin and acid on the particular protein used in these experiments?

If time permits, other proteins may be tested in the same way. Try a hard-boiled egg grated or minced into fine particles, shreds of lean meat, or cheese. Also, the acid may be omitted, in order to show that pepsin alone will not digest proteins.

(D) The action of the rennin may be illustrated by adding some commercial liquid rennet or junket tablets to milk. Place some of the curds on filter-paper, wash with water, place in a test-tube with some water, add strong nitric acid, heat. What nutrient is abundant in the curds? Pepsin and acid will digest them.

Summarizing, gastric juice can digest proteins, coagulate the proteins of milk, and then digest them, and digest a

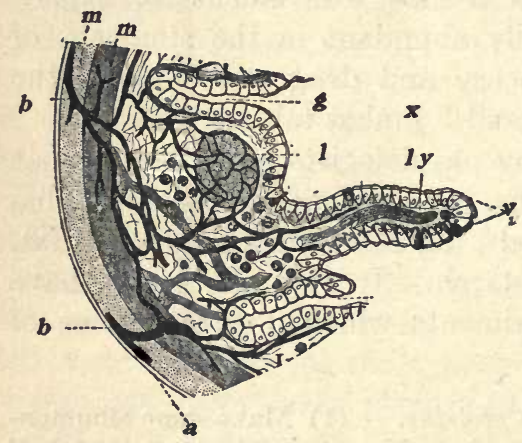

Fig. 158. Part of a cross section of intestine of a mammal. $x$, marks center of cavity of intestine; $g$, intestinal gland between villi ; $l$, lymph gland; $v$, villus ; $m$, muscle layers, the outer being longitudinal and the inner circular in arrangement of fibers (cells); $b$, blood-vessels ; $l y$, a lacteal or lymph-vessel in center of a villus $(v) ; a$, peritoneum. Compare with Fig. 19 of frog. (From Wiedersheim.) may be in the form of needle-
shaped crystals at the ordinary temperatures. If a small piece of fat meat be soaked for a time in ether to dissolve the fat, and then placed in iodine-eosin or other stains, the nuclei of the fat-cells will be stained. it does not digest starch. Fat meat breaks up extensively when kept for some hours in gastric juice, but only the protein walls of the cells have been digested, allowing the contained oil to escape undigested.

\section{Fat-cells. - (D) Place a} small piece of fat meat or suet on an object-slide in a drop of glycerine, and with a pair of needles tease the meat into as small particles as possible. Put on coverglass, and examine with low and high power of a microscope. Note the spherical cells filled with fat, which

small amount of fat; but

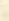


400. Digestion in the Intestine. - While the gastric juice has power to digest protein foods, the fact is that most of them do not remain in the stomach long enough for complete digestion ready for absorption. At intervals the muscular ring of the pylorus relaxes and allows partially digested foods to escape into the intestine, there to undergo final digestion and absorption into the blood. This food which escapes into the intestine from the stomach is water containing (1) much starch not digested by the saliva, (2) sugar which was eaten as such and much of the sugar formed by the digestion of starch by the saliva, (3) dissolved proteins digested in the stomach, (4) many small particles of undigested proteins, (5) fat in liquid (oil) condition. The work of the intestine is to complete the digestion of the proteins, fats, and starch;

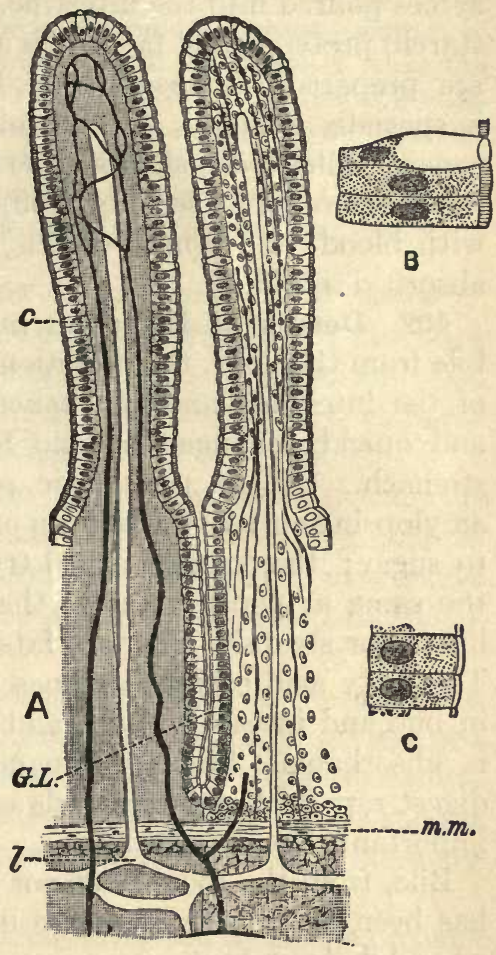

Fig. 159. $A$, two villi from intestine. Blood-vessels represented by black lines. $g . l$, intestinal gland; $l$, lacteal or lymphvessel ; $B$, three cells from covering of villi ; $C$, two cells from lining of glands. (After Hardy.) and to absorb the products of digestion into the blood.

401. Secretions in the Intestine. - These are formed by the pancreas, the liver, and the glands of the intestinal walls. The pancreatic secretion contains enzymes able to digest 
any kind of food, and consequently any food not made ready for absorption while in the stomach is digested by the enzymes poured into the intestine. The final result is that the starch, proteins, and fat which enter the intestine undigested are prepared for absorption. For this work the intestine is specially adapted, because of the numerous delicate processes (called villi, singular is villus) which extend from the lining membrane (see Fig. 159). These are richly supplied with blood- and lymph-vessels, into which digested food is absorbed rapidly.

402. Details of Digestion in Intestine (Optional). - The bile from the liver, the secretion from the glands in the walls of the intestine, and the pancreatic secretion are alkaline, and quickly change the acid food which arrives from the stomach. Of the pancreatic secretion, the enzyme called amylopsin acts like the ptyalin of the saliva and digests starch to sugar; the enzyme called trypsin acts on proteins much the same as pepsin does in the stomach; and the enzyme lipase (or steapsin) changes fats to fatty acid and glycerine. The fatty acid easily combines with the alkaline substances in bile and intestinal juice and forms a kind of soap which is absorbable. Since the pancreatic secretion is able to digest rapidly the three kinds of food, it is by far the most important digestive secretion.

Bile, from the liver, does not itself digest any food, but it has been shown that fats are digested and absorbed better when bile is present.

The intestinal juice, from the glands of the intestine, has a number of enzymes of which the most important change cane-sugar, milk-sugar, and malt-sugar into glucose or similar sugars.

(D) It is possible to demonstrate the effect of pancreatic secretion upon proteins and starch by performing experiments similar to the previous ones ( $\$ 3398$ and 399 ) with saliva and gastric juice, but substituting in this case pancreatic secretion. This may be 
obtained by erushing some fresh pancreas and soaking in glycerine as suggested in $\S 399$ for extracting pepsin; or commercial preparations of pancreas extract may be obtained at drug-stores. Add either the glycerine extract or the commercial pancreatic extract to water in test-tubes and also enough sodium carbonate to make the mixture alkaline (as shown by red litmus paper). Starch or protein may be digested in this solution for one or more hours, with temperature between 35 and $40^{\circ} \mathrm{C}$., and then tested for osmosis as suggested in $\$ 398$. Extract of pancreas does not furnish very active lipase, and hence it is difficult to demonstrate the action on fats.

403. Summary of Digestion and Absorption of Foods. Grape-sugar dissolved in water may be very slightly absorbed in mouth-cavity and esophagus, some in stomach, but chiefly in intestine. Cane and other sugars are changed before absorption in the intestine.

Starch - some digestion to sugar (maltose) in mouthcavity and in stomach by salivá, but chiefly digested to maltose in intestine by amylopsin of the pancreatic secretion. Resulting sugar may be absorbed as stated for sugar above.

Proteins - digested to absorbable form in stomach by pepsin and in intestine by pancreatic secretion. Absorbed to slight extent from stomach, but chiefly from intestine. Milk proteins are coagulated in stomach by rennin as a special preparation for digestion by action of pepsin, or later by pancreatic secretion.

Fats. - Chiefly digested in the intestine by lipase of pancreatic secretion, aided by bile and intestinal juice. Slightly digested by lipase in the stomach. The protein part of tissues containing fat (e.g., bacon or suet) is digested away in stomach by pepsin, thus freeing the liquid fat.

Mineral foods. - Those that, like common salt, are soluble in water require no change. Others present in various animal and vegetable foods which we commonly use are easily dissolved in the acid gastric juice. All the mineral foods are ready for absorption when dissolved in water. 
404. Effect of Cooking Foods. - Cooking not only renders foods more palatable, but also more digestible. It is easily observed that meats and vegetables are softened by cooking processes, and this aids the penetration of digestive juices. See effect of boiling upon starch ( $\$ 398)$. Roasting, broiling, boiling, and steaming best prepare foods for digestion; while frying in lard, butter, or oil causes the oily materials to penetrate the food and thus render the penetration of digestive juices more difficult. In general, a similar result comes from any heating of starch and fat together; and hence pastries, such as pie-crust, are more indigestible than would be the same amount of the ingredients taken into the stomach without heating together. Various methods of making starchy foods porous, such as the action of baking-powder and yeast, favor rapid penetration by the digestive juices.

405. Transportation of Digested Food to All Cells. - It has been stated in a general way that foods are required by all the living cells of the human body. The study of digestion and absorption has shown how foods get into the blood, and now we want to know how foods get from the blood into the cells. This requires (1) that blood should be moved from the capillaries in the walls of the digestive organs to the capillaries in all the other organs of the body, and (2) then absorption from the blood by cells which need food. Before one can understand how food is transported to all parts of the body, it is necessary to make some study of the general plan of structure of the organs concerned with the movement of blood and lymph, and also of the nature of these two liquids. Hence our next lesson deals with these topics.

\section{BLOOD AND LYMPH}

406. Structure of Blood. - If examined with the microscope, blood is found to be a liquid, called plasma, in which float numerous small bodies, blood-corpuscles or blood-cells. 
About 90 per cent of the plasma consists of water, which has been absorbed from the digestive organs, and more than 9 per cent consists of absorbed foods. There is also a small amount of excretions which have been absorbed from cells and are on their way to being eliminated by the excretory organs.

There are two kinds of corpuscles or cells visible with the microscope; namely, the red and the white. The red ones are bi-concave discs, about $\frac{1}{3200}$ of an inch in diameter. They are often seen in rolls like piles of coins. The number of them is astounding, for there are about five millions in a cubic millimeter of blood. (A millimeter is approximately $\frac{1}{25}$ of an inch long; com-

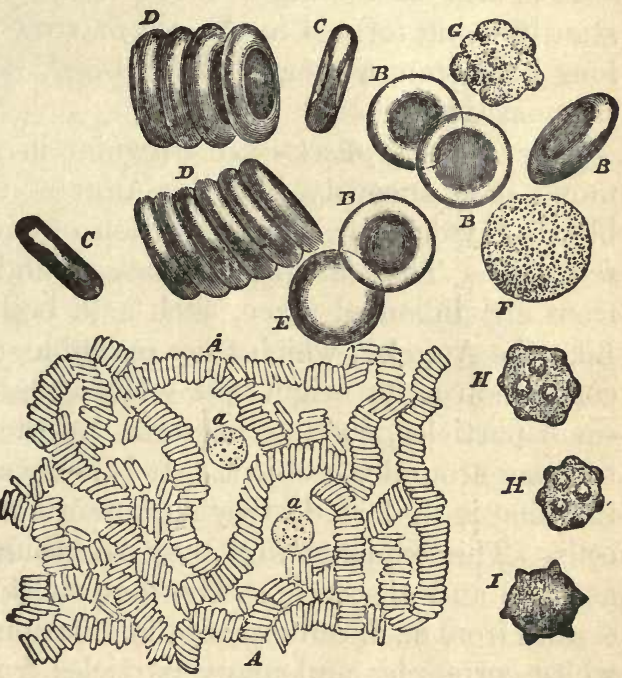

Fig. 160. Human blood-cells. $A$, rolls of red cells with two white cells $a$. $B, C, D, E$, various views of red cells. $F$, white cell magnified same as D. $H, I$, red cells covered with little knobs (abnormal, due to changes after blood is placed on object-slide). (From Huxley.)

pute how many red corpuscles in a cubic inch of blood.) In the diseased condition known as anæmia, which is characterized by the whiteness of the skin, the number of red corpuscles is greatly reduced.

The red color of these blood-cells is due to a substance called hoomoglobin, which is most important in the blood's work of carrying oxygen to the cells of the body. 
The red corpuscles of man and other mammals have no nuclei, except in embryonic stages ; but all lower vertebrates - birds, reptiles, amphibia, and fishes - have nuclei in all red corpuscles, even in the adult animals. Scientists have not yet found any reason for this difference.

It is also interesting to know that red corpuscles are constantly being formed in the red marrow of bones. Obtain a long bone from a meat-market, break it open, and examine the marrow.

The white corpuscles are irregular in shape, because they move spontaneously like the Amœba ( $\$ 270)$. In human blood they occur in the proportion of one white to 700 of the red cells. They are exceedingly abundant in blood taken from any inflamed place, such as a boil, pimple, or wound. Like the Amœba, which they resemble, although there is no connection as to origin, the white cells can engulf (" eat") small particles, such as bacteria, by the flowing of the protoplasm around the object. It is believed that their work or function is that of destroying bacteria and particles of dead cells. This explains why they are abundant in such places as boils and abscesses. The material known as pus, which exudes from such centers of inflammation, contains numerous white corpuscles and many particles from the dead cells of the inflamed tissue.

(D) Examine a drop of human blood spread on an object-slide and protected with a cover-glass. A drop may be obtained by wrapping a piece of string tightly around a finger near its end and then pricking the skin with a needle which is first made sterile by passing several times through a flame. One small drop of blood should be placed in a drop of normal salt solution (common salt 7 grams in a liter of water, which gives 0.7 per cent). The red corpuscles appear faint yellow in color when not in masses. The rarer white cells are difficult to find, but may be located by turning the micrometer screw about half a revolution so as to throw the red corpuscles slightly out of clear focus, and then a few white cells may be seen as glistening points of light in the field. They are somewhat larger than the red cells. 
Blood from a frog, fish, or bird, or permanent preparations of these, may also be examined. Nuclei will be seen in the red cells which are much larger than those in human blood.

Blood from an earthworm, erayfish, insect, or other invertebrate animal will show only white cells.

407. Coagulation of Blood. - Every one knows how blood will flow from a cut for a time and then stop because some of it has thickened into a jelly-like mass, which closes over the cut and forms a scab. This process of thickening is known in physiology as coagulation, or clotting. Examination of blood allowed to clot in a drop of normal salt solution will show that coagulation is due to the formation of delicate fibers which bind the corpuscles into a semi-solid mass. These fibers are composed of a substance called fibrin, which is simply the solid form of a protein called fibrinogen. This is dissolved in the plasma of the blood and becomes hardened into threads when blood escapes from a blood-vessel. If fresh blood of any animal be stirred with a feather or camel'shair brush, the fibrin will form on the feather and the defibrinated blood will not coagulate, thus proving that the formation of fibrin causes coagulation.

The use of coagulation is obviously to prevent excessive bleeding. If blood did not coagulate quickly, a slight injury to even a small artery might cause the death of animals or of men before surgical aid could be secured.

408. Lymph. - This is a liquid very much like blood, but without red corpuscles. In fact, it is chiefly blood-plasma which has osmosed out through the capillaries into the very small spaces which exist between the cells in all tissues. Thus lymph comes into direct contact with the cells, giving to them food and oxygen, which are dissolved in plasma, and receiving from the cells some excretions. These small lymph-spaces between the cells are united together into larger lymph-capillaries, which in turn connect with the large lymph-vessels. These pour their lymph into the veins 
in which blood goes back to the heart. Lymph, then, osmoses from blood-capillaries into lymph spaces, then flows into lymph-capillaries, and through larger vessels back into the blood. The arrangement of blood and lymph-vessels in any organ might be compared with the irrigation systems for watering agricultural land. The blood-capillaries correspond to the ditches from which water seeps out into the spaces between particles of soil. These spaces in the soil correspond to lymph-spaces, while the lymph-capillaries and larger tubes correspond to the drain tiles which carry excess water away from irrigated soil. In short, the lymph-spaces in any organ constitute a sort of combined irrigation and drainage system for cells which are not directly reached by the main canal system of the blood-capillaries.

Lymph contains white corpuscles, which are able to squeeze through the walls of blood-capillaries into lymph-spaces. They are also formed in great numbers in the lymphatic glands through which lymph flows on its way back to the blood in the large veins. In fact, all the white corpuscles in blood are lymph-cells washed into the blood from the lymphatic glands. The spleen and the tonsils are examples of large lymphatic glands, and there are hundreds of small ones in various parts of the body.

\section{CIRCULATION OF THE BLOOD}

409. Need of Movement of Blood. - In order that blood may serve its purpose as a distributor of food and oxygen to the cells and in removing excretions from the cells to the excretory organs, it is necessary that it should be kept constantly in motion. This is accomplished by the circulation of the blood from the heart through the arteries and capillaries into the veins, which conduct it back to the heart. That the blood thus goes around in a circuit was unknown until 1621, when Dr. Harvey, of England, proved that in 
man and other vertebrates the blood always flows in one direction from the heart in blood-vessels, and that these are so arranged that blood ultimately comes back to the heart, i.e., makes a complete circuit. This circulation is remarkably rapid; it is believed that blood goes from the heart through the capillaries in such a distant organ as a foot and returns to the heart in about half a minute. (How many times would the blood complete its circuit in a day?)

410. Structure of the Heart. - $(D$ or $L)$ Procure from the market a sheep's heart with the lungs attached, and with the membrane (pericardium) surrounding the heart. Insert a large tube in the trachea and inflate the lungs by blowing into them. Note the relation of lungs and heart. Carefully dissect away (with forceps and scissors) the fat which adheres to the heart, taking care not to cut off any arteries or the thin-walled veins. It is best to stuff the veins with cotton or insert a small roll of paper.

The general form and external structure of the sheep's heart is similar to that described and illustrated for the human heart in many books on anatomy and physiology. Examine pictures in such books; note positions of the two auricles and two ventricles of the sheep's heart. Notice that a probe (e.g., a rounded stick) inserted into a vein enters an auricle. The connection of the arteries with the ventricles can be seen later (next paragraph). Cut across the heart transversely about an inch from the pointed end (apex). This will open the two ventricles. The left one is a rounded cavity, the right is crescentic in outline. Note the relative thickness of the muscular walls of the two ventricles. Now, take a blunt stick about the size of a pencil, and inserting it into the left ventricle, probe carefully until it emerges out of the largest artery. This is the aorta, whose branches are arteries leading to all the organs except the lungs. Insert a similar stick into the right ventricle and out through its artery. This is the pulmonary artery, whose branches conduct blood to the lungs.

The action of the valves in the aorta or pulmonary artery in preventing blood from flowing back into the ventricles can be demonstrated on a heart with the apex removed, as follows. Insert a large glass tube (about $\frac{1}{2}$ " or $\frac{3}{4}$ " caliber) into one of these arteries held upright, and fill it with water. Or connect the artery with a large funnel. If the valves are still in good order, the water will remain in the tube or funnel. 
To demonstrate the action of the auriculo-ventricular valves, cut away the auricles from a heart with the apex removed, and then plunge it with the ventricle held downward into water so as to float the valves into the closed position.

Slit open the aorta to expose its valves (semilunar); and also cut the side of a ventricle to show attachment of the auriculo-ventricular valves.

411. The cause of circulation is the constant, rhythmic beating of the heart, which is a muscular force-pump. Its general plan of structure is illustrated by an ordinary rubber bulb such as is used for atomizers and syringes. In such a bulb there are two valves arranged so that when the bulb is filled with water and then compressed, one valve (inlet) closes and prevents the water from flowing outward, while the other valve (outlet) remains open and allows the water to escape into the outlet tube. Then if the bulb be allowed to expand, the outlet valve is closed by the back pressure of the water in the outlet tube, and water rushing in from the supply. tube opens the inlet valve. The next compression will again arrange the valves as first described above. (Draw diagrams to illustrate positions of valves and with arrows show direction of flow through a two-valved bulb.)

In a similar manner, there are valves arranged in the heart in two places, one valve to prevent a flow of the blood back into the veins (inlet) when contraction occurs, the other to prevent the return flow from the arteries (outlet tube) when relaxation or dilation takes place. And, as can be easily demonstrated with a rubber bulb having one inlet and one outlet valve, repeated contraction and expansion will cause fluids to flow through always in the same direction, which is determined by the arrangement of the valves. Hence, the blood circulates because the heart is a pump with valves so arranged as to force the blood to flow in only one direction, out through one valve, and thence around through tubes which lead back to the inlet valve. 
The valves (Fig. 161) in all but the largest veins also aid in the circulation of blood, especially when contracting muscles press upon them between two valves. The region so pressed acts like a syringe-bulb which has outlet and inlet valves, each compression driving blood towards the heart.
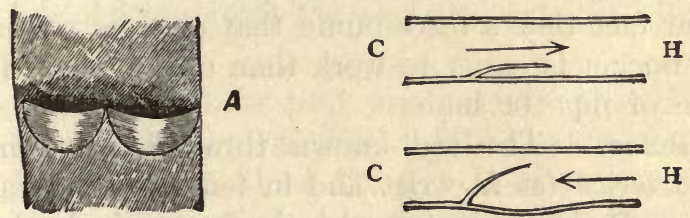

FIG. 161. $A$, pocket-like valves of veins. Arrow pointing from $c$ to $h$ shows valves opening towards the heart, while the lower figure shows how the valve would float up and close if blood started to flow backward towards the capillaries through which it came.

412. Heart a Duplex Pump. - Like some pumping-machines with two pumps united side by side, the heart of man and other mammals is double. To illustrate it correctly with the rubber bulb mentioned in above paragraph, it would be necessary to place two bulbs side by side. One bulb would represent the right and the other the left ventricle of the heart. The right ventricle pumps blood into arteries which extend to the lungs, and the left pumps blood to all the other organs. Moreover, the blood pumped from the right side of the heart to the lungs comes back in veins which connect with the left side, and the blood from the left ventricle comes back to the right side of the heart. In other words, the two sides are constantly supplying each other. Both right and left ventricles contract at the same time, the right one forcing blood into the lungs, and thence into the left collecting chamber (left auricle), while the left ventricle forces blood into all other organs, from which the blood returns to the right collecting chamber (right auricle).

It is evident from the above that the two sides of the heart 
must pump equal amounts of blood, for they beat (contract) together, and each supplies the other with blood. Why then should the walls of the right ventricle be so much thinner than those of the left? The answer is that the right ventricle pumps to the lungs, which are near the heart, while the left ventricle pumps to all the distant parts of the body. It is a familiar fact that a force-pump that empties water into a near-by bucket is easier to work than one connected with a long line of pipe or hose.

413. Pulse. - The well-known throbbing movement of certain arteries (as in wrist and in temple) which are near enough to the skin to be felt with the fingers, is due to a rush of blood through the arteries and a sudden expansion of the walls.

(D) It may be imitated with a soft rubber tube (5 or 6 feet long) connected to a pump or syringe-bulb. While pumping water, steadily press upon the tube with a finger, and note the pulsations which follow each stroke of the pump. Then insert a glass tube with a small opening into the end of the rubber tube, and pump rapidly enough to make the jet of water a steady stream. Now note that the rubber tube is somewhat expanded under internal pressure, and that the "pulse" is still discernible.

This simple experiment imitates the conditions in arteries. Their walls are elastic, the capillaries exert resistance and prevent the sudden flow of blood through them (just as the small opening at the end of the rubber tube does); the blood in the elastic arteries is then under pressure, and the elasticity of the arteries steadily forces blood through the capillaries.

If the arteries were rigid like glass or iron, there would be no pulse, and blood would be sent in sudden spurts through the capillaries.

(D) To illustrate this replace rubber tube in experiment above with a tube of glass of same caliber and length. (Any desired length may be made by joining chemical glass tubing with short pieces 
of rubber tubing.) When the pump is worked as before, jets of water will issue from the end of the glass tube, no matter how large or small an opening is present. The same thing happens in an ordinary force-pump, and in order to get a steady flow of water in our city water systems an air-chamber near the pump is used. (Explain action of air-chamber.) Obviously the elastic arteries afford a simple way to get the effect of an air-chamber on a force-pump; namely, a steady flow of fluid through the small branches (capillaries) of the system of tubes.

In $\S 414$ it is shown that another advantage of elastic arteries is in regulating the flow of blood to the organs.

414. Regulation of Blood Flow. - It often happens that an increased activity of the organs demands a greater movement of blood for carrying more food, oxygen, or excretions; or a decreased activity demands a lessened circulation. In short, there is need of a regulated blood flow from the heart through the organs. This is brought about in two ways; (1) by control of the heart, and (2) control of the arteries.

Control of the heart is through the nervous system, certain nerves carrying to the heart impulses or "messages " which increase the rate of its beat, while other nerves inhibit or diminish the beat.

Control of the arteries is likewise the function of certain nerves. The elastic small arteries in all living organs are usually somewhat constricted, thus offering some resistance to arterial flow, and keeping up a continuous stream through the capillaries and veins back toward the heart. This constriction or reduced caliber is produced by a contraction of the muscle-fibers which are in the walls of arteries, and this contraction is under control of nerves.

When an organ needs an increased blood-supply (e.g., the stomach in digestion), it may be obtained by a nervous reaction leading to a relaxation of the muscles of smaller gastric arteries and an increased diameter. Similarly, the application of hot water or alcohol to the skin leads reflexly to an increased blood flow; but cold water produces the 
reverse effect of contraction of the arteries and a reduced circulation of blood (see bathing, $\S 458$ ). Also see account of heat regulation by the skin ( $\$ 447,453)$.

With the exception of the heart itself and the lungs and brain, the larger organs of the body are well supplied with nerves able to regulate the size of the smaller arteries and consequently the amount of blood-supply.

Whenever there is a great increase in caliber of the bloodvessels, the heart's beat is increased in order to keep up the pressure. In this way, certain drugs used by physicians may increase the action of the heart by reducing the pressure in dilated arteries.

\section{USE OF FOODS IN THE CELLS}

415. We have now studied the preparation and distribution of fouds to the cells. Naturally we now ask questions regarding the use which the cells make of the food supplied to them. In studying the frog, we have noted that foods are used in part for supplying energy and in part for repair and growth. In order to apply this fact to human life we need to consider more carefully the kinds of foods in relation to the supply of energy and materials for repair and growth. Are all foods equally valuable? Should we give any attention to the selection of our daily diet? Such questions are common nowadays, and show that there is a widespread popular interest in the uses of various foods. We shall first consider foods for energy (especially heat and muscular work), and later, foods for repair and growth. This is only a convenient division into topics, for some foods can serve all purposes.

416. Energy and its Conservation. - Some general considerations regarding energy will make clear the use of foods as a source of power in the human body.

We are familiar with the fact that stored energy in coal 
may be changed into mechanical energy by a steam-engine; and this into electrical energy by a dynamo; and the electrical energy into heat energy by an electric-stove, or into light by an electric-lamp, or into mechanical energy by a motor. Thus the stored energy of coal may be changed into various other forms of energy which are known as mechanical energy, heat, light, and electricity.

One of the important discoveries of the last century was that in such changes no energy is lost to the world. At first this statement will be puzzling to one who has read that 85 per cent of the energy stored in a ton of coal does not appear in the work of the best steam-engines in common use, and hence seems to be lost energy. Also, there appears to be a loss in transforming to electricity, for a 20-horse-power steam-engine driving a dynamo cannot generate enough electricity to drive a 20-horse-power electric-motor. However, this loss of energy from the practical standpoint is, after all, not a real loss, for the same amount of energy remains in the universe. This could be proved by placing a small steam-boiler and its furnace, an engine, a dynamo, and a motor together in a room with walls which would prevent loss of heat. Then if some fuel were burned and the machinery set to work, generating steam and electricity, the explanation of the apparent loss of energy would be found chiefly in the heat given off to the air in the room. Adding this to the energy of the engine, dynamo, and motor, the sum would equal the amount of energy which a chemist can demonstrate by burning the same amount of coal in a calorimeter ( $\$ 418$ ). In short, from 85 to 90 per cent of the energy stored in coal is given off from the machinery as heat, 10 to 15 per cent appears in the mechanical energy of the steam-engine, some less in the electrical energy of the dynamo, still less in the energy manifested in the electric-lamp, heater, or motor. But each apparent loss is represented by heat given off to the surrounding air. The fact is, then, that 
energy can be transformed from one form into another without loss, or reducing the amount of energy in the universe; that is, energy cannot be destroyed. The total amount of energy in the universe is always the same.

Another important discovery is that energy is not now being created anywhere. The most wonderful machines can do nothing but transform energy. For example, the steam-engine is a machine for changing heat energy into motion or kinetic energy. A water-wheel changes the potential energy of water stored at an elevation into motion. A watch changes into motion the energy stored in a wound-up spring. An electric-battery changes the energy stored in chemicals into electricity. And so we might go through the whole list of known physical changes in the universe, and in each case find evidence that transformation of energy, and not destruction and creation, is constantly occurring in the universe. Science has found no positive evidence concerning either creation or destruction of energy in all its forms.

The facts regarding changes of energy are now embodied in the Law of Conservation of Energy, which is, that energy can be transformed from one form into another, but cannot be created or destroyed.

One of the most interesting phases of science study is that of the changes of energy as presented in the science of physics. The law of conservation of energy has numerous practical applications in machinery. For example, a physicist would never spend his time in trying to invent a "perpetualmotion machine," for that would be completely opposed to the established law of conservation of energy.

417. Energy of the Human Body. - This is manifested externally in the form of heat, and muscular and nervous activity. The source of this energy is the stored or potential energy of food, and numerous experiments have demonstrated that the law of conservation of energy applies to the human body (and all other living things), just as it does to a steam- 
engine. Food for the human engine and fuel for a steamengine are both necessary because they contain stored energy. It is interesting to recall ( $\$ 116)$ that in both cases the original source of the stored energy was sunlight.

418. Foods for Energy. - The energy value of the three important nutrients (proteins, carbohydrates, and fats) is easily computed by chemists. Samples of such nutrients are dried and then quickly burned inside an apparatus known as a calorimeter (heat-measurer), and the heat thus generated is measured in terms of the amount of heat necessary to raise 1000 grams of water one degree Centigrade. This amount of heat is a calorie, the standard unit for heat measurements ;* and since heat may be converted into other forms of energy ( $\$ 416)$, it is convenient for measuring the total energy of foods.

The heat value of a gram of food measured in this way is as follows : for dry protein, about 5.6 calories (i.e., 5600 grams of water $1^{\circ}$ ); for carbohydrates, about 4.1 ; and for fats, about 9.3. It has been shown by a method described in the next paragraph that a man or a higher animal obtains 9 calories from fats, and 4 from carbohydrate foods; but protein is less completely oxidized in the living body, so that one gram of protein gives only about 4 calories of the 5.6 it contains. Obviously proteins are not economical as foods for heat or muscular energy, while carbohydrates and fats give almost as much energy in the body as when they are completely burned in a chemist's calorimeter. The chemical result for fats and carbohydrates is the same in both cases, for these foods produce carbon dioxide $\left(\mathrm{CO}_{2}\right)$ and water $\left(\mathrm{H}_{2} \mathrm{O}\right)$ in both the living body and the calorimeter.

Many substances which will produce heat energy in a

* Some authors use the "small calorie," which is the amount of heat necessary to raise one gram of water one degree C. It has an advantage in measuring small quantities of heat less than enough to raise 1000 grams of water one degree. 
calorimeter could not be burned in an animal body. For example, a gram of pure carbon (charcoal) gives 8.08 calories, and a gram of hydrogen would give 34.5 calories; but neither of these could be oxidized at the temperature suitable protoplasm. This shows the importance of determin whether foods give as much heat in an animal body as ir calorimeter; and this has been tested with a special app ratus as follows :

419. Calorimeter for Living Animals. - This consists a small room with walls arranged to prevent heat conductio as far as possible, with coils of metallic pipes arranged so that circulating water will absorb heat from the air in the room, and with machinery for ventilation. Delicate apparatus attached to the pipes for supplying air and water recor? changes in temperature, and chemical analyses are made $\dot{f}$ foods, breathed air, and excretions. There is also inside $t^{t}$. room a foot-power machine connected with a small dynamo or otherwise arranged so that the man may treadle the machine and thus do easy, moderate, or hard work for eight hours per day. Thus it is possible to determine the effect of different amounts of work upon food requirements.

Inside such a special calorimeter men have often lived constantly for several days at a time; and chemists have carefully compared the heat value of the foods oxidized with the heat given off from their bodies to the air of the room and measured by the apparatus.

The results of many such experiments have shown that foods are oxidized as stated in $\S 418$ above. The apparatus has proved of great value in cases where the value of a food to a living animal or to man was unknown. For example, the dispute as to whether alcohol is a food ( $\$ 472)$ has been settled by showing that in very small quantities alcohol serves as a food for energy; and that the amount so usable is so small as to be of no interest in the ordinary daily diet of healthy persons. 
Many experiments with different combinations of foods have led to the conclusion that men of about 150 pounds $\checkmark$ ght engaged in sedentary work require food yielding from 0 to 2400 calories per day of 24 hours. Men doing hard vsical work for eight hours per day require food furnishing m 3000 to 4000 calories; and very hard work may require ure than 5000 calories. There are individual variations ong men of equal weight doing the same work. Some idea the meaning of these figures will be gained from the statement that 100 grams of protein food giving 400 calories, 100 grams of fat giving 900 calories, and 250 grams of carbohydrates giving 1000 calories makes a total of 2300 . This would suffice for a professional man or a shoemaker, but a t.urmer, mason or carpenter would require more fat and rbohydrate food daily.

420. Foods for Muscular Work. - It was formerly upposed that protein foods are necessary for muscular work, and we frequently hear that "laborers must eat much meat in order to get strength to work." This is not true. It has been shown that muscular exercise does not require more protein food, for fats or carbohydrates can furnish the necessary extra energy. Two physiologists who used a mixed diet of proteins and the other foods have proved that their bodies oxidized as much protein on a day of rest as on a day when they climbed a mountain over 6000 feet high; and also that the amount of energy in the protein they oxidized was not sufficient to lift their bodies so high. Hence the other foods (fats and carbohydrates) eaten must have supplied the necessary extra energy. But if these men had eaten more pure protein, they could have gotten from it the necessary energy.

The conclusion from many such experiments is that large amounts of protein are not needed for ordinary work. The necessary amount is approximately the same for every day, whether keeping quiet or working, and is from 60 to 100 2 K 
grams of protein for an average 150-pound man. Most well-to-do people eat on the average more than 100 grams of protein in meat daily, and also they get much additional protein in milk, eggs, bread, all vegetables - in fact, there is some in most common foods.*

While the amount of protein foods needed daily is quite constant for the ordinary adult, the amount of the other foods should vary with the amount of energy required. A student certainly needs less fats and carbohydrates than a laborer does. So long as digestion is good, variation in weight is a good index to the amount of such foods needed; for if taken in excess and digested, there will likely be increased weight due to food storage in the fat tissues of the body. Loss in weight by an adult who daily eats as much as 100 grams of protein indicates a need of more fats and carbohydrates; and whether starch, sugar, or fats should be used depends upon availability, digestibility, and taste of the individual.

Growing animals and children require a higher proportion of protein (see $\$ 422$ ) than do adults, who alone have been considered in the above discussion.

421. Foods for Heat. - As previously stated, birds and mammals must maintain a constant body temperature. To a large extent they do this by preventing excessive loss from the skin $(\$ 447)$. The production of heat is chiefly the result of muscular activity. We all know how easily we can keep warm by exercise on a cold day, but how uncomfortably cold we often feel if we simply eat our regular meals and keep quiet. For this reason we require more clothing or covering when sleeping, for then only the circulatory and respiratory muscles and chemical changes in the digestive organs are the chief sources of heat production.

* Per cent of protein in foods: lean meat 15-22; eggs 15 ; white bread 9 ; dried beans 22 ; potatoes 2 ; peanuts 25 ; butter 1 ; outmeal 16 ; olive oil 0 ; sugar 0 ; milk 3 ; green corn 3 ; fish $15-20$; apples 0.4 ; rice 8 . 
More food is required in cold weather. The non-nitrogenous foods are most economically increased; but whether fats or carbohydrates should be used for increased heat largely depends upon availability and digestibility. The inhabitants of very cold regions can easily get fat from animals, while those in temperate regions can store plant food, containing large amounts of carbohydrates, for winter use. When both kinds of foods are available, there is great individual variation in taste for and digestion of carbohydrates and fats; and any desired combination of the two may be made provided that we remember the energy value of fats as 9 calories, while sugars and starches have 4 per gram.

422. Foods for Repair and Growth of Protoplasm. - Attention has several times been directed to the fact that living substance (protoplasm) is composed of protein. Also, it has been stated that animal cells can make new protoplasm only from protein foods, while plant cells can make protein from the elements furnished by carbohydrates and the nitrogen-containing substances absorbed from the soil. There is abundant evidence that in the human body protein food is required for making new protoplasm in repair and growth; and that the foods lacking nitrogen (i.e., carbohydrates and fats) cannot answer this purpose.

The amount of protein food required daily for repair of the protoplasm in the cells of an adult man is believed to be from 60 to 100 grams (28 grams to an ounce). However, it should be said that some physiologists hold that 100 is nearly twice the amount which is absolutely necessary; but many conservative authorities regard 60 grams of protein as too little for a regular daily diet intended for repair and the maintenance of good health.

If 200 grams of protein be eaten daily, the nitrogen excretion will be double that from the usual 100 grams. This indicates (1) that protein is easily disintegrated in the living cells, (2) that excess protein in the food is not stored 
for possible future use, and (3) that if excess protein goes to cells they use the necessary amount for making new protoplasm and disintegrate into excretions the remainder, which may serve as a source of energy. In other words, at least one-half of 200 grams of protein might furnish heat or muscular energy as other foods do.

423. Why limit Protein Food? - Since protein is necessary for repair and growth, and may also supply energy, we may properly ask why physiologists so often recommend a limited protein diet, e.g., 60 grams per day. There are several answers :

(1) Protein as a source of energy is physiologically wasteful, for the human body can obtain only about 4 calories per gram, which in the chemist's calorimeter shows 5.6 calories. This is because nitrogen excretion leaves the body incompletely oxidized.

(2) Protein for energy-supply forces the body to handle the useless nitrogen which it contains, for energy comes from oxidation of the carbon and hydrogen. A hard-working man getting about 2880 calories from a daily diet of 120 grams of protein, 100 of fat, and 375 of carbohydrates would have to eat about 720 grams of protein daily to get the same amount of energy if he took food other than clear lean meat. This would require the kidneys to excrete 200 extra grams of urea which would come from the nitrogen content of the extra 600 grams of protein. Such an excess of nitrogen excretions is injurious and tends to cause disease of kidneys and other organs. Hence it is better to get the necessary energy from 100 grams of fats and 375 of carbohydrates $(100 \times 9+375 \times 4=2400$ calories) rather than from 600 of extra protein $(600 \times 4=2400$ calories $)$.

(3) Protein is not economical in a pecuniary sense. It costs much more than common carbohydrates (starch in vegetables, or even sugar) which have the same energy value, - namely, 4 calories per gram ; and clear lean meat is much more 
expensive than fat meat or butter when we consider that fat has 9 calories per gram, and hence has more than double the energy value of protein, which has 4 calories per gram.

424. Mixed Diet. - The three reasons given above have led all physiologists to advocate a mixed daily diet containing (1) the protein necessary for daily repair, and for growth in early life; and (2) enough non-nitrogenous foods to furnish the necessary heat and muscular energy. Whether these foods for energy should be fat or carbohydrates depends upon availability and digestibility. One man may digest and use 100 grams of fat and 300 of starch as well as another could use 50 grams of fat and 412 of starch; but the energy obtained is nearly the same.

Special foods are not known for special organs. That celery is a "nerve" food, fish a "brain" food, lean meat a " muscle" food, etc., are popular beliefs which are entirely without scientific foundation.

It is not yet known whether proteins from plants are equally valuable with those from meat. Certainly many people have lived well on a vegetarian diet; but those who adopt it in its strictest form without milk, cheese, and eggs should eat more than 60 grams of protein, because it is probable that the human cells select only certain kinds of plant proteins for use in repair and growth of protoplasm.

It also should be noted that with a strict vegetarian diet there may be difficulty in getting enough protein without an excess of carbohydrates, because there is relatively little protein in many plant tissues. However, certain seeds (especially beans, peas, lentils, wheat, rye, and oats) contain much more protein than the foods obtained from" green vegetables" (roots, stems, and leaves of plants). Hence, it is possible by using such seeds to make a strict vegetarian diet with proper proportions of protein and other food. However, the safest way for most people is to add milk and its products and eggs to plant foods, if one has tastes or principles 
opposed to the use of meats. On scientific grounds there is no known objection to the proper use of meats, but simply an objection to meats in excess of actual protein requirements (i.e., above 60 to 100 grams a day).

References: Those who are interested in questions of diet should read the chapters on " Nutrition," and "Hygiene of Feeding" in Hough and Sedgwick's "Human Mechanism." Also obtain from the Department of Agriculture, at Washington, the bulletins on the nutritive values of foods.

\section{OXYGEN-SUPPLY}

425. Respiration. - This has already been defined as including the functions of obtaining oxygen and eliminating carbon dioxide. In some lower animals (e.g., earthworm) the skin is the respiratory organ; in fishes and others there are gills; amphibians breathe with both skin and lungs; but in the vertebrates higher than the amphibians lungs are the sole respiratory organs. In all these cases the membranes which take up oxygen also give out or excrete carbon dioxide. For greater convenience in study, we shall in this lesson confine our attention to the supplying of oxygen to cells in the human body; and deal with the excretion of carbon dioxide in the next lesson.

The respiratory organs consist of nasal passages, pharynx, larynx, trachea (windpipe), bronchi (right and left branches of the trachea), bronchial tubes (branches of bronchi), airchambers at ends of smallest bronchial tubes, diaphragm, and wall of the thorax- or chest-cavity.

426. Respiratory Passages. - We commonly think of the nose as an organ for the sense of smell; but the fact is that only a limited amount of epithelium (lining membrane) in some of the upper nasal cavities has nerve-endings connected with the olfactory part of the brain, and hence has the power of perceiving odors. Most of the cavities in the 
nose are respiratory, and serve the purpose of warming the inrushing air by contact with the warm membranes; and also the membranes and hairs in the nose collect much of the dust from the air which enters.

The passages connecting those of the nose with the pharynx are called post-nasal. They lie back of the soft palate. Into the same passages open the Eustachian tubes from the ears. Some of the soft tissues in the lining of the postnasal chamber may enlarge and form adenoids (\$449), which interfere with free breathing through the nose and cause injurious breathing through the mouth.

427. Lungs. - $(D$ or $L)$ Examine sheep's lungs obtained from a meat-market. Notice the trachea and the arrangement of its cartilaginous rings, incomplete on the side next to the esophagus. Follow the two branches (bronchi) to the lungs. Try inflating the lungs by forcing air through a large glass or wooden tube inserted into the trachea. Why do the lungs collapse and force out air when pressure is released? Compare action with that of a rubber bag or balloon. Note that there is air remaining in all parts of the lungs after their complete return to the original size before inflation. In human lungs after ordinary breathing out or expiration of air, the amount of air still left is about 200 cubic inches, and one-half of this can be forced out by "blowing hard" (i.e., forcibly expiring air, which is accomplished by muscular compression of the lungs). This additional discharge of air from sheep's lungs can be demonstrated by pressing upon them with the hands. Of course, the lungs inside an animal cannot be inflated larger than the chest-cavity.

The delicate outer membrane of the lungs is the pleura, and similar tissue lines the chest-cavity. These membranes are lubricated by a secreted liquid which reduces friction between the lungs and the chest-wall during the movements of respiration. Inflammation of these membranes, which often occurs in connection with severe "colds," is termed pleurisy.

Cut into one bronchus and then split it open as you work toward the smaller branches. In this way follow up and lay open a series of successively smaller branches leading out toward the surface of the lung. The smallest branches end in air-chambers. Notice the numerous blood-vessels in the tissues of the lungs. The arteries can be distinguished by having thicker walls than the veins. 
428. Breathing Movements. - Expansion of the chestcavity will result in reduced pressure so that the external air will enter through the trachea until the pressure in the air-chambers of the lungs balances that between the lungs and walls of the chest-cavity. This is exactly what happens in the following experiment.

(D) Action of Diaphragm. Use apparatus constructed as follows : A sheet of dental rubber is stretched and tied over the base of an open-top bell-jar, or lamp-chimney that is wide at base and narrow at top. A short glass tube is tied into the mouth of a delicate rubber bag (e.g., toy balloon), and the free end of this tube is then inserted through a hole in a cork which will fit tightly into the top of the bell-jar. When the cork is placed, the rubber bag should hang near the center of the jar. The glass jar represents the walls of the chestcavity, the glass tube represents trachea, the rubber bag stands for elastic lungs, and the rubber sheet at the bottom acts as a diaphragm. Note that when this is made convex by pressure, the rubber bag (imitating lungs) collapses. Why? Why is air forced out of any toy balloon when free to escape? When the rubber diaphragm becomes flat (imitating the downward or posterior movement of the human diaphragm), the rubber "lungs" expand. Why? Why does water rush into a pump when the piston is raised? This apparatus illustrates the mode of respiratory action; but is not exact, for the lungs fit and fill the chest-cavity, except that the heart lies in a space between them.

The expansion of the human chest-cavity is due to breathing movements, which are of two kinds: (1) those of the diaphragm, whose positions, due to muscular movements, may be imitated by the rubber sheet across the mouth of the jar used in the preceding demonstration; and (2) to expansion of the side-walls, increasing the diameter in a horizontal plane. This latter is due to raising the ribs, and can be demonstrated by measuring with a tape the circumference of the chest before and after inspiration. Young children expand the chest-cavity chiefly by the movements of the diaphragm; in many men the diaphragm does most of the breathing work; in some women, particularly when tight 
clothing is worn, the diaphragm is but little used, and expansion of the chest-cavity is due chiefly to movements of the ribs. The ideal breathing movements combine to use both ribs and diaphragm. Note effect of forced breathing, or after rapid exercise.

429. Changes in Breathed Air. - Analysis of air which has been taken into the lungs and then expired shows that it has lost oxygen. This means that the blood circulating in the capillaries of the lungs has absorbed some oxygen from the air in the air-chambers around which the blood-capillaries are arranged.

The average adult person can expel from his lungs after the deepest possible inspiration about 230 cubic inches of air. In ordinary breathing a man takes in about 30 cubic inches, and expels about the same amount. An additional 100 (total 130) cubic inches can be taken in by forced inspiration (" a long breath"), and this much plus about 100 not usually expired (total 230) can be expelled by forced expiration, as stated above.

After the deepest possible expiration of 230 cubic inches of air, about 100 cubic inches of air remain in the lungs. It is obvious that under ordinary breathing conditions the amount of air inspired ( 30 cubic inches) is not sufficient to fill the lungs, which contain 130 cubic inches, for it is not quite onethird of the amount left in the lungs. The fact is that the 100 cubic inches of air usually left in the lungs is mixed and diluted with the 30 of fresh air.

430. Absorption of Oxygen by Blood in Lungs. - The blood-supply to the lungs is venous, from the right side of the heart, which in turn receives it from all the organs except the lungs. This blood passes through the capillaries of the lungs and leaves by way of the pulmonary veins to the left side of the heart, thence to all the organs except the lungs. On the way through the lungs the blood is changed from venous to arterial, by losing part of the carbon dioxide ( $\$ 433)$ and 
absorbing oxygen from air in the air-chambers through the delicate walls of the chambers and into adjoining bloodcapillaries.

We must guard against the common error of supposing that venous blood coming back to the lungs from all other organs is devoid of oxygen. The fact is that every $100 \mathrm{cc}$. of venous blood coming to the lungs has about $10 \mathrm{cc}$. of oxygen dissolved in the blood, and when it leaves as arterial blood it has about $20 \mathrm{cc}$. In other words, the amount of oxygen is doubled in arterial blood. This additional oxygen becomes combined with the hæmoglobin in the red corpuscles, and results in the change of color. Blood without the red cells could not contain so much oxygen, and hence vertebrates have a great advantage over the lower animals.

431. Distribution of Oxygen to Cells. - Blood carrying oxygen received in the lungs passes from the left auricle to the left ventricle of the heart, and thence to all the organs except the lungs. As the blood flows through the capillaries in organs, some of its oxygen is absorbed by near-by cells and some by the lymph, which distributes it to cells that are distant from blood-capillaries. Recall the statement that all living cells are continually using oxygen, and it is obvious that they as constantly must demand a fresh supply. If the blood were to stop flowing for even a short time, the available oxygen in the lymph and blood in every organ would soon be used by the oxidation going on in protoplasm; and the result would be asphyxiation of the cells. But under normal conditions the flow of blood is so rapid that only approximately one-half of its contained oxygen is extracted while it flows from the arteries through the capillaries into the veins. Hence venous blood returning to the lungs has about one-half the oxygen of the arterial blood leaving the lungs. 


\section{EXCRETION}

432. Purpose of Excretion. - The products of oxidation in the cells of the human body are of no further use, and when accumulated may be injurious. Hence special organs have as their work the elimination of excretions (1) from the blood and lymph which absorbs them from the cells, and (2) from the body. Most important of the excretions are, as in the case of all other organisms we have studied, carbon dioxide $\left(\mathrm{CO}_{2}\right)$, water $\left(\mathrm{H}_{2} \mathrm{O}\right)$, and nitrogenous excretions. The carbon dioxide is chiefly excreted by the lungs, the water by the kidneys (with some unnecessary help by the skin), the nitrogenous excretions chiefly by the kidneys.

433. Excretion of Carbon Dioxide. - Venous blood entering the lungs contains about 46 cc. of carbon dioxide in 100 cc. of blood, and the arterial blood leaving the lungs has only about $40 \mathrm{cc}$. Thus it appears that approximately one-eighth of the contained carbon dioxide is excreted as blood flows through the capillaries in the lungs.

The chief difference between arterial and venous blood is that the arterial has twice as much oxygen and seven-eighths as much carbon dioxide. The small amount of nitrogen dissolved in the blood is always the same ( 1 to 2 per cent), for free nitrogen takes no part in the activities of living cells in animals.

Evidently it is incorrect to state that "venous blood is purified in the lungs" and that "arterial blood is pure." The loss of only one-eighth of the carbon dioxide is not making "pure." We should not say that we have made muddy water "pure" if only one-eighth of the mud is extracted. The words "pure" and "purify" should never be used in connection with respiration of blood in the lungs. It is easy to remember that blood doubles its oxygen and loses about one-eighth of its carbon dioxide in changing from venous to arterial blood in the lungs. 
(D) Carbon Dioxide in Expired Air. - Connect a bellows, bicyclepump, or atomizer bulb to a glass tube, and blow air into some limewater (or barium-water). Note the effect. If much cloudiness (precipitate) appears, compare with air pumped out-of-doors directly into the lime-water.

Now, exhale air from the lungs through a glass tube into limewater. Compare with the lime-water mixed with fresh air in above experiment.

434. Excretion of Nitrogen and Water. - This work of the kidneys is carried on by the tubules which are numerous in these organs. A section of a kidney prepared for microscopic study -shows tubules cut transversely, longitudinally, and obliquely; and surrounding the tubules are abundant blood-capillaries. The cells composing each tubule extract nitrogenous excretions and water from the blood, and then eliminate them into the cavity or lumen of the tubule. The water washes the excretion out of the tubule into the ureter or kidney-duct, which in all mammals extends from each kidney to the bladder. This is a reservoir for the temporary storage of the excretions (urine) of the kidneys, and in all mammals is connected with the exterior by a duct known as the urethra.

435. Skin in Excretion. - Under conditions of high temperature the human skin-glands eliminate water ("sweat" or "perspiration") in which is dissolved salt and small quantities of other substances commonly excreted by the kidneys. However, all the water and other substances could be excreted by the kidneys, and so the skin is not a necessary organ of excretion. It will be explained in $\S 447$ that the skin eliminates water in order to get rid of excess heat, and hence excretion of water is not the primary work of the skin-glands.

\section{SUMMARY}

436. Functions Serving the Cells. - (1) Digestion and absorption of foods are functions for getting dissolved foods into the blood, which directly or through the lymph distributes 
it to the cells of the entire body. (2) The lungs are adapted to supplying the blood with oxygen, which is then distributed to the cells. (3) The excretory organs (chiefly lungs and kidneys) are adapted to remove from the blood the various excretions found in the cells. (4) The circulating liquids (blood and lymph) serve the functions of food-supply, oxygen-supply, and excretion, in that food and oxygen must be distributed to the cells and excretions taken away to the excretory organs.

These four great functions, involving the organs of digestion, respiration, excretion, and circulation, serve the cells. We have noted $(\S 268)$ that a one-celled organism does not need such a complicated mechanism, for with respect to food, oxygen, and excretions it can deal directly with the external world. The vast number of cells in a higher animal has made necessary the complicated organs of digestion, respiration, excretion, and circulation for serving the cells.

\section{NERVOUS ACTIVITY}

437. Need of Coördination. - The functions which serve the cells named in the preceding paragraph must work together or in harmony. For instance, if muscles are working faster, there is need of more food and oxygen. This requires more rapid circulation, greater oxygen-supply, and increased digestion in order to supply and transport the necessary food and oxygen. And faster work results in more excretions and consequently greater activity of the circulatory and excretory organs in removing them. Thus increased activity of certain organs may demand a corresponding increase in work of many others; and there must be coördinated activity.

The function of coördination is part of the work of the nervous system. This is parallel with the case of a frog's functions ( $\$ 54)$; but also the human nervous system has a vast amount of other work arising from mental activities. 
438. Reflex Action. - How the nervous system exerts its coödinating power on other organs may be clearer after a brief account of some simple cases of control.

If by accident one touches a finger to a hot stove, a sudden contraction of the muscles will cause the hand to be jerked away before the brain becomes conscious of the burn. The explanation of such an action is that the hot stove stimulated sensory nerve-endings in the finger, the stimulus was transmitted to nerve-cells in the spinal cord between the shoulders, and at once turned back as a motor impulse, which, transmitted along other nerve-fibers back to the finger, caused the muscles to contract. Since the impulse originating in the stimulated sensory nerve-endings appears to be reflected back to the muscles by the spinal cord, the process is called reflex action.

Headless frogs and other animals will make the same reflex movements if their toes are stimulated. This proves that reflex action is quite independent of consciousness (knowing, feeling), for all observations on injured men and animals indicate that the brain is the organ of consciousness. For example, a man with the spinal cord seriously injured, say in the middle of the back, would feel no pain in the legs and could not move them voluntarily; that is, by conscious action from the brain. Such facts, of which many have been recorded in medical books, prove that there must be uninjured nerve-fibers extending to the brain in order to have conscious control of organs. Hence, a headless animal or one with the spinal cord cut off just back of the head could not feel or be conscious of changes occurring in the body.

In the case of instantly closing an eyelid to escape a threatened injury, the stimulation of the nerve of sight (optic nerve) causes an unconscious reflex in the brain back to muscles which move the eyelids. There are many other possible reflexes through nerves in the head which are directly connected with the brain. 
The above are simple examples of unconscious reflex actions which are constantly occurring. We learn to do a large number of things reflexly, in addition to the fundamental processes such as breathing, heart-beat, digestion, etc., which naturally and necessarily are subject to reflex control. In walking, playing musical instruments, using various tools, etc., we learn by long practice to act more or less reflexly or automatically, and with little or no exercise of the will (conscious control).

439. Conscious or Voluntary Action. - Touching a hot stove used above as an illustration of reflex action also affords a case of conscious action. Soon after the hand is jerked away by reflex action, one becomes conscious of being burned. Obviously, there are nerve-fibers for transmitting the sensory impulse from the tip of the burned finger to the brain. There a conscious action may occur, and it may be reasoned that it is dangerous to keep the hand anywhere near a hot stove, and so on, with the result that it is decided or willed to take the hand far away. This is accomplished by a conscious motor impulse from the brain to the muscles of the arm, causing them to move as the will dictates.

Moreover, the brain may also control the direction of the movement; that is, it may coördinate the contraction of the various muscles. A splendid example of such coördinated voluntary or conscious control of muscles is that of baseball pitchers who can will to throw a ball to a given place and at the same time consciously control the contraction of the muscles of the arm so that the ball will be given the twirling or curving motion so much desired by experts in ball-playing. Learning to do this by long practice means training the nervecells in the brain so that they will come to control the muscular contractions and so cause the desired muscular movements.

440. Spinal Cord and its Nerves. - The human spinal cord is usually described as lying in a cavity in the backbone (vertebral column). Examination of some of the segments of 
any backbone which may be obtained at a meat-market will show that the cord lies dorsal to the central axis. Moreover, the cord is not completely covered by bone, and at the uncovered places are the spinal nerves. These are arranged in pairs (thirty-one pairs altogether). Each nerve is divided near the cord, and one branch or root joins the dorsal side of the cord, while the other joins the ven-

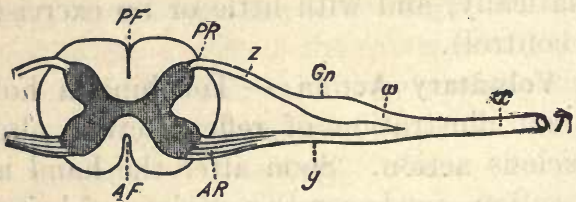

FIG. 162. Diagram showing relations of a cross section of spinal cord to spinal nerves. The $\mathrm{X}$-shaped central mass is gray matter, surrounded by white matter. $P F$, posterior or dorsal fissure; $A F$, anterior or ventral fissure ; $P R$, dorsal root: $A R$, ventral root of spinal nerve $(x) ; G n$, spinal ganglion. (From Huxley-Lee.) tral side. On each dorsal root is a thickening called spinal ganglion, which contains nerve-cells whose fibers extend into the cord and also out through the nerves (Fig. 162). The cells whose fibers constitute the ventral root lie inside the cord.

A cross section of a spinal cord shows an X- or H-shaped figure in the center. In a fresh cord this is silvery gray, and is called the gray matter (Fig. 162). The surrounding whitish tissue (white matter) is chiefly composed of connective tissue and covered nerve-fibers. Many of these fibers extend to the brain and others to the regions of the cord where other spinal nerves are attached. The gray matter contains many nerve-cells, especially those whose fibers extend out through the ventral root into the spinal nerve and thence to muscles and other organs.

The spinal cord is surrounded by membranes which separate it from the hard walls of the backbone. These contain numerous blood-vessels, some of which extend into the cord. Similar membranes are between the brain and the skull.

Deep furrows or fissures extend longitudinally on both 
the dorsal and ventral sides of the cord and partially divide it into right and left halves. The membranes which surround the cord extend into these fissures and supply the inner parts of the cord with blood-vessels.

It is known that the sensory nerve-fibers (e.g., those of touch in the skin) are connected with the dorsal root of the spinal nerves, and that the fibers which carry motor impulses from the cord to the muscles and other organs are in the ventral root. Since the dorsal and ventral roots unite into a spinal nerve, it is clear that each nerve is a bundle of both motor and sensory fibers, each one connected with a nervecell in a spinal ganglion or in the spinal cord.

In reflex action $(\$ 438)$ the sensory impulse travels from the skin to the cord by way of the dorsal root, and then in the gray matter is "reflected" to the motor cells whose fibers leave the cord in the ventral root and extend out to the muscles. How this "reflecting" is done is unknown,we only know that events occur as stated. As a parallel case, we know that electricity will "travel " in certain substances according to definite laws; but scientists are very much in the dark as to how and why it does so.

441. The Brain. - The human brain is a very complicated mass of nerve-cells, fibers, blood-vessels, and connective tissue. The following brief account includes the most important facts regarding structure of the brain.

The convoluted mass of the brain in the upper part of the skull is the cerebrum, which is divided by a deep furrow into right and left cerebral hemispheres. Beneath the back part of the skull and just above the neck lies the next largest part of the brain, the cerebellum. Beneath the cerebrum are various small structures, such as the two olfactory lobes from which nerves extend to the nose, two optic lobes with a nerve to each eye, masses of white tissue through which nerve-fibers connect opposite sides of the brain, several pairs of nerves, and the spinal bulb or expanded connection of the 
spinal cord with the brain. Most of these structures can be identified on a sheep's brain, obtained from a meat-market, and hardened in strong alcohol or formalin solution.

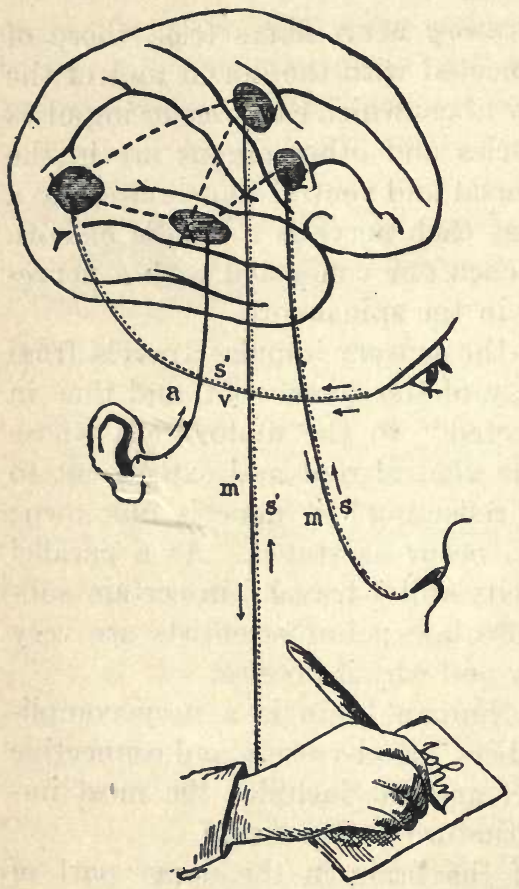

Fig. 163. Diagram showing nerve connections of ear, eye, lips, and hand with centers in the cerebrum of the brain. The ear and eye have only sensory fibers which carry impulses to the brain, while the lips and hand have both sensory and motor fibers $(m, s)$ carrying impulses as shown by the arrows. (From Landois and Stirling.)

Microscopic study of sections shows that most of the nerve-cells of the brain are near the surface (cortex), while inside are the fibers connecting with the spinal cord and also connecting various parts of the brain. Hence a cut across a fresh brain shows gray matter (with nerve-cells) on the outside and white matter (nerve-fibers) inside, just the reverse of the spinal cord.

The cerebral hemispheres are the center of mental life, consciousness, and voluntary action. The cerebellum is the center of coördination, causing muscles to work in definite and controlled ways. The nervecells which control the respiratory movements and the circulatory organs are in the spinal bulb.

Localized centers in the cerebrum are known to exist. So far our knowledge is limited, but there are areas in the 
surface layers where the nerve-cells are concerned with special organs; for example, there are such centers for muscular movements concerned in speech, for hearing, taste and smell, sight, touch, and voluntary movements of various organs. This discovery of localized centers in the cerebrum is already valuable to surgeons. For instance, if an injury to a brain interferes with any of the functions named above, the surgeon knows approximately where to look for broken bloodvessels and other injuries.

The discovery of the centers controlling certain functions has no bearing upon the once-popular pseudo-science called phrenology. That pretended to locate certain general mental powers by the contour of the skull. It was utterly unscientific because (1) there is no evidence of such localization as phrenology claimed, and (2) there is much evidence against the idea that the outer surface of the skull indicates the degree of development of the brain beneath.

\section{ORGANS OF SPECIAL SENSES}

442. Structure of Eye. - Use a hand-mirror and examine your own eyes after reading the next two paragraphs. Refer frequently to Fig. 164.

The center of the outer coat of the eyeball in front is the transparent cornea, through which one can look into the interior of the eye. All the remainder of the outer coat of the eyeball is hard, white, and opaque (the sclerotic coat). One might imitate the external appearance of an eyeball by painting a glass ball white, excepting a circular spot to represent the transparent cornea.

Inside the cornea, and of nearly the same size, is the black, gray, or blue part of the eye with a hole in the center. The color pigment is in a sort of thin membrane (called iris), and the hole in the membrane is the pupil. Just back of the pupil is the lens, which is bi-convex, transparent, and elastic, 
so that by pressure its shape can be changed. Back of the lens is the sensitive membrane (retina), which is closely

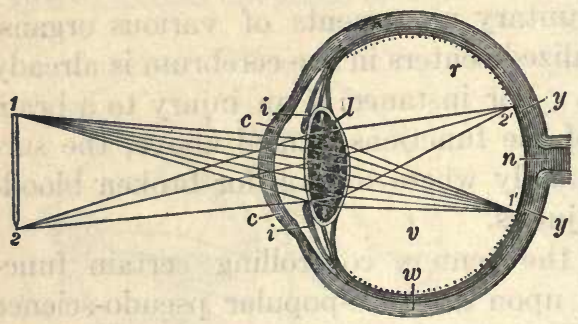

Fig. 164. Diagram of human eye. $w$, outer or sclerotic wall; $y$, inner or choroid wall; $r$, retina (dotted line) ; $c$, cornea ; $i$, iris ; $l$, lens with its suspending capsule; $v$, vitreous humor; $n$, optic nerve ; $1-2$, an object, and $1^{\prime}-2^{\prime}$, its image on the retina. (From Hough and Sedgwick.)

lens and the cornea is filled with a watery fluid (aqueous humor). If an eyeball, obtained from a meat-market, be punctured, these humors escape, and the eyeball collapses. They are so transparent as not to interfere with the passage of light from the cornea to the retina.

Between the retina and the outer white coat (sclerotic) is a layer of tissue (choroid) with abundant blood-vessels and black pigment. The pigment in the choroid and iris prevents light from entering the eye except through the pupil and the lens. In the same way black pigment in a photographic camera prevents light from reaching the sensitive plate or film except through the lens and the opening of the diaphragm which corresponds in use to the iris and its opening.

In both a camera and an eye the whole structure is essentially a light-proof box with a diaphragm or iris for regulating the amount of light, and a lens for focusing the rays of light upon a sensitive plate or retina. The chief differences be- 
tween a camera and an eye are: (1) the eye is living tissue; (2) the sensitive plate or retina of the eye contains living nerve-cells connected to the brain by nerve-fibers of the eye-nerve or optic nerve; and (3) the lens of the eye is focused, not as in a camera by moving the sensitive plate nearer to or farther from the lens, but by muscles which change the shape of the eye-lens.

The adjustment of the lens to suit different distances of objects seen is called accommodation. It is accomplished as follows: The lens when not compressed is very bi-convex, as may be seen in a lens cut from an eye obtained at a meatmarket. When an eye is resting in sleep or is looking at faraway objects, the lens is much flattened (or made less convex) by the pull of the elastic choroid upon the transparent capsule which incloses the lens and attaches it to the choroid. In order to see clearly near-by objects the lens must be focused by being made more convex. This is simply the elastic return of the lens toward its natural very bi-convex shape, and this return is permitted by a sheet-like circular muscle which opposes the elastic pull of the choroid and thereby eases the tension upon the lens.

It is evident from the above that the feeling of strain when we look at very small objects is due to the pull of muscles against the constant elastic pull of the choroid upon the capsule that incloses the lens.

(D) In order to study the effect of change of shape of the lens upon the focus of the eye, first set up a photographic camera and focus upon near and distant objects by moving the lens. If one had lenses for different distances (very bi-convex for near-by, and less so for far-away) the distance from the lens to the sensitive plate might be kept stationary in a camera. In the case of an eye the distance to objects seen varies, and there is need of many lenses of different curvatures; or better still, of one elastic lens whose shape can be changed to fit objects at any distance.

Eyes that cannot see distant objects clearly are said to be "near-sighted," and one with such eyes must hold print 
very near in order to bring it into focus. This is due to the lens being abnormally distant from the retina. Concave glasses should be worn in order to change the direction of rays of light and cause them to focus on the retina. Other eyes are "far-sighted," and only by constant strain can the lens be kept convex enough to focus the rays on the retina, which is abnormally near the lens. Even when straining to the utmost some persons must hold books at arm's length in order to read. Convex glasses correct such difficulty and relieve the excessive strain. In old age the lens loses some of its elasticity and fails to become convex enough when trying to read, and hence a book must be held at a distance, unless convex glasses are used. Astigmatism is a very common congenital or inborn defect due to irregular curvature of the cornea or lens, making it impossible to see equally well lines which run in different directions, as on a clock-face. For such eyes so-called "cylindrical" glasses should be used constantly to avoid eye-strain.

443. The Ear. - The human organ popularly known by this name is the external ear, from which a tube leads inward to the tympanic membrane, or ear-drum. Beyond this is a cavity known as the middle ear, and from it the Eustachian tube leads to the pharynx. Still deeper in the head than the middle ear is the inner ear, a complicated membranous structure lying in bony cavities of corresponding shape. The lower part (cochlea) is shaped like the cavity in a snail's shell (Fig. 165), and the upper part has three ring-like canals (semi-circular canals). Branches of the auditory nerve connect the sensitive membranes of the inner ear with the brain.

The inner ear is filled with a fluid. Sound vibrations enter the outer tube, and throw the tympanic membrane into vibration. This moves a chain of three bones which extend across the cavity of the middle ear and touch 
the membranous wall of the inner ear. Through this membrane vibrations are passed on to the fluid of the inner ear, and its vibrations stimulate the sensory endings of the auditory nerve which are sensitive to sound. The semicircular canals give us the sense of equilibrium, of which we are aware even when we are blindfolded.

444. S m e11, Taste, Tou ch, Temperature. These senses are connectedwith special nerves.

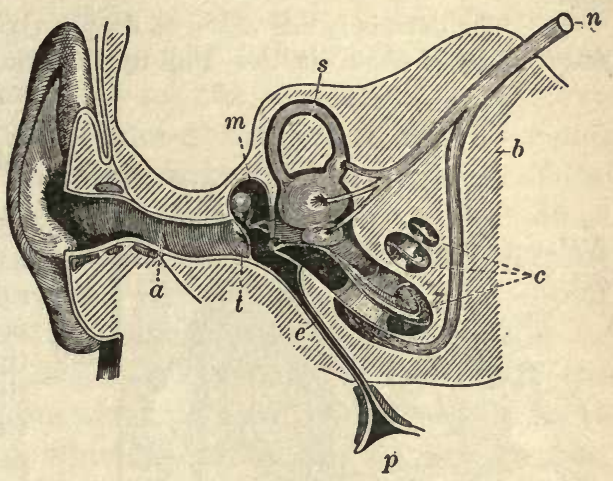

Fig. 165. Diagram of human ear. $a$, canal from external ear; $t$, tympanum; $m$, middle ear with small bones extending from tympanum to inner ear; $s$, one of the semicircular canals of inner ear; $c$, cochlea of inner ear; $n$, auditory nerve; $b$, bone surrounding middle and inner ears; $e$, Eustachian tube, and $p$, its opening to pharynx.

Those of smell have endings in the epithelium which lines certain upper cavities of the nose. The nerves of taste end in the little projections (papilla) on the tongue. Those of touch and temperature are widely distributed in the skin of all parts of the body.

\section{THE SKIN AND ITS WORK}

445. Human Skin. - Microscopic preparations show that the surface of the skin is made up of closely set cells, while the lower side next to the muscles and bones is made up of connective tissue (Fig. 166). The cellular layer is the epidermis, and the connective tissue is the dermis.

(D) A piece of leather tanned without the hair will give a good view of the intricately interlaced fibers of the dermis, the epidermis 
having been removed in the process of tanning. Soften such a piece of leather by soaking in hot water, and then examine by tearing it apart.

(L) The dermis is tied down to deeper tissues by connective-tissue fibers, many of them elastic. Pull up the skin on back of your hand,

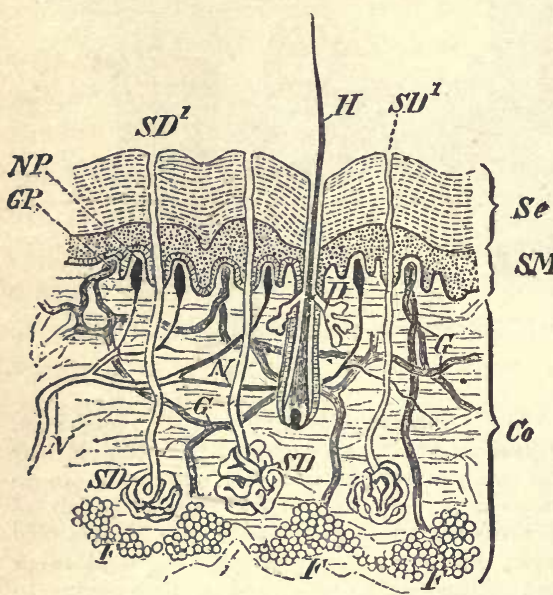

Frg. 166. Section of human skin. Se, outer hard layer of dry cells; $S M$, layer of living cells; $\mathrm{Co}$, the dermis (connective tissue). Se and $S M$ together constitute the epidermis. $G$, blood-vessels ; $N$, nerves ; $N P$, nerve-endings; $F$, fat; $S D$, sweat-glands and ducts; $H$, hair. (From Wiedersheim.)

in solutions of caustic-potash or washing-soda, and pieces scraped from a calloused spot on the palm may be so treated in preparing for microscopic examination. This swelling when wet explains why the hands get so soft when kept for a time in water, especially if soapy.

A microscopic section shows that the scaly cells at the surface are many rows deep, and that deeper down in the epidermis are several rows of rounded or cubical cells, which are usually brightly stained in preparations. These are living cells, while the hard scaly ones at the surface are dead. The 
scaly cells together constitute the horny layer, and the lower cells of the epidermis form the Malpighian layer, or living layer. The horny dead cells at the surface are continually wearing off, and the living layer cells are by division forming new cells, which are pushed toward the surface.

In warts, corns, and callouses the horny layer becomes greatly thickened. The cause is growth and division of the lower living cells faster than the hard cells at the surface wear off. Friction, pressure, and introduction of some foreign substance through a cut or scratch are some of the well-known causes of these thickenings of epidermis.

In the ordinary healing of a cut or burn, the cells of the living layer quickly form new cells to fill the gap beneath the blood-clot or "scab" which forms on the surface. In extensive burns it is sometimes necessary to resort to skingrafting. This means taking healthy pieces of skin from other persons and applying them where the epidermis has been completely destroyed. The cells of these healthy bits of grafted skin soon become firmly attached and by repeated division grow over the injured surface.

Nails and hairs are specialized masses of horny cells. The so-called "roots" of nails and hairs are deep-lying masses of living cells which grow and divide rapidly. The pit in which each hair is attached is called a hair-follicle, and in it is a conical elevation (papilla) from which the hair grows as cells are formed and pushed outward. Oil glands (sebaceous glands) open into the hair-follicles. Cutting or shaving hair, contrary to popular belief, does not increase the number of hairs, for the follicles are formed in embryonic life.

$(L)$ Examine a hair with microscope, and note that it is composed of overlapping scales (cells). The center is also filled with cells. The idea of barbers that hairs are hollow and require singeing to prevent the escape of oil is absurd - but profitable to the barber.

Sweat-glands are abundant over the whole human skin. They are most numerous on palms and soles, which also have 
no hairs. Each sweat-gland is a tube extending from the socalled "pore " down into the dermis, where it is much coiled and surrounded by blood-capillaries.

446. Functions of the Skin. - Next to protection, the most important function of the human skin is heat regulation.

While the soft skin of frogs and other lower animals is important for respiration, the dry hard skin of mammals and man is of little use in this way. It has been shown by putting a man's body in a rubber bag which was tightly fitted around the neck that the lungs give off nearly two hundred times more carbon dioxide than the skin does. Hence, skin respiration is of no practical importance to us.

Absorption by the skin is of little importance.

Oily materials are often rubbed into the skin, and small quantities appear to be absorbed. Probably the massage effect is most important. It is known that some drugs can be absorbed when thus rubbed into the skin.

Secretion of Sweat.- Under ordinary temperature conditions the amount of secretion does not attract attention, because it is evaporated as rapidly as formed. Large quantities of water taken into the stomach are absorbed, raise the blood-pressure, and increase perspiration.

Certain drugs bring about dilation of blood-vessels and cause profuse sweating; others act in an opposite manner.

Normal sweat is nearly 99 per cent water. The one per cent of dissolved substances are chiefly mineral excretions similar to those in the urine. If the skin were varnished, the same excretion would be easily eliminated by the kidneys. Sweat, then, is not necessary as an excretion, but as an aid to heat regulation (see next section).

447. Skin as Heat-Regulator. - The fact that the noticeable activities of the sweat-glands are usually associated with great internal heat suggests that the chief function of these glands is not so much to get rid of sweat (which the kidneys could manage) as to discharge heat. 
The muscular system is the chief source of increased heat production, while the other organs probably generate the heat which is more or less constant, as during sleep or complete rest. When these organs are unable to supply the necessary heat, shivering, which is involuntary activity of muscles, may begin and thus increase the internal supply of heat.

Loss of heat is partly by respiration. This is very important for dogs, which "pant" when overheated because their skin perspires little except on the pads of the feet. They also lose heat rapidly from the evaporation that occurs on the surface of the protruded tongue. Chickens are often seen breathing rapidly with the mouth open; and thus birds lose heat from their lungs and air-sacs. In man, however, the skin is the one great heat-regulator.

The loss of heat from the human skin is controlled by nerves, some of which regulate $(\$ 414)$ the flow of blood to the skin and sweat-glands, and some stimulate these glands into activity. Rapid exercise causes the sweat-glands to become active. The effect of the sweat is illustrated by the familiar cooling of bottles of water wrapped with wet towels exposed to warm air; of water allowed to evaporate from the hands or face on a summer's day; or of the porous waterjugs and canvas bags which the inhabitants of some hot countries use for their drinking water because the small amount of oozing water is evaporated and cools the water in the jug. We also know that following a bath on a hot day the body cools rapidly, and the explanation is that the heat of the skin was used to evaporate the water. Now, the sweat-glands are simply mechanisms for covering the skin with water ready for evaporation at all times when the skin is warmed by blood circulating rapidly.

In addition to the heat lost by evaporation of water on the surface, there is much loss by radiation, especially in cold weather when the skin gets warm after exercise which is not often active enough to cause moistening the skin with sweat. 
Certainly at such times the warmer skin must radiate more heat to the air, just as a hot-water pipe does when the temperature of the circulating water increases.

The importance of the evaporation of sweat as a method of cooling depends upon the temperature and humidity of the air, which determine the amount of water the air can absorb. Dry cold air can take up little watery vapor, while dry warm air may contain much more water than the cold air. Hence in hot weather loss of heat by the evaporation of sweat becomes more important. Obviously, hot dry winds would favor evaporation; while hot moisture-laden air prevents rapid heat loss both by radiation and by evaporation. This is the usual condition on oppressively hot days when heat prostrations and sunstrokes are common. The moist hot air prevents proper loss of heat from the skin, and the internal temperature rises too far above $99^{\circ} \mathrm{F}$., which is best for human protoplasm. Obviously, reduced heat production will help avoid excessive internal heat ; and this means keeping as quiet as possible and eating sparingly, thus reducing the activity of muscles and the digestive organs. (Why do people in tropical climates with hot sun and moisture-laden winds suffer from the heat more than do the inhabitants of equally hot but dry regions?)

Fever is due to increased production of heat (caused by toxins of disease), and is usually accompanied with dry skin and inactive sweat-glands; but occasionally even great perspiration does not discharge the heat fast enough. The so-called "wasting" indicated by loss of weight during prolonged fever is due to the rapid oxidation of tissues when little or no food is available. Drugs act upon fevers by reducing oxidation in tissues or by promoting perspiration and consequent heat loss from the skin.

The effect of cold baths upon heat regulation by the skin is discussed in $\$ 458$. 


\section{CHAPTER XVIII}

\section{PRINCIPLES OF BIOLOGY APPLIED TO HEALTHFUL LIVING}

\section{PERSONAL HYGIENE}

448. Hygiene. - In this chapter it will be shown that very many of the facts and principles of biology are of great value when applied to making the human body freer from disease and a more efficient mechanism for carrying on a useful and happy life.

That department of biological science which deals with the established principles of human health is commonly known as hygiene. It is simply a phase of applied biology. Whenever hygiene deals with the health of individuals, how to take care of oneself, as in rules for eating, breathing, sleeping, etc., it is called personal hygiene. This is chiefly the principles of biology, particularly of physiology, applied with a good proportion of common sense. By this we mean, for example, that one who has studied the structure and working of the lungs will need only common sense to enable him to see that tight clothing will interfere with the natural movements, and then the hygienic rule "do not wear tight clothing" is seen to be a scientific application of the biological principles relating to human breathing. The same is true with regard to every organ; and we do well to examine every proposed new rule of hygiene from the standpoint of applied biology. Thousands of foolish rules of hygiene have been published, but it is usually possible to select the good ones because they are obviously based upon the principles of biology. For example, if some one advised prolonged mastication of fat 
meat, we should reject this absurd rule at once, because biology teaches that such food is not digested by saliva. We should keep a sharp lookout for such applications of biology to the unscientific rules of personal hygiene which so many people accept without question.

Public hygiene or sanitation means principles of biology applied to increasing the health of a community of people. Problems relating to clean streets, pure food-supply, infectious diseases, sewerage, water-supply, and others under health officers, belong to public hygiene.

\section{RESPIRATORY ORGANS}

449. Habits of Breathing. - The effect of tight clothing is referred to in the preceding section. All authorities in medicine and hygiene unite in condemning the fashion of wearing any clothing which interferes with breathing movements.

Exercise in deep breathing is important, for it trains the respiratory muscles so that fuller expansion of the lungs occurs regularly. This means that fresh air goes deeper into the air-tubes (see $\$ \S 428,429$ ). Consult your physical-training teacher as. to the advantage of training in breathing for athletics.

Breathing through the nose is the natural way, because the air is properly warmed and much dust is stopped in the nasal passages. Mouth-breathing is abnormal, but very common. Children who breathe habitually through the mouth probably have enlarged adenoids in the upper part of the pharynx back of the soft palate. If not removed at once by a competent surgeon, they may seriously interfere with the health or cause deafness, or even deformation of the upper jaw. They commonly disappear after childhood, but then the damage has been done. Hence any special difficulty with natural breathing through the nose should be referred at once to a doctor for advice. 


\section{BIOLOGY APPLIED TO HEALTHFUL LIVING 527}

450. Ventilation. - Frequent renewal of the air of buildings is required; because the breathing of human beings and the combustion in stoves, lamps, etc., use oxygen and add carbon dioxide; and because bacteria are carried in the dust of the air. All systems of ventilation should provide for the exit of the lighter warm air near the ceiling and the entrance of fresh air (preferably warmed previously by heaters) near the floor. At night when the body is properly protected in bed the supply of fresh air may safely be very cold. The important rule is to get as much fresh air as possible without chilling any part of the body by drafts. That is far more harmful than poor ventilation, for "colds" are often produced ( $(453)$. Good ventilation does not require that a high wind constantly blow through a house.

For accounts of the best methods of ventilation, see books of hygiene and household science. The subject is so extensive that only the great principles can be suggested here.

451. Avoiding Dust. - Owing to the fact that dust often carries dangerous bacteria and that there is a harmful effect of accumulated dust in the lungs, dust in the air should be eliminated as far as possible from homes, factories, and public buildings. Old-fashioned sweeping and dusting are dangerous unless windows are opened and the wind allowed to blow out the dust and bacteria. Carpet-sweepers and especially vacuum-cleaners are better. Damp cloths should be used for wiping dust from furniture ; brushes and featherdusters are relies of the dark ages and should never be used. If brooms must be used indoors, damp sawdust will help keep dust from rising into the air. The most sanitary modern dwellings have no carpets which are not easily taken outdoors for cleaning, while the floors are painted, varnished, or waxed so as to make washing easy and sweeping unnecessary.

452. Artificial Breathing. - This means causing the lungs to respire after normal breathing has been stopped by immersion in water or by gas asphyxiation. This can be done 
by placing the patient on his back and regularly (fifteen times a minute) raising the arms above his head and then gently lowering them to the sides, making the chest-walls move as in natural breathing. For details of this and other methods, see special chapters on "accidents" in books on hygiene. The teacher should give practical lessons on this important topic, selecting one student as patient and another as operator.

453. Colds in Resuiratory Organs. - Severe "colds" which are liable to lead to bronchitis (inflammation of bronchial tubes), pleurisy (inflammation of pleura of lungs), or pneumonia (congestion of blood, with certain poisonous bacteria in the lungs) require medical advice. Chronic congestion of nasal membranes leading to the condition known as catarrh should receive medical attention, for a slight operation or special treatment may be necessary to effect a cure.

For preventing colds see $\$ 458$ on bathing. One whose skin has been chilled should hasten to restore the normal circulation of the skin by exercise, friction, hot drinks, hot bath (with great precaution against more chilling), and in extreme cases by certain drugs which physicians advise. It is dangerous to neglect a " cold," especially in its incipient stages.

\section{DIGESTIVE ORGANS}

454. Teeth. - The proper care of the teeth is commonly emphasized in the books on "physiology" written for elementary schools, and need not be repeated here. The fact that bacteria are largely responsible for dental decay suggests the daily use of antiseptic tooth-powders and mouth-washes.

455. Hygiene of Eating. - The practicable hygiene of digestion for most people is that which suggests the time for eating, and the amount and kind of food.

The question of the time of meals depends upon other physiological demands. A light breakfast and a light lunch are best 
for busy people, whose muscles or nerves are hard-worked. Then, after the day's work and a brief rest is the best time for dinner, the chief meal of the day. The reason for this is that intellectual activity leads to a marked increase in the amount of blood in the vessels of the brain, and physical work affects muscles similarly. The result must be a withdrawal of blood from the vessels of the digestive organs and a consequent retardation of the digestive process.

The above plan suits most people; ; $_{2}$ but there are numerous individual exceptions, and each one must experiment with himself if he would find the most satisfactory time for meals. Work and habits of human beings vary so greatly that there can be no universal law of eating. Certain rules for general application have been established by the experience of thousands of people, and probably most important for remembering are the following: (1) There should be regularity in meals. (2) Physical and mental fatigue interfere with digestion. (3) Overeating acts likewise.

The selection of diet and its amount is similarly variable. Hard physical exertion and exposure to cold demand abundant food for energy ( $\$ \$ 420,421$ ), and the question of easy digestibility is of minor importance. On the other hand, persons of sedentary habits should avoid unnecessary amounts of all kinds of foods; and also observe well their own peculiarities as to digestibility of carbohydrates and fats.

Eating anything between meals is, as a rule, inadvisable; but here again there are individual exceptions, and knowledge of the possible harm to digestion will lead to caution.

Some water (not iced) should be taken at every meal, for it is needed in liquefying the contents of the stomach in preparation for escape into the intestine $(\S 400)$. But water should not be taken at the same moment with solid food, for it "washes down" the food and thus prevents mastication. The old idea that water dilutes the gastric juice does not now seem to be very significant, for it has been discovered 
recently that water soon passes from the stomach into the intestine and gastric juice is secreted rapidly.

Overeating is the chief point on which many people need physiological advice. Scientific studies have often shown that men need no more food than stated in $\S 419$, and yet a large number of people take more daily. Especially is it true that we use too much protein $(\$ 423)$, thus unnecessarily overworking all organs without any gain. The other foods are also used to excess by many whose daily activities do not require so much stored energy; and the common result is fat-storage, which often becomes so excessive as to be uncomfortable or even a danger to the heart and other organs. In the majority of cases excessive fat storage is due to the overeating of foods containing carbon, hydrogen, and oxygen (sugar, starch, butter, and fat meat). The over-fat condition, once established, is difficult to change; and hence young people should guard against an excessive increase in stored fat due to intemperate eating.

An excess of meat diet is more harmful than an excess of other foods, for the reason that most proteins eaten are oxidized and excreted within a day ( $\$ 422)$. The nitrogen excretions thus formed from proteins may play an important part in the development of gouty and rheumatic conditions.

The value of mastication has been the subject of much discussion, and is still uncertain; for there are some people who masticate little and have perfectly healthy digestion, and there are others who masticate extensively and claim to have thereby cured indigestion. The truth is that it is largely a question of the kind and amount of food and the habits of the individual. Certainly one who eats an excessive amount of starchy food, or who has starch-indigestion in the intestine, will do well to masticate starchy food and allow the saliva to exert its digestive influences as long as possible, for thereby he may cure a form of indigestion. But this does not prove a rule for all people or for all articles of diet; and 
with no more than the necessary amount of properly cooked food the average individual can safely follow his natural instincts as to the amount of mastication. There are those who point to the rumination of cows and sheep for evidence that naturally animals masticate food for a long time; but this gives no rule for human guidance. In the first place, a cow's natural food is uncooked and otherwise unprepared; second, her digestive organs are quite unlike the human; and, third, dogs more closely resemble man in structure and in foods and they never masticate. Obviously, it cannot be concluded that man should chew his food long because cows and sheep do. The only scientific conclusion must be based upon individual human experience; and this indicates that some people with weak digestion of starch need to give special attention to mastication, but that most people may safely forget their jaws while eating (i.e., masticate instinctively) provided that they do not eat too rapidly or in excess. However, it is well for each person to experiment upon himself, and thus determine how far special attention to mastication is important for himself.

One point in favor of thorough mastication deserves mention, namely, that it tends to prevent overeating. When food is rapidly swallowed, there may be an excess taken before the gastric nerves give us warning of too much food. Prolonged mastication tends to avoid this result, possibly because the sugar obtained from the salivary digestion acts upon the gastric nerves just as sweets before a meal "take away the appetite " or reduce it. However, it is well never to eat until hunger is completely satisfied, and this rule would avoid most overeating.

Stimulants. - Should digestive stimulants be avoided, is a much-discussed question, usually with regard to alcoholic drinks. It also should apply to spices, condiments, coffee, tea, cocoa, carbonated water, and even hot food; for these all have some stimulating effect upon the digestive organs. 
It is argued that stimulants are not natural, for animals do not require such things; but it may be answered that animals do not lead sedentary lives, undergo intense nervous strain, and do similar things which in civilized man tend to interfere with proper digestion. Certainly we can often gain by the temperate use of some stimulants; but harm will always come from overstimulation, which is most likely to happen in the use of tea and coffee $(\$ 480)$ and alcohol ( $\$ 468)$. Probably the most useful and safest of all stimulants are hot foods and hot drinks, such as hot bouillon, hot milk, or even hot water.

456. Psychology of Digestion. - We have already defined psychology as the science of the mind ; and here it is important to note that the mind greatly influences digestion. It is well known that the thought, sight, or smell of savory food causes the "mouth to water," that is, stimulates the salivary glands; and there is a similar effect on the gastric glands. We also know how certain disagreeable mental states may cause loss of appetite and even nausea. In fact, the gastric glands may fail to secrete, and indigestion and other disturbances may be caused by the direct influence of the mind.

Such well-known facts suggest the importance of pleasant surroundings while one is eating. Here is the secret of the good digestive influence of music, jolly company, and other things which make for a happy state of mind during the meal hour. That is a time when every one should drop his cares and troubles.

On the other hand, there is a certain real danger in pleasant accompaniments of meals. It is simply that fine fare and surroundings which are agreeable to all the senses tend to feasting or overeating, a result often capable of unhealthful consequences. The only safety is in learning self-control so that one's stimulated appetite may not lead on and on to gluttony. 


\section{SKIN}

457. Skin Cleanliness. - The scientific reason for cleaning the skin, especially by the use of soap in warm water, is that it removes dirt, which is objectionable primarily because it is unæsthetic and therefore disagreeable to refined people, and secondarily, because dirt contains micro-organisms which may produce disease. In fact, dirty hands have often caused typhoid and other dangerous diseases by leaving bacteria on dishes and on foods. Formerly it was supposed that a third reason for cleansing the skin with soap was to remove substances which " $\mathrm{clog}$ the pores of the skin and keep in excretions"; but, as we have seen in $\$ 446$, the skin has little to do with excretions except as water is incidentally eliminated while the skin is reducing the internal heat, hence "keeping the pores open" is a weak argument for cleaning the skin. The fact is that the pores "open " quickly, even on the dirtiest skin, when exercise develops excess of heat. However, we do not need the unscientific theory of " keeping pores open"; for the æsthetic and bacteriological reasons named above are sufficient to convince any civilized person, and especially any one who sees the force of the rule, "use soap because refined people do so and the barbarians do not." This is more sensible than trying to show that soap is necessary for hygienic reasons, for there are healthy people who never or rarely use soap. Such health is not surprising, for soap has very much to do with æsthetics and little with health, except in possible bacterial infections.

The abuse of soap by many refined people deserves attention. Exposed parts of the body must be washed very frequently with soap, and preferably with warm water; but a complete bath with soap and warm water is taken too frequently by many persons. Soap removes the oily secretions and the warm water dilates the blood-vessels of the skin, increasing liability to colds. A warm soapy tub-bath once 
a week should be followed by cold water, which causes contraction of the blood-vessels and a subsequent reaction (§ 414).

As far as is consistent with cleanliness, soap with cold water, and especially cold water without soap should be used daily on all parts of the body not exposed. The special reason for cold instead of warm water is given in the next paragraph.

458. Bathing as a Skin Tonic. - Above we have considered bathing for cleanliness only; but here we are interested in bathing for health. While only in removing bacteria does skin cleanliness appear to be necessary for health, we have fortunately a very strong argument for daily bathing as a means of leading to healthy action of the skin blood-vessels, and indirectly of the whole body. For this purpose water should be much colder than the temperature of the body, and is best applied as sponge-bath, shower-bath, or plungebath (as in sea-bathing). Contact with the cold water first causes a reflex action leading to a reduced caliber of the skin arteries, and the skin quickly becomes pallid. A reaction follows brief exposure to the cold water, and the blood-vessels expand, the skin glows, and the bather feels stimulated. No such good effect comes from too long exposure, or when the water is too cold for some people.

The explanation of the value of this cold bathing is that it gives the skin practice in readjusting its blood-supply when exposed to a low temperature. Many persons, especially those of sedentary habits, have skins which are not accustomed to react quickly to changes of temperature; and hence if chilled their skin arteries remain contracted, blood which ought to circulate in the skin is congested in some internal organ, where inflammation develops, and a "cold" follows. Frequent practice in readjusting the blood-vessels, as given by cold bathing, will make the skin more likely to react and continue to receive its fair share of blood whenever exposed 
to low temperature, thus tending to avoid the dangerous internal congestions known as "colds." It should be noted that "colds" are not confined to the lungs and respiratory passages, for one may have an unrecognized "cold" from congestion of blood in other organs, especially in stomach, intestine, and kidneys.

If in spite of systematic cold bathing one's skin sometimes gets so chilled that it does not soon react, the normal circulation should be restored by ways suggested in $\S 453$. To allow the skin to remain chilled for hours is dangerous.

Cold baths are best taken before breakfast, never within two hours after a meal, because the rush of blood to the skin interferes with the proper supply in the digestive organs.

\section{NERVOUS SYSTEM}

459. Overwork. - Unhealthy conditions of the nervous system are frequently the result of overwork, both mental and physical. Hence it is important that brain-workers should consider the hygiene of the nervous organs.

Regular mental work, as well as physical work, should be limited to a number of hours per day; and these should be the hours before late in the afternoon, when the maximum exhaustion of nervous force occurs. Nervous exhaustion from mental overwork is most often due to neglect of this rule; and the brain-worker should limit his regular day's work to a reasonable number of hours per day, and those when the brain is at its best. Too often mental overwork simply means such long days at intellectual tasks that exercise, recreation, and sleep are neglected. Sooner or later, this means the inevitable penalty of nervous disturbance, if not serious breakdown.

There come times in the lives of many brain-workers when some important work demands temporary nervous strain; but the man who is wise in the laws of hygiene will try to re- 
duce the necessary strain to the minimum and to follow it with as much recreation as possible.

The importance of fads and avocations as a means to mental and physical recreation is great. Every man and woman should cultivate at least one hobby. Even collecting postagestamps, coins, and natural-history specimens may be made an important daily relief to a nervous system tired by the regular day's work; but best of all avocations are those which are as far different as possible from the regular work, e.g., a greenhouse, a garden, or a work-shop for a man engaged in mental work.

But overwork is not all due to excessive exercise of the nervous organs directly, for physical work may lead to nervous disturbance. This is obviously due to the fact that muscular contraction occurs only as the result of nervous action. Moreover, there may be the added effect of the wearisome monotony of uninteresting toil.

The close relation of muscular and nervous work points to the important hygienic law that mental work should not be forced after physical exhaustion, or physical work after becoming mentally tired. The time for hard physical exercise is not near the close of a day of such intense mental strain that the tired nervous system seems to rebel at lashing the muscles into action. That is certainly a time to rest or recreate in any way which is not approached as an unpleasant duty. Conversely, the time for hard study is not at the close of a day of exhausting physical work. Whether mental work may safely succeed physical activity, or vice versa, usually depends upon whether one finds it possible to take up the change of work without constantly goading oneself against a feeling of exhaustion. Ambitious workers will not meet with the one great danger in this advice; namely, that of confusing real exhaustion and mere laziness.

460. Sleep. - Probably more important than any other rules of hygiene are those concerning sleep. Loss of sleep 
does far more damage than starvation. Men have voluntarily fasted 30, 40 and even 50 days, and afterward quickly regained their normal weight and health; but it is certain that a normal healthy man could not go without sleep so long. Sleep is a period of rest, repair, and growth, and is especially important for growing children. Much of the physical harm to children in crowded tenements is due to a combination of late retiring to rest, uncomfortable bedroom, disturbed sleep, and early rising. Many a child in the poorer regions of great cities is anæmic, nervous, haggard in face, listless in school, sluggish at play; and the real trouble may be that he is not getting enough good sound sleep. This is likewise true of some adults, who should average seven to nine hours of sleep in twenty-four. As far as possible, this should be taken during the quietest hours (10 P.M. to 6 A.M.) when there are the fewest external stimuli tending to cause awakening.

\section{MUSCULAR SYSTEM}

461. Exercise for Health. - Those whose business or pleasure leads them to sedentary habits of life need to consider most seriously the question of physical exercise. The scientific reason for exercise is to be found in the coördination of muscular activity with all the other organs of the body, rather than in the development of the muscular system itself. In short, most people should exercise primarily in order to get reactions of the digestive, circulatory, respiratory, and nervous organs, while secondarily and incidentally they may develop their muscular systems. However, it is very doubtful in the opinion of many qualified physiologists whether excessive development of the muscular system is best for the general health of those who are not professional athletes or laborers. The well-known aphorism, "Mens sana in corpore sano" (a sound mind in a sound body), means that giant intellects ought to be located in healthy 
bodies in which all the functions are properly coördinated, and it should not be understood to mean that only one with the muscles of a champion athlete can hope to do great intellectual work. On the contrary, it is a remarkable fact that some of the greatest work in literature, art, and science has been accomplished by men and women who suffered from lifelong physical weakness. In such examples there is hope for all who are physically weak by nature. Athletic constitutions commonly originate congenitally and not in gymnasia.

The average man and woman, then, should exercise for health, deriving it from renewed activity of the organs that are closely coördinated with muscles, and from rest and recreation for the brain. This reference to the association of exercise and recreation is important, for we certainly derive most benefit from exercise which is at the same time pleasurable and recreative. Herein is one great advantage of many forms of outdoor exercise over gymnasium work.

462. Excessive exercise is not beneficial when it leads to exhaustion; and severe over-strain may lead to injury of the heart, blood-vessels, or lungs. Athletic enthusiasts often answer this medical criticism against certain extra-strenuous games, such as football and rowing, by claiming that the moral gain from severe athletic contests overbalances the recognized danger of great physical harm from excessive exhaustion. That there is moral gain worth while in compelling tired muscles to obey to the point of exhaustion is extremely doubtful; and those who are fond of quoting that "Waterloo was won on the playing-fields of Rugby" should re-read history and note the victories in peace, and even in battles scarcely less strenuous than Waterloo, and by men whose moral fiber was certainly not directly traceable to previous athletic training on any school-playground. Moral qualities which make men great are inherent, not originated by any one form of activity; and hence we are not justified 
in excusing dangerously excessive exercise because one or more famous generals or other great men happened to play football, or some other game, when they were boys. There is no scientifically proved moral effect of physical strain which in the slightest degree militates against the hygienic rule that exercise for health should never be carried to extreme exhaustion. The world would have far more healthy and efficient men if this rule for muscular activity were more often applied both in work and in play.

\section{PHYSIOLOGICAL EFFECTS OF STIMULANTS AND NARCOTICS *}

463. Introduction. - Man has long been more or less accustomed to take into the body certain substances (alcohol, tobacco, tea, coffee, certain drugs, etc.) which are not properly classed with the ordinary food materials, for their value as sources of energy and materials for repair is so slight as to be negligible. Ail these substances are conveniently grouped under the heading "Stimulants and Narcotics," which indicates that their action in the human body is either to excite or stimulate greater activity of certain organs, or to reduce their activity and tend to produce stupor or sleep (narcosis). Both the exciting action of stimulants and the quieting effect of narcotics are pleasurable to most people, and it is solely for this peculiar pleasure that mankind has adopted the habit of using the various substances which afford stimulating and narcotic effects.

464. Are Stimulants and Narcotics Needed? - It is interesting to note that no animal naturally makes use of any of the stimulants and narcotics; and hence it is often argued that the human species ought to be natural and avoid them. However, this is a rather weak argument, for

* To Teachers: See "Teachers' Manual" for notes concerning the use of this section. 
in many other ways man has ceased to be natural (e.g., cooking food is certainly unnatural for animals), and with advantage to himself. Clearly the use of stimulants and narcotics must be judged by their good or bad effects upon men, and not rejected simply because animals do not use them. The experience of animals indicates that man does not absolutely need stimulants and narcotics; but it has no bearing whatever on the question of whether man may or may not profitably make use of such substances. This will be discussed at various places in the following sections. The general conclusion is that under some conditions there is need of stimulation, or of quieting organs by narcotics ; but that, on the whole, stimulants and narcotics are easily used in excess.

465. Examples of Stimulants and Narcotics. - Alcohol in small quantities is a stimulant which increases the activities of many organs. It is well known that alcohol in large quantities produces a narcotic effect, leading to the complete stupor of intoxication. Opium is well known as a powerful narcotic which quiets active organs, and in large doses leads to a fatal sleep. Most users of tobacco in any form claim that it has a soothing effect, i.e., is a narcotic. Tea and coffee contain substances which are usually stimulating to most persons. Many drugs used by physicians (e.g., strychnine, nitro-glycerine) are powerful stimulants, and are given in exceedingly small quantities. When powerful narcotics are demanded as relievers of pain, physicians commonly use opium and its products (laudanum, morphine).

466. Alcohol and Common Alcoholic Fluids. - The formation of alcohol from sugar has already been described in $\$ \S 250,251$, which deal with fermentation caused by the yeastplant. Practically any natural substance which contains starch or sugar may undergo fermentation. Thus juices expressed from grapes, apples, and other fruits, and the carbohydrates in grains of rye, corn, and barley, and in 
potatoes, are commonly used in producing alcoholic liquors, of which the chief varieties are mentioned below.

Malt liquors (beer, ale, and porter) are made from malt, which is generally sprouted barley grains. This is ground in water, and allowed to ferment. Hops are added to give a bitter flavor. Such a fermented liquor consists chiefly of water, 1 to 8 per cent alcohol, and small quantities of other substances derived from the grains used.

Wines are juices of grapes which have fermented and produced 6 to 12 per cent of alcohol. Some wines are stronger because brandy or strong alcohol has been added when bottling.

Distilled liquors (whisky, gin, brandy, rum) contain 30 to 50 per cent alcohol, and are made thus strong by distilling the fermented fluids (water with rye, corn, oats, or potatoes for gin and whisky; molasses in water for rum; wines for brandy). Various flavoring and coloring materials are added to the distilled liquors. They differ essentially only in color, flavor, and proportions of alcohol. Some of the substances used to color and flavor are harmful, but are used in such small quantities that the alcohol is chiefly responsible for the physiological injury done by the distilled liquors.

Alcohol in a more or less pure state can be made by redistilling and otherwise purifying any fluid in which fermentation has occurred. Since distilled liquors are nearly half alcohol, it is easily obtained from them. The grain alcohol of commerce is usually from 91 to 95 per cent pure; i.e., it contains 5 to 9 per cent of water. A special quality for scientific purposes is about 99 per cent pure, and is very expensive to make.

Commercial alcohol is usually called "grain-alcohol," or, in chemical terms, ethyl alcohol. Wood-alcohol, or methyl alcohol, is commonly made from wood. In all the discussions in this lesson, the word "alcohol" is used to mean grain or ethyl alcohol, for this is the characteristic constituent of 
the alcoholic liquors whose physiological effects are under consideration. We shall see that the effect of alcoholic liquors is largely in proportion to the amount of contained alcohol, and so it is justifiable and convenient to deal directly with the effects of alcohol and neglect, temporarily, the minor fact that alcohol, as commonly taken in wine, beer, whisky, etc., is diluted with water and variously flavored.

467. Is Alcohol a Poison? - In popular usage, the word "poison" is associated with such powerful substances as arsenic, strychnine, snake-venom, and others which, when introduced into the human body, produce marked and even fatal disturbances. In scientific usage the term is applied to many substances which cause demonstrable disturbance of any function of the body. There is no substance which is always a poison, for even strychnine and ricin may be diluted so as to produce no noticeable disturbance. A cup of coffee is not poisonous to an average adult, and yet it contains a greatly diluted dose of caffein, which in large amounts is a poison. Tea, coffee, ginger, pepper, and many other things taken with food contain small quantities of substances which in large amounts are poisons. Even common salt in very large quantities has proved a fatal poison. Evidently the word "poison" has a relative significance, and involves the quantity. In general, we apply it only to substances which in very limited quantity are harmful. The question, then, "Is alcohol a poison?" can be answered only by reference to the amount of alcohol and to the constitution of the individual who drinks it. That alcohol in large and intoxicating doses has proved fatally poisonous is well known, and that it commonly produces profound disturbances of various organs when used excessively and habitually is also common knowledge; but whether alcohol in very small quantities is a poison, is a difficult scientific question which only physiologists can answer by experimental studies made with animals and men. 
A liter (nearly a quart) of whisky, gin, or rum given in one dose would kill any animal weighing 67 kilograms.

(How many pounds?) Evidently alcohol in large quantities is a poison. However, even a victim of the alcoholic habit would not drink a quart of whisky within a short time. The question is whether in the smaller amounts, such as are commonly used by drinkers, alcohol should be classed as a poison. The next four sections discuss this question with reference to the organs of digestion, circulation, and respiration, and the nervous system; and it is pointed out that it is impossible to show by scientific methods that ordinary small amounts of alcohol produce effects comparable to those of the substances which druggists label " poison." Hence it must be concluded that, so far as we now know, alcohol in small amounts is not harmful enough to warrant labeling it "poison." We do not so label common salt, although a strong solution taken into the stomach has caused death; and while coffee contains a dangerous poison, a pot of the beverage should not be labeled as dangerous. Most people would be misled by such a label, for they know well that in ordinary quantities common salt and coffee produce no symptoms of poisoning. Likewise, in very limited quantity alcohol is not a poison in the sense that we understand various drugs to be poisons.

468. Effect of Alcoholic Liquors on Digestion. - When taken into the stomach, alcoholic fluids cause a marked increase in the flow of gastric juice from the glands of the stomach wall. There is also an increase in the amount of the constituents of gastric juice: namely, pepsin and hydrochloric acid. Wine, alcohol, beer, whisky, brandy, and wines - all stimulate the gastric glands in this way. The alcohol quickly leaves the stomach, being absorbed into the blood, leaving the gastric juice in concentrated form. Whether such an effect of alcoholic fluids upon gastric secretion is directly harmful or not seems to depend upon the 
amount of alcohol present. Thus strong beverages, like brandy, gin, and whisky, with 40 to 50 per cent of aleohol, retard the digestive action of pepsin on proteids; but this effect depends upon the amount of alcohol taken, the amount of food in the stomach, the strength of the gastric juice, and the health of individuals. Hence it is impossible to lay down any general rule as to the minimum quantity of alcohol which will harmfully affect digestion in the stomach. It is certain, however, that intoxicating doses do impede gastric digestion even in healthy individuals.

But it should be emphatically stated that the effect of alcoholic drinks upon digestion is not solely due to the amount of alcohol in them. Thus sherry wine with 20 per cent of alcohol retards digestion much more than does an equal quantity of 20 per cent pure alcohol. Large amounts of claret wines have a similar, but less, effect. The same is true of ale, beer, and other malt liquors. When any of the wines and malt liquors are used freely with meals, there is likely to be a considerable retardation of the digestive processes.

Concerning the effect of moderate amounts of alcoholic fluids upon gastric digestion, it appears from experiments made by competent investigators that the greater secretion of gastric juice is counterbalanced by the retarding effect; and hence, as a rule, there is no reason for or against using small amounts with meals, so far as the effect on gastric digestion is concerned. But we shall see later $(\$ \$ 471,473)$ that alcohol has much more decided effects upon other organs which help us to decide for or against its use.

469. Effect of Alcohol on Blood-System. - Small quantities, as used by physicians in cases of great depression of the heart, stimulate that organ reflexly through the nervous system. In large and intoxicating quantities alcohol is a direct and powerful depressant, weakening the beat, distending the cavities, and diminishing the pumping of blood. 
Herein is the scientific reason for the remark in $\S 353$ concerning the use of alcoholic fluids for snake-bites.

It is well known that alcohol in small amount causes flushing of the face and a sense of heat over the skin. This is due to the dilation of blood-vessels.

The combined effect of large quantities of alcohol on heart and blood-vessels is to lower the pressure of the blood.

Whether these effects are harmful or not depends upon conditions, especially of organs other than the blood-system. It is a significant fact that the modern physician is careful in prescribing alcohol, even in moderate doses, when he desires stimulating effects on the heart and blood-vessels; for the effects on other organs may more than counterbalance any possible good done to the organs of circulation.

470. Alcohol and Respiration. - For a short time after drinking alcoholic fluids there is an increase in the rate of respiration. This is probably due to an increased loss of heat from the dilated blood-vessels of the skin ( $\$ 447)$. In other words, the respiratory organs must work faster in order to supply oxygen for the increased internal oxidation needed to supply heat in place of that lost. Such a chain of events leads many physicians to question seriously whether good or harm will come from a dose of alcohol, as in pneumonia, with weakened heart and lungs already congested with blood. At any rate, it is exceedingly doubtful whether in conditions of health any useful purpose is served by increasing respiration by means of alcohol.

471. Alcohol and Nervous Organs. - The general influence of large amounts of alcohol on the nervous system is well known to all who have observed the actions of drunken men.

Large quantities of alcohol lessen all mental activities. Careful experiments have shown that even a pint of wine diminishes acuteness of smell and touch and interferes with the power of the eye to estimate measurements. Psychologists have failed to prove that alcohol increases the quantity 
and vigor of mental operations; on the contrary even small doses tend to lessen reasoning power. Larger quantities affect the power of attention, judgment, and reason, render the senses less acute, and exert an anæsthetic action which leads to the sleep characteristic of intoxication. Study of many such conditions leads eminent experts on drugs to the opinion that, even in moderate quantities, alcohol tends to have a sedative or narcotic action on the brain. In connection with this statement, may be cited the undoubted fact that alcohol regularly used during the day's work diminishes the amount and quality of work done. This has been experimentally proved by tests with type-setters and others whose work is of such a nature that it is easy to compute both speed and accuracy. Those who command armies and large groups of men engaged in physical labor agree that the use of alcohol during work decreases effectiveness. This is probably because of the sedative action above mentioned.

It has not yet been possible for investigators to collect and collate the facts as to the effect of small amounts of alcoholic liquors upon large numbers of brain-workers (lawyers, teachers, clergymen, business men, physicians), for the reason that many will not or cannot answer questions concerning their own experience with alcoholic drinks. One list of 892 brain-workers in the United States showed 167 total abstainers, 579 occasional drinkers, and 146 moderate drinkers. A large number of the moderate drinkers expressed the opinion that the use of alcoholic drinks gives bad results as stimulants to mental work, and many also stated that they used alcohol from habit, and with no expectation of being enabled to do more or better mental work.

In spite of the fact that we cannot say definitely how little alcohol will seriously interfere with the normal functions of the nervous system, it is clear that the brain-worker acts most wisely by avoiding alcohol during his mental work. Whether he will gain or not by avoiding alcohol altogether 
cannot yet be demonstrated, because the possible accumulative effects of small doses of alcohol are still unknown to science. 472. Nutritive Value of Alcohol. - "Is alcohol a food?" The answer depends upon what we understand by food. It is not food in the sense that bread and meat are foods, for it cannot support life. It lacks the nitrogen and necessary mineral elements for growth and repair. But it may, in small quantities, take the place of foods used for fuel or energy, for some is oxidized in the human body.

However, it is of little moment that alcohol has a slight food value, for against it there are great objections as follows: (1) Only small amounts can be used as food, and it is easy to overestimate the amount which is safe. (2) There is a peculiar tendency to excessive and habitual use. (3) Its action as a harmful drug may overbalance its value as food. (4) It is a very expensive food as compared with carbohydrates and fats, which can supply equivalent energy.

Such grave objections to alcohol in any form make it necessary to regard its food value as of little importance. One of the best of advanced books on physiology well summarizes the whole matter as follows: "Only in very exceptional cases can alcohol have any practical importance as a nutriment. It is especially in the case of acute diseases accompanied by diminished digestive power that alcohol seems to serve as a valuable nutriment."

After all, no one regularly uses alcohol as food, but rather for its peculiar taste and stimulating effect. The use or disuse of alcohol must depend upon the answer to the question, "Is the stimulating effect of alcohol injurious?" Except in small quantities it certainly is an injurious stimulant; and no one can safely estimate the quantity which may not lead to accumulated effects, or to habits of excess.

473. Disease Effects of Alcohol. - Many organs are known to become diseased as the result of long-continued excessive use of alcoholic drinks. Liver, kidneys, heart, 
blood-vessels, and nervous organs are frequently involved in disease changes. All medical men recognize that alcoholic intemperance leads to an immense amount of sickness. A large number of deaths are due to the diseases known as chronic alcoholism and delirium tremens; and many cases of Bright's disease of the kidneys, paralysis, pneumonia, tuberculosis, and other diseases are believed by eminent physicians to have been hastened to a fatal issue by the previous use of alcohol.

It should be noted that no reputable physician claims that even excessive use of alcohol will always lead to diseased conditions. There are exceptional individuals who are almost constantly intoxicated, and yet show no external evidences of diseased organs. However, no sane person who has learned of the great liability of excessive drinkers to diseases will care to take his chances of being one of the few who appear to escape the most serious consequences. Moreover, it should be noted that in recent years there have been found many cases of diseases in " moderate drinkers" which are probably due to long use of alcoholic liquors.

It is well known that the excessive use of alcohol leads to obesity or storing of fat. This is most dangerous when in the muscles of the heart. Gout is often, not always, caused by alcoholic liquors.

474. "Pure" Alcoholic Beverages. - Manufacturers of alcoholic liquors often advertise that their products have been "purified" so that they have no poisonous action. This is an absolutely false claim, for scientific studies have shown that while the so-called "impurities" in alcoholic drinks are poisonous, they are present in such small amounts that their effect is slight, and that the poisonous effect of alcoholic liquors is chiefly due to the alcohol they contain. Even absinthe and other highly flavored French liqueurs, containing extracts of wormwood, anise, and other aromatic herbs, certainly owe most of their decidedly poisonous action 
to their large amount of alcohol (50 to 80 per cent). Alcohol in large quantities has been demonstrated to be poisonous enough to account for most of the physiological evils ascribed to alcoholic drinks. So long as people will drink alcoholic fluids, there should be "pure food " laws aimed at making them as free from harmful substances as possible; but no one should be deceived by claims that a given brand of liquor is harmless. Whenever alcohol is present in considerable amount, there is a substance which, in quantity varying with individuals and conditions, is certain to be harmful in its effect upon the essential life-processes. Even the "best" alcoholic beverages have the power to do this.

The common opinion that the cheap artificial whisky sold in some saloons for three cents a glass is especially injurious to health as compared with " high grade" natural whisky made from corn and rye is not supported by chemical analysis. This cheap whisky consists of 30 to 50 per cent alcohol with caramel, sugar, and flavoring essences. Its harmfulness depends chiefly upon the alcohol contained. Perhaps the chief reason why such cheap liquors appear harmful is that the low price leads to the use of more alcohol. Certain it is, however, that the chief danger in cheap whisky, and in all whisky, lies in the 30 to 50 per cent of alcohol which it contains.

475. Alcoholic "Temperance Drinks."-Very many people who hold strictly to temperance principles are unaware that many fluids sold at drug-stores contain large amounts of alcohol. In general, all "tonics," " bitters," " malt-extracts," " celery compounds," and other similar fluids advertised as givers of strength, vigor, etc., contain alcohol. Certain much-advertised medicines called "sarsaparilla" contain at least 25 per cent of alcohol. Many "bitters" and "tonics" contain from 15 to 45 per cent alcohol. Some of these are advertised as containing no alcohol, or as "temperance" medicines. According to the labels on the bottles, 
doses as large as a wineglassful four times daily are sometimes advised. This means a large amount of alcohol.

Root-beers, ginger ale, and fermented milk contain very small amounts of alcohol, usually less than one per cent. So-called "sweet-cider" sold by all dealers may have more alcohol than the average beer, and frequently contains 0.2 to 3.5 per cent. "Hard" or fermented cider contains 4 to 8 per cent of alcohol, and therefore compares with mild wines and strong beer.

476. Alcohol as Medicine. - Concerning the value of alcohol in treating diseases, great authorities on medicine do not yet agree. There are many eminent physicians who never prescribe it, but prefer to use drugs whose stimulating action is more definite and certain than that of alcohol. Other equally eminent doctors hold that alcohol is of great value in certain acute diseases where there is a tendency toward general and heart weakness.

477. Alcohol and Growth. - It is universally admitted by physiologists that all alcoholic drinks are deleterious to growing individuals, and this means the first eighteen or twenty years of life. It is absolutely pernicious to young children. And in addition to its direct physiological injury to young people, there is the oft-demonstrated tendency to excessive use. The vast majority of habitual drinkers of alcholic liquors begin in early life.

478. Summary of Effects of Alcohol. - Professor Atwater, the famous chemist, who contributed much to our knowledge of foods and their uses, wrote that in his personal opinion "people in health, and especially young people, act most wisely in abstaining from alcoholic beverages."

A committee of five prominent American physiologists has thus summarized the facts regarding the use of alcohol:

(1) While in moderate quantities beer and wine may be in a certain sense a food, they are a very imperfect and expensive kind of food, and are seldom used for food purposes; 
(2) they are not needed by young and healthy persons, and are dangerous to them in so far as they tend to create a habit; (3) in certain cases of disease and weakness they are useful in quantities to be prescribed by physicians ; (4) when taken habitually, it should be only at meals; and, as a rule, only with the last meal of the day, or soon after it; (5) alcoholic drinks of all kinds are worse than useless to prevent fatigue or the effects of cold, although they may at rare times be useful as restoratives; (6) they are almost always a useless expense ; (7) their use in excess is the cause of much disease, suffering, and poverty, and of many crimes; excessive use is sometimes the result, rather than the cause, of disease.

479. Effects of Tobacco. - Concerning the effect of tobacco in its various forms upon health, there has been much discussion. As is well known, the stem and leaves of the tobacco plant contain a poisonous substance known as nicotine, which, in concentrated doses, quickly kills small animals. However, this proves nothing regarding the effect of smoking tobacco, or of the disgusting habit of chewing it, which is now almost unknown among the better classes of people; for in both of these ways of using tobacco the nicotine is exceedingly diluted, as is the poison found in tea and coffee. The result is that the effects of tobacco are not marked, and so even physicians are not always certain as to its influence upon their patients. The best established knowledge we now have is that indigestion, irritation of the respiratory organs, and heart and nervous disturbance may in some people result from the use of tobacco, while others show no apparent effect.

All this refers to healthy adult men, for all medical authorities agree that tobacco is always harmful to growing boys, and interferes with their physical and mental development. All schoolboys know how rigidly most athletic trainers forbid the use of tobacco by those in training. 
The direct effect of tobacco is narcotic, and many smokers say that it "soothes their nerves." It is very doubtful whether the nerves would need "soothing" if the tobacco habit had not been established. Opium also has such an effect; but it is well known that the craving for the soothing is the result of the established habit, and those who never used opium do not need to be soothed by that drug. Likewise those who have never acquired the tobacco habit appear to have no need for its narcotic or soothing effect. The difference between the opium and tobacco habits is in degree, not in kind. Both create a demand or craving for their peculiar narcotic effects.

Some few people have their eyes seriously affected by even small amounts of tobacco, while many find that tobacco smoke irritates the eye-membranes and causes some blurring of vision.

The whole physiological truth about tobacco so far as now known is that: (1) no one needs it except to satisfy an established habit; (2) many adults are injured by it, and no one knows just how much will do harm to a particular person; (3) some adults are apparently not harmed by limited use ; (4) it is decidedly injurious to growing boys ; (5) those who avoid establishing the habit in youth do not as a rule care to learn later, for there are no physiological reasons why any one should deliberately set out to learn the use of tobacco in any form.

480. Effects of Tea, Coffee, and Cocoa. - The first two are most important because they are so widely used as beverages. It is now well known to physicians that many people drink too much tea and coffee, and that temperance is needed in use of these beverages no less than with alcoholic drinks. Their stimulating effect is due to the presence of a powerful drug (caffein), which has a stimulating action on the nervous system. Nervousness, insomnia, headache, and indigestion are common symptoms arising from exces- 
sive use of tea or coffee; and disturbances of other organs may follow. One who uses even small amounts of tea and coffee and who does not "feel well" for no apparent reason, should try the effect of complete abstaining for a few days occasionally. By so experimenting with themselves, many people have learned that tea and coffee harm them. Other people are certainly benefited by a limited use of these beverages.

Tea and coffee should never be given to young children. They may be easily harmed by such stimulants.

Cocoa and chocolate are made from the seeds of the cacaoor chocolate-tree, a native of tropical America. The seeds are rich with 50 per cent of fat, some of which is extracted in the process of making commercial cocoa or chocolate. Both of these contain a substance similar to the caffein of tea and coffee, but milder in its effects. However, one who has difficulty from the use of tea and coffee will do well to experiment with himself and thus learn whether chocolate or cocoa produces marked effects.

481. Effect of Narcotic Drugs. - We are here concerned with the effect of such drugs as opium, morphine, cocaine, laudanum, chloroform, chloral hydrate, and various patent or secret preparations, all of which are habitually used by some people. In most cases, such drugs are used first to narcotize the nerves and thus relieve pain, and their frequent use finally becomes a habit even more powerful than the alcoholic habit. It is unnecessary to go into an extensive discussion of narcotic drugs, for most intelligent people now understand that only on a physician's advice is it safe to use any drug to relieve pain; and also that no drug should be used so frequently as to offer the grave risk of establishing a habit.

It should be noted that the narcotic drugs do not cure diseases. For example, patent headache powders and pills simply dull the sensory nerves. The headache may be due to a disordered stomach, eye-strain, constipation, or other 
causes; and hence the narcotics give only temporary relief. Obviously, it is wiser to consult a doctor, who may be able to find the cause and prescribe treatment.

The remarks made above concerning narcotic drugs might well be applied to habitual taking of any kind of medicine without a physician's advice. An immense amount of harm is done by the thousands of patent medicines.

\section{BACTERIOLOGY APPLIED TO HUMAN HEALTH}

482. Bacteriology and Health. - The principles of bacteriology which have been discovered within the past thirty years are not only of interest in connection with the cause and cure of many diseases, as stated in $\S 259$, but are also of much greater importance in that they are capable of being applied so as to maintain health. The relation of bacteriology to hygiene is already a vast subject, and we can take time for only a few of the most important points. We shall consider (1) how to avoid the disease germs which are widespread, and (2) how to prevent the wide distribution of disease germs.

483. Avoiding Disease Germs. - The methods of avoiding the introduction of disease germs into one's body depend upon the nature of the disease and the causative organism. As a rule, the germs are introduced into the alimentary canal with food and water, into the respiratory organs, into the blood by insect bites, or into wounds.

484. Infection through Alimentary Canal. - Typhoid fever and Asiatic cholera are good examples of intestinal diseases caused by germs which are spread by excreta. Imperfect sewerage and insects may lead to contamination of various foods (milk, vegetables, fruits, raw oysters) and drinking water. In places where these diseases are common the only safety for the individual is in the use of cooked foods and served hot or at least guarded from contamination by flies, 
dirty human hands, and dust. Salads and other vegetables not cooked should at least be carefully washed in water which has been boiled.

If there is reason to doubt the purity of drinking water, heat it to the boiling temperature and pour into sterile closed containers (such as earthen jugs). Longer boiling is unnecessary and undesirable. These rules will protect individuals. How to check the spread of intestinal diseases and thus protect the public is a problem of sewerage, and of insect control (see $\$ 330$ ).

Intestinal diseases of children, especially in warm weather ("summer complaint"), are largely due to stale milk. If very clean, fresh milk is not available, then the milk should be pasteurized ( $\$ 256)$. Thoroughly wash and sterilize ( $\$ 256)$ daily all milk-bottles.

It should be remembered that some bacteria of decay may develop in foods and cause unhealthful conditions. This and the fact that some disease germs will multiply in milk and other foods should lead to caution. Foods showing signs of decomposition should, of course, be rejected; but more important is the protection of foods from dust, insects, and growth of bacteria. Remembering that bacteria do not flourish when very cold, one thinks of the ice-box as best for preventing growth; but rarely is an ice-box cold enough to preserve highly decomposable foods (such as soup, gelatin, milk) for many days. Best of all methods is heating to the boiling point daily the foods to be preserved temporarily, and also keep them in an ice-box from day to day. Thus disease germs, if present, will be killed; and ordinary decay, which might produce ptomaines (poisons in foods) is prevented.

485. Infection through Respiratory Organs. - Tuberculosis is a good example. Tubercle bacilli may get into the lungs (1) by close contact of healthy with tuberculous persons (e.g., by kissing, and using common drinking cups or 
towels); (2) from dust arising from the dried sputum of a tuberculous victim; (3) possibly from milk of tuberculous cows; (4) by flies which carry the bacteria from sputum. The first line of danger is easily avoided. Especially should those suffering from tuberculosis or whose friends are afflicted get from the Society for the Prevention of Tuberculosis, in New York City, the free circulars which give rules for preventing the spread of the disease, and follow them carefully. Doubtful milk should always be pasteurized, or boiled. The danger from dust is impossible of avoidance by individuals so long as the spitting nuisance continues. Fortunately, drying and sunlight kill most of the germs out of doors, and good ventilation and cleaning reduce the danger indoors.

So far as personal hygiene is concerned, the most important preventive measure against pulmonary tuberculosis is keeping in general good health by good food, outdoor exercise, fresh air, good sleep, and avoiding colds. So long as the body is in good condition there is little danger of the bacteria getting a chance to flourish in the lungs; and even if the disease has begun to develop, a cure may be effected by careful attention to the rules of hygiene which physicians and special books and pamphlets prescribe.

Many children's diseases (whooping cough, measles, mumps, chicken-pox, etc.) are believed to be contracted through the respiratory organs. The best protection is (1). keeping away from cases of such diseases, and (2) avoiding the use of pencils, toys, towels, handkerchiefs, drinking cups, etc., which may have been in contact with the mouths of other children, who may carry the germs although apparently well. These and the biological rules given in $\S 488$ will prevent most epidemics of children's diseases.

486. Infection by Insects. - The relations of mosquitoes and flies to disease have been stated in $\$ \S 329,330$. Constant warfare should be waged against these insects. 
487. Infection in Wounds. - Proper healing without inflammation depends upon keeping out bacteria. Wash all cuts with weak antiseptics obtained from druggists (e.g., carbolic acid one part to 40 of water, or mercuric chloride tablets in water as directed on bottle. Listerine for slight cuts). Deep cuts or punctures should be dressed by a surgeon, for bacteria may have been pushed in too deep to be reached without special instruments, and there is danger of tetanus or septicæmia (blood poisoning). Protect wounds with sterilized cotton and medicated gauze. Adhesive tape should not be put on so closely as to keep out the air; and collodion solution is of questionable value for any except shallow and well-cleaned cuts. Always consult a doctor at once if any marked inflammation develops in any wound. Delay in such cases often proves serious.

488. Preventing Distribution of Disease Germs. - This may be accomplished (1) by scientifically dealing with cases of germ diseases, which we discuss below under " the bacteriology of sick-rooms" ; and (2) by public sanitary control of food-supplies, water, sewage, quarantines, and other matters of public hygiene.

489. Bacteriology of Sick-rooms. - Since it often happens, especially with children, that germ diseases must be treated at home, it is important that every citizen should understand the scientific principles which doctors prescribe. All the rules depend upon the fact that micro-organisms are numerous in the secretions and excretions of patients, and must be destroyed by the methods of bacteriology.

(1) Isolation. - As soon as a person becomes ill, he should remain in one room until the doctor is sure of the diagnosis. If it proves to be a contagious disease, complete isolation will be required by the health officers. Even in cases of diseases not commonly isolated it is best that only the nurse come into contact with the patient, for there is always possible danger of spreading the germs to healthy people. 
In the cases of all germ diseases, the doorway to the patient's room should be kept covered with a damp sheet to prevent as far as possible the exit of dust particles.

(2) Disinfection. - Remember that all articles in contact with a patient may bear the germs. To reduce the amount of disinfection which will be necessary, remove all useless carpets, curtains, and furniture as soon as the disease is diagnosed; and thereafter remove nothing from the room except under the strictest germicidal precautions. Hence all clothing, bedding, handkerchiefs, etc., should be placed in a tight receptacle, such as a wash-boiler, covered with water, and boiled for twenty minutes before they can safely be sent to a laundry or washed in tubs at home. The washboiler should be kept in the patient's room, and not opened until after boiling. Its outside should be wiped with a cloth wet with strong washing-powder solution, or other germicide, if necessary to take it to another room for boiling.

Spoons, dishes, tumblers, etc., should be placed in a bucket of water (preferably hot and containing much washingpowder) before removing from the patient's room, and then boiled for 20 to 30 minutes. Food left by the patient should also be boiled or burned. Playthings used by children should be such as can be washed in germicide solutions, boiled, or burned after convalescence. A doll or a "teddy bear," if not burned, might be a very dangerous carrier of germs. Books or magazines should be of little value, never from libraries, and finally carried (in a closed bucket) to a stove or furnace.

The one who attends the patient should thoroughly wash her hands frequently with hot water and strong soap, or germicide solutions, and before leaving the room. Neglect of this simple precaution has often spread germ diseases. The habit of expert nurses of wearing washable dresses should be imitated as far as possible. 
All excreta should be treated with strong chloride of lime or other disinfectants sold by the druggists.

No sweeping should be allowed in a sick-room. Dust should be wiped up with a damp cloth which should afterward be washed out in boiling water with washing-powder.

After recovery of the patient, fumigate (disinfect by fumes or gas) the room with formaldehyde $(\$ 256, f)$, leaving in the room all furniture, pictures, matresses, books, etc., and spread the articles, open the drawers, wardrobe, etc., so as to allow free circulation of the gas. Do not remove rugs, curtains, clothing or any article from the room until after thorough disinfection.

These precautions may seem extremely detailed, but bacteriologists have shown that they are necessary in order to safeguard against distribution of dangerous germs. It is the duty of every citizen to aid in popularizing and putting into practice knowledge by which some of the most dangerous diseases may be made extremely rare in occurrence.

490. Public Hygiene. - Since a large number of people are ignorant of scientific principles or have no regard for the health of other people, it has become necessary to institute public sanitary control of foods, water, sewage, quarantines, etc., in all cities and to a certain extent in the country at large. Thus the official representatives of the government are charged with the duty of protecting citizens against disease in cases where the individuals cannot exert control. For example, a city board of health can force dealers to sell only clean milk, but without such public control the individual citizen must accept the impure milk which the dealer offers for sale. Likewise, under public sanitary control, meats and other foods can be inspected, and made to meet the requirements of the law; water can be obtained from the purest possible sources; sewage systems can be arranged to avoid danger from disease germs; streets and other public places kept clean; quarantine of dangerous diseases 
established; vaccination required; spitting on walks prohibited; and still other measures in the interest of public health put into legal operation. Such is the field of public hygiene and the work of health officials, who aim to apply biological principles and ${ }^{7}$ protect the citizens in numerous cases where individuals ea anot protect themselves. Every citizen should get acquairted with the health laws in his locality and then coöperate with the officials whose duty it is to get the laws enforced. 


\section{PART V \\ CHAPTER XIX \\ EVOLUTION AND HEREDITY OF ANIMALS AND PLANTS}

491. Definition of Evolution. - Many times in the preceding chapters we have had occasion to point out that similarity of structures suggests relationship of animals and plants, and that all organisms have undergone more or less change in adaptation to the environments in which they live. A remarkable set of examples are the adaptations of roots, stems, leaves, flowers, and fruits described in Chapter VIII. In all these cases the similarity of structure suggests that the adapted organs (roots, stems, etc.) have been derived from the typical forms of these organs as seen in other plants. For example, fleshy roots have developed from plants with ordinary tap-roots, irregular flowers adapted to insects probably descended from regular flowers; and so in all special adaptations of plants close examination suggests the line of modificat on in making special adaptations and points out the relationship.

As an example on the animal side, it has been shown that the various forms of feet of hoofed mammals appear to have been derived from ancestors with five toes ( $\$ 361)$, and that general similarity in structure of all parts of their bodies points to relationship between all the ungulates. Such facts of similarity which suggest relationship are fundamental to the study of Organic Evolution, by which is meant the theory 
that all organisms which are now on the earth have been derived from and consequently are directly related to organisms which previously existed in the past history of the earth; and that similarities or homologies of structure are due to common descent from earlier forms. To give a concrete illustration: the similarities of existing vertebrates are believed to mean that they have descended from an ancient type of animal which had the general plan of body ( $\$ 344$ ) now common to all existing vertebrates; the similarity of existing birds that they are the direct descendants of an ancient type; and so on for all groups of animals. Hence, all vertebrates are more or less closely related to each other, the degree of relationship being indicated by the closeness of similarity (e.g., a fish and a mammal distantly related; a dog and a wolf closely).

492. History of Evolution. - This idea that animals have been derived from other animals is a very old one dating back to some philosophers among the ancient Greeks, but it was not developed in a thoroughly scientific form before the nineteenth century. In 1859 Charles Darwin, of England, published a book entitled "The Origin of Species," in which he marshalled such a convincing array of facts to prove the evolution of animals and plants that the theory was soon accepted by most scientific men. Following Darwin's suggestions, hundreds of scientific men began to investigate all the facts which seemed to be connected with evolution, and the result is that the theory has been universally accepted in the scientific world as the only explanation for the remarkable similarity of organisms which has so often attracted our attention in previous chapters. To-day there is no famous living botanist or zoölogist who does not believe in the theory of evolution; that is, that species of animals and plants have originated by descent with modification of pre-existing forms. The entire scheme of classification of animals and plants which is now in use by all biologists is based on the idea of 
relationship through descent. Thus we classify together the fishes, amphibians, reptiles, birds, and mammals because the general plan of structure is so similar in all these vertebrates as to suggest that they have descended from some common ancestors or primitive vertebrates which lived in the fardistant ages with which geology deals.

493. Evidences of Evolution. - In this book we can consider only briefly the lines of evidence which led Darwin and his followers to accept the idea of evolution as a true statement of the facts observed in study of animals and plants. These evidences will be outlined under the following paragraphs: Structural Evidences; Embryological Evidences; Geological Evidences; Distribution Evidences; and Experimental Evidences.

494. Structural Evidences of Evolution. - In almost every lesson of this book we have had occasion to refer to similarity of structures in organisms. Such similarity is the most remarkable fact in biology; and the only scientific explanation is that organisms have been evolved or have developed from common ancestors.

Especially striking are the facts in the case of many adaptations. Whales have become adapted to aquatic life, but the lost hind-legs are still represented by rudimentary bones far beneath the skin. Snakes appear to have been derived from ancestors with legs, but only a few species (python, boa) show the rudiments of the bones of the hindlegs. Wingless birds (ostrich, Apteryx) have small rudiments of useless wings. Horses still show the rudimentary bones of two lost toes. And in special books one can find records of thousands of such cases of rudimentary structures which point to relationship with forms in which the organs are fully developed and functional. In short, every known adaptation of animals and plants gives evidence of evolution having occurred, for the adapted structures obviously have originated by change and modification of previously existing structures. 
We cannot explain the presence of a rudimentary organ (such as an Apteryx wing, or whale hind limb) except as an adaptation or change of a corresponding organ in closely similar animals. Hence the Apteryx must have descended from birds with wings, and whales from mammals with hind legs. Such is the line of interpretation which biologists now apply everywhere in the animal and plant kingdoms.

495. Embryological Evidences of Evolution. - In $\S 364$ we have noted that gill-slits appear in the embryos of all vertebrates, although only fishes and young amphibians make use of them. The appearance of embryonic organs which never develop is very common in every group of animals, and also in plants. The case of gill-slits is only one of many such cases among higher vertebrates.

For such appearance of useless embryonic organs there is only one satisfactory explanation; namely, that they are evidences of ancestral history. Zoölogists now agree in believing that the presence of gill-slits in embryos of all vertebrates means that all vertebrates were derived from fishlike ancestors which needed gill-slits for respiration. But why gill-slits should still persist in the embryology of every vertebrate is a mystery. We can simply point to the facts indicating that it is inherited from an ancestral condition, an heirloom from the long-past history of the race of backboned animals.

In still another way does the embryology of animals suggest evolution from common ancestors, and that is in the similar development. In formation of egg-cells and spermcells, in fertilization of eggs, in cell-division, and in formation of organs there is a surprising similarity among animals; and in case of animals whose structure shows close relationship the similarity extends into great detail. Such facts lead to only one interpretation; namely, that all animals have been developed from the first forms of life. The facts from embryology are so convincing as to relationship that classification 
of an animal or plant is not considered absolutely decided until its embryology has been investigated and compared with that of its allies. Animals and plants which are often highly modified when adult are usually much like their near relatives when in embryonic stages. By this means it has been determined that some wingless insects are related to others which have wings ; that the sac-like barnacle ( $\$ 316)$ and the goose-barnacle are crustaceans; and so in hundreds of cases study of embryos of animals and plants have disclosed hidden resemblances which mean relationship.

496. Geological Evidences of Evolution. - If present animals and plants have descended from ancient ones, we ought to find some evidence of changes in the fossils. This is exactly what has been learned from study of the past history of organisms preserved in the rocks. A vast number of species of many groups of animals and plants have now been collected and studied, and all the facts learned make biologists more than ever convinced that the theory of evolution or descent is true. One illustration must suffice for our present study, and that is the fossils of ancestral horses showing gradual reduction of toes to one on each foot which have been found $(\$ \S 361,363)$. In the case of thousands of other species of organisms, the great museums now contain fossils which help in the study of relationships of both past and present organisms. Popular books in this line are Lucas's "Animals of the Past " or Lancaster's " Extinct Animals." Similar popular books on plant fossils have not been written because there is not such widespread knowledge and interest in ancient plants as in the animals. However, the fossil plants are to biologists no less interesting and convincing than the animals.

497. Distribution Evidences of Evolution. - Briefly we may state the central fact in this line as follows: Organisms which appear in their similar structure and embryonic development to be clearly related are commonly found in regions 
that were easily accessible to the descendants of their common ancestors. The animals and plants on islands near continents are very like those of the mainland. Within any continent, the species show a correlation with the possibility of distribution. For example, the crayfishes of eastern North America, though of many species, belong to one genus. On the west coast there is another genus. As might be expected, the high mountains have kept the two genera apart. There are thousands of known cases in which mountains, rivers, and seas have been barriers confining groups of animals or plants to a given territory, with the result that their descendants show close relationship.

The fact that there are many species of both animals and plants which occur in both Asia and America and in Europe and America suggests that there was once some land connection over which the ancestors of existing forms might have passed to America. That such a connection once existed in the region of Behring Sea seems probable to geologists.

Another interesting series of facts of distribution which point to evolution are those relating to introduction of new species into regions where they do not live naturally. In America we have numerous weeds, insects, English sparrows and other Old World organisms which are certainly well adapted to the conditions here. Why then were they not here naturally? The answer is that they were developed in limited geographical territory where their direct ancestors and near allies lived. This is why hundreds of species of organisms occur only in one part of the world, often on a single island, in a valley, river, or other region isolated by natural barriers.

498. Experimental Evidences. - The most convincing evidence of descent is obtained from study of domesticated animals and plants, with which for thousands of years man has, consciously and unconsciously, been experimenting. That organisms are changeable is proved beyond doubt by 
the hundreds of varieties of animals and plants which are raised under domestication. For example, we have dozens of varieties of chickens at the poultry shows and most of them are known to have descended from certain breeds; and probably all of them came from one or few wild species, one of which was certainly the East Indian jungle-fowl. Several hundred varieties of pigeons have developed from one (or possibly a few) species of wild pigeon. Numerous varieties of many common plants cultivated for food, ornament, or other use have been selected and improved by horticulturists. In short, any one who looks through books describing the numerous breeds or varieties of common domesticated animals and plants will not doubt the fact that organisms do change. And it must be remembered that most of these changes under domestication have occurred within a few hundred years; while tens of thousands of years have been available for changes in wild animals and plants.

It should be noted that man has affected animals and plants under his care chiefly by selecting peculiar individuals. When we speak of making a new variety of corn or a new breed of cattle we simply mean that we have selected for propagation certain individuals that by nature were better than their relatives. For example, breeds of hornless cattle have within recent years been started by selecting individuals born without power of growing horns and able to transmit this characteristic to their progeny. Likewise, horticulturists select every year certain extra-valuable plants and from them obtain seed of new varieties. With new varieties of either animals or plants improvements may be made with each new generation by selecting the best individuals as parents. For example, the hornless cattle are now being improved rapidly by selecting for propagation only those which are entirely hornless and which are also excellent for milk or meat. This is an illustration of the kind of selection which scientific agriculturists are constantly applying to all kinds of animals 
and plants under domestication. It is often called artificial selection to distinguish from natural selection ( $\$ 500)$.

499. Struggle for Existence. - Long before Darwin wrote the "Origin of Species" (1859) it was recognized by scientific men that vastly more individual animals and plants came into the world than can possibly survive, for there is not room and food for them all. To illustrate: Probably not one oyster embryo in a million grows to maturity; and if none of them perished for a very few generations, the oceans would be solid with oysters.

Elephants multiply slower than all other animals, but if all elephants lived one hundred years and produced but six young per pair, there would be after 800 years about 19,000,000 living descendants of one pair. Imagine each of these descendants $(9,500,000$ pairs) reproducing at the same rate for another 800 years! And yet elephants have been on this earth many times 800 years and living elephants are not very numerous anywhere. Evidently the vast majority do not live long enough to produce six young per pair. In fact, most of them do not live through the 30 years required to reach maturity.

Among plants, we have only to watch a patch of weeds crowding each other and the cultivated plants in a garden in order to see how severe is the struggle resulting from overpopulation. Suppose an annual plant this year produces 100 seeds, that each of these next year will form a plant with 100 seeds, and so on for many years. There would be 100 plants next year, 10,000 the second year, $1,000,000$ the third year, and so on in geometrical ratio multiplied by 100 each year. Evidently the world would soon be full of that kind of plant. But although many plants produce far more than 100 seeds annually, none of them increase at such a rate. Obviously, the vast majority of seeds do not develop into mature plants. Only a very small proportion of the sum total of all kinds of seeds formed in any one year could ever 
develop beyond the seedling stage, for there is not space and food enough. The vast majority will be eaten by animals, destroyed by unfavorable weather, or killed by crowding such as one can see in any place where plant seedlings are numerous.

Turn where we will among animals and plants, the facts are essentially as stated for the oyster and weeds: the total number of young organisms is so enormous that there must be a struggle for existence and destruction of the vast majority. This leads to many complicated relations between organisms. Food must be obtained, and this means competition between those which require the same kind of food, between carnivora and their prey, and between animals and plants. Also, there is a constant struggle with the environment (e.g., crowded plants struggling for light and water; and various animals against adverse climatic conditions). These are only suggestions of some ways in which nature subjects organisms to struggle in an intense competition, which is caused chiefly by the fact that all forms of life reproduce more rapidly than necessary to maintain a constant number of individuals of each species.

There are reasons for believing that the struggle for existence does not result in indiscriminate destruction of individuals. Some are better fitted than others to withstand the adverse conditions. For example, plant twenty sunflower seeds in a flower-pot, and gradually one or two of the seedlings will grow above the others. They are best fitted and therefore win in the struggle for existence. The same thing is constantly happening in all species of organisms. Those which survive and reproduce are the fittest. The weakest and least adapted soon perish. Thousands of forms of animals and plants which once lived on the earth have disappeared because they were not properly fitted for the struggle with changed environment and improved competitors. The effect of all this upon origin of new species will be discussed in the next paragraph. 
500. Natural selection, meaning selection by nature, is a term applied to the preservation of the favored or best adapted individuals in the struggle for existence ( $\$ 499)$. For example, a grasshopper with legs adapted for jumping farther than can other individuals of the same kind has a better chance of surviving by escaping enemies. Hence, it is said that nature selects such best fitted individuals. These will have a chance to propagate; and, according to the laws of heredity ( $\$ 501)$, will tend to transmit their own peculiar structure to descendants.

Darwin's "Origin of Species," was designed to show how natural selection working for tens of thousands of years and generations might have led to great changes in organisms. For example, the best jumping grasshoppers surviving in each generation would lead, in the course of long series of years, to the perfectly adapted legs of existing grasshoppers. Thus a new kind or species might have arisen.

Only one organ (leg) has been considered above, but it should be noted that any important organ might affect the question of survival in the same way.

It also should be noted that only useful things could be selected by nature. For example, a tumbler-pigeon, which occasionally turns somersaults while flying, would not have been preserved by natural selection. In fact, the habit is not only useless, but in nature probably would lead to destruction by birds of prey which could easily capture a pigeon during the delay in tumbling. But under human care and artificial selection, these peculiar pigeons have been preserved as curiosities or freaks, and allowed to multiply.

Numerous peculiarities of structure and color in many domesticated animals and plants have been preserved by artificial selection; but not being useful would not have developed under natural selection.

Seedless plants and double flowers tending to become seedless could not have developed except under artificial 
selection. The fact that natural selection acts only by survival of the fittest in the struggle for existence, limits its effect to things which make animals and plants better adapted to their environment. On the other hand, man can control conditions so as to select for preservation under domestication any peculiar individuals which interest him. This is why we have such vast numbers of varieties of cultivated plants and domesticated animals.

501. Heredity. - It is a well-known fact that offspring tend to resemble their parents closely. We commonly speak of characteristics which previously appeared in the parents or even earlier progenitors as inherited or due to heredity.

What may be Inherited. - We have previously noted that "like tends to produce like," which means that each animal and plant has the power of transmitting its own general characteristics to its offspring, e.g., a frog transmits frog characteristics, a bird those of its own species, and so on. Such characteristics which are part of the constitution of organisms are often called germinal, which means, in the germ (i.e., ova and sperm cells).

Characteristics developed during the lifetime of any individual, dating from the fertilized egg, are said to be acquired. Any change in structure due to use or accident, e.g., development of muscles by exercise, or loss of organs by accidents or surgical operations, produces an acquired characteristic.

Stating briefly the essential facts of heredity as now known, characteristics acquired during the lifetime of individuals are not transmitted in heredity, while germinal ones are capable of inheritance. For example, the horns have been removed from many cattle, the appendix from many human individuals, tails from sheep and certain breeds of dogs; and in no case has the removal of any organ from a parent caused the birth of offspring without the organ. In short, such acquired characteristics are not inherited. On the other hand, a cow which was germinally hornless (i.e., born without ability to 
grow horns) would probably transmit the tendency towards absence of horns to a considerable percentage of her offspring, and these, in turn, would tend to produce hornless offspring. In fact, the breeds of hornless cattle, which are now becoming popular among farmers, have been developed by selecting for breeding certain individuals born without the beginnings of horns. Likewise, dogs and cats born with short tails are likely to transmit that germinal characteristic, which in some unknown way is carried in the reproductive cells from parent to offspring.

The above paragraph states the facts verified by careful observation in hundreds of cases. In fact, the numerous varieties of domesticated animals and plants have originated by man's selection of individuals which during their embryonic history began to develop peculiarities. If these peculiarities make the individual decidedly different from its parents, a new breed might be originated, as from the first hornless cow from horned ancestors. Usually, however, the peculiarities are little things which, if selected by man, will make an improvement in the breed. Hence the scientific farmer is continually watching for young animals which show some slight improvement over their parents; and he goes through his fields in search of corn and other plants which are better than the others. This, in brief, is the secret of the remarkable improvement in almost all kinds of farm animals and cultivated plants in the past fifty or one hundred years.

502. Heredity and the Germ-cells. - Since an offspring resembles each of its parents (a fact especially striking when the two parents belong to different varieties and the offspring is a hybrid), it is evident that the egg-cell and the sperm-cell which united to produce the new individual were the bearers of heredity. In fact, it has been shown that the chromosomes in the nuclei of the egg-cell and spermcell contain the hereditary substance, the nature of which is unknown. Since each germ-cell contributes one-half of the 
chromatin of the fertilized egg-cell in all animals and plants, it follows that the new individual inherits from the two parents. However, there may be a more striking resemblance to one parent. For example, the offspring of a pair of pigs of which one is white and one black are often some white, some black, and some spotted black and white. But the white and the black ones have also inherited from each parent, although in color they show relationship to only one parent. It often happens that a pig which in color resembles one parent will, in shape of head and body, or other characteristics resemble the other.

503. Law and Order in Biology. - One who has never studied biology might look upon a vast museum of natural history as a chaotic mass of specimens; but biologic science has reduced them to order. There are many hundred thousand kinds or species of living things which can be distinguished from one another; but after all, they are remarkably similar, for they are dependent upon the same fundamental substance, protoplasm, which must perforce carry on the same essential life-processes in all plants and animals.

And what we find in biology is true in every other natural science. Everywhere in nature there is law and order. Planets and comets move in definite orbits, light and heat and electricity are subject to unchanging laws, elements unite and separate according to fixed principles - in short, all things in nature are conducted in accordance with law.

It has been one aim of this book to make the reader realize that the whole organic world, the field of biology, is subject to definite laws to which the human species is by no means an exception. Man is certainly an integral part of organic nature, and it behooves him to study and apply the discovered laws of biology upon which the continued advancement of the human race will, in no small measure, depend. 



\section{INDEX}

[The figures' given refer to pages, not to sections.]

A

Absorption, 50, 473, 479; by roots, $88,91$.

Accommodation, 517.

Adaptation, 28; birds, 429; flowers, 199-202; insects, 398 ; leaves, 191 ; mammals, 437 ; roots, 159 ; seed-plants, 228; stems, $180,184$. Adenoids, 503.

Asophagus, see esophagus.

Age, of animals, 17; plants, 20.

Air, breathed, 505. See oxygen.

Akene, 218.

Albuminoids, 463.

Alcohol, 269, 273, 275, 531, 539-553.

See also yeast, and fermentation. Algæ, 245-252.

Amœba, 307-311.

Amphibia, 65 ; 424-426.

Anaërobes, 286.

Anatomy, definition of, 21. See structure. .

Annuals, 177.

Angiosperms, 213.

Annelids, 347-353.

Anterior, 26.

Antitoxins, 293.

Ants, raising mushrooms, 266.

Appendages, of crayfish, 361 .

Appendix, human, 470.

Aptera, 387.

Aquarium, balanced, 128.

Arachnids, 376-380.

Arteries, 31; control of, 491.

Arthropods, 358-403.

Assimilation, 15, 109, 120, 304. See also nutrition, and metabolism.

B

Bacteria, 276-298; in hygiene, 554560.

Bacteriology, and health, 554-560.
Barnacle, 374-376.

Bathing, 533, 534.

Bean plant, classification, 121 ; physiology, 85-120 ; reproduction, 7685 ; structure, 67-85.

Bees, 386.

Biennial, 156, 177.

Biogenesis, 346.

Biology, definition of, 2 ; applied, 3 ; animal, 23-65 ; plant, 66-121 ; human, 457-560.

Birds, 428-436.

Bladder-worm, 342 .

Blights, 260.

Blood, uses of, $50,53-55$; animals with, 353 ; human, 482 . See also circulation.

Blood-cells, 312, 483.

Body-cavity, 330 ; frog, 29 ; human, 458 ; worm, 350.

Body-wall, human, 458.

Bone, 42, 417, 457.

Botany, definition of, 2.

Brain, human, 513.

Branching, of stems, 165.

Bread making, 273, 274.

Breathing, animal, 15; chemical tests for, 16, 19; human, 504; hygiene, 526-528; plant, 19 . See also respiration.

Bronchi, 503.

Bryophytes, 245.

Budding, 175, 227 ; corals, 337 ; hydra, 328; hydroids, 331 ; worm, 349 (Fig. 112).

Buds, 71,164 . See also budding and reproduction.

Bulblets, 226.

Bulbs, 183.

Butterfly, 382.

Cactus, stem, 183.

Calorie, 495. 
Calorimeter, 495, 496.

Cambium, 71, 174.

Capillaries, 32, 485.

Carbohydrates, 100-106 ; 460-462.

Carbon, 12; cycle of, 126.

Carbon dioxide, in plants, 113 ; in animals, 54; in human, 507. See also excretion.

Castor-oil seed, 148.

Caulicle, 83.

Cecropia, 383.

Cell-body, 41.

Cell-division, 59, 198.

Cells, $39-44,69,71,108-110,122$; use of foods, 492 ; relation to nutritive processes, 509 .

Cell-wall, 41.

Centipede, 379.

Cephalopods, 414.

Cephalothorax, 359.

Cerebellum, 513.

Change, physical, 5 ; chemical, 6 ; of matter, $5 \mathrm{ff}$.

Characteristics, 139.

Chemistry, 6.

Chlorophyll, 75 ; and plant food, $99-$ 102 ; in Hydra, 325.

Chloroplast, 75.

Chordata, 417.

Chromosomes, 59.

Cicada, 382, 385.

Cilia, 301 ; Fig. 16.

Circulation, animal, 55 ; plant, 130 ; simplest animals, 305; crayfish, 366; earthworm, 351 ; Hydra, 327; human, 486-492. See also under blood, and lymph.

Clam, 405.

Class, definition, 136.

Classification, principles and tables, 133-144; frog, 64; bean, 121; ferns, 240 ; mosses, 245 ; sporeplants, 233; protozoans, 319 ; cœlenterates, 339 ; "worms," 354 ; insects, 387-390 ; echinoderms, 357 ; mollusks, 416; vertebrates, 417 ; fishes, 422; amphibians, 426; reptiles, 428; birds, 433; mammals, 436 ; man, 455 .

Cloaca, 33,
Coagulation of blood, 485 .

Cocoon, of worm, 352 ; insect, 383 , 384.

Cod fish, 422.

Cœlenterata, 324-339.

Colome, 330.

Coffee, 552.

Colds, 528.

Coleoptera, 389.

Colon, human, 470.

Colony, protozoan, 316; hydroid, 331 ; Volvox, 318.

Coloration, protective, of frog, 27 ; of insects, 399-403.

Commensalism, 371.

Composition, chemical, of living matter, 8, 10-13.

Compounds, chemical, 7.

Conifers, 214.

Conjugation, mold, 254; Paramecium, 303 ; Spirogyra, 249.

Conservation, of energy, 492-494.

Control, of blood flow, 491.

Cooking, effect on foods, 482.

Coördination, 56, 509. See also nervous activity.

Copepod, 374.

Coral-animals, 336-339.

Cord, spinal, 511.

Corn, grain, 149 ; stalk, 168.

Corpuscles, blood, 483, 484 .

Cotyledons, 83 ; 146-150 ; number of, 152 ; work of, 153.

Cover crops, 162.

Crab, 370.

Crayfish, 358-370.

Crustaceans, 358-376.

Cryptogams, 144, 233.

Ctenophore, 335.

Cultures, bacteria, 280 ; molds, 255258 ; yeast, 270.

Cycle, of carbon, 126; of nitrogen, 128 ; of organic matter, 126.

Cyst, 310.

Cytoplasm, see protoplasm of cellbody.

\section{D}

Daphnia, 374.

Death, animal, 17 ; plant, 20. 
Decapods, 369.

Decomposition, by bacteria, 289 .

Dermis, 519.

Development, embryonic, of frog, 5763 ; of plant, 198. See also embryology.

Diaphragm, human, 469, 504.

Diastase, 105. ,

Dicotyledon, stem, 169-173.

Diet, 529 ; mixed, 501.

Diffusion, see osmosis.

Digestion, animal, 46-49; crayfish, 366 ; frog, 46-49 ; human, 471481 ; Hydra, 326 ; hygiene of, 528533 ; plant, 105, 120.

Diœcious, 243.

Diptera, 389.

Disease, bacterial, 291, 554-560 ; human, 262; and insects, 393-398; of plants, 259-261; and protozoans, 291, 312-315.

Disinfection, 285, 558.

Dispersal, of fruits and seeds, 223.

Dissection, of animals, 25.

Division, Amøba, 310 ; Paramecium, 302. See also cell-division.

Dorsal, 26.

Drugs, 553.

Dust, 527.

\section{E}

Ear, 518.

Earthworm, 349-353.

Eating, hygiene of, 528 .

Echinoderms, 355-357.

Economics, of seed-plants, 231; ferns, 241 ; fungi, $246-263$; mushrooms, 267; algæ, 251; yeasts, 275 ; bacteria, 286-291 ; protozoans, 312-316; cœlenterates, 339 ; worms, 340-345, 350 ; echinoderms, 356 ; decapods, 372 ; arachnids, 378 ; insects, $390-$ 398; mollusks, 414-416 ; fishes, 422-424; amphibians, 425,426 ; reptiles, 427, 428; mammals, 441.

Ectoderm, 322, 325.

Egg-cell, animal, 58; plant, 79; fern, 236.
Elements, chemical, 6.

Embryo, animal, 59-62 ; bean, 83 ; and evolution, 564 ; fish, 443 ; frog, 59-62; fern, 238; mammal, 449-453; plant, 198 . See also reproduction.

Embryology, definitions, 17. See embryo, and reproduction.

Endoderm, 322, 325.

Endosperm, 149.

Energy, 124 ; in animals, 45 ; conservation of, 492-494; of human body, 494; in foods, 495-497.

Entomology, 380.

Enzyme, 110, 129, 274. See also under digestion.

Epicotyl, 83, 153.

Fpidermis, 37 ; plant, 174. See also skin.

Epithelium, 37.

Erosion, and roots, 161.

Esophagus, human, 469.

Eustachian tube, human, 468, 519.

Evaporation, 93, 95.

Evolution, 561-571.

Excess, in food, in exercise, 538.

Excretion, animal, 53; animal and plant compared, 126 ; frog, 53 ; human, 507; Hydra, 328; nitrogen, 499 ; plant, 113-115; protozoans, 126 ; worm, 352.

Exercise, 537.

Exoskeleton, 359.

Eye, human, 515.

\section{F}

Fairy-rings, 265.

Fats, 463.

Feather, 431.

Fehling's reagent, 461.

Fermentation, 268.

Ferns, 233-241.

Fertilization, animal, 59 ; plant, 79 , 197, 216, 236.

Fertilizers, for soils, 99.

Fibrin, 485.

Fishes, 419-424.

Flagella, 248, 322.

Flea, 387, 389. 
Flies, 397.

Flowers, 76, 196-213 ; functions of, 196.

Flower-clock, 117.

Flower-clusters, 208.

Fluids, digestive, 471.

Fœetus, 453.

Foods, of animal, 14, 123; energy of, 495-497; human, 460-465; need of, 44 ; uses of, 46 ; of plant, 19 , $98,108,119,123$; of protozoan, 304,309 . See also digestion, and metabolism.

Food-storage, in roots, 158 ; in stems, 174 ; in leaves, 192.

Food-supply, human, 125.

Forestry, 187.

Frog, 14-17 ; anatomy, 25-37 ; histology, 37-44; physiology, 4457 ; embryology, 57-64 ; classification, 65.

Fruits, 79-81, 216-225.

Functions, see life-activities.

Fungi, 245, 252-276 ; food of, 99101.

\section{G}

Gametes, 238.

Gametophyte, 239.

Gametospore, 255.

Ganglion, 352, 512.

Gases, from organic matter, 11. See also carbon dioxide.

Gasteropods, 411.

Gastrula, 349.

Gelatin plates, 280.

Generation, spontaneous, 346.

Generations, alternation of, in fern, 238 ; in seed-plants, 239 ; in moss, 244 ; in cœlenterates, 334, 335.

Geology, and evolution, 565.

Germ-cells, in heredity, 572. See also egg-cell, sperm-cell.

Germicides, 285.

Germination, 84 ; 146-156.

Germs, 135 . See bacteria.

Gestation, 450.

Gills, clam, 407 ; crayfish, 361 ; embryos, 444; fishes, 419; tadpole, 62 .
Gill-slits, 444.

Girdling, 107.

Glands, salivary, 467; liver and pancreas, $470 ; 471$.

Grafting, 175, 227.

Grains, 218.

Grasshopper, 381, 392.

Growth, in living matter, 44 ; foods for, 499; secondary in stem, 169. Gullet, see esophagus.

Gymnosperms, 213.

\section{H}

Hæmatococcus, 249.

Hæmoglobin, 483.

Hair, 521.

Head, of flowers, 210.

Heart, 487-490.

Heat, animal, 52 ; effect on bacteria, 283 ; in foods, 495,498 ; in germination, 155 ; from skin, 522-524.

Hemiptera, 388.

Heredity, 571-573.

Hermaphroditism, 328.

Hermit-crab, 370.

Histology, definition of, 22; of frog, $37-44$.

Horse, fossil, 439.

Horse hair worm, 345 .

Human biology, 455.

Hydra, 324-329.

Hydranth, 332.

Hydroids, 330-335.

Hydrophobia, 296.

Hygiene, 525-561.

Hymenoptera, 389.

Hyphæ, 252, 264.

Hypocotyl, 83, 154.

\section{I}

Ichneumon-fly, 391.

Imago, 383, 384.

Immunity, 294.

Incubation, chick, 448.

Inflorescence, 208.

Infusoria, 319.

Inheritance, 571.

Inoculation, 256; protective, 296 . 
Insects, $380-403$; in pollination, 199.

Instincts, birds, 434; insects, 403. Inter-cellular substance, 41.

Interdependence of animals and plants, 128.

Intestines, human, 470 .

Irritability, 129 ; -Hydra, 328 ; Paramecium, 305; plants, 116-119. See also nervous activity.

\section{J}

Jelly-fishes, 335.

\section{K}

Kidneys, frog, 35; human, 508.

King-crab, 378.

Koch, Dr., 314.

\section{L}

Labor, division of, 306, 329 .

Lamellibranchs, 408.

Larva, barnacle, 375; butterfly, 383; frog, 63; mosquito, 396, 399 ; worm, 349 .

Leaf, $73-76$; 188, 196 ; arrangement, 189 ; variegated, 103.

Leaf-scars, 163.

Lenticel, 112.

Lepidoptera, 388.

Lichens, 266.

Life-activities, animal, 13-18, 4457 ; in cells, 41 ; human, 457-523 ; plant, 18-20, 119-121.

Light, artificial, on plant growth, 104; on oxygen-supply of plants, 111 ; in photosynthesis, $102,111$. Limulus, 378.

Liquors, see alcohol.

Liver-fluke, 343.

Liver, human, 470.

Lobster, 358.

Locomotion, animal, 14 ; plant, 18.

Locusts, 382, 385.

Lungs, 503.

Lymph, 32,485 . See also circulation.

\section{M}

Machinery, of life, 21.

Maize, 149.

Malaria, 312-314, 394-396.

Mammals, 436-442.

Man, biology and classification, 455, 456 ; structure and life-activities, 457-523.

Manures, and bacteria, 287.

Mastication, $471,530$.

Matter, organic, 2; three states of, 5 ; gray, 512, 514; white, 502.

Medusa, 332, 335.

Mesentery, 33.

Metabolism, 53, 109, 120, 125, 499. See also growth, repair, assimilation.

Metamere, see segment.

Metamorphosis, frog, 62 ; insect, 384 ; worm, 349.

Metazoa, 320.

Microbes, see bacteria.

Micro-organisms, see bacteria, and protozoa.

Migration, bird, 434.

Mildew, 259.

Milk, 473; bacteria in, 288.

Millipede, 379.

Mimicry, 400.

Minerals, in organic matter, 12 ; as foods, 464 ; in soil, 97.

Molds, 252.

Mollusca, 405-416.

Molting, btrd, 431 ; crustacean, 368 .

Monocotyledon, 152 ; stem, 167.

Monœcious, 243.

Morphology, definition of, 21.

Mosquitoes, 312, 393-396.

Mosses, 241-245.

Moth, 382.

Mouth-cavity, human, 465.

Movement, animal, 14 ; blood, 486 ; plant, 18 ; protozoan, 305,308 ; respiratory, 504; stomach, 472.

Mulching, 87.

Muscle, cells, Figs. 14, 15; hygiene, 537-539.

Mushroom, 263-268.

Mycelium, 252, 264.

Myriopods, 379. 


\section{N}

Nails, 521.

Names, scientific, 133, 142.

Narcotics, 539, 553.

Nature-study, of frog, 24.

Nautilus, 413.

Nereis, 348.

Nerve-endings, 510.

Nerves, 511.

Nervous activity, frog, 56, 57; human, 509-519; worm, 352 ; reflex, 510; voluntary, 511. See also irritability.

Nervous organs, 57, 509-519; hygiene, 535-537.

Nettle-cells, 325 .

Neuroptera, 388.

Nitrogen, cycle, 128; as excretion, 508 ; in foods, 499 ; in soils, 99.

Nucleus, 40.

Nutrients, 460.

Nutrition, animals, 56; human, 492-502.

Nuts, 218.

Octopus, 413.

Organisms, 2.

Organs, 27; of frog, 36 ; plant, 67.

Orthoptera, 388.

Osmosis, 89.

Ovary, in flowers, 204.

Overwork, 535.

Oviparous development, 63.

Ovipositor, 381.

Ovum, see egg-cell.

Ovules, 78, 197.

Oxidation, 7, 51, 125, 304.

Oxygen, in animals, 52,125 ; in blood, 505 ; in cells, 506 ; in germination, 155; in plants, 110,119 , 125 ; in photosynthesis, 112.

Oxygen-supply, animal, 52,55 ; crayfish, 366; human, 502-506; Hydra, 327 ; plant, 110-125.

Oyster, 357, 409.

\section{$\mathbf{P}$}

Palate, human, 465.

Palisade cells, 75 .
Pancreas, human, 470.

Paramecium, 300-307.

Parasites, barnacle, 375 ; insect, 390 ; malarial, 312 ; plant, 100, 259, 267 ; protozoan, $312-315$; worms, 341-345.

Parthenogenesis, 196, 386.

Pasteur, 269, 284.

Pasteurization, 284.

Pepsin, 477.

Perianth, 203.

Pericardium, 30, 487.

Perennial, 177.

Petal, 203.

Petri dish, 257.

Phanerogams, 233.

Pharynx, human, 468.

Photosynthesis, 102.

Phylum, 137.

Physics, definition of, 6 .

Physiology, 22 ; animal, 44-57 ; human, 457-524; plant, 85-120. See also under descriptions of types of animals and plants in Parts II and III.

Pistil, 205.

Pith-rays, 170.

Placenta, animal, 452 ; plant, 80 .

Planaria, 341.

Plant, biology of, 66-121.

Plant-lice, 386.

Plants, group of, 144.

Pleura, 503.

Pleurisy, 503.

Pleurococcus, 237.

Plumule, 83.

Pod, 79-81.

Pollen-grain, 197, 216.

Pollination, $77,78,197,200,201$, 216, 390.

Polyp, 332.

Porifera, 320-324.

Posterior, 26.

Pregnancy, 450.

Propagation, from roots, 162 ; from stems, 180 ; of seed-plants, 225227. See also reproduction.

Proteins, 104, 497-500, 530.

Prothallium, 235.

Protonema, 243. 
Protoplasm, 43, 69, 71, $122,499$.

Protozoa, 300-319.

Pruning, 176.

Pseudopod, 309.

Psychology, of digestion, 532.

Pteridophytes, 240.

Ptyalin, 476.

Puff-ball, 268.

Pulse, 490.

Pupa, 383, 384.

Pylorus, 33.

Q

Quarter-sawing, 186.

$\mathbf{R}$

Radicle, 83.

Reflexes, 510.

Regeneration, of Hydra, 329; of starfish, 357.

Rennin, 477.

Repair, 44, 499.

Reproduetion, 16, 27; asexual plant, 225-227; asexual animal (see budding) ; frog, $57-65$; bean, 7685; animal and plant compared, 131 ; seed-plants, 146-156, 196227 ; fern, 235-240 ; moss, 243245 ; various spore-plants, 246254 ; Paramecium, 302, 305 ; amœba, 310 ; sponge, 322 ; Hydra, 328 ; hydroids, 334 ; corals, 337 ; earthworm, 351-353; insects, 384-387; vertebrates, 442-453; fishes, 443 ; birds, 446 ; reptiles, 447 ; mammals, 447-453.

Reptiles, 426-428.

Resemblances, in elassification, 138.

Respiration, 55; human, 502-508; plant, 110. See also oxygen-supply, and excretion of $\mathrm{CO}_{2}$.

Retina, 516.

Rhizoids, 236.

Rings, annual, 170.

Root-cap, 70.

Root-hairs, 69, 89.

Root-pressure, 89 .

Roots, 68-70; 88; 156-163.
Rootstock, 182 ; fern, 233.

Root-tubereles, 69, 159, 287.

Rudiments, 563.

Rusts, 259.

\section{S}

Saliva, human, 467.

Salmon, 422.

Sandworm, 348.

Sanitation, 526.

Sap, 130.

Saprophyte, 100.

Scale-leaves, 193.

Sciences, 2.

Scorpion, 377.

Sea-anemone, 336.

Sea-fans, 338.

Sea-urchin, 355.

Sea-weeds, 246, 251.

Secretions, 48; digestive, 474 ; salivary, 474 ; gastric 477 ; intestinal, 479 ; pancreatic, 480 ; bile, 480.

Seed-distribution, 210.

Seedlings, 146-156.

Seed-plants, 146-231.

Seeds, bean, $81-83$; various, 146156.

Segment, crayfish, 360 ; insects, 381 ; worm, 347.

Sense-organs, 27, 362.

Senses, special, 515-519.

Sensitive plant, 18.

Sepal, 203.

Sewage, bacteria in, 289.

Shad, 423.

Sieve-tubes, work of, 106.

Silver-moth, 387.

Skeleton, coral, 337 ; crustacean, 359 ; frog, 30 ; human, 457; insect, 381.

Skin, human, 508, 519, 521-524; hygiene, 533-535.

Sleep, of plants, 118; human, 536.

Sleeping sickness, 314 .

Slugs, 411.

Smallpox, 295.

Smut, 259.

Snail, 410.

Soap, in hygiene, 533 . 
Soils, and bacteria, 286 ; plant foodmaterials in, 99 ; and worms, 350 ; water in, 87.

Solutions, 47.

Sow-bug, 373.

Species, 135.

Spermaphyta, see seed-plants.

Sperm-cell, 58; plant, 197; fern, 236.

Sphærella, 247.

Spider, 376.

Spiracle, 381.

Spirogyra, 249.

Sponge-animals, 320-324.

Sporangia, see spore-case.

Spore-case, 215, 235, 253.

Spore-plants, 232-298.

Spores, 232, 235, 243, 279, 283, 312.

Sporophyll, 215, 235.

Sporophyte, 239.

Squid, 413.

Stamens, 207.

Starch, in leaves, 104 ; test for, 102 ; formula, 111; as food, 462 .

Starfish, 355.

Stem, 70-72; 163-188; functions, 179 ; as leaves, 184 ; adaptations, 191.

Sterilization, 256, 284.

Sterilizer, 256.

Stimulants, 531, $53-553$.

Stimuli, effect on plants, 116-119.

Stipules, 193.

Stoma, 74, 75; work of, 96.

Structure, see descriptions of various animals and plants.

Struggle for existence, 568 .

Sugar, test for, 105; as food, 461 , 462.

Swallowing, $471,472$.

Sweat-glands, 521,522 .

Symbiosis, 329.

Symmetry, bilateral, and radial, 26.

Systems, of organs, 27, 36 .

\section{$\mathbf{T}$}

Tadpole, 62.

Tape-worm, 340.

Taste, 519.
Tea, 552.

Teeth, 466 ; hygiene of, 528 .

Temperature, sense, 519 .

Tendrils, 180.

Tentacle, 325 .

Tests, for foods, 460-462.

Thallophytes, 246.

Thorns, 181, 193.

Tissues, animal, $37-43$; plant, 69 , $71,72,74,123$.

Toad, 24.

Tobacco, 551.

Tongue, human, 467.

Tonsil, 468.

Touch, 519.

Toxins, 293.

Tracheæ, human, 502; of insects, 381.

Transpiration, 97.

Trichina, 344.

Tropisms, 118.

Tubers, 182.

Twigs, 163.

Tyndall, 347.

\section{U}

Unicellular animals, 300-307.

Univalves, 411.

Ureter, 508.

Use of animals and plants (see economics); in science, 24.

Uterus, 450.

\section{V}

Vaccination, 295.

Vacuole, contractile, 302, 309; food, 302 ; yeast, 269.

Valves, heart, 487 ; veins, 489.

Varieties, dog, 138; beans, 81 .

Veining, of leaves, 194.

Veins, 31, 489.

Ventilation, 527.

Ventral, 26.

Venus fly-trap, 18.

Vermes, 340.

Vertebra, 457.

Vertebrates, 417-453.

Vinegar, 276, 289. 
Vinegar-eel, 344.

Viviparous development, 63.

Vivisection, 25.

Volvox, 318.

Vorticella, 317.

Water-flea, 374.

Wood, 184.

Wood-lice, 373.

Work, see energy.

Worms, 340-354.

$$
\because \mathrm{W}
$$

Waste, in living matter, 44. See also excretions.

Water, 8; as excretion, 114, 509; in Yolk, 447. germination, 154; hygiene, 529; in leaf, 95 ; need of, 95 ; in organisms, 10,44 ; in roots, 92 ; in soil, 87 ; in stems, 94 ; use of in plant, 97 ; in transportation, 130.

Zoölogy, definition of, 2.

Zygospore, 255. 




RETURN TO the circulation desk of any

University of California Library

or to the

NORTHERN REGIONAL LIBRARY FACILITY

Bldg. 400, Richmond Field Station

University of California

Richmond, CA 94804-4698

ALL BOOKS MAY BE RECALLED AFTER 7 DAYS

2-month loans may be renewed by calling

(415) 642-6753

1-year loans may be recharged by bringing books to NRLF

Renewals and recharges may be made 4 days prior to due date

DUE AS STAMPED BELOW 


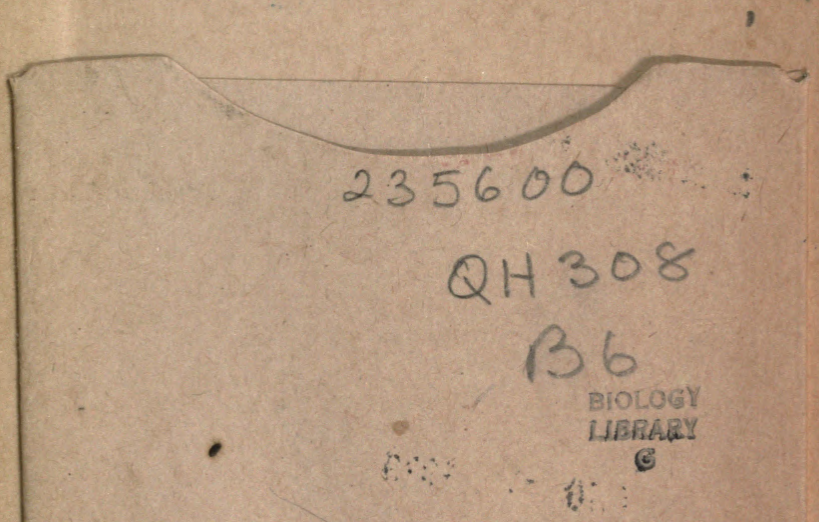

UNIVERSITY OF CALIFORNIA LIBRARY 
